\title{
A Multi-Regional Computable General Equilibrium Model for New Zealand
}

\author{
by \\ Nathaniel Robson
}

\begin{abstract}
A thesis
submitted to the Victoria University of Wellington in fulfilment of the requirements for the degree of Doctor of Philosophy
\end{abstract}

Victoria University of Wellington 2012 



\begin{abstract}
Although New Zealand has had an active CGE modelling community since the 1980's, a multi-regional CGE model for the country has not been developed until now. This thesis presents a prototype multi-regional CGE model to demonstrate the feasibility of developing a comprehensive model that captures the benefits of modelling agent behaviour with a bottom-up approach. The prototype model is built upon bottom-up regional micro-foundations and New Zealand data is used to operationalise a particular implementation of the model. The thesis fills an important gap in the New Zealand CGE modelling literature as none of the models in current use have a structure involving bottom-up regional modelling. The method of implementation is also a key contribution, utilising a maximum-entropy approach to overcome data shortages. An illustrative simulation of a natural disaster that strikes the Wellington central business district demonstrates the strengths of the bottomup multi-regional approach — that the model can capture differential effects across regions of shocks that occur at the regional level, and incorporate flowon and feedback effects between regions. Sensitivity testing of the substitution elasticity between domestic sources of products reinforces the importance of empirically-estimated parameters in CGE models.

The basic model is extended in two ways. The first is to introduce modelling of distribution services as has been done in the ORANI and subsequently FEDERAL models. The key structural difference here is that products identified as distribution services are required to facilitate movement of other products from seller to buyer. Thus there are no opportunities to substitute away from these services if they become relatively more expensive. To implement the additional structure, sets of coefficients are specified to control technical
\end{abstract}


possibilities in the usage of the distribution services. These include switches that can dictate, for example, that wholesale trade is only involved in the delivery of tangible products, that retail trade is only used by in-region purchasers, and that transport is required for moving physical products across regional borders or to exporters. That these assumptions can be integrated seamlessly into the database highlights the strength of the maximum-entropy approach used to generate the multi-regional input-output database. Simulations of an oil price shock show that the regional assumptions surrounding the distribution networks are material to the results.

The second extension to the model is the addition of a module to control the degree of inter-regional labour mobility. Essentially the user is given the ability to specify the extent to which households respond to regional real wage differences by moving to regions with relatively higher rates. Therefore, in short-run simulations labour can be made more mobile than capital, while in the long-run it can be less mobile than capital. The module also introduces additional structure to link populations, households, and labour market components. One important element of this new structure is a link back to the endogenous labour supply theory of the basic model. Publicly available demographic and labour market data are used to implement the mobility module. The importance of a mobility response to relative real wage changes is explored in an illustrative application looking at the impact of regionally-concentrated immigration flows. The simulations suggest that population movements can work to dissipate the welfare effects of such migration inflows. 


\section{Acknowledgements}

In achieving the completion of this thesis, I owe a debt of gratitude to my supervisors, Professor Viv Hall and Dr. Stephen Burnell, for their continuous support. Their excellent academic advice and direction in the research process was essential, along with their kind assistance in securing financial support.

I gratefully acknowledge the following sources of financial assistance provided during various stages of this research:

- School of Economics and Finance PhD Scholarship

- Philpott-BERL Scholarship

- G. G. G. Watson Award (on two separate occasions)

- Department of Labour Graduate Research Award

In addition, the School of Economics and Finance (SEF) and Business and Economics Ltd. (BERL) have provided fixed-term employment opportunities which have extended my range of professional experience and complemented my research.

I wish to express my thanks to Dr. Ganesh Nana of BERL for very helpful discussions and the provision of data which aided this research. The data provision was funded through the Department of Labour Graduate Research Award.

The New Zealand Institute of Economic Research (NZIER) invited me to join a CGE modelling workshop they had organised, and later to make a presentation to the Institute. Both of these provided valuable opportunities for discussion and very useful feedback.

Professor John Madden of the Centre of Policy Studies, Monash University generously supplied me with a copy of his doctoral thesis and Dr. Adolf Stroombergen of Infometrics kindly granted me permission to use his input-output tables. I wish to also thank all 
participants of the presentations I have made to SEF, the Post-Graduate Students Association (PGSA), and the New Zealand Association of Economists (NZAE). Professor Jacques Poot of the National Institute of Demographic and Economic Analysis, University of Waikato gave particularly useful advice following my NZAE conference presentation. I acknowledge the contribution of Dr. Peter Chang, formerly of SEF, who provided the initial impetus and supervision during the early stages of this research. Members of the SEF staff have provided valuable assistance on various matters throughout. A special thanks to Dr. Paul Calcott for the $\mathrm{AT}_{\mathrm{EX}}$ help.

Complementing the assistance elucidated above has been the fantastic support I have received from family and friends. First and foremost has of course been that of my wife Mayumi and daughter Jennifer. Thank you for your endless support and patience, standing by while I pursued my dream. Thanks are also due to the wider family, whose encouragement and assistance have been vital to our survival in the interim.

And last but certainly not least, my appreciation to all my friends who endured the awkwardly constructed descriptions of my research when they asked "what's your topic?" and incomprehensible rambling when I encountered difficulties. Thanks for providing the opportunities I needed to restore my sanity. My thanks go to one friend especially, for being there as a source of strength when I needed it most.

In all affairs it's a healthy thing now and then to hang a question mark on the things you have long taken for granted.

Bertrand Russell

花は盛りに、月は隈なきをのみ、見るものかは。

吉田 兼好 [徒然草第 137 段]

(Translation) Why only when flowers are in full bloom and the moon is shining in spotless perfection should they be looked upon?

Yoshida Kenkō [Idle time essay \#137] 


\section{Contents}

Glossary $\quad$ Xv

1 Introduction 1

1.1 Bottom-up Micro-foundations . . . . . . . . . . . . . . . . . . 4

1.2 Multi-regional IO Data and Information Theory . . . . . . . . . . . . . 6

1.3 Implementation in GAMS . . . . . . . . . . . . . . . . . . . . . . . 8

1.4 Regional Focus of Applications . . . . . . . . . . . . . . . . . . . 10

2 The Basic Model 13

2.1 Introduction . . . . . . . . . . . . . . . . . . . . 13

2.2 Basic Description . . . . . . . . . . . . . . . . . . 13

2.3 Theoretical Structure . . . . . . . . . . . . . . . . . . . . . 18

2.4 Model Implementation . . . . . . . . . . . . . . . . . . . . . . . . . . 63

2.5 Simulations Using the JENNIFER Model . . . . . . . . . . . . . . . . 102

3 Distribution Services $\quad 109$

3.1 Introduction . . . . . . . . . . . . . . . . . . . . . 109

3.2 Modelling Distribution Services . . . . . . . . . . . . . . . . 110

3.3 Additional Model Structure . . . . . . . . . . . . . . . . . . . . . . . . . 114

3.4 Implementation of the Margins Modelling . . . . . . . . . . . . . . . 119

3.5 An Illustrative Application . . . . . . . . . . . . . . . . . . . . . . . . . . . 139

4 Labour Mobility $\quad 147$

4.1 Introduction . . . . . . . . . . . . . . . . . . . 147

4.2 Modelling Labour Mobility . . . . . . . . . . . . . . . . . . . . . 148

4.3 Additional Model Structure . . . . . . . . . . . . . . . . . . . 150 
4.4 Implementation of the Labour Mobility Modelling . . . . . . . . . . . . . . 158

4.5 An Illustrative Application . . . . . . . . . . . . . . . . . . . . . 165

5 Conclusion $\quad 181$

5.1 The JENNIFER Model . . . . . . . . . . . . . . . . . . . . . . . . . . 181

5.2 The Geographic Nature of Distribution . . . . . . . . . . . . . . . . 182

5.3 Partial Labour Mobility . . . . . . . . . . . . . . . . . . . . . . 182

5.4 Future Development . . . . . . . . . . . . . . . . . . 183

$\begin{array}{ll}\text { Appendices } & 185\end{array}$

A CGE Models in the Literature . . . . . . . . . . . . . . . . 187

B Selected RPEP Papers . . . . . . . . . . . . . . . . . . . 191

C List of Model Variables . . . . . . . . . . . . . . . . . . . . . . . . . 195

D List of Model Equations . . . . . . . . . . . . . . . . . . . 199

E Solutions to Constrained Optimisation Problems . . . . . . . . . . . . . . 211

F Linearisation of Demand Functions . . . . . . . . . . . . . . . . 215

G Labour Supply Functions . . . . . . . . . . . . . . . . . . . . . . 219

$\mathrm{H}$ Endogenous Investment Allocation . . . . . . . . . . . . . 221

I Regional Consumption and Propensities to Save . . . . . . . . . . . . . 225

J Conversion Factors . . . . . . . . . . . . . . . . . . . . . . . 227

K Calibration of Demand Functions . . . . . . . . . . . . . . . . 231

L Walras' Law . . . . . . . . . . . . . . . . . . . . . . . . 233

M Margins Demands: A Worked Example . . . . . . . . . . . . . 235

N The Extended MRIO Matrix . . . . . . . . . . . . . . . . . . 239

O Simulation Results - Disaster Scenario . . . . . . . . . . . . . 269

P Simulation Results - Oil Price Rise Scenario . . . . . . . . . . . . . 293

Q Simulation Results — Short-Run Immigration Scenarios . . . . . . . . . . 309

R Simulation Results - Long-Run Immigration Scenarios . . . . . . . . . . . 327 


\section{List of Figures}

2.1 Household agents' decision nest . . . . . . . . . . . . . . . . . . 27

2.2 Industry agents' decision nest for current production . . . . . . . . . . . . 33

2.3 Industry agents' decision nest for capital formation . . . . . . . . . . . 38

2.4 Government agent's decision nest for consumption . . . . . . . . . . . . 46

3.1 Formation of delivered products . . . . . . . . . . . . . . . . 117 


\section{List of Tables}

2.1 Indices and Default Sets . . . . . . . . . . . . . . . . . . . 19

2.2 Other Sets . . . . . . . . . . . . . . . . . . . . . . . . 19

2.3 Variable Notation Scheme . . . . . . . . . . . . . . . . . . . 20

2.4 Equation Blocks . . . . . . . . . . . . . . . . . . . . 64

2.5 Industries . . . . . . . . . . . . . . . . . . . . 67

2.6 Input-Output Table . . . . . . . . . . . . . . . . . . . . . 70

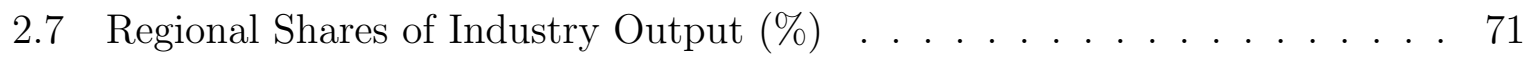

2.8 MRIO Data Matrix . . . . . . . . . . . . . . . . . . . . . . . . 72

2.9 Industry by Region Shares of Total Capital (\%) . . . . . . . . . . . . 77

2.10 Local Industries . . . . . . . . . . . . . . . . . . . . . . . . . . . . . 79

2.11 Industry by Region Shares of Total Investment $(\%) \quad \ldots . . . .87$

2.12 Investment Data and Solution Values . . . . . . . . . . . . . . . . 88

2.13 Regional Time Endowment (IOunits) . . . . . . . . . . . . . . . . . . 91

2.14 Average Propensities to Save $(\%)$. . . . . . . . . . . . . . . . . . . . 92

2.15 Source Substitution Elasticities . . . . . . . . . . . . . . . . . . 95

2.16 Short-run Substitution Elasticities . . . . . . . . . . . . . . 96

2.17 A Short-run Closure . . . . . . . . . . . . . . . . . . . . 98

2.18 Sets of Elasticities for Simulations . . . . . . . . . . . . . . . 107

3.1 Additional Equations . . . . . . . . . . . . . . . . . . . . . . . 119

3.2 Margin-use Proportions . . . . . . . . . . . . . . . . . . . . . 120

3.3 Margins Required to Deliver Products . . . . . . . . . . . . . . . . . . . 122

3.4 Destination-type Proportions of Margin Demands . . . . . . . . . . . . . 123

3.5 Export Columns of the Expanded MRIO Matrix . . . . . . . . . . . . . . . 124

3.6 Product Flows in the Extended MRIO Matrix . . . . . . . . . . . . . . 126 


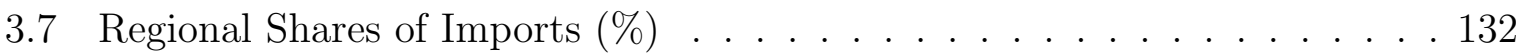

3.8 A Long-run Closure . . . . . . . . . . . . . . . . . . . . . . . . . . 138

4.1 Demographic Variables . . . . . . . . . . . . . . . . . . . . . . 152

4.2 Labour Market Variables . . . . . . . . . . . . . . . . . . . . . . . . . . 152

4.3 Demographic and Labour Market Data (000s) . . . . . . . . . . . . . . 159

4.4 Employment Data $(000 \mathrm{~s}) \ldots \ldots$. . . . . . . . . . . . . . 160

4.5 Population Projections . . . . . . . . . . . . . . . . . . . . 168

4.6 Simulations . . . . . . . . . . . . . . . . . . . 170

J.1 Conversion Factors - FTEs $\rightarrow$ persons . . . . . . . . . . . . . . 228

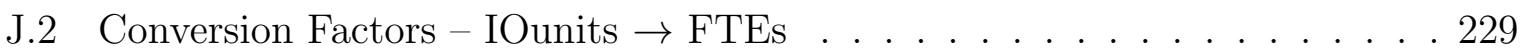

N.1 The Extended MRIO Matrix . . . . . . . . . . . . . . . . . . . . . . 240

O.1 Regional Capital Stocks _ . . . . . . . . . . . . . . . . . . . 269

O.2 Regional Output . . . . . . . . . . . . . . . . . 270

O.3 Regional Output Price Index . . . . . . . . . . . . . . . . . 270

O.4 Industry Capital Stocks . . . . . . . . . . . . . . . . . . 271

O.5 Industrial Output . . . . . . . . . . . . . . . . . . . 272

O.6 Output By Industry - Region WLG . . . . . . . . . . . . . . 273

O.7 Output By Industry - Region AKL . . . . . . . . . . . . . . . . . . . 274

O.8 Output By Industry - Region CAN . . . . . . . . . . . . . . . . 275

O.9 Industry Employment . . . . . . . . . . . . . . . . . . . 276

O.10 Employment By Industry - Region WLG . . . . . . . . . . . . . 277

O.11 Industrial Investment . . . . . . . . . . . . . . . . . . . . 278

O.12 Investment By Industry — Region WLG . . . . . . . . . . . . . 279

O.13 F.O.B. Exports . . . . . . . . . . . . . . . . . . . 280

O.14 C.I.F. Imports . . . . . . . . . . . . . . . . . . . . . 281

O.15 Industry Output Price Index . . . . . . . . . . . . . . . . . . . . 282

O.16 Output Prices - Region WLG and ONI . . . . . . . . . . . . . 283

O.17 Industry Capital Rents Index . . . . . . . . . . . . . . . . . . . 284

O.18 Capital Rental Rates - Regions WLG and ONI . . . . . . . . . . . 285

O.19 Industry Average Net Rates of Return . . . . . . . . . . . . . . 286

O.20 Current Net Rates of Return - Region WLG . . . . . . . . . . . . . 287 
O.21 Regional Nominal After-Tax Wage . . . . . . . . . . . . . . . . 288

O.22 Regional Consumer Price Index . . . . . . . . . . . . . . . . . 288

O.23 Regional Real After-Tax Wage . . . . . . . . . . . . . . . . . . . 288

O.24 Household Real Disposable Income ． . . . . . . . . . . . . . . . . 289

O.25 Household Real Spending . . . . . . . . . . . . . . . . . . 289

O.26 Household Real Saving (After-Tax) _ . . . . . . . . . . . . . 290

O.27 CV and EV Measures . . . . . . . . . . . . . . . . . . . . 290

O.28 Macro Measures . . . . . . . . . . . . . . . . . . . . . . . 291

O.29 Economy-wide Price Measures . . . . . . . . . . . . . . . . . 291

P.1 Regional Capital Stocks . . . . . . . . . . . . . . . . . . 293

P.2 Regional Output — Selected Industries and Total . . . . . . . . . . . . . . 294

P.3 Regional Output Price Index — Selected Industries and Total . . . . . . . 295

P.4 Industrial Output . . . . . . . . . . . . . . . . . 296

P.5 Industry Output Price Index . . . . . . . . . . . . . . . . . 297

P.6 F.O.B. Exports . . . . . . . . . . . . . . . . . . . . . . . . . . . . . 298

P.7 F.O.B. Exports — benchmark differences . . . . . . . . . . . . . . . 299

P.8 C.I.F. Imports . . . . . . . . . . . . . . . . . . . . . . . . 300

P.9 Industrial Investment . . . . . . . . . . . . . . . . . . 301

P.10 Industry Capital Stocks . . . . . . . . . . . . . . . . . . . 302

P.11 Industry Capital Rents Index . . . . . . . . . . . . . . . . . 303

P.12 Industry Employment . . . . . . . . . . . . . . . . . . . . . . . 304

P.13 Regional Employment . . . . . . . . . . . . . . . . . 305

P.14 CV and EV Measures . . . . . . . . . . . . . . . . . . . . . . . . . . . . . . . . . . . . . . . . . . .

P.15 Household Real Disposable Income . . . . . . . . . . . . . . . . 306

P.16 Household Real Spending . . . . . . . . . . . . . . . . 306

P.17 Household Purchase Prices — Commodity PETR . . . . . . . . . . . 306

P.18 Macro Measures . . . . . . . . . . . . . . . . . . . . 307

P.19 Economy-wide Price Measures . . . . . . . . . . . . . . . . . 307

Q.1 Number of Households . . . . . . . . . . . . . . . . . . . 309

Q.2 Total Population . . . . . . . . . . . . . . . . . 310

Q.3 Working-Age Population . . . . . . . . . . . . . . . . 310

Q.4 Non-Working Age Population . . . . . . . . . . . . . . . . 310 
Q.5 Regional Employment . . . . . . . . . . . . . . . . 311

Q.6 Regional Capital Stocks . . . . . . . . . . . . . . . . . 311

Q.7 Regional Output . . . . . . . . . . . . . . . . . . . . . . . 312

Q.8 Regional Investment . . . . . . . . . . . . . . . . . . . . . . . . . . . . 312

Q.9 Regional Output Price Index . . . . . . . . . . . . . . . . . . . . . . . . 312

Q.10 Regional Investment Price Index . . . . . . . . . . . . . . . . . 313

Q.11 Regional Capital Rents Index . . . . . . . . . . . . . . . . . . 313

Q.12 Regional Average Net Rates of Return . . . . . . . . . . . . . . . . . 313

Q.13 Industry Employment . . . . . . . . . . . . . . . . . . . . . . 314

Q.14 Industry Capital Stocks . . . . . . . . . . . . . . . . . . . 315

Q.15 Industrial Output . . . . . . . . . . . . . . . . . . 316

Q.16 Industrial Investment . . . . . . . . . . . . . . . . . 317

Q.17 Industry Output Price Index . . . . . . . . . . . . . . . . . . 318

Q.18 Industry Investment Price Index . . . . . . . . . . . . . . . . . . . 319

Q.19 Industry Capital Rents Index . . . . . . . . . . . . . . . . . 320

Q.20 Industry Average Net Rates of Return . . . . . . . . . . . . . . . . . . . . 321

Q.21 Macro Measures . . . . . . . . . . . . . . . . . . . . . . . . . . . 322

Q.22 Economy-wide Price Measures . . . . . . . . . . . . . . . . . . . . 322

Q.23 Regional Nominal After-Tax Wage . . . . . . . . . . . . . . . . . . 323

Q.24 Regional Consumer Price Index . . . . . . . . . . . . . . . . . . . 323

Q.25 Regional Real After-Tax Wage . . . . . . . . . . . . . . . . . . . 323

Q.26 Welfare Measures . . . . . . . . . . . . . . . . . . . . . . 324

Q.27 Unemployment . . . . . . . . . . . . . . . . . . . . . 325

Q.28 Labour Force Participation Rate . . . . . . . . . . . . . . . . . . . 325

Q.29 CV and EV Measures . . . . . . . . . . . . . . . . . . 326

R.1 Number of Households . . . . . . . . . . . . . . . . . . . . . . 327

R.2 Total Population . . . . . . . . . . . . . . . . . . . . . 328

R.3 Working-Age Population . . . . . . . . . . . . . . . . . 328

R.4 Non-Working Age Population . . . . . . . . . . . . . . . . . . . . 328

R.5 Regional Employment . . . . . . . . . . . . . . . . . . . . . 329

R.6 Regional Capital Stocks _. . . . . . . . . . . . . . . . . . . . . 329

R.7 Regional Output . . . . . . . . . . . . . . . . . . 330 
R.8 Regional Investment . . . . . . . . . . . . . . . . . . . . 330

R.9 Regional Output Price Index . . . . . . . . . . . . . . . . . . . . . 330

R.10 Regional Investment Price Index . . . . . . . . . . . . . . . . . . 331

R.11 Regional Capital Rents Index . . . . . . . . . . . . . . . . . . 331

R.12 Regional Average Net Rates of Return . . . . . . . . . . . . . . . . . 331

R.13 Industry Employment . . . . . . . . . . . . . . . . . . . . . . . 332

R.14 Industry Capital Stocks . . . . . . . . . . . . . . . . . . . 333

R.15 Industrial Output . . . . . . . . . . . . . . . . . . . . 334

R.16 Industrial Investment . . . . . . . . . . . . . . . . . . . . 335

R.17 Industry Output Price Index . . . . . . . . . . . . . . . . . 336

R.18 Industry Investment Price Index . . . . . . . . . . . . . . . . 337

R.19 Industry Capital Rents Index . . . . . . . . . . . . . . . . . 338

R.20 Industry Average Net Rates of Return . . . . . . . . . . . . . . . . . . 339

R.21 Macro Measures . . . . . . . . . . . . . . . . . . . . . . 340

R.22 Economy-wide Price Measures . . . . . . . . . . . . . . . . . . 340

R.23 Regional Nominal After-Tax Wage . . . . . . . . . . . . . . . . . . 341

R.24 Regional Consumer Price Index . . . . . . . . . . . . . . . . . . . . . 341

R.25 Regional Real After-Tax Wage . . . . . . . . . . . . . . . . . . 341

R.26 Welfare Measures . . . . . . . . . . . . . . . . . . . . . . . 342

R.27 Unemployment . . . . . . . . . . . . . . . . . . . . . 343

R.28 Labour Force Participation Rate . . . . . . . . . . . . . . . . . . . 343

R.29 CV and EV Measures . . . . . . . . . . . . . . . . . . . . . 344 


\section{Glossary}

Bottom-up A modelling approach that begins with components of an economy (e.g. regions) and sums them up to obtain an aggregate description of the economy (e.g. a nation); the opposite of 'top-down'.

BERL Business and Economics Research Ltd.: an economics consultancy based in Wellington which operates a CGE model descended from JOANNA. See http://www. berl.co.nz/ for details.

Benchmark equilibrium A model solution consistent with the database to which the model is calibrated. The calibrated model replicates the benchmark equilibrium when solved with no shock specified.

CES Constant Elasticity of Substitution: a functional form used typically for utility or production functions; the single substitution elasticity parameter captures the preferences (in the case of utility) or technology (in the case of production functions) over the inputs that generate the utility or composite product respectively.

CGE Computable General Equilibrium: an applied method of economic modelling, useful for investigating the medium to long-term effects of changes in policy, technology, or the external environment.

CoPS Centre of Policy Studies: a research unit based at Monash University. See http://www.monash.edu.au/policy/for details.

CRESH Constant Ratio of Elasticity of Substitution Homothetic: a functional form for utility or production functions that is more general than CES; it requires pairwise substitution elasticities between each of the inputs to describe the preferences or technology. 
Default set A set for which an associated index is assumed to vary over unless otherwise indicated. For example the default set for $r$ is $R E G$ so any time $r$ is used as an index, it can be assumed that $r \in R E G$ unless an alternative is given such as $r \in N Z R E G$ (which is the same as $R E G$ except that it also includes the national 'region' NZ.

FTE Full-Time Equivalent: a measure of labour hours, deflated by what is considered average for an individual working full-time. The Statistics NZ definition is that 1 FTE is equivalent to 30 hours of employment per week.

GAMS General Algebraic Modelling System: a software package capable of finding numerical solutions to a wide range of model types, including CGE models expressed in levels or linear form.

GEMPACK General Equilibrium Modelling PACKage: a specialised suite of programs designed for solving CGE models by linearisation.

IFPRI International Food Policy Research Institute: a U.S. based agricultural research centre.

Infometrics An economics consultancy based in Wellington which operates a CGE model descended from JULIANNE. See http://www.infometrics.co.nz/ for details.

IO table Input-Output table: a table that records (value or volume) flows of goods and services (including those of factors) between and within different sectors of an economy.

Landcare Research A Crown Research Institute (CRI) with staff spread across 10 locations throughout NZ, and focused on sustainable resource management, including biodiversity, ecosystems, the biosphere, and land use. This organisation has recently developed an environmental CGE model for NZ: the New Zealand Climate-Economy Model (NZCEM). See http://www.landcareresearch.co.nz/ for details.

LEaP Centre for Land, Environment, and People: a research unit based at Lincoln University which has recently developed a New Zealand CGE model focusing on tourism (NZTGEM). See http://www.lincoln.ac.nz/leap for details. 
Linearisation Conversion of a non-linear equation into a linear equation in the proportional deviations (or growth rates) of the variables in the original equation by total differentiation (i.e. log-linearising using a first-order Taylor approximation).

Market Economics Ltd. An economics consultancy based in Auckland which has developed a SAM and regional CGE model for the Auckland region. See http://www.marketeconomics.co.nz/ for details.

Mixing function A function, in this thesis either Leontief, Cobb-Douglas, or CES, that takes product or factor varieties as inputs, and outputs a composite of those varieties (or utility); a discrete version of the Dixit-Stiglitz aggregator function.

MPSGE Mathematical Programming System for General Equilibrium (analysis): a programming environment in GAMS that automates the algebraic representation of CGE models to the program.

MRIO table Multi-Regional Input-Output table: a table that records (value or volume) flows of goods and services (including those of factors) between and within different sectors and regions of an economy.

NZIER New Zealand Institute of Economic Research: an economics consultancy based in Wellington which operates NZ versions of ORANI and MONASH. See http://nzier.org.nz/ for details.

Product A (model) good or service; i.e. a composite of real-world goods or real-world services. The degree of similarity in the real-world products combined to form the model product depends on the level of disaggregation in the model - low levels of disaggregation can lead to quite distinct real-world products being lumped together into a single model product category.

ROW Rest Of the World: a catch-all for the portion of the world economy external to a given model.

RPEP Research Project on Economic Planning: a research unit based at Victoria University of Wellington from 1970 to around 2000 (although its main period of activity was during the 1980's). 
SAM Social Accounting Matrix: a table that records value flows of goods and services (including those of factors) between and within different sectors of an economy. Similar to IO tables but contains more detail concerning the flows between the non-industry elements of the economy. For example, transfers from government to households are recorded in the SAM. Every column total must equal the corresponding row total in a balanced SAM, not just those pertaining to product market equilibrium as in IO tables.

TABLO A program component of GEMPACK that translates the algebraic representation of a CGE model into a form usable by the computer for solving the system. Traditionally the model had to be coded in TABLO in its linearised form but recent versions of GEMPACK enable to user to enter some or all the equations in the levels, with the linearisation performed automatically.

Top-down A modelling approach that begins with an aggregate economy (e.g. a nation) and uses data and assumptions to split the economy into component parts (e.g. regions); the opposite of 'bottom-up'.

Working-age population The number of normally-resident persons aged 15 years and over (Statistics NZ definition). 


\section{Chapter 1}

\section{Introduction}

Computable general equilibrium (CGE) models are tools that help us think about the likely effects on the structure of an economy of a wide range of shocks, including changes in government policy, external markets, technology, the environment, and population. The goal of CGE modelling, in common with other types of economic modelling, is to capture the important aspects of the economy while suppressing those that are either not especially relevant to the matter of concern or too complex to be modelled tractably. The closer a CGE model comes to achieving this goal, the more useful it will be for the purposes of analysis and simulation.

The field of CGE modelling has grown enormously since its beginnings in the groundbreaking work of Johansen (1960). ${ }^{1}$ Internationally, the current state of the art is perhaps most visibly represented by MONASH, the multi-period national CGE model from the Centre of Policy Studies (CoPS) at Monash University, and GTAP, the multi-region global CGE model from the Center for Global Trade Analysis at Purdue University. ${ }^{2}$ Within New Zealand at present, CGE modelling is primarily the domain of the professional consultancies. ${ }^{3}$ Academic research in the field has waned since the 1990's; as far as the

\footnotetext{
${ }^{1} \mathrm{~A}$ list of models relevant to this thesis can be found in appendix A, with a brief description of each along with citations for the reader who wishes to investigate them further.

${ }^{2}$ There are a great number of other CGE models that have characteristics similar to, and are arguably as sophisticated as these models, but these two are perhaps the most well known, at least amongst practitioners in New Zealand.

${ }^{3}$ Organisations that the author knows of that undertake CGE modelling in New Zealand are BERL, NZIER, Infometrics, Market Economics, LEaP, and Landcare Research. See the Glossary for details of each of these.
} 
author is aware, the most recent New Zealand $\mathrm{PhD}$ thesis in CGE modelling at the time of writing is Nana (1999). Nevertheless, the consultancies have maintained close links with the universities and there have been signs during the preparation of this thesis of renewed academic interest flowing from the increasing usage of CGE in contracted-out policy analysis.

All the CGE models in current use in New Zealand have a core theoretical structure based on the modelling of activity within a single domain, such that the model simulates the behaviour of the New Zealand economy as a whole, or that of one of its regions. ${ }^{4}$ Some of the models have a top-down regional extension module, which will be discussed shortly, but they nevertheless are primarily national models. A Trans-Tasman regional model was developed in New Zealand in the late 1980's — the JOANI model, which linked the JOANNA national model of New Zealand with the ORANI national model of Australia. JOANI was therefore a two-region model, with an external sector consisting of the rest of the world outside the Australia-New Zealand system. The last published work that made use of JOANI was Nana \& Poot (1996).

Until this point, therefore, there has been no fully multi-regional CGE model for New Zealand. Our national CGE models were developed independently at roughly the same time as ORANI was being developed in Australia. ${ }^{5}$ However, while multi-regional modelling in Australia began to gain traction with the publishing of the FEDERAL model (Madden 1990), there has been no parallel development here. A report was prepared for the Ministry of Works and Development by Burton (1985) that set out a broad framework for policy-relevant multi-regional modelling in New Zealand. ${ }^{6}$ At the heart of the

\footnotetext{
${ }^{4}$ Anything outside the geographical area covered by the model is the external sector, with essentially exogenous behaviour. The external sector is the rest of the world (ROW) in national models and the rest of the country combined with the ROW in sub-national models.

${ }^{5}$ The main ones being JOANNA and JULIANNE, developed under the auspices of Professor Philpott within the Research Project on Economic Planning (RPEP) at Victoria University of Wellington. Both of these models have an associated academic thesis lodged with the Victoria University Library - see the list of models in appendix A for their citations. Wells \& Easton (1986) review these models (amongst others) and provide a guide to the historical RPEP papers in which their development is documented. Hall (1992) discusses the RPEP models in the context of long-run equilibrium concepts, the macroeconomic models of the time, and model interfacing including that of JOANI. A selection of RPEP papers relevant to this thesis are cited in appendix B. For a broad, retrospective view of the 30 years of RPEP work, see Philpott (2000).

${ }^{6}$ The Ministry was subsequently abolished in 1988.
} 
framework was to be a suite of interfaced models "to simulate the effects of policy and development scenarios" (Burton 1985, p. xi). Models were suggested that described one or two sectors of the economy each ${ }^{7}$ and involved a variety of modelling approaches. ${ }^{8}$ The family of Johansen-type multi-sectoral models was reviewed in the New Zealand context and it was concluded that JOANNA or a set of regional versions (one for each region) could be integrated into the module. The author acknowledged that the wider framework was ambitious not only due to the data collection and processing requirements, but also to the need to co-ordinate a large group of researchers, policy analysts, and specialists in its implementation. Since the report was published, it appears that there has been no further development of this broad framework. A modern multi-regional CGE model placed at the centre of such a framework would significantly reduce the complexity inherent in its implementation. Perhaps the primary reason that such a model has not emerged here is that New Zealand lacks the kind of data exploited in FEDERAL: regional input-output (IO) tables obtained through survey techniques that capture interregional flows - exports are disaggregated by destination and imports are disaggregated by source including domestic regions and the foreign sector. There are, however, several non-survey techniques that can be used to obtain estimates of these items.

The first multi-regional model of Australia was MRSMAE (Liew 1981), developed prior to the availability of Australian state-level IO tables. As Madden (1990, p. 10) notes, this meant the multi-regional input-output database of MRSMAE had to be constructed using "very mechanical methods" — namely, the application of Leontief \& Strout's (1963) gravity model. It might seem that for a New Zealand CGE model with many regions, one might be able to do no better than MRSMAE due to the lack of data. A New Zealand version of MRSMAE would also suffer the same drawbacks as the original. In particular, it could be argued that the assumptions underlying the database construction are equally as "ad hoc" as those used to drive top-down regional extension modules such as that of ORANI, named ORES (ORANI Regional Equation System). Such an approach would enable many regions to be modelled, but introduces an unknown amount of spurious information.

\footnotetext{
${ }^{7}$ For example, one model would describe fiscal and monetary policy, another would determine prices and wage rates, and yet another would be an interregional population and income/expenditure model.

${ }^{8}$ Amongst the model types suggested were regional input-output, linear programming, econometric, system dynamic, and micro-analytic models.
} 
There have, however, been significant advances in the theory and practice of CGE modelling since the time of MRSMAE. Important aspects include the standardisation of modelling theory and techniques, new insights emerging from information theory, and the ubiquitousness and power of modern computers. At the same time, the application of CGE to regional policy issues has become increasingly popular internationally, reflecting the increased attention being given to welfare of subgroups within populations and the distribution of shock impacts across those groups. ${ }^{9}$

A key objective of this thesis then is to build a multi-regional CGE model exploiting recent advances in the field. To develop a fully-fledged model of this type takes many person-years due to the complex nature of CGE and its heavy data requirements. This thesis makes an initial contribution towards this through the presentation of a prototype model, named JENNIFER, and two extensions to it. Illustrative applications are used to highlight the important aspects of the model and the over-arching framework, but should not be interpreted as providing "real world" predictions of "what will happen" in the face of a particular shock. ${ }^{10}$

The remainder of this chapter will show how the JENNIFER model fits into the evolution of CGE modelling in New Zealand by expanding on those developmental aspects mentioned above and discussing how they are manifested in the proposed framework.

\subsection{Bottom-up Micro-foundations}

There are two broad approaches to multi-regional modelling: handling regions either 'topdown' or 'bottom-up'. The terms are attributed to Klein \& Glickman (1977) and refer, in the first case, to regional economies being treated as 'satellites' of the national economy, with only a one-way interface (from the nation to the region), and in the second case, to regions being the building blocks of the national economy, so feedbacks run in both directions. In CGE models, the top-down approach manifests as a regional disaggregation module being attached to a model of the national economy. Regional shares data and assumptions regarding sourcing and usage of products drive the disaggregation. This is

\footnotetext{
${ }^{9}$ For a broad international survey of the multi-regional CGE modelling literature, see Donaghy (2009).

${ }^{10}$ Nevertheless, paraphrasing Johansen $(1960$, p. 3), it can also be said that the quantitative analysis does not solely serve the purpose of illustrating a method. The numerical results can give a broadly indicative description of some important economic relationships in the New Zealand reality.
} 
the approach taken in ORANI and MONASH to derive regional results from simulations using those models. The bottom-up approach, on the other hand, requires agent behaviour and institutions (markets, government, etc.) to be described at the regional level. A significant amount of structure needs to be added to a national CGE model to make it multi-regional, as evidenced by MRSMAE and then FEDERAL and its cousins. ${ }^{11}$ From a theoretical point of view, the bottom-up approach is preferred as it allows supply-side differences and feedbacks from regions to the national economy. That is, since activity is modelled at the regional level and regulated through regional market prices, it is possible for technology, household behaviour, etc. to differ across them. Firms in the same industry but different regions can be restricted in their access to factors to only those available within the region. Households can face different wage rates and consumer prices across regions and behave differently as a result. By building the economy bottom-up in this fashion, the model can be subjected to shocks originating at the regional level. Simulation results will indicate the extent that regional interdependencies serve to propagate the shock through the economy and the impact observed at the national level.

At a minimum, the requirements of a bottom-up multi-regional model are the addition of a regional dimension to many variables and equations, and the incorporation of a regional sourcing level at the bottom of agents' decision nests. For this thesis, the functions used for mixing product types, factors, etc. are limited to the CES (Constant Elasticity of Substitution) level of generality. This includes mixing of products of a given type from different regional sources to form a composite domestic product. Not all sectors of the economy are modelled at the regional level - the public and trade sectors are not given a regional dimension, although they source products from the regions as the regionally-located agents (households and firms) do. This is partly for simplicity of the prototype model, but also because arguably many aspects of these sectors are determined at the national level (e.g. tax policy, foreign currency export prices). Giving a regional dimension to the public sector, for instance, would require the splitting of the sector into central government and local government so as to regionalise only the local government

\footnotetext{
${ }^{11}$ Namely FEDERAL-F (Giesecke \& Madden 2003), MMRF (Centre of Policy Studies 2008), and TERM (Horridge, Madden \& Wittwer 2005). The comprehensive treatment of MRSMAE (Liew 1981) is currently available only on microfilm from Monash University library. A shorter but more accessible description is found in Liew (1984a). There is also a useful comparison of the top-down and bottom-up approaches in Liew (1984b).
} 
components. In a sense then, JENNIFER is a hybrid model since not all decisions are made at the regional level and sum up to an aggregate picture. In the case of government, exporters, and importers, behaviour essentially occurs at the national level and is disaggregated top-down as required.

Along with Burton (1985) and the Australian multi-regional models, it is useful to take guidance from JOANI and GTAP even though they are multi-country models and therefore involve structure not relevant here such as bilateral exchange rates. In particular they suggest approaches to dealing with transport costs and factor mobility in a bottomup context that will become important in chapters 3 and 4 .

\subsection{Multi-regional IO Data and Information Theory}

The data requirements for implementing a bottom-up multi-regional model come under three broad headings: multi-regional input-output (MRIO) data, substitution and demand elasticities, and other data concerning factors, investment, fiscal policy settings etc. Since New Zealand does not have an official set of integrated regional input-output tables, but a considerable amount of regional information is nevertheless available, we aim to supply the MRIO data to the model using a hybrid method - that is, using a combination of a non-survey method along with survey data where possible. There are two broad hybrid approaches ${ }^{12}$ : the GRIT method or the RAS / entropy method. Each approach requires a national IO table and some regional data. With the GRIT method, regional IO tables are estimated separately using location quotients and then a gravity model is used to estimate the interregional flows. Throughout, the method requires the use of "superior data" and professional judgement where possible. ${ }^{13}$ TERM and MRSMAE essentially use this approach although MRSMAE used a method more similar to

\footnotetext{
${ }^{12}$ See Miller \& Blair (2009) for comprehensive treatment of the components of these approaches.

${ }^{13}$ On this point see Jensen \& West (1989) and Lahr (2001). The single-region version of GRIT, which doesn't use the gravity model, is described in Jensen, Mandeville \& Karunaratne (1979) and West (1980). The method was extended for interregional analysis in West, Morison \& Jensen (1984). Market Economics Ltd. have recently developed a Social Accounting Matrix (SAM) for the Auckland region using the single-region GRIT method. The SAM is described in Zhang, McDonald, Nixon \& Smith (2008) and the development of an Auckland region CGE model that uses the SAM is discussed in Yeoman, Kim, McDonald \& Zhang (2009). Reports that make use of the SAM are Auckland Regional Council (2009) and Enterprise North Shore (2010).
} 
the LMPST approach of ORES to obtain the intra-regional technical coefficients, rather than location quotients.

The RAS and entropy techniques are usually thought of as matrix adjustment methods but they are amenable to matrix-filling as well. That is, given borders (row and column sums) of a matrix and initial estimates of the cells, both these methods can determine consistent cell values. In the case of entropy methods, it is explicit in the algorithm that an information criterion is being optimised. The simplest entropy method is maximum entropy which, intuitively speaking, maximises the disorder of the matrix. A completely disordered matrix would be uniform across all cells. When new information is introduced (for example, new column totals) the maximum entropy algorithm scales the matrix as needed for consistency but minimises any other information gain. Cross-entropy is a generalisation of maximum entropy - it minimises the distance between the solution matrix and a given prior, not necessarily uniform matrix. (Shore \& Johnson 1980) It turns out that proportional allocation is equivalent to maximum entropy and Bacharach's (1970) RAS method is equivalent to cross-entropy for specific objective functions of entropy. Along with sketching a proof of these, McDougall (1999) argues that proportional allocation / RAS should be the method of choice for most cases of matrix filling / balancing over other entropy-theoretic methods. ${ }^{14}$

In this thesis, the cross-entropy formulation of RAS is used as the starting point for deriving a MRIO table. This is in contrast to the GRIT method, where RAS is used at the end just to restore balance to the system of regional IO tables. This does not prevent elements of GRIT being implemented within the information-theoretic framework. Especially where superior data is available or professional judgement can be used, the framework is designed to incorporate those seamlessly. Although not pursued in this thesis, selective use of location quotients or gravity adjustments could also be made and the optimising approach would enable the loss of entropy from each change to be calculated for comparison. GRIT is applied in spirit then, especially with regard to its notion of "holistic accuracy" of the database as opposed to "partitive accuracy". (Jensen 1980) Augmentation of the initially-derived MRIO database should be made if, and only if, it improves the accuracy of the model output in a real-world context.

\footnotetext{
${ }^{14}$ For discussions of cases where the information-theoretic framework can be used to extend RAS, see for example Batten (1982) and Robinson, Cattaneo \& El-Said (2001).
} 


\subsection{Implementation in GAMS}

GAMS and GEMPACK are currently the two main software packages used for CGE modelling. ${ }^{15}$ Each has strengths and weaknesses, and the choice of package ultimately depends on the programmer's and/or the user's requirements and preferred style of implementation. GAMS is a general-purpose modelling package used for finding numerical solutions to a wide variety of problems, including linear, non-linear, and mixed-integer programming, and mixed complementarity problems (MCP). A CGE model can be implemented in GAMS in the levels as a non-linear programme or a constrained non-linear system a special case of MCP. ${ }^{16}$

GEMPACK on the other hand is a purpose-built CGE modelling package that typically solves models in percentage changes. When originally developed, GEMPACK solved models using Johansen's method and therefore required the model equations to be entered in linearised form. Early enhancements saw the linearisation errors inherent in the one-step Johansen solution reduced through the use of multi-step methods with extrapolation. It was still necessary to linearise the model equations by hand and update statements were required to facilitate multi-step solutions. Such manual operations are not required when implementing CGE models in GAMS and solving using non-linear techniques. These points of difference between the two software packages have since disappeared. In the latest version of GEMPACK - release 11 at the time of writing models can be entered as a mixture of linearised and levels equations and solved in such a way that linearisation errors are arbitrarily small. Equations entered in levels form are automatically linearised and corresponding update statements are not required. From the perspective of obtaining simulation results as percentage changes of variables from one model equilibrium to another, the only difference is how they are typically obtained. ${ }^{17}$

\footnotetext{
${ }^{15}$ GAMS documentation (GAMS Development Corp. 2011) is available at http://www.gams.com/ docs/document.htm while the GEMPACK Manual (Harrison, Horridge, Jerie \& Pearson 2012) can be found at http://www.monash.edu.au/policy/gpdoc.htm. MPSGE (Rutherford 1999) is often cited as a third package although it is actually a subsystem of GAMS. Horridge \& Pearson (2011) provide a useful review of GEMPACK, GAMS, and MPSGE and compare their performance with a simple CGE model implemented in all three.

${ }^{16} \mathrm{An}$ MCP is a system of non-linear equations, inequalities, and complementarity pairs (slackness conditions). If no complementarity pairs are specified, the MCP reduces to a constrained system of non-linear equations.

${ }^{17}$ It is now possible for a CGE model to be implemented entirely in levels and solved using Newton's
} 
What remains is that GAMS is a more flexible tool due to its general-purpose nature while GEMPACK automates many routine programming tasks and is more user-friendly to non-technicians. In light of this, it was decided to implement JENNIFER in GAMS, as this package seemed more appropriate for the task of model development. Porting the core model to GEMPACK would be relatively straightforward when the need arises to make it accessible to other users or to increase its dimensionality. ${ }^{18}$

There are, nevertheless, GAMS-specific features of the way JENNIFER is implemented that would not translate so well to GEMPACK. One is that an object-oriented approach was taken in programming the model. This stands in contrast to the traditional blockby-block, subroutine approach used in GAMS reference works such as Löfgren, Harris \& Robinson (2002), Gillig \& McCarl (n.d.), and Hosoe (2004). Keyzer (1997) approaches the issue with discussion of some simple macros but these are still of the subroutine type rather than interacting with program objects. Taking this approach simplifies program development significantly. Variables can be added to the variable list, for example, which acts as an object that various parts of the program interact with. In doing so the program writes its own code for the simulation and reports. The model can be run from the DOS command line via a batch file, which enables multiple runs with different levels of industry and regional disaggregation, different closure, elasticities, and simulation settings and tables of results to be produced all without the need for user input. Regarding the reporting facility, tables are produced pre-formatted for immediate use in $\mathrm{AT}_{\mathrm{E}} \mathrm{X}$. None of these features are directly replicable in GEMPACK.

GEMPACK has a side program for performing a RAS balance but if one wanted to use a different objective function, a new side program would have to be written in FORTRAN by the user or the developers on their behalf. ${ }^{19}$ However, since GAMS is primarily an

method in GEMPACK, and it has always been possible to input a model in linearised form and solved in GAMS, but each of these works against the advantages of the respective packages. For a general discussion of levels vs. linearisation, see Hertel, Horridge \& Pearson (1992).

${ }^{18}$ One of the disadvantages of implementing the model in levels form is that it takes considerably more computer resources to solve a model of given size non-linearly than when using even the multistep linearised method. Models with more regions and industries are therefore more efficiently solved in GEMPACK.

${ }^{19}$ Unless such a program was already available. There is, for example, a program available on the Internet that uses Kuroda's method but it is unclear how it would interface with GEMPACK. See Wilcoxen's website at http://wilcoxen.maxwell.insightworks.com/pages/764.html. 
optimisation tool, implementing the entropy-theoretic form of RAS (or indeed any entropy method) is easily done within the GAMS programming environment. ${ }^{20}$ The formulation of the MRIO database as discussed above is therefore integrated fully within the program code.

\subsection{Regional Focus of Applications}

The JENNIFER model can now be described as a prototype multi-regional CGE model with bottom-up micro-foundations and implemented in GAMS on top of a MRIO database derived using information-theoretic principles. Such a model can be used to investigate the regional effects of external events (to the region or nation) or changes in fiscal policy. On a deeper level however, the model can serve as a framework to consider many of the issues raised in the review by Partridge \& Rickman (2010) of the use of CGE for regional development analysis. For example, how do population movements affect relative regional performance and what implications are there of feedbacks from regional performance to population movements? What are the important channels through which a shock permeates through New Zealand's regions or what are the reasons that it does not? That is, what are the drivers of regional differences and similarities?

In this thesis, the model is used to investigate three questions alluded to in the Partridge \& Rickman review. In chapter 2, which presents the core prototype model, the ability of the model to simulate regional supply-side shocks is demonstrated through an application involving a natural disaster. Sensitivity testing is used to investigate how the regional implications of the shock depend on the values of the substitution elasticities between domestic sources of products.

Chapter 3 introduces ORANI-style modelling of distribution services. ${ }^{21}$ Long-run simulations of an (imported) oil price rise are conducted under various modelling assumptions regarding the distribution networks. The idea under investigation here is that the function of distribution, especially transport, is important in a regional context. Even in the longrun when opportunities for factor substitution exist, one would expect the distribution network to continue to constrain regions' ability to adjust to shocks.

One issue that is given considerable attention in the Partridge \& Rickman article,

\footnotetext{
${ }^{20}$ See for example Fofana, Lemelin \& Cockburn (2005)

${ }^{21}$ See Dixon, Parmenter, Sutton \& Vincent (1982, section 17).
} 
and increasingly elsewhere in the literature, is that of interregional labour mobility. This is the topic of chapter 4. Usually the issue of factor mobility is handled by way of a short-run/long-run closure decision whereby factors are assumed regionally immobile in the short-run and fully mobile in the long-run. Here, allowance is also made for partial mobility of labour across regions relative to that of capital through an extension module, such that labour can be more regionally mobile than capital in the short-run but less mobile than capital in the long-run. The suggested approach makes such partial mobility operational without needing the explicit introduction of dynamic adjustment processes. An application of the extended model investigates how the impact of higher immigration flows depends on the regional dispersion of the flows and the degree of labour mobility in the short-run and long-run.

Rather than focus on one broad scenario throughout this thesis, it was decided to apply the model to separate scenarios in each chapter in order to demonstrate the flexibility of the model, indicate the range of possibilities, and emphasise the importance of the modelling done in each given chapter. While interregional labour mobility as discussed in chapter 4 may have an important bearing on the results of the natural disaster scenario of chapter 2, for example, the implications of the distribution network as discussed in chapter 3 are less relevant without a higher degree of regional disaggregation.

The key contribution of this thesis, therefore, is a methodological approach to developing a New Zealand multi-regional CGE model, and the prototype JENNIFER model presented herein is the first step in that development. 


\section{Chapter 2}

\section{The Basic Model}

\subsection{Introduction}

This chapter shows how the methodological approach summarised in the previous chapter is applied to construct JENNIFER, a prototype New Zealand multi-regional CGE model. The basic underlying theoretical structure is set out along with the key aspects of the model implementation. Two extensions to this basic variant of the model are then described in the chapters that follow.

\subsection{Basic Description}

The key characteristic of the JENNIFER model is that the aggregate economy is not only modelled as a group of linked sectors, but also as a group of interdependent geographical regions. It separately describes the economic behaviour within each region of those sectors deemed to have important region-specific characteristics. The model is designed with application to the New Zealand economy in mind, but this does not preclude the general principles underlying the model design, or indeed the model itself, being used for another country.

\subsubsection{Sectors}

The model has five sectors - the household, production, trade, public, and foreign sectors.

The household sector primarily provides labour services to producers and consumes 
goods and services made by them. The labour supply choice is endogenous, arising from a decision of how to split the household's time endowment between labour and leisure. Households also own the stock of currently installed capital and so receive its rental income. Any part of after-tax household income that is not spent becomes private saving. The share of income that is saved is usually exogenously imposed.

The production sector produces goods and services (hereafter grouped together as 'products') for consumption by households, the government, and foreigners (i.e. exports), and for use within the sector as an input into production. It relies on currently installed capital as well as labour and intermediate inputs (domestically produced or imported) for production. As capital by definition takes time to build (that is, more time than production of consumption goods and intermediate inputs), capital formation takes place within the sector concurrently with production for current usage. The associated investment spending is financed by private and public saving and allocated to industries and regions according to a user-selected criterion which is discussed in section 2.3.4.

The trade sector acts as a conduit for products between the domestic economy and the foreign sector. Essentially this sector serves the function of transforming domestically produced products into exports, and transforming foreign products into imports, ready for domestic use.

The public sector purchases products for consumption and derives revenue from four types of taxation: tax on labour income, Goods and Services Tax (GST, borne primarily by domestic households and international tourists), other commodity taxes (borne by all purchasers), and import duties. Provision is also made for some domestic purchases to be subsidised via negative commodity taxes, and some foreign purchases to be subsidised via export subsidies. Any excess of government revenue over spending becomes public saving.

The foreign sector demands domestic products (exports) and supplies the domestic economy with its own products (imports). A trade surplus is equivalent to borrowing by foreigners, funded by net positive domestic saving.

\subsubsection{Industries}

Producers are classified by industry to allow for differences in production technology between them. Each industry is also differentiated by region and produces one unique 
type of product for current use. The basic price of the product of a given industry in adjacent regions may differ due to different production techniques. Each industry also engages in capital formation in each region and the new capital thereby produced is also unique to the industry and region of production.

\subsubsection{Regions}

The national economy is divided up into a set of regional economies, each one tied to a geographic region of the country. Activities of the household and production sectors are given a regional dimension while those of the trade, government and foreign sectors are not. Households and industries can be considered located within regions but the trade and government sectors are national in nature while the foreign sector is external.

\subsubsection{Sources}

From a domestic user's point of view, the sources of products are the domestic regions and importers that supply them. This highlights the role of the trade sector in making foreign products available to the domestic economy. The price relevant to the purchaser is the import price (not the foreign price) which may include duty and trade margins. Similar reasoning holds for the foreign purchaser: as far as they are concerned, the source of products is the exporter; the region of origin is irrelevant because the export price is the same regardless.

\subsubsection{Agents}

The behaviour of the sectors of the economy is captured by modelling the behaviour of representative agents assigned to them as follows:

Household sector: one household agent per region which represents the regional population of actual households

Production sector: one industry agent per region for each industry engaged in current production and/or capital formation; one investor agent which allocates the investment budget across industries and regions 
Trade sector: one exporter agent and one importer agent per product type (i.e. per industry)

Public sector: one government agent which decides the pattern of government consumption and taxation

Foreign sector: one foreign agent which decides how much of each product to buy (export) and sell (import)

Specifying that separate activities are undertaken by separate agents amounts to assuming that decisions regarding one activity are made independently of decisions regarding another. For example, the composition of investment expenditure is decided by the investor agent, independently from the decisions by industries of how to form the units of capital demanded by the investor agent.

The reason why exports' region of origin is unimportant to the foreign sector as stated above is that the agents in the trade sector operate at the national level - where they are physically located is of no consequence in the model. This is also the case for the other agents that lack a regional dimension, namely the investor, the government, and the foreign agent. For example, importers pay the same price for a given product regardless of its source country, and it is assumed that the location of government consumption of products is irrelevant (although the source region of those products is not).

\subsubsection{Endowments}

Each region is assumed to have industry-specific capital stock endowments ${ }^{1}$ and an endowment of time. Both endowments are owned by the household agent located within the region, and rented out only to local industry agents for the purpose of current production. The rental price of the time endowment is the wage rate, given in exchange for labour services.

\subsubsection{Markets}

The microeconomic foundations of the model dictate that agents interact via markets, through which any quantity traded must pass. As such, each traded quantity has an

\footnotetext{
${ }^{1}$ If a region does not have a particular industry operating in it, then it is assumed to have no capital stock specific to that industry
} 
associated supply, demand, and price. Except where prices are exogenously imposed, the market mechanism works to determine the general equilibrium price vector. The important sets of markets in the model are summarised below:

Product markets: one market for each type of product coming from each source, including imports; an industry agent is on the supply side of each domestic product market while household, industry, government, and exporter agents are on the demand side; in the case of imports, an importer agent is on the supply side and exporter agents are excluded from the demand side; the market determines the basic price of the relevant regional product or import

Export markets: one market for each export product; an exporter agent is on the supply side of each market and the foreign agent is on the demand side; determines the quantity of exports - each exporter supplies perfectly elastically at the foreign currency price of their export (which is determined by domestic cost conditions)

Import markets: one market for each import product; the foreign agent is on the supply side of each market and an importer agent is on the demand side; determines the quantity of foreign product to be imported - the domestic economy is assumed a price taker for foreign products

Investment markets: one market for each type of new capital to be installed in each region; an industry agent is on the supply side of each market while the investor agent is on the demand side; determines the construction cost of the relevant industry and region-specific capital

Capital markets: one market for each type of currently installed capital in each region; a household agent is on the supply side of each market while an industry agent (in the same region) is on the demand side; determines the rental rate of the relevant industry and region-specific capital

Labour markets: one market for each region; a household agent is on the supply side of each market while industry agents (in the same region) are on the demand side; determines the wage rate for labour within the region 
Saving market: one market where finance for investment and trade surpluses (lending to foreigners) is available; this is the market that is ignored due to Walras' Law see section 2.4.5 - so modelling it is unnecessary

\subsection{Theoretical Structure}

This section begins by describing the behaviour of the agents of the JENNIFER model and sets out the equations that formalise that behaviour. The conditions required to generate a competitive general equilibrium are then explained and expressed mathematically. While some issues surrounding closure of the model are discussed here, they are mainly matters of model implementation and so are discussed in more detail in section 2.4 especially sections 2.4.4 and 2.4.5.

Notation will be introduced as needed although guidance for notation can be found in tables 2.1 to 2.3. A reference list of all the variables in the core model can be found in appendix C. To simplify the presentation, equations will occasionally be stated with less dimensionality than they are given in the list of equations found in appendix D. Many equations that are straight-forward, such as those that define a national measure as a sum of the regional measures, will not be explicitly stated in the text but are nevertheless included in the equation list.

\subsubsection{Households}

\section{Saving, Labour Supply, and Consumption}

Before addressing the optimising choice of households, we need to consider the issue of private saving. Standard microeconomic theories of the consumption / savings trade-off are necessarily intertemporal. As this CGE model is of the static variety, such a theory is not incorporated. The usual approach in static CGE models is to fix the level of household 


\begin{tabular}{|c|c|c|}
\hline Index & Default Set & Elements \\
\hline agent & $A G E N T S$ & $\begin{array}{l}\text { Agents: household (hsh), industry (ind), investor } \\
(\text { inv), government (gov), exporter (exp), importer } \\
\text { (imp), foreign (for) }\end{array}$ \\
\hline$c$ & $C U R$ & Currency denomination: domestic $(\mathbf{d} \$)$ or foreign $(\mathbf{f} \$)$ \\
\hline$g, h$ & $C O M$ & Products $(=I N D)$ \\
\hline$j, i$ & $I N D$ & Industries (user-specified) \\
\hline$o$ & $O R G$ & Origins: domestic (dom), imported (imp) \\
\hline$p$ & $P P S$ & $\begin{array}{l}\text { Purposes: production for current consumption }\left({ }^{(\mathbf{Q})}\right) \text {, } \\
\text { capital formation }\left({ }^{(\mathbf{K})}\right)\end{array}$ \\
\hline$r, x, y$ & $R E G$ & Regions (user-specified) \\
\hline$s$ & $S R C$ & Sources $(=R E G \cup\{\mathbf{i m p}\})$ \\
\hline$v$ & $V A L$ & $\begin{array}{l}\text { Valuations: current prices and quantities - nominal } \\
(\mathbf{c} \$) \text {, base-year prices and current quantities - real } \\
(\mathbf{b} \$) \text {, current prices and base-year quantities - for } \\
\text { Laspeyres calcuations }(\mathbf{b Q})\end{array}$ \\
\hline$w$ & $T A X V A L$ & $\begin{array}{l}\text { Tax status of income measure: before tax (btax), after } \\
\operatorname{tax}(\operatorname{atax})\end{array}$ \\
\hline
\end{tabular}

Table 2.1: Indices and Default Sets

Set Elements

$N Z R E G \quad$ All regions including the national 'region' $(=R E G \cup\{\mathbf{N Z}\})$

$E N D I N D$ Industries for which investment is allocated endogenously to equalise expected future rates of return $(\subseteq I N D$, user-specified)

TRDVAL Trade flow valuations: basic prices (bas), f.o.b. prices (fob), c.i.f. prices (cif)

PINDEX Price index: Paasche $(\mathbf{P})$, Laspeyres $(\mathbf{L})$, Fisher $(\mathbf{F})$, geometric Laspeyres $(\mathbf{G})$ 


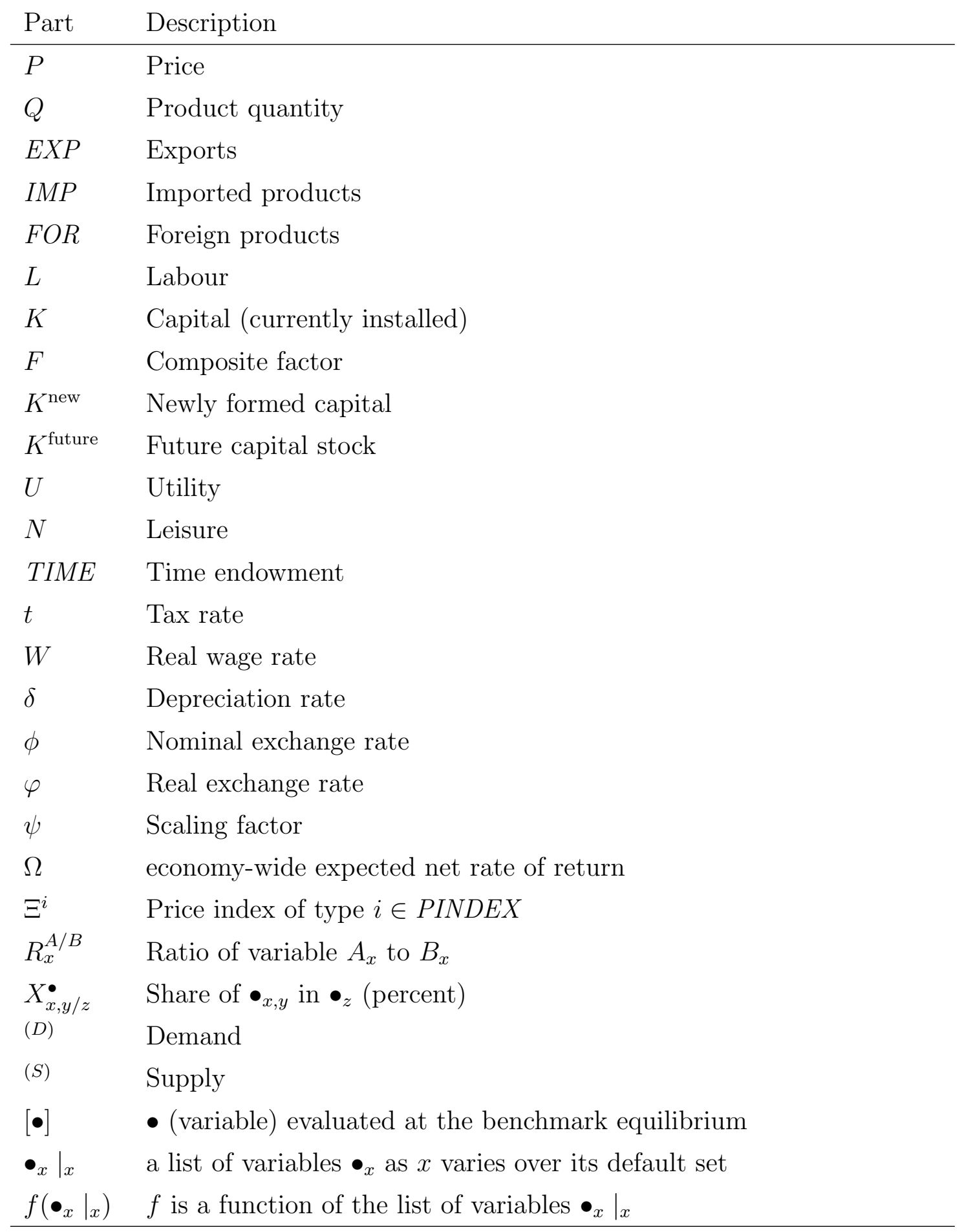

Examples

$Q_{g, s, r}^{\mathbf{h s h}(D)} \quad$ Demand for product $g$ from source $s$ by hsh in region $r$

$L_{r}^{(S)} \quad$ Supply of labour by hsh in region $r$

$P_{g, s, j, r}^{Q, \text { ind(K) }}$ Price paid by ind $_{j, r}$ for purchases of $Q_{g, s}$ for the purpose of capital formation 
expenditure as a proportion of household income, and is followed here: ${ }^{2}$

$$
S P N_{r}^{\mathbf{c} \$}=\frac{1}{100} \cdot A P C_{r}^{\text {atax }} \cdot I N C_{r}^{\text {atax }, \mathbf{c} \$}
$$

with variable descriptions:

$A P C_{r}^{\text {atax }} \quad$ average propensity to consume of $\mathbf{h s h} \mathbf{h}_{r}$ (out of after-tax income) and $0<A P C_{r}^{\text {atax }}<100 \%$

$S P N_{r}^{\mathbf{c} \$}$ nominal expenditure of $\mathbf{h s h}_{r}$

$I N C_{r}^{\text {atax,c\$ }} \$$ nominal after-tax income of $\mathbf{h s h}_{r}$

To fix the level of spending relative to income, the average propensity to save of each household agent is set exogenously, ${ }^{3}$ so essentially the consumption propensities are exogenous, since:

$$
A P C_{r}^{\text {atax }}+A P S_{r}^{\text {atax }}=100
$$

Nominal after-tax income and saving are defined as follows:

$$
\begin{aligned}
I N C_{r}^{\text {atax }, \mathbf{c} \$} & =P_{r}^{L, \text { atax }} \cdot L_{r}^{(S)}+\sum_{j} P_{j, r}^{K} \cdot K_{j, r}^{(S)} \\
S A V_{r}^{\text {atax }, \mathbf{c} \$} & =I N C_{r}^{\text {atax }, \mathbf{c} \$}-S P N_{r}^{\mathbf{c} \$}
\end{aligned}
$$

with variable descriptions:
$K_{j, r}^{(S)}$
capital endowment specific to production by ind $_{j, r}$
$L_{r}^{(S)}$ supply of labour by $\mathbf{h s h}_{r}$
$S A V_{r}^{\text {atax,c } \$}$ nominal after-tax saving of $\mathbf{h s h}_{r}$
$P_{r}^{L, \text { atax }} \quad$ after-tax wage rate paid to $\mathbf{h s h}_{r}$
$P_{j, r}^{K} \quad$ rental rate on $K_{j, r}$ paid to $\mathbf{h s h}_{r}$

The assumption underlying the definition of household income is that the household agent of each region only receives the income earned by the capital located within their region, and only supply labour to the firms within their own region.

\footnotetext{
${ }^{2}$ The equation that is included in the computer model is slightly more broad to allow for measurement of propensities to consume and save out of gross income and their national averages. The actual equation, as listed in appendix D, is:

$$
S P N_{r}^{\mathbf{c} \$}=\frac{1}{100} \cdot A P C_{r}^{w} \cdot I N C_{r}^{w, \mathbf{c} \$} \quad \forall r \in N Z R E G
$$
}

${ }^{3}$ This has important implications for macroeconomic balance which are addressed in section 2.4.5. 
Labour supply is an endogenous choice resulting from a consumption / leisure tradeoff, which implies the household agent has a time endowment to divide between labour and non-labour activities. To facilitate incorporating the trade-off into the agent's optimisation problem, we define endowment income and labour supply as follows:

$$
\begin{aligned}
\operatorname{ENDINC}_{r}^{\text {atax }, \mathbf{c} \$} & =P_{r}^{L, \text { atax }} \cdot \operatorname{TIME}_{r}+\sum_{j} P_{j, r}^{K} \cdot K_{j, r}^{(S)} \\
L_{r}^{(S)} & =T I M E_{r}-N_{r}^{\mathbf{h s h}(D)}
\end{aligned}
$$

with variable descriptions:

$T I M E_{r} \quad$ time endowment of $\mathbf{h s h}_{r}$

$N_{r}^{\mathbf{h s h}(D)}$ quantity of leisure demanded by $\mathbf{h} \mathbf{s} \mathbf{h}_{r}$

Since a region's household agent represents all the actual households located within the region, an easy way of quantifying the time endowment is to define it as the number of full-time equivalents (FTEs) in the working-age population. ${ }^{4}$

Given the relationships between endowment income, expenditure, and saving, the behaviour of the household agent in region $r$ is summarised in a set of demand equations derived from optimisation. The equations have a nested form so that for example the decision of how much of each product to consume is separated from the decision of how much of each given product is to come from each source.

The top level of the decision nest consists of an optimising choice over the level of total composite product $Q_{r}^{\mathbf{h s h}(D)}$ and a level of leisure $N_{r}^{\mathbf{h s h}(D)}$ to consume. That is, the utility function

$$
U_{r}=U_{r}\left(Q_{r}^{\mathbf{h s h}(D)}, N_{r}^{\mathbf{h s h}(D)}\right)
$$

\footnotetext{
${ }^{4}$ FTEs are a measure of labour hours, deflated by what is considered average for an individual working full-time. The Statistics NZ definition is that 1 FTE is equivalent to 30 hours of employment per week. The ratio of FTE employment to persons employed is proportional to average hours worked per person. To convert the working-age population to FTEs, we assume that the unemployed and non-labour force (but still of working age) would work the same number of hours on average as those currently employed if they were to enter employment. That is, the conversion rate from people to FTEs is the same for all components of the working-age population. How this is applied can been seen in section 2.4.3.

However it is measured, the size of the time endowment relative to capital and employment determines the labour supply elasticity — see appendix G — so care needs to be taken that the resulting elasticity is reasonable.
} 
is maximised subject to the definition equations set out above and

$$
P_{r}^{Q, \mathbf{h s h}} \cdot Q_{r}^{\mathbf{h s h}(D)}+P_{r}^{L, \mathbf{a t a x}} \cdot N_{r}^{\mathbf{h s h}(D)}=E N D I N C_{r}^{\text {atax }, \mathbf{c} \$}-S A V_{r}^{\text {atax }, \mathbf{c} \$}
$$

where $P_{r}^{Q, \text { hsh }}$ is the price of composite product paid by $\mathbf{h s h}$. The righthand side of the constraint above is the expenditure of after-tax endowment income (valued at current prices), which is determined indirectly by the exogenous average propensity to save.

This thesis restricts the function $U_{r}(\bullet)$ to be of the Constant Elasticity of Substitution (CES) class, such that the function's shape depends primarily on the value of the elasticity of substitution parameter $\sigma_{r}^{\text {hsh }}$, which dictates the degree of substitutability between composite product and leisure. ${ }^{5}$ In general $\sigma_{r}^{\text {hsh }}$ is non-negative but there are two special cases with specific values of $\sigma_{r}^{\text {hsh }}$ : Leontief utility $\left(\sigma_{r}^{\text {hsh }}=0\right)$ and Cobb-Douglas utility $\left(\sigma_{r}^{\text {hsh }}=1\right)$. The solution to the above utility maximisation problem provides the household demand functions, and the form of the demand functions depends on whether $U_{r}$ is one of these special cases or is the general CES case $\left(0<\sigma_{r}^{\text {hsh }}<\infty\right.$ and $\left.\sigma_{r}^{\text {hsh }} \neq 1\right)$. The main difference is which prices appear in each demand function. For example, the demand functions arising from maximisation of Cobb-Douglas utility do not involve the cross-prices. Then if $U_{r}$ is Cobb-Douglas $P_{r}^{L, \text { atax }}$ will not be an argument in the expression for $Q_{r}^{\mathbf{h s h}(D)}$ and $P_{r}^{Q \text {,hsh }}$ will not be an argument in the expression for $N_{r}^{\mathbf{h s h}(D)}$. It is convenient here to express the demand function in general, without assuming a particular functional form (that is, a value of $\sigma_{r}^{\text {hsh }}$ ) for $U_{r}$, so that all possible prices are listed as arguments of the functions. Thus the household demand functions are written as:

$$
\begin{aligned}
& Q_{r}^{\mathbf{h s h}(D)}=Q_{r}^{\mathbf{h s h}}\left(P_{r}^{Q, \mathbf{h s h}}, P_{r}^{L, \text { atax }}, E N D I N C_{r}^{\text {atax }, \mathbf{c} \$}, S A V_{r}^{\text {atax }, \mathbf{c} \$} ; \sigma_{r}^{\mathbf{h s h}}\right) \\
& N_{r}^{\mathbf{h s h}(D)}=N_{r}^{\mathbf{h s h}}\left(P_{r}^{Q, \mathbf{h s h}}, P_{r}^{L, \mathbf{a t a x}}, E N D I N C_{r}^{\text {atax, } \mathbf{c} \$}, S A V_{r}^{\text {atax }, \mathbf{c} \$} ; \sigma_{r}^{\mathbf{h s h}}\right)
\end{aligned}
$$

This approach of expressing agents' objective functions and resulting demand functions in general and listing all potential arguments will be followed throughout this chapter. Appendix E shows how the functions would be written algebraically for each of the cases discussed above. The expressions found there are in levels form, which are difficult to

\footnotetext{
${ }^{5}$ Using this general class of functions implies that there are no scale effects $\left(U_{r}\right.$ is homothetic). We also restrict $\sigma_{r}^{\mathbf{h s h}}$ to be finite so that composite product and leisure are not perfect substitutes $\left(U_{r}\right.$ is not linear). This ensures that household demand functions are well-defined and continuous (since preferences are strictly convex). These properties are needed for existence of a unique competitive general equilibrium.
} 
interpret. ${ }^{6}$ The functions can however be expressed in linearised form so that the equilibrium effects of changes in prices, endowment income, or saving can be seen more easily. The linearised equations also reveal the implications of the functional form assumption (i.e. the value assigned to $\sigma_{r}^{\text {hsh }}$ ). Using a simplified notation and following the convention of using lowercase letters for percentage changes, ${ }^{7}$ Appendix $\mathrm{F}$ shows that for a given value $\sigma \geq 0$, the above demand functions can be written in linearised form as:

$$
\begin{array}{r}
q=e-\left(\left[X_{Q}\right] \cdot p_{Q}+\left[X_{N}\right] \cdot p_{L}\right)-\sigma\left(p_{Q}-\left(\left[X_{Q}\right] \cdot p_{Q}+\left[X_{N}\right] \cdot p_{L}\right)\right) \\
n=e-\left(\left[X_{Q}\right] \cdot p_{Q}+\left[X_{N}\right] \cdot p_{L}\right)-\sigma\left(p_{L}-\left(\left[X_{Q}\right] \cdot p_{Q}+\left[X_{N}\right] \cdot p_{L}\right)\right)
\end{array}
$$

with variable descriptions:

$q \quad$ percentage change in total composite product demanded

$n \quad$ percentage change in leisure demanded

$p_{Q} \quad$ percentage change in purchase price of total composite product

$p_{L} \quad$ percentage change in after-tax wage rate

$e \quad$ percentage change in nominal after-tax endowment income net of saving

$\left[X_{Q}\right]$ weight on total composite product

$\left[X_{N}\right]$ weight on leisure

The weights $\left[X_{Q}\right]$ and $\left[X_{N}\right]$ are constant expenditure shares - in this case the shares

\footnotetext{
${ }^{6}$ Appendix E states that for the general CES utility maximisation case, the solution is given by:

$$
Q_{i}=\frac{a_{i}{ }^{\sigma} P_{i}{ }^{-\sigma}}{\sum_{j=1}^{n} a_{j}{ }^{\sigma} P_{j}{ }^{1-\sigma}} \cdot E \quad \forall i \in\{1, \ldots, n\}
$$

The algebraic form of the demand functions at the top of the household agent's decision nest would therefore be:

$$
\begin{aligned}
Q_{r}^{\text {hsh }(D)} & =\frac{\left(a_{r}^{Q, \text { hsh }}\right)^{\sigma_{r}^{\text {hsh }}}\left(P_{r}^{Q, \text { hsh }}\right)^{-\sigma_{r}^{\text {hsh }}}}{\left(a_{r}^{Q, \mathbf{h s h}}\right)^{\sigma_{r}^{\text {hsh }}}\left(P_{r}^{Q, \text { hsh }}\right)^{1-\sigma_{r}^{\text {hsh }}}+\left(a_{r}^{N}\right)^{\sigma_{r}^{\text {hsh }}}\left(P_{r}^{L, \text { atax }}\right)^{1-\sigma_{r}^{\text {hsh }}} \cdot \operatorname{ENDXX} P_{r}^{\text {atax }, \mathbf{c} \$}} \\
N_{r}^{\mathbf{h s h}(D)} & =\frac{\left(a_{r}^{N}\right)^{\sigma_{r}^{\text {hsh }}}\left(P_{r}^{L, \text { atax }}\right)^{-\sigma_{r}^{\text {hsh }}}}{\left(a_{r}^{Q, \mathbf{h s h}}\right)^{\sigma_{r}^{\text {hsh }}}\left(P_{r}^{Q, \text { hsh }}\right)^{1-\sigma_{r}^{\text {hsh }}}+\left(a_{r}^{N}\right)^{\sigma_{r}^{\text {hsh }}}\left(P_{r}^{L, \text { atax }}\right)^{1-\sigma_{r}^{\text {hsh }}} \cdot E N D E X P_{r}^{\text {atax }, \mathbf{c} \$}}
\end{aligned}
$$

where $E N D E X P_{r}^{\text {atax, } \mathbf{c} \$}=E N D I N C_{r}^{\text {atax }, \mathbf{c} \$}-S A V_{r}^{\text {atax }, \mathbf{c} \$}$.

${ }^{7}$ For levels variable $Z$, the variable in the linearised equation is $z=d Z / Z$, so the equations are approximately true for small percentage changes from the benchmark equilibrium.
} 
of consumption and leisure in expenditure out of endowment income. ${ }^{8}$ The linearised expressions make it clear that a change in endowment income net of saving will result in the same proportional change in total composite product demanded - the expenditure elasticity is 1 . The effect of single price changes can be described by expressions for own-price and cross price elasticities ( $\varepsilon$ and $\eta$ respectively) derived from the above: ${ }^{9}$

$$
\begin{array}{ll}
\varepsilon_{Q}=-\sigma-(1-\sigma)\left[X_{Q}\right] & \varepsilon_{N}=-\sigma-(1-\sigma)\left[X_{N}\right] \\
\eta_{Q}=-(1-\sigma)\left[X_{N}\right] & \eta_{N}=-(1-\sigma)\left[X_{Q}\right]
\end{array}
$$

If $\sigma>1$ the own-price elasticities will be greater than one in absolute value and the cross-price elasticities will be positive, regardless of the relative sizes of the expenditure shares.

More generally the linearised equations help us see the influence of endowment expenditure, own-price, and weighted-average price on demand for consumption and leisure. For instance, if endowment expenditure rises proportionately more than the average price but less than the wage rate, then leisure demanded will fall (labour supplied will increase) if $\sigma$ is large enough (i.e. the substitution effect dominates). Regardless of the size of $\sigma$, total composite product demanded will rise.

The effects of changes in endowment income and saving can be separated in the linearised demand functions by replacing $e$ with $i \cdot\left[X_{I}\right]-s \cdot\left[X_{S}\right]$ where $i$ and $s$ are the percentage changes in endowment income and saving while $\left[X_{I}\right]$ and $\left[X_{S}\right]$ are the constant ratios of those to endowment expenditure. ${ }^{10}$ Then for example a $1 \%$ increase in endowment income with no change in saving would produce a $\left[X_{I}\right] \%$ rise in both consumption and leisure demand.

For similar reasons, a $1 \%$ rise in leisure demand will imply a less than $1 \%$ decrease in labour supply for a given time endowment. The expression for labour supply in equation (2.6) implies that $l=t \cdot\left[X_{T}\right]-n \cdot\left[X_{N(L)}\right]$ where $l$ and $t$ are the percentage changes in labour supply and time endowment while $\left[X_{T}\right]$ and $\left[X_{N(L)}\right]$ are the constant ratios of the

\footnotetext{
${ }^{8}$ As usual for linearised equations, the weights are constants evaluated from the base-period data. The square brackets are used to signify that they are benchmark equilibrium values.

${ }^{9}$ For a given proportional price change $p_{i}$, set $p_{-i}$ (all other price changes) and e to zero and divide through by $p_{i}$

${ }^{10}$ This is from the definition of endowment expenditure, which would be, in terms of our simplified notation here, $E=I-S$. The ratio of endowment income to endowment expenditure is of course greater than one, and $\left[X_{I}\right]-\left[X_{S}\right]=1$.
} 
time endowment and leisure to labour supply. Thus if leisure demanded increases $1 \%$ for whatever reason, labour supplied will fall by $\left[X_{N(L)}\right] \%$.

While (2.8) could be replaced by a labour supply equation

$$
L_{r}^{(S)}=L_{r}^{(S)}\left(P_{r}^{Q, \mathbf{h s h}}, P_{r}^{L, \text { atax }}, E N D I N C_{r}^{\text {atax }, \mathbf{c} \$}, S A V_{r}^{\text {atax }, \mathbf{c} \$} ; \sigma_{r}^{\mathbf{h s h}}\right)
$$

such that leisure demand is determined by (2.6), the interpretation of such an equation is more difficult than (2.8). Appendix G derives labour supply functions for the different functional forms of $U_{r}$.

\section{The Composition of Consumption}

Once households have decided how much of their endowment income to put towards consumption, it remains to be decided how much of each type of product to consume, and how much of each type to buy from each source. These represent the second and third levels of the households' decision nest respectively. The source choice is divided into two parts: a choice between the domestic and imported varieties, and then a choice between the domestic regions' varieties. This division is made so that the substitution elasticity involved at each stage may differ. We may assume, for example, that the elasticity between domestic and imported varieties of a product is lower than that between the domestic regions' varieties. ${ }^{11}$ The full decision nest of the household agent in each region $r$ is shown in figure 2.1 .

Each optimising choice is an expenditure minimisation problem except the top-level decision, which is the utility maximising choice discussed above. Once the optimal level of total composite product $Q_{r}^{\text {hsh }}$ has been decided, the household agent seeks the minimum cost combination of the different types of product to form the composite product subject to a CES mixing function (including the special cases of Leontief and Cobb-Douglas). That is, minimise

$$
\sum_{g} P_{g, r}^{Q, \mathbf{h s h}} \cdot Q_{g, r}^{\mathbf{h s h}(D)}
$$

\footnotetext{
${ }^{11}$ This could be extended further by having a choice between the local variety and a composite out-ofregion variety, and then a choice between the out-of-region sources. We could then assume, for example, a higher substitution elasticity between the out-of region varieties than between the local variety and the out-of-region varieties. This would serve as a proxy for transport costs if they are not explicitly included in the model. If they were, such an assumption would imply pure home-bias in households' preferences - there would still be some quality of the local variety that makes it preferable to out-of-region varieties.
} 


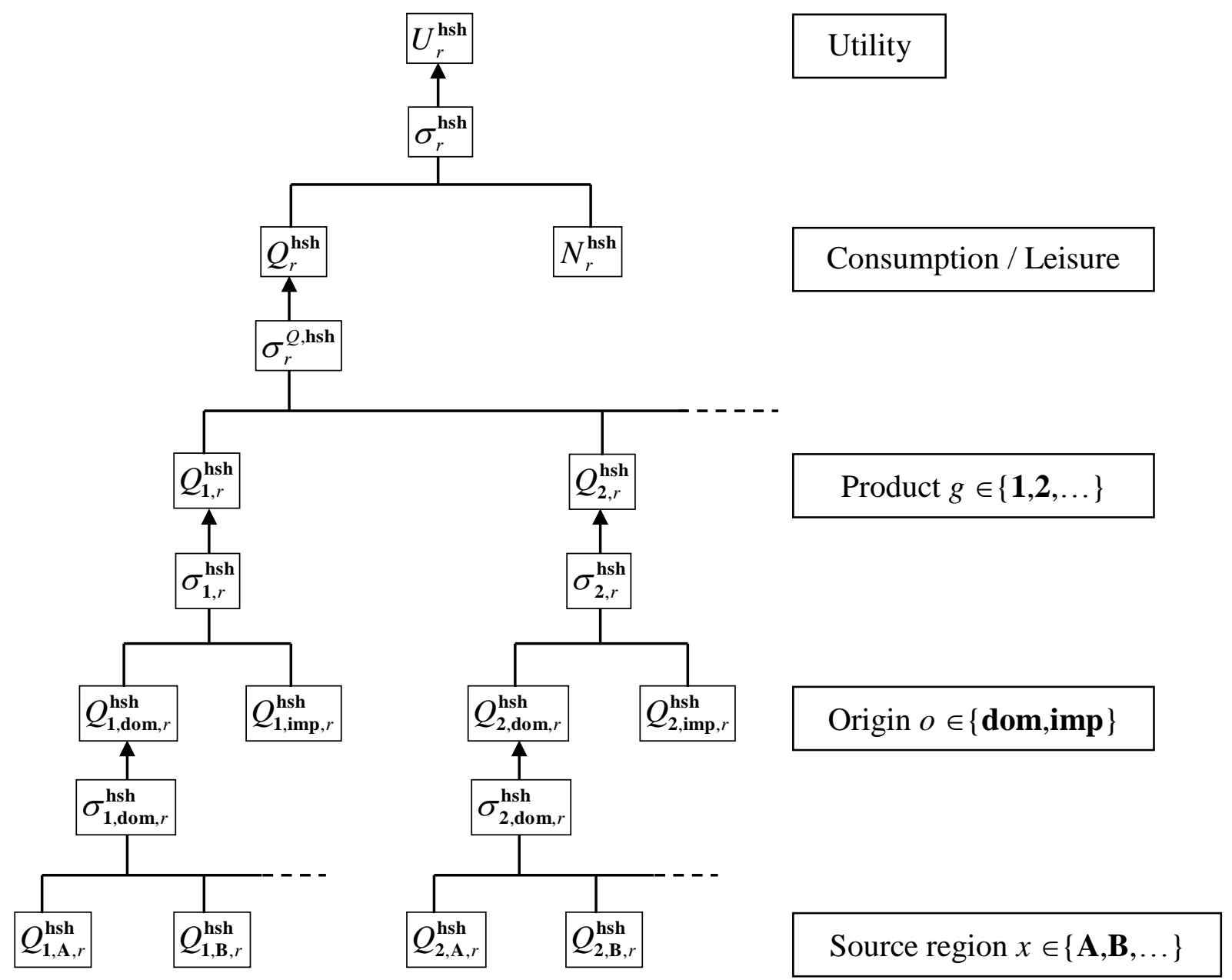

Figure 2.1: Household agents' decision nest

subject to

$$
Q_{r}^{\mathbf{h s h}(D)}=Q_{r}^{\mathbf{h s h}}\left(\left.Q_{g, r}^{\mathbf{h s h}(D)}\right|_{g}\right)
$$

with variable descriptions:

$P_{g, r}^{Q, \mathbf{h s h}} \quad$ price of composite product $g$ paid by $\mathbf{h s h} \mathbf{h}_{r}$

$Q_{g, r}^{\mathbf{h s h}(D)} \quad$ composite product $g$ demanded by $\mathbf{h s h} \mathbf{h}_{r}$

The notation $Q_{r}^{\mathbf{h s h}}\left(\left.Q_{g, r}^{\mathbf{h s h}(D)}\right|_{g}\right)$ is a shorthand for saying that $Q_{r}^{\mathbf{h s h}(D)}$ is a function of $Q_{\mathbf{1}, r}^{\mathbf{h s h}(D)}, Q_{\mathbf{2}, r}^{\mathbf{h s h}(D)}, \ldots$ with $g \in C O M=\{1,2, \ldots\}$. Currently no provision is made in the prototype model for industries to produce multiple product types, so COM and IND always contain the same elements. 
The same assumptions are made for the mixing technology $Q_{r}^{\mathbf{h s h}}(\bullet)$ as were made for the utility function - it is of the CES class which includes Cobb-Douglas and Leontief functions as special cases, and has an associated elasticity of substitution, in this case $\sigma_{r}^{Q, \text { hsh }}$. Thus it is possible for the substitution elasticity for product types to differ from that for the consumption / leisure trade-off, and these can also vary across the regional household agents. ${ }^{12}$

The demand functions that solve the above expenditure minimisation problem can be written as:

$$
Q_{g, r}^{\mathbf{h s h}(D)}=Q_{g, r}^{\mathbf{h s h}}\left(Q_{r}^{\mathbf{h s h}(D)},\left.P_{h, r}^{Q, \mathbf{h s h}}\right|_{h} ; \sigma_{r}^{Q, \mathbf{h s h}}\right)
$$

As above, the demand functions have different algebraic forms depending on the value of $\sigma_{r}^{Q, \text { hsh }}$, and their interpretation is made easier by considering their linearised form. ${ }^{13}$ Appendix $\mathrm{F}$ shows that for a given $\sigma \geq 0$, the linearised equations are:

$$
q_{g}=q-\sigma\left(p_{g}^{Q}-\sum_{h}\left[X_{h}^{Q}\right] \cdot p_{h}^{Q}\right)
$$

with variable descriptions:

$q_{g} \quad$ percentage change in demand for composite product $g$

$p_{g}^{Q} \quad$ percentage change in price of composite product $g$

$\left[X_{g}^{Q}\right] \quad$ weight on composite product $g$

The weights $\left[X_{g}^{Q}\right]$ are the constant shares of product $g$ in total expenditure. As before we can derive expressions for own-price and cross-price elasticities:

$$
\begin{aligned}
\varepsilon_{g}^{Q} & =-\sigma\left(1-\left[X_{g}^{Q}\right]\right) \\
\eta_{g, h}^{Q} & =\sigma \cdot\left[X_{h}^{Q}\right]
\end{aligned}
$$

where $\varepsilon_{g}^{Q}$ is the own-price demand elasticity of composite product $g$ and $\eta_{g, h}^{Q}$ is the crossprice elasticity of demand of composite product $g$ with respect to the price of composite

\footnotetext{
${ }^{12}$ While the model design allows for such flexibility, it is reasonable to assume they do not vary over regions unless information is available that suggests otherwise. Indeed, this should be the default position.

${ }^{13}$ Readers familiar with ORANI (Dixon et al. 1982) or FEDERAL (Madden 1990) will see that these are of a similar form to ORANI's equation (14.11) and FEDERAL's equation (2.16) except those equations concern choices over sources whereas the equations here are for choices over products. The two models mentioned employ Klein-Rubin (Stone-Geary) utility at their top level rather than the simpler CES utility used here, and their household decision nest top level is the choice over products rather than the consumption / leisure trade-off modelled above.
} 
product $h$. The cross-price elasticities will always be positive, but whether demand is more than unit elastic with respect to its own-price depends jointly on the size of the substitution elasticity and the expenditure share. The linearised demand functions imply that in general if the price of composite product $g$ rises relative to a share-weighted index of all the composite product prices, ceteris paribus, the demand for composite product $g$ will fall by an amount determined by the value of $\sigma$. The higher the value of $\sigma$ is above 1 , the more elastic demand is to changes in own-price relative to the weighted-average price. $^{14}$

\section{The Source Choice for Consumption}

The remainder of the nested demand functions for household agents are derived in a similar manner to the above. Referring back to the diagram in figure 2.1, the choice over the domestic and imported product varieties, and then the domestic regions' varieties are each expenditure minimisation problems analogous to that seen directly above. We therefore omit the statements of the constrained optimisation problems and list the demand functions directly: ${ }^{15}$

$$
\begin{aligned}
Q_{g, \mathbf{d o m}, r}^{\mathbf{h s h}(D)} & =Q_{g, \mathbf{d o m}, r}^{\mathbf{h s h}}\left(Q_{g, r}^{\mathbf{h s h}(D)}, P_{g, \mathbf{d o m}, r}^{Q, \mathbf{h s h}}, P_{g, \mathbf{i m p}, r}^{Q, \mathbf{h s h}} ; \sigma_{g, r}^{\mathbf{h s h}}\right) \\
Q_{g, \mathbf{i m p}, r}^{\mathbf{h s h}(D)} & =Q_{g, \mathbf{i m p}, r}^{\mathbf{h s h}}\left(Q_{g, r}^{\mathbf{h s h}(D)}, P_{g, \mathbf{d o m}, r}^{Q, \mathbf{h s h}}, P_{g, \mathbf{i m p}, r}^{Q, \mathbf{h s h}} ; \sigma_{g, r}^{\mathbf{h s h}}\right) \\
Q_{g, x, r}^{\mathbf{h s h}(D)} & =Q_{g, x, r}^{\mathbf{h s h}}\left(Q_{g, \mathbf{d o m}, r}^{\mathbf{h s h}(D)},\left.P_{g, y, r}^{Q, \mathbf{h s h}}\right|_{y} ; \sigma_{g, \mathbf{d o m}, r}^{\mathbf{h s h}}\right)
\end{aligned}
$$

with variable descriptions:

\footnotetext{
${ }^{14}$ Demand is inelastic in this sense for $\sigma<1$. The interpretation of the linearised demand function given here is due to Dixon, Parmenter, Powell \& Wilcoxen (1992, p. 126).

${ }^{15}$ Equations (2.10) and (2.11) can be written more compactly as:

$$
Q_{g, o, r}^{\mathbf{h s h}(D)}=Q_{g, o, r}^{\mathbf{h s h}}\left(Q_{g, r}^{\mathbf{h s h}(D)},\left.P_{g, a, r}^{Q, \mathbf{h s h}}\right|_{a \in O R G} ; \sigma_{g, r}^{\mathbf{h s h}}\right)
$$
}

For clarity these origin demands are written separately, due to the inclusion of $Q_{g, \mathbf{d o m}, r}^{\mathbf{h s h}}$ in equation (2.12). Later we will also find it useful to combine variables like $Q_{g, \mathbf{i m p}, r}^{\mathbf{h s h}(D)}$ with $Q_{g, x, r}^{\mathbf{h s h}(D)}$ as $Q_{g, s, r}^{\mathbf{h s h}(D)}$ with $s \in S R C$, source demands for products from all domestic regions plus imports. 


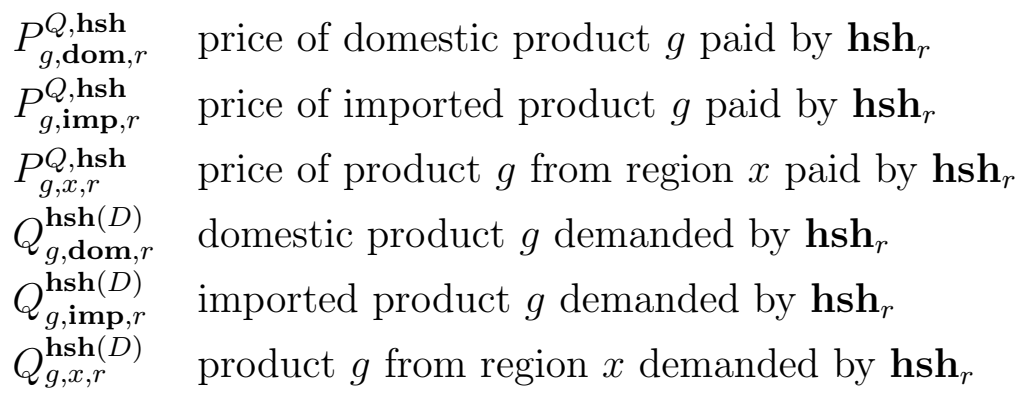

The demand functions can be interpreted by considering their linearised form, analogous to those directly above. For example, they imply that if the purchase price of a product $g$ from region $x$ that the household agent in region $r$ faces rises relative to the weighted-average price of that product from all regions, the agent will substitute away from region $x$ 's variety towards the other regions' varieties, with the strength of the substitution determined by the value of $\sigma_{g, \mathbf{d o m}, r}^{\text {hsh }}$.

\section{Summary}

This section has introduced a block of equations that describe the behaviour of households. Equations (2.1) - (2.6) set out definitions of household income, saving, endowment income, labour supply, and average propensities to consume and save. A system of demand equations were then derived in (2.7) - (2.12). The decision nest shown in figure 2.1 may appear to have many tiers (levels) compared to other CGE models such as ORANI and FEDERAL. However, this is simply because the form of the utility and mixing functions have been restricted to CES. FEDERAL in contrast uses CRESH functions, which are a generalisation of the CES form that allows the substitution elasticity between any two sources to differ from that between any other two sources. While this makes for a more elegant and complicated model, for this thesis the choice was made to assume constant elasticities over the domestic regions. This assumption is reasonable until New Zealand data becomes available to support such an extension of the household decision nest. It also enhances the transparency and simplicity of the model.

\subsubsection{Industries - Current Production}

Within the production sector the following activities take place:

- production of goods and services for consumption by households, the government, 
and the foreign sector,

- production of goods and services for use as an intermediate input by other firms,

- construction of new capital, and

- investment in new capital

Each industry agent is assumed to engage only in the first three of these activities - i.e. making products for current use and construction of capital. This latter activity involves deciding the best mixture of inputs to produce a given quantity of new capital. The decision of how much new capital is desired by each industry in each region is the fourth activity in the list above and is undertaken by a separate agent. The implication of dealing with these activities separately is that the optimising choice involved in one activity is made independently of decisions regarding the other activities. For example production decisions are made independently of investment decisions. ${ }^{16}$ This section will set out the behaviour of the industry agents with respect to current production and the following section will deal with capital formation decisions.

\section{Factors, Inputs, and Output}

For each industry there is one agent in each region that produces a single unique product. Each industry is therefore named according to its product. Industry $j$ in region $r$ makes product $g$ and $g=j$. Intermediate inputs and factors of production are used in the production process. The full decision nest of the industry agent ind $_{j, r}$ in the production of $Q_{g, r}$ is shown in figure 2.2 .

The industry agent ind $_{j, r}$ aims to minimise costs of production by choosing the optimal combination of total composite product $Q_{j, r}^{\operatorname{ind}(D)}$ and composite factor $F_{j, r}^{\operatorname{ind}(D)}$ to produce a given level of output of its product $Q_{g, r}^{(S)}$. That is, ind $_{j, r}$ minimises:

$$
P_{j, r}^{Q, \text { ind }} \cdot Q_{j, r}^{\text {ind }(D)}+P_{j, r}^{F, \text { ind }} \cdot F_{j, r}^{\text {ind }(D)}
$$

subject to its production function

$$
Q_{g, r}^{(S)}=Q_{g, r}\left(Q_{j, r}^{\operatorname{ind}(D)}, F_{j, r}^{\operatorname{ind}(D)}\right)
$$

\footnotetext{
${ }^{16}$ Naturally the outcome of investment decisions will affect production decisions. The point is that the decisions are made separately so that agents' optimisation problems only involve a single objective function.
} 
with variable descriptions:

$P_{j, r}^{Q, \text { ind }} \quad$ price of total composite product paid by ind $_{j, r}$

$P_{j, r}^{F, \text { ind }} \quad$ price of composite factor paid by ind $_{j, r}$

The production function $Q_{g, r}(\bullet)$ is of the CES class of functions so the form of the resulting demand functions depends on the assumed value of the elasticity of substitution parameter $\sigma_{j, r}^{\text {ind }}$. The approach taken in section 2.3.1 of expressing the demands using function notation and interpreting them using linearised equations is followed here.

The demand functions that solve the above cost minimisation problem are:

$$
\begin{aligned}
& Q_{j, r}^{\text {ind }(D)}=Q_{j, r}^{\text {ind }}\left(Q_{g, r}^{(S)}, P_{j, r}^{Q, \text { ind }}, P_{j, r}^{F, \text { ind }} ; \sigma_{j, r}^{\text {ind }}\right) \\
& F_{j, r}^{\text {ind }(D)}=F_{j, r}^{\text {ind }}\left(Q_{g, r}^{(S)}, P_{j, r}^{Q, \text { ind }}, P_{j, r}^{F, \text { ind }} ; \sigma_{j, r}^{\text {ind }}\right)
\end{aligned}
$$

As discussed above in the context of the household agents' decision nest, the form of the demand functions for specific values of $\sigma_{j, r}^{\text {ind }}$ can be seen from appendix E and can be interpreted by considering their linearised form. Linearising the above demand functions for a given value of $\sigma \geq 0$ gives: ${ }^{17}$

$$
\begin{aligned}
& q=s-\sigma\left(p_{Q}-\left(\left[X_{Q}\right] \cdot p_{Q}+\left[X_{F}\right] \cdot p_{F}\right)\right) \\
& f=s-\sigma\left(p_{F}-\left(\left[X_{Q}\right] \cdot p_{Q}+\left[X_{F}\right] \cdot p_{F}\right)\right)
\end{aligned}
$$

with variable descriptions:

$q \quad$ percentage change in total composite product demanded

$f \quad$ percentage change in composite factor demanded

$p_{Q} \quad$ percentage change in purchase price of total composite prod-

uct

$p_{F} \quad$ percentage change in price of composite factor

$s \quad$ percentage change in output (supply)

$\left[X_{Q}\right] \quad$ weight on total composite product

$\left[X_{F}\right] \quad$ weight on composite factor

\footnotetext{
${ }^{17}$ These linearised functions are analogous to ORANI's equation (12.23) and FEDERAL's equation (2.1) (See Dixon et al. 1982, Madden 1990); the forms differ as ORANI and FEDERAL derive their demands from CRESH rather than CES functions and include technology coefficients which are treated here as structural parameters for simplicity. Introducing technology coefficients would make it possible to run simulations of unbalanced growth or technology shocks. A few simple changes to the program code would be all that is required to reassign the parameters as exogenous variables.
} 


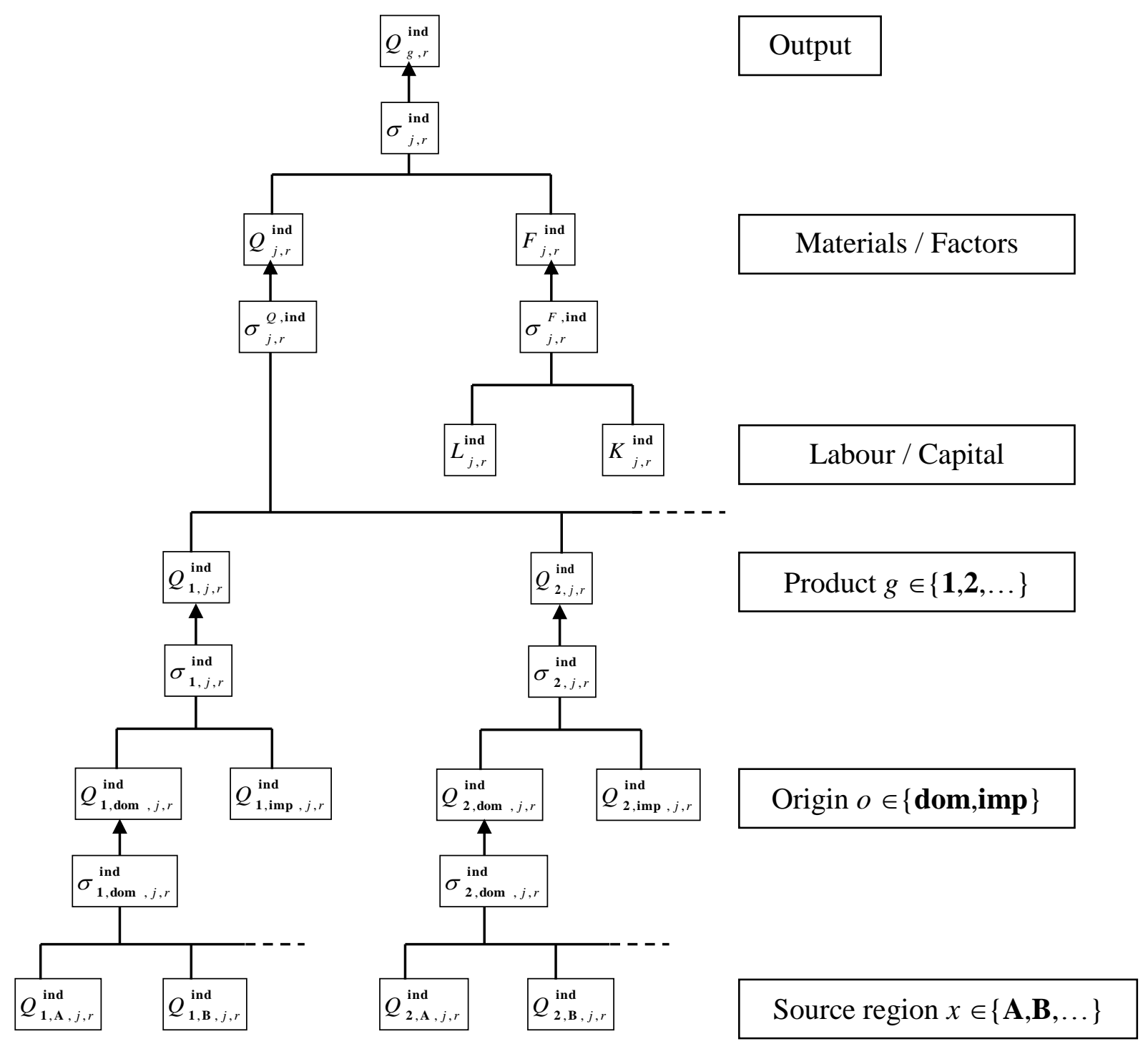

Figure 2.2: Industry agents' decision nest for current production

The weights $\left[X_{Q}\right]$ and $\left[X_{F}\right]$ are product and factor shares in total costs. If the price of composite factor rises (price of composite product falls) relative to a weighted average price index constructed using these weights, the industry agent will substitute away from the use of factors towards the use of material input, to the degree allowed by the value of $\sigma$. In order to increase output by a certain proportion, the industry agent needs to increase its use of composite product and factor by the same proportion in the absence of input price changes. This confirms the constant returns to scale property of the production 
function.

To this point, all prices that have been introduced have been purchase prices - the price paid by the purchasing agent for the product itself plus any auxiliary charges such as taxes, transport costs etc. Thus there is a distinction between these prices that are relevant for demands, and the prices that the seller receives - the basic price. There is a basic price associated with the industry agent's output, $P_{g, r}^{Q}$ and we will see this is determined by the market clearing condition of the relevant product market. In this basic version of the prototype model, the only items that may put a wedge between the purchase and basic prices are ad valorem product subsidies and taxes (such as GST, import duties, and excise taxes). Purchase prices will be discussed fully in section 2.3.10.

\section{The Composition of Input Demand}

The industry agent's choice of how to form its composite intermediate input from the set of available products and sources is analogous to the choice by household agents over the composition of their consumption discussed in sections 2.3.1. With appropriate adjustments to notation, it follows that the demand functions that describe each industry agent's input product mix are:

$$
Q_{g, j, r}^{\operatorname{ind}(\mathbf{Q})(D)}=Q_{g, j, r}^{\operatorname{ind}(\mathbf{Q})}\left(Q_{j, r}^{\operatorname{ind}(D)},\left.P_{h, j, r}^{Q, \mathbf{i n d}(\mathbf{Q})}\right|_{h} ; \sigma_{j, r}^{Q, \mathbf{i n d}(\mathbf{Q})}\right)
$$

with an interpretation analogous to that of equation (2.9) for households. The quantity variables carry a superscript ${ }^{(\mathbf{Q})}$ to distinguish these demands for inputs to current production from demands for inputs to capital formation, which are denoted with ${ }^{(\mathbf{K})}$ instead. Similarly, the purchase prices carry the superscript ${ }^{(Q)}$ to allow for the possibility that the industry agent pays a different price for the same composite product depending on its use. It may be that purchases of some products are subsidised if they are used for capital formation, for example. It is usual to assume Leontief mixing (no substitution, $\sigma_{j, r}^{Q, \operatorname{ind}(\mathbf{Q})}=0$ ) at this level of the industry agent's decision nest but here the technology is specified for any given positive substitution elasticity so that this assumption may be relaxed if desired. ${ }^{18}$

\footnotetext{
${ }^{18}$ That is, the Leontief technology is not hard-coded into the program; the user may change the elasticity by simply altering the appropriate number.
} 


\section{The Source Choice for Inputs}

For each product demanded as an input to production, the industry agent decides how much of the product will come from each source in a fashion identical to how household agents made this choice as detailed in section 2.3.1, so the demand functions that describe each industry agent's source choice are:

$$
\begin{aligned}
& Q_{g, \mathbf{d o m}, j, r}^{\operatorname{ind}(\mathbf{Q})(D)}=Q_{g, \mathbf{d o m}, j, r}^{\operatorname{ind}(\mathbf{Q})}\left(Q_{g, j, r}^{\operatorname{ind}(\mathbf{Q})(D)}, P_{g, \mathbf{d o m}, j, r}^{Q, \operatorname{ind}(\mathbf{Q})}, P_{g, \mathbf{i m p}, j, r}^{Q, \operatorname{ind}(\mathbf{Q})} ; \sigma_{g, j, r}^{\operatorname{ind}(\mathbf{Q})}\right) \\
& Q_{g, \mathbf{i m p}, j, r}^{\operatorname{ind}(\mathbf{Q})(D)}=Q_{g, \mathbf{i m p}, j, r}^{\operatorname{ind}(\mathbf{Q})}\left(Q_{g, j, r}^{\operatorname{ind}(\mathbf{Q})(D)}, P_{g, \mathbf{\operatorname { i n d }}, j, r}^{Q, \mathbf{Q})}, P_{g, \mathbf{i m p}, j, r}^{Q, \mathbf{i n d}(\mathbf{Q})} ; \sigma_{g, j, r}^{\operatorname{ind}(\mathbf{Q})}\right) \\
& Q_{g, x, j, r}^{\operatorname{ind}(\mathbf{Q})(D)}=Q_{g, x, j, r}^{\operatorname{ind}(\mathbf{Q})}\left(Q_{g, \mathbf{d o m}, j, r}^{\operatorname{ind}(\mathbf{Q})(D)},\left.P_{g, y, j, r}^{Q, \mathbf{i n d}(\mathbf{Q})}\right|_{y} ; \sigma_{g, \mathbf{d o m}, j, r}^{\operatorname{ind}(\mathbf{Q})}\right)
\end{aligned}
$$

Here again the purchase prices may differ across uses for a given industry agent, and any differences will feed through to higher levels in the decision nest. In general any auxiliary cost (or benefit) accrued in acquiring products for one purpose but not the other can cause the purchase prices to differ.

\section{The Composition of Factor Demand}

As indicated in figure 2.2, industry agents demand a combination of labour and capital to form their composite factor input. The important feature of this choice is that they can only demand labour and capital from within their own region, and indeed this is what gives the industry agents their regional characteristic. Additionally, they can only use capital specific to their own industry. ${ }^{19}$ The model implicitly assumes that labour is perfectly mobile between industries. Therefore additional labour requirements can be met by acquiring employees from other industries. The extent they also may be met by acquiring employees from other regions depends on the regional labour mobility assumption, which is a closure issue in this basic version of the model. Similarly, the only way additional current capital requirements may be met is if the closure allows capital mobility between industries and/or regions. ${ }^{20}$ Consider the industry agent's factor composition problem:

\footnotetext{
${ }^{19}$ This is why the labour supply variable $L_{r}^{(S)}$ only has a region subscript but the capital stock variable $K_{j, r}^{(S)}$ has both industry and region subscripts.

${ }^{20}$ Additional capital formation cannot be used because it is assumed that new capital only comes online after the current period - an essential element of the comparative-static framework.
} 
Minimise

$$
P_{r}^{L, \text { btax }} \cdot L_{j, r}^{\operatorname{ind}(D)}+P_{j, r}^{K} \cdot K_{j, r}^{\operatorname{ind}(D)}
$$

subject to

$$
F_{j, r}^{\operatorname{ind}(D)}=F_{j, r}^{\operatorname{ind}}\left(L_{j, r}^{\operatorname{ind}(D)}, K_{j, r}^{\operatorname{ind}(D)}\right)
$$

with variable descriptions:

$$
\begin{array}{ll}
L_{j, r}^{\operatorname{ind}(D)} & \text { demand for labour by } \text { ind }_{j, r} \\
K_{j, r}^{\operatorname{ind}(D)} & \text { demand for capital by } \text { ind }_{j, r}
\end{array}
$$

Notice the relevant wage rate for the industry agent is the before-tax (gross) rate while it was the after-tax rate that entered the household agent's optimisation problem. There is therefore provision for direct tax to be imposed on labour income, but no such provision has been made at this stage for tax on capital income.

The solution to the factor-cost minimisation problem is:

$$
\begin{aligned}
& L_{j, r}^{\operatorname{ind}(D)}=L_{j, r}^{\operatorname{ind}}\left(F_{j, r}^{\operatorname{ind}(D)}, P_{r}^{L, \text { btax }}, P_{j, r}^{K} ; \sigma_{j, r}^{F, \text { ind }}\right) \\
& K_{j, r}^{\text {ind }(D)}=K_{j, r}^{\text {ind }}\left(F_{j, r}^{\operatorname{ind}(D)}, P_{r}^{L, \text { btax }}, P_{j, r}^{K} ; \sigma_{j, r}^{F, \text { ind }}\right)
\end{aligned}
$$

Analogous to the demand function for composite factor and its linearised form seen above, the linearised demands for labour and current capital are:

$$
\begin{aligned}
& l=f-\sigma\left(p_{L}-\left(\left[X_{L}\right] \cdot p_{L}+\left[X_{K}\right] \cdot p_{K}\right)\right) \\
& k=f-\sigma\left(p_{K}-\left(\left[X_{L}\right] \cdot p_{L}+\left[X_{K}\right] \cdot p_{K}\right)\right)
\end{aligned}
$$

If demand for composite factor rises but levels of capital stocks are fixed (under a shortrun closure assumption), there must be a rise in the rental rate relative to the wage rate for market clearing, and a subsequent fall in the $\mathrm{K} / \mathrm{L}$ ratio.

\section{Summary}

This section has presented the block of equations (2.13) - (2.20) that describe the behaviour of industry agents with respect to production for current usage. These equations are a set of nested demand functions that formalise the composition of production inputs as summarised in figure 2.2. As with the household agent's decision nest, the degree of nesting employed here allows different substitution elasticities to be used at each level of the nest. 


\subsubsection{Industries - Capital Formation}

At the same time as producing products for current usage, industry agents construct capital for future use. Their behaviour with regard to product and source composition of inputs for capital formation is analogous to that for current production. The decision nest is essentially the same except the top-level choice between materials and factors, and the composition of factor demand, are not present - see figure 2.3. As per common convention, no labour or capital are directly employed in the construction of new capital; the only costs are purchases of goods and services which have already used labour and capital in their production. It is assumed that industry agents decide how a given quantity of new capital is formed while the issue of how much should be constructed is determined by behaviour of the investor agent, discussed in the next section. For a given level of new capital $K_{j, r}^{n e w(S)}$ constructed by the industry agent ind in $_{j, r}$, the product and source composition is described by the following demand functions:

$$
\begin{aligned}
& Q_{g, j, r}^{\operatorname{ind}(\mathbf{K})(D)}=Q_{g, j, r}^{\operatorname{ind}(\mathbf{K})}\left(K_{j, r}^{n e w(S)},\left.P_{h, j, r}^{Q, \mathbf{i n d}(\mathbf{K})}\right|_{h} ; \sigma_{j, r}^{Q, \mathbf{i n d}(\mathbf{K})}\right) \\
& Q_{g, \mathbf{d o m}, j, r}^{\operatorname{ind}(\mathbf{K})(D)}=Q_{g, \mathbf{d o m}, j, r}^{\operatorname{ind}(\mathbf{K})}\left(Q_{g, j, r}^{\operatorname{ind}(\mathbf{K})(D)}, P_{g, \mathbf{d o m}, j, r}^{Q, \mathbf{i n d}(\mathbf{K})}, P_{g, \mathbf{i m p}, j, r}^{Q, \mathbf{i n d}(\mathbf{K})} ; \sigma_{g, j, r}^{\operatorname{ind}(\mathbf{K})}\right) \\
& Q_{g, \mathbf{i m p}, j, r}^{\operatorname{ind}(\mathbf{K})(D)}=Q_{g, \mathbf{i m p}, j, r}^{\operatorname{ind}(\mathbf{K})}\left(Q_{g, j, r}^{\operatorname{ind}(\mathbf{K})(D)}, P_{g, \mathbf{i n d}(\mathbf{K}), j, r}^{Q, \mathbf{i n d}}, P_{g, \mathbf{i m p}, j, r}^{Q, \mathbf{i n d}(\mathbf{K})} ; \sigma_{g, j, r}^{\operatorname{ind}(\mathbf{K})}\right) \\
& Q_{g, x, j, r}^{\mathbf{i n d}(\mathbf{K},(D)}=Q_{g, x, j, r}^{\mathbf{i n d}(\mathbf{K})}\left(Q_{g, \mathbf{d o m}, j, r}^{\operatorname{ind}(\mathbf{K})(D)},\left.P_{g, y, j, r}^{Q, \mathbf{i n d}(\mathbf{K})}\right|_{y} ; \sigma_{g, \mathbf{d o m}, j, r}^{\operatorname{ind}(\mathbf{K})}\right)
\end{aligned}
$$

The meaning of the variables in equations (2.21) - (2.24) are the same as those in equations (2.15) - (2.18) except that they are for capital formation instead of current production. This is indicated by the variables having ${ }^{(K)}$ superscripts instead of $(Q)$ 's. Note that the substitution elasticities also carry the ${ }^{(K)}$ superscripts to indicate that the mixing technology may differ from the current production counterpart, although without good reason to assume so, the default position of invariance should be taken. 


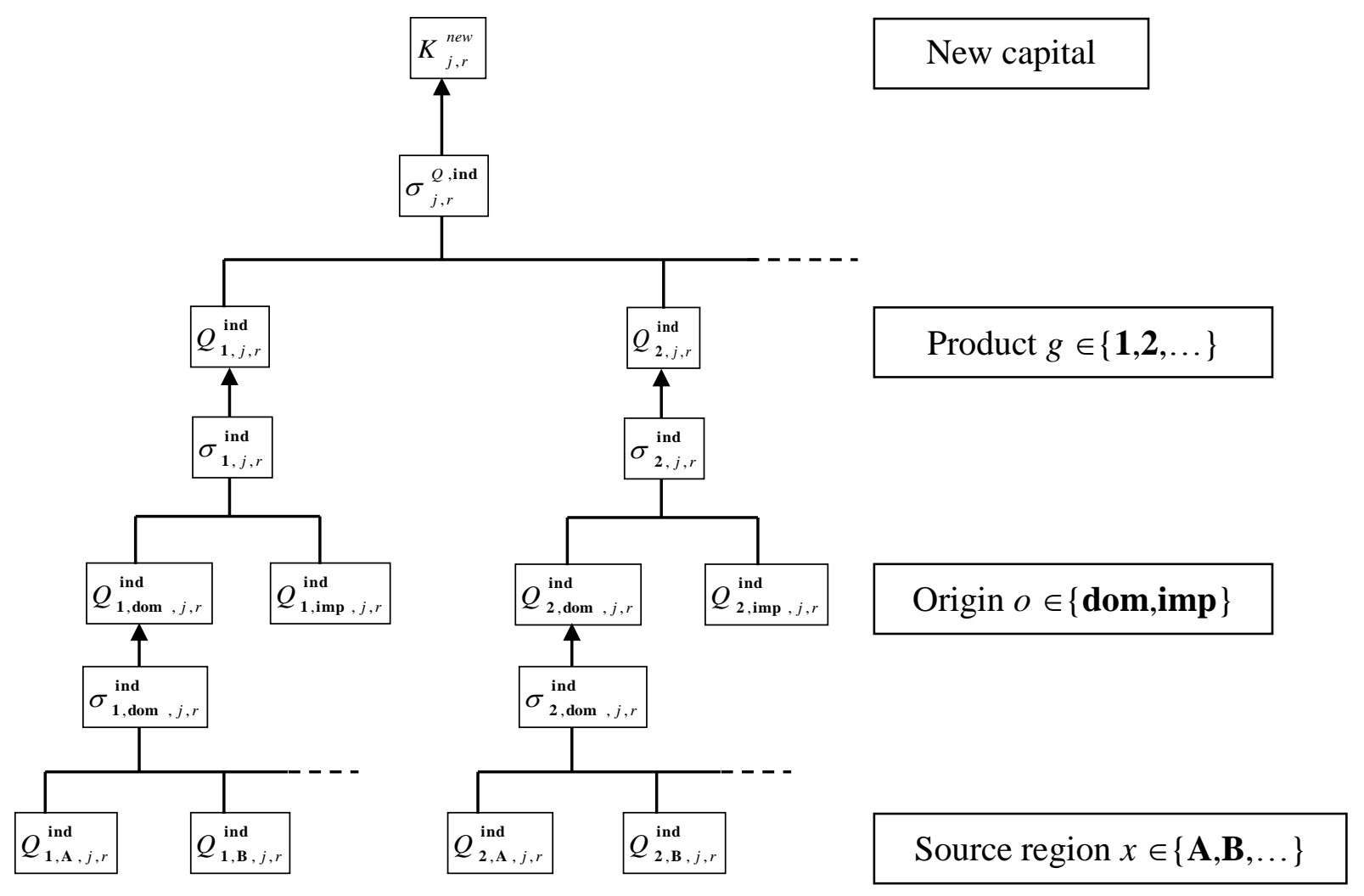

Figure 2.3: Industry agents' decision nest for capital formation

\subsubsection{Investment Allocation}

The specification of an investor agent inv allows the separation of the investment allocation decision from the capital formation process. It also allows capital construction costs to be established, which can be compared to rental rates to determine rates of return on investment in new capital. The investor agent takes the level of aggregate gross investment as given and decides how to allocate that investment between the industries and regions. That is, the agent decides the level of gross investment demand $I_{j, r}^{\text {ind }(D)}$ for each industry $j$ in each region $r$. There are two possible options for the investment agent's behaviour:

exogenous investment demands are fixed at exogenously determined proportions of, and 
therefore mimic changes in, real aggregate investment. The latter is also usually exogenously specified, either its level or its share in absorption or GDP.

endogenous investment demands respond to relative rates of return, such that expected rates of return on investment are equalised. This option is based on the ORANI / FEDERAL endogenous allocation module.

Regardless of which option is chosen, in equilibrium $I_{j, r}^{\operatorname{ind}(D)}=K_{j, r}^{\text {new }(S)} \forall j, r$. That is, the set of market clearing conditions for the investment markets will establish that the supply of new capital for each industry in each region will equal the respective investment demand. The market clearing price, $P_{j, r}^{K, \text { new }}$, is the construction cost of the new capital.

In order to facilitate the endogenous investment allocation mechanism we first define a set of variables:

$$
\begin{aligned}
G R O R_{j, r} & =100 \times \frac{P_{j, r}^{K}}{P_{j, r}^{K, \text { new }}} \\
N R O R_{j, r} & =G R O R_{j, r}-\delta_{j, r} \\
R_{j, r}^{G R O R / N R O R} & =\frac{G R O R_{j, r}}{N R O R_{j, r}} \\
R_{j, r}^{K \text { new } / K \text { future }} & =100 \times \frac{K_{j, r}^{\text {new }(S)}}{K_{j, r}^{\text {future }}} \\
K_{j, r}^{\text {future }} & =\left(1-\frac{\delta_{j, r}}{100}\right) K_{j, r}^{(S)}+K_{j, r}^{\text {new }(S)}
\end{aligned}
$$

\begin{tabular}{|c|c|}
\hline$G R O R_{j, r}$ & gross rate of return \\
\hline$N R O R_{j, r}$ & net rate of return \\
\hline$R_{j, r}^{G R O R / N R O R}$ & ratio of gross to net returns \\
\hline$R_{j, r}^{K \text { new } / K \text { future }}$ & ratio of new to future capital stock \\
\hline$\delta_{j, r}$ & depreciation rate \\
\hline$K_{j, r}^{\text {future }}$ & future capital stock \\
\hline
\end{tabular}

with variable descriptions:

Of the sets of new variables listed above, one set of $J \times R$ variables needs to be exogenously specified, where $J$ is the number of industries and $R$ is the number of regions. In this thesis, depreciation rates are the exogenous components. Depending on what data is available, in general the user may wish to exogenously specify new to future capital 
ratios (a measure of capital growth) or gross to net rates of return ratios instead, hence the inclusion of their defining equations to facilitate this.

As discussed later in section 2.4, since the model is implemented in levels, base-period or benchmark equilibrium values of all model variables have to be established. This enables real measures to simply be defined in constant prices. Real aggregate investment is therefore defined as:

$$
I^{\mathbf{b} \$}=\sum_{j} \sum_{r}\left[P_{j, r}^{K, \text { new }}\right] \cdot I_{j, r}^{\mathrm{ind}(D)}
$$

where $\left[P_{j, r}^{K, \text { new }}\right]$ is the benchmark equilibrium value of $P_{j, r}^{K, \text { new }}$ and $I^{\mathbf{b} \$}$ is real aggregate investment - investment measured at constant construction-cost prices. Shares of real aggregate investment $X_{j, r / \text { tot }}^{I, \mathbf{b} \$}$ are defined by the equation:

$$
X_{j, r / \text { tot }}^{I, \mathbf{b} \$}=100 \times \frac{\left[P_{j, r}^{K, \text { new }}\right] \cdot I_{j, r}^{\mathrm{ind}(D)}}{I^{\mathbf{b} \$}}
$$

To allow for equalisation of expected rates of return on capital, we adapt the FEDERAL (Madden 1990, eq. 2.54) expected net rate of return schedule so that:

$$
N R O R_{j, r}^{\text {future }}=\left(\frac{K_{j, r}^{\text {future }}}{K_{j, r}^{(S)}}\right)^{-\beta_{j, r}} \cdot N R O R_{j, r}
$$

with variable descriptions:

$N R O R_{j, r}^{\text {future }}$ expected net rate of return on ind $_{j, r}$ 's capital

$\beta_{j, r} \quad$ elasticity of expected/current rate of return ratio with respect to future/current capital ratio - the marginal efficiency of investment $\left(\beta_{j, r}>0\right)$

When the investment shares are exogenous, this equation causes the expected rates of return consistent with those shares to be reported as part of the equilibrium solution, and simulation results will report how the specified shock causes the expected rates to diverge. To implement endogenous allocation, all that is additionally needed is a set of equations to tie all the expected rates of return together: ${ }^{21}$

$$
N R O R_{j, r}^{\text {future }}=\Omega \quad \forall j \in E N D I N D
$$

\footnotetext{
${ }^{21}$ In terms of the system of equations that constitute the model, these equations replace those that are used to set $X_{j, r / \text { tot }}^{I, \mathbf{b} \$}$ exogenously.
} 
where $\Omega$ is the economy-wide average expected net rate of return on capital. The equation holds for those industries included by the user in the set ENDIND which is a subset of all industries IND. Thus the user can exclude some industries from endogenous allocation, in which case their investment level will be as required to meet the exogenously specified real investment share. For those industries for which endogenous allocation is allowed, investment levels can only adjust in a way consistent with (2.32) and (2.33). It can be shown analytically that this means: ${ }^{22}$

$$
\frac{X_{j, r / \text { tot }}^{I, \mathbf{b}}}{100}=\frac{1}{I^{\mathbf{b} \$}}\left(\left(\frac{N R O R_{j, r}}{\Omega}\right)^{\frac{1}{\beta_{j, r}}}-\left(1-\frac{\delta_{j, r}}{100}\right)\right) K_{j, r}^{(S)} \quad \forall j \in \text { ENDIND }
$$

The higher the current net rate of return for an industry agent's capital, the more investment will be allocated to it. Industries and regions that have relatively more capital or higher depreciation rates will also be allocated proportionately more gross investment (for given rates of return). ${ }^{23}$

\subsubsection{Exporters}

The exporter agents act as simple conduits between the domestic and foreign sectors. They purchase domestic products and transform them into exports. The main purpose of identifying these agents at this stage is to provide for the possibility of export subsidies, which would place a wedge between the domestic (basic) prices and foreign (purchase) prices. Their inclusion also makes it possible to introduce model enhancements that for example relax the assumption of costless transformation of domestically produced goods into exports.

There is one exporter agent $\exp _{g}$ for each product $g$ and the costless transformation assumption is embodied in the following set of demand functions:

$$
Q_{g}^{\exp (D)}=E X P_{g}^{(S)}
$$

\footnotetext{
${ }^{22}$ See the derivation of this expression in appendix $\mathrm{H}$.

${ }^{23}$ The implications for the level of investment demand $I_{j, r}^{\text {ind }(D)}$ depends on the closure assumption with respect to $\Omega$. If real aggregate investment is fixed exogenous as usual, then $\Omega$ endogenously adjusts to reflect any shock to real investment. On the other hand $\Omega$ could be fixed exogenously instead, but this has implications for the operation of Walras' Law since real investment would then be endogenous. This issue is addressed later in section 2.4.5.
} 
with variable descriptions:

$Q_{g}^{\exp (D)} \quad$ composite product $g$ demanded by $\exp _{g}$

$E X P_{g}^{(S)} \quad$ supply of exports of product $g$ by $\exp _{g}$

The interpretation of (2.34) is that the exporter will demand exactly the same volume of composite products from the domestic economy as it is going to supply to the foreign sector. Inclusion of (2.34) also establishes a purchase price $P_{g}^{Q, \exp }$ that can be compared to the basic price of exports $P_{g}^{E X P}$. Only with costless transformation will the two be identical. Note that neither of these prices will necessarily equal the f.o.b. export price of product $g$, which is the price paid by the foreign sector ( for ) for exports. For those products whose exporter receives a subsidy, the f.o.b. export price and the basic export price will differ. $^{24}$

Once the demand for composite product $g$ has been determined, all that remains is a decision of how much of product $g$ to purchase from each domestic region. This takes the same form as the domestic regions part of the source choice for household as detailed in 2.3.1. The first part of the source choice is excluded as the usual assumption is made that imported products are not immediately exported again without any value-added from the domestic economy. Each exporter agent $\exp _{g}$ then has a set of demand functions analogous to (2.12) for households:

$$
Q_{g, x}^{\exp (D)}=Q_{g, x}^{\exp }\left(Q_{g}^{\exp (D)},\left.P_{g, y}^{Q, \exp }\right|_{y} ; \sigma_{g}^{\exp }\right)
$$

The implication of this formation process for exports is that the foreign sector faces the same f.o.b. export price for a given product $g$ regardless of the port of export. In this basic version of the model, there are no delivery costs involved in moving products from their region of production to the port. This assumption will be relaxed in the next chapter but it is done in such a way that the above implication still follows.

\subsubsection{Importers}

Similar to the exporter agents, the importer agents have the simple task of transforming foreign products into imported products and selling them onwards to domestic users.

\footnotetext{
${ }^{24}$ The nominal exchange rate $\phi$, to be introduced shortly, also has a role here. We assume the exporter's basic price is in domestic currency but the price paid by the foreign sector is in foreign currency. Equation (2.45) converts the f.o.b. price into domestic currency for comparison with other domestic currency prices.
} 
Import duties and potentially other costs incurred in the process of importation and delivery to market can be passed on to users by modelling this transformation.

The transformation itself is assumed costless here, so the importer agent $\mathbf{i m p}_{g}$ for each product $g$ has the following demand for foreign product when it is supplying a given amount of imported products to the domestic economy:

$$
F O R_{g}^{\operatorname{imp}(D)}=I M P_{g}^{(S)}
$$

with variable descriptions:

$F O R_{g}^{\operatorname{imp}(D)}$ foreign product $g$ demanded by $\operatorname{imp}_{g}$

$I M P_{g}^{(S)} \quad$ supply of imported product $g$ by $\operatorname{imp}_{g}$

Any import duty is included in the domestic currency $(\mathbf{d} \$)$ purchase price paid by the importer for foreign products, $P_{g}^{F O R, \mathbf{d} \$ \text {,imp }}$, and is passed on to domestic users in the basic price of imported products $P_{g}^{I M P}$ that the importer receives. With costless transformation both of these prices are the landed duty-paid import price while the price the foreign agent receives (the basic price of foreign products) is the c.i.f. import price. ${ }^{25}$

For convenience we introduce alternative notation for the supply of imports and their basic price: ${ }^{26}$

$$
\begin{aligned}
Q_{g, \mathrm{imp}}^{(S)} & \equiv I M P_{g}^{(S)} \\
P_{g, \mathrm{imp}}^{Q} & \equiv P_{g}^{I M P}
\end{aligned}
$$

The basic model assumes that there is no cost to deliver an imported product from its port of entry to the region of usage. Users therefore face the same basic price of an imported product regardless of the port of entry. The next chapter introduces delivery costs but this implication continues to hold.

\subsubsection{Government}

The activities of the public sector that are incorporated into the model are government consumption of goods and services, subsidisation of production and exports, and taxa-

\footnotetext{
${ }^{25}$ Here again the nominal exchange rate has the role of converting the c.i.f. import price, which we measure in foreign currency, into domestic currency for comparison to other domestic currency prices. The currency conversion is done by equation (2.64).

${ }^{26}$ For an example of the use of the alternative notation for supply and basic price of imports, see $(2.46)$ and (2.59) respectively.
} 
tion of labour income and purchases of goods and services. These latter taxes consist of GST, import duties, and general product taxes. Provision is also made for the inclusion of region-specific lump-sum transfers, but transfers that are related to other model variables (such as unemployment) and corporate income tax are not modelled. Government policy with respect to all these items is usually assumed exogenous. That is, the level and composition of government expenditure is usually fixed (perhaps as a proportion of absorption or GDP) along with the various tax and subsidy rates.

The modelled components of government expenditure and revenue are discussed below, while the definition of fiscal balance will be specified later, in section 2.3.12.

\section{The Composition of Government Consumption}

The government agent gov decides on its composition of consumption in an analogous way to how household agents choose their consumption composition. The government agent takes the level of real government consumption expenditure as given and decides how much of each product to purchase and then from where to source those products see figure 2.4. The following demand functions summarise the consumption behaviour of the government:

$$
\begin{aligned}
& Q_{g}^{\text {gov }(D)}=Q_{g}^{\text {gov }}\left(G^{\mathbf{b} \$},\left.P_{h}^{Q, \text { gov }}\right|_{h} ; \sigma^{Q, \text { gov }}\right) \\
& Q_{g, \text { dom }}^{\text {gov }(D)}=Q_{g, \text { dom }}^{\text {gov }}\left(Q_{g}^{\text {gov }(D)}, P_{g, \text { dom }}^{Q, \text { gov }}, P_{g, \text { imp }}^{Q, \text { gov }} ; \sigma_{g}^{\text {gov }}\right) \\
& Q_{g, \text { imp }}^{\text {gov }(D)}=Q_{g, \text { imp }}^{\text {gov }}\left(Q_{g}^{\text {gov }(D)}, P_{g, \text { dom }}^{Q, \text { gov }}, P_{g, \text { imp }}^{Q, \text { gov }} ; \sigma_{g}^{\text {gov }}\right) \\
& Q_{g, x}^{\text {gov }(D)}=Q_{g, x}^{\text {gov }}\left(Q_{g, \text { dom }}^{\text {gov }(D)},\left.P_{g, y}^{Q, \text { gov }}\right|_{y} ; \sigma_{g, \text { dom }}^{\text {gov }}\right)
\end{aligned}
$$

where $G^{\mathbf{b} \$}$ is real aggregate government consumption expenditure and the remainder of the variables have meanings analogous to those of equations (2.9) - (2.12).

Exogenously specifying the level of $G^{\mathbf{b} \$}$ or its share in absorption or GDP is the standard way of dealing with government expenditure in CGE models. It is usual not to allow any substitution at least at the top level of the decision nest to reflect the idea that the government is very unresponsive to price changes when considering its composition of expenditure. It also seems reasonable that the government will be less willing than firms to substitute between sources of products. 


\section{Tax on Labour Income}

The government is assumed to receive a fixed proportion of gross wages as income tax. This average labour income tax rate $t^{L}$ places a wedge between the wage rate paid by firms $P_{r}^{L, \text { btax }}$ and the wage rate received by households $P_{r}^{L, \text { atax. }}$

$$
P_{r}^{L, \text { atax }}=P_{r}^{L, \text { btax }}\left(1-t^{L}\right)
$$

For a given labour income tax rate, the nominal revenue from income tax is then:

$$
\operatorname{INCTAX} X^{\mathbf{c} \$}=t^{L} \cdot \sum_{j} \sum_{r} P_{r}^{L, \mathbf{b t a x}} \cdot L_{j, r}^{\mathrm{ind}(D)}
$$

As mentioned above when discussing the measurement of real aggregate investment in section 2.3.4, real aggregates are calculated using constant prices. Real income tax revenue is therefore defined by:

$$
\operatorname{INCTAX} X^{\mathbf{b} \$}=t^{L} \cdot \sum_{j} \sum_{r}\left[P_{r}^{L, \mathbf{b t a x}}\right] \cdot L_{j, r}^{\operatorname{ind}(D)}
$$

\section{Import Duties and Export Subsidies}

The government can receive duty on any product imported and pay subsidies for exports. Duty is paid by importer agents and is passed on to users through the price of imported products. That is, the basic price of imported products includes duty while the c.i.f. import price does not. Similarly, export subsidies are received by exporter agents and place a wedge between their basic price (the price they receive, including the subsidy) and the f.o.b. export price paid by the foreign sector. By virtue of the duty being paid by importers, all users indirectly pay the same ad valorem duty rate for a given product. Similarly, products from each domestic region indirectly receive the same ad valorem export subsidy rate. ${ }^{27}$ The equations that show the relationship between these rates and the relevant purchase and basic prices are listed in section 2.3.10 and the defining equations that measure duty revenue and export subsidies are given in section 2.3.12.

\footnotetext{
${ }^{27}$ In common with ORANI, the duty and export subsidy rates can be interpreted broadly as the tariff-equivalents of import protection and export assistance respectively but for simplicity the additional options in ORANI of specifying these in real or specific-tax form are not modelled here. See Dixon et al. (1982, eq. 18.9 and eq. 18.13) for how these may be introduced.
} 


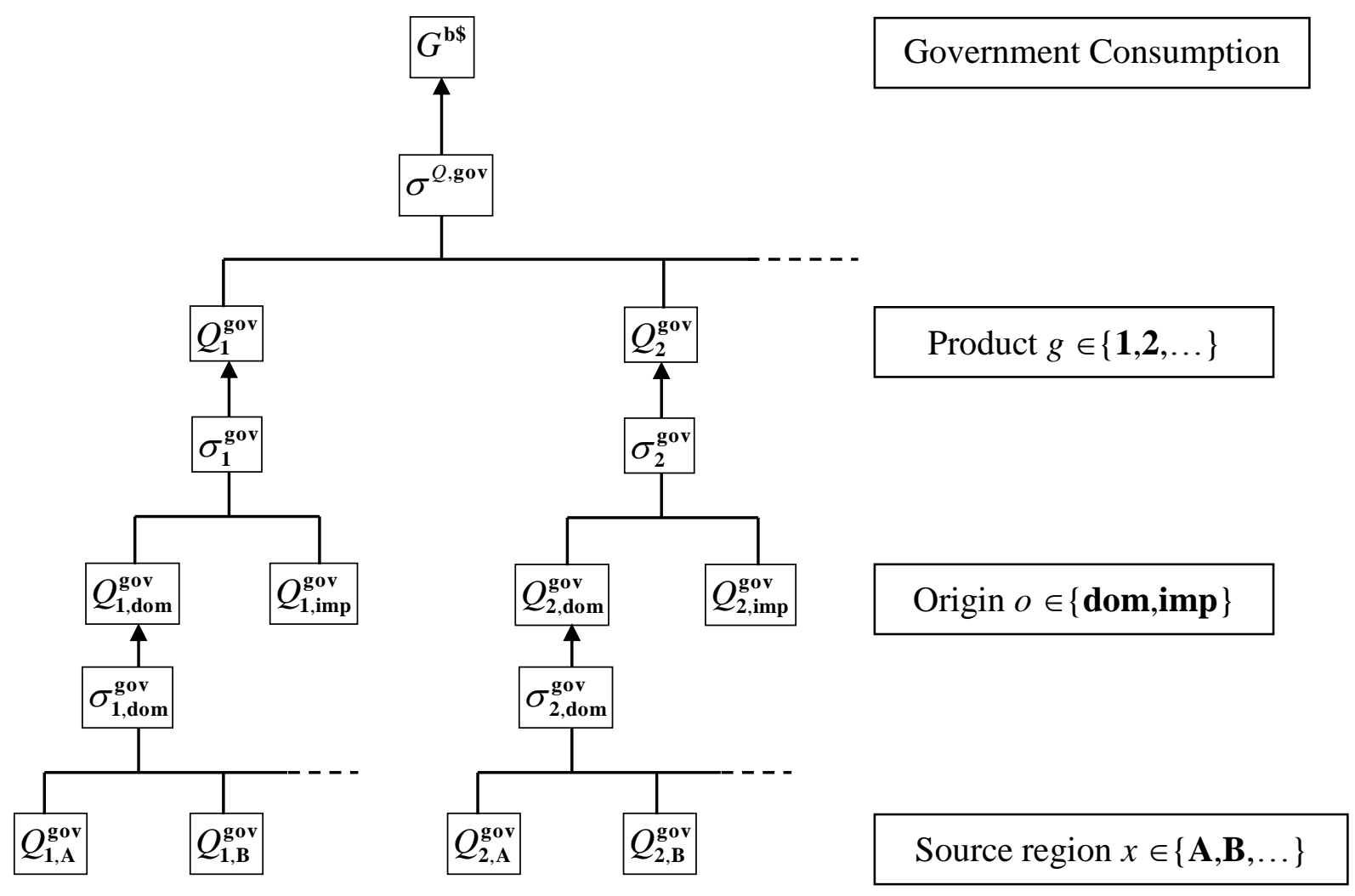

Figure 2.4: Government agent's decision nest for consumption

\section{Commodity Taxes}

The government can in general collect commodity tax on any product from any source purchased by any domestic user. These taxes are modelled using ad valorem commodity tax rates that are not source-specific but are given a regional dimension for those users that are located in regions. Thus for example households in different regions may face different tax rates for the same product from the same source, but an industry agent pays the same tax rate on material inputs of the same type from different sources. ${ }^{28}$ The model includes provision for the government to pay commodity tax, to reflect the non-zero flow

\footnotetext{
${ }^{28}$ Although if one of the sources was imports, then duty could also be payable, and the commodity tax paid is calculated using the duty-paid price.
} 
of tax recorded in the input-output data. However, the user has the option of forcing all the tax rates the government faces to zero if desired by ignoring that tax flow. Exporters do not have a regional dimension and only demand their own product so only face one commodity tax rate each. ${ }^{29}$

Naturally the commodity tax rates are exogenous to the model and have values assigned during the closure phase of model implementation. As their direct effect is on purchase prices, their implications are discussed in section 2.3.10 and the measures of government revenue from commodity tax are listed in section 2.3.12.

\section{Goods and Services Tax}

The main commodity tax households pay is GST, so this is given separate treatment. Unlike the general commodity tax rates, the rate of GST cannot vary across the regions but can potentially vary across products. Provision is also made for GST to be payable on exports. While most commodity exports are exempt, services such as those typically purchased by tourists accrue GST revenue. The rate of GST adds to the wedge between basic and purchaser prices for households and exporters as discussed in section 2.3.10. The equations for measuring the government's GST revenue are set out in section 2.3.12.

\section{Lump-Sum Transfers}

Autonomous levels of transfer (tax if negative) between the government and regional household agents can be specified by the addition of the variable $L S T_{r}$ to the right-hand side of equations (2.3) and (2.5) and the appropriate adjustment made to the government fiscal balance equation listed in section 2.3.12. ${ }^{30}$ The inclusion of these variables would aid simulations of fiscal policy and welfare. For example, the regional employment effects of certain lump-sum transfers could be investigated, or the lump-sum transfers required for there to be no change in regional welfare (as measured by compensating or equivalent variation) could be calculated, when the economy is subject to a given shock.

\footnotetext{
${ }^{29} \mathrm{As}$ for import duties and export subsidies, the commodity tax rates can be thought of as taxequivalents of other policies that discourage or (if negative) encourage usage.

${ }^{30}$ The value of $L S T_{r}$ would have to be deflated by some price index for calculations of real measures. The lump-sum transfer is included in the set of equations listed in appendix D.
} 


\subsubsection{Foreign Sector}

The foreign sector agent for supplies each product perfectly elastically at the foreign currency world price $P_{g}^{F O R, \mathbf{f} \$}$ - the c.i.f. price. The supply is therefore determined by the demand of the importer in equilibrium - see equation (2.51).

The foreign agent also demands exports of each product given foreign currency export prices $P_{g}^{E X P, \mathbf{f} \$ \text {,for }}$ according to the (inverse) export demand curve:

$$
P_{g}^{E X P, \mathbf{f} \$ \text {,for }}=\left(E X P_{g}^{\text {for }(D)}\right)^{-1 / \varepsilon_{g}^{E X P}} \cdot \psi_{g}^{E X P}
$$

with variable descriptions:

$$
\begin{array}{ll}
E X P_{g}^{\text {for }(D)} & \text { demand for exports of product } g \text { by for } \\
\varepsilon_{g}^{E X P} & \text { (absolute value of the) elasticity of export demand for product } \\
\psi_{g}^{E X P} & \text { co-efficient reflecting the height of the export demand curve } \\
& \text { for product } g
\end{array}
$$

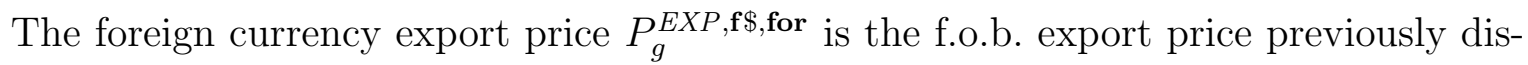
cussed in relation to the basic price of exports, the latter of which includes any export subsidy received by the exporter. The f.o.b. price can be expressed in domestic rather than foreign currency terms by using the equation:

$$
P_{g}^{E X P, \mathbf{d} \$, \text { for }}=\phi \cdot P_{g}^{E X P, \mathbf{f} \$, \text { for }}
$$

where $\phi$ is the nominal exchange rate (domestic $\$$ per foreign $\$$ ). The elasticities of export demands $\varepsilon_{g}^{E X P}$ and the demand curve height co-efficients $\psi_{g}^{E X P}$ need to be exogenously specified during the model closure process.

The option is available to the user to exclude the export demand curve for any product for which the downward-sloping relationship is considered inappropriate from the model. In those cases, the quantity variable is set exogenous and the corresponding height coefficient $\psi_{g}^{E X P}$ is endogenous but of no consequence to the rest of the model.

While foreign sector demand and supply have been discussed here, the definition of the trade balance is left until section 2.3.12. 


\subsubsection{Conditions for Competitive General Equilibrium}

Having set out the key relationships that govern the behaviour of the agents, we now turn to the constraints imposed on them by the assumption of competitive equilibrium. The constraints fall into one of two groups of conditions:

1. Market clearing conditions - there is no excess demand (or supply) in any market.

2. Zero pure profit conditions - there is no excess of revenue over costs in any productive activity.

\section{Market Clearing}

Market clearing is assumed in all markets. Total demand for each product (differentiated by source $s$ ) must equal its supply, and total demand of each factor (differentiated by region $r$ ) must equal its supply. These assumptions are embodied in the following equations:

$$
\begin{aligned}
\breve{Q}_{g, s}^{(S)} & =\sum_{r} \breve{Q}_{g, s, r}^{\mathbf{h s h}(D)}+\sum_{p} \sum_{j} \sum_{r} \breve{Q}_{g, s, j, r}^{\operatorname{ind}(p)(D)}+\breve{Q}_{g, s}^{\operatorname{gov}(D)}+\breve{Q}_{g, s}^{\exp (D)} \\
K_{j, r}^{(S)} & =K_{j, r}^{\operatorname{ind}(D)} \\
L_{r}^{(S)} & =\sum_{j} L_{j, r}^{\operatorname{ind}(D)}
\end{aligned}
$$

Note that equation (2.46) is indexed over the source set $(s \in S R C)$ so it covers market clearing of imported products along with those domestically produced. ${ }^{31}$ The above three sets of market clearing conditions therefore effectively establish basic prices for each product type from each source $\left(P_{g, s}^{Q}\right)$, each type of currently installed capital $\left(P_{j, r}^{K}\right)$, and regional after-tax wage rates $\left(P_{r}^{L, \text { btax }}\right)$. Equation $(2.46)$ also introduces the notation $\breve{Q}$ to signify quantities of product before they leave their point of production 'undelivered products' . The difference between $\breve{Q}$ and $Q$ ('delivered products') is made explicit in section 2.3.10, although the difference is only minor in this basic version of the model. Notice also that there is no provision for idle capacity or unemployment in (2.47) and (2.48) for simplicity. The assumption of no (involuntary) unemployment is relaxed in chapter 4 .

\footnotetext{
${ }^{31}$ This is facilitated by the equivalence of the two forms of notation for supply of imports, $Q_{g, \text { imp }}^{(S)}$ and $I M P_{g}^{(S)}$ noted in section 2.3.6.
} 
The following market clearing conditions are also required for equilibrium due to the separate specification of investment, exporting, and importing activities as discussed in sections 2.3.4, 2.3.5, and 2.3.6:

$$
\begin{aligned}
& K_{j, r}^{\text {new }(S)}=I_{j, r}^{\operatorname{ind}(D)} \\
& {E X P_{g}^{(S)}}^{\text {nafor }(D)} \\
& F O R_{g}^{(S)}=F O R_{g}^{\operatorname{imp}(D)}
\end{aligned}
$$

These equations allow the model to determine the basic prices of new capital (the construction $\operatorname{cost} P_{j, r}^{K, \text { new }}$ ), exports (the subsidy-inclusive domestic currency price $P_{g}^{E X P}$ ), and foreign products (the c.i.f. foreign currency price $\left.P_{g}^{F O R}\right) .{ }^{32}$ The role of the importer and exporter agents as conduits between the domestic economy and foreign sector can now be seen by looking at equations (2.51) and (2.36) for importing, and equations (2.50) and (2.34) for exporting.

\section{Zero Pure Profits in Equilibrium}

Along with market clearing, competitive general equilibrium requires zero pure profits in all production activities. These include the production of products for current usage, the production of new capital, and the transformation processes that convert domestic products into exports and foreign products into imports. Assumptions of zero pure profits are specified by the following equations:

$$
\begin{aligned}
P_{g, r}^{Q} \cdot \breve{Q}_{g, r}^{(S)} & =P_{j, r}^{Q, \text { ind }} \cdot Q_{j, r}^{\text {ind }(D)}+P_{j, r}^{F, \text { ind }} \cdot F_{j, r}^{\text {ind }(D)}
\end{aligned} \quad \forall g=j
$$

Equations (2.54) and (2.36) imply that the basic price of an imported product is equal to the price of the foreign product paid by the importer, which includes any duty payable. Therefore both changes in world prices and changes in duty rates are transmitted to the

\footnotetext{
${ }^{32}$ Although, the last of these is usually set exogenous, in which case equation (2.51) determines the supply of foreign products instead. By fixing the world price, the implicit assumption is made that the foreign sector will meet any domestic demand at that price.
} 
domestic economy through this channel. Similarly, equations (2.55) and (2.34) imply that the basic price of exports is equal to the price paid by the exporter for the domestic product, so domestic price changes are passed on to the foreign sector through this channel.

\subsubsection{Purchase Price Definitions}

In a sense, the definitions of purchase prices are zero profit conditions. They simply state that the expenditure by a given agent at one level in their decision nest equals the sum of the expenditures at the level below. There is no value gained or lost by forming composite products or factors. This implies that the purchase price at one level is a weighted sum of the purchase prices of the level below, where the weights are expenditure shares. For example, for each household agent $\mathbf{h} \mathbf{h}_{r}$ there is an equation:

$$
P_{r}^{Q, \mathbf{h s h}} \cdot Q_{r}^{\mathbf{h s h}(D)}=\sum_{g} P_{g, r}^{Q, \mathbf{h s h}} \cdot Q_{g, r}^{\mathbf{h s h}(D)}
$$

This defines the purchase price of total composite product $P_{r}^{Q, \text { hsh }}$ as a weighted sum of the purchase prices of each product $g, P_{g, r}^{Q, \mathbf{h s h}}$, where the sum weights are the proportions of household expenditure that goes to each product.

The purchase prices $P_{g, r}^{Q, \text { hsh }}$ are in turn defined by the following set of equations:

$$
P_{g, r}^{Q, \mathbf{h s h}} \cdot Q_{g, r}^{\mathbf{h s h}(D)}=\sum_{o} P_{g, o, r}^{Q, \mathbf{h s h}} \cdot Q_{g, o, r}^{\mathbf{h s h}(D)}
$$

while the purchase price of each domestic composite product $P_{g, \mathbf{d o m}, r}^{Q, \mathbf{h s h}}$ is defined by:

$$
P_{g, \mathbf{d o m}, r}^{Q, \mathbf{h s h}} \cdot Q_{g, \mathbf{d o m}, r}^{\mathbf{h s h}(D)}=\sum_{x} P_{g, x, r}^{Q, \mathbf{h s h}} \cdot Q_{g, x, r}^{\mathbf{h s h}(D)}
$$

What remains to be determined are the purchase prices $P_{g, x, r}^{Q, \mathbf{h s h}}$ and $P_{g, \mathbf{i m p}, r}^{Q, \mathbf{h s h}}$. It is useful here to refer to these together as the purchase price of product $g$ from source $s$ with $s \in S R C$ with $S R C=R E G \cup\{\mathbf{i m p}\}$ - i.e. $P_{g, s, r}^{Q, \mathbf{h s h}}$. By assuming there are no costs involved in delivering each product from each source to the household agent in each region, the only thing that puts a wedge between the price paid by the household and the price received by the producer are commodity taxes (including GST). It would seem then that we could define the purchase prices $P_{g, s, r}^{Q, \text { hsh }}$ as follows:

$$
P_{g, s, r}^{Q, \mathbf{h s h}}=P_{g, s}^{Q} \cdot\left(1+t_{g, r}^{Q, \mathbf{h s h}}+t_{g}^{G S T, \mathbf{h s h}}\right) \quad \forall s \in S R C
$$


with variable descriptions:

$t_{g, r}^{Q, \mathbf{h s h}} \quad$ commodity tax rate faced by $\mathbf{h s h} \mathbf{h}_{r}$ on purchases of product $g$ from any source

$t_{g}^{G S T, \text { hsh }}$ GST rate faced by all household agents on purchases of product $g$ from any source

For computational reasons, rather than use equations such as the above to describe the relationship between purchase prices and basic prices, equations analogous to (2.56), (2.57), and (2.58) are specified: ${ }^{33}$

$$
P_{g, s, r}^{Q, \mathbf{h s h}} \cdot Q_{g, s, r}^{\mathbf{h s h}(D)}=P_{g, s}^{Q} \cdot\left(1+t_{g, r}^{Q, \mathbf{h s h}}+t_{g}^{G S T, \mathbf{h s h}}\right) \cdot \breve{Q}_{g, s, r}^{\mathbf{h s h}(D)}
$$

where $\breve{Q}_{g, s, r}^{\mathbf{h s h}(D)}$ can be thought of as a quantity of 'undelivered' products while $Q_{g, s, r}^{\mathbf{h s h}(D)}$ is the 'delivered' version. One can think of an undelivered product being the assembled item sitting at the factory door while a delivered product is the same item in the hands of the user. It makes no difference to simulation results (i.e. percentage changes) if the benchmark levels of $Q_{g, s, r}^{\mathbf{h s h}(D)}$ and $\breve{Q}_{g, s, r}^{\mathbf{h s h}(D)}$ are equal and benchmark cost wedges are reflected in the level differences between purchase and basic prices, or the benchmark levels of $P_{g, s, r}^{Q, \text { hsh }}$ and $P_{g, s}^{Q}$ are equal and cost wedges are reflected in the level differences between delivered and undelivered quantities. As will be outlined in section 2.4.3, the latter option was taken. In simulations, the quantity variables still move in unison and cost-wedge changes open gaps between purchase and basic prices. In chapter 3, where delivery costs are not necessarily zero, the distinction between delivered and undelivered products becomes more meaningful.

Most purchase price definitions for the other agents follow a similar pattern to that above with adjustments as necessary for the different decision nest structure. Only those that do not follow immediately from the above are made explicit below. The full list of purchase price equations can be found in appendix D.

The definition of the composite factor purchase price reflects the assumption that industries only use factors from their own region:

$$
P_{j, r}^{F, \text { ind }} \cdot F_{j, r}^{\text {ind }(D)}=P_{r}^{L, \text { btax }} \cdot L_{j, r}^{\text {ind }(D)}+P_{j, r}^{K} \cdot K_{j, r}^{\text {ind }(D)}
$$

\footnotetext{
${ }^{33}$ The equations can be written in this compact form by exploiting the equivalence of the two forms of notation for basic prices of imports, $P_{g, \text { imp }}^{Q}$ and $P_{g}^{I M P}$ noted in section 2.3.6.
} 
Since exporter agent $\exp _{g}$ only demands product $g$, and it sources this product only from the domestic sources (i.e. the regions), its purchase price equations are:

$$
\begin{aligned}
& P_{g}^{Q, \exp } \cdot Q_{g}^{\exp (D)}=\sum_{x} P_{g, x}^{Q, \exp } \cdot Q_{g, x}^{\exp (D)} \\
& P_{g, x}^{Q, \exp } \cdot Q_{g, x}^{\exp (D)}=P_{g, x}^{Q} \cdot\left(1+t_{g}^{Q, \exp }+t_{g}^{G S T, \exp }\right) \cdot \breve{Q}_{g, x}^{\exp (D)}
\end{aligned}
$$

The inclusion of GST in equation (2.62) implies that the exporter pays GST on behalf of, and passes the cost on to the foreign agent. The superscript exp is added to $t^{G S T}$ to indicate that exports of product $g$ can be subject to a different GST rate from the domestic version. $t_{g}^{G S T, \exp }$ is essentially an effective rate: if the user wished to specify that only half the exports of $g$ are subject to GST, $t_{g}^{G S T, \exp }$ can be set to half the value

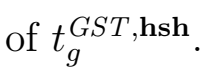

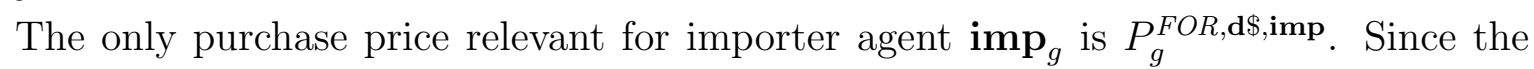
basic price of foreign products $P_{g}^{F O R}$ is a foreign currency price, we define the foreign currency purchase price first and then convert it into domestic currency using the nominal exchange rate $\phi$ :

$$
\begin{aligned}
P_{g}^{F O R, \mathbf{f} \$ \mathbf{i m p}}=\left(1+t_{g}^{F O R}\right) \cdot P_{g}^{F O R} \\
P_{g}^{F O R, \mathbf{d} \$ \mathbf{i m p}}=\phi \cdot P_{g}^{F O R, \mathbf{f} \$ \mathbf{i m p}}
\end{aligned}
$$

where $t_{g}^{F O R}$ is the duty rate on imports of product $g$. The implication of (2.63) is that the importer pays duty on behalf of domestic users and passes the cost on to them through its import price (as distinct from the foreign product price).

Finally, the foreign agent for is concerned with the set of foreign currency f.o.b. export prices $P_{g}^{E X P, \mathbf{f} \$ \text {,for }}$, as discussed in section 2.3.8. These are usually exogenously set or determined by the export demand curve (2.44) when the respective export volume is fixed. The relationship between the f.o.b. export price and the basic price of exports is:

$$
P_{g}^{E X P}=\left(1-t_{g}^{E X P}\right) \cdot P_{g}^{E X P, \mathbf{d} \$, \text { for }}
$$

where $t_{g}^{E X P}$ is the rate of export tax on product $g$ (negative for an export subsidy) and $P_{g}^{E X P, \mathbf{d} \$ \text {,for }}$ is converted to foreign currency by equation (2.45).

\subsubsection{Numeraire}

The model focuses on the real economy and as such contains no monetary theory of price determination. The level of each price variable in the model must then be interpreted as 
that price relative to some undetermined price level - the numeraire. The usual approach is to exogenously specify one of the price variables, so that all other prices are relative to it. The nominal exchange rate $\phi$ is commonly used for this purpose, although other useful candidates include the economy-wide average wage, the consumer price index, or the GDP deflator. The standard implementation of the prototype model has the nominal exchange rate specified as the numeraire by the equation:

$$
\phi=1
$$

\subsubsection{Defining Equations for Macro Aggregates, Indices, etc.}

These equations define various aggregate measures and indices that facilitate different closure assumptions and provide useful summary measures. A number of nominal and real measures have already been introduced in previous sections. Since the model is solved in levels form, we have levels of base-period (benchmark equilibrium) and current-period (new equilibrium) prices. Nominal measures value quantities at current prices while real measures use base-period prices. The current prices are model variables while the baseperiod prices are constants. To distinguish these in equations, base-period prices are enclosed in square brackets as we have already seen a number of times above.

For brevity only the key building blocks of the aggregate measures are listed here. For example, many variables that are New Zealand totals of regional measures are not separately defined here. In other cases where definitions are obvious, equations explicitly stating the definitions are omitted. All variable definitions can however be found in appendix D. 


\section{Household Endowment Income, Expenditure, Income, and Saving}

Nominal measures of household endowment income, expenditure, income, and saving were introduced in section 2.3.1. The real counterparts to those equations are:

$$
\begin{aligned}
E N D I N C_{r}^{\mathbf{a t a x}, \mathbf{b} \$} & =\left[P_{r}^{L, \mathbf{a t a x}}\right] \cdot T I M E_{r}+\sum_{j}\left[P_{j, r}^{K}\right] \cdot K_{j, r}^{(S)} \\
I N C_{r}^{\mathbf{a t a x}, \mathbf{b} \$} & =\left[P_{r}^{L, \mathbf{a t a x}}\right] \cdot L_{r}^{(S)}+\sum_{j}\left[P_{j, r}^{K}\right] \cdot K_{j, r}^{(S)} \\
S P N_{r}^{\mathbf{b} \$} & =\left[P_{r}^{Q, \mathbf{h s h}}\right] \cdot Q_{r}^{\mathbf{h s h}(D)} \\
S A V_{r}^{\mathbf{a t a x}, \mathbf{b} \$} & =I N C_{r}^{\mathbf{a t a x}, \mathbf{b} \$}-S P N_{r}^{\mathbf{b} \$}
\end{aligned}
$$

The last of these equations can be listed more generally using $v \in V A L$ :

$$
S A V_{r}^{\operatorname{atax}, v}=I N C_{r}^{\operatorname{atax}, v}-S P N_{r}^{v}
$$

For $v=\mathbf{c} \$$ and $v=\mathbf{b} \$$, the above defines nominal and real measures as discussed. The other element of $v, \mathbf{b Q}$ denotes current price, constant quantity measures such as:

$$
I N C_{r}^{\text {atax, } \mathbf{b Q}}=P_{r}^{L, \text { atax }} \cdot\left[L_{r}^{(S)}\right]+\sum_{j} P_{j, r}^{K} \cdot\left[K_{j, r}^{(S)}\right]
$$

Such measures are used for Laspeyres index calculations, which are discussed later. For variables where the $\mathbf{b Q}$ components are not used elsewhere (such as for the above), those components can be ignored.

National aggregates of the above measures are calculated as summations of the regional variables — their defining equations are listed in appendix D.

\section{Domestic Expenditure on GDP}

These are the measures of aggregate household consumption, investment, and government consumption. Real investment was defined equation (2.30). An equation defining real government consumption expenditure cannot be included in the model because this would cause the model to be over-identified, due to its inclusion in the demand functions of 
equation $(2.37) \cdot{ }^{34}$

$$
\begin{aligned}
C^{v} & =\sum_{r} S P N_{r}^{v} \\
I^{\mathbf{c} \$} & =\sum_{j} \sum_{r} P_{j, r}^{K, \text { new }} \cdot I_{j, r}^{\text {ind }(D)} \\
I^{\mathbf{b Q}} & =\sum_{j} \sum_{r} P_{j, r}^{K, \text { new }} \cdot\left[I_{j, r}^{\text {ind }(D)}\right] \\
G^{\mathbf{c} \$} & =\sum_{g} P_{g}^{Q, \text { gov }} \cdot Q_{g}^{\operatorname{gov}(D)} \\
G^{\mathbf{b Q}} & =\sum_{g} P_{g}^{Q, \text { gov }} \cdot\left[Q_{g}^{\operatorname{gov}(D)}\right]
\end{aligned}
$$

The current price, constant quantity measures of these items will be useful later for constructing expenditure price indices.

\section{Trade Flows and the Trade Balance}

Imports and exports are valued in a number of ways - in domestic and foreign currency, in nominal and real terms, and in basic and c.i.f. or f.o.b. prices.

$$
\begin{aligned}
E X P^{\mathbf{c} \$, \mathbf{d} \$, \mathbf{b a s}} & =\sum_{g} P_{g}^{E X P} \cdot E X P_{g}^{(S)} \\
E X P^{\mathbf{c} \$, \mathbf{f} \$, \mathbf{b a s}} & =\frac{1}{\phi} \cdot E X P^{\mathbf{c} \$, \mathbf{d} \$, \text { bas }} \\
E X P^{\mathbf{c} \$, c, \text { fob }} & =\sum_{g} P_{g}^{E X P, c, \text { for }} \cdot E X P_{g}^{\mathbf{f o r}(D)} \\
I M P^{\mathbf{c} \$, c, \text { bas }} & =\sum_{g} P_{g}^{F O R, c, \mathbf{i m p}} \cdot F O R_{g}^{\mathbf{i m p}(D)} \\
I M P^{\mathbf{c} \$, \mathbf{f} \$, \mathbf{c i f}} & =\sum_{g} P_{g}^{F O R} \cdot F O R_{g}^{(S)} \\
I M P^{\mathbf{c} \$, \mathbf{d} \$, \mathbf{c i f}} & =\phi \cdot I M P^{\mathbf{c} \$, \mathbf{f} \$, \mathbf{c i f}}
\end{aligned}
$$

\footnotetext{
${ }^{34}$ Real government consumption is determined by the CES mixing function constraint in the expenditure minimisation problem at the top of the government agent's decision nest. The constraint becomes one of the first-order conditions of the solution. This is also why nominal household expenditure is defined in terms of the average propensity to consume rather than the product of the purchase price and quantity of total composite product, analogous to the definition of real household expenditure listed above - the expenditure constraint becomes a first-order condition to the solution of the utility maximisation problem.
} 
The corresponding real measures are defined in the usual way for $v=\mathbf{b} \$$. The balance of trade can therefore be measured in nominal and real terms as follows:

$$
T R D B A L^{v, c}=E X P^{v, c, \text { fob }}-I M P^{\mathbf{c} \$, c, \mathbf{c i f}}
$$

\section{Government Revenue and the Fiscal Balance}

Measures of nominal and real income tax revenue were given in section 2.3.7. The other components of government revenue and the fiscal balance are defined below:

$$
\begin{aligned}
\operatorname{DUTY} Y^{\mathbf{c}}= & \sum_{g} t_{g}^{F O R} \cdot \phi \cdot P_{g}^{F O R} \cdot F O R_{g}^{\operatorname{imp}(D)} \\
E X P S U B^{\mathbf{c} \$}= & -\sum_{g} t_{g}^{E X P} \cdot P_{g}^{E X P, \mathbf{d} \$, \text { for }} \cdot E X P_{g}^{(S)} \\
C O M T A X^{\mathbf{c} \$}= & \sum_{g} \sum_{s} \sum_{p} \sum_{j} \sum_{r}\left(P_{g, s}^{Q} \cdot t_{g, r}^{Q, \mathbf{h s h}} \cdot \breve{Q}_{g, s, r}^{\mathbf{h s h}(D)}\right. \\
& \left.+P_{g, s}^{Q} \cdot t_{g, j, r}^{Q, \text { ind }(p)} \cdot \breve{Q}_{g, s, j, r}^{\operatorname{ind}(p)(D)}+P_{g, s}^{Q} \cdot t_{g}^{Q, \text { gov }} \cdot \breve{Q}_{g, s}^{\operatorname{gov}(D)}+P_{g, r}^{Q} \cdot t_{g}^{Q, \mathbf{e x p}} \cdot \breve{Q}_{g, r}^{\mathbf{e x p}(D)}\right) \\
G S T^{\mathbf{c} \$}= & \sum_{g} \sum_{s} \sum_{r}\left(P_{g, s}^{Q} \cdot t_{g}^{G S T, \mathbf{h s h}} \cdot \breve{Q}_{g, s, r}^{\mathbf{h s h}(D)}+P_{g, r}^{Q} \cdot t_{g}^{G S T, \exp } \cdot \breve{Q}_{g, r}^{\mathbf{e x p}(D)}\right)
\end{aligned}
$$$$
G O V R E V^{v}=I N C T A X^{v}+D U T Y^{v}+C O M T A X^{v}+G S T^{v}-E_{X P S U B}
$$$$
G O V B A L^{v}=G O V R E V^{v}-G^{v}
$$

\section{Labour Market Measures}

In this basic version of the model, the only component of the labour market that is modelled is employment. Full employment is assumed in equilibrium - unemployment rates are fixed at zero. Without measures of population, participation rates cannot be calculated. These items will be introduced in chapter 4 to give a richer description of the labour market. ${ }^{35}$ At this stage, we only define the employment measures:

\footnotetext{
${ }^{35}$ In chapter 4, non-participation in the labour market is directly linked to consumption of leisure such that participation rates may be calculated. Unemployment is then measured as the residual between the labour force and employment. To facilitate the calculation of unemployment rates, an unemployment variable will be added to the righthand side of (2.48).
} 


$$
\begin{aligned}
E M P_{j, r} & =\left[P_{r}^{L, \mathbf{b t a x}}\right] \cdot L_{j, r}^{\operatorname{ind}(D)} \\
E M P_{j, \mathbf{N Z}} & =\sum_{r} E M P_{j, r} \\
E M P_{r} & =\sum_{j} E M P_{j, r} \\
E M P_{\mathbf{N Z}} & =\sum_{r} E M P_{r}
\end{aligned}
$$

Sets of employment shares can be calculated from these measures as necessary. The definition of these are obvious from the notation. For example, $X_{j, r / \mathbf{N Z}}^{E M P}$ is industry $j$, region $r$ 's share of total employment, $X_{j, r / \mathbf{r}}^{E M P}$ is industry $j$ 's share of region $r$ 's employment, and $X_{r / \mathbf{N Z}}^{E M P}$ is region $r$ 's share of total employment.

\section{Factor Incomes and Gross Value Added}

A selection of wage and capital income measures are defined followed by measures of gross value added (GVA):

$$
\begin{aligned}
W A G E S_{j, r}^{w, \mathbf{c} \$} & =P_{r}^{L, w} \cdot L_{j, r}^{\operatorname{ind}(D)} & & \\
W A G E S_{r}^{w, v} & =\sum_{j} W A G E S_{j, r}^{w, v} & & \\
K R E N T S_{j, r}^{\mathbf{c}} & =P_{j, r}^{K} \cdot K_{j, r}^{\operatorname{ind}(D)} & & \\
K R E N T S_{r}^{v} & =\sum_{j} K R E N T S_{j, r}^{v} & & \\
G V A_{j, r}^{v} & =W A G E S_{j, r}^{\mathbf{b t a x}, v}+K R E N T S_{j, r}^{v} & & \forall r \in N Z R E G \\
G V A_{r}^{v} & =W A G E S_{r}^{\mathbf{b t a x}, v}+K R E N T S_{r}^{v} & & \forall r \in N Z R E G
\end{aligned}
$$

\section{Output, Investment, Capital Stocks, and Net Returns}

Aggregate investment measures have already been defined above. Industrial and regional output, investment, capital stocks, and net returns can be measured along similar lines 
to the factor incomes above.

$$
\begin{aligned}
I_{j, r}^{\mathbf{c} \$} & =P_{j, r}^{K, \text { new }} \cdot I_{j, r}^{\text {ind }(D)} \\
\operatorname{OUTPUT}_{j, r}^{\mathbf{c} \$} & =P_{g, r}^{Q} \cdot \breve{Q}_{g, r}^{(S)} \\
\operatorname{KSTOCK}_{r} & =\sum_{j} K_{j, r}^{(S)} \\
\operatorname{NRTRN}_{j, r} & =K R E N T S_{j, r}^{\mathbf{c} \$}-\frac{\delta_{j, r}}{100} \cdot P_{j, r}^{K, \text { new }} \cdot K_{j, r}^{(S)}
\end{aligned}
$$

Along with useful shares such as $X_{r / \mathbf{N Z}}^{O U T P}$, these measures also facilitate the calculation of average $\mathrm{K} / \mathrm{L}$ ratios such as $R_{r}^{K S T O C K / E M P}$ and average rates of return such as:

$$
N R O R_{r}=100 \cdot \frac{N R T R N_{r}}{\sum_{j} P_{j, r}^{K, \text { new }} \cdot K_{j, r}^{(S)}}
$$

\section{Measures of GDP and Domestic Saving}

Gross Domestic Product (GDP) is measured in nominal and real terms using the three common approaches of expenditures, incomes, and value added.

$$
\begin{aligned}
G D P E X P^{v} & =C^{v}+I^{v}+G^{v}+T R D B A L^{v, \mathbf{d} \$} \\
G D P I N C^{v} & =\sum_{r} I N C_{r}^{\operatorname{atax}, v}+G O V R E V^{v} \\
G D P V A^{v} & =G V A^{v}+G O V R E V^{v}-I N C T A X^{v}
\end{aligned}
$$

Using the above definitions, shares of GDP components such as $X_{C / \text { tot }}^{G D P}, X_{I / \text { tot }}^{G D P}$, etc. are defined though straightforward equations.

Aggregate domestic saving is defined as:

$$
S A V^{v}=\sum_{r} S A V_{r}^{\text {atax }, v}+G O V B A L^{v}
$$

\section{Price Indices}

For the purpose of measuring average price levels, this thesis uses the Fisher price index, defined for a given price deflator $\Xi$ by the equation:

$$
\Xi^{\mathbf{F}}=\sqrt{\Xi^{\mathbf{P}} \cdot \Xi^{\mathbf{L}}}
$$


where $\Xi^{\mathbf{F}}$ is the Fisher index and $\Xi^{\mathbf{P}}$ and $\Xi^{\mathbf{L}}$ are the arithmetic Paasche and Laspeyres indices respectively. ${ }^{36}$ A well-known problem with Paasche indices is that they fail to adequately account for substitution effects within the basket of goods over which they index prices, and therefore under-estimate average price changes. The Laspeyres index on the other hand over-estimates inflation so movements of the two indices can be seen as lower and upper bounds respectively of true average price changes (IMF 2004, page 9). The Fisher index seeks to offset the biases of each of these indices by taking their geometric average.

For example, regional consumption price deflator indices are specified as follows:

$$
C P I_{r}^{\mathbf{F}}=\sqrt{C P I_{r}^{\mathbf{P}} \cdot C P I_{r}^{\mathbf{L}}}
$$

where the Paasche and Laspeyres consumption price deflators are:

$$
\begin{aligned}
& C P I_{r}^{\mathbf{P}}=\sum_{g} \frac{P_{g, r}^{Q, \mathbf{h s h}}}{\left[P_{g, r}^{Q, \mathbf{h s h}}\right]} \cdot X_{g, r / r}^{\mathbf{P}} \\
& C P I_{r}^{\mathbf{L}}=\sum_{g} \frac{P_{g, r}^{Q, \mathbf{h s h}}}{\left[P_{g, r}^{Q, \mathbf{h s h}}\right]} \cdot X_{g, r / r}^{\mathbf{L}}
\end{aligned}
$$

with weights:

$$
\begin{aligned}
X_{g, r / r}^{\mathbf{P}} & =\frac{\left[P_{g, r}^{Q, \mathbf{h s h}}\right] \cdot Q_{g, r}^{\mathbf{h s h}(D)}}{\sum_{h}\left[P_{h, r}^{Q, \mathbf{h s h}}\right] \cdot Q_{h, r}^{\mathbf{h s h}(D)}} \\
X_{g, r / r}^{\mathbf{L}} & =\frac{\left[P_{g, r}^{Q, \mathbf{h s h}}\right] \cdot\left[Q_{g, r}^{\mathbf{h s h}(D)}\right]}{\sum_{h}\left[P_{h, r}^{Q, \mathbf{h s h}}\right] \cdot\left[Q_{h, r}^{\mathbf{h s h}(D)}\right]}
\end{aligned}
$$

It can be easily shown that the price deflator definitions above are equivalent to:

$$
\begin{aligned}
C P I_{r}^{\mathbf{P}} & =\frac{\sum_{g} P_{g, r}^{Q, \mathbf{h s h}} \cdot Q_{g, r}^{\mathbf{h s h}(D)}}{\sum_{g}\left[P_{g, r}^{Q, \mathbf{h s h}}\right] \cdot Q_{g, r}^{\mathbf{h s h}(D)}} \\
C P I_{r}^{\mathbf{L}} & =\frac{\sum_{g} P_{g, r}^{Q, \mathbf{h s h}} \cdot\left[Q_{g, r}^{\mathbf{h s h}(D)}\right]}{\sum_{g}\left[P_{g, r}^{Q, \mathbf{h s h}}\right] \cdot\left[Q_{g, r}^{\mathbf{h s h}(D)}\right]}
\end{aligned}
$$

\footnotetext{
${ }^{36}$ The Fisher index is an alternative to the geometric Laspeyres index, the latter being commonly used in linearised models — see IMF (2004, page 10) for details and FEDERAL's equation (2.73) for an example of their usage. The use of such weighted geometric mean price indices is problematic in a levels-form model because of non-linearity and issues such as how to construct trade price and GDP deflator indices arise. Hence the Fisher index is a more natural choice of price index in this kind of model, and behaves approximately the same as the geometric Laspeyres index in any case.
} 
We can therefore define the deflators more succinctly in terms of $S P N_{r}^{v}$ (with $v \in V A L$ ) as follows: ${ }^{37}$

$$
\begin{aligned}
C P I_{r}^{\mathbf{P}} & =\frac{S P N_{r}^{\mathbf{c} \$}}{S P N_{r}^{\mathbf{b} \$}} \\
C P I_{r}^{\mathbf{L}} & =\frac{S P N_{r}^{\mathbf{b Q}}}{\left[S P N_{r}^{\mathbf{c} \$}\right]}
\end{aligned}
$$

All price deflator indices are calculated in this way in the model. The GDP deflator $G D P D E F$ for example is calculated by the set of equations:

$$
\begin{aligned}
G D P D E F^{\mathbf{P}} & =\frac{G D P E X P^{\mathbf{c} \$}}{G D P E X P^{\mathbf{b}}} \\
G D P D E F^{\mathbf{L}} & =\frac{G D P E X P^{\mathbf{b Q}}}{\left[G D P E X P^{\mathbf{c}}\right]} \\
G D P D E F^{\mathbf{F}} & =\sqrt{G D P D E F^{\mathbf{P}} \cdot G D P D E F^{\mathbf{L}}}
\end{aligned}
$$

Appendix D lists these along with analogous equations for price deflator indices, for:

- Expenditure components of GDP - private consumption $(C P I)$, investment $(I P I)$, government consumption $(G P I)$, exports (XPI, both at basic and f.o.b. prices), imports (MPI, both at basic and c.i.f. prices), and the trade balance (TPI)

- Industrial and regional investment $\left(I P I_{j}\right.$ and $\left.I P I_{r}\right)$

- Industrial, regional, and national output $\left(O P I_{j}, O P I_{r}\right.$, and $O P I_{\mathbf{N Z}}$ - average basic output prices)

- Industrial, regional, and national nominal labour costs $\left(L C I_{j}, L C I_{r}\right.$, and $L C I_{\mathbf{N Z}}$ average nominal wage rates before and after tax)

\section{Real Prices}

The above price indices are used to calculate regional and economy-wide average real wage rates, and the real exchange rate:

$$
\begin{array}{rlrl}
W_{r}^{w} & =\frac{L C I_{r}^{\mathbf{F}, w}}{C P I_{r}^{\mathbf{F}}} & & \\
\varphi & =\phi \cdot \frac{M P I^{\mathbf{F}, \mathbf{c i f}}}{G D P D E F^{\mathbf{F}}} & N Z R E G
\end{array}
$$

\footnotetext{
${ }^{37}$ The denominator of the second equation here is $S P N_{r}^{\mathbf{c} \$}$ evaluated for benchmark equilibrium values, so what $v$ is does not matter: $\left[S P N_{r}^{\mathbf{c} \$}\right]=\left[S P N_{r}^{\mathbf{b} \$}\right]=\left[S P N_{r}^{\mathbf{b Q}}\right]$.
} 
In the terminology of Easton (1990, p. 5), $W_{r}^{w}$ is the real income wage as opposed to the real product wage, which would measure the real labour cost to a given industry. Such a measure could easily be specified by dividing the labour cost index by a variable that measures production cost — one candidate would be $P_{j, r}^{Q \text {,ind }}$.

The real exchange rate measures domestic trade competitiveness and as such is a proxy for (the reciprocal of) the terms of trade. The measure is appropriate insofar as the GDP deflator and c.i.f. import prices capture domestic and world prices (exclusive of any tariffs or export subsidies).

\section{Household Welfare}

Common measures of welfare changes are compensating variation (CV) and equivalent variation $(\mathrm{EV})$, as these are measurable bounds on changes in consumer surplus. The EV is the income change required at initial prices to give a consumer the equivalent utility change. The $\mathrm{CV}$ is the income change required at final prices to compensate the consumer for a utility change (i.e. to restore their initial level of utility). Since this model is solved in levels, these can easily be calculated using the evaluated levels of utility: ${ }^{38}$

$$
\begin{aligned}
& C V_{r}=\frac{U_{r}-\left[U_{r}\right]}{U_{r}} \cdot\left(E N D I N C_{r}^{\text {atax }, \mathbf{c} \$}-S A V_{r}^{\operatorname{atax}, \mathbf{c} \$}\right) \\
& E V_{r}=\frac{U_{r}-\left[U_{r}\right]}{\left[U_{r}\right]} \cdot\left(\left[E N D I N C_{r}^{\text {atax }, \mathbf{c} \$}\right]-\left[S A V_{r}^{\text {atax }, \mathbf{c} \$}\right]\right)
\end{aligned}
$$

\footnotetext{
${ }^{38}$ Given demand functions (in vector form) $\mathbf{Q}=\mathbf{f}(\mathbf{P}) \cdot E$, the indirect utility function can be written $v(\mathbf{P}, E)=F(\mathbf{P}) \cdot E$. Varian (1992) defines $\mathrm{CV}$ and $\mathrm{EV}$ using the expenditure function $E(\mathbf{P}, v)$ as:

$$
\begin{aligned}
& C V=E(\mathbf{P}, v)-E(\mathbf{P},[v]) \\
& E V=E([\mathbf{P}], v)-E([\mathbf{P}],[v])
\end{aligned}
$$
}

with notation adapted for consistency here — square brackets indicate benchmark values; variables without square brackets are the solution (final) values. Evaluated as such, we can write:

$$
\begin{aligned}
& C V=E-\frac{[v]}{F}=E-\frac{[v]}{v / E}=\frac{v-[v]}{v} \cdot E \\
& E V=\frac{v}{[F]}-[E]=\frac{v}{[v] /[E]}-[E]=\frac{v-[v]}{[v]} \cdot[E]
\end{aligned}
$$

Both the $\mathrm{CV}$ and EV are therefore measures of proportional utility change, and carry the same sign as that change. 


\subsection{Model Implementation}

The prototype JENNIFER model is implemented in GAMS and solved for the levels of all endogenous variables. A 25-industry, 5-region implementation is described in this section. The three key steps involved in the model implementation are calibration, closure, and shock. Each of these will be discussed in detail but first we express the model in compact form and consider its dimensions.

\subsubsection{Matrix Form Representation of the Whole Model}

The above sections 2.3.1 - 2.3.11 have presented and discussed the core equations of the model. The defining equations of section 2.3.12 are required for implementation but do not significantly add to the difficulty of the computational problem to be solved. It is essentially the number of industries and regions, and in turn the number of core equations that determine the model size and complexity.

If $J$ is the number of industries (and therefore products) and $R$ is the number of regions specified, then the number of equations in the core model are as shown in table 2.4. ${ }^{39} \mathrm{In}$ comparison, the defining equations summarised in section 2.3.12 only add around 5,000 equations to the model for this implementation. ${ }^{40}$

All the equations of sections 2.3.1 - 2.3.12 can be represented in matrix form as:

$$
\mathbf{F}(\mathbf{V})=0
$$

where $\mathbf{F}$ is a vector function of $e$ equations

$\mathbf{V}$ is a vector of $v$ variables

\footnotetext{
${ }^{39}$ The table shows the maximum number of core equations. If some industries do not produce in some regions, or some agents do not consume every product, or do not demand each product from every source, then the number of equations is reduced. Also, if the investment allocation is exogenously rather than endogenously determined as discussed in section 2.3.4, the set of $J \times R$ equations that equalise expected net rates of return (equation (2.33)) are replaced by non-core exogenising equations.

${ }^{40}$ In general, the number of non-core equations only increases linearly in $J$ and $R$ while the number of core equations grows exponentially so the latter set will come to dominate as $J$ and/or $R$ is increased towards double-digits.

${ }^{41}$ Equation (2.41) is counted as a purchase price equation and equations (2.42) and (2.43) are not part of the core model.

${ }^{42}$ Equation (2.45) is counted as a purchase price equation.
} 


\begin{tabular}{|c|c|c|}
\hline Equation block & General case & $J=25, R=5$ \\
\hline Households & $8 R+3 J R+J R^{2}$ & 1,040 \\
\hline Current production & $4 J R+3 J^{2} R+J^{2} R^{2}$ & 25,500 \\
\hline Capital formation & $3 J^{2} R+J^{2} R^{2}$ & 25,000 \\
\hline Investment allocation & $8 J R$ & 1,000 \\
\hline Exporters & $J+J R$ & 150 \\
\hline Importers & $J$ & 25 \\
\hline Government $^{41}$ & $3 J+J R$ & 200 \\
\hline Foreign sector ${ }^{42}$ & $J$ & 25 \\
\hline Market clearing & $R+3 J+3 J R$ & 455 \\
\hline Zero pure profits & $2 J+2 J R$ & 300 \\
\hline Purchase prices & $2 R+8 J+7 J R+J R^{2}+6 J^{2} R+2 J^{2} R^{2}$ & 51,710 \\
\hline Numeraire & 1 & 1 \\
\hline TOTAL & $\begin{array}{l}1+11 R+19 J+29 J R+2 J R^{2}+12 J^{2} R+ \\
4 J^{2} R^{2}\end{array}$ & 105,406 \\
\hline
\end{tabular}

Table 2.4: Equation Blocks

The number of model variables $V$ exceeds the number of equations $E$, so some of the variables need to be exogenously determined. ${ }^{43}$ In order to make the system square, we add $V-E$ equations to the system, with each new equation setting one variable equal to a particular value. For example if the capital endowment specific to industry $j$ in region $r, K_{j, r}^{(S)}$, is to be fixed exogenously at the level determined in the base-period data, $\left[K_{j, r}^{(S)}\right]$, for all industries and regions then the following $J \times R$ equations are appended to the system:

$$
K_{j, r}^{(S)}-\left[K_{j, r}^{(S)}\right]=0
$$

The model can then be written as:

$$
\mathbf{D}(\mathbf{V})=\left(\begin{array}{l}
\mathbf{F}(\mathbf{V}) \\
\mathbf{E}(\mathbf{V})
\end{array}\right)=\left(\begin{array}{c}
\mathbf{F}(\mathbf{Y}, \mathbf{X}) \\
\mathbf{E}(\mathbf{0}, \mathbf{X})
\end{array}\right)=0
$$

\footnotetext{
${ }^{43}$ Matching of variables to explaining equations reveals that $3+2 R+8 J+3 J R+2 J^{2} R$ variables remain undetermined in general, or 6,838 in the 25-industry, 5-region version (assuming investment is endogenously allocated across all industries and regions).
} 
where the vector function $\mathbf{E}(\mathbf{V})$ consists of the equations that exogenously set $\mathbf{X}$, the undetermined component of $\mathbf{V}$. The remaining component $\mathbf{Y}$ are endogenous variables explained by $\mathbf{F}(\mathbf{V})$.

In terms of our key steps of model implementation, calibration involves fitting the system $\mathbf{F}(\mathbf{V})$ to data, closure is the choice of which variables to assign to each of $\mathbf{X}$ and $\mathbf{Y}$, and a shock is the choice of levels $\mathbf{X}_{\mathbf{0}}$ at which to fix the exogenous variables.

\subsubsection{Implementing The Model In GAMS}

All operations concerning the model take place within GAMS. ${ }^{44}$ The program takes input from suitably formatted text and spreadsheet files and outputs tables formatted for direct compilation in $\mathrm{LT}_{\mathrm{E} X}$. A model run generally involves executing one program module to perform the calibration and another module to perform the simulation. Multiple simulations can however be run via a batch file after a single run of the calibration module. A third program component is then executed to produce reports on a user-selected subset of the simulations. The program code incorporates elements of object-oriented programming to automate model coding and generation of reports; details are omitted here but available from the author upon request. In the following sections we focus on the calibration, closure, and solution of the 25-industry, 5-region version of JENNIFER.

\subsubsection{Calibrating the Model}

The model is calibrated using the following procedure:

1. National input-output data is loaded and used to construct a model-consistent economy-wide input-output data matrix

2. Regional GDP figures are used to construct a regional shares data matrix

\footnotetext{
${ }^{44}$ The official GAMS users guide, GAMS Development Corp. (2011), is available at http://www . gams . com/dd/docs/bigdocs/GAMSUsersGuide.pdf. Other useful references for GAMS in general is the McCarl Guide (McCarl \& GAMS Development Corp. 2011) at http://www.gams.com/mccarl/mccarlhtml/and other materials at http://agecon2.tamu.edu/people/faculty/mccarl-bruce/. References that deal specifically with the implementation of CGE models in GAMS include Löfgren et al. (2002), Hosoe (2004), and Keyzer (1997).
} 
3. The regional shares are used to disaggregate the national input-output data matrix to obtain a multi-regional input-output (MRIO) data matrix.

4. The MRIO matrix is then used to establish the initial solution to the model - that is, the benchmark equilibrium

5. Finally, the benchmark equilibrium and assumed elasticity parameters are used to calibrate the agents' demand functions

\section{Construction Of The Economy-wide Input-output Data Matrix}

A national inter-industry input-output table for 2005-6 provided by BERL is used to establish the economy-wide data matrix for the model. ${ }^{45}$ The raw data has industrial production disaggregated to 53 industries. For the purposes of this thesis, the table was aggregated to 25 industries. ${ }^{46}$ The industry classification is shown in table 2.5.

A different set of industries can be specified by providing the program with a different mapping from the raw data to model industries. This is useful for running a smaller model when implementing new features for debugging or separating out certain industries of interest for particular simulations.

The derived economy-wide input-output database is shown in table 2.6. Cells in this table are expenditure flows valued at basic prices and will be referred to as $\mathrm{IO}(\mathrm{row}, \mathrm{col})$. The EXP column cells IO(IMP- $g$,EXP) are zeros due to the assumption that no imports are immediately exported again without any value-added. ${ }^{47}$ The raw data records export and import values at f.o.b. and c.i.f. prices respectively. Any export subsidies and import duties should be added to IO(DOM-g, EXP) and IO(IMP-g, col) $\forall c o l, g \in C O M$ as

\footnotetext{
${ }^{45}$ The latest official input-output data at the time of writing is for 1995-6 (Statistics NZ 2001). The BERL data is based on Statistics NZ supply and use tables for 2002-3 that were updated for 2005-6.

${ }^{46}$ In the BERL table, oil and gas was combined with petrol refining. For the purposes of simulations in Chapter 3, these were separated into one industry for oil and gas and another for petrol using data from the Infometrics IO table available at www.motu.org.nz/docs/I02005-06.xls. The compilation of the Infometrics table is documented in Stroombergen (2008). Therein, the author requests that anyone who downloads the data send notification to info@motu.org.nz. The BERL data is essentially derived from the same source, but with a different classification of industries.

${ }^{47}$ If the raw data did have some import flows recorded as going to exports, one way of handling this would be to shift the flows from the imported row to the corresponding domestic row. This was not an issue with the BERL data, however.
} 


\begin{tabular}{lll} 
No. & Label & Description \\
\hline 1 & AGRI & Agriculture \\
2 & FOLO & Forestry and logging \\
3 & FISH & Fishing \\
4 & MINE & Mining and quarrying \\
5 & OIGA & Oil and gas \\
6 & PETR & Refined petrol \\
7 & FDBT & Food, beverages, and tobacco \\
8 & TWPM & Textiles, wood, paper, and media \\
9 & CHNM & Chemicals and non-metallic minerals \\
10 & METL & Basic and fabricated metal \\
11 & EQFO & Equipment, furniture, and other manufacturing \\
12 & UTIL & Electricity, water, and waste services \\
13 & CONS & Construction \\
14 & ACCR & Accommodation, restaurants, and bars \\
15 & CMIF & Communications, insurance, and finance \\
16 & PROP & Real estate and equipment hire \\
17 & RBUS & Research and business services \\
18 & GOVT & Government administration \\
19 & EDUC & Education \\
20 & HEAL & Health \\
21 & CUPE & Cultural and personal services \\
22 & OWND & Ownership of owner-occupied dwellings \\
23 & WHOL & Wholesale trade \\
24 & RETT & Retail trade \\
25 & TRAN & Transport \\
\hline & &
\end{tabular}

Table 2.5: Industries 
needed so that cells record these flows at basic prices. For the purposes of this thesis however, both of these items were assumed zero for simplicity, so no adjustments were made. ${ }^{48}$ The original data had a separate column for inventory changes. For simplicity this was combined with gross fixed capital formation in the INV column. ${ }^{49}$

\section{Construction Of The Regional Shares Data Matrix}

Estimates of industrial contribution to the output of five New Zealand regions were also provided by BERL. The five regions are Auckland (AKL), Wellington (WLG), Canterbury $(\mathrm{CAN})$, Other North Island (ONI), and Other South Island(OSI)..$^{50}$ The raw regional data had industries classified into 54 groups. This has been aggregated to be consistent with the 25 industries of the model. An issue that arose was that petrol refining was combined with coal and chemical manufacturing in the raw data. This has been separated out exploiting the assumption that domestic petrol refining only occurs in Other North Island, since it

\footnotetext{
${ }^{48}$ New Zealand has no explicit export subsidies at present, although in some cases it could be argued that government industry assistance amounts to effective export subsidisation. Since this thesis is not primarily concerned with trade issues, and New Zealand tariffs are very low or zero on most imports at present, duty was not separated out from the rest of commodity taxes included in the IO(TAXES, $\bullet$ ) row.

${ }^{49} \mathrm{In}$ a few cases, the resulting entry in the INV column cell was a small negative number. The sign of those cells was changed so that all investment demands are positive. This inevitably leads to the table becoming unbalanced. In order to restore balance, the CON column was adjusted as needed. While a RAS is the appropriate procedure for rebalancing, the required adjustment was quite small so the simpler approach was taken. The most significant change was an increase in IO(DOM-FDBT,CON) of $3 \%$.

${ }^{50}$ Higher levels of regional detail would be possible if regional employment data were used to further split national industry output, but either some industry detail would have to be sacrificed or the model streamlined to reduce the rate that its size rises with the number of regions since, at the current level of disaggregation, the model with the extensions of chapters 3 and 4 approaches the limits of what a standard desktop computer can handle without special regard given to memory management.

Hall \& McDermott (2007) observed a broad dichotomy in the behaviour of urban vs. rural regions over the business cycle and in later work (Hall \& McDermott 2011) found it useful to aggregate their original 14 regions of analysis to the equivalent of our 5 regions for the purpose of considering the influence of fiscal and monetary policy, and external shocks, on the regional cycles. The current level of regional detail therefore appears sufficient in that context.

An experimental set of regional GDP figures was published in Statistics NZ (2006) for 16 industries across 15 regions, for the years 2000-3. These figures are based on the summation of gross value added (GVA) measured in current prices. Since the BERL data is in constant prices and for the same year as the national IO table, it is preferred to the Statistics NZ data for our purpose here.
} 
is in fact only done in Northland. The data has then been used to derive the regional shares data matrix shown in table 2.7.

This is the primary data set used to disaggregate economic activity across the regions. Where necessary, cells in this table will be referred to as $\left[X_{j, r / j}^{O U T P U T}\right]$ to indicate that cell $(j, r)$ is region $r$ 's share of the output of industry $j$. The square brackets are used to indicate that these raw regional shares actually become the benchmark equilibrium values of $X_{j, r / j}^{O U T P U T}$.

\section{Deriving a Multi-Regional Input-Output Data Matrix}

A balanced multi-regional input-output (MRIO) database of the form shown in table 2.8 is required for calibration of the model. To obtain this database we appeal to principles of information theory. ${ }^{51}$

\footnotetext{
${ }^{51}$ The approach used is supported in principle by Batten (1982, p. 54):
}

"[B]y adopting a statistical or information-theoretic approach it is possible to overcome some of [the] deficiencies in information and at the same time avoid some of the difficulties in working with causal relations between location and trade at the multi-regional level. Certain elements of information theory provide a consistent means of estimating interregional flows which are minimally biased, subject to whatever flow information is available." 
具一十

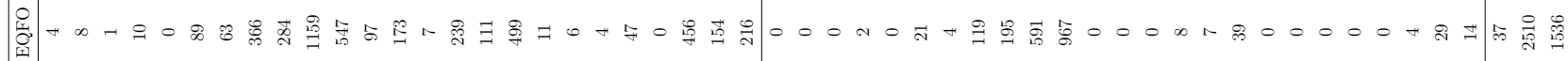

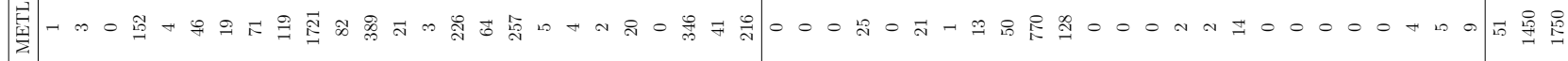

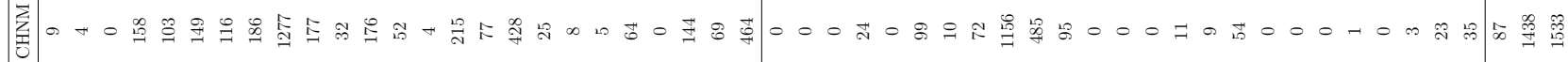

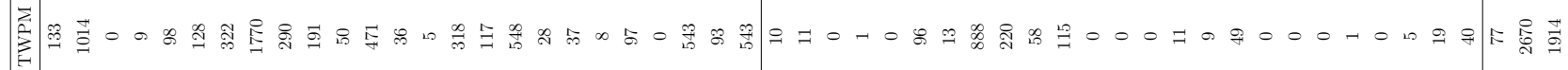

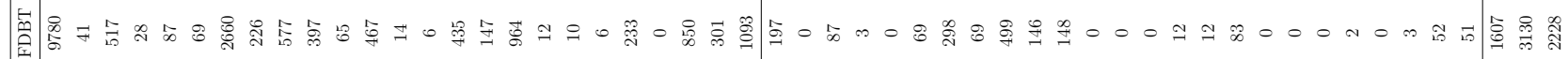

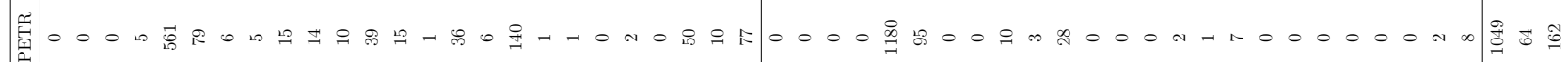

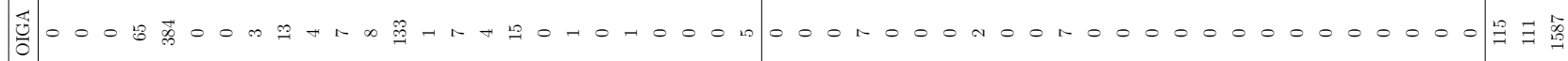
罢

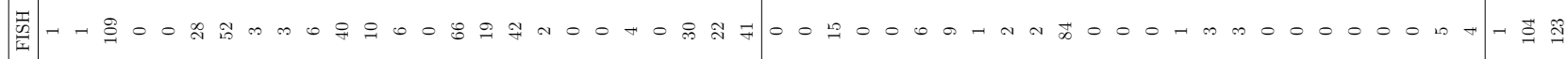

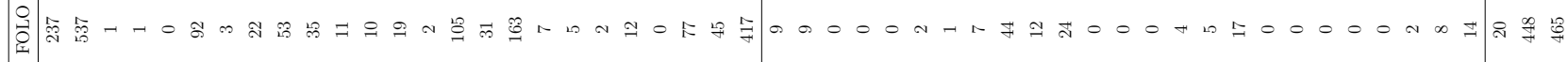

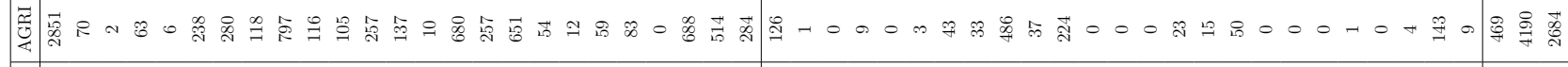

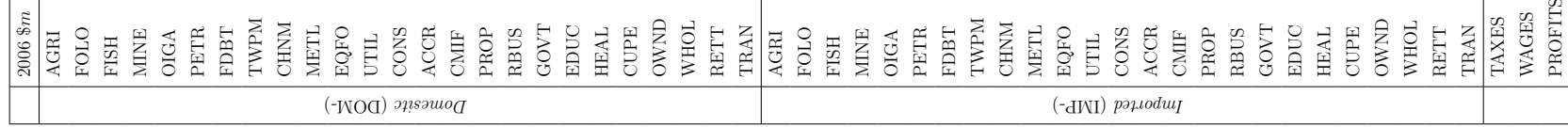




\begin{tabular}{|l|ccccc|}
\hline \multicolumn{5}{|c|}{ Regional Shares of Industry Output (\%) } \\
\hline \multirow{2}{*}{ Industry } & \multicolumn{5}{c|}{ Region } \\
\cline { 2 - 6 } & AKL & WLG & ONI & CAN & OSI \\
\hline AGRI & 4.4 & 1.0 & 58.8 & 6.1 & 29.7 \\
FOLO & 5.7 & 1.9 & 63.7 & 4.3 & 24.4 \\
FISH & 9.6 & 1.9 & 32.0 & 2.6 & 53.9 \\
MINE & 8.1 & 1.0 & 49.9 & 9.8 & 31.2 \\
OIGA & 0.6 & 9.4 & 90.0 & 0.0 & 0.0 \\
PETR & 0.0 & 0.0 & 100.0 & 0.0 & 0.0 \\
FDBT & 20.4 & 3.5 & 39.5 & 9.2 & 27.5 \\
TWPM & 30.9 & 6.8 & 37.1 & 11.1 & 14.0 \\
CHNM & 58.9 & 11.7 & 2.3 & 18.2 & 8.9 \\
METL & 38.5 & 6.1 & 31.1 & 13.4 & 11.0 \\
EQFO & 45.5 & 8.0 & 24.1 & 13.9 & 8.6 \\
UTIL & 24.6 & 17.2 & 39.8 & 6.8 & 11.6 \\
CONS & 30.8 & 9.8 & 34.9 & 10.8 & 13.7 \\
ACCR & 28.8 & 10.1 & 30.8 & 10.9 & 19.4 \\
CMIF & 45.2 & 20.4 & 16.9 & 11.2 & 6.4 \\
PROP & 37.7 & 9.7 & 28.9 & 12.2 & 11.5 \\
RBUS & 45.1 & 16.0 & 21.6 & 9.8 & 7.5 \\
GOVT & 22.7 & 35.7 & 25.5 & 8.7 & 7.5 \\
EDUC & 33.8 & 11.1 & 31.5 & 10.9 & 12.7 \\
HEAL & 28.8 & 11.1 & 33.8 & 12.5 & 13.8 \\
CUPE & 36.2 & 14.4 & 26.3 & 11.0 & 12.2 \\
OWND & 34.5 & 12.0 & 30.0 & 10.7 & 12.8 \\
WHOL & 51.6 & 9.4 & 18.6 & 11.7 & 8.7 \\
RETT & 32.3 & 9.8 & 33.0 & 11.1 & 13.8 \\
TRAN & 42.9 & 9.9 & 21.2 & 13.0 & 13.0 \\
\hline
\end{tabular}

Table 2.7: Regional Shares of Industry Output (\%) 


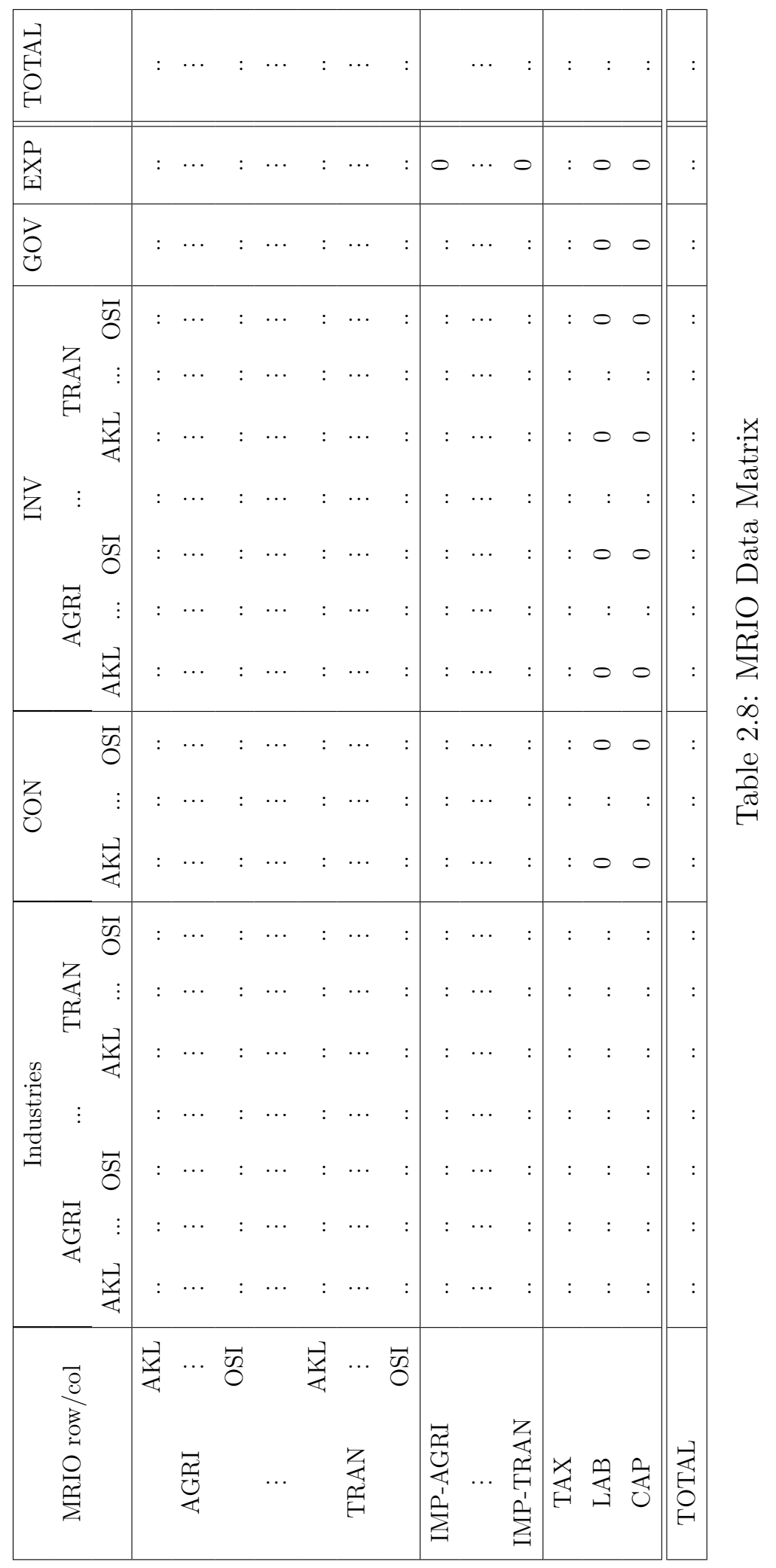


We refer to cells in the MRIO table as MRIO(row, col) where row $\in$ AGRI-AKL, AGRI-WLG, ... IMP-AGRI, IMP-FOLO, ... TAX, LAB, CAP $\}$ and col $\in\{$ AGRIAKL, AGRI-WLG, ... CON-AKL, CON-WLG, ... INV-AGRI-AKL, INV-AGRI-WLG, ... GOV, EXP\}. McDougall (1999) demonstrates that biproportional allocation is a maximum entropy solution to the matrix-filling problem. In other words, if we have estimates of all the row and column totals of the MRIO matrix but no other information, then an entropy maximising solution is:

$$
\operatorname{MRIO}(\text { row }, c o l)=\frac{\operatorname{MRIO}(\text { row }, \text { TOTAL }) \times \text { MRIO }(\text { TOTAL }, c o l)}{\text { MRIO }(\text { TOTAL }, \text { TOTAL })} \quad \forall \text { row }, c o l
$$

We could then derive an initial estimate of the MRIO matrix using the regional shares and the borders of the national IO table. To do so however would be ignoring the information in the cells of the IO table - that the intra- and inter-regional flows from one industry to another sum up to the value of the relevant IO cell. It is appropriate then to apply (2.120) for each MRIO block and its corresponding single IO table cell. To see how this is done, we will consider the blocks within each group of MRIO columns separately.

Industry Columns Since the value in $\mathrm{IO}(\mathrm{DOM}-g, j)$ is the sum of product flows from industry $g$ in every region to industry $j$ in every region, a straightforward estimate of the flow sum from industry $g$ to industry $j$ in region $r$ is given by:

$$
\sum_{x} \operatorname{MRIO}(g-x, j-r)=\left[X_{j, r / j}^{\text {OUTPUT }}\right] \times \mathrm{IO}(\mathrm{DOM}-g, j)
$$

The flow sum from industry $g$ in region $x$ to industry $j$ can similarly be estimated:

$$
\sum_{r} \operatorname{MRIO}(g-x, j-r)=\left[X_{g, x / g}^{\text {OUTPUT }}\right] \times \mathrm{IO}(\mathrm{DOM}-g, j)
$$

Applying (2.120) yields an estimate of the flow of products from industry $g$ in region $x$ to industry $j$ in region $r$ :

$$
\operatorname{MRIO}(g-x, j-r)=\left[X_{g, x / g}^{O U T P U T}\right] \times\left[X_{j, r / j}^{O U T P U T}\right] \times \mathrm{IO}(\mathrm{DOM}-g, j)
$$

The larger the presence of an industry $g$ in a region $x$, and the larger the presence of an industry $j$ in a region $r$, the larger will be the flow of products between those regions as a proportion of all flows between industry $g$ and $j$. 
The calculation of (2.121) amounts to splitting the rows and columns of the domestic intermediate inputs section of the IO table (the top-left quadrant) using the regional shares. By estimating MRIO(IMP- $g, j-r)$, MRIO(TAX, $j-r)$, MRIO(LAB, $j-r)$, and MRIO $(\mathrm{CAP}, j-r)$ in a similar way, the entire IO table column for each industry $j$ is effectively split by the regional shares to become the $j-r$ columns of the MRIO table. Note that these latter rows are not split, so only the industry $j$ regional shares are required:

$$
\begin{aligned}
\operatorname{MRIO}(\mathrm{IMP}-g, j-r) & =\left[X_{j, r / j}^{\text {OUTPUT }}\right] \times \mathrm{IO}(\mathrm{IMP}-g, j) \\
\mathrm{MRIO}(\mathrm{TAX}, j-r) & =\left[X_{j, r / j}^{\text {OUTPUT }}\right] \times \mathrm{IO}(\mathrm{TAXES}, j) \\
\mathrm{MRIO}(\mathrm{LAB}, j-r) & =\left[X_{j, r / j}^{\text {OUTPUT }}\right] \times \mathrm{IO}(\text { WAGES }, j) \\
\mathrm{MRIO}(\mathrm{CAP}, j-r) & =\left[X_{j, r / j}^{\text {OUTPUT}}\right] \times \mathrm{IO}(\mathrm{PROFITS}, j)
\end{aligned}
$$

This specification is due to simplifying assumptions in the model design. Imports for example are not modelled bottom-up — importers receive the same price regardless of which port products arrive at. The LAB and CAP rows do not need splitting since we assume that labour and installed capital are only employed within the region where they are located. The TAX row is also not split since the public sector is not divided into central and local government in the present model.

Consumption Column A similar approach to that taken with the industry columns is used to split the consumption column. Analogous to before we assume that the flow from industry $g$ in region $x$ to private consumption is:

$$
\sum_{r} \operatorname{MRIO}(g-x, \mathrm{CON}-r)=\left[X_{g, x / g}^{\text {OUTPUT }}\right] \times \mathrm{IO}(\mathrm{DOM}-g, \mathrm{CON})
$$

However, we use shares of labour demand (employment) to distribute consumption demand for product $g$ over the regions:

$$
\sum_{x} \operatorname{MRIO}(g-x, \mathrm{CON}-r)=\left[X_{r / t o t}^{L A B O U R}\right] \times \mathrm{IO}(\mathrm{DOM}-g, \mathrm{CON})
$$

such that the flow of product $g$ from region $x$ to private consumption in region $r$ is estimated as:

$$
\operatorname{MRIO}(g-x, \mathrm{CON}-r)=\left[X_{g, x / g}^{\text {OUTPUT }}\right] \times\left[X_{r / t o t}^{L A B O U}\right] \times \mathrm{IO}(\mathrm{DOM}-g, \mathrm{CON})
$$


The imports cells IO(IMP- $g, \mathrm{CON})$ and tax cell IO(TAXES,CON) are similarly split using the labour demand shares:

$$
\begin{aligned}
\mathrm{MRIO}(\mathrm{IMP}-g, \mathrm{CON}-r) & =\left[X_{r / t o t}^{L A B O U R}\right] \times \mathrm{IO}(\mathrm{IMP}-g, \mathrm{CON}) \\
\mathrm{MRIO}(\mathrm{TAX}, \mathrm{CON}-r) & =\left[X_{r / t o t}^{L A B O U R}\right] \times \mathrm{IO}(\mathrm{TAXES}, \mathrm{CON})
\end{aligned}
$$

The labour demand shares are calculated by: ${ }^{52}$

$$
\left[X_{r / t o t}^{L A B O U R}\right]=\sum_{j} \frac{\mathrm{IO}(\mathrm{WAGES}, j)}{\sum_{i} \mathrm{IO}(\mathrm{WAGES}, i)} \times\left[X_{j, r / j}^{\text {OUTPUT }}\right]
$$

Thus the distribution of labour demand over regions depends on the distribution of labour demand across industries and the distribution of each industry's production across the regions. The implication of splitting the consumption column using these employment shares is that regions that have concentrations of labour-intensive industries, and therefore a relatively high labour income share in total household income, will have average consumption propensities above the national average. The converse is that in regions where the share of capital rents in household income is above the national average, so will be the average propensity to save. ${ }^{53}$

Investment Column The splitting of the investment column is analogous to the above but requires estimates of industry-by-region shares of investment. The BERL input-out table was accompanied by data on industry capital stocks, depreciation, and estimates of the marginal efficiency of investment capital (the $\beta_{j, r}$ parameters). A simple assumption we can make therefore is that the industry-by-region allocation of investment follows the industry-by-region distribution of capital stocks.:

$$
\left[X_{j, r / \mathrm{tot}}^{I, \mathbf{b}}\right]=\frac{\left[K S T O C K_{j, r}\right]}{\left[K S T O C K_{\mathbf{N Z}}\right]}
$$

\footnotetext{
${ }^{52}$ Again, the square brackets indicate that these labour demand shares are consistent with the benchmark equilibrium that is established below from the MRIO matrix.

${ }^{53}$ To impose the assumption that average propensities were constant across all regions instead, the regional labour demand shares in these calculations could be replaced by regional total income shares. Appendix I establishes the relationship between the propensities and the distribution of consumption in the benchmark equilibrium.
} 
where each industry's capital stock is assumed to be distributed over regions in line with their output. That is,

$$
\left[X_{j, r / \text { tot }}^{K S T O C K}\right]=\frac{\left[K^{\prime} S T O C K_{j, r}\right]}{\left[K S T O C K_{\mathbf{N Z}}\right]}=\left[X_{j, r / j}^{O U T P U T}\right] \times \frac{\left[K S T O C K_{j, \mathbf{N Z}}\right]}{\left[K S T O C K_{\mathbf{N Z}}\right]}
$$

and the values $\left[X_{j / \text { tot }}^{K S T O C K}\right]=\frac{\left[\text { KSTOCK }_{j, \mathbf{N Z}}\right]}{\left[K_{\text {STOCK }} \mathrm{NZ}\right]}$ come from the BERL data. For the 25-industry implementation under consideration here, the industry-by-region shares of capital are given in table 2.9 .

If the user plans to specify the investment allocation exogenously during simulations as discussed in section 2.3.4 then the above assumption is fine. However, if the user wishes investment to be allocated endogenously to equalise expected net rates of return in equilibrium, then the industry-by-region shares used to split the IO investment column must be consistent with this equalisation in the benchmark equilibrium. That is, all the equations (2.25) - (2.33) must be satisfied. The model-consistent values of $\left[X_{j, r / \text { tot }}^{I, \mathbf{b} \$}\right]$ will depend on the gross rates of return and depreciation rates along with the capital stock levels for each industry in each region, and can only be determined numerically. How this is done will be discussed shortly; at present assume that we have obtained values for $\left[X_{j, r / \text { tot }}^{I, \mathbf{b} \$}\right]$ that are consistent with the benchmark equilibrium. The MRIO investment column cell values are then given by:

$$
\operatorname{MRIO}(g-x, \mathrm{INV}-j-r)=\left[X_{g, x / g}^{O U T P U T}\right] \times\left[X_{j, r / \mathrm{tot}}^{I, \mathbf{b} \$}\right] \times \mathrm{IO}(\mathrm{DOM}-g, \mathrm{INV})
$$

and similar formulae, analogous to (2.127) and (2.128) above.

The Government and Exports Columns As the government and exporters are not modelled bottom-up, these columns do not need to be split. ${ }^{54}$ The evaluation of the MRIO cells for government and exports is similar to that of consumption. For example:

$$
\begin{aligned}
\operatorname{MRIO}(g-x, \mathrm{GOV}) & =\left[X_{g, x / g}^{\text {OUTPUT }}\right] \times \mathrm{IO}(\mathrm{DOM}-g, \mathrm{GOV}) \\
\operatorname{MRIO}(g-x, \mathrm{EXP}) & =\left[X_{g, x / g}^{\text {OUTPUT }}\right] \times \mathrm{IO}(\mathrm{DOM}-g, \mathrm{EXP})
\end{aligned}
$$

and so forth.

\footnotetext{
${ }^{54}$ If they were to be given a regional dimension, the government column could be split by regional shares of employment in the public sector, and exports could be split if one had sufficiently detailed data on commodity arrivals at each New Zealand port.
} 


\begin{tabular}{|l|ccccc|c|}
\hline \multicolumn{1}{|c}{ Industry by Region Shares of Total Capital $(\%)$} \\
\hline \multirow{2}{*}{ Industry } & \multicolumn{5}{c|}{ Region } \\
\cline { 2 - 7 } & AKL & WLG & ONI & CAN & OSI & NZ \\
\hline AGRI & 0.1 & 0.0 & 1.8 & 0.2 & 0.9 & 3.1 \\
FOLO & 0.0 & 0.0 & 0.2 & 0.0 & 0.1 & 0.3 \\
FISH & 0.0 & 0.0 & 0.1 & 0.0 & 0.1 & 0.2 \\
MINE & 0.0 & 0.0 & 0.1 & 0.0 & 0.1 & 0.2 \\
OIGA & 0.0 & 0.1 & 0.9 & 0.0 & 0.0 & 0.9 \\
PETR & 0.0 & 0.0 & 0.1 & 0.0 & 0.0 & 0.1 \\
FDBT & 0.5 & 0.1 & 1.0 & 0.2 & 0.7 & 2.4 \\
TWPM & 0.5 & 0.1 & 0.6 & 0.2 & 0.2 & 1.7 \\
CHNM & 0.6 & 0.1 & 0.0 & 0.2 & 0.1 & 1.1 \\
METL & 0.3 & 0.0 & 0.2 & 0.1 & 0.1 & 0.7 \\
EQFO & 0.6 & 0.1 & 0.3 & 0.2 & 0.1 & 1.3 \\
UTIL & 1.2 & 0.8 & 2.0 & 0.3 & 0.6 & 4.9 \\
CONS & 0.4 & 0.1 & 0.4 & 0.1 & 0.2 & 1.2 \\
ACCR & 0.3 & 0.1 & 0.3 & 0.1 & 0.2 & 1.1 \\
CMIF & 1.5 & 0.7 & 0.5 & 0.4 & 0.2 & 3.2 \\
PROP & 6.7 & 1.7 & 5.1 & 2.2 & 2.1 & 17.8 \\
RBUS & 0.6 & 0.2 & 0.3 & 0.1 & 0.1 & 1.4 \\
GOVT & 2.1 & 3.3 & 2.3 & 0.8 & 0.7 & 9.2 \\
EDUC & 1.2 & 0.4 & 1.1 & 0.4 & 0.4 & 3.4 \\
HEAL & 0.7 & 0.3 & 0.8 & 0.3 & 0.3 & 2.3 \\
CUPE & 0.9 & 0.3 & 0.6 & 0.3 & 0.3 & 2.4 \\
OWND & 12.0 & 4.2 & 10.5 & 3.7 & 4.5 & 34.8 \\
WHOL & 0.9 & 0.2 & 0.3 & 0.2 & 0.2 & 1.8 \\
RETT & 0.5 & 0.2 & 0.5 & 0.2 & 0.2 & 1.5 \\
TRAN & 1.3 & 0.3 & 0.6 & 0.4 & 0.4 & 3.0 \\
\hline All & 32.8 & 13.3 & 30.7 & 10.5 & 12.6 & 100.0 \\
\hline
\end{tabular}

Table 2.9: Industry by Region Shares of Total Capital (\%) 


\section{Making Adjustments to the MRIO Matrix}

By splitting each cell of the IO table as required using regional shares, we have maximised the entropy of each associated block of the MRIO table. For each row-split we have used the same share across all the columns of $\mathrm{IO}(\mathrm{DOM}-g, \bullet)$, and for each column split we have used the same share down all the rows, so the MRIO table remains balanced. This method of deriving the MRIO table can also be thought of as maximising the entropy of the whole matrix, subject to the condition that each block adds up to the respective IO table cell.

We may have superior data or wish to make an assumption such that the allocation of some IO cell values are not biproportional across the relevant blocks of cells in the MRIO table. For instance, we may want to assume some product types are local as per the regional extension to ORANI. This means they are products that can only be purchased and used in the region where they are produced. The producers of local products are therefore local industries because they only sell to their local region (and the government and exporters). The implication is that there is no inter-regional trade in local products. This assumption is made here for the industries listed in table 2.10.

Adjustments are required to the allocation of IO cell values over the relevant MRIO blocks to reflect the local product assumption. For example, since (the product/industry) EDUC is assumed local, the flows to MRIO column CON-r are specified as: ${ }^{.5}$

$$
\mathrm{MRIO}(\mathrm{EDUC}-x, \mathrm{CON}-r)=\left[X_{r / t o t}^{L A B O U R}\right] \times \mathrm{IO}(\mathrm{DOM}-\mathrm{EDUC}, \mathrm{CON}) \times \mathbb{1}^{x=r}
$$

Note that the local product assumption only affects domestic product flows; the imported variety of the product can co-exist. If there is some characteristic of the domestic variety that suggests it is predominantly local, but imports of the foreign variety are recorded in the IO data, then the two types must be significantly different. The elasticity of substitution between the two ought to be fairly low to reflect this.

These kinds of adjustments inevitably disturb the balance of the MRIO matrix. It is almost certain that the totals of the domestic product rows will no longer equal the totals

\footnotetext{
$55 \mathbb{1}$ is the indicator function:

$$
\mathbb{1}^{\text {condition }}= \begin{cases}1 & \text { if condition }=\text { true } \\ 0 & \text { if condition }=\text { false }\end{cases}
$$
}


Local Industries

\begin{tabular}{ll}
\hline EDUC & Education \\
HEAL & Health \\
CUPE & Cultural and personal services \\
OWND & Ownership of owner-occupied dwellings \\
WHOL & Wholesale trade \\
RETT & Retail trade \\
\hline
\end{tabular}

Table 2.10: Local Industries

of the respective industry columns and the MRIO will not be consistent with equilibrium. To enforce consistency and restrict the information gain to those parts of the MRIO table that we directly manipulate, we seek a cross-entropy solution to re-balancing the matrix. It turns out the traditional RAS (biproportional adjustment) method is ideal for this purpose. The RAS is commonly used to scale the rows and columns of a matrix to conform to given row and column totals. The seminal treatment of the RAS method is Bacharach (1970) while McDougall (1999) links the RAS to cross-entropy.

The essence of the cross-entropy method is that an objective function quantifying the distance of a solution matrix from an initial given matrix is minimised subject to specified constraints. ${ }^{56}$ In our case we wish to find a new MRIO matrix that has the same row and column totals as the initial matrix (so that balance is maintained and output shares etc. do not change), has some cell blocks specified for local products, and has the remainder of the blocks as close to biproportional as possible (so as to minimise spurious information gain). The task can also be framed as a RAS problem: we wish to update the initial matrix using the same row and column totals and some newly specified cells. ${ }^{57}$ Re-balancing using RAS therefore ensures that cross-entropy is minimised.

Let the elements of an unbalanced MRIO matrix of dimension $i \times j$ be denoted $A_{i j}$. We wish to find a new balanced matrix with elements $B_{i j}$ as close to $A_{i j}$ as possible but which has the same row totals $A_{i \bullet}$ and same column totals $A_{\bullet j}$ as the original balanced

\footnotetext{
${ }^{56}$ Maximum entropy is a special case of minimum cross entropy which minimises the distance between the solution matrix and a uniform matrix (where the data is spread evenly over the matrix cells.)

${ }^{57}$ This is quite different to how the RAS is traditionally used - to find cell values that conform to newly specified row and column totals.
} 
MRIO matrix (i.e. before adjustments). The RAS method involves iteratively searching for a set of row scaling factors $R_{i}$ and a set of column scaling factors $S_{j}$ such that:

$$
B_{i j}=R_{i} \cdot A_{i j} \cdot S_{j} \quad \forall i, j
$$

The solution is obtained in GAMS by a subroutine minimising the quadratic loss function: ${ }^{58}$

$$
\text { Loss }=\sum_{i} R_{i}^{2}+\frac{1}{R_{i}^{2}}+\sum_{j} S_{j}^{2}+\frac{1}{S_{j}^{2}}
$$

subject to (2.135) and the constraints:

$$
\begin{array}{lll}
\sum_{j} B_{i j}=A_{i \bullet} & & \forall i \\
\sum_{i} B_{i j}=A_{\bullet j} & \forall j
\end{array}
$$

The RAS procedure is only performed on part of the MRIO matrix - an additional set of constraints ensure that the TAX, LAB, and CAP rows do not change because otherwise the assumptions used to construct the MRIO in the first place may no longer hold. For example the regional distribution of labour demand could be changed by the RAS adjustment, in which case the consumption column split would be incorrect. The GAMS program also outputs the sum of squared proportionate deviations:

$$
D e v=\sum_{i} \sum_{j}\left(\frac{B_{i j}-A_{i j}}{A_{i j}}\right)^{2}
$$

For the particular implementation we are discussing here, Dev $=42.2$ although this measure is only really useful in a relative sense.

The important point about this use of biproportional adjustment is that it guarantees a balanced, model-consistent MRIO matrix with minimal adjustment. Where the RAS algorithm makes adjustments, it is only the regional allocation that is affected; the economy-wide input-output table is not affected. The formulation of the MRIO, including

\footnotetext{
${ }^{58}$ The GAMS code is based on a posting by Rutherford (n.d.) to the GAMS-User List. Other objective functions can be used instead to explicitly minimise the cross-entropy or the sum of squared deviations — see Rutherford (2003) and Fofana et al. (2005) for details. The form of the objective function used here makes the RAS adjustment explicit and probably facilitates quick convergence since it is a quadratic (as opposed to logarithmic) function.
} 
the initial split of columns and rows, is consistent with the principles of information theory - the MRIO embodies all of the available information (the IO matrix and the regional shares data), but only that information. Any results from a model based on the MRIO will then be based on that information, rather than on spurious information that could be created by estimating the MRIO in a different way. In particular, the RAS method guarantees that the ordering of the input intensities is preserved. If one industry uses a particular product from a given region more intensively than another industry initially, applying the RAS method will not change this ordering. ${ }^{59}$

\section{Establishing the Benchmark Equilibrium Solution}

This section describes the general procedure, having derived a balanced MRIO table as per the above, for specifying the benchmark values for all model variables (including exogenous variables) that are consistent with competitive general equilibrium, using the 25-industry, 5-region implementation as an example. ${ }^{60}$

Domestic Prices The model is homogenous of degree zero in prices so we are free to choose the absolute price level. The flows in the MRIO data matrix are basic value flows - they have basic price and quantity components. If we assume all basic prices (the price received by the seller) are equal to one then the flows can be interpreted as quantity flows instead. Purchase prices were defined in section 2.3.10, using equations such as (2.59) to define purchase prices at the lowest level of agents' decision nests. The introduction of delivered and undelivered quantities $(Q$ and $\breve{Q}$ respectively) allows the associated benchmark purchase prices to also be set equal to one. ${ }^{61}$ The result is that

\footnotetext{
${ }^{59}$ This is not true for cross-entropy methods in general — see McDougall (1999, proposition 5, page 10).

${ }^{60}$ Appendix $\mathrm{N}$ presents the MRIO matrix but the version found there incorporates elements introduced later, in chapter 3.

${ }^{61}$ As discussed in section 2.3.10, the benchmark purchase price levels relative to those of basic prices are immaterial to simulation results. The absolute levels of all prices are not meaningful anyway - they are after all only relative prices (relative to the numeraire). During model development it was simpler to initialise purchase prices at 1 - it made nominal homogeneity testing and double-checking of benchmark product demand values easier. The use of delivered and undelivered products also lends itself to an analogy with value-added that becomes more obvious in chapter 3 - taxes and other costs of delivery add value to undelivered products as they move towards the purchasing agent.
} 
almost all the benchmark equilibrium prices in the model are equal to one (or more generally, the level of the numeraire). There are three important sets of prices that are exceptions, which we now turn to.

Foreign Prices We saw in equation (2.65) that export subsidies put a wedge between the price exporters receive and the f.o.b. price the foreign sector pays. Rather than introduce a distinction between delivered and undelivered exports, we simply define the base-period purchase price of export $g$ as: ${ }^{62}$

$$
\left[P_{g}^{E X P, \mathbf{d} \$, \text { for }}\right]=\frac{\left[P_{g}^{E X P}\right]}{1-\left[t_{g}^{E X P}\right]}=\frac{1}{1-\left[t_{g}^{E X P}\right]}
$$

Similarly for imports, we saw in equation (2.63) that import duties put a wedge between the price importers pay and the c.i.f. price the foreign sector receives. Rather than introduce a distinction between delivered and undelivered imports, we simply define the base-period foreign currency price of import $g$ as: ${ }^{63}$

$$
\left[P_{g}^{F O R}\right]=\frac{\left[P_{g}^{F O R, \mathbf{f} \$, \mathbf{i m p}}\right]}{1+\left[t_{g}^{F O R}\right]}=\frac{1}{1+\left[t_{g}^{F O R}\right]}
$$

For simplicity in the current implementation of JENNIFER, the export subsidy and import duty rates are assumed equal to zero.

After-Tax Wage Rates We also saw in equation (2.41) that income tax separates the gross and net wage rates received by households. It is convenient to assume gross wage rates equal one in the base-period, which implies:

$$
\left[P_{r}^{L, \mathbf{a t a x}}\right]=1-\left[t^{L}\right]
$$

In BERL's recent study of the impact of immigration (Nana, Sanderson \& Hodgson 2009), an average direct income tax rate of $21.1 \%$ was used and we adopt this figure here.

\footnotetext{
${ }^{62}$ We already have $\breve{Q}_{g, x}^{\exp (D)}$ and $Q_{g, x}^{\exp (D)}$ which capture the difference between product $g$ at the factory door and the same product in the hands of the exporter.

${ }^{63}$ We already have $I M P_{g}^{(S)}$ and $Q_{g \text {,imp }}^{\text {agent }(D)}$ which capture the difference between product $g$ at the door of the importer's warehouse and the same product in the hands of agent where agent is a household or industry agent, or the government
} 
Capital Rental Rates Capital construction costs $\left[P_{j, r}^{K, \text { new }}\right]$ and rental rates $\left[P_{j, r}^{K}\right]$ should not both be normalised to 1 because this would imply $100 \%$ gross rates of return for all industries by equation (2.25). For each industry $j$ we can derive the rental rates from the BERL data:

$$
\left[P_{j, r}^{K}\right]=\frac{\mathrm{IO}(\mathrm{PROFITS}, j)}{\left[K S T O C K_{j, \mathbf{N Z}}\right]}
$$

The gross rates of return will therefore be $100 \times\left[P_{j, r}^{K}\right]$ when $\left[P_{j, r}^{K, \text { new }}\right]$ is normalised to 1. The calculation above implies that industry gross rates of return will not vary across regions. The economy-wide rates of return can be found in table 2.12 .

Product and Factor Demands Each value flow in the product rows of the MRIO matrix is a basic price multiplied by an undelivered quantity, while each flow in the factor rows is a basic (gross) price multiplied by a factor quantity. Assuming all the basic prices equal one implies that all the cells are interpreted as quantities of either $\breve{Q}, L$, or $K$. Evaluating benchmark equilibrium values for product and factor demand variables is therefore straightforward, although since the capital rental price is an exception to the basic price equals one assumption, we divide the cell value by that price to measure capital in the appropriate units. For current production, for example:

$$
\begin{aligned}
{\left[\breve{Q}_{g, x, j, r}^{\mathbf{i n d}(\mathbf{Q})(D)}\right] } & =\operatorname{MRIO}(g-x, j-r) \\
{\left[\breve{Q}_{g, \mathbf{i m p}(\mathbf{i}, r)(D)}^{\operatorname{ind}(\mathbf{Q})}\right] } & =\operatorname{MRIO}(\mathrm{IMP}-g, j-r) \\
{\left[L_{j, r}^{\operatorname{ind}(D)}\right] } & =\operatorname{MRIO}(\mathrm{LAB}, j-r) \\
{\left[K_{j, r}^{\operatorname{ind}(D)}\right] } & =\frac{\operatorname{MRIO}(\mathrm{CAP}, j-r)}{\left[P_{j, r}^{K}\right]}
\end{aligned}
$$

To obtain the delivered quantities, a portion of commodity tax is added on to each undelivered quantity. How much is added depends on the assumed commodity tax rates - how these are determined will be discussed shortly. For given tax rates, the purchase

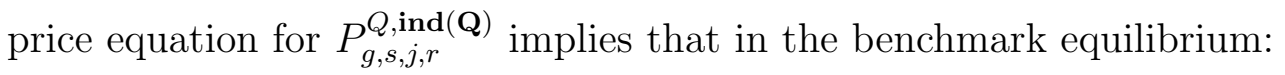

$$
\begin{aligned}
{\left[P_{g, s, j, r}^{Q, \mathbf{i n d}(\mathbf{Q})}\right] \cdot\left[Q_{g, s, j, r}^{\operatorname{ind}(\mathbf{Q})(D)}\right] } & =\left[P_{g, s}^{Q}\right] \cdot\left(1+\left[t_{g, j, r}^{Q, \mathbf{i n d}(\mathbf{Q})}\right]\right) \cdot\left[\breve{Q}_{g, s, j, r}^{\operatorname{ind}(\mathbf{Q})(D)}\right] \\
\Rightarrow\left[Q_{g, s, j, r}^{\operatorname{ind}(\mathbf{Q})(D)}\right] & =\left(1+\left[t_{g, j, r}^{Q, \mathbf{i n d}(\mathbf{Q})}\right]\right) \cdot\left[\breve{Q}_{g, s, j, r}^{\mathbf{i n d}(\mathbf{Q})(D)}\right]
\end{aligned}
$$

The purchase price definitions discussed in section 2.3 .10 can similarly be used to evaluate the composite quantities as we move up the agent's decision nest. For example, 
the base-period values of $Q_{g, \mathbf{d o m}, j, r}^{\operatorname{ind}(\mathbf{Q})(D)}, Q_{g, j, r}^{\operatorname{ind}(\mathbf{Q})(D)}, Q_{j, r}^{\operatorname{ind}(D)}$, and the composite factor $F_{j, r}^{\operatorname{ind}(D)}$ are given by:

$$
\begin{aligned}
{\left[Q_{g, \mathbf{d o m}, j, r}^{\mathbf{i n d}(\mathbf{Q})(D)}\right] } & =\sum_{x}\left[Q_{g, x, j, r}^{\mathbf{i n d}(\mathbf{Q})(D)}\right] \\
{\left[Q_{g, j, r}^{\mathbf{i n d}(\mathbf{Q})(D)}\right] } & =\sum_{o}\left[Q_{g, o, j, r}^{\mathbf{i n d}(\mathbf{Q})(D)}\right] \\
{\left[Q_{j, r}^{\operatorname{ind}(D)}\right] } & =\sum_{g}\left[Q_{g, j, r}^{\operatorname{ind}(\mathbf{Q})(D)}\right] \\
{\left[F_{j, r}^{\operatorname{ind}(D)}\right] } & =\left[L_{j, r}^{\operatorname{ind}(D)}\right]+\left[K_{j, r}^{\operatorname{ind}(D)}\right]
\end{aligned}
$$

The base-period product demands for the other agents can be evaluated along similar lines to the above:

$$
\begin{aligned}
{\left[\breve{Q}_{g, x, j, r}^{\mathbf{i n d}(\mathbf{K})(D)}\right] } & =\operatorname{MRIO}(g-x, \mathrm{INV}-j-r) \\
{\left[\breve{Q}_{g, \mathbf{i m p}, j, r}^{\mathbf{i n d}(\mathbf{K})(D)}\right] } & =\operatorname{MRIO}(\mathrm{IMP}-g, \mathrm{INV}-j-r) \\
{\left[\breve{Q}_{g, x, r}^{\mathbf{h s h}(D)}\right] } & =\operatorname{MRIO}(g-x, \mathrm{CON}-r) \\
{\left[\breve{Q}_{g, \mathbf{i m p}, r}^{\mathbf{h s h}(D)}\right] } & =\operatorname{MRIO}(\mathrm{IMP}-g, \mathrm{CON}-r)
\end{aligned}
$$

and so on.

Product Supplies Equilibrium requires the supply of each product from each source to equal its demand. The MRIO matrix can be used directly to find the product supplies as the total of row $\operatorname{MRIO}(g-x, \bullet)$ equals $\left[P_{g, x}^{Q}\right] \cdot\left[\breve{Q}_{g, x}^{(S)}\right]$. Similarly, the total of row MRIO(IMP$g, \bullet)$ equals $\left[P_{g, \text { imp }}^{Q}\right] \cdot\left[\breve{Q}_{g, \text { imp }}^{(S)}\right]$. Since $\left[P_{g, s}^{Q}\right]=1 \forall s$ the row totals give the benchmark values of $\breve{Q}_{g, s}^{(S)}$. Alternatively, having obtained all the product demand quantities above, we can find values of $\breve{Q}_{g, s}^{(S)}$ consistent with the product market clearing equation (2.46):

$$
\left[\breve{Q}_{g, s}^{(S)}\right]=\sum_{r}\left[\breve{Q}_{g, s, r}^{\mathbf{h s h}(D)}\right]+\sum_{p} \sum_{j} \sum_{r}\left[\breve{Q}_{g, s, j, r}^{\operatorname{ind}(p)(D)}\right]+\left[\breve{Q}_{g, s}^{\operatorname{gov}(D)}\right]+\left[\breve{Q}_{g, s}^{\exp (D)}\right]
$$

The two approaches should result in the same values, so this serves as a useful check that the MRIO is in fact consistent with equilibrium. ${ }^{64}$

\footnotetext{
${ }^{64}$ Tiny discrepancies of the order $1 \times 10^{-16}$ do show up due to GAMS being limited by 32-bit machine accuracy. These do not affect the operation of the model in any material way.
} 
Factor Supplies The base-period factor demands and the market clearing conditions (2.48) and (2.47) are used to calculate the benchmark factor supplies:

$$
\begin{aligned}
{\left[L_{r}^{(S)}\right] } & =\sum_{j}\left[L_{j, r}^{\operatorname{ind}(D)}\right] \\
{\left[K_{j, r}^{(S)}\right] } & =\left[K_{j, r}^{\operatorname{ind}(D)}\right]
\end{aligned}
$$

Export and Import Quantities The export agents' demand functions (2.34) and the market clearing equations (2.50) imply:

$$
\left[E X P_{g}^{(S)}\right]=\left[E X P_{g}^{\text {for }(D)}\right]=\left[Q_{g}^{\exp (D)}\right]
$$

while the import agents' demand functions (2.36) and the market clearing equations (2.51) imply:

$$
\left[F O R_{g}^{(S)}\right]=\left[F O R_{g}^{\operatorname{imp}(D)}\right]=\left[I M P_{g}^{(S)}\right]
$$

Investment Parameters and Quantities Of the variables in the endogenous investment allocation module (equations (2.25) - (2.32), with the addition of (2.33) for those industries we wish to have investment allocated endogenously, and the market clearing condition (2.49)), we have seen that the per-unit capital construction cost is normalised to 1 and the rental rates are derived from data on gross returns (from the IO table) and industry capital stocks. The remainder of the variables' benchmark equilibrium values have to be found numerically by virtue of the inclusion of (2.33) in the system. Assuming for simplicity that investment is to be allocated endogenously for all industries, the system consists of $9 J R+1$ equations in $12 J R+2$ unknowns where $J$ is the number of industries and $R$ is the number of regions. With the values of $\left[P_{j, r}^{K}\right],\left[P_{j, r}^{K, \text { new }}\right],\left[K_{j, r}^{(S)}\right]$, and $\left[I^{\mathbf{b} \$}\right]$ supplied by the data, $3 J R+1$ unknowns are removed and the system becomes square. All the unknowns are then solved for simultaneously in GAMS. We are specifically interested in the net rates of return and allocation of investment that is consistent with the endogenous allocation theory. Since we do not have data to support differences between regions in industry rental rates or depreciation rates in the benchmark equilibrium, the numerically obtained values for $\left[N R O R_{j, r}\right],\left[R_{j, r}^{K \text { new } / K \text { future }}\right]$, etc. do not vary over the regions. It is shown in appendix $H$ that despite this, the values of $\left[X_{j, r / \text { tot }}^{I, \mathbf{b} \$}\right]$ will vary over regions for a given industry $j$ insofar as its capital is regionally concentrated. Industry by 
region shares of total $\mathrm{NZ}$ capital stock $\left(\left[K S T O C K_{j, \mathbf{N Z}}\right]=\$ 470 b\right)$ were shown in table 2.9 while the shares of total investment $\left.\left(\left[I^{\mathbf{c}}\right]\right]=\$ 38.3 b\right)$ obtained through solving the system of equations are found in table 2.11. Table 2.12 lists the gross rates of return and the marginal efficiency parameter values derived from the BERL data, and the net rates of return and investment / future capital ratios that result from solving the system using those numbers. All the items of this latter table do not vary across regions for each given industry. The $\left[N R O R_{j, r}^{\text {future }}\right]$ column indicates expected net rates of return equalisation for most industries. Those that are concerned primarily with the provision of public services (GOVT, EDUC, and HEAL), and the housing proxy sector (OWND), are excluded from the endogenous investment allocation equation (2.33), so their expected net rates of return are calculated consistent with investment being allocated according to their capital shares.

Indirect Tax Rates The TAXES row of the raw input-output data includes sales taxes, import duties, and GST. Given import duty and GST rates, duty and GST are removed from the TAX row of the MRIO matrix to be handled separately, and what remains is general 'commodity tax'. This can be dealt with in a variety of ways; the simplest approach is to pro-rate the tax over the MRIO flows of products from all sources. For example, the tax flow in MRIO(TAX, $j, r)$ can be split so that the tax paid by industry $j$ in region $r$ on the flow of product $g$ (summed over all sources) is:

$$
\begin{aligned}
\operatorname{TAX}(g, j, r) & =\operatorname{MRIO}(\operatorname{TAX}, j-r) \\
& \times \frac{\sum_{x} \operatorname{MRIO}(g-x, j-r)+\operatorname{MRIO}(\operatorname{IMP}-g, j-r) \times \pi_{g, j}^{\text {ind }}}{\sum_{h}\left(\sum_{x} \operatorname{MRIO}(h-x, j-r)+\operatorname{MRIO}(\operatorname{IMP}-h, j-r) \times \pi_{h, j}^{\text {ind }}\right)}
\end{aligned}
$$

where $\pi_{g, j}^{\text {ind }}$ is a pro-rating switch set by the user: $\pi_{g, j}^{\text {ind }}=1$ means industry $j$ pays tax on product $g$, and not if $\pi_{g, j}^{\text {ind }}=0$. 


\begin{tabular}{|l|ccccc|c|}
\hline \multirow{2}{*}{ Industry by Region Shares of Total Investment $(\%)$} \\
\hline \multirow{2}{*}{ Industry } & \multicolumn{7}{|c|}{ Region } \\
\cline { 2 - 7 } & AKL & WLG & ONI & CAN & OSI & NZ \\
\hline AGRI & 0.1 & 0.0 & 1.8 & 0.2 & 0.9 & 3.0 \\
FOLO & 0.0 & 0.0 & 0.2 & 0.0 & 0.1 & 0.3 \\
FISH & 0.0 & 0.0 & 0.0 & 0.0 & 0.1 & 0.1 \\
MINE & 0.0 & 0.0 & 0.2 & 0.0 & 0.2 & 0.5 \\
OIGA & 0.0 & 0.2 & 1.9 & 0.0 & 0.0 & 2.1 \\
PETR & 0.0 & 0.0 & 0.2 & 0.0 & 0.0 & 0.2 \\
FDBT & 0.5 & 0.1 & 1.0 & 0.2 & 0.7 & 2.5 \\
TWPM & 0.7 & 0.2 & 0.8 & 0.3 & 0.3 & 2.3 \\
CHNM & 1.0 & 0.2 & 0.0 & 0.3 & 0.1 & 1.7 \\
METL & 0.6 & 0.1 & 0.5 & 0.2 & 0.2 & 1.7 \\
EQFO & 0.6 & 0.1 & 0.3 & 0.2 & 0.1 & 1.3 \\
UTIL & 0.5 & 0.3 & 0.8 & 0.1 & 0.2 & 1.9 \\
CONS & 0.6 & 0.2 & 0.7 & 0.2 & 0.3 & 2.1 \\
ACCR & 0.3 & 0.1 & 0.3 & 0.1 & 0.2 & 0.9 \\
CMIF & 5.3 & 2.4 & 2.0 & 1.3 & 0.8 & 11.8 \\
PROP & 1.8 & 0.5 & 1.4 & 0.6 & 0.5 & 4.7 \\
RBUS & 2.4 & 0.8 & 1.1 & 0.5 & 0.4 & 5.3 \\
GOVT & 2.1 & 3.3 & 2.3 & 0.8 & 0.7 & 9.2 \\
EDUC & 1.2 & 0.4 & 1.1 & 0.4 & 0.4 & 3.4 \\
HEAL & 0.7 & 0.3 & 0.8 & 0.3 & 0.3 & 2.3 \\
CUPE & 0.8 & 0.3 & 0.6 & 0.2 & 0.3 & 2.2 \\
OWND & 12.0 & 4.2 & 10.5 & 3.7 & 4.5 & 34.8 \\
WHOL & 1.3 & 0.2 & 0.5 & 0.3 & 0.2 & 2.5 \\
RETT & 0.9 & 0.3 & 0.9 & 0.3 & 0.4 & 2.8 \\
TRAN & 0.2 & 0.0 & 0.1 & 0.1 & 0.1 & 0.4 \\
\hline All & 33.6 & 14.2 & 30.0 & 10.4 & 11.9 & 100.0 \\
\hline
\end{tabular}

Table 2.11: Industry by Region Shares of Total Investment (\%) 


\begin{tabular}{|c|c|c|c|c|c|}
\hline Industry & $\beta_{j, r}$ & {$\left[G R O R_{j, r}\right](\%)$} & {$\left[N_{R O R}, r\right](\%)$} & {$\left[N R O R_{j, r}^{\text {future }}\right](\%)$} & {$\left[R_{j, r}^{K \text { new } / K \text { future }}\right](\%)$} \\
\hline AGRI & 30.2 & 18.7 & 10.4 & 11.1 & 8.1 \\
\hline FOLO & 92.7 & 34.4 & 25.9 & 11.1 & 9.4 \\
\hline FISH & 21.8 & 12.8 & 4.9 & 11.1 & 4.4 \\
\hline MINE & 10.1 & 35.7 & 21.5 & 11.1 & 19.6 \\
\hline OIGA & 10.1 & 35.7 & 27.3 & 11.1 & 16.2 \\
\hline PETR & 10.1 & 35.7 & 27.3 & 11.1 & 16.2 \\
\hline FDBT & 9.1 & 19.4 & 10.6 & 11.1 & 8.3 \\
\hline TWPM & 20.9 & 23.8 & 14.1 & 11.1 & 10.7 \\
\hline CHNM & 16.8 & 30.9 & 22.3 & 11.1 & 12.4 \\
\hline METL & 14.9 & 51.3 & 41.8 & 11.1 & 17.2 \\
\hline EQFO & 30.0 & 26.1 & 19.5 & 11.1 & 8.3 \\
\hline UTIL & 10.1 & 14.3 & 10.7 & 11.1 & 3.2 \\
\hline CONS & 139.9 & 70.1 & 57.2 & 11.1 & 14.0 \\
\hline ACCR & 34.1 & 20.0 & 14.5 & 11.1 & 6.3 \\
\hline CMIF & 8.2 & 50.3 & 35.7 & 11.1 & 26.0 \\
\hline PROP & 108.6 & 8.4 & 5.6 & 11.1 & 2.2 \\
\hline RBUS & 11.6 & 79.4 & 64.1 & 11.1 & 27.2 \\
\hline GOVT & 95.5 & 2.8 & -0.7 & 0.0 & 7.8 \\
\hline EDUC & 95.5 & 3.3 & 0.0 & 0.0 & 7.8 \\
\hline HEAL & 95.5 & 16.9 & 12.0 & 0.5 & 7.9 \\
\hline CUPE & 108.8 & 21.4 & 13.9 & 11.1 & 7.7 \\
\hline OWND & 100.0 & 5.0 & 3.4 & 0.0 & 7.7 \\
\hline WHOL & 34.1 & 31.9 & 22.5 & 11.1 & 11.3 \\
\hline RETT & 34.1 & 54.5 & 44.0 & 11.1 & 14.1 \\
\hline TRAN & 4.8 & 15.6 & 8.0 & 11.1 & 1.1 \\
\hline
\end{tabular}

Table 2.12: Investment Data and Solution Values 
Other possibilities allowed for are that the tax flows are split according to specified shares, or split to enforce certain tax rates, but for this implementation pro-rating is used, with the $\pi_{g, \bullet}^{a g e n t}$ switches set such that all domestic agents except exporters may pay commodity tax on all products except OWND, WHOL, and RETT. Only exporters of ACCR, CUPE, OIGA, PETR, WHOL, RETT, and TRAN pay commodity tax, and then only on the product they export. The purpose of this assumption is to capture any commodity tax paid by the foreign sector for these items not included in GST.

The TAX matrix is used to calculate the commodity tax rates as follows:

$$
\begin{aligned}
{\left[t_{g, r}^{Q, \mathbf{h s h}}\right] } & =\frac{\mathrm{TAX}(g, \mathrm{CON}-r)}{\operatorname{MRIO}(g, \mathrm{CON}-r)+\mathrm{MRIO}(\mathrm{IMP}-g, \mathrm{CON}-\mathrm{r})} \\
{\left[t_{g, j, r}^{Q, \mathbf{i n d}(\mathbf{Q})}\right] } & =\frac{\mathrm{TAX}(g, j, r)}{\operatorname{MRIO}(g, j-r)+\mathrm{MRIO}(\mathrm{IMP}-g, j-r)} \\
{\left[t_{g, j, r}^{Q, \mathbf{i n d}(\mathbf{K})}\right] } & =\frac{\mathrm{TAX}(g, \mathrm{INV}-j, r)}{\operatorname{MRIO}(g, \mathrm{INV}-j-r)+\mathrm{MRIO}(\mathrm{IMP}-g, \mathrm{INV}-j-r)} \\
{\left[t_{g}^{Q, \mathbf{g o v}}\right] } & =\frac{\mathrm{TAX}(g, \mathrm{GOV})}{\operatorname{MRIO}(g, \mathrm{GOV})+\mathrm{MRIO}(\mathrm{IMP}-g, \mathrm{GOV})} \\
{\left[t_{g}^{Q, \mathbf{e x p}}\right] } & =\frac{\mathrm{TAX}(g, \mathrm{EXP})}{\operatorname{MRIO}(g, \mathrm{EXP})}
\end{aligned}
$$

A consequence of the way these tax rates are calculated is that a given agent will pay the same tax rate on all products. There are slight differences in the tax rates faced by household and industry agents in different regions, but these are typically less than half a percentage point. The levels of the tax rates are not so important - the useful aspect is that in simulations they can be made to diverge across regions, for example if one region has an additional tax imposed while the others do not.

All duty and export subsidy rates are set at zero for this implementation. Although the GST rate consistent with the BERL data is $12.5 \%$, the values of $t_{g}^{G S T \text {, hsh }}$ and $t_{g}^{G S T, \mathbf{e x p}}$ are not all set to this level. Adjustments are made to reflect that GST is not paid on OWND, GOVT, and some components of CMIF, EDUC, HEAL, CUPE. Most exports are exempt from GST; the primary exceptions to this are ACCR and TRAN, although not all purchases of these are subject to GST. The GST rates in the model therefore are effective rates, as opposed to the legislated rate of the time. ${ }^{65}$

\footnotetext{
${ }^{65}$ As no simulations were undertaken in this thesis involving changes to commodity tax or effective GST rates, their values are not reported here for brevity. They are available from the author upon request.
} 
Direct Tax Rates The raw input-output data records gross payments to factors (before income taxes are deducted). To obtain tax-paid labour income measures, we adopt the average income tax rate used in the BERL impact of immigration report (Nana et al. 2009, p. 61) of $21.1 \%$. While the BERL figure would include direct tax on unearned income, we place the tax entirely on labour income for simplicity.

Time Endowments and Leisure The idea was introduced in section 2.3.1 that the time endowment can be measured in terms of the number of Full-Time Equivalents (FTEs) available from the working age population. This will include those classified as unemployed or non-labour force as well as the employed. ${ }^{66}$ Data concerning these demographic and labour market measures will be introduced in Chapter 4. At this stage, given estimates of the working age population for our five regions, we can convert these to numbers of FTEs. Statistics NZ data measures employment by industry and region in terms of persons and FTEs, so we can derive average conversion factors from persons to FTEs. The factors vary across regions and industries but average around 1.1 persons to $1 \mathrm{FTE}^{67}$

Labour demand and supply are not measured in this core model in terms of FTEs, but rather units from the IO table. For example, the total of the WAGES row of the IO table is 70121 . Since the nominal wage rate is set at 1 in the benchmark equilibrium, we interpret the value as a quantity - 70121 units of labour demanded and supplied. A unit of labour supply, referred to here as an IOunit, is the quantity of person-hours required to earn $\$ 1$ million (since the IO table is in these units). For consistency between these labour measures and the time endowment, especially to facilitate equation (2.6), the time endowment needs to be measured in IOunits. After converting the working age population from persons to FTEs, we need to convert this to IO units. Using the Statistics NZ data on employment FTEs and the WAGES row of the IO table, we obtain conversion factors from IOunits to FTEs. Converting the working age population measured in FTEs to IOunits by dividing through, the value of the time endowment for each regional household agent is shown in table 2.13 .

Having determined values of $\mathbf{h s h}_{r}$ 's time endowment and labour supply with consistent

\footnotetext{
${ }^{66}$ The implication of this definition therefore is that these groups are undertaking FTEs of activities that somehow generate utility so the meaning of leisure is quite broad, including child-raising and study. The working age population will have much more physical time than the time endowment of FTEs — on average 138 hours per person per week — which cannot be used for any utility-enhancing activity.

${ }^{67}$ For the full list of conversion factors, see appendix J.
} 


\begin{tabular}{|l|c|}
\hline Region & Time Endowment \\
\hline AKL & 34257 \\
WLG & 11926 \\
ONI & 29047 \\
CAN & 10181 \\
OSI & 12189 \\
\hline NZ & 97600 \\
\hline
\end{tabular}

Table 2.13: Regional Time Endowment (IOunits)

units, we calculate the benchmark equilibrium level of leisure consumption using equation $(2.6)$ :

$$
\left[N_{r}^{\mathbf{h s h}(D)}\right]=\left[T I M E_{r}\right]-\left[L_{r}^{(S)}\right]
$$

As neither the level of consumption nor the level of leisure depends on the level of utility, we are free to choose this arbitrarily as 100 in the benchmark equilibrium.

Average Propensities to Consume and Save The average consumption propensities are calculated as consistent with equation (2.1) while the saving propensities are according to equation $(2.2)$ :

$$
\begin{array}{ll}
{\left[A P C_{r}^{w}\right]=100 \times \frac{\left[S P N_{r}^{\mathbf{c}}\right]}{\left[I N C_{r}^{w, \mathbf{c}}\right]}} & \forall r \in N Z R E G \\
{\left[A P S_{r}^{w}\right]=100-\left[A P C_{r}^{w}\right]} & \forall r \in N Z R E G
\end{array}
$$

The resultant benchmark equilibrium values of the saving propensities are shown in table 2.14 .

Export Demand Parameters The demand curves for exports of product $g$ were specified in equation (2.44). This equation involves two parameters for each product which need to be assigned values for the benchmark equilibrium. The first is the absolute value of the export demand elasticity $\varepsilon_{g}^{E X P}$. For this implementation of the JENNIFER model these are all set at 4.0 except that for AGRI, which is set at 3.0. The authors of MONASH (Dixon \& Rimmer 2002) make a case for assuming elasticities of these sorts 


\begin{tabular}{|l|c|}
\hline Region & Propensity to Save (\%) \\
\hline AKL & 22.95 \\
WLG & 20.28 \\
ONI & 24.25 \\
CAN & 21.71 \\
OSI & 20.41 \\
\hline NZ & 22.57 \\
\hline
\end{tabular}

Table 2.14: Average Propensities to Save (\%)

of magnitude, much lower than those used in ORANI. While lower elasticities suggest more market power (hence the lower value for AGRI here), they also suggest difficulty in market access due to transport costs etc. In New Zealand's case the market access issue is probably more significant and so reasonably low elasticities are justified although the country has very little influence on world prices. These values are also in the range found in the RPEP papers and Stroombergen (1986) of (negative) 2.0 to 6.0. The advantage of being conservative in setting the export demand elasticities is that shocks should not cause unjustifiably large changes in the composition of exports and the terms of trade, which can lead to the model reporting unrealistic welfare effects.

The second item for which we need a benchmark equilibrium value is the coefficient $\psi_{g}^{E X P}$, the demand curve height - in model experiments it can be shocked to simulate changes in foreign demand for domestic products. The values of this coefficient that are consistent with (2.44) in the benchmark equilibrium are calculated by:

$$
\left[\psi_{g}^{E X P}\right]=\frac{\left[P_{g}^{E X P, \mathbf{f} \$, \mathbf{f o r}}\right]}{\left[E X P_{g}^{\text {for }(D)}\right]^{-1 / \varepsilon_{g}^{E X P}}}
$$

Other Variables The remainder of the variables' benchmark equilibrium values can be established using the aggregate measures and indices defined in section 2.3.12 along with the values already determined above. In the base-period, all real and nominal measures coincide, so for example to evaluate the total base-period level of income tax received by government, we use equation (2.42) to find:

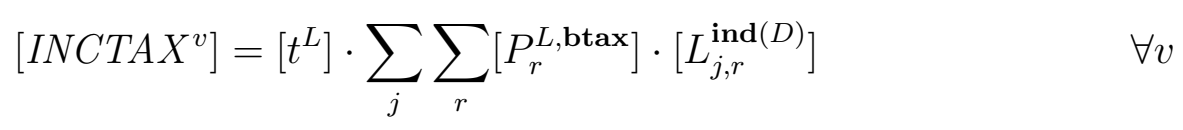


For the same reason, all the price indices $C P I, I P I$, etc. listed in section 2.3.12 are equal to one in the base-period.

\section{Calibration of the Demand Functions}

Having established a benchmark solution to the model, values of the demand function parameters that are consistent with that solution can now be determined. This is easily done with analytical solutions for the parameters of demand functions obtained from optimisation problems involving Leontief, Cobb-Douglas, or CES functions. For userspecified substitution elasticities, the program calculates the coefficients according to the formulae set out in appendix K. For example, the household product demand functions (2.9) derived from expenditure minimisation subject to a Cobb-Douglas mixing function are:

$$
Q_{g, r}^{\mathbf{h s h}(D)}=\frac{Q_{r}^{\mathbf{h s h}(D)}}{v_{r}^{Q, \mathbf{h s h}}} \cdot \prod_{h}\left(\frac{a_{g, r}^{Q, \mathbf{h s h}} \cdot P_{h, r}^{Q, \mathbf{h s h}}}{a_{h, r}^{Q, \mathbf{h s h}} \cdot P_{g, r}^{Q, \mathbf{h s h}}}\right)^{a_{h, r}^{Q, \mathbf{h s h}}}
$$

The program interprets the setting $\sigma_{r}^{Q, \text { hsh }}=1$ as specifying the above demand function and calibrates it according to:

$$
\begin{aligned}
a_{g, r}^{Q, \mathbf{h s h}} & =\frac{\left[P_{g, r}^{Q, \mathbf{h s h}}\right]\left[Q_{g, r}^{\mathbf{h s h}(D)}\right]}{\left[Q_{r}^{\mathbf{h s h}(D)}\right]} \\
v_{r}^{Q, \mathbf{h s h}} & =Q_{r}^{\mathbf{h s h}(D)} \cdot\left(\prod_{g}\left(Q_{g, r}^{\mathbf{h s h}(D)}\right)^{a_{g, r}^{Q, \mathbf{h s h}}}\right)^{-1}
\end{aligned}
$$

The distribution parameters (the $a$ 's) are set equal to the expenditure shares and the scale parameter (the $v$ ) is set to ensure the agent is on the constraint boundary. This is the usual procedure to calibrate functions derived from Leontief or Cobb-Douglas technology. Calibration for the general CES case is similar but more complicated since the distribution parameters depend on the substitution elasticity.

As a double-check, the program also calibrates the functions numerically. Any failure to do so will indicate a coding error or will be an early warning of convergence difficulties. The method of numerical calibration follows that of solving any square system of nonlinear equations. The equation set consists of the demand functions for a given agent and 
a constraint that the distribution parameters add up to 1 . This last equation effectively determines the scale parameter. ${ }^{68}$

For the purposes of this thesis, a number of sets of elasticities were prepared based on different behavioural assumptions. BERL has provided a set of suggested domestic/imported substitution elasticities which has been adapted for the 25-industry implementation here. These elasticities have also been used, with modifications, to describe substitutability between domestic regional versions of products. This primary set of source substitution elasticities is shown in table 2.15. A notable feature is that the elasticities for many of the products on the left-hand side of the table, mainly being primary and manufactured goods, are higher than those on the right-hand side, which are primarily service products. This reflects the idea that services tend to be more closely associated with the location where they are produced and used. It is reasonable to assume that the substitution elasticities between domestic varieties are higher than between the domestic and imported varieties. ${ }^{69}$ Table 2.16 presents a suggested set of elasticities for use when running short-run simulations. The vector of source substitution elasticities of table 2.15 are referred to there as $\vec{\Sigma}$. The substitution elasticities between regional varieties are $\vec{\Sigma}$, scaled-up by a common factor. To make an assumption that the government is less willing or able to substitute between varieties than other agents, lower scaling factors were applied for their source choice. The remainder of the elasticities reflect the short-run assumption: no substitution between product types is possible except by households, who have some limited ability to substitute between them as well as between overall consumption and leisure. Firms within industries have only limited possibilities to shift between material and factor inputs, and between factor types. For long-run simulations, all the elasticities apart from those linked to $\vec{\Sigma}$ are increased to 1.0 - i.e. Cobb-Douglas functions are used. $^{70}$

\footnotetext{
${ }^{68}$ In the case of demand functions derived from utility maximisation, the $v$ parameters have no role except to scale the utility function to the arbitrary level set in the benchmark equilibrium.

${ }^{69}$ See for example the comments made on this matter in Jones \& Whalley (1989, p. 385).

${ }^{70}$ The non- $\vec{\Sigma}$ elasticities are generally similar to those used by ORANI and the RPEP models. Dixon et al. (1982, p. 189) cite empirical evidence for capital/labour substitution of 0.5 in the short-run and 1.0 in the long-run. Recent empirical work for New Zealand broadly supports this notion. Regressions reported by Tipper (2011) indicate capital/labour substitution elasticities by broad industry group of 0.30 (primary), 0.55 (goods-producing), and 0.44 (services) although in the goods-producing case the Cobb-Douglas specification could not be rejected. These regressions included a one-year lag variable and the elasticities were termed long-run although they are closer in meaning to our short-run definition.
} 
Source Substitution Elasticities

\begin{tabular}{lcllc}
\hline Industry & Elasticity & & Industry & Elasticity \\
\cline { 1 - 2 } \cline { 5 - 5 } AGRI & 2.2 & & UTIL & 2.7 \\
FOLO & 2.8 & & CONS & 1.5 \\
FISH & 2.2 & & ACCR & 2.0 \\
MINE & 2.8 & & CMIF & 1.9 \\
OIGA & 0.0 & & PROP & 1.9 \\
PETR & 4.0 & & RBUS & 1.9 \\
FDBT & 2.5 & & GOVT & 1.5 \\
TWPM & 2.9 & & EDUC & 1.5 \\
CHNM & 1.9 & & HEAL & 1.5 \\
METL & 2.8 & & CUPE & 1.9 \\
EQFO & 2.9 & & OWND & 0.0 \\
\cline { 1 - 1 } & & & WHOL & 2.0 \\
& & & RETT & 2.0 \\
& & & TRAN & 2.0 \\
\hline
\end{tabular}

Table 2.15: Source Substitution Elasticities

A key underlying assumption of this set of elasticities is that for a given industry there is no variation in technology across regions except as required by adjustments to the MRIO matrix. All the parameters of the CES functions (the $a$ 's, $v$ 's, and $\sigma$ 's) are identical at the sourcing levels for each industry $j$ except for local products. ${ }^{71}$ There is some slight variation at the product-type level due to the RAS adjustment but recall the input intensity is preserved across industries. There is a similar lack of variation in the demand parameters across the household agents. This uniformity reflects the information theoretic approach whereby information gain is minimised when assumptions are made. Simulation results are therefore driven by those transparent assumptions (and the structure implied by the input-output table and regional shares) rather than differences

Tipper also estimated elasticities at a more disaggregated industry level, and it would be interesting to investigate the effect of using these elasticities in our simulations. This task has been put aside for future research.

${ }^{71}$ If $g$ is a local product then the associated $a$ parameter for $g$ from region $x$ will be 1 for industry $j$ from region $r$ when $x=r$ and zero otherwise. 
Short-run Substitution Elasticities

\begin{tabular}{llc}
\hline \multicolumn{2}{c}{ Households } & \\
Level & Choice between... & Elasticity \\
\hline 1 & consumption \& leisure & 0.5 \\
2 & product types & 0.5 \\
3 & domestic \& imported & $\vec{\Sigma}$ \\
4 & regional sources & $\frac{3}{2} \vec{\Sigma}$ \\
\hline & & \\
& $\quad$ Government & \\
Level & Choice between... & Elasticity \\
\hline 1 & product types & 0.0 \\
2 & domestic \& imported & $\frac{3}{4} \vec{\Sigma}$ \\
3 & regional sources & $\vec{\Sigma}$ \\
\hline
\end{tabular}

Industries - current production

\begin{tabular}{|c|c|c|c|c|c|}
\hline Level & Choice between... & Elasticity & Level & Choice between... & Elasticity \\
\hline 1 & consumption \& leisure & 0.5 & 1 & materials \& factors & 0.5 \\
\hline 2 & product types & 0.5 & $2=$ & product types & 0.0 \\
\hline 3 & domestic \& imported & $\vec{\Sigma}$ & $2=$ & labour \& capital & 0.5 \\
\hline 4 & regional sources & $\frac{3}{2} \vec{\Sigma}$ & 3 & domestic \& imported & $\vec{\Sigma}$ \\
\hline & & & 4 & regional sources & $\frac{3}{2} \vec{\Sigma}$ \\
\hline
\end{tabular}

\begin{tabular}{llc}
\multicolumn{3}{c}{ Industries - capital formation } \\
Level & Choice between... & Elasticity \\
\hline 1 & product types & 0.0 \\
2 & domestic \& imported & $\vec{\Sigma}$ \\
3 & regional sources & $\frac{3}{2} \vec{\Sigma}$ \\
\hline
\end{tabular}

Exporters

\begin{tabular}{llc} 
Level & Choice between... & Elasticity \\
\hline 1 & regional sources & $\frac{3}{2} \vec{\Sigma}$ \\
\hline
\end{tabular}

Table 2.16: Short-run Substitution Elasticities

in demand parameters generated by the application of more complex techniques such as gravity calculations and location quotients.

\subsubsection{Model Closure}

We saw in section 2.4.1 that the model is closed - i.e. turned into a square system - by adding equations that effectively constrain some variables to be equal to given numbers. In GAMS the user specifies for which variables are such exogenising equations added to the system. The closure choice is important because simulation results can only be interpreted with respect to that choice. A typical short-run closure for the model is shown in table 2.17. The closure is interpreted as short-run because the endowments of time and capital are fixed - these cannot move between regions, nor between industries in the case of capital, to seek out the best rate of return. The notation used in table 2.4 is used again here $-J$ is the number of industries and $R$ is the number of regions in the model. The 
number of industries subject to exogenous investment allocation is potentially up to $J{ }^{72}$ For our simulations there are only four: the GOVT, EDUC, HEAL, and OWND industries. The rest of the items are commonly included in the standard short-run closure list of CGE models as usually there is no modelling of the determination of tax and depreciation rates and the levels of real investment and government consumption expenditure. ${ }^{73}$ The small country assumption naturally leads to exogenous world prices and export demand curves, and the comparative-static nature of the model implies saving needs to be exogenously determined. Many variations of the closure are possible depending on the policy question. As with ORANI, alterations to the closure are made by swaps of variables between the exogenous and endogenous lists. The trade balance could be made exogenous, swapped with some component of domestic absorption, for simulations where no deterioration of the current account is desired. The level of real aggregate investment could be made endogenous by swapping it for the economy-wide average expected rate of return $\Omega$ so that simulations return the level of investment consistent with no change (or an exogenously specified change) in the latter variable. The closure treatment of investment also has implications for the determination of aggregate savings and macroeconomic balance, to which we now turn.

\footnotetext{
${ }^{72}$ When adding the exogenous and endogenous equations together to obtain a total equation count for the core model, $J \times R$ should be subtracted to avoid double-counting since each equation that sets $X_{j, r / \text { tot }}^{I, \mathbf{b} \$}$ exogenous replaces one equation from (2.33). Table 2.17 shows the maximum number of exogenous variables under this closure including all $J \times R$ investment shares. This differs from the number of unexplained variables referred to in footnote 43 , which was calculated based on the assumption that all industries were subject to endogenous investment allocation. That is, $J \times R$ variables were counted as explained by (2.33) and therefore excluded from the calculation in footnote 43.

${ }^{73}$ Powers of taxes $(1+t)$ are usually set exogenous in CGE models rather than tax rates for computational reasons. Simulations often involve increasing some tax rates from zero to some positive number; the percentage change in the tax rates are undefined in this case while the percentage change in the tax powers are not, so it is the latter that are useful for computing model solutions. Specifying a simulation is also easier with powers of tax since an $x \%$ increase in the power of a tax is equivalent to an $x$ percentage point increase in the tax rate. With the separate treatment of GST in equation (2.59), it can be seen that only one of $\left(1+t_{g, r}^{Q, \mathbf{h s h}}\right)$ and $\left(1+t_{g}^{G S T, \mathbf{h s h}}\right)$ can be shocked in a single simulation.In the event that both commodity tax components needed to be shocked, either a new GST rate could be declared or equation (2.59) could be altered to become:
}

$$
P_{g, s, r}^{Q, \mathbf{h s h}} \cdot Q_{g, s, r}^{\mathbf{h s h}(D)}=P_{g, s}^{Q} \cdot\left(1+t_{g, r}^{Q, \mathbf{h s h}}+1+t_{g}^{G S T, \mathbf{h s h}}-1\right) \cdot \breve{Q}_{g, s, r}^{\mathbf{h s h}(D)}
$$




\begin{tabular}{|c|c|c|c|}
\hline \multicolumn{2}{|c|}{ Exogenous Variables } & \multicolumn{2}{|c|}{ Dimension } \\
\hline Symbol & Description & General case & $J=25, R=5$ \\
\hline$K_{j, r}^{(S)}$ & capital stocks & $J R$ & 125 \\
\hline$T I M E_{r}$ & time endowments & $R$ & 5 \\
\hline$X_{j, r / \text { tot }}^{I, \mathbf{b} \$}$ & real investment shares & $J R$ & 125 \\
\hline$P_{g}^{F O R}$ & c.i.f. import prices & $J$ & 25 \\
\hline$A P S_{r}^{\text {atax }}$ & savings propensities & $R$ & 5 \\
\hline$\delta_{j, r}$ & depreciation rates & $J R$ & 125 \\
\hline$t^{L}$ & labour income tax rate & 1 & 1 \\
\hline $1+t_{g, \bullet}^{Q, a g e n t}$ & powers of commodity tax & $2 J^{2} R+J R+2 J$ & 6,245 \\
\hline $1+t_{g}^{G S T, \text { agent }}$ & powers of GST & $2 J$ & 50 \\
\hline $1+t_{g}^{F O R}$ & powers of import duty & $J$ & 25 \\
\hline $1+t_{g}^{E X P}$ & powers of export subsidy & $J$ & 25 \\
\hline$\psi_{g}^{E X P}$ & levels of export demands & $J$ & 25 \\
\hline$X_{I / t o t}^{G D P, \mathbf{b} \$}$ & $\begin{array}{l}\text { investment share of real } \\
\text { GDP }\end{array}$ & 1 & 1 \\
\hline$X_{G / t o t}^{G D P, \mathbf{b} \$}$ & $\begin{array}{l}\text { government consumption } \\
\text { share of real GDP }\end{array}$ & 1 & 1 \\
\hline TOTAL & & $\begin{array}{l}3+2 R+8 J+ \\
4 J R+2 J^{2} R\end{array}$ & 6,963 \\
\hline
\end{tabular}

Table 2.17: A Short-run Closure

\subsubsection{Walras' Law and Macro Balance}

When specifying the closure, or making changes to it, careful thought has to be given to the implications of Walras' Law. To prevent the model from being over-identified, the equilibrium condition for the saving market was excluded from the set of market clearing equations listed in section 2.3.9. On one side of the saving market stand the household and government agents with funds available for lending. On the other side stand the investor agent demanding finance for capital formation and the foreign agent demanding finance for their trade deficit (a domestic trade surplus). ${ }^{74}$ If all the other markets are

\footnotetext{
${ }^{74}$ If the government runs a budget deficit they are on the demand side of the market, and if the foreign sector runs a trade surplus (a domestic trade deficit) they are on the supply side.
} 
in equilibrium, then the equation system automatically implies that the saving market is in equilibrium too, for any vector of equilibrium prices. Evidence that Walras' Law is satisfied by the JENNIFER model can be found in appendix L.

Walras' Law can be seen as the source of the macroeconomic balancing identity domestic saving $\equiv$ investment + trade balance

Since Walras' Law only applies at the current equilibrium price vector, the identity is only satisfied for nominal measures. That is,

$$
S A V^{\mathbf{c} \$} \equiv I^{\mathbf{c} \$}+T R D B A L^{\mathbf{c} \$, \mathbf{d} \$}
$$

Once one side of the identity is determined, the other side is determined as well. When we make a closure assumption, we have to decide which component will adjust to the others. The standard short run closure above, for example, implicitly assumes that changes in the trade balance will be reflected in changes in nominal investment. The exogenous saving propensities and components of fiscal policy lock down domestic saving, so changes in the trade balance lead to offsetting changes in nominal investment. With real investment also fixed, either in absolute terms or as a proportion of GDP, the offsetting adjustments will manifest in changes in construction costs. Alternatives include making the economy-wide average saving propensity endogenous (and locking down the regional propensities relative to that) so that domestic private saving reflects changes in the trade balance, or fixing the trade balance to force nominal investment to adjust to changes in saving (perhaps accompanied by a swapping-in of $\Omega$ so that real investment also adjusts).

Note that since Walras' Law only holds at current equilibrium prices, the measures of real GDP using the different measurement approaches will typically diverge since the price movements that satisfy the identity do not flow through consistently into the deflator indices associated with each measurement method.

\subsubsection{Obtaining Solutions to the Model}

There are two key approaches to solving CGE models written as a system of equations $\mathbf{F}(\mathbf{V})=0$ :

1. using a numerical algorithm for solving systems of ordinary differential equations (ODEs) such as the Euler Method 
2. using a numerical algorithm for solving systems of non-linear equations such as the Newton Method

The first method emerged from the contribution of Johansen and is mainly associated with the Australian / CoPS / GTAP school of CGE modelling who primarily use GEMPACK to solve their models. The main characteristic of this method is that the model is typically written down and coded in linearised form, and the solution consists of percentage changes in the variables. For details on this method see for example Dixon et al. (1992).

The second method comes from the American / World Bank / IFPRI school of CGE modelers who mainly use GAMS for model solving. The models are usually expressed and programmed in levels of variables, such that the model solution is also in the levels. This is the method followed for solving the JENNIFER model.

Provided an initial solution $\mathbf{V}_{0}$ to the square system $\mathbf{D}(\mathbf{V})=0$ (equation (2.119)), the Newton algorithm involves iterations according to:

$$
\mathbf{V}_{n+1}=\mathbf{V}_{n}-\mathbf{J}_{n}^{-1} \mathbf{D}\left(\mathbf{V}_{n}\right)
$$

where $\mathbf{J}$ is the Jacobian matrix of first partial derivatives:

$$
\mathbf{J}_{n}=\frac{\partial}{\partial \mathbf{V}_{n}^{\prime}} \mathbf{D}(\mathbf{V})
$$

GAMS compiles the JENNIFER model as a Constrained Non-linear System (CNS) and computes its solution using the PATH solver. This means that bounds are put on $\mathbf{V}$, an initial solution $\mathbf{V}_{0}$ is specified, and the solver performs pivots to solve the system of linear equations:

$$
\mathbf{J}_{n}\left(\mathbf{V}_{n+1}-\mathbf{V}_{n}\right)+\mathbf{D}\left(\mathbf{V}_{n}\right)=0
$$

The solver terminates after $N+1$ iterations if

$$
\begin{aligned}
& \quad \mathbf{J}_{n}\left(\mathbf{V}_{n+1}-\mathbf{V}_{n}\right)+\mathbf{D}\left(\mathbf{V}_{n}\right)<\epsilon \\
& \text { or } N+1>\text { Iterlim }
\end{aligned}
$$

where $\epsilon$ is the convergence tolerance level and Iterlim is the iteration limit. The default PATH values are $\epsilon=1 \times 10^{-6}$ and Iterlim $=10000$. PATH uses an advanced merit function 
to guide the iterations so convergence should occur well before the default iteration limit is reached. ${ }^{75}$

Although the field of CGE modelling developed along the two delineated strands noted above, recent years have seen some convergence in the competing schools. Johansen's method of model-solving using a single matrix inversion was appropriate given the level of computer technology of his time. With modern, fast computers iteration is not so costly so accurate solutions can be easily obtained with either method. When running simulations, the changes in variables are our primary interest rather than their levels. GEMPACK has the advantage of providing percentage changes immediately; for GAMS to do so, additional coding is required. The object-oriented approach used in the coding of JENNIFER removes much of that additional burden. ${ }^{76}$ Since GEMPACK is based on solving models with a single matrix inversion, iterative methods such as Euler require the program to be told how to update the database at each step of the algorithm. Recent versions of GEMPACK allow model equations to be input in levels form which obviates the need for such updating statements. For large and complex models, GAMS iterations require more physical memory and take longer because of the evaluation of the structural model at each step, something which GEMPACK does not do. For that reason however, GAMS is able to solve a wider range of model types, such as those that involve non-homogeneity or discontinuities in the demand functions and those that require optimisation of an objective function.

There is therefore no superior approach to CGE modelling - the approach depends on the purpose of the modelling and the requirements of the user. Where flexibility and transparency are useful, such as during model development, GAMS has a substantial advantage. For the deployment of a large standardised model and timely production of

\footnotetext{
${ }^{75}$ See GAMS Development Corp. (2008) for details of the PATH solver. GAMS has the advantage of double-precision accuracy so rounding errors do not accumulate as quickly as in standard 32-bit GEMPACK (such that accuracy is only to 5-6 significant figures). Very small numbers therefore do not create convergence problems although in economic modelling the difference between such tiny numbers and zero are unimportant. See Centre of Policy Studies (2010) for details on numerical accuracy in GAMS and GEMPACK.

${ }^{76}$ Essentially, the variable list was treated as a program object that different parts of the code could interact with. Then for example, an instruction of calculating percentage changes was interacted with the list. This meant that new variables could be added to the list during program development without the need for additional manual inputting of code for the percentage change of each new variable.
} 
results, which in turn can be easily interpreted by users not involved in the programming of the model, GEMPACK is the package of choice. ${ }^{77}$

\subsection{Simulations Using the JENNIFER Model}

Once the model has been calibrated by establishing a benchmark equilibrium solution and the parameters of the demand functions, and a closure assumption has been chosen, the model is ready for running simulations. ${ }^{78}$ A shock is applied to the exogenous variables either by direct replacement (e.g. replacing the GST rate of $12.5 \%$ with the value $15 \%$ ) or expressing as a change or percentage change from the benchmark value. GAMS then solves the model as discussed above using the benchmark equilibrium as the starting point for the algorithm and calculates the changes and percentage changes of all the variables from their benchmark equilibrium values.

The JENNIFER model is deployed in GAMS in such a way as to maximise its flexibility. Each program component runs from a command line that takes items such as the number of industries and regions, the chosen sets of elasticities, closure assumptions, and exogenous shocks as options. New versions of the model with more or less industries and regions can be implemented easily with the creation of two auxiliary files: one specifying the sets (of industries, regions, etc.) and the other specifying the data (mappings from the raw data to the model industries and regions, etc.) ${ }^{79}$ Similarly, new elasticities, closures, and shocks can be specified by creation of small text files and referred to in the command line when running program components. The output of each simulation is saved in a

\footnotetext{
${ }^{77}$ Some of the user-friendly aspects of GEMPACK can be emulated by applications in GAMS. Rutherford's MPSGE system is one example. (See Rutherford 1999, Paltsev 1999)

${ }^{78}$ Three different diagnostic tests can also be run at this point. They are tests for replication, convergence, and nominal homogeneity. The replication test runs the model with no shock to check for system squareness and all percentage changes should be zero (with allowance for rounding errors of the order $10^{-6}$ ). The convergence test perturbs the algorithm starting point but still applies no shock to check that the same solution is obtained within its neighbourhood. Runs of the homogeneity test change the value of the numeraire and checks that all domestic prices and current price measures change proportionately, but there is no change in real measures or foreign prices. It was checked that the JENNIFER model passed these tests prior to running any simulations.

${ }^{79}$ As such, files for 6-industry, 5-region and 6-industry, single-region versions were prepared during model development.
} 
separate database and is portable to Microsoft Excel, Matlab, and even GEMPACK. ${ }^{80}$ The capability of GAMS to produce text files containing results is exploited to generate report tables in $\mathrm{HT}_{\mathrm{E}} \mathrm{X}$ mark-up ready for inclusion in documents that are typeset using that system, such as this thesis.

\subsubsection{An Illustrative Application}

To demonstrate the usefulness of the JENNIFER model relative to the national models currently in use within New Zealand, the results of a region-specific supply-side shock are discussed in this section. The specified shock simulates the short-run effects of an unexpected event that causes the destruction of, or otherwise renders inoperative, a portion of the currently installed capital stock of the Wellington region. ${ }^{81}$ It is assumed that the Central Business District (CBD) of Wellington is the area most affected, so industries concentrated within it are disproportionately affected - see the table below.

\begin{tabular}{lcclc}
\multicolumn{4}{c}{ Shock to WLG Capital Stock } \\
\cline { 1 - 2 } Industry & $\%$ change & & Industry & $\%$ change \\
\cline { 1 - 2 } \cline { 5 - 5 } UTIL & -1 & & EDUC & -10 \\
CONS & -1 & & HEAL & -10 \\
ACCR & -5 & & CUPE & -5 \\
CMIF & -10 & & OWND & -5 \\
PROP & -10 & & WHOL & -1 \\
RBUS & -10 & & RETT & -5 \\
GOVT & -15 & & TRAN & -10 \\
\hline
\end{tabular}

With the elasticities and closure as specified in tables 2.16 and 2.17 respectively, the model is solved and the results are shown in the sim101 column of the tables found in appendix $\mathrm{O}$.

\footnotetext{
${ }^{80}$ The data is saved as a GDX, a native format to GAMS, but there are conversion tools available in the public domain.

${ }^{81}$ The source of the shock remains unspecified. It could be imagined that it is due to a natural disaster or terrorist attack but such events would entail many other effects which we wish to ignore here for simplicity. The results do not take into account any effects of insurance pay-outs or government spending in preparation for rebuilding, for example.
} 
The effect of the unexpected event on the region's total capital stock is a fall of about $8 \%$, equivalent to a loss of about $1 \%$ of the national stock (table O.1). Due to the short-run immobility of capital, the sudden shortage of capital causes Wellington rental rates to rise (table O.18). With limited opportunities to replace the lost capital with labour or materials, output falls and output (basic) prices rise (tables O.6 and O.16). ${ }^{82}$ All these effects are concentrated within those industries most severely affected by the shock. Those industries that do not lose capital see an expansion of output although not enough to offset the output reduction in the service industries. There is an accompanying expansion of employment in those industries (table O.10). Interestingly, employment also expands in GOVT and the local industries (see table 2.10), and their output does not fall by as much and output prices do not rise by as much as in CMIF, PROP, RBUS, and TRAN. In the case of GOVT, this is because Wellington has such a large share of the industry and it also faces no competition from imports. Similar reasoning holds for the local industries. Agents are not able to substitute towards varieties from other regions and imports of these services are insignificant.

The higher capital rental rates translate to higher net rates of return because construction costs are virtually unaffected by the shock (tables O.19 and O.20). ${ }^{83}$ The endogenous investment allocation mechanism leads to an interesting, but somewhat extreme effect on the pattern of investment: the vast majority of reallocated investment goes to the transport industry in Wellington, such that investment in this industry increases more than $400 \%$ (tables O.11 and O.12). The large increases in the rates of return to the capital of GOVT, EDUC, HEAL, and OWND should be ignored because these industries are excluded from endogenous investment allocation. This leaves TRAN in WLG with an increase in net rate of return of $29 \%$, well above that of the industry with the next highest increase, RBUS, whose rate of return increases $18 \%$. That the value of $\beta_{j, r}$ for $j=$ TRAN is the lowest out of all industries also has a role in this producing result — see section 2.3.4 and appendix $\mathrm{H}$.

The macroeconomic results are unremarkable (tables 0.28 and 0.29); as expected

\footnotetext{
${ }^{82}$ In all simulation results reported throughout this thesis, 'output' is used in the sense of 'gross real output'. Results for regional output, for example, correspond to $O U T P U T_{r}^{\mathbf{b} \$}=\sum_{g}\left[P_{g, r}^{Q}\right] \cdot \breve{Q}_{g, r}^{(S)}$.

${ }^{83}$ Looking at table 2.6, we see that the service industries subjected to the majority of the shock do not have a very significant role in capital formation. Tables similar to O.19 and O.20 for the price of new capital units are not given in appendix $\mathrm{O}$ because most of the numbers therein are zero to one decimal place and otherwise no more than $0.2(\%)$.
} 
from a supply-side shock, GDP falls and there is an increase in the price level, but the effects are small at the national level because the shock only directly affects one region. There is a slight worsening of the trade balance due to the change in the pattern of trade. ${ }^{84}$ Nationally, some substitution occurs towards the domestic variety of goods that are otherwise key imports (table O.14), and there is a slight expansion in key exports (table O.13), but these are more than offset by decreases in exports from those industries disproportionately affected by the shock. Notice for example that CMIF exports falls $6 \%$ while imports of the same increases $2 \%$.

Those relatively benign macroeconomic effects hide a significant distributional impact across the regions. Wellington bears the brunt of the short-run impact. While there is virtually no change in Wellington employment (table O.10), the nominal wage rate falls $3 \%$ (table O.21). This suggests that the labour supply curve is relatively steep at the benchmark equilibrium level of labour supply. Higher product purchase prices also erode the real wage rate in Wellington (tables O.22 and O.23). Since the household agent is assumed to receive capital returns only from in-region capital, the Wellington agent sees a drop in income from both wage and profit sources (table O.24). To maintain consumption spending as a share of nominal income, the household agent in Wellington reduces their level of saving by $12 \%$ (table O.26).

The other regions are only affected by the shock indirectly, through their interdependence with Wellington, and through feedbacks from the macroeconomy (such as trade effects). The higher cost of inputs from Wellington leads to higher basic prices and lower output in the other regions (tables O.2 and O.3). The effect on other regions of lower product demand in Wellington is offset by additional export demand for their products. The household agents in the regions outside Wellington see a slight dip in the purchasing power of their wage as lower demand for their labour manifests as lower wage rates. Since they are assumed to not own any Wellington capital, their real income is mostly unaffected. There is a slight decrease in their spending and increase in their saving.

The results discussed above suggest that the welfare effects of the shock are mostly borne by the directly impacted region. Calculations of CV and EV are used to quantify

\footnotetext{
${ }^{84}$ For variables that can be negative, the percentage changes have been adjusted to properly reflect the direction of change: when the trade balance is negative and the percentage change is also negative, this is interpreted as the trade balance becoming more negative. The change reported in table 0.28 is equivalent to an increase in the trade deficit of $\$ 8.4$ million.
} 
the magnitude of the welfare change for all regions (table O.27). According to those estimates, households in Other North Island collectively experience a loss of welfare about half the size of those in Wellington. ${ }^{85}$ This highlights a key strength of the JENNIFER model and more generally the bottom-up modelling approach: by taking regional linkages into account, we are able to estimate the regional effects of shocks, and these suggest distributional consequences that are not observable in a national model. Neither can the indirect effects be ignored - the shock has effects beyond the borders of the region it directly impacts upon. The nature of the distributional consequences are also suggestive: the welfare loss rankings are Wellington (worst affected), then Other North Island, followed by Other South Island and Auckland, and then Canterbury. These may reflect the degree of closeness and therefore interdependence between Wellington and these regions, at least in terms of those industries primarily affected by the shock. ${ }^{86}$

Since our results are likely to be sensitive to some of our elasticity assumptions, it is important to check how the results change when these are altered. We saw in table 2.15 that all our assumed source elasticities were above 1.0 except for OWND and OIGA, so specifying substitution elasticities for agents as in table 2.16 means that their responsiveness to price changes, relative to both imported prices and other-region prices, is higher than that implied by Cobb-Douglas mixing functions. In order to test the implications of assuming such responsiveness, we run the same simulation with alternative sets of elasticities for agents' source choice, progressively scaling down the elasticities. Table 2.18 shows the values of the elasticities used for SIM102 and SIM103, besides those of our initial simulation, SIM101, for comparison. Lower substitution elasticities can be justified on the grounds that (1) the ability to substitute between sources may be more limited in the short-run, and (2) higher levels of aggregation imply less commonality between varieties of the same product, and so less substitutability. ${ }^{87}$ The results of the simulations with the lower source substitution elasticities are reported in the tables of appendix $O$, in the SIM102 and SIM103 columns.

\footnotetext{
${ }^{85}$ Using real spending (per household) as a welfare criterion would suggest the welfare loss was about one quarter that of Wellington's. Whichever criterion is used, the ranking in terms of welfare loss remains the same.

${ }^{86}$ Closeness at this stage carries no implication of geographical proximity as this basic version of the model has no information on the configuration of the regions.

${ }^{87}$ The source substitution elasticities were averaged from more disaggregated data, so some downward adjustment may be appropriate to account of the loss of "sameness" when aggregating.
} 
Sets of Elasticities for Simulations

\begin{tabular}{llccc}
\hline Agents & Choice between... & SIM101 & SIM102 & SIM103 \\
\hline \multirow{2}{*}{ Households } & domestic \& imported & $\vec{\Sigma}$ & $\frac{2}{3} \vec{\Sigma}$ & $\frac{1}{3} \vec{\Sigma}$ \\
& regional sources & $\frac{3}{2} \vec{\Sigma}$ & $\vec{\Sigma}$ & $\frac{1}{2} \vec{\Sigma}$ \\
Industries & domestic \& imported & $\vec{\Sigma}$ & $\frac{2}{3} \vec{\Sigma}$ & $\frac{1}{3} \vec{\Sigma}$ \\
- current production & regional sources & $\frac{3}{2} \vec{\Sigma}$ & $\vec{\Sigma}$ & $\frac{1}{2} \vec{\Sigma}$ \\
Industries & domestic \& imported & $\vec{\Sigma}$ & $\frac{2}{3} \vec{\Sigma}$ & $\frac{1}{3} \vec{\Sigma}$ \\
- capital formation & regional sources & $\frac{3}{2} \vec{\Sigma}$ & $\vec{\Sigma}$ & $\frac{1}{2} \vec{\Sigma}$ \\
& domestic \& imported & $\frac{3}{4} \vec{\Sigma}$ & $\frac{1}{2} \vec{\Sigma}$ & $\frac{1}{4} \vec{\Sigma}$ \\
Government & regional sources & $\vec{\Sigma}$ & $\frac{2}{3} \vec{\Sigma}$ & $\frac{1}{3} \vec{\Sigma}$ \\
Exporters & regional sources & $\frac{3}{2} \vec{\Sigma}$ & $\vec{\Sigma}$ & $\frac{1}{2} \vec{\Sigma}$ \\
\hline
\end{tabular}

Table 2.18: Sets of Elasticities for Simulations

The role of the source substitution elasticities in inter-regional feedbacks is central to understanding these results. When the elasticities are high as in SIM101, agents substitute away from the Wellington variety of CMIF, PROP, and RBUS (compare table O.6 with tables O.7 and O.8). The goods producing industries of Wellington (AGRI - EQFO) face lower composite factor costs (since their rental rates do not increase by much and the wage rate falls significantly) which feeds into lower output prices and higher output. Agents substitute towards the products of those industries further, increasing their output and reinforcing the reallocation of Wellington labour towards those industries (table O.10). With the lower degrees of substitution assumed in SIM102 and SIM103, the CMIF, PROP, and RBUS service industries of Wellington do not see such a large fall in demand and the goods producing industries' demand effect is dampened. The reallocation of labour is therefore less pronounced and the variation in output response across industries within the region is reduced. The higher costs associated with the higher capital rental rates come through more strongly in the industries' output prices (table O.16).

Less source substitutability means the CMIF, PROP, and RBUS service industries in the other regions see less of an increase in demand and therefore output and capital rental rates do not increase by as much (tables O.17 and O.18). Although this leads to dampened output price increases within those regions, the Wellington price effect dominates (tables O.15 and O.16). The nominal wage rate in Wellington does not fall as much while there are partially offsetting drops in the other regions' wage rates (table O.21). 
The outcome of the rental rate and wage rate differences across the simulations is that the household agent in Wellington has higher nominal income and those of the other regions less nominal income in SIM103 than SIM101. Since it is assumed that a constant proportion of nominal income is saved, spending actually increases in Wellington in sim103 while it falls elsewhere (even in real terms - see table O.25). This leads to a counter-intuitive result in terms of welfare - the household agent in Wellington is better-off and those outside the region worse-off as a result of the shock (table O.27).

The sensitivity of our simulation results, particularly with regards the welfare effects across regions, signals the need for empirical estimation of the source substitution elasticities. With a single set of elasticities we can see that distributional consequences are significant, but to predict what those consequences would be requires a robust set of estimates.

Two issues that could be raised with our simulation results are that the predicted regional real wage rate differences could lead to population movements even in the shortrun, and that the treatment of the distribution services WHOL, RETT, and TRAN is simplistic, with these industries treated just like any other. For example, no account has been taken of the fact that destruction of capital stock of TRAN may make delivery of Wellington products to other regions difficult. These two issues are addressed by the model extensions presented in the following two chapters. 


\section{Chapter 3}

\section{Distribution Services}

\subsection{Introduction}

The basic model outlined in the previous chapter contained no special treatment of distribution services such as wholesale and retail trade and transport services. The demands for these were derived in the same way as for the other products of the model. This approach leads to unrealistic predictions from simulations that involve relative price changes

- if some product becomes more expensive relative to a distribution service then users substitute away from that product and increase their demand for the distribution service. A more sensible modelling setup would ensure that, when relative prices change, agents change their demands for distribution services in the same direction as their demands for products that are delivered using those services. Special treatment of distribution services is especially relevant to bottom-up multi-regional models where regional prices vary. For example, if the price of a region's variety of a certain product rises relative to others then the ability of agents to substitute towards other varieties is constrained by the capacity of associated distribution services to accommodate the additional demand.

This chapter sets out enhancements to the basic model that allow for such realism in our treatment of these services. The structure of the chapter reflects that of chapter 2 : after a discussion of the role that distribution services play and how they are modelled, the additional equations required will be summarised followed by details of implementing the changes to the model. Illustrative simulations using the 25-industry, 5-region version of the model are discussed at the end of the chapter. 


\subsection{Modelling Distribution Services}

The role of services such as wholesaling, retailing, and transportation is of great importance in a multi-regional CGE model, since that role is essentially to facilitate distribution of products to users who may be domiciled in a different region from the producer. In classifying agents by region, we introduce the idea of "distance" between them and the issue immediately arises of how distribution within a region differs from that between regions. Our treatment must capture in some way the relationship between producer-user distance and delivery costs. Each type of distribution service ought to be considered separately to capture their unique characteristics, but first we need to look at the way the use of these services is recorded in the input-output table and how we can model that usage in general.

When distribution services are used to deliver products, their cost is usually builtin or otherwise attached to the purchase price. For this reason, they are referred to as 'margins' when discussing their usage for delivery, as distinct from 'distribution services' which refers to the set of services supplied by the distribution industries, irrespective of their usage.

Interpreting Input-Output Value Flows Recall that the flows shown in the inputoutput table in chapter 2 are valued at basic prices. Cell IO(DOM-g, CON) is the value flow of domestically-produced product $g$ to household consumption and equivalent to the value received by the producer. The value of any margin service $m$ used to deliver the product is recorded in row $\mathrm{IO}(\mathrm{DOM}-m, \bullet)$. Agents purchased distribution services just like any other product in chapter 2. In this chapter we link delivery transactions to product transactions so that they can be seen as one joint purchase - hence the 'margin' terminology. The product rows of the input-output table then show the portion of the value of purchases received by producers while the portion received by the distribution services are included in the margin rows.

One Purchase, Many Transactions As consumers we are familiar with retail services. The retailer facilitates trade between producers and consumers by bringing products to market. Prior to this the products may pass through many hands on the way from the place of production (e.g. the farm or factory) to the purchaser. It is common 
for produced goods to be transferred to a wholesaler who oversees their distribution to retailers. Transportation and insurance services are usually required for each movement of the goods. Each pair of hands the products pass through adds a markup on to their purchase price. Ultimately the consumer pays a price that reflects distribution markups on top of the production or importation cost and markup (normal profits). Each purchase is modelled in this chapter as a set of transactions: one transaction between the purchaser and each of the producer, the wholesaler, the retailer, and the transporter. To understand the nature of these transactions, we need to look at each type of distribution service in turn.

Retail Trade The primary function of the retail trade is to provide a location where products that are in a ready-to-use form can be purchased. Rational purchasers would optimally choose to visit retail outlets nearby rather than far away if they both offer similar products at similar retail prices. Provided there are not many purchasers nor retail outlets close to the borders of the regions in our model, we can make the simplifying assumption that retail services are a local product as was done in the previous chapter. The volume of retail service demanded in a region would then be related to the volume of products demanded within that region, regardless of the source of the products.

Wholesale Trade Wholesalers facilitate the delivery of products from producers to retailers or directly to other producers if they are to be used as an intermediate input into current production or capital formation. The wholesale margin is ultimately paid by the purchaser, even though a retailer may pay the margin on behalf of the purchaser along with the producer's cost and markup. These are simply passed through into the retail price. The wholesale margins recorded in the input-output table as paid by retail service suppliers are therefore margins on their purchases of inputs into production, not the margins on the goods they sell. Associating demands for wholesale services with demands for products is more complicated than it was for retail. One approach is that demands for wholesale services from a region are related to the product demands of agents in that region analogous to the retail services. An alternative is to associate wholesale demands with demands for products from each region by agents in all destinations. The first approach is appropriate for wholesalers located close to purchasers while the latter is for when they are close to producers. The model gives control of the regional configuration 
of producers, wholesalers, and purchasers to the user by allowing either of these, or a mixture of both, to be assumed.

Transportation Including transportation as a separate margin in the model is complicated by the fact that transport can be demanded for delivery of goods as well as simply a service to be consumed (e.g. commuting to work) or used in production (e.g. business trips). The input-output table unfortunately does not make a distinction between these uses so the model user must make an assumption over what proportion of each flow of transport service is for margin use and otherwise. To do so, a number of complexities need to be taken into account. The first of these is the way that transport is measured in the table. Purchases of transport are only recorded when they are separately invoiced. In many cases the transport costs of delivery have been included in the wholesale margin flows. That in turn means that wholesalers are making significant use of transport as an input into production so only some of the transport they purchase is margin-type. For example, the cost of delivering forklifts from the port to the wholesaler is transport margin on the forklifts purchase. When the wholesaler is contracted to deliver fresh produce to a supermarket retailer however, the services of a trucking firm to deliver the produce is a direct input into the wholesaler's production. The composition of the product we call transport here is also important. The 25-industry aggregation used in this thesis necessarily combines all types of transport — road, rail, water, and air — and includes both passenger and freight services. ${ }^{1}$ It is left to the user to set the proportion of each flow of transport service that is for direct use as opposed to margin use. Transport margins are handled in a similar way to wholesale margins in that demands for a region's transport can be associated with product demand flows from that region, or demands by agents within that region, or a mixture of the two.

Use of Margins by Government and Exporters Since the model assumes no regional dimension to government and exporters, they can be viewed as destinations that cover all regions for our purposes. Their margin demands are dealt with in a similar way to those of the regionally domiciled agents. The main difference is that local products

\footnotetext{
${ }^{1}$ The raw data is sufficiently detailed to separate road passenger and freight services but not for the other transport types, and making the strong assumption that the usage of these can be split according to the shares of passenger and freight in road transport does not seem appropriate.
} 
lose their meaning for the government and exporters. Exporters' demands for retail trade for example only depend on the source distribution of their product demand, not where they plan to export the goods from, which is an aspect not described by the model. Note that all margins on exports are paid by the exporter and subsequently included in the export price that is paid by the foreign sector. Since wholesale and retail services are used only as delivery margins, there is no export demand curve for each of these. However, the model does include an export demand curve for transport in the case when the user specifies a positive proportion of the flow of transport services to exporters as non-margin usage.

Use of Margins to Deliver Imports Analogous to the exporting agents, importers also lack a regional dimension. They are treated as a single extra source of products on top of the domestic regions. This complicates the linking of margins to source product flows as was suggested for wholesale and transport services above. How this issue is addressed will be discussed in the section below detailing the model implementation enhancements. The key extra ingredient required is an assumption on the regional distribution of imports, to effectively disaggregate import flows top-down across regions.

Use of Margins to Deliver Services Naturally some products do not require all types of distribution service to facilitate delivery. There are no costs involved in delivering 'Ownership of owner-occupied dwellings' to households, for example. Wholesale and transport services are usually only needed to deliver physical products. ${ }^{2}$ Many service providers supply their own retail service, so the separation of their product value (e.g. a haircut) from their retail margin (e.g. the delivery of the haircut to the market) needs to be done during the compilation of the input-output table. For example in the 1996 IO table, the retail components of the accommodation, restaurants, and bars industry were recorded in the retail trade row rather than in the relevant product row. ${ }^{3}$ For our purposes it is presumed that this separation is carried over to the BERL 2006 table such that the

\footnotetext{
${ }^{2}$ Exceptions may arise depending on what services are aggregated under these headings in the inputoutput table. For example, wholesaling of services such as passenger flights and travel insurance could come under the banner of wholesale trade. Communication services could also be treated as margins since they are becoming increasingly important in the delivery of many services (e.g. banking), but such an enhancement was not investigated for this thesis.

${ }^{3}$ See Statistics NZ (2001, page 7).
} 
services rows only contain the value flows of service product, with the retail components shifted to the retail trade row. In general the model user can account for these kinds of issues by setting, for each type of margin, the subset of products which require that margin for delivery to each given purchaser.

Imports of Margin Services Consistent with the assumption that margin services are not exported but simply used to prepare products for export, any imports of wholesale, retail, or transport services are treated as products for non-margin use. ${ }^{4}$

Undelivered and Delivered products The specific assumptions surrounding how a product is delivered are captured in the model by distinguishing between 'delivered' and 'undelivered' products. In general delivered product $g$ from source $s$ is a package of the undelivered product (on the factory floor or in the hands of the importer) and all the services used in its delivery. Payment of commodity tax is also required to complete delivery so this is an additional component of the delivered product. The model user has control over how each product is packaged. For example it could be assumed (given a sufficiently disaggregated IO table) that each motor vehicle purchased by households in Wellington is in fact a package of the actual (duty-paid) imported vehicle, some wholesale margin from Auckland, and some retail service from Wellington.

\subsection{Additional Model Structure}

The most significant additional structure required to introduce our modelling of margins is a set of equations for each relevant agent that describes their demand for each distribution service from each region required to deliver each product from source to destination. ${ }^{5}$

The product set COM is used to refer to direct (non-margin) usage of products only. A separate set $M A R$ is specified for the margin services, with an associated index $m$. Thus 'transport' as an element of $C O M$ is distinguished from 'transport' as an element of $M A R$. The distribution industries remain included in $I N D$ and non-margin uses of distribution

\footnotetext{
${ }^{4}$ The input-output data indicates that imports of these only account for small proportions of total demands anyway $-0.5 \%$ for wholesale, $15 \%$ for retail, and $12 \%$ for transport.

${ }^{5}$ Note that importing agents pay all delivery costs to port as part of the c.i.f. import prices they face and the investment agent does not require any margins to convert new capital into investment.
} 
services are indexed over $C O M$, so most of the core model structure described in chapter 2 and made explicit in appendix D generalises without alteration, although in many cases the interpretation changes. This is because there are some alterations made to the equilibrium conditions and purchase price definitions, discussed below. ${ }^{6}$

\subsubsection{Margin Demands}

Each agent's demand equations for margins are derived from optimisation in a fashion consistent with chapter 2. However, it is assumed that there are no opportunities for substitution between margins of different types or margins of a given type from different regions, so the solution is relatively straightforward. Formally, each agent minimises costs in obtaining a certain quantity of delivered product $g$ from source $s$, given that each unit of delivered product is a Leontief combination of units of the undelivered product and all required margin services $m \in M A R$ sourced from any region(s) $y \in R E G$. Using a generalised notation, the agent's problem is written: ${ }^{7}$

Minimise

$$
P_{g, s}^{Q} \cdot\left(1+t_{g}^{Q, a g e n t}+t_{g}^{G S T, a g e n t}\right) \cdot \breve{Q}_{g, s}^{\text {agent }(D)}+\sum_{m} \sum_{y} P_{m, y}^{Q} \cdot Q_{m, y, g, s}^{a g e n t(D)}
$$

subject to: 8

$$
Q_{g, s}^{\text {agent }(D)}=\text { Leontief }{ }_{g, s}^{\text {agent }}\left(\breve{Q}_{g, s}^{\text {agent }(D)},\left.Q_{m, y, g, s}^{\text {agent }(D)}\right|_{m, y}\right)
$$

with variable descriptions:

\footnotetext{
${ }^{6}$ Take the definition of the regional consumption price deflator index $C P I_{r}^{\mathbf{F}}$ for example: the purchase price of a delivered product now includes all the delivery costs, not just commodity tax - compare equation (3.3) with (2.59) — so changes in margin prices will have a stronger effect on $C P I_{r}^{\mathbf{F}}$ than in chapter 2, ceteris paribus.

${ }^{7}$ The notation abstracts from the variations required to write the expressions for specific agents. For example we would change $Q_{m, y, g, s}^{a g e n t(D)}$ to $Q_{m, y, g, s, r}^{\mathbf{h s h}(D)}$ for households and $Q_{m, y, g, s, j, r}^{\operatorname{ind}(\mathbf{Q})(D)}$ for current production

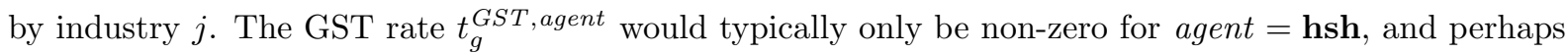
also for agent $=\exp$ for those products usually purchased by international visitors (tourists, students, etc.)

${ }^{8}$ The notation Leontief ${ }_{j}\left(A_{j},\left.B_{i j}\right|_{i \in\{1, \ldots, n\}}\right)$ is a shorthand for $v \cdot \min \left(\frac{A_{j}}{a_{j}}, \frac{B_{1 j}}{b_{1 j}}, \ldots, \frac{B_{n j}}{b_{n j}}\right)$ where the $v$, $a_{j}$ 's, and $b_{i j}$ 's are parameters.
} 

$\breve{Q}_{g, s}^{\text {agent }(D)} \quad$ quantity of undelivered product $g$ from source $s$ demanded by agent
$Q_{g, s}^{\text {agent }(D)}$ quantity of delivered product $g$ from source $s$ demanded by agent
$Q_{m, y, g, s}^{a g e n t(D)} \quad$ quantity of margin $m$ from region $y$ required to deliver product $g$ from source $s$ to agent

The demand functions derived by solving this optimisation problem are:

$$
\begin{aligned}
& \breve{Q}_{g, s}^{\text {agent }(D)}=a_{g, s}^{\breve{Q}, \text { agent }} \cdot \frac{Q_{g, s}^{\text {agent }(D)}}{v_{g, s}^{Q, \text { agent }}} \\
& Q_{m, y, g, s}^{\text {agent }(D)}=a_{m, y, g, s}^{Q, \text { agent }} \cdot \frac{Q_{g, s}^{\text {agent }(D)}}{v_{g, s}^{Q, \text { agent }}}
\end{aligned}
$$

Equations (3.1) and (3.2) imply that margin services are required in fixed proportion to the volume of products they are used to deliver. The parameters $v_{g, s}^{Q, a g e n t}$ and $a_{m, y, g, s}^{Q, a g e n t}$ embody all the assumptions made by the user surrounding delivery costs as discussed above - the $v$ 's depend on the commodity tax assumptions while the a's capture the distribution technology. The values of these parameters are determined by calibration, which is discussed in the next section.

The addition of these demand equations to the model amounts to attaching an extra layer of decision nodes to the bottom of each agent's decision nest. Just as each agent's demand for product $g$ was represented as a composite of demands from each source, their demands for each delivered product from each source is a composite of the undelivered product and the margins. Figure 3.1 shows how a given agent's demand for delivered product $g$ from source $s$ is formulated.

\subsubsection{Prices of Delivered Products}

The discussion of purchase prices in section 2.3.10 continues to be relevant in this chapter. However, the expressions that define purchase prices of delivered products, such as equation (2.59), need to be altered to account for distribution costs. Using the generalised notation from before, the purchase price paid by agent for product $g$ from source $s$ is defined by:

$$
\begin{aligned}
P_{g, s}^{Q, \text { agent }} \cdot Q_{g, s}^{\text {agent }(D)} & =P_{g, s}^{Q} \cdot\left(1+t_{g}^{Q, \text { agent }}+t_{g}^{G S T, \text { agent }}\right) \cdot \breve{Q}_{g, s}^{\text {agent }(D)} \\
& +\sum_{m} \sum_{y} P_{m, y}^{Q} \cdot Q_{m, y, g, s}^{\text {agent }(D)}
\end{aligned}
$$




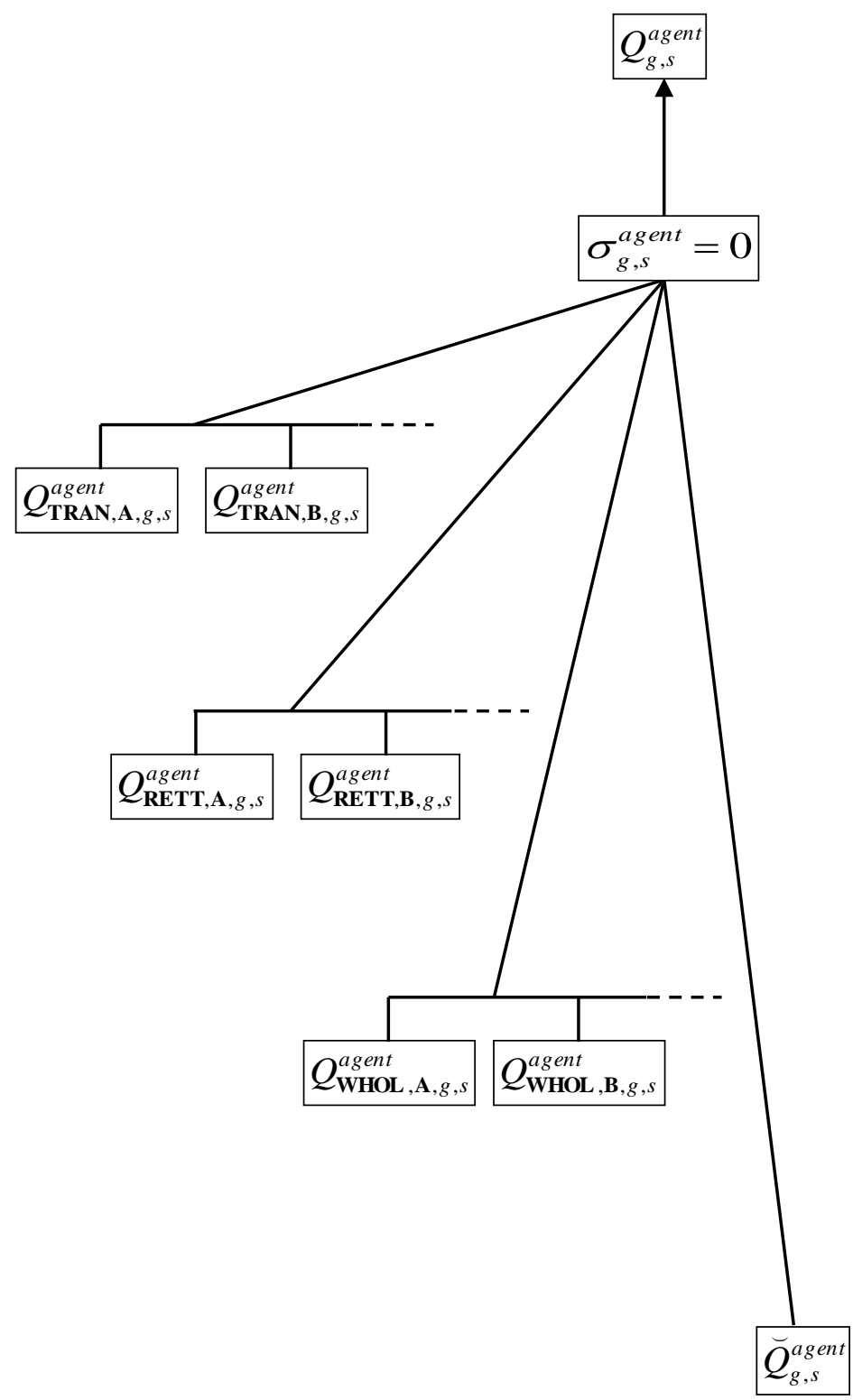

Delivered product $g \in\{\mathbf{1}, \mathbf{2}, \ldots\}$

from source $s \in\{\mathrm{A}, \mathrm{B}, \ldots, \mathbf{i m p}\}$

Leontief mixing function

Transport margin from

region $y \in\{\mathrm{A}, \mathrm{B}, \ldots\}$

Retail margin from region $y \in\{\mathrm{A}, \mathrm{B}, \ldots\}$

Wholesale margin from

region $y \in\{\mathrm{A}, \mathrm{B}, \ldots\}$

Undelivered product $g \in\{\mathbf{1}, \mathbf{2}, \ldots\}$

from source $s \in\{\mathrm{A}, \mathrm{B}, \ldots, \mathbf{i m p}\}$

Figure 3.1: Formation of delivered products

Note the implication that no commodity tax or GST is payable on margins. Nonmargin use of distribution services may however be subject to such taxes due to their inclusion in the product set $C O M$. 


\subsubsection{General Equilibrium Conditions}

The product market clearing equations of chapter 2 need to be altered to take into account the margin usage of domestically-produced distribution services as introduced in this chapter. The set of equations have to be split into those concerning the distribution services and those that are not. For this purpose it is useful to specify a set of products $P R O D$ that excludes the distribution services. That is, $P R O D=C O M \backslash M A R$. We can then write the market clearing conditions for the products $g \in P R O D$ as:

$$
\breve{Q}_{g, s}^{(S)}=\sum_{r} \breve{Q}_{g, s, r}^{\mathrm{hsh}(D)}+\sum_{p} \sum_{j} \sum_{r} \breve{Q}_{g, s, j, r}^{\operatorname{ind}(p)(D)}+\breve{Q}_{g, s}^{\operatorname{gov}(D)}+\breve{Q}_{g, s}^{\exp (D)}
$$

That is, identical to equation (2.46) but indexed over the restricted set. The market clearing conditions for the domestic distribution services have two components on the right-hand side - direct usage demands and margin demands — and can be written compactly as: ${ }^{9}$

$$
Q_{m, y}^{(S)}=\sum_{\text {agent }}\left(\breve{Q}_{h, y}^{\text {agent }(D)} \cdot \mathbb{1}^{h=m}+\sum_{g} \sum_{s} Q_{m, y, g, s}^{\text {agent }(D)}\right)
$$

It remains to specify the market clearing conditions for imported distribution services, which are assumed to be for direct usage. The equations are analogous to equation (3.4) and written in the compact form of (3.5):

$$
Q_{m, \mathbf{i m p}}^{(S)}=\sum_{\text {agent }} \breve{Q}_{h, \mathrm{imp}}^{\text {agent }(D)} \cdot \mathbb{1}^{h=m}
$$

The zero pure profit conditions (2.52) can similarly be divided into expressions for margin and non-margin use of products but this is not strictly necessary. The equations (2.52) continue to be valid for all $g \in C O M .^{10}$

${ }^{9} \sum_{\text {agent }} Q_{\bullet}^{\text {agent }(D)}$ is a shorthand for $\sum_{r} Q_{\bullet, r}^{\mathbf{h s h}(D)}+\sum_{p} \sum_{j} \sum_{r} Q_{\bullet, j, r}^{\mathbf{i n d}(p)(D)}+Q_{\bullet}^{\operatorname{gov}(D)}+Q_{\bullet}^{\exp (D)}$ and $\mathbb{1}$ is the indicator function defined in section 2.4.3.

${ }^{10}$ The alternative, partitioned form of (2.52) is obtained by restricting $g \in P R O D$ and writing:

$$
\begin{aligned}
P_{g, r}^{Q} \cdot \breve{Q}_{g, r}^{(S)} & =P_{j, r}^{Q, \text { ind }} \cdot Q_{j, r}^{\operatorname{ind}(D)}+P_{j, r}^{F, \text { ind }} \cdot F_{j, r}^{\text {ind }(D)} & & \forall g=j \\
P_{m, y}^{Q} \cdot Q_{m, y}^{(S)} & =P_{j, y}^{Q, \text { ind }} \cdot Q_{j, y}^{\operatorname{ind}(D)}+P_{j, y}^{F, \text { ind }} \cdot F_{j, y}^{\text {ind }(D)} & & \forall m=j
\end{aligned}
$$




\begin{tabular}{llr} 
Equation block & General case & $J=25, R=5, M=3$ \\
\hline Households & $J R+J R^{2}+M J R^{2}+M J R^{3}$ & 12,000 \\
Current production & $J^{2} R+J^{2} R^{2}+M J^{2} R^{2}+M J^{2} R^{3}$ & 300,000 \\
Capital formation & $J^{2} R+J^{2} R^{2}+M J^{2} R^{2}+M J^{2} R^{3}$ & 300,000 \\
Exporters & $J R+M J R^{2}$ & 2,000 \\
Government & $J+J R+M J R+M J R^{2}$ & 2,400 \\
\hline TOTAL & $J+3 J R+J R^{2}+2 J^{2} R+2 J^{2} R^{2}+M J R+$ & 616,400 \\
& $3 M J R^{2}+2 M J^{2} R^{2}+M J R^{3}+2 M J^{2} R^{3}$ &
\end{tabular}

Table 3.1: Additional Equations

\subsection{Implementation of the Margins Modelling}

To facilitate the implementation of the modelling of distribution services discussed above, a number of changes are made to the multi-regional input-output (MRIO) matrix and various coefficients are introduced to give the user control over how products are delivered within and between regions.

Before describing these changes, it is useful to look at how the overall model implementation is enhanced to handle the margins theory. The addition of the demand functions (3.1) and (3.2) to the model increases the number of equations significantly. ${ }^{11}$ While they are linear and therefore do not place much extra burden on the solver, their dimensionality does mean that computer memory gets used up rapidly as the dimension of the model increases. The user therefore faces a trade-off between the realism the margins modelling provides and the additional detail that would come from higher disaggregation of industries, regions, etc. Given $J$ industries (and products), $M$ of which are distribution services, and $R$ regions, the maximum increase in the number of model equations is set out in table 3.1 above. $^{12}$

\footnotetext{
${ }^{11}$ The alterations to the market clearing, zero pure profit, and purchase price equations discussed above make no difference to the overall number of equations.

${ }^{12}$ Trivial equations that set components of $Q_{m, y, g, s}^{a g e n t(D)}$ and $\breve{Q}_{g, s}^{\text {agent }(D)}$ equal to zero due to user assumptions can be dropped to reduce the size of the system if necessary. Other programming tweaks are possible to use memory more efficiently and recent versions of GAMS have a grid computing facility to directly control how memory is allocated to computational tasks. The 25-industry, 5-region, 3-margin implementation is close to the maximum model dimension a standard 32-bit PC can handle without resorting to these measures. The solution time is still only a matter of minutes, however.
} 


\begin{tabular}{|c|c|c|c|}
\hline \multirow{2}{*}{ Agents } & \multicolumn{3}{|c|}{ Distribution Service } \\
\hline & WHOL & RETT & TRAN \\
\hline all $\operatorname{ind}(\mathbf{Q})_{j, r}$ except $j=\mathrm{WHOL}$ & 1 & 1 & 1 \\
\hline all $\operatorname{ind}(\mathbf{Q})_{\mathbf{W H O L}, r}$ & 1 & 1 & 0.5 \\
\hline $\operatorname{all} \operatorname{ind}(\mathbf{K})_{j, r}$ & 1 & 1 & 1 \\
\hline all $\mathbf{h s h}_{r}$ & 1 & 1 & 0.1 \\
\hline gov & 1 & 1 & 1 \\
\hline all $\exp _{g}$ & 1 & 1 & 0.5 \\
\hline
\end{tabular}

Table 3.2: Margin-use Proportions

\subsubsection{Margin Coefficients}

The onus is on the model user to inspect and alter as needed the following coefficients which are used during and after the estimation of the MRIO matrix. The first set of coefficients are the proportions of each agent's demand for each distribution service $m$ that is for margin usage, denoted $\zeta_{m}^{a g e n t}$. These coefficients are useful in addressing issues surrounding the usage of transport by households, wholesalers, and exporters. A suggested set of values for $\zeta_{m}^{a g e n t}$, used for the 25-industry, 5-region, 3-margin implementation of JENNIFER, is shown in table 3.2.

The suggested values imply that $50 \%$ of the usage of transport by wholesalers and $90 \%$ of the usage by households is non-margin. A large part of the service wholesalers provide involves delivery, so they use a significant amount of transport as a direct input into production - thus the 50/50 split of their transport usage. Households' transport demand is mainly for consumption - flights, public transport, etc. They are rarely billed separately for delivery of the products they buy: it is usually part of the wholesale margin they pay. ${ }^{13}$ This is reflected in the relatively low share of transport demand that is assumed to be for margin use.

Setting the margin share of demand for transport by exporters less than 1 captures the idea that some transport services are purchased by foreigners for consumption. The definition of transport is important when choosing the appropriate proportions. A sig-

\footnotetext{
${ }^{13}$ Delivery costs could also be built into retail margins, but then the production of retail services would need some transport and/or wholesale as direct input.
} 
nificant proportion of the exports of transport may be purchases by tourists, and that transport may partly involve sightseeing, and partly involve delivery — in this case delivery of the tourist to the location where they can consume some other products such as accommodation or a cultural performance. Nevertheless, the model definition of an exported physical product is the finished good, delivered to port and loaded on the boat (i.e. Free On Board). So exporters of these face significant delivery costs, whether captured as wholesale, retail, or transport margins. Table 3.2 shows a suggested proportion of $50 \%$ of exporters' usage of transport that is for delivery, so the remaining share is interpreted as exports of transport services for consumption by non-residents.

From the above discussion it can be seen that consideration needs to be given to what types of products each margin service delivers. While transport may be involved in the delivery of services to tourists (actually by moving the tourists), the services demanded by domestic residents and firms usually do not require transport, or indeed wholesale services either. The user can control whether margin $m$ is required to deliver product $g$ to each agent by adjusting the binary switch $\chi_{m, g}^{a g e n t}$ - a value of 1 implies that $m$ is required. For simplicity here we assume values of $\chi_{m, g}^{\text {agent }}$ that do not vary across agents, and therefore the delivery requirements can be represented as the matrix shown in table $3.3 .^{14}$

The final set of margin coefficients are the $\gamma_{m}^{a g e n t}$ coefficients, which set the proportion of each agent's demand for margin $m$ that is 'destination-type' as opposed to 'sourcetype'. If $\gamma_{m}^{a g e n t}=1$ then the agent's demand for $m$ depends only on the size of the flow of products coming to the destination while $\gamma_{m}^{\text {agent }}=0$ implies that it depends only on the flow size of products coming from the source. A suggested split of margin demands into these two types is given in table 3.4. For simplicity we assume the values of $\gamma_{m}^{\text {agent }}$ for given $m$ are the same across all agents. ${ }^{15}$

\footnotetext{
${ }^{14}$ See table 2.5 for descriptions of products. $1=$ required, blank $=$ not required (the parameter has a value of zero). There are in fact three exceptions to all agents having the same $\chi_{m, g}^{\text {agent }}$ for given $m$ and $g$ : only hsh agents pay retail margin on OIGA, PETR, and TRAN. These are indicated in the table with asterisks $(*)$.

${ }^{15}$ Sometimes there are cases where the user setting needs to be over-ridden. One such case is where a product that is exported is not produced in all regions. Source-type margins can only be demanded from a region if there is a non-zero product flow from that region to which it can be attached. The margins demanded from regions which do not produce the product are forced to be destination type in this case. For example, since refined petrol (PETR) is only produced in region ONI, the value of $\gamma_{m, \mathbf{P E T R}}^{\exp }$ is fixed at
} 


\begin{tabular}{|c|c|c|c|c|c|c|c|}
\hline \multirow{2}{*}{ Product } & \multicolumn{3}{|c|}{ Distribution Service } & \multirow{2}{*}{ Product } & \multicolumn{3}{|c|}{ Distribution Service } \\
\hline & WHOL & RETT & TRAN & & WHOL & RETT & TRAN \\
\hline AGRI & 1 & 1 & 1 & UTIL & & 1 & \\
\hline FOLO & 1 & 1 & 1 & CONS & & 1 & \\
\hline FISH & 1 & 1 & 1 & ACCR & & 1 & \\
\hline MINE & 1 & 1 & 1 & CMIF & & 1 & \\
\hline OIGA & 1 & $1^{*}$ & 1 & PROP & & 1 & \\
\hline PETR & 1 & $1^{*}$ & 1 & RBUS & & 1 & \\
\hline FDBT & 1 & 1 & 1 & GOVT & & 1 & \\
\hline TWPM & 1 & 1 & 1 & EDUC & & 1 & \\
\hline CHNM & 1 & 1 & 1 & HEAL & & 1 & \\
\hline METL & 1 & 1 & 1 & CUPE & & 1 & \\
\hline \multirow[t]{3}{*}{ EQFO } & 1 & 1 & 1 & OWND & & & \\
\hline & & & & WHOL & & & \\
\hline & & & & RETT & & & \\
\hline \multicolumn{4}{|c|}{$*$ only for agent $=\mathbf{h s h}$} & TRAN & & $1^{*}$ & \\
\hline
\end{tabular}

Table 3.3: Margins Required to Deliver Products

\subsubsection{Extending The Multi-Regional Input-Output Data Matrix}

The process described in chapter 2 of establishing an economy-wide input-output (IO) matrix and splitting the rows and columns of that matrix using regional shares to obtain an initial estimate of the MRIO matrix is applicable in this chapter as well. Before discussing matrix adjustments to take into account our margin assumptions, two useful extensions are made to the MRIO table to obtain a more detailed matrix, denoted MRIO'.

\section{Usage of Distribution Services by Exporters}

A consequence of our margins treatment is that distribution services are used to deliver products to exporters. In chapter 2 we treated the flows of these services to exporters as though they were product exports. In order to easily associate margin flows with product one when calculating $\mathrm{MRIO}_{d s t}^{\prime}(m-y, \mathrm{EXP}-\mathrm{PETR})$ and $\mathrm{MRIO}_{s r c}^{\prime}(m-y, \mathrm{EXP}-\mathrm{PETR})$ for all regions $y$ other than ONI. 


\begin{tabular}{lc} 
Margin & Proportion \\
\hline WHOL & 0.5 \\
RETT & 1 \\
TRAN & 0
\end{tabular}

Table 3.4: Destination-type Proportions of Margin Demands

flows to exporters in this chapter, the EXP column of table 2.8 is expanded into a set of columns, one for each exported product, including non-margin usage of distribution services - see table 3.5. ${ }^{16}$ We can then spread the margin flows (and commodity tax) across those columns using our assumptions. ${ }^{17}$ For the non-distribution service products, the expanded MRIO matrix records the flow of $g$ from region $x$ to the exporter of $g$ as:

$$
\operatorname{MRIO}(g-x, \mathrm{EXP}-g)=\left[X_{g, x / g}^{\text {OUTPUT }}\right] \times \mathrm{IO}(\mathrm{DOM}-g, \mathrm{EXP}) \quad \forall g \in P R O D
$$

That is, analogous to equation (2.132) except valid only over the restricted set PROD.

Exports (i.e. non-margin usage) of a distribution service $m$ sourced from region $x$ is separated from exporters' margin usage by:

$$
\operatorname{MRIO}(m-x, \mathrm{EXP}-m)=\left(1-\zeta_{m, m}^{\mathbf{e x p}}\right) \times\left[X_{m, x / m}^{\text {OUTPUT }}\right] \times \mathrm{IO}(\mathrm{DOM}-m, \mathrm{EXP})
$$

To consider the margin usage of distribution service $m$ to deliver a product $g$ to its corresponding exporter $\left(\mathbf{e x p}_{g}\right)$, we first define a pro-rating share $\pi_{m, g}^{\mathbf{e x p}}$ as: ${ }^{18}$

$$
\pi_{m, g}^{\exp }=\frac{\chi_{m, g}^{\exp } \times \mathrm{IO}(\mathrm{DOM}-g, \mathrm{EXP})}{\sum_{h} \chi_{m, h}^{\exp } \times \mathrm{IO}(\mathrm{DOM}-h, \mathrm{EXP})}
$$

\footnotetext{
${ }^{16}$ The '..' indicate data values while all other (blank) cells are zero. Since it is assumed that wholesale and retail services are used only as margins, they are not part of the set of exported products. The OWND industry is given an export column since it is part of the PROD set but the value of OWND exports is zero.

${ }^{17}$ Note the implications of the $\zeta_{m, g}^{\mathbf{e x p}}$ and $\chi_{m, g}^{\mathbf{e x p}}$ coefficients set out in tables 3.2 and 3.3. Some transport service is exported, but it is assumed no margins are required to make the transport service available to the export market. Taking a deeper look at the tourism sector might lead us to adding retail and other margin requirements for the provision of transport exports.

${ }^{18}$ The symbol $\pi$ was used in chapter 2 for pro-rating tax values. In this chapter it is exclusively used for pro-rating margin flow values.
} 


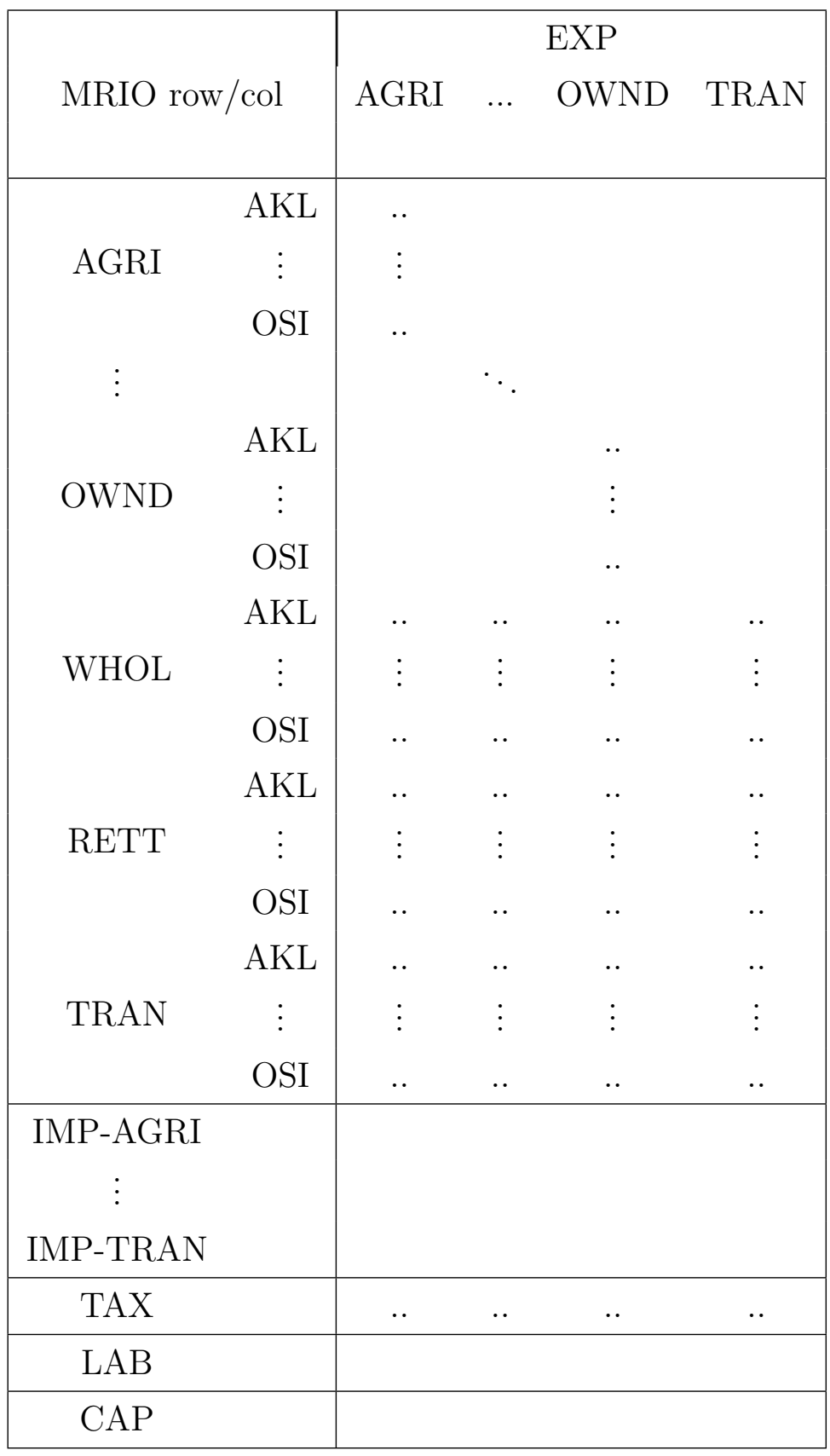

Table 3.5: Export Columns of the Expanded MRIO Matrix 
$\pi_{m, g}^{\mathbf{e x p}}$ is the share of product $g$ in the total flow of products to exporters that need margin $m$ for delivery. The binary switch in the numerator ensures that if $m$ is not required to deliver $g, \pi_{m, g}^{\exp }=0$.

The usage of margin service $m$ from region $y$ is pro-rated over the export columns by:

$$
\operatorname{MRIO}(m-y, \mathrm{EXP}-g)=\zeta_{m, g}^{\mathbf{e x p}} \times \pi_{m, g}^{\mathbf{e x p}} \times\left[X_{m, y / m}^{\text {OUTPUT }}\right] \times \mathrm{IO}(\mathrm{DOM}-m, \mathrm{EXP})
$$

\section{Direct Usage, Destination- and Source-Type Margins}

We know from tables 3.2 and 3.3 that all the transport usage recorded in the MRIO(TRAN- $x$,EXP-TRAN) cells is direct (i.e. non-margin) and the usage in all the other cells MRIO(TRAN- $x, \mathrm{EXP}-g$ ) is margin. For our purposes, it is useful to shift the margin flows in all columns of the MRIO table into rows apart from the direct flows. At the same time the margin flows are divided into separate destination-type and sourcetype rows. The product flow rows of the MRIO table (with the expanded export column discussed above) are therefore replaced with three blocks of rows: $\mathrm{MRIO}_{\text {dir }}^{\prime}, \mathrm{MRIO}_{\mathrm{dst}}^{\prime}$, and $\mathrm{MRIO}_{\text {src }}^{\prime}$ as shown in table 3.6 to obtain the MRIO' matrix.

The row blocks of MRIO' are derived from the MRIO table using the $\zeta_{g}^{\text {agent }}$ coefficients to split demands into direct and margin demands, and the $\gamma_{m}^{a g e n t}$ coefficients to split margin demands into destination-type and source-type as shown below. ${ }^{19}$ The reason for this latter partition is so that they can be treated differently when making adjustments to MRIO' for local margins, for example, and when pro-rating the margin flows over product flows to establish the benchmark equilibrium.

The flow of product $g$ from region $x$ to a given $\mathrm{MRIO}^{\prime}$ column, where $g$ is a product other than a distribution service, is simply transferred from the MRIO matrix:

$$
\mathrm{MRIO}_{\text {dir }}^{\prime}(g-x, c o l)=\operatorname{MRIO}(g-x, c o l) \quad \forall g \in P R O D
$$

The non-margin usage of a distribution service $m$ from region $x$ is extracted from the appropriate row of the MRIO using $\zeta_{m}^{a g e n t}$ :

$$
\operatorname{MRIO}_{\text {dir }}^{\prime}(m-x, c o l)=\left(1-\zeta_{m}^{\text {agent }}\right) \times \operatorname{MRIO}(m-x, c o l)
$$

The remaining usage in that MRIO row, the margin usage of distribution service $m$ from region $y$, is split between destination-type and source-type margin usage using $\gamma_{m}^{\text {agent }}$ as

\footnotetext{
${ }^{19}$ The obvious mapping of agents to columns of MRIO' is suppressed for simplicity.
} 


\begin{tabular}{|c|c|c|c|c|c|c|c|c|c|c|c|c|c|}
\hline & \multirow{2}{*}{\multicolumn{2}{|c|}{$\begin{array}{l}\text { MRIO' }^{\prime} \\
\mathrm{row} / \mathrm{col}\end{array}$}} & \multicolumn{3}{|c|}{ Industries } & \multirow{2}{*}{$\begin{array}{c}\text { CON } \\
\text { AKL...OSI }\end{array}$} & \multicolumn{3}{|c|}{ INV } & \multirow[t]{2}{*}{ GOV } & \multicolumn{3}{|c|}{ EXP } \\
\hline & & & $\begin{array}{c}\text { AGRI } \\
\text { AKL...OSI }\end{array}$ & $\cdots$ & $\begin{array}{c}\text { TRAN } \\
\text { AKL...OSI }\end{array}$ & & $\begin{array}{c}\text { AGRI } \\
\text { AKL...OSI }\end{array}$ & $\cdots$ & $\begin{array}{c}\text { TRAN } \\
\text { AKL...OSI }\end{array}$ & & AGRI & $\ldots$ & TRAN \\
\hline \multirow{13}{*}{ 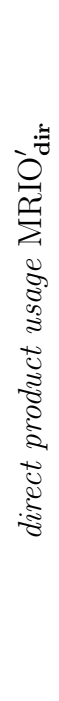 } & & AKL & .. & .. & .. & .. & .. & .. & .. & .. & .. & .. & .. \\
\hline & AGRI & $\vdots$ & $\vdots$ & $\vdots$ & & $\vdots$ & $\vdots$ & $\vdots$ & $\vdots$ & $\vdots$ & $\vdots$ & $\vdots$ & $\vdots$ \\
\hline & & OSI & .. & .. & .. & .. & .. & .. & .. & .. & .. & .. & .. \\
\hline & $\vdots$ & & $\vdots$ & $\vdots$ & $\vdots$ & $\vdots$ & $\vdots$ & $\vdots$ & $\vdots$ & $\vdots$ & $\vdots$ & $\vdots$ & $\vdots$ \\
\hline & & AKL & .. & .. & .. & .. & .. & .. & .. & .. & .. & .. & .. \\
\hline & WHOL & $\vdots$ & $\vdots$ & $\vdots$ & $\vdots$ & $\vdots$ & $\vdots$ & $\vdots$ & $\vdots$ & $\vdots$ & $\vdots$ & $\vdots$ & $\vdots$ \\
\hline & & OSI & .. & & & .. & .. & .. & .. & .. & .. & .. & .. \\
\hline & & AKL & .. & .. & .. & .. & .. & .. & .. & .. & .. & .. & .. \\
\hline & RETT & & $\vdots$ & $\vdots$ & $\vdots$ & $\vdots$ & & & & $\vdots$ & $\vdots$ & & \\
\hline & & OSI & .. & .. & .. & .. & .. & .. &.. & .. & .. & .. & .. \\
\hline & & AKL & .. & .. & .. & .. & .. & .. & .. & .. & .. & .. & .. \\
\hline & TRAN & $\vdots$ & $\vdots$ & $\vdots$ & & $\vdots$ & $\vdots$ & $\vdots$ & $\vdots$ & $\vdots$ & $\vdots$ & $\vdots$ & $\vdots$ \\
\hline & & OSI & .. & .. & .. &.. &.. & .. &.. & .. & .. & .. &.. \\
\hline \multirow{9}{*}{ 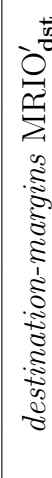 } & & AKL & .. & .. & & .. & .. & .. & .. & .. & .. & .. & .. \\
\hline & WHOL & $\vdots$ & $\vdots$ & $\vdots$ & & $\vdots$ & $\vdots$ & $\vdots$ & $\vdots$ & $\vdots$ & $\vdots$ & $\vdots$ & $\vdots$ \\
\hline & & OSI &.. & .. &.. &.. &.. & .. &.. & .. & .. & .. &.. \\
\hline & & AKL & .. & .. & .. & .. & .. & .. & .. & .. & .. & .. & .. \\
\hline & RETT & $\vdots$ & & $\vdots$ & & $\vdots$ & $\vdots$ & $\vdots$ & $\vdots$ & & $\vdots$ & $\vdots$ & $\vdots$ \\
\hline & & OSI & .. & & & .. & .. & .. & .. & .. & .. & .. & .. \\
\hline & & AKL & .. & .. & .. & .. & .. & .. & .. & .. & .. & .. & .. \\
\hline & TRAN & $\vdots$ & $\vdots$ & $\vdots$ & $\vdots$ & $\vdots$ & & $\vdots$ & & $\vdots$ & $\vdots$ & $\vdots$ & \\
\hline & & OSI & .. & .. & .. & .. &.. &.. & .. & .. & .. & .. &.. \\
\hline \multirow{9}{*}{ 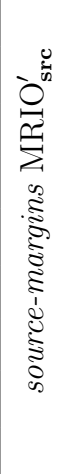 } & & AKL &.. & .. & .. &.. &.. &.. &.. &.. & .. & .. &.. \\
\hline & WHOL & $\vdots$ & $\vdots$ & $\vdots$ & & $\vdots$ & $\vdots$ & $\vdots$ & $\vdots$ & $\vdots$ & $\vdots$ & $\vdots$ & $\vdots$ \\
\hline & & OSI & .. & .. & .. & .. & .. & .. & .. & .. & .. & .. & .. \\
\hline & & AKL & & & &.. &.. & .. &.. & .. & .. & &.. \\
\hline & RETT & $\vdots$ & $\vdots$ & $\vdots$ & $\vdots$ & $\vdots$ & $\vdots$ & $\vdots$ & $\vdots$ & $\vdots$ & $\vdots$ & $\vdots$ & $\vdots$ \\
\hline & & OSI & .. & .. & .. & .. & .. & .. & .. & .. & .. & .. & .. \\
\hline & & AKL & .. & .. & .. &.. & .. & .. & .. & .. & .. & .. &.. \\
\hline & TRAN & $\vdots$ & $\vdots$ & $\vdots$ & & $\vdots$ & $\vdots$ & $\vdots$ & $\vdots$ & $\vdots$ & $\vdots$ & $\vdots$ & $\vdots$ \\
\hline & & OSI & .. & .. & .. & .. & .. & .. &.. &.. &.. & .. &.. \\
\hline
\end{tabular}

Table 3.6: Product Flows in the Extended MRIO Matrix 
follows: ${ }^{20}$

$$
\begin{aligned}
& \operatorname{MRIO}_{\mathbf{d s t}}^{\prime}(m-y, c o l)=\zeta_{m}^{\text {agent }} \times \gamma_{m}^{\text {agent }} \times \operatorname{MRIO}(m-y, c o l) \\
& \operatorname{MRIO}_{\text {src }}^{\prime}(m-y, c o l)=\zeta_{m}^{\text {agent }} \times\left(1-\gamma_{m}^{\text {agent }}\right) \times \operatorname{MRIO}(m-y, c o l)
\end{aligned}
$$

Flows of distribution services into the $\mathrm{MRIO}^{\prime}$ export columns are calculated slightly differently, however:

$$
\begin{aligned}
\operatorname{MRIO}_{\text {dir }}^{\prime}(m-x, \mathrm{EXP}-m) & =\operatorname{MRIO}(m-x, \mathrm{EXP}-m) \\
\mathrm{MRIO}_{\mathbf{d s t}}^{\prime}(m-x, \mathrm{EXP}-m) & =\mathrm{MRIO}_{\mathbf{s r c}}^{\prime}(m-x, \mathrm{EXP}-m)=0 \\
\mathrm{MRIO}_{\mathbf{d s t}}^{\prime}(m-y, \mathrm{EXP}-g) & =\gamma_{m, g}^{\mathbf{e x p}} \times \mathrm{MRIO}(m-y, \mathrm{EXP}-g) \\
\mathrm{MRIO}_{\mathbf{s r c}}^{\prime}(m-y, \mathrm{EXP}-g) & =\left(1-\gamma_{m, g}^{\mathbf{e x p}}\right) \times \mathrm{MRIO}(m-y, \mathrm{EXP}-g)
\end{aligned}
$$

\section{Adjusting The Extended MRIO Matrix}

Rich detail regarding the usage of margins can now be added to the model by making adjustments to the extended MRIO matrix and applying the information-theoretic approach discussed in chapter 2. This allows us to make significant changes to the underlying assumptions surrounding margin usage without having to worry about introducing spurious information into the model database. Two adjustments are described in this section: adjusting for local margins, and adjusting for border margins.

Local margins These are similar to the local products of chapter 2 but distinct since they are services used for delivery. In our implementation for this chapter, WHOL and RETT continue to be local in nature. The interpretation in the context of these being margins is that regionally domiciled agents only use the local variety for delivery of products from all sources. ${ }^{21}$ The way that local margins are pro-rated over product demands is consistent with the way that destination-type margins will be pro-rated when we establish the benchmark equilibrium. This is why it was suggested in table 3.4 that retail should be treated as a destination-type margin. In general all local margins should be designated as destination-type, and adjustments are made in the $\mathrm{MRIO}_{d s t}^{\prime}$ block of rows to make the

\footnotetext{
${ }^{20}$ The region counters have been switched from $x$ to $y$ to consistently use $y$ for the origin region of a given margin.

${ }^{21}$ This restriction does not apply to exporters and the government, but is of little consequence since these agents do not make significant use of WHOL and RETT.
} 
margin local. For example, the following makes $m$ a local margin for the household agent in region $r$ :

$$
\mathrm{MRIO}_{\mathbf{d s t}}^{\prime}(m-y, \mathrm{CON}-r)=\mathbb{1}^{y=r} \times \zeta_{m, r}^{\mathbf{h s h}} \times \gamma_{m, r}^{\mathbf{h s h}} \times \sum_{x} \operatorname{MRIO}(m-x, \mathrm{CON}-r)
$$

These adjustments move all destination-type flows of margin $m$ to $\mathbf{h s h}_{r}$ into the region $r$ cell out of the other regions' cells, so that all margin $m$ demanded by $\mathbf{h s h}_{r}$ is from region $r .^{22}$

Border margins In order to introduce additional costs in procuring products out-ofregion, we may wish some margin flows to be required to deliver products only when they are crossing the receiving agent's regional border. For our implementation here, transport is specified as a border margin, meaning that transport (as a margin) is only used to deliver products between regions, not within them. Then an increase in transport costs will cause agents to substitute away from out-of-region products, ceteris paribus, if such substitution is possible. Usage of border margins will be pro-rated over product demands consistent with the way that source-type margins will be when we establish the benchmark equilibrium. Thus the suggestion in table 3.4 that transport be treated as source-type. All border margins should be designated as source-type, and adjustments are made in the $\mathrm{MRIO}_{s r c}^{\prime}$ block of rows. For example, to specify that $m$ is a border margin for the household agent in region $r$, we first define a pro-rating share:

$$
\pi_{m, y, r}^{\mathrm{hsh}}=\frac{\operatorname{MRIO}(m-y, \mathrm{CON}-r)}{\sum_{x} \operatorname{MRIO}(m-x, \mathrm{CON}-r)}
$$

The intra-region flow of $m$ is shifted out of the relevant cell of the MRIO table and spread proportionally over the inter-region flows using the pro-rating share as follows:

$$
\begin{aligned}
\operatorname{MRIO}_{\mathbf{s r c}}^{\prime}(m-y, \mathrm{CON}-r) & =\mathbb{1}^{y \neq r} \times \zeta_{m, r}^{\mathbf{h s h}} \times \gamma_{m, r}^{\mathbf{h s h}} \\
& \times\left(\pi_{m, y, r}^{\mathrm{hsh}} \times \operatorname{MRIO}(m-r, \mathrm{CON}-r)+\operatorname{MRIO}(m-y, \mathrm{CON}-r)\right)
\end{aligned}
$$

\footnotetext{
${ }^{22}$ This effectively reverses the row splitting of the IO matrix to obtain the MRIO. Making a product local can be thought of as exempting it from the row-splitting of the IO matrix, but computationally it is easier to split all the rows and then add back up those cases which are determined to be exemptions. This process does create some rounding errors, and the RAS of the extended MRIO table propagates the rounding error throughout the matrix, but the effects only appear beyond eight decimal places.
} 
Mixtures of margin types In general, for each margin, the user decides how much of the margin flow to each agent is destination-type and source-type. Of the destinationtype flows, the user decides whether they are local margins or not, and of the source-type flows, whether they are border margins or not. In our implementation of JENNIFER with margins modelling, we assume that all retail flows are local destination-type margins and transport margin flows are border source-type margins. Our treatment of wholesale margins shows that mixtures of the different types are possible. According to table 3.4, half of all wholesale margins are destination-type and therefore the other half are sourcetype. Assuming that WHOL is a local margin only affects those flows allocated to the $\mathrm{MRIO}_{\text {dst }}^{\prime}$ rows. This provides a setup where some wholesaling is done close to purchasers and some is done close to producers.

\subsubsection{Obtaining the Benchmark Solution}

Once adjustments have been made to the extended MRIO matrix and it has been rebalanced using the RAS algorithm, the database is consistent with the conditions for competitive general equilibrium and is used to obtain the benchmark equilibrium values in a manner analogous to that of chapter 2. This section describes how the values of $\left[Q_{m, y, g, s}^{a g e n t(D)}\right]$ are calculated. The reader may find it useful to look through the simple numerical example found in appendix $\mathrm{M}$ prior to or in combination with the general presentation here. The reader is also referred to appendix $\mathrm{N}$ which presents the extended MRIO matrix as derived according to the above methodology and used in our implementation of the JENNIFER model.

Destination-type margin flows are pro-rated over all product flows while source-type margins are spread only over product flows coming from the same region. For exposition we define these separately as $\left[Q_{m, y, g, s}^{(\mathbf{d s t}) \operatorname{agent}(D)}\right]$ and $\left[Q_{m, y, g, s}^{(\mathbf{s r c}) \operatorname{agent}(D)}\right]$, and these add up to $\left[Q_{m, y, g, s}^{\text {agent }(D)}\right]$. That is, we determine the benchmark value of $Q_{m, y, g, s}^{a g e n t}(D)$ as the sum of these two components.

Destination-type Margin Demands We first define a pro-rating share $\pi_{m, g, s}^{\text {agent }}$ to allocate each agent's demand for margin $m$ from each region according to the relative importance of their demand for product $g$ from source $s$ in their total demand for products that require $m$ for delivery:

$$
\pi_{m, g, s}^{\text {agent }}=\frac{\chi_{m, g}^{\text {agent }} \times\left[\breve{Q}_{g, s}^{\text {agent }(D)}\right]}{\sum_{h} \sum_{z \in S R C} \chi_{m, h}^{\text {agent }} \times\left[\breve{Q}_{h, z}^{\text {agent }(D)}\right]}
$$


The demand for each margin from each region is then spread over the product demands to obtain the agent's destination-type demand for margin $m$ from region $y$, for delivery of product $g$ from source $s$ :

$$
\left[Q_{m, y, g, s}^{(\mathbf{d s t}) \operatorname{agent}(D)}\right]=\pi_{m, g, s}^{a g e n t} \times \mathrm{MRIO}_{\mathbf{d s t}}^{\prime}(m-y, c o l)
$$

Note that the pro-rating factor $\pi_{m, g, s}^{a g e n t}$ does not depend on the source region $y$ of the margin $m$. This is what makes the margin demand 'destination-type'. The value $\left[\breve{Q}_{g, s}^{\text {agent }(D)}\right]$ is the appropriate value from $\mathrm{MRIO}^{\prime}-\mathrm{MRIO}_{\mathbf{d i r}}^{\prime}(g-x, c o l)$ for domestic product $g$ and $\mathrm{MRIO}^{\prime}(\mathrm{IMP}-g, \mathrm{col})$ for imported $g$. Any assumption that $m$ is a local margin is already built into the $m$ rows of $\mathrm{MRIO}_{\mathbf{d s t}}^{\prime}$. If $m$ is local for an agent located in region $r$, the value of $\mathrm{MRIO}_{\mathbf{d s t}}^{\prime}(m-y, \bullet)$ will be zero for all $y \neq r$. Provided the agent demands some margin $m$ and some imported product $g$, some of the margin will be used to deliver the imported product. No reference is made to the port of entry of imports, and the amount of margin required is therefore invariant to it.

Source-type Margin Demands The basic idea of source-type margins is that some margin service is attached to products in their region of production to facilitate their delivery. The margin requirements then depend on where the product is coming from, not where it is going to (which is the destination-margin case). A problem immediately arises: how can there be source-margin requirements on imported products when importers are treated as a single source separate to the regions? This compatibility problem between imports and source-margins is addressed by regionalising the import flows top-down. The import flows are distributed across regions without introducing separate importing agents in each region. As such there is still a single economy-wide market and basic price for each imported product. The import flow of product $g$ is allocated to region $x$ using the regional import share $X_{g, x / g}^{I M P}$; these shares enter the model as coefficients so do not add to the number of equations or variables. ${ }^{23}$ Regional share values adopted for

\footnotetext{
${ }^{23}$ The solution returns consistent regional import values but these appear nowhere else in the model. Essentially the demand-side of the importing sector is split but the supply-side described by the importing agents remains economy-wide. If source-type (especially border) margins are used to deliver imported products, the purchase prices that agents in different regions face may diverge after a shock that significantly affects the distribution industries. Nevertheless, the top-down regionalisation imposes a fixed importing sector structure which is unresponsive to shocks. There is no regional supply-side response by importers to shocks, even in the long-run. A similar top-down regionalisation of the exporting sector
} 
our implementation are shown in table $3.7 .{ }^{24}$ Pro-rating shares for source-type margin demands used to deliver imported products can then be defined as:

$$
\pi_{m, y, g}^{(\mathrm{imp}) \text { agent }}=\frac{\chi_{m, g}^{\text {agent }} \times\left[X_{g, y / g}^{I M P}\right] \times\left[\breve{Q}_{g, \text { imp }}^{\text {agent }(D)}\right]}{\sum_{h}\left(\left[\breve{Q}_{h, y}^{\text {agent }(D)}\right]+\chi_{m, h}^{\text {agent }} \times\left[X_{h, y / g}^{I M P}\right] \times\left[\breve{Q}_{h, \mathbf{i m p}}^{\text {agent }(D)}\right]\right)}
$$

$\pi_{m, y, g}^{(\mathbf{i m p}) a g e n t}$ captures the importance of imported product $g$ in all products delivered from region $y$ to the agent using margin $m$. The source-margin demands that facilitate that delivery are:

$$
\left[Q_{m, y, g, \mathbf{i m p}}^{(\mathbf{s r c}) \operatorname{agent}(D)}\right]=\pi_{m, y, g}^{(\mathbf{i m p}) \text { agent }} \times \mathrm{MRIO}_{\mathbf{s r c}}^{\prime}(m-y, \text { col })
$$

Since the rows of $\mathrm{MRIO}_{\text {src }}^{\prime}$ have been adjusted to account for any assumption that $m$ is a border margin, the value of $\left[Q_{m, y, g, \text { imp }}^{(\mathbf{s r c}) \operatorname{agent}(D)}\right]$ would be zero if that were the case and the agent was located in region $y$. Agents located in other regions will face distribution costs for imports that are landed in region $y$. The more an agent is dependent on imports from a region other than their own, the higher the distribution costs will be that they face.

The source-margins required for delivery of domestically produced products follows analogously:

$$
\begin{aligned}
\pi_{m, y, g}^{(\text {dom }) \text { agent }} & =\frac{\chi_{m, g}^{\text {agent }} \times\left[\breve{Q}_{g, y}^{\text {agent }(D)}\right]}{\sum_{h}\left(\left[\breve{Q}_{h, y}^{\text {agent }(D)}\right]+\chi_{m, h}^{\text {agent }} \times\left[X_{h, y / g}^{I M P}\right] \times\left[\breve{Q}_{h, \mathbf{i m p}}^{\text {agent }(D)}\right]\right)} \\
{\left[Q_{m, y, g, x}^{(\mathbf{s r c}) \text { agent }(D)}\right] } & =\mathbb{1}^{y=x} \times \pi_{m, y, g}^{(\mathbf{d o m}) \text { agent }} \times \operatorname{MRIO}_{\mathbf{s r c}}^{\prime}(m-y, \text { col })
\end{aligned}
$$

would suffer from the same limitation in feedbacks to the aggregate economy.

${ }^{24}$ These are based roughly on Statistics NZ figures for cargo imports available at http://www.stats . govt.nz/tools_and_services/tools/TableBuilder/exports-and-imports-tables.aspx. The data indicates the overall regional distribution of cargo imports for 2006 was: AKL 59\%, WLG 6\%, ONI 24\%, CAN 7\%, and OSI 4\%. There is some variation amongst the different commodity categories, but due to difficulties in matching the Statistics NZ classification to our product groups, accurate product-by-region shares cannot be obtained. For example neither crude oil nor refined petrol are separately identified in the data. The figures given here therefore set regional shares of physical products according to the overall average, except for OIGA. For this product it was assumed the majority of imports were crude oil imported directly to the petrol refinery. No data was available to determine the regional shares of services imports. It was assumed that these roughly followed the regional shares of tangibles although the urban centres probably take larger shares, hence the different allocation for service product imports. 


\begin{tabular}{|l|ccccc|}
\hline \multicolumn{5}{|c|}{ Regional Shares of Imports $(\%)$} \\
\hline \multirow{2}{*}{ Product } & \multicolumn{5}{c|}{ Region } \\
\cline { 2 - 6 } & AKL & WLG & ONI & CAN & OSI \\
\hline AGRI & 59 & 6 & 24 & 7 & 4 \\
FOLO & 59 & 6 & 24 & 7 & 4 \\
FISH & 59 & 6 & 24 & 7 & 4 \\
MINE & 59 & 6 & 24 & 7 & 4 \\
OIGA & 10 & 10 & 70 & 5 & 5 \\
PETR & 59 & 6 & 24 & 7 & 4 \\
FDBT & 59 & 6 & 24 & 7 & 4 \\
TWPM & 59 & 6 & 24 & 7 & 4 \\
CHNM & 59 & 6 & 24 & 7 & 4 \\
METL & 59 & 6 & 24 & 7 & 4 \\
EQFO & 59 & 6 & 24 & 7 & 4 \\
UTIL & 60 & 10 & 10 & 10 & 10 \\
CONS & 60 & 10 & 10 & 10 & 10 \\
ACCR & 60 & 10 & 10 & 10 & 10 \\
CMIF & 60 & 10 & 10 & 10 & 10 \\
PROP & 60 & 10 & 10 & 10 & 10 \\
RBUS & 60 & 10 & 10 & 10 & 10 \\
GOVT & 60 & 10 & 10 & 10 & 10 \\
EDUC & 60 & 10 & 10 & 10 & 10 \\
HEAL & 60 & 10 & 10 & 10 & 10 \\
CUPE & 60 & 10 & 10 & 10 & 10 \\
OWND & 60 & 10 & 10 & 10 & 10 \\
WHOL & 60 & 10 & 10 & 10 & 10 \\
RETT & 60 & 10 & 10 & 10 & 10 \\
TRAN & 60 & 10 & 10 & 10 & 10 \\
\hline
\end{tabular}

Table 3.7: Regional Shares of Imports (\%)

As noted above, we obtain the agent's total demand for margin $m$ from region $y$ to facilitate delivery of product $g$ from source $s$ by adding the destination and source 
components together:

$$
\left[Q_{m, y, g, s}^{\text {agent }(D)}\right]=\left[Q_{m, y, g, s}^{(\text {dst }) \operatorname{agent}(D)}\right]+\left[Q_{m, y, g, s}^{(\text {src }) \operatorname{agent}(D)}\right]
$$

All other benchmark equilibrium values are obtained by analogous extension of the material of section 2.4.3. For example, $\left[Q_{m, y}^{(S)}\right]$ can be obtained by adding up all the margin demands across all agents.

It can be seen from the purchase price equations (3.3) that the initial equilibrium values of delivered and undelivered quantities are wedged apart by distribution costs and commodity taxes. Since $\left[P_{g, s}^{Q, a g e n t}\right],\left[P_{g, s}^{Q}\right]$, and $\left[P_{m, y}^{Q}\right]$ are all set to one, the benchmark equilibrium value of $Q_{g, s}^{\text {agent }(D)}$ is evaluated as:

$$
\left[Q_{g, s}^{\text {agent }(D)}\right]=\left(1+\left[t_{g}^{Q, \text { agent }}\right]+\left[t_{g}^{G S T, \text { agent }}\right]\right) \cdot\left[\breve{Q}_{g, s}^{\text {agent }(D)}\right]+\sum_{m} \sum_{y}\left[Q_{m, y, g, s}^{\text {agent }(D)}\right]
$$

While the levels of the delivered and undelivered quantities may differ for this reason, they will move in unison in simulations. That is, the percentage change in $\breve{Q}_{g, s}^{\text {agent }(D)}$ will equal the percentage change in $Q_{g, s}^{a g e n t(D)}$. This follows intuitively from the form of the demand functions (3.1). Similarly $Q_{m, y, g, s}^{a g e n t(D)}$ will move in line with $Q_{g, s}^{\text {agent }(D)}$ due to the form of equation (3.2).

\subsubsection{Calibration of the Demand Functions}

The determination of the model parameters (the $a_{\bullet}$ 's and $v_{\bullet}$ 's) proceeds as set out in section 2.4.3. For the implementation of the enhanced model of this chapter, this involves the calibration of the demand equations (3.1) and (3.2) in addition to those of chapter 2 .

It is inferred from (3.1) and (3.2) that, when $a_{g, s}^{\breve{Q}, \text { agent }}+\sum_{m} \sum_{y} a_{m, y, g, s}^{Q, \text { agent }}=1$,

$$
\left.v_{g, s}^{Q, \text { agent }}=\frac{\left[Q_{g, s}^{\text {agent }(D)}\right]}{\left[\breve{Q}_{g, s}^{\text {agent }(D)}\right]+\sum_{m} \sum_{y}\left[Q_{m, y, g, s}^{a g e n t}(D)\right.}\right]
$$

such that

$$
\begin{aligned}
& a_{m, y, g, s}^{Q, a g e n t}=\left[Q_{m, y, g, s}^{\text {agent }(D)}\right] \cdot \frac{v_{g, s}^{Q, \text { agent }}}{\left[Q_{g, s}^{\text {agent }(D)}\right]} \\
& a_{g, s}^{\breve{Q}, \text { agent }}=\left[\breve{Q}_{g, s}^{\text {agent }(D)}\right] \cdot \frac{v_{g, s}^{Q, \text { agent }}}{\left[Q_{g, s}^{\text {agent }(D)}\right]}
\end{aligned}
$$


The $v_{g, s}^{Q, a g e n t}$ parameters capture commodity tax mark-ups and we can interpret $\left[Q_{g, s}^{\text {agent }(D)}\right] / v_{g, s}^{Q, \text { agent }}$ as pre-tax delivered product $g$ - the delivery margins have been attached but not the commodity tax. Then $a_{m, y, g, s}^{Q, a g e n t}$ is the requirement of margin $m$ from region $y$ per unit of pre-tax delivered product $g$ from source $s$ for delivery to the agent. ${ }^{25}$ The $a_{g, s}^{\breve{Q}, a g e n t}$ parameter can be similarly interpreted as the part of a unit of undelivered $g$ required for one unit of pre-tax delivered $g .{ }^{26}$

To provide an example, the tables on page 135 show some of the calibrated values in the parameter array $a_{m, y, g, s, r}^{Q, \mathbf{h s h}}$, namely the components for $m=$ WHOL, RETT, and TRAN, $g=$ PETR, and $s=(\mathrm{ONI}$, imp). They are the margin requirements per unit of delivered refined petrol, either the domestic variety (from ONI) or the imported variety, for delivery to the household agent in region $r$. The figures can be interpreted as percentages by multiplying them by 100 - for example, domestic petrol purchased in AKL includes $12 \%$ local wholesale margin and $14 \%$ local retail margin along with $5 \%$ wholesale margin from ONI. The tables reflect our margin assumptions: retail is a local margin; transport is a border margin; wholesale is a mixture of local and (non-border) source-type margins. ${ }^{27}$

\footnotetext{
${ }^{25}$ To obtain ORANI-style margin requirements per unit of undelivered product $b_{m, y, g, s}^{Q, a g e n t}$, we can simply divide the first set of parameters by the second:$$
b_{m, y, g, s}^{Q, \text { agent }}=\frac{\left[Q_{m, y, g, s}^{\text {agent }(D)}\right]}{\left[\breve{Q}_{g, s}^{\text {agent }(D)}\right]}=\frac{a_{m, y, g, s}^{Q, \text { agent }}}{a_{g, s}^{Q, \text { agent }}}
$$

${ }^{26}$ Note that $a_{g, x}^{Q, a g e n t}$ is an entirely separate set of parameters that captures the distribution of an agent's demand for product $g$ over the domestic regions $x$. They govern the choice in the decision nest that is one level higher than what we are concerned with here.

${ }^{27}$ The transport margin requirements are relatively low because of our assumption that households only pay transport costs indirectly as part of wholesale margins.
} 

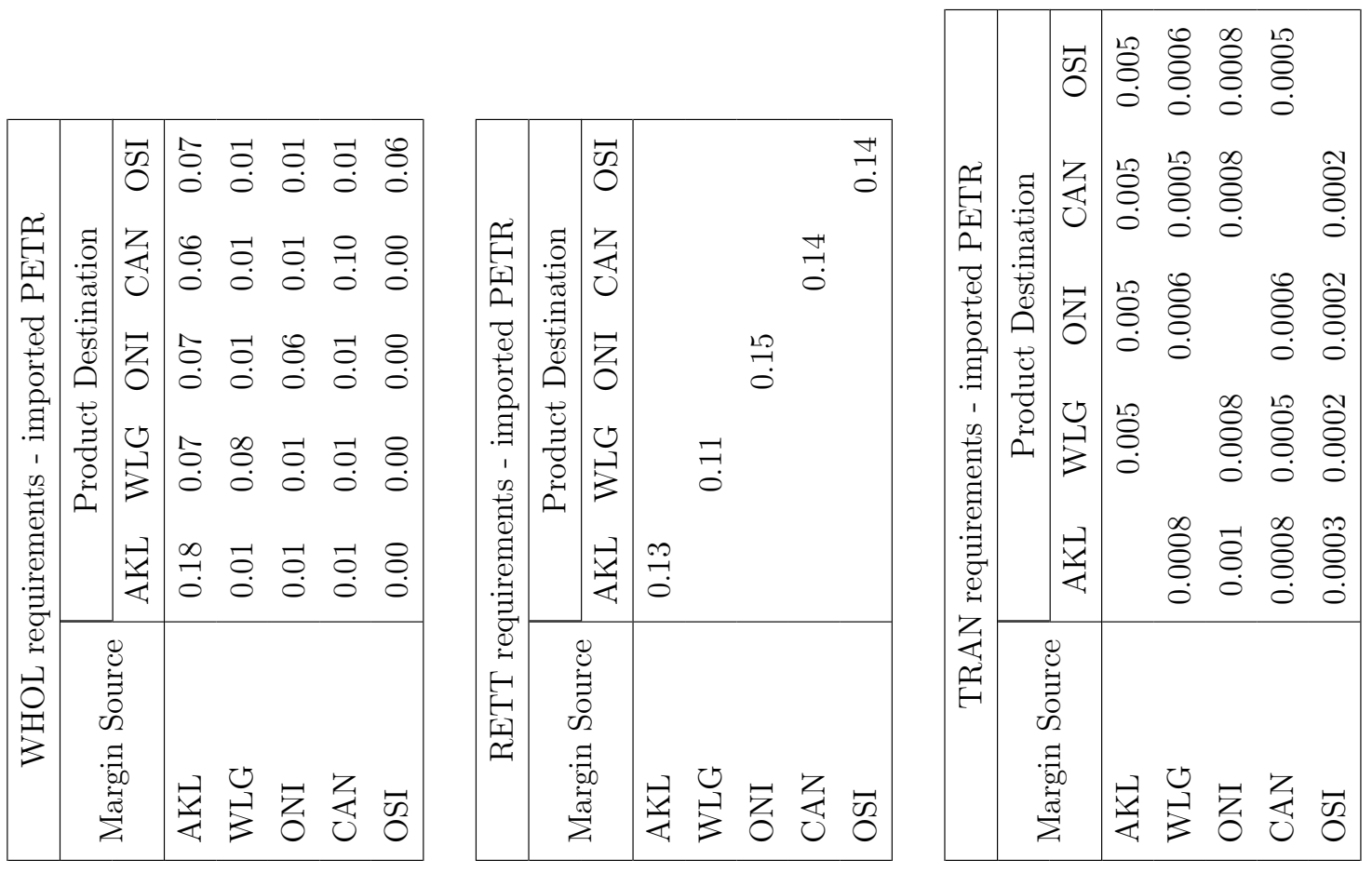

0
0
0
0
0
0
0
0
0
0
0
0
.7
0
0
0
0
0
0
0
0
0
0
0
4
0
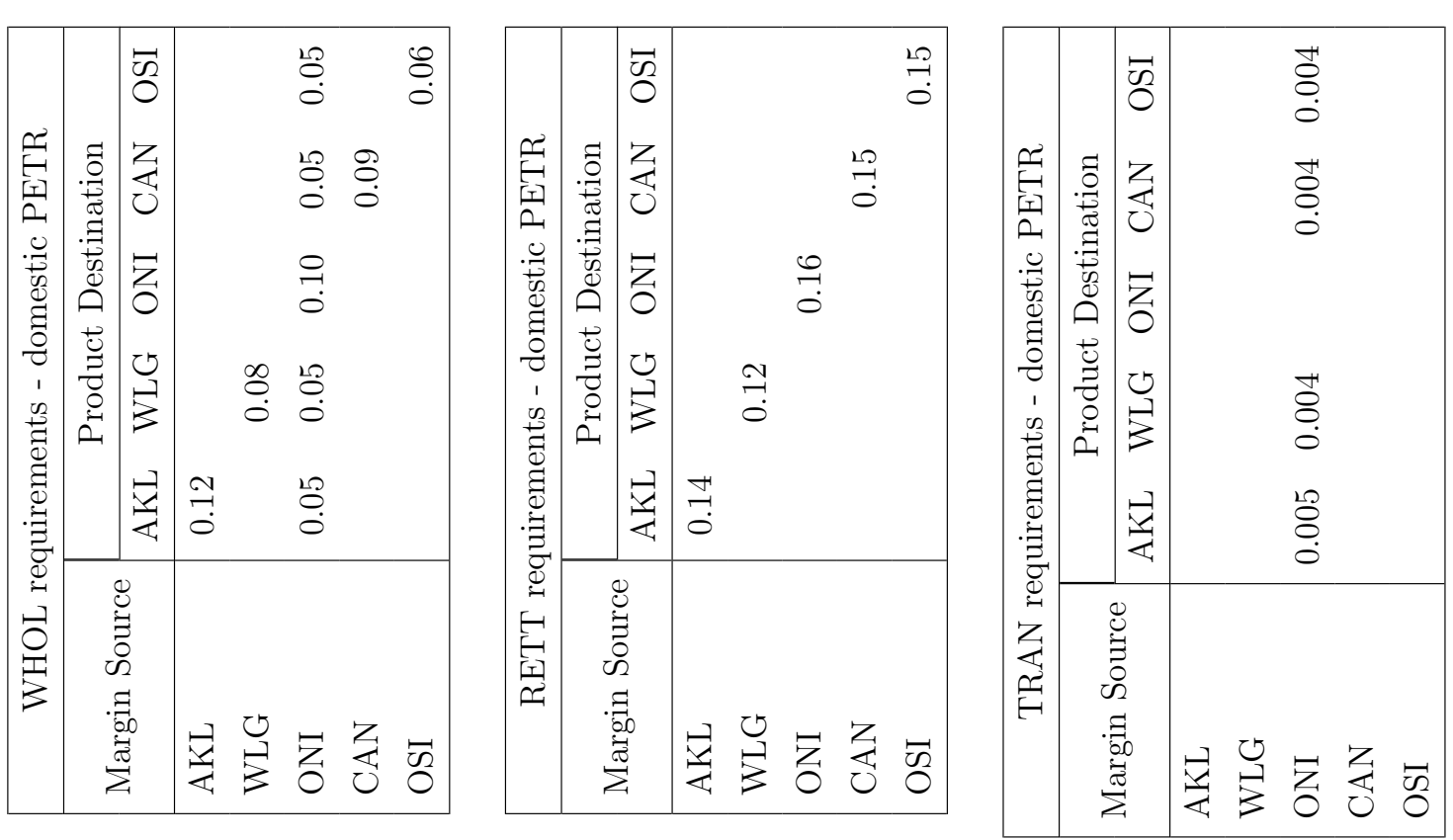

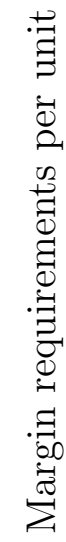


The parameter values are important since they control how changes in the prices of margin services feed into agents' purchase prices. This can be seen by considering the linearised form of (3.3). Assuming no change in commodity tax rates, it can be shown that: ${ }^{28}$

$$
\begin{aligned}
\frac{\mathrm{d} P_{g, s}^{Q, \text { agent }}}{P_{g, s}^{Q, \text { agent }}} & =\left(a_{\breve{Q}_{g, s}^{\text {agent }(D)}} \cdot\left(1+\left[t_{g}^{Q, \text { agent }}\right]+\left[t_{g}^{G S T, \text { agent }}\right]\right) \cdot \frac{\mathrm{d} P_{g, s}^{Q}}{P_{g, s}^{Q}}\right. \\
& \left.+\sum_{m} \sum_{y} a_{m, y, g, s}^{Q, a g e n t} \cdot \frac{\mathrm{d} P_{m, y}^{Q}}{P_{m, y}^{Q}}\right)
\end{aligned}
$$

An increase in the basic price of a margin service from a given region may cause one agent's product purchase price to increase by more than another. Even if both agents have the same elasticity of substitution over products, their behavioural response will differ and this will have flow-on effects to the economic outcome for their respective regions. For example, looking at the table on page 135, it can be seen that the household agent in AKL faces higher wholesale margin costs for domestic PETR than the other regions. If a shock

${ }^{28}$ The equation is linearised by firstly substituting in the demand functions (3.2) and (3.1) to obtain:

$$
\begin{aligned}
P_{g, s}^{Q, \text { agent }} \cdot Q_{g, s}^{\text {agent }(D)} & =P_{g, s}^{Q} \cdot\left(1+t_{g}^{Q, \text { agent }}+t_{g}^{G S T, \text { agent }}\right) \cdot a_{\widetilde{Q}_{g, s}^{\text {agent }(D)}} \cdot \frac{Q_{g, s}^{\text {agent }(D)}}{v_{g, s}^{Q, \text { agent }}} \\
& +\sum_{m} \sum_{y} P_{m, y}^{Q} \cdot a_{m, y, g, s}^{Q, \text { agent }} \cdot \frac{Q_{g, s}^{\text {agent }(D)}}{v_{g, s}^{Q, \text { agent }}}
\end{aligned}
$$

or, using a shorthand

$$
P \cdot Q=P_{b} \cdot(1+t) \cdot a \cdot \frac{Q}{v}+\sum_{i} P_{i} \cdot a_{i} \cdot \frac{Q}{v}
$$

Eliminating $Q$ (i.e. $\left.Q_{g, s}^{a g e n t(D)}\right)$ and totally differentiating gives:

$$
\mathrm{d} P=\frac{1}{v}\left(a \cdot P_{b} \cdot \mathrm{d}(1+t)+a \cdot(1+t) \cdot \mathrm{d} P_{b}+\sum_{i} a_{i} \cdot \mathrm{d} P_{i}\right)
$$

Assuming no change in the commodity tax rate and some manipulation of terms provides:

$$
\frac{\mathrm{d} P}{P}=\left(a \cdot \frac{P_{b} \cdot(1+t)}{P} \frac{\mathrm{d} P_{b}}{P_{b}}+\sum_{i} a_{i} \cdot \frac{P_{i}}{P} \frac{\mathrm{d} P_{i}}{P_{i}}\right)
$$

At the benchmark equilibrium, the linearisation implies:

$$
\frac{\mathrm{d} P}{P}=\left(a(1+[t]) \cdot \frac{\mathrm{d} P_{b}}{P_{b}}+\sum_{i} a_{i} \cdot \frac{\mathrm{d} P_{i}}{P_{i}}\right)
$$


causes a uniform increase in the basic price of wholesale services from all regions, the purchase price that the AKL household agent faces for petrol will rise disproportionately higher than that of other regions, ceteris paribus. There will be a larger fall in demand for domestic PETR in AKL than elsewhere, even though the household agents have the same substitution elasticities (between PETR varieties and between product types). Overall AKL might see a larger fall in consumption spending than elsewhere due to the shock, and this will lead to different regional output and employment outcomes.

\subsubsection{Simulations with the Margins Extension}

The extension described in this chapter has been overlayed on top of the core JENNIFER model of chapter 2. Command-line options can be used to control whether the margins modelling is imposed, or only partially implemented (e.g. just local destination-type margins). At a deeper level, the user can control the way distribution services are used by editing the margin coefficients file.

No additions to the closure list of table 2.17 were required so this remains relevant for short-run simulations. A long-run view is especially useful when considering the distribution networks. While in the long-run we may assume capital is perfectly mobile, our modelling implies distributive connections remain fixed. We can observe the implications of those connections by running a given long-run simulation with and without the margins extension activated. For this purpose, a suggested long-run closure is given in table 3.8. ${ }^{29}$

To obtain the long-run closure, capital stocks have been been removed from the exogenous variable list, replaced by the current net rates of return. ${ }^{30}$ The implication is that capital is mobile between industries and regions. This mobility can occur either in the form of machinery etc. being physically moved, or capital in one industry/region being allowed to depreciate without replacement while investment builds up the stock of another industry/region. Our concept of mobility therefore requires that it is possible to actually shift the capital or sufficient time is allowed for the capital to shrink and grow

\footnotetext{
${ }^{29} \mathrm{As}$ for table 2.17, the investment shares are included on the list to show the maximum possible number of exogenous variables. If investment for all industries was endogenously determined by the model to equalise expected net rates of return, $X_{j, r / \text { tot }}^{I, \mathbf{b} \$}$ would be removed from the list and the number of exogenous variables would fall by $J \times R$.

${ }^{30}$ In cases where the capital stock is zero in the benchmark equilibrium, the variable should not be swapped because the system will not converge in most cases.
} 


\begin{tabular}{|c|c|c|c|}
\hline \multirow[b]{2}{*}{ Symbol } & \multirow{2}{*}{$\begin{array}{l}\text { Exogenous Variables } \\
\text { Description }\end{array}$} & \multicolumn{2}{|c|}{ Dimension } \\
\hline & & General case & $J=25, R=5$ \\
\hline$N R O R_{j, r}$ & net rate of return on $K_{j, r}$ & $J R$ & 125 \\
\hline$R_{r / \mathbf{N Z}}^{W^{\text {atax }}}$ & $\begin{array}{l}\text { real wage relative to national av- } \\
\text { erage }\end{array}$ & $R-1$ & 4 \\
\hline$T I M E_{\mathrm{NZ}}$ & aggregate time endowment & 1 & 1 \\
\hline$X_{j, r / \text { tot }}^{I, \mathbf{b} \$}$ & real investment shares & $J R$ & 125 \\
\hline$P_{g}^{F O R}$ & c.i.f. import prices & $J$ & 25 \\
\hline$A P S_{r}^{\text {atax }}$ & saving propensities & $R$ & 5 \\
\hline$\delta_{j, r}$ & depreciation rates & $J R$ & 125 \\
\hline$t^{L}$ & labour income tax rate & 1 & 1 \\
\hline $1+t_{g, \bullet}^{Q, \text { agent }}$ & powers of commodity tax & $2 J^{2} R+J R+2 J$ & 6,245 \\
\hline $1+t_{g}^{G S T, \text { agent }}$ & powers of GST & $2 J$ & 50 \\
\hline $1+t_{g}^{F O R}$ & powers of import duty & $J$ & 25 \\
\hline $1+t_{g}^{E X P}$ & powers of export subsidy & $J$ & 25 \\
\hline$\psi_{g}^{E X P}$ & levels of export demands & $J$ & 25 \\
\hline$X_{I / t o t}^{G D P, \mathbf{b} \$}$ & investment share of real GDP & 1 & 1 \\
\hline$X_{G / t o t}^{G D P, \mathbf{b} \$}$ & $\begin{array}{l}\text { government consumption share of } \\
\text { real GDP }\end{array}$ & 1 & 1 \\
\hline TOTAL & & $\begin{array}{l}3+2 R+8 J+ \\
4 J R+2 J^{2} R\end{array}$ & 6,963 \\
\hline
\end{tabular}

Table 3.8: A Long-run Closure 
as needed.

The other closure swap in table 3.8 is that the regional time endowments have been swapped for $R-1$ relative real wage rates and the aggregate time endowment, $R-1$ real wage rates are fixed relative to the national average so that labour market adjustment is by employment changes. The $R^{\text {th }}$ relative real wage rate must be endogenous to avoid overidentification of the system. Therefore the $R^{\text {th }}$ regional time endowment is replaced by the aggregate endowment. This implies that the aggregate stock of working-age population is static. Here as well, mobility can either take the form of persons actually shifting between regions, looking for better opportunities, or demographic changes - changes in fertility, regional population age structure, etc. By closing the model in this way, labour is assumed to be perfectly mobile between regions.

\subsection{An Illustrative Application}

In this section we present a selection of results from three simulations involving an identical shock but with different assumptions concerning distribution services to highlight the effect of those assumptions. The shock that is applied is a $100 \%$ increase in the c.i.f. import prices of oil \& gas (OIGA) and a $50 \%$ rise in the c.i.f. import price of refined petrol (PETR), to simulate the long-run effects of a significant increase in the world price of crude oil relative to other tradeable products. Since the model does not determine foreign prices, any effect that the higher oil price has on import prices needs to be exogenously specified. While it is likely a higher world oil price would feed into higher prices of many imported products, for simplicity only the effect on oil and petrol import prices is taken into account - hence the shock specified above. ${ }^{31}$

This shock is applied using the long-run closure set out in table 3.8 under three alternative environments with respect to the distribution services. The first simulation, SIM201, provides results from the basic model of chapter 2. That is, the margins modelling is switched off. In the second simulation, sIM301, the margins treatment is present but all margin demands are destination-type, with wholesale (WHOL) and retail (RETT)

\footnotetext{
${ }^{31}$ The assumption of a $50 \%$ increase in the petrol import price is made to reflect the notion that increases in oil prices impact most strongly on petrol prices, but petrol production also involves other inputs (capital, other chemical compounds, etc.) so petrol supply prices will rise less than one-for-one with oil prices, ceteris paribus.
} 
being purely local margins. By comparing that second simulation with the third, SIM401, which has source-margins included, we can see how adding inter-regional transport costs affects the results. Tables of results can be found in appendix P. ${ }^{32}$ For all the simulations, substitution elasticities are assumed as in table 2.16 except that since these are long-run simulations, the non-source substitution elasticities are assigned values of 1.0 as noted on page 94 .

Before looking at the results, it is useful to consider the role oil and petrol (both domestic and imported) play in the domestic economy as indicated by the IO table. ${ }^{33}$ The primary user of imported oil is the petrol industry. Some domestic oil is also used for petrol production but quality differences between New Zealand and imported crude are such that the two varieties are not substitutes - the Marsden Point refinery was not designed to refine the grade of oil found in New Zealand. ${ }^{34}$ The majority of oil extracted in New Zealand is instead sent offshore (exported) for refining. ${ }^{35}$ Households are the most

\footnotetext{
${ }^{32}$ Although the results of each simulation are presented together as percentage changes from a single benchmark equilibrium, each one actually has its own benchmark and hence the numbering SIM201, SIM301, SIM401 rather than SIM201, SIM202, SIM203. However, for almost all the tables found in appendix $\mathrm{P}$, the benchmark equilibrium values are identical, so the percentage change from the 'no margins' benchmark is the same as the percentage change from their own benchmark. The only exception is the table of f.o.b. exports. Since WHOL and RETT and some of TRAN were reassigned from direct export flows to margin flows on products to exporters, the benchmark export values are different. Table P.7 shows how the benchmark equilibria differ. For example, the benchmark value of AGRI exports for SIM301 and SIM401 are 20.1\% higher than for SIM201. (The baselines, BSLN3 and BSLN4, are replication equilibria of the respective benchmarks.) The results in the f.o.b. exports table (P.6) therefore need to be looked at in combination with table P.7. It can be shown that if the percentage change from $A$ to $B$ is $x$, and from $A$ to $C$ is $y$, then the percentage change from $C$ to $B$ is $(x-y) \frac{100}{100+y}$ or approximately $x-y$ for small $y$. Therefore, to know the (approx.) percentage changes in SIM301 and SIM401 from their own benchmarks, subtract the BSLN numbers from the sIM numbers. For example, AGRI exports are down approximately $1.9 \%$ and $2.0 \%$ from their own benchmarks in SIM301 and SIM 401 . When comparing to the SIM201 results, it is advisable to use the exact calculation to see the correct pattern across simulations. The exact calculations corresponding to the approximations for AGRI exports given directly above are -1.6 and -1.7 , for example.

${ }^{33}$ Useful background information on New Zealand oil and petrol can be found at http://www.teara. govt.nz/en/oil-and-gas (The Encyclopedia of New Zealand).

${ }^{34}$ This is why OIGA is given a source substitution elasticity of zero in table 2.15. On the other hand, domestic and imported refined petrol are virtually identical to the user so a relatively high substitution elasticity of four is used for PETR.

${ }^{35}$ One suspects that most of the non-export usage of domestic OIGA recorded in the IO table is in fact
} 
significant user of both imported and domestic petrol, followed by the transport (TRAN) industry, and then other industries such as construction (CONS) and utilities (UTIL). The transport industry is characterised by significant intra-industry trade but also has important downstream links to wholesalers, households, and exporters. It is this set of interdependencies between oil, petrol, transport, wholesaling, and final users that makes the way delivery services are modelled important for this simulation.

According to the SIM201 results, the deterioration of the terms of trade (an increase in the real exchange rate) has a contractionary effect on the economy (tables P.18 and P.19). All components of domestic absorption and real GDP fall. There is an improvement in the trade deficit because the volume of exports falls less than that of imports. ${ }^{36}$ Output falls in all regions with a larger fall in Other North Island than elsewhere, which is understandable since the petrol refinery is located in that region (table P.2). With imported oil being by far the most important intermediate input for the petrol industry (PETR), opportunities to substitute when its price rises are limited. In this long-run scenario, substitution of factors for intermediate inputs is allowed and does occur in the PETR industry - compare the $42 \%$ fall in imported OIGA in table P. 8 with the much smaller decreases in usage of capital and labour by PETR reported in tables P.10 and P.12. Petrol output is nevertheless cut back and the domestic petrol price rises significantly (table P.3). ${ }^{37}$ Coincidentally, the endogenous rise in the domestic petrol price almost matches the exogenously specified increase in the import price of petrol. While this is to be expected with the high degree of substitutability assumed between domestic and imported petrol, it appears that only a small amount of substitution has actually occurred (towards domestic PETR - compare the fall in domestic output of PETR with

\footnotetext{
gas usage.

${ }^{36}$ The percentage change in the trade balance is large because the size of the deficit is small relative to the size of the trade volumes. The real value of exports falls by $\$ 1,321 \mathrm{~m}$ while real imports fall by $\$ 2,064 \mathrm{~m}$, implying a $\$ 743 \mathrm{~m}$ decrease in the deficit.

${ }^{37}$ One noticeable but minor effect is a very large percentage decrease in petrol exports seen in table P.6. The input-output data records a small amount of petrol export receipts, most likely being for petrol and diesel sent to the nearby Pacific Islands. The large fall in petrol exports is due to the rise in the domestic petrol price, which pushes up the export price, and the sensitivity of foreign export demand to changes in that price. If importers of New Zealand petrol face prohibitive costs in sourcing the product from elsewhere, the assumed export price elasticity is perhaps too high. This is an empirical issue and, in any case, for these simulations unimportant since petrol export demand is very small relative to domestic demand.
} 
the fall in imported PETR). This suggests that it is mainly supply-side factors driving the domestic petrol price rise rather than demand-side factors.

Faced with higher petrol prices from both domestic and imported sources, the transport industry (TRAN) cuts output and the basic price of transport services rises (tables P.4 and P.5). As the price of domestic petrol relative to transport still rises, and under the model settings of SIM201 where transport is treated only as a product for direct usage, agents substitute away from petrol towards transport. Their demand for petrol falls much more than their demand for transport, so the larger decrease in petrol output compared to transport output is partly due to that substitution. ${ }^{38}$

In SIM301, most transport usage is treated as destination-type margin, with the exceptions being allowances for direct usage by wholesalers, households, and exporters discussed in section 3.4.1. Under this setting, agents can only substitute between petrol and direct usage of transport when their relative price changes. The substitution away from petrol towards transport seen in SIM201 is therefore dampened in SIM301. Output of the transport industry is lower and the output price is higher. An analogous substitution away from petrol towards retail and wholesale trade seen in SIM201 is also not present in SIM301. This, coupled with the higher transport price and lower economic activity generally (for example real GDP is slightly lower - see table P.18) causes the wholesale and retail industries to cut output further than in SIM201 as well.

Due to the substitution away from petrol being dampened in SIM301, imports of petrol and oil do not fall by as much as in SIM201. However, since there are transport costs associated with imports in SIM301, imports of most other products fall further for a lower level of total imports compared to sIM201 (table P.8). Exports do not fall by as much, so consistent with a lower terms of trade (relative to that of SIM201), there is a greater improvement in the trade deficit (table P.18).

The difference in output effects between SIM201 and SIM301 are reflected in the differences in employment and capital stock changes between the two scenarios - compare tables P.4, P.10 and P.12. Under the long-run closure, capital is mobile between industries and regions and therefore there is a shift towards PETR in SIM301 relative to SIM201. This capital shift primarily benefits the household agent of Other North Island, as does

\footnotetext{
${ }^{38}$ Petrol is of course a significant input into the production of some types of transport. The demand shift could take the form of relatively higher usage of mass-passenger transport by households and industries switching to non-petrol types of transport, for example.
} 
the employment shift, because they are the assumed owners of the PETR capital stock and the only supplier of labour to that industry. While aggregate household income and spending are falling in both these scenarios, and the decrease is larger in sIM301, the factor mobility favouring Other North Island causes the other regions to be disproportionately worse off in this latter scenario compared to the former (tables P.15 and P.16). ${ }^{39}$

Generally the basic version of JENNIFER from chapter 2 understates the negative effects of the terms of trade shock if the margins modelling used in SIM301 is a better representation of reality. If one were to rank the outcomes across industries in terms of output and investment, there are several notable changes in rankings between sIM201 and sim301 (tables P.4 and P.9). WHOL and RETT, for example, have better outcomes for output than AGRI, FDBT, METL, CMIF, and GOVT in SIM201 but in SIM301 the outcomes are worse. Similarly, PETR has an outcome for investment in sIM201 worse than that of several other industries but in sim301 the outcome is better. In terms of output at least, the industries most affected after oil and petrol are still the mining industry (MINE) followed by transport. The same kind of ranking applied to the regions indicates that Other North Island remains the region with the largest fall in overall output (table P.2).

The most significant factor in this illustrative application that causes the SIM401 results to differ from those of SIM301 is that it is assumed that delivery of petrol requires some wholesale service from the product source as well as some from the destination region in SIM401. By comparing the wholesale margin requirements between the simulations shown in the tables on page 145, it can be seen that in SIM401 the wholesale industry in Other North Island and Auckland are relatively more important for delivery of domestic and imported petrol respectively to households across the regions. ${ }^{40}$ We might expect this to be an additional source of variation in regional economic outcomes. The different assumptions regarding how margins are used to deliver petrol result in purchase prices that are mainly higher for most agents in sim401 compared to SIM301. In particular, household agents in Auckland, Wellington, and Canterbury face higher prices for petrol — see table P.17. ${ }^{41}$ Some substitution occurs away from domestic petrol towards the

\footnotetext{
${ }^{39}$ For example, real spending in ONI is 0.1 percentage points lower in SIM301 relative to sIM201 while for the other regions it is at least 0.3 percentage points lower.

${ }^{40}$ There is little difference in the retail and transport requirements between the simulations.

${ }^{41}$ Detailed simulation results, available from the author, show that the household agents of these regions generally face higher purchase prices for the products that are important components of their
} 
imported variety (see tables P.2 and P.8). This mainly has implications for output and employment in the PETR industry and other industries closely connected to it, such as OIGA and TRAN (tables P.4 and P.12). Due to the relatively small size of the domestic petrol industry, and partly owing to the perfect factor mobility assumption underlying the simulations, these effects do not significantly change the results for regional household income and macro measures such as real GDP (tables P.15 and P.18).

We have seen that a simple margins treatment of distribution services (in SIM301) and enhancements to that treatment (in SIM401) changes the magnitudes of results from an oil price shock simulation in the basic JENNIFER model (SIM201), particularly those for the petrol industry and sectors that have important forward and backward linkages to it. The broad national and regional effects are not altered by the different treatments but these simulations demonstrate how the margin assumptions allow the model to produce more sensible results at a deeper level. The use of "superior data" to inform the setting of the margin coefficients is therefore worthwhile, especially for investigations concentrating on certain groups of industries or regions.

consumption. The same is the case for Other South Island although the effect is less pronounced. Purchase prices in Other North Island are slightly lower owing to this being the source region for petrol. The petrol purchase price faced by the household agent of Other South Island is slightly lower due to a fall in their purchase price of transport - table P.3 indicates a fall in the basic price of TRAN from Other North Island and Other South Island (the source and destination respectively of the petrol). 

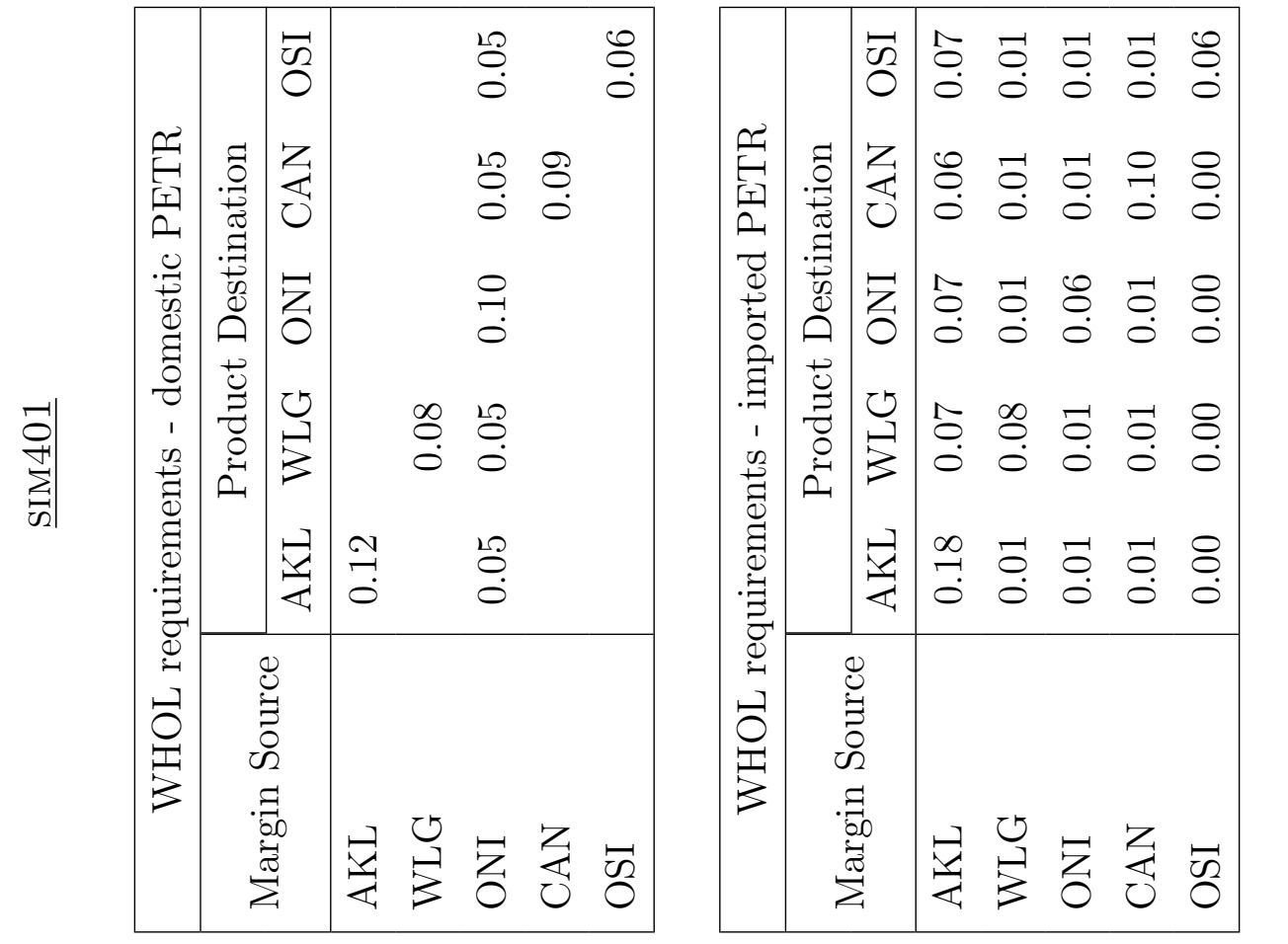

0
0
0
0
0
0
0
0
0
0
0
0
0
.0
0
0
0
0
0
0
0
0
0
0
0
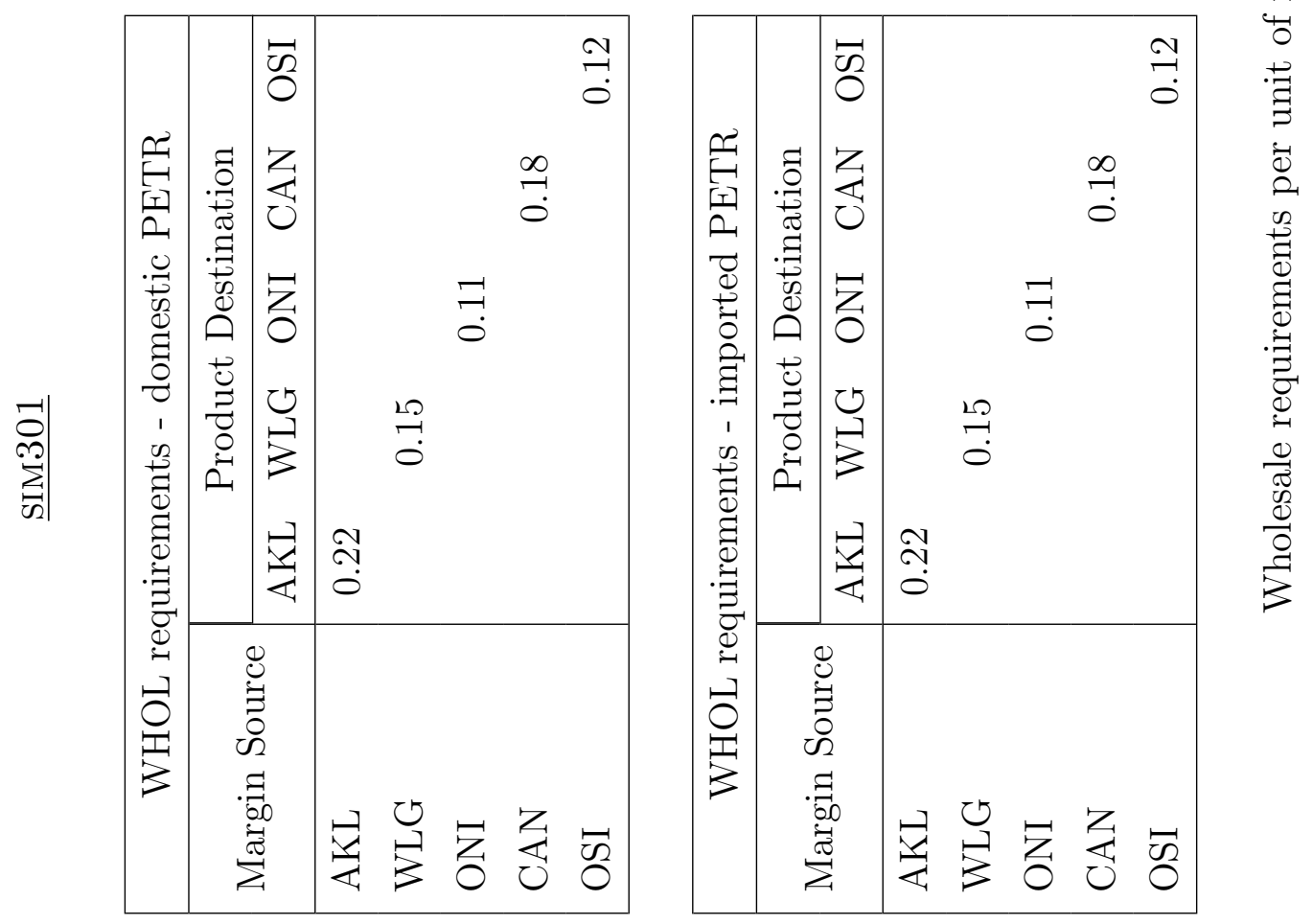


\section{Chapter 4}

\section{Labour Mobility}

\subsection{Introduction}

The focus of this chapter is factor mobility — in particular how simulation results depend on the assumptions made concerning inter-regional factor mobility. In the previous two chapters, discussion of factor mobility has been restricted to that of closure assumptions with respect to endowments. In the short-run closure shown in table 2.17 , each industryspecific, regional capital stock and each regional time endowment were fixed (exogenous), while these were swapped for corresponding current net rates of return and regional real wage rates (relative to the national average) for the suggested long-run closure of table 3.8. These closure options represent a stark choice between no mobility of endowments (between regions, and between industries as well in the case of capital) and perfect mobility. Capital mobility is very much a dynamic matter, and is best investigated using a time-staged model as was done in Nana (1999). In this chapter we continue to confine the issue to that of closure assumption so as to not depart from the comparative-static CGE framework used thus far.

The nature of labour mobility was briefly touched on in chapter 3, in the last paragraph of section 3.4.5. In simulations of certain shocks, such as the disaster scenario simulated in chapter 2, labour mobility responses can significantly alter the impact of the shock. Considering the issue of labour mobility in this context raises a number of important questions:

- What does labour mobility mean? An increase in a region's labour supply could 
be the result of those currently employed in the region working more hours, people already living within the region entering the labour force, or people entering the region to work.

- What is the effect of labour mobility? Each of the sources of regional labour supply expansion listed above may have different effects on the region and national economy.

- What are the drivers of labour mobility? What is the role of economic factors such as regional differences in wage rates and unemployment rates? How do we explain persistence of these regional differences?

This chapter sets out a framework within which these questions can be investigated. Rather than simply using the closure swap mentioned above, a more detailed treatment of the supply side of the labour market is introduced and an algorithm is used to control labour mobility.

\subsection{Modelling Labour Mobility}

The starting point for modelling the mobility of labour is the recognition that households supply labour in response to economic incentives, and changes in these incentives may lead them to supply more or less labour in their home region, or even shift to another region entirely if the economic incentives are strong enough. The CGE model framework is ideal for investigating the implications of this household behaviour since there are clear interdependencies. An inflow of households into a region affects the potential labour market outcomes of those households already residing within the region, resulting in a behavioural change for those households. ${ }^{1}$ The nature of this feedback loop makes computing a solution in a single run of the CGE model difficult. The main point of difficulty is that the household flows and the behaviour change cannot be simultaneously determined because households are not homogenous across regions. Homogeneity of labour is an implicit assumption in closures such as our long-run closure of chapter 3. This sort of assumption is common and reasonable in national models with labour divided into occupation/skill groups; ${ }^{2}$ with the individual labour force member as the unit of analysis,

\footnotetext{
${ }^{1}$ There are also reciprocating implications for outcomes in the migrants' source region.

${ }^{2}$ See for example Philpott \& Stroombergen (1986) and Philpott (1990b).
} 
it is reasonable to assume that in the long-run they up-skill such that they are equally as productive as members of the occupation they wish to enter (due to excess demand at the long-run fixed wage relativities). The average productivity level and other characteristics of the occupation/skill group do not change due to mobility being allowed between groups. For inter-regional labour mobility, the household is a more appropriate unit of analysis but it is not reasonable to assume that when households move between regions they spontaneously adopt the characteristics of the destination region's households, for example in terms of age-structure or labour market status. Their arrival will result in changes to such regional average household characteristics.

This chapter describes a method of manually controlling the second part of the feedback loop to overcome this problem. Essentially the model is solved twice, once to obtain the solution when there is no mobility of households between regions, and then again to take into account the behaviour change implied by the first solution. The second solution of the model is not a dynamically efficient equilibrium: the household behaviour change is only partially successful in eliminating household incentives to relocate. ${ }^{3}$

Additions are made to the structure of the core JENNIFER model of chapter 2 to facilitate two types of regional demographic and labour market changes:

- level changes: changes in the number of households, working age and non-working age persons, employed, unemployed, and non-labour force; changes in employment hours by industry

- composition changes: the characteristics of the average household may change. That is, the number of working age and non-working age persons in the household may change along with the number of employed, unemployed, and non-labour force in the household. Changes in the labour market characteristics reflect changes in regional unemployment and labour force participation rates.

These changes may come about due to an exogenous shock, such as imposing an increase in the number of non-working age persons per household to simulate aging regional populations. They may also be the result of endogenous behaviour change, including movements of households between regions. For example, if a given shock caused a flow of

\footnotetext{
${ }^{3}$ The solution is also not a dynamic equilibrium due to the static nature of the model, in the sense that there is no investment-capital link or forward-looking expectations. These items are left for future research.
} 
households from a region with a high participation rate to one with a low participation rate, we should see the participation rate of the receiving region rise ceteris paribus.

An interesting micro-foundation for partial labour mobility between regions is suggested by Jones \& Whalley (1989). ${ }^{4}$ They use intensity of location preference to control the degree of labour mobility — individuals (who are otherwise homogenous) suffer a utility penalty if they shift between regions so relative incomes have to rise sufficiently for migration to become optimal. It is useful to have such a theoretical underpinning of household mobility in mind here, but formal development of a micro-foundation is left for future research. The Jones \& Whalley model is also subject to the feedback problem they only go as far as determining the migration response, with migrants still consuming the old-region bundle and facing old-region prices at the point of model solution.

We assume here that household mobility occurs as a response to regional real wage rate differences. It might be desirable to have a mobility response to regional real income differences instead, but then we would need to consider the implications of our simplifying assumption that the residents of a region receive all the returns from capital installed in their region. An alternative setting where households respond to regional differences in unemployment rates instead - a "Keynesian mode" — is discussed in section 4.4.5.

\subsection{Additional Model Structure}

Three elements are introduced to operationalise inter-regional household mobility:

- Equations that define demographic and labour market measures, to enable their associated variables to be solved for in model runs

- A formula for determining households' behavioural response to real wage rate differences across regions, post-solution

- An algorithm for re-running the model taking into account that behavioural response.

\footnotetext{
${ }^{4}$ They argue that neither perfect mobility nor immobility are particularly useful assumptions because perfect mobility casts doubt on measured regional effects of shocks since "regions, as such, are not defined", and perfect immobility allows distributional consequences to be captured, but ignores efficiency implications of labour mobility, which are "heavily stressed in recent literature on fiscal federalism" (page $371)$.
} 
This section details the first two of these items and sets out the theoretical background for the third — the algorithm is more fully discussed in the model implementation section. The defining equations are fairly straightforward and notation is introduced as needed.

\subsubsection{Demographic Measures}

The set of demographic variables added to the model (with $r \in N Z R E G$, where NZREG is the set of all domestic regions $R E G$ plus the national region NZ) are shown in table 4.1. Other than the effect household mobility has on these items, the model lacks a description of how they are determined. That is, they are naturally exogenous to the model. A number of equations are required however to ensure that summation identities are satisfied in the computed solution. The first set ensures that a region's total population is the sum of its working age and non-working age populations:

$$
P O P_{r}=W A P_{r}+N W A P_{r}
$$

Other equations dictate that regional demographic measures add up to national demographic measures:

$$
\begin{aligned}
& H S H_{\mathbf{N Z}}=\sum_{r} H S H_{r} \\
& P O P_{\mathbf{N Z}}=\sum_{r} P O P_{r}
\end{aligned}
$$

and similarly for $W A P_{\mathrm{NZ}}$ and $N W A P_{\mathrm{NZ}}$. Household demographic characteristics are defined in equations such as:

$$
C_{r}^{P O P}=\frac{P O P_{r}}{H S H_{r}} \quad \forall r \in N Z R E G
$$

These measure the number of persons per household in each region. There are analogous equations for $C_{r}^{W A P}$ and $C_{r}^{N W A P}$. We will see later how these characteristics are useful in determining composition effects of household mobility. ${ }^{5}$

\subsubsection{Labour Market Measures}

The labour market variables (with $r \in N Z R E G$ ) listed in table 4.2 are added to the model to enrich the description of the supply side of the labour market. They also allow

\footnotetext{
${ }^{5}$ We could use the symbols $R_{r}^{P O P / H S H}$ etc. instead but $C$ is used to remind the reader these are $[\mathrm{C}]$ haracteristics.
} 


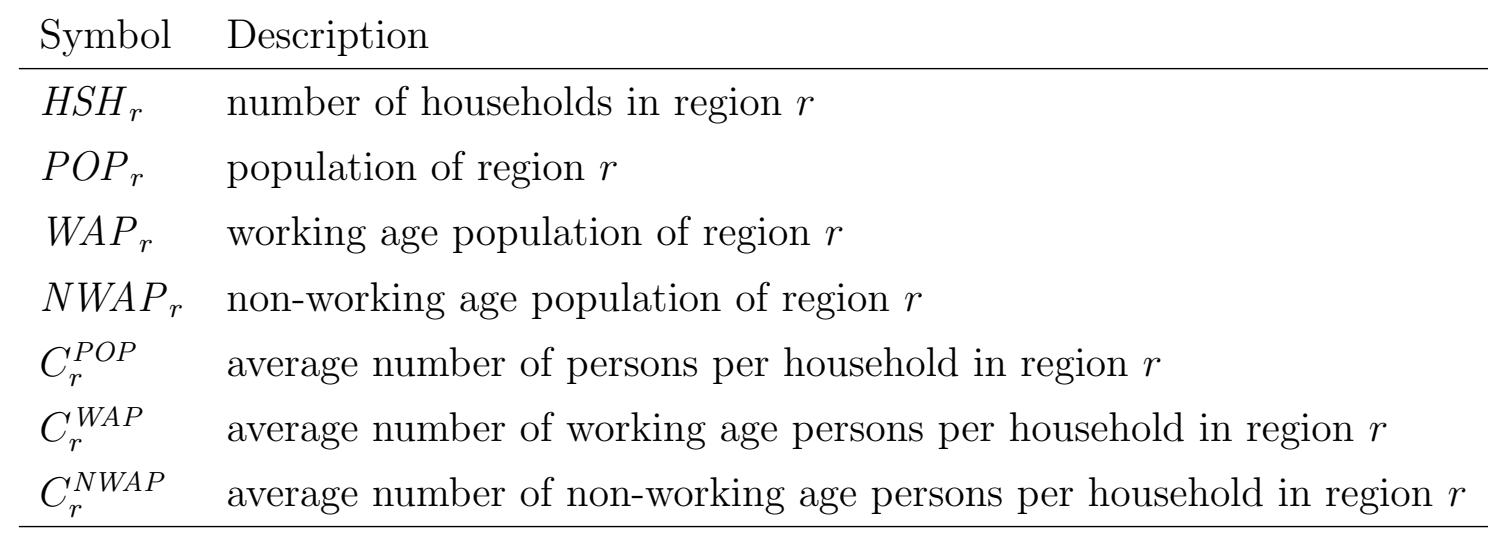

Table 4.1: Demographic Variables

\begin{tabular}{ll} 
Symbol & Description \\
\hline$E M P_{u, j, r}$ & employment measured in $u$ units in industry $j$ in region $r$ \\
$U N E M P_{r}$ & unemployed persons in region $r$ \\
$L F_{r}$ & persons in the labour force in region $r$ \\
$N L F_{r}$ & persons not in the labour force in region $r$ \\
$C_{u, j, r}^{E M P}$ & average employment in $u$ units in industry $j$ per household in region $r$ \\
$C_{r}^{U N E M P}$ & average unemployed per household in region $r$ \\
$C_{r}^{N L F}$ & average non-labour force per household in region $r$ \\
$U R_{r}$ & unemployment rate in region $r$ \\
$L F P R_{r}$ & labour force participation rate in region $r$ \\
\hline
\end{tabular}

Table 4.2: Labour Market Variables

household mobility to have implications for labour market outcomes. These variables are introduced in equations that either link them to variables in the core model, or in defining equations that ensure summation identities hold. Several elements related to our labour market measures were incorporated in the basic model of chapter 2: the time endowment of a region is proportional to its working age population (section 2.3.1); labour market variables can be measured in terms of persons, full-time equivalents (FTEs), and units consistent with the input-output data (IOunits) (section 2.4.3); employment measured in IOunits is equivalent to before-tax wage bills (equations $(2.89)-(2.92)$ ).

For this chapter employment is measured in the three different units $u \in U N I T=$ \{persons, ftes, iounits\}. FTEs give a measure of labour hours while IOunits represent real labour costs. Employment in industry $j$ in region $r$ is equivalent to the labour demand 
of the appropriate agent. This can be obtained from the core model as the real pre-tax wage bill: 6

$$
E M P_{\text {iounits }, j, r}=\left[P_{\text {pretax }, r}^{L}\right] \cdot L_{j, r}^{d}
$$

To obtain employment in terms of persons and FTEs, the conversion factors introduced in chapter 2 are used: ${ }^{7}$

$$
\begin{aligned}
E M P_{\mathrm{ftes}, j, r} & =E M P_{\text {iounits }, j, r} \cdot F_{j, r}^{\text {iounits } \rightarrow f t e s} \\
E M P_{\text {persons }, j, r} & =E M P_{\mathrm{ftes}, j, r} \cdot F_{j, r}^{f t e s \rightarrow \text { iounits }}
\end{aligned}
$$

The conversion factors are coefficients of proportionality derived from the base data and will be further discussed in section 4.4.2. Note that the factors $F_{\bullet}^{\mathbf{a} \rightarrow \mathbf{b}}$ are used to convert from $\mathbf{a}$ to $\mathbf{b}$ units in multiplication and vice versa in division.

The level of unemployment is determined as a residual in the model. With the unemployment rate for each region $r \in R E G$ included in the closure list of exogenous variables, the regional level of unemployment is solved consistent with the definitions of the unemployment rate and labour force:

$$
\begin{array}{ll}
U R_{r}=\frac{U N E M P_{r}}{L F_{r}} \times 100 & \forall r \in N Z R E G \\
L F_{r}=\sum_{j} E M P_{\text {persons }, j, r}+U N E M P_{r} & \forall r \in N Z R E G
\end{array}
$$

Participation in the labour force can be endogenously determined within the model via operation of the labour-leisure choice, or exogenously controlled. The modelling of the labour-leisure choice of chapter 2 continues to be relevant here. The definition of regional labour supply, equation (2.6), remains unchanged:

$$
L_{r}^{s}=T I M E_{r}-N_{r}^{d}
$$

This equation is consistent with the working age population being the sum of all labour force and non-labour force persons and that population's time (in FTEs) being divided between labour force and non-labour force (utility-increasing) activities (using appropriate

\footnotetext{
${ }^{6}$ This equation replaces (2.89).

${ }^{7}$ Recall, values derived from Statistics NZ data for our 25 industry, 5 region implementation are listed in appendix $\mathrm{J}$
} 
conversion factors). The time endowment of region $r$ is converted from working age persons into IOunits by:

$$
T I M E_{r}=\frac{W A P_{r}}{F_{r}^{\text {iounits } \rightarrow \text { ftes }} \cdot F_{r}^{\text {ftes } \rightarrow \text { persons }}}
$$

The factors used here are averages across industries of the factors associated with employment (the $F_{j, r}^{\bullet}$ 's).

The non-labour force population of a region is similarly linked to its consumption of leisure:

$$
N L F_{r}=N_{r}^{d} \cdot F_{r}^{\text {iounits } \rightarrow \text { ftes }} \cdot F_{r}^{f t e s \rightarrow \text { persons }}
$$

Finally, the addition of defining equations for labour force participation rates facilitates the closure swap that turns the labour-leisure choice on and off:

$$
L F P R_{r}=\frac{L F_{r}}{W A P_{r}} \times 100 \quad \forall r \in N Z R E G
$$

Turning off the labour-leisure choice involves dropping the top-level demand functions of the representative household agents (equations (2.7) and (2.8) of chapter 2) and adding the following equation to the system:

$$
P_{r}^{Q, \mathbf{h s h}} \cdot Q_{r}^{\mathbf{h s h}(D)}+P_{r}^{L, \mathbf{a t a x}} \cdot N_{r}^{\mathbf{h s h}(D)}=E N D I N C_{r}^{\text {atax, } \mathbf{c} \$}-S A V_{r}^{\text {atax }, \mathbf{c} \$}
$$

This is simply the expenditure constraint that was used in the household agent's utility maximisation problem used to derive the top-level demands. ${ }^{8}$

As with the demographic measures, we require some equations to ensure the appropriate summation identities hold:

$$
\begin{aligned}
E M P_{u, j, \mathbf{N Z}} & =\sum_{r} E M P_{u, j, r} \\
U N E M P_{\mathbf{N Z}} & =\sum_{r} U N E M P_{r} \\
N L F_{\mathbf{N Z}} & =\sum_{r} N L F_{r}
\end{aligned}
$$

\footnotetext{
${ }^{8}$ The inclusion of this equation can be thought of as replacing the equation for $Q_{r}^{\mathbf{h s h}(D)}$ while the exogenous setting of $L F P R_{r}$ can be thought of as replacing the equation for $N_{r}^{\mathbf{h s h}(D)}$.
} 
The labour market characteristics of households are now straightforward to define:

$$
\begin{array}{rlrl}
C_{u, j, r}^{E M P} & =\frac{E M P_{u, j, r}}{H S H_{r}} & & \forall r \in N Z R E G \\
C_{r}^{U N E M P} & =\frac{U N E M P_{r}}{H S H_{r}} & & \forall r \in N Z R E G \\
C_{r}^{N L F} & =\frac{N L F_{r}}{H S H_{r}} & \forall r \in N Z R E G
\end{array}
$$

\subsubsection{Other Measures}

The introduction of the demographic and labour market variables discussed above allow a number of useful measures to be calculated. These include real GDP per capita, real income per household, capital stock per household, and capital-employment ratios. ${ }^{9}$ The last two measures are particularly useful when choosing a long-run closure, since either could be set exogenous instead of the current net rates of return to provide a different interpretation of the long-run.

\subsubsection{Inter-regional Household Flows}

Household mobility is introduced by assuming that divergence in regional real wage rates gives households an incentive to shift between regions. To the extent that they are able, households would respond to these incentives. If there is some trade-off in making the choice to shift, such as a moving cost or utility penalty, households may not be perfectly mobile even in the long-run. ${ }^{10}$ The regional return to labour is but one of a range of factors that households consider when making stay-or-leave decisions. There may be financial as well as social barriers to a household leaving its current region of residence. Even if these barriers are overcome, moving to another region takes time and effort.

It is unlikely that there is a satisfactory way to model such partial mobility of households within the equation system, because there would be simultaneous feedbacks from households to real wages at the same time that real wages affect households. The problem would be compounded by the model attempting to treat households simultaneously

\footnotetext{
${ }^{9}$ The defining equations for these are straightforward. For example, real GDP per capita is calculated

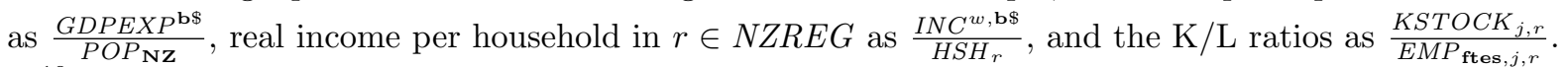

${ }^{10}$ That is, in the long-run of the model, however long that is. There is no reason to believe for example that regional real wage differences disappear at the same speed as differences in current net rates of return on capital.
} 
as different (for mobility purposes) and the same (represented by a single agent in each region). To avoid these difficulties, the issue of household mobility is addressed once the model has been solved. The general approach is that the model is solved for a given shock, and then the solution is used to calculate the mobility response of households. The model is then re-run with the shock updated to take the mobility into account.

Given solution values (in angle brackets \langle\rangle ) and benchmark equilibrium values (in square brackets []), the flows of households between regions due to changes in real wage relativities are calculated by the formula:

$$
\frac{H S H_{x \rightarrow r}}{\left\langle H S H_{x}\right\rangle}=\max \left\{\frac{\theta_{x, r}}{100}\left(\frac{\left\langle W_{r}\right\rangle}{\left\langle W_{x}\right\rangle}-\frac{\left[W_{r}\right]}{\left[W_{x}\right]}\right), 0\right\}
$$

where $H S H_{x \rightarrow r}$ is the flow of households from region $x$ to region $r$

$$
\begin{array}{ll}
W_{r} & \text { is the pre-tax real wage rate in region } r \\
\theta_{x, r} & \text { is a parameter that represents the sensitivity of } \\
& \text { households in region } x \text { to changes in the real wage rate } \\
& \text { of region } r \text { relative to their own }
\end{array}
$$

In words, the proportion of households that move from region $x$ to region $r$ equals the product of $\theta_{x, r} / 100$ and the increase in the real wage rate of $r$ relative to $x{ }^{11}$ This lends a useful interpretation to $\theta_{x, r}$ : if $\theta_{x, r}=10$, a doubling of the real wage of $r$ relative to $x$ ceteris paribus will cause $10 \%$ of the households in $x$ to move to $r$. Further discussion of the $\theta_{x, r}$ parameters is left until the section on model implementation.

Having obtained the household flows, the number of households is updated to take those flows into account as follows (with' used to indicate updated values):

$$
\left\langle H S H_{r}\right\rangle^{\prime}=\left\langle H S H_{r}\right\rangle+\sum_{x \in R E G}\left(H S H_{x \rightarrow r}-H S H_{r \rightarrow x}\right)
$$

The shock to $H S H_{r}$ for the second run is then such that the solution value is $\left\langle H S H_{r}\right\rangle^{\prime}$ :

$$
\text { shock }=100 \times \frac{\left\langle H S H_{r}\right\rangle^{\prime}-\left[H S H_{r}\right]}{\left[H S H_{r}\right]}
$$

\footnotetext{
${ }^{11}$ Either pre-tax or post-tax real wage rates could be used. It is convenient to use the pre-tax rates since they are normalised to one in the benchmark equilibrium. Post-tax rates would only need to be used if simulations entailed direct tax rates on labour income that changed by different proportions across regions.
} 
The issue arises that, if there are movements of households between regions, and if households in different regions have different characteristics on average, the household flows may cause those average characteristics to change. For example, if a region receives an inflow of households from another region that has a relatively higher unemployment rate, there is likely to be a change in the receiving region's (and sending region's) unemployment rate. To account for this possibility, we assume that there is no bias in the self-selection of households that move between regions. That is, the migration flows consist of households with average characteristics of the source region. ${ }^{12}$ For our example of differing unemployment rates across regions, the shock is adjusted so that the solution of the second model run has:

$$
\begin{aligned}
\left\langle U N E M P_{r}\right\rangle^{\prime} & =\left\langle U N E M P_{r}\right\rangle+\sum_{x \in R E G}\left\langle C_{x}^{U N E M P}\right\rangle \times H S H_{x \rightarrow r} \\
& -\left\langle C_{r}^{U N E M P}\right\rangle \times \sum_{x \in R E G} H S H_{r \rightarrow x}
\end{aligned}
$$

The inflow of unemployed from all other regions is added to unemployment in region $r$ and the outflow of unemployed is subtracted. Similar adjustments can be made to the other demographic and labour market measures as needed.

A flow of households from one region to another causes the household characteristics of the two to converge, ceteris paribus. If there are also household flows between those two regions and others, the overall effect depends on the size of all the flows and the differences in characteristics between all the regions. Specifically, if $C_{r-q}$ is the difference in a given household characteristic $C$ between two regions $r$ and $q$, the effects of household

\footnotetext{
${ }^{12} \mathrm{~A}$ simpler approach is to assume instant assimilation of incoming households to the characteristics of the receiving region. One could make the case for a quick improvement in unemployment outcomes for households coming from regions with higher unemployment rates. It is more difficult to argue for immediate assimilation of other characteristics however, particularly the demographic characteristics.
} 
flows on $C_{r-q}$ can be described by the formula: ${ }^{13}$

$$
\left\langle C_{r-q}\right\rangle^{\prime}-\left\langle C_{r-q}\right\rangle=\sum_{x \in R E G}\left(\left\langle C_{x-r}\right\rangle \frac{H S H_{x \rightarrow r}}{\left\langle H S H_{r}\right\rangle^{\prime}}-\left\langle C_{x-q}\right\rangle \frac{H S H_{x \rightarrow q}}{\left\langle H S H_{q}\right\rangle^{\prime}}\right)
$$

Regions may therefore converge or diverge in characteristics, but in general household flows act to pull the characteristics towards the national average. This can be seen by considering the example of the regions with the highest and lowest number of persons per household (PPH) - any household flow will necessarily cause the PPH of the highest $\mathrm{PPH}$ region to fall and the PPH of the lowest PPH region to rise.

\subsection{Implementation of the Labour Mobility Modelling}

The procedures required to implement the household mobility modelling discussed above involve establishing the benchmark equilibrium values of the demographic and labour market variables, choosing an appropriate model closure, and running the algorithm that adjusts the shock to account for inter-regional household flows.

${ }^{13}$ This is derived from formulae such as (4.23) as follows:

$$
\begin{aligned}
\left\langle C_{r}\right\rangle^{\prime}\left\langle H S H_{r}\right\rangle^{\prime} & =\left\langle C_{r}\right\rangle\left\langle H S H_{r}\right\rangle+\sum_{x \in R E G}\left(\left\langle C_{x}\right\rangle H S H_{x \rightarrow r}-\left\langle C_{r}\right\rangle H S H_{r \rightarrow x}\right) \\
\Rightarrow\left\langle C_{r}\right\rangle^{\prime}-\left\langle C_{q}\right\rangle^{\prime} & =\left\langle C_{r}\right\rangle \frac{\left\langle H S H_{r}\right\rangle}{\left\langle H S H_{r}\right\rangle^{\prime}}-\left\langle C_{q}\right\rangle \frac{\left\langle H S H_{q}\right\rangle}{\left\langle H S H_{q}\right\rangle^{\prime}}+\sum_{x \in R E G}\left\langle C_{x}\right\rangle\left(\frac{H S H_{x \rightarrow r}}{\left\langle H S H_{r}\right\rangle^{\prime}}-\frac{H S H_{x \rightarrow q}}{\left\langle H S H_{q}\right\rangle^{\prime}}\right) \\
& -\left(\left\langle C_{r}\right\rangle \frac{\sum_{x \in R E G} H S H_{r \rightarrow x}}{\left\langle H S H_{r}\right\rangle^{\prime}}-\left\langle C_{q}\right\rangle \frac{\sum_{x \in R E G} H S H_{q \rightarrow x}}{\left\langle H S H_{q}\right\rangle^{\prime}}\right) \\
\Rightarrow\left\langle C_{r-q}\right\rangle^{\prime}-\left\langle C_{r-q}\right\rangle & =\sum_{x \in R E G}\left\langle C_{x}\right\rangle\left(\frac{H S H_{x \rightarrow r}}{\left\langle H S H_{r}\right\rangle^{\prime}}-\frac{H S H_{x \rightarrow q}}{\left\langle H S H_{q}\right\rangle^{\prime}}\right) \\
& +\left\langle C_{r}\right\rangle \frac{\left\langle H S H_{r}\right\rangle-\sum_{x \in R E G} H S H_{r \rightarrow x}-\left\langle H S H_{r}\right\rangle^{\prime}}{\left\langle H S H_{r}\right\rangle^{\prime}} \\
& -\left\langle C_{q}\right\rangle \frac{\left\langle H S H_{q}\right\rangle-\sum_{x \in R E G} H S H_{q \rightarrow x}-\left\langle H S H_{q}\right\rangle^{\prime}}{\left\langle H S H_{q}\right\rangle^{\prime}} \\
\Rightarrow\left\langle C_{r-q}\right\rangle^{\prime}-\left\langle C_{r-q}\right\rangle & =\sum_{x \in R E G}\left\langle C_{x}\right\rangle\left(\frac{H S H_{x \rightarrow r}}{\left\langle H S H_{r}\right\rangle^{\prime}}-\frac{H S H_{x \rightarrow q}}{\left\langle H S H_{q}\right\rangle^{\prime}}\right) \\
& -\left(\left\langle C_{r}\right\rangle \frac{\sum_{x \in R E G} H S H_{x \rightarrow r}}{\left\langle H S H_{r}\right\rangle^{\prime}}-\left\langle C_{q}\right\rangle \frac{\sum_{x \in R E G} H S H_{x \rightarrow q}}{\left\langle H S H_{q}\right\rangle^{\prime}}\right)
\end{aligned}
$$

This last formula simplifies to (4.24). 


\begin{tabular}{|l|ccccc|}
\hline \multicolumn{1}{|c|}{ Demographic and Labour Market Data (000s) } \\
\hline \multirow{2}{*}{ Variable } & \multicolumn{5}{|c|}{ Region } \\
\cline { 2 - 6 } & AKL & WLG & ONI & CAN & OSI \\
\hline$\left[\right.$ POP $\left._{r}\right]$ & 1371 & 466 & 1348 & 540 & 459 \\
{$\left[H S H_{r}\right]$} & 434 & 167 & 482 & 200 & 172 \\
{$\left[\right.$ UNEMP $\left._{r}\right]$} & 37 & 13 & 36 & 11 & 9 \\
{$\left[N L F_{r}\right]$} & 219 & 65 & 198 & 78 & 66 \\
\hline
\end{tabular}

Table 4.3: Demographic and Labour Market Data (000s)

\subsubsection{Labour Market and Demographic Data}

Figures from publicly available census data for 2006 have been added to the model database. The raw employment data consists of numbers of persons employed, either full-time or part-time, by industry and region for 86 industries and 16 regions. The remainder of the raw data are figures for unemployment, non-labour force, households, and population for the 16 regions.

The labour market counts are of those 15 years and older. This means that in the data, all persons 65 years and older that are neither employed nor unemployed are counted as non-labour force. It is appropriate to treat at least part of these 65+'s as non-working age rather than non-labour force, especially given the labour-leisure choice mechanism in the model. The official non-labour force figures have therefore been reduced by a regionspecific proportion of the number of non-labour force 65+'s. For example, the figures show that in 2006, 16\% of all 65+'s in Auckland were either employed or unemployed. We assume then that only $16 \%$ of those counted as non-labour force are actually of that category, with the remainder being non-working age. The proportions do not differ much over the regions. They range from $14 \%$ for Otago to $19 \%$ for Gisborne, and this reflects the general pattern overall: North Island regions have on average slightly higher proportions of working 65+'s than South Island regions.

The raw data is mapped to the model variables to obtain the benchmark equilibrium values as shown in table 4.3 above and table 4.4 below. ${ }^{14}$

\footnotetext{
${ }^{14}$ These figures were obtained from the Statistics NZ website http://www.stats.govt.nz on $7 / 12 / 2010$. Note that the population figures are estimates of usually-resident population based on 2006 regional boundaries. These figures differ slightly from the 2006 census night counts.
} 


\begin{tabular}{|l|ccccc|}
\hline \multicolumn{5}{|l}{ Employed Persons $(000$ s $)-\left[E M P_{\text {persons }, j, r}\right]$} \\
\hline \multirow{2}{*}{ Industry } & \multicolumn{5}{|c|}{ Region } \\
\cline { 2 - 6 } & AKL & WLG & ONI & CAN & OSI \\
\hline AGRI & 8.8 & 4.2 & 69.8 & 18.0 & 26.9 \\
FOLO & 0.3 & 0.3 & 3.1 & 0.4 & 1.4 \\
FISH & 0.1 & 0.1 & 0.7 & 0.4 & 0.7 \\
MINE & 0.4 & 0.2 & 1.8 & 0.2 & 1.2 \\
OIGA & 0.0 & 0.0 & 0.2 & 0.0 & 0.0 \\
PETR & 0.0 & 0.0 & 6.9 & 0.0 & 0.0 \\
FDBT & 12.4 & 3.1 & 22.7 & 10.0 & 11.6 \\
TWPM & 24.9 & 7.1 & 20.7 & 9.9 & 8.0 \\
CHNM & 10.8 & 2.6 & 2.7 & 4.1 & 1.7 \\
METL & 10.3 & 2.0 & 8.2 & 3.4 & 3.0 \\
EQFO & 20.2 & 3.5 & 14.0 & 9.6 & 4.7 \\
UTIL & 2.6 & 1.0 & 3.8 & 1.2 & 1.1 \\
CONS & 45.4 & 15.9 & 50.3 & 19.6 & 18.2 \\
ACCR & 31.0 & 12.3 & 33.4 & 16.9 & 17.5 \\
CMIF & 40.4 & 16.6 & 17.2 & 11.2 & 6.3 \\
PROP & 19.1 & 6.0 & 16.1 & 7.6 & 5.7 \\
RBUS & 89.5 & 36.0 & 50.9 & 26.2 & 17.8 \\
GOVT & 20.1 & 22.9 & 22.9 & 8.7 & 6.6 \\
EDUC & 44.6 & 17.5 & 46.1 & 18.2 & 15.6 \\
HEAL & 54.5 & 21.5 & 65.0 & 27.8 & 24.0 \\
CUPE & 29.0 & 12.9 & 23.9 & 11.5 & 9.7 \\
OWND & 0.0 & 0.0 & 0.0 & 0.0 & 0.0 \\
WHOL & 45.5 & 8.9 & 19.4 & 13.5 & 7.6 \\
RETT & 59.2 & 21.9 & 62.9 & 28.2 & 23.9 \\
TRAN & 23.6 & 6.3 & 17.4 & 10.8 & 7.8 \\
\hline
\end{tabular}

\begin{tabular}{|l|ccccc|}
\hline \multicolumn{5}{|c|}{ Employed FTEs $(000 \mathrm{~s})-\left[E M P_{\text {ftes }, j, r}\right]$} \\
\hline \multirow{2}{*}{ Industry } & \multicolumn{5}{c|}{ Region } \\
\cline { 2 - 6 } & AKL & WLG & ONI & CAN & OSI \\
\hline AGRI & 7.7 & 3.7 & 62.3 & 16.1 & 24.3 \\
FOLO & 0.3 & 0.3 & 3.0 & 0.4 & 1.3 \\
FISH & 0.1 & 0.1 & 0.6 & 0.3 & 0.7 \\
MINE & 0.4 & 0.2 & 1.8 & 0.2 & 1.2 \\
OIGA & 0.0 & 0.0 & 0.2 & 0.0 & 0.0 \\
PETR & 0.0 & 0.0 & 6.5 & 0.0 & 0.0 \\
FDBT & 11.6 & 2.8 & 21.5 & 9.4 & 11.0 \\
TWPM & 23.1 & 6.6 & 19.3 & 9.0 & 7.3 \\
CHNM & 10.3 & 2.5 & 2.6 & 3.9 & 1.6 \\
METL & 9.9 & 1.9 & 7.9 & 3.2 & 2.9 \\
EQFO & 19.3 & 3.4 & 13.3 & 9.2 & 4.4 \\
UTIL & 2.4 & 1.0 & 3.6 & 1.2 & 1.0 \\
CONS & 42.9 & 15.1 & 47.7 & 18.6 & 17.3 \\
ACCR & 24.9 & 9.7 & 26.3 & 13.4 & 14.1 \\
CMIF & 37.8 & 15.5 & 15.2 & 10.0 & 5.5 \\
PROP & 17.0 & 5.2 & 14.2 & 6.6 & 5.0 \\
RBUS & 81.1 & 32.8 & 45.0 & 23.2 & 15.7 \\
GOVT & 19.3 & 22.0 & 21.8 & 8.3 & 6.1 \\
EDUC & 38.1 & 14.9 & 38.9 & 15.2 & 13.1 \\
HEAL & 47.5 & 18.4 & 55.4 & 23.2 & 19.9 \\
CUPE & 24.7 & 11.1 & 20.2 & 9.7 & 8.2 \\
OWND & 0.0 & 0.0 & 0.0 & 0.0 & 0.0 \\
WHOL & 42.8 & 8.3 & 18.0 & 12.6 & 7.0 \\
RETT & 49.1 & 18.1 & 52.6 & 23.1 & 19.8 \\
TRAN & 22.2 & 6.0 & 16.1 & 10.1 & 7.3 \\
\hline
\end{tabular}

Table 4.4: Employment Data (000s) 


\subsubsection{Benchmark Equilibrium Values}

The figures above and the MRIO data matrix are used to obtain benchmark equilibrium values for all the demographic and labour market variables. Benchmark values of $E M P_{\text {iounits }, j, r}$ are given by:

$$
\left[E M P_{\text {iounits }, j, r}\right]=\operatorname{MRIO}(\mathrm{LAB}, j-r)
$$

The conversion factors used in section 4.3.2 are calculated as follows: ${ }^{15}$

$$
\begin{aligned}
F_{j, r}^{f t e s \rightarrow \text { persons }} & =\frac{\left[E M P_{\text {persons }, j, r}\right]}{\left[E M P_{\mathrm{ftes}, j, r}\right]} \\
F_{j, r}^{\text {iounits } \rightarrow \text { ftes }} & =\frac{\left[E M P_{\mathrm{ftes}, j, r}\right]}{\left[E M P_{\text {iounits }, j, r}\right]} \\
F_{r}^{f t e s \rightarrow \text { persons }} & =\frac{\sum_{j \in I N D}\left[E M P_{\text {persons }, j, r}\right]}{\sum_{j \in I N D}\left[E M P_{\mathrm{ftes}, j, r}\right]} \\
F_{r}^{\text {iounits } \rightarrow \text { ftes }} & =\frac{\sum_{j \in I N D}\left[E M P_{\mathrm{ftes}, j, r}\right]}{\sum_{j \in I N D}\left[E M P_{\text {iounits }, j, r}\right]}
\end{aligned}
$$

Although these conversion factors only have a benign role in the model, they have important economic interpretations. The $F^{f t e s \rightarrow \text { persons }}$ factors measure persons per FTE in the base data, and so capture work intensity (inversely). A value of one implies each employee is working full-time - one employee is one FTE - and higher values imply more part-time workers. ${ }^{16}$ The $F^{\text {iounits } \rightarrow \text { ftes }}$ coefficients are the reciprocal of the absolute level of wages per FTE in the base data. This recovers information on actual industry and regional wage differences lost when wage rates are normalised for model calibration.

A few further points should be noted concerning the use of these conversion factors. Since they are exogenous to the model, simulations assume the economic features they represent are independent of the introduced shock. For example, the shock makes no difference to the work intensity in any industry. Also, the way the factors are used in (4.11) and (4.12) imply identical convertibility for all components of the working age population. This amounts to an assumption that, for example, as people move from

\footnotetext{
${ }^{15}$ The conversion factors thus evaluated for our 25 industry, 5 region implementation are listed in appendix J.

${ }^{16}$ The Statistics NZ definition of one FTE is 30 hours per week i.e. 1560 hours per year.
} 
unemployment or non-labour force status to employment, there is no change in the fulltime/part-time mix of employees in the workforce.

The remaining elements of the demographic and labour market variables are given benchmark values in accordance with the equations listed in section 4.3.2. For example, $\left[L F_{r}\right]$ is evaluated using the data from tables 4.3 and 4.4 in equation (4.9). [UR $]$ can then be found using $\left[L F_{r}\right]$ in (4.8). The evaluation of $\left[W A P_{r}\right]$ requires the use of the following identity, which is implied by the system of equations used to solve the model: ${ }^{17}$

$$
W A P_{r} \equiv \sum_{j} E M P_{\text {persons }, j, r}+U N E M P_{r}+N L F_{r}
$$

Establishing the benchmark equilibrium values of the demographic and labour market variables as discussed above are the main adjustments required to calibrate this enhanced version of the JENNIFER model. Benchmark values also need to be calculated for the measurement-type variables listed in section 4.3.3, and this is done according to their defining equations.

\subsubsection{Model Closures}

The short-run and long-run alternatives for model closure discussed in the previous chapters remain relevant here. Capital stocks are fixed exogenous for a short-run closure while current net rates of return are exogenously set for a long-run closure. However, rather than replacing regional time endowments on the short-run exogenous list with real wage relativities for a perfect labour mobility long-run closure as was done for the chapter 3 simulations, in this chapter they are replaced by the regional number of households $\left(H S H_{r}\right)$ for both short-run and long-run simulations. This facilitates the use of the household mobility algorithm discussed below to control labour mobility. The equations of section 4.3 link the regional number of households with regional time endowments, and thus the model core. Given $R$ model regions, $3 R$ more variables than equations have been introduced into the model. To complete the model closure for runs in either the short-run or long-run mode, the following items are added to the list of exogenous variables: ${ }^{18}$

\footnotetext{
${ }^{17}$ Since it is an identity it should not be added to the system of equations. This is tedious to prove analytically but is easily confirmed numerically. In the illustrative simulations conducted for this chapter, discrepancies between the LHS and RHS of (4.31) were no more than $0.002 \%$.

${ }^{18}$ There are of course many alternative sets of variables that could be used instead. For example $C_{r}^{P O P}$ instead of $P O P_{r}, U N E M P_{r}$ instead of $U R_{r}$, etc.
} 
- $P O P_{r}$ - number of persons in each region $r$

- $N W A P_{r}$ - number of non-working age persons in each region $r$

- $U R_{r}$ - unemployment rate in each region $r$

As discussed in section 4.3.2. if the labour-leisure choice is deactivated, we also need to add the labour force participation rates $L F P R_{r}$ to the exogenous list.

Sometimes the user may wish to swap variables between the exogenous and endogenous list between model runs if results from the first run are used in subsequent runs. For example, we might wish to observe the long-run effects of a shock holding the capitalemployment ratios constant. To do so, after a baseline run with exogenous current net rates of return, we would replace these with the regional capital-employment ratios and shock them according to the baseline results in the simulation run. ${ }^{19}$

\subsubsection{The Household Mobility Algorithm}

The first step of the algorithm used to simulate household mobility effects was specified in section 4.3.4. Once the model has been solved once, inter-regional household flows are calculated by the mobility formula (4.20). The size of the flows depend on the values assigned to $\theta_{x, r}$. Recall their interpretation: if $\theta_{x, r}=10$ and the real wage of $r$ relative to $x$ doubles ceteris paribus, $10 \%$ of the households in $x$ will shift to $r$. If the $\theta_{x, r}$ values are high enough (and/or the change in real wage relativities are large enough), a region may end up with a negative number of households. Although not strictly necessary, a simple rule-of-thumb might be to set values such that $\sum_{r} \theta_{x, r} \leq 100$ for each $x$.

The simplest assumption is that all the sensitivity parameters are equal to some value $\theta$. How the strength of the household mobility response affects model results can then be investigated by running the model repeatedly with different values for $\theta$. Alternatively, $\theta_{x, r}$ could be assumed to vary across source region $x$ and destination region $r$ to look at the implications of various asymmetries in the response of households to regional differences in labour market outcomes, such as:

- households are more likely to move to an adjacent region than one far away

\footnotetext{
${ }^{19}$ In an industry that uses no labour (e.g. OWND), the capital-employment ratio is undefined. Options are therefore to fix that industry's capital stock or capital per household exogenous.
} 
- households are more likely to move to a region similar to their own

- households are more likely to move to urban areas than away from them.

This thesis focuses on the effects of the overall level of sensitivity $\theta$; investigation of asymmetries is left for future research, which could potentially include empirical work towards estimating the parameters $\theta_{x, r}$.

The household flows calculated from the solution to the first model run are used to update the exogenous shock, to reflect the implied household mobility. As discussed in section 4.3.4, the solution values of the relevant demographic and labour market variables are adjusted and the shock required to generate those values is calculated. The computations required depend on the closure assumption (including whether the labour-leisure choice is operating and if the model is being run in Keynesian mode) but are relatively straightforward. ${ }^{20}$ Having computed the adjusted shock, this is then used to run the model a second time. The solution of this run will reflect not only the direct impact of whatever policy change is being investigated, but also the impact of any household movements thereby induced. This enables us to observe whether the assumed sensitivity of households to relative regional labour market conditions exacerbates or dampens the simulated economic shock.

We have so far considered just one iteration of the algorithm; the results at this point indicate the implications of households being mobile to a degree implied by $\theta_{x, r}$. It is easy to repeat the algorithm many times - the solution of one model run is used to update the shock of the next run. Each iteration will involve smaller household flows than the previous one as regional real wage relativities are squeezed. After enough iterations, the algorithm will not be making any significant adjustments to the shock and the model solution can be viewed as an equilibrium consistent with perfect mobility of households. The size of the $\theta_{x, r}$ values will determine the number of iterations required to reach such an equilibrium. Iterating the algorithm can be seen as manually controlling a tâtonnement process to obtain equilibrium.

\footnotetext{
${ }^{20}$ For example, if unemployment rates are exogenously fixed we need adjusted levels of regional employment and unemployment to update the exogenous shock. The level adjustments are found using formulae such as (4.23) and then updated labour force levels and unemployment rates are calculated using their definitions (equations (4.8) and (4.9)).
} 


\subsubsection{The Keynesian Mode and Mobility}

In the spirit of the Keynesian Mode of ORANI, we may wish to use a short-run model closure where the labour market is slack - increases in labour demand are reflected in falls in unemployment rates rather than increases in real wages. The closure swap needed to operationalise this is the replacement in the exogenous list of the regional unemployment rates with the real wage rates. Our mobility algorithm requires some adjustment to be compatible with such a Keynesian mode closure. Such compatibility would come from assuming that households respond to differences in regional unemployment rates rather than regional real wage rates. ${ }^{21}$ We therefore model the mobility response such that households are attracted to regions with relatively lower unemployment rates, replacing (4.20) with:

$$
\frac{H S H_{x \rightarrow r}}{\left\langle H S H_{x}\right\rangle}=\max \left\{\frac{\mu_{x, r}}{100}\left(\frac{\left\langle U R_{x}\right\rangle}{\left\langle U R_{r}\right\rangle}-\frac{\left[U R_{x}\right]}{\left[U R_{r}\right]}\right), 0\right\}
$$

The $\mu_{x, r}$ parameters have a similar interpretation to the $\theta_{x, r}$ parameters except that they concern households' sensitivity to changes in regional unemployment rate relativities.

The household mobility algorithm proceeds as for the usual case (the 'Classical Mode'), although the computation of the updated shock is complicated by the fact that the unemployment rates are endogenous in this mode. The main issue is how the shock on the real wage rates should be updated. The model as it stands only allows this to be addressed in an ad-hoc manner so there is scope for further research in this area. ${ }^{22}$

\subsection{An Illustrative Application}

In the period 2005-10 a significant programme of research was conducted under the auspices of the Department of Labour into the impact of immigration into New Zealand on the domestic economy. The output of the programme was synthesised into a final report

\footnotetext{
${ }^{21}$ This household mobility response to regional unemployment rate differences is made in recognition that real wage rate differences are not the only drivers of internal migration. Poot (1984) provides an in-depth introduction to this topic in a New Zealand context.

${ }^{22} \mathrm{~A}$ suggested approach is to shock the model in Keynesian Mode, using (4.32) to prepare a shock for a second run, which is done in Classical Mode. The first run calculates changes in regional unemployment rates to use for the second run, which then calculates changes in real wage rates.
} 
by Hodgson \& Poot (2010). A component of the research was a CGE analysis, simulating a number of different immigration scenarios which were then delineated in Nana et al. (2009). With the labour market modelling and household mobility extension of this chapter, the JENNIFER model can be used to obtain simulation results that are complementary to the outcomes of the research programme. The scenarios investigated here are however primarily to illustrate the use of the model and are not directly comparable to the results of Nana et al. (2009) and the wider body of research. ${ }^{23}$

The results of six simulations are presented for each of the short-run and long-run modes of the model. The first (in each mode) is a baseline simulation of demographic change. The following two simulations present alternative scenarios where the level of immigration is higher than in the baseline. The first of these assumes that the immigration increase occurs entirely in Auckland (AKL), while the second divides the additional immigrant households evenly between Canterbury (CAN) and Other South Island (OSI). The differences between the results of these alternative scenarios (relative to the baseline) reflect the regional and sectoral significance of the geographic distribution of immigration. For each mode, the baseline and immigration scenarios are then repeated under assumptions of partial household mobility. A comparison of the latter three simulations with the first three under the short-run mode illustrates the implications of a somewhat constrained ability of resident households to move in the short-run, while the same comparison of simulations under the long-run mode indicates the effect of a moderate degree of household mobility as opposed to the standard long-run perfect mobility assumption. ${ }^{24}$

The input for the baseline simulation is taken from population projections by Statistics New Zealand. ${ }^{25}$ They include projections of regional populations by age group and number of households from a base of 2006 out to 2031. Three different series are available: low, medium, and high, each representing certain assumptions regarding fertility, mortality,

\footnotetext{
${ }^{23}$ This is partly due to different data being fed into the respective CGE models, differences in the structure of the models themselves, and quite different tools such as econometric analysis being used in other components of the research to inform the overall outcomes. All the research reports produced under this programme are available at http://www.dol.govt.nz/research/migration/economics.asp.

${ }^{24}$ The first set of three simulations under each mode assume no household mobility. The results of these simulations, especially those for the long-run, should be viewed as interim results only. Their primary purpose is to generate the regional real wage relativities used in the household mobility algorithm.

${ }^{25}$ Downloaded from http://www.stats.govt.nz/browse_for_stats/population/estimates_and_ projections.aspx on April 1, 2011
} 
and migration. For our purposes, the 'medium' projection figures are used to obtain baseline percentage changes from 2006 of households, total population, and non-working age population across the five regions of the model. ${ }^{26}$ These percentage changes are shown in table 4.5 below. The projections reveal three basic trends:

- falling household sizes across regions

- increasing share of non-working age population in total across regions

- more rapid population growth in Auckland and other urban centres than elsewhere

For our short-run baseline simulation, we use the projected percentage changes for 2016 while for the long-run baseline the 2031 figures are used. In doing so, it is not intended to ascribe a time period to our definition of short-run and long-run; the projections are used purely as a source of hypothetical numbers.

We will see shortly that the imbalance in baseline population growth across regions leads to diverging economic outcomes. By comparing the results from the other simulations to the baseline, we can investigate how different immigration scenarios and household mobility assumptions affect these measured outcomes. The details of the simulations are summarised in table 4.6. A selection of the quantitative results are given in appendices $\mathrm{Q}$ and $\mathrm{R}$ to accompany our discussion. Further tables of results are available from the author upon request.

\subsubsection{Short-run Simulations}

The main driver of the short-run simulation results is employment change resulting from the simulated demographic change. In the baseline simulation, BSLN5, the capital stock used by each industry in each region is assumed to grow in line with their usage of labour so that there is no change in capital intensity. ${ }^{27}$ For the rest of the short-run simulations, the percentage changes in capital stocks are kept at their baseline levels. The implication is that neither household mobility nor immigration shocks affect how much regional capital is installed in the short-run.

\footnotetext{
${ }^{26}$ The definition of non-working population used here is all persons aged 0-14 and $65+$.

${ }^{27}$ It does not follow however that capital intensity does not change at the regional, sectoral, or aggregate level. The sum of the fractions do not always equal the fraction of the sums - an implication of Jensen's Inequality.
} 


\begin{tabular}{|l|c|c|c|c|c|}
\hline \multicolumn{5}{|c|}{ Households } \\
\hline \multirow{2}{*}{ Region } & \multicolumn{5}{|c|}{ Projected $\% \Delta$ from 2006} \\
\cline { 2 - 6 } & 2011 & 2016 & 2021 & 2026 & 2031 \\
\hline AKL & 10 & 21 & 33 & 44 & 55 \\
WLG & 7 & 13 & 19 & 24 & 28 \\
ONI & 6 & 12 & 17 & 21 & 25 \\
CAN & 7 & 14 & 20 & 26 & 31 \\
OSI & 6 & 10 & 14 & 17 & 20 \\
\hline \multicolumn{5}{|c|}{ Population } \\
\hline \multirow{2}{*}{ Region } & \multicolumn{7}{|c|}{ Projected $\% \Delta$ from 2006} \\
\cline { 2 - 6 } & 2011 & 2016 & 2021 & 2026 & 2031 \\
\hline AKL & 9 & 17 & 25 & 34 & 42 \\
WLG & 5 & 9 & 11 & 14 & 16 \\
ONI & 4 & 7 & 9 & 11 & 12 \\
CAN & 6 & 10 & 14 & 18 & 21 \\
OSI & 4 & 6 & 7 & 8 & 8 \\
\hline \multicolumn{7}{|c|}{ Non-working Age Population } \\
\hline \multirow{2}{*}{ Region } & 2011 & 2016 & 2021 & 2026 & 2031 \\
\cline { 2 - 6 } AKL & 8 & 20 & 32 & 45 & 59 \\
WLG & 5 & 14 & 21 & 29 & 36 \\
ONI & 5 & 12 & 20 & 26 & 33 \\
OSI & 7 & 17 & 26 & 35 & 44 \\
\hline \multirow{2}{*}{ OAN } & 5 & 13 & 22 & 29 & 35 \\
\hline
\end{tabular}

Table 4.5: Population Projections 
BSLN5: In this baseline simulation, the regions can be ranked into three groups in terms of increase in working age population. Auckland (AKL) has the largest increase, followed by Wellington (WLG) and Canterbury (CAN), and then Other North Island (ONI) and Other South Island (OSI) (table Q.3). Employment and output follow the same pattern of regional ranking (tables Q.5 and Q.7). Those industries whose production is concentrated in Auckland see larger increases in output than others - notably CHNM, METL, and EQFO (table Q.15). The increase in output for OWND is also relatively high since its capital stock in each region is assumed to grow in line with the number of households.

The closure requires that aggregate real investment increases in line with real GDP. The pattern of investment across industries depends partly on the pattern of capital growth and partly on the changes in net rates of return - compare tables Q.14, Q.16, and Q.20. The manufacturing industries have relatively high capital growth (due to being concentrated in Auckland where the population growth is the highest) and therefore receive a larger share of the expanded investment budget. The results for FISH and TRAN are due to a rise and fall respectively in each industry's net rate of return (averaged over regions) and a relatively high sensitivity to those changes (the $\beta_{j, r}$ values are relatively low) ${ }^{28}$ The regional allocation of investment reflects the bias in capital growth towards Auckland (table Q.8).

The macroeconomic effects of the baseline demographic change include an increase in real GDP, but this is not enough to sustain constant real GDP per capita (tables Q.21 and Q.26). This result is consistent with the predictions of simple growth models productivity improvements (exogenous in our model) are required to maintain constant real GDP per capita with population growth.

\footnotetext{
${ }^{28}$ The results for EDUC in table Q.20 at first glance may appear extreme. Recall that along with GOVT, HEAL, and OWND, this industry is excluded from endogenous investment allocation — see pages 86 and 97. Investment in EDUC in each region is therefore restricted to increase in line with aggregate investment. The net rate of return variable adjusts as required to satisfy the system of equations but otherwise has no bearing on the simulation results. Calibration of the model based on the investment parameters given in table 2.12 resulted in EDUC having a low average net rate of return of $0.015 \%$ initially - it ranged from $-0.16 \%$ in Auckland to $+0.17 \%$ in Other South Island. Since this number is so close to zero, even very large percentage changes are insignificant. If the average rate of return increases $500 \%$ for example, the new rate of return would only be $0.09 \%$, less than 0.1 percentage points higher.
} 


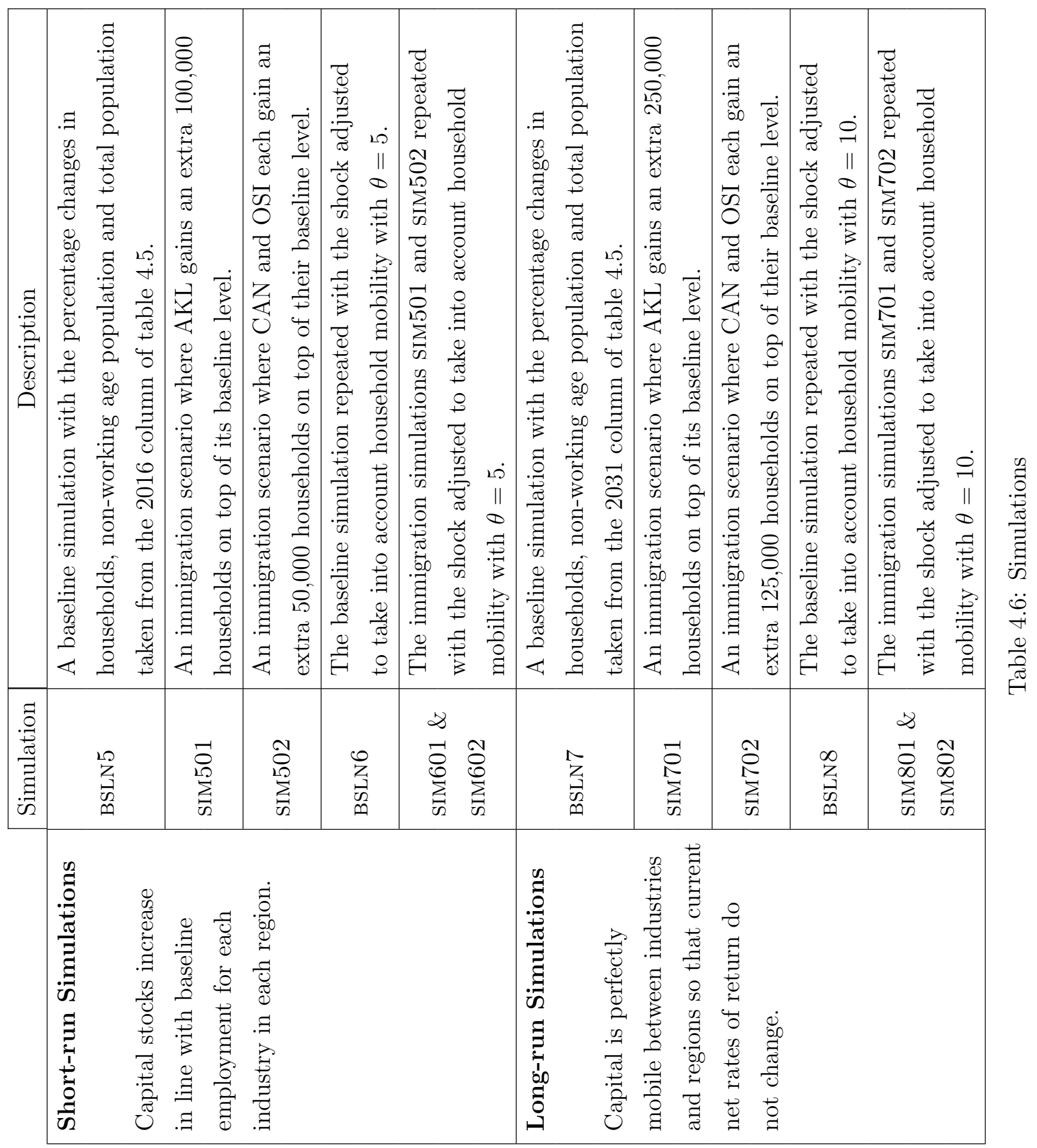


When considering the regional differences in household welfare as measured by real disposable income, it is interesting to note that households in Other North Island are (marginally) the worst off even though their region sees higher employment and capital growth than Other South Island (tables Q.5, Q.6, and Q.26). The source of this effect is that the nominal wage rate and capital rental rates in the latter region are higher (relative to the regional consumption price index) than in the former. This finding highlights the important role regional prices play in the model.

SIM501 \& SIM502: To investigate the economic effects of immigration, we look at alternative immigration scenarios where the immigration inflows are biased towards particular regions. In SIM501, the BSLN5 shock is altered so that Auckland ends up with 100,000 more households, while in SIM502 Canterbury and Other South Island receive 50,000 additional households each instead. While we interpret these as due to immigration, it should be noted that the additional households are assumed to have the same composition as those of the receiving region for simplicity. For example, in each simulation the immigration shock has no effect on any region's trend in average working age persons per household — it continues to fall as in the baseline. ${ }^{29}$ More realistic results would be obtained if the immigrant households' composition were adjusted to match what the typical immigrant household composition has been in NZ's recent experience.

The additional labour supply that immigration brings to the respective regions in each simulation boosts those regions' employment and output above that of BSLN5 (tables Q.5 and Q.7). Output in the other regions is also higher although there has been a slight fall in their employment (due to participation rate reductions - see table Q.28) and no change in capital on the baseline (due to assumption). Their higher output is then driven by lower input prices and higher consumption demand, especially from the regions receiving the immigrants. ${ }^{30}$ Notably, the primary industries (AGRI, FOLO, FISH, and MINE) and food processing (FDBT) benefit more in terms of output and investment expansion from

\footnotetext{
${ }^{29} \mathrm{An}$ implication is that the macro effects differ between the scenarios partly due to the differences in assumed household composition. For example aggregate employment rises more in SIM501 partly because Auckland has higher average working age persons per household than the South Island regions. In comparing the results, the focus should therefore be on the pattern across industries etc. rather than aggregate effects.

${ }^{30}$ Compare the magnitudes of the percentage falls in spending per household with those of the percentage increases in households, for instance, in tables Q.26 and Q.1.
} 
immigration into the South Island than into Auckland (tables Q.15 and Q.16). Aggregate investment is higher in these scenarios than the baseline owing to higher real GDP and generally the allocation of that investment becomes strongly biased towards the regions receiving the immigrants in each simulation (tables Q.21 and Q.8). The welfare measures indicate lower real GDP per capita in each scenario and the negative effects on real income and spending are borne entirely by households in the receiving regions - positive and negative short-run spill-over effects to other regions broadly cancel in the absence of inter-regional household mobility (table Q.26).

In these scenarios there is a significant divergence in regional real after-tax wage rates (table Q.25). The immigration-receiving regions see a fall in their real wage both in absolute terms and relative to that of the other regions. We might doubt that such real wage differences persist even in the short-run, so our next task is to investigate the implications of allowing a household mobility response to these differences.

BSLN6: When considering the implications of household mobility for our immigration scenarios, the appropriate baseline to use for comparisons is one that takes that mobility into account. The shock of BSLN5 is therefore adjusted for mobility and then used for this new simulation. Since we are currently considering short-run scenarios, we assume that households are relatively constrained in their ability to move between regions $(\theta$ is assigned a value of 5). The BSLN5 results for regional real wage rates suggest that if mobility is allowed, the largest household flows will be out of Auckland and into Other South Island. This is reflected in the regional pattern of household growth in BSLN6 compared to BSLN5 (table Q.1). ${ }^{31}$ The household flows serve to shift some of the growth in working age population from Auckland to the other regions (table Q.3). The employment and output effects are slightly more balanced across regions as a result (tables Q.5 and Q.7). The household flows have also caused the regional real wage differences to be slightly compressed (table Q.25). The allocation of investment shifts somewhat towards Other South Island due to changes in relative capital rental rates (tables Q.8 and Q.11). ${ }^{32}$

\footnotetext{
${ }^{31}$ The size of the net household flows into a given region can be calculated using the percentage-point difference between BSLN 6 and BSLN5. For example, the net flow into Other South Island is $1.7 \% \times 172000 \approx$ 3000 while for Other North Island it is $0.4 \% \times 482000 \approx 2000$. The percentage-point difference between the simulations captures the size of the household flow relative to the initial regional population of households.

${ }^{32}$ The effect of capital construction cost changes is relatively benign — see table Q.10.
} 
Although allowing household mobility has made very little difference to the macro effects of this demographic change, it has shifted the regional effects on real income and spending per household in Auckland's favour (tables Q.21, Q.22, and Q.26).

SIM601 \& SIM602: The demographic shocks used for the immigration scenarios SIM501 and SIM502 are adjusted to take into account household mobility in line with BSLN6 (the assumed value of $\theta$ is still 5). Immigration into Auckland results in an outflow of households that favours Wellington and Other South Island over the other regions while immigration into the South Island regions induces household movements favouring Wellington and Other North Island over Auckland (compare the percentage point differences between SIM501/2 and SIM601/2 across the regions in table Q.1). ${ }^{33}$ Comparing SIM601 with SIM501, the region-rankings for employment and output in Other North Island and Other South Island switch due to mobility favouring the latter over the former (tables Q.5 and Q.7). An analogous comparison of SIM602 and SIM502 indicates that Wellington and Other North Island receive most of the benefits from household mobility in terms of output and employment. Generally the simulations that account for household mobility indicate that such mobility causes the gains in investment and output from regionally-biased immigration to be distributed across all regions more evenly relative to the no-mobility case (tables Q.8 and Q.7). The negative effects of the simulated immigration flows on real income and spending per household are more balanced across regions when the scenario includes adjustments for inter-regional household mobility (table Q.26).

Discussion It is primarily the working age population component of short-run demographic change that determines the effect of immigration on regional economic activity,

\footnotetext{
${ }^{33}$ The household flows could consist of a mixture of immigrant and "native" households; since we assume there is no difference in household characteristics between the two types the distinction is immaterial to the results. Introducing such a distinction to the model would raise many empirical questions. Here the question is how to determine the mix of natives and immigrants in inter-regional household flows. A propensity to cluster in enclaves may make immigrants less sensitive to regional wage differences. On the other hand, immigrants may be more sensitive than natives if they don't have familial roots in any particular region. They are also more mobile by virtue of being self-selected as immigrants. Other issues that would need to be addressed are differences between natives and immigrants in consumption, labour supply, and saving behaviour, and immigrants' impact on trade and investment. Many of these issues are discussed in the New Zealand context in Hodgson \& Poot (2010).
} 
and this depends on whether there are household flows between regions in response to emerging real wage differences. Without household mobility, the positive effects of immigration on output, employment, and investment accrue mainly to the region(s) that receive the bulk of the immigrants. Household mobility allows those effects to be spread more evenly over the regions. In either case, immigration stimulates output in non-receiving regions through price effects. Our household welfare change calculations indicate that the immigrant-receiving regions experience most of the negative effects associated with the immigration shock even with household mobility. One possible implication for policy from these simulations is that immigration remains a regional issue in the short-run even when household mobility is taken into account.

\subsubsection{Long-run Simulations}

These simulations are similar to those discussed above for the short-run case, but the underlying assumptions differ in three important respects:

- the current net rates of return on capital are exogenously fixed instead of the stocks of capital in each industry in each region

- the assumed demographic change is larger than that used in the short-run simulations

- the assumed sensitivity of households to regional real wage differences is higher than in the short-run simulations to capture the idea that households are more mobile in the long-run

Each of these changes are made to reflect a longer time period between the base year and the solution year. The length of the long-run is not specified but by definition is long enough for currently installed capital to respond to changes in rental rates and construction costs. Capital stocks are endogenously determined to ensure no change in current rates of return. ${ }^{34}$

\footnotetext{
${ }^{34}$ Recall that in BSLN5, the capital stock of each industry in each region was assumed to change in line with employment. This restriction is not imposed for BSLN7 — current net rates of return are exogenous instead of $\mathrm{K} / \mathrm{L}$ ratios. As for the short-run baseline scenarios, the capital of OWND (Ownership of Occupied Dwellings) is assumed to grow in line with the number of households in each region. Unlike the short-run simulations however, capital (of all industries) is not then restricted to grow at the baseline rate for the immigration scenarios — the closure for BSLN7 is maintained throughout.
} 
BSLN7: The population change in this simulation generates a similar pattern for output and employment over the regions as in BSLN5: the outcomes are just more skewed towards Auckland away from Other North Island and Other South Island, even to the extent that employment has fallen in these latter two regions (tables R.7 and R.5). ${ }^{35}$ The simulation results indicate that capital increases less than employment in Auckland but more so in the other regions (tables R.6 and R.5). Since current rates of return are fixed and expected rates of return are equalised, the industry results for current capital are broadly reflected in the investment results - more capital requires more replacement investment (tables R.14 and R.16). An interesting exception is UTIL, which has a relatively lower increase in current capital but higher increase in investment compared to other industries. Important factors behind this result are a relatively low depreciation rate for this industry and a net rate of return close to the expected future rate. Overall the industry results follow a similar pattern to that of BSLN5 in terms of output and employment, although primary and lower value-added manufacturing industries (AGRI - FDBT) see smaller increases in these despite the higher aggregate increases (compare tables R.15 and R.13 with Q.15 and Q.13). The long-run welfare consequences as measured by percentage changes in real GDP per capita and real income and spending per household are considerably worse in the long-run than the short-run and indicate that Auckland becomes the worst-off region in terms of income per household instead of least worse-off (compare table R.26 to table Q.26).

SIM701 \& SIM702: These immigration scenarios are analogous to those of SIM501 and SIM502 except that the immigration shock is 250,000 households rather than 100,000. As before, they either add to the population of Auckland or are divided equally between Canterbury and Other South Island. An implication of the long-run closure used for these scenarios is that the aggregate capital stock can change and capital can also shift between regions. ${ }^{36}$ While capital stocks increase in each of our model regions relative to the baseline, there has been substitution toward labour in the regions that receive the immigration inflows and substitution toward capital in the rest (tables R.5 and R.6). As

\footnotetext{
${ }^{35}$ Falling employment in Other North Island and Other South Island reflects the projected decrease in working age population of those regions embodied in the Statistics NZ estimates - see tables 4.5 and R.3.

${ }^{36}$ See page 137 of chapter 3 for a description of the form that such capital mobility may take.
} 
in BSLN7, the additional capital generates additional gross investment and the increase in capacity allows output to expand (tables R.8 and R.7). Although the rankings of output and investment outcomes across regions and industries are broadly similar to those of the short-run simulations, there are some interesting exceptions. One example is Canterbury, which has the second highest increase in output and investment in SIM701 compared to third and fourth (equal) respectively in SIM501. OWND and RBUS have better output outcomes (relative to other industries) in SIM701 while METL has better and FISH has worse investment results in SIM702, all compared to the relevant short-run scenario (SIM501 and SIM502). Capital growth is biased towards the immigrant-receiving regions, with a significant component of that growth being of OWND capital stock (because it comprises a large share of the total and increases the most — see tables R.6 and R.14). The increase in after-tax income for the immigrant-receiving regions, however, is still such that they see the largest fall in income per household (table R.26). There are some notable differences between the short-run and long-run income per household results for the other regions. For example, there is less variation in the percentage decrease of this measure in SIM701 compared to SIM501 for the regions outside Auckland. In SIM702, Auckland has a better result relative to the baseline (BSLN7) while it was slightly worse in SIM502 compared to BSLN5. Generally the regions that don't receive immigrants are better off in terms of income and spending per household in SIM701 and SIM702 compared to the baseline. This was not the case for the short-run immigration scenarios, at least in terms of income per household.

For each scenario, the real wage rate that prevails in each immigrant-receiving region falls significantly while it increases relative to the baseline in the other regions (table R.25). ${ }^{37}$ We therefore run the long-run immigration scenarios again, taking into account households' endogenous mobility response to these real wage differences.

BSLN8: For the present long-run case, we assume a level of sensitivity to regional real wage differences that is higher than that of the short-run simulations, to imply that households are more mobile over this longer time frame $(\theta=10)$. As was seen in the short-run baseline with household mobility (BSLN6), households flow out of Auckland, and Other South Island sees the largest percentage-point increase in households (table

\footnotetext{
${ }^{37}$ Recall that Auckland is the immigrant-receiving region in SIM701 while Canterbury and Other South Island are the regions that receive the immigrant inflows in SIM702.
} 
R.1). However, unlike in the short-run simulations, household mobility has implications for the distribution of capital - since it is mobile here, capital has moved with labour away from Auckland (tables R.5 and R.6). The regional outcomes for output reflect this shift of resources; the outcome for Other South Island is significantly better (relative to BSLN7) compared to its outcome in BSLN6 (relative to BSLN5) — see tables Q.7 and R.7. The regional shift of capital induced by household mobility also affects the allocation of investment such that the percentage increases in regional investment are closer to the national average than in BSLN7 (table R.8). Labour-intensive industries whose production is relatively concentrated in Other South Island (e.g. AGRI, FISH) have higher levels of output and investment than in BSLN7 while capital-intensive industries concentrated in Auckland (e.g. CHNM and EQFO) have lower levels (tables R.15 and R.16). Along with the welfare effects being more negative in the long-run than the short-run, household mobility affects the results more in the long-run than the short-run (tables Q.26 and R.26). For example, real income per household in Other South Island is 2.4 percentage points lower, and in Auckland 2.1 percentage points higher in BSLN8 than BSLN7 while the analogous comparison between BSLN6 and BSLN5 show percentage point differences of only 0.7 for these regions.

SIM801 \& SIM802: This final pair of simulations estimate the long-run effects of our immigration shocks while households are partially mobile between regions (theta $=10$ ). The household flows out of the immigration-receiving regions favour Wellington and Other South Island in SIM801 and Wellington and Other North Island in SIM802 when compared to their no-mobility simulation counterparts, sim701 and SIM702 (table R.1). This is a similar result to that seen in SIM601 and SIM602. ${ }^{38}$ The moderate degree of household mobility, combined with perfect capital mobility, leads to different regional rankings of employment, output, and investment for SIM801 (it does not appear to have this effect for sim802). For example, Canterbury had the second-best outcome (after Auckland) and Other South Island had the worst outcome for all of these measures in SIM701 but

\footnotetext{
${ }^{38} \mathrm{~A}$ minor difference arises regarding the direction of household flows between Canterbury (CAN) and Other South Island (OSI) when comparing SIM802 with sIm602: in the long-run case, the real wage rate falls more in CAN than OSI in SIM702, so there is a flow of households from CAN to OSI in SIM802. On the other hand, households flow from OSI to CAN in SIM602 because the real wage falls more in OSI than CAN in SIM502 - compare tables Q.25 and R.25.
} 
Wellington has the second-best and Other North Island the worst outcome in SIM801 (tables R.5, R.7, and R.8). As seen in the comparison to SIM601 and SIM602 to their no-mobility counterparts, assuming partial household mobility (this time with capital mobility as well) results in a balancing-out of the effects of our immigration shocks on industrial and regional measures of economic activity and the distribution of the welfare effects across the regions. When the percentage changes in regional and industrial output are above the national average in the no-mobility simulations $(22.6 \%$ in SIM701, $20.6 \%$ in SIM702), generally the corresponding results in SIM801 and SIM802 are lower and when they are below the national average, the results from the household mobility simulations are higher. Two notable exceptions are the CONS and GOVT industries which have higher output in both SIM801 and SIM802 even though they already have above-average increases in output in SIM701 and sIM702 (table R.15). Similarly, when a region's real income or spending per household is higher than the national average in the no-mobility simulations, it is lower in SIM801 and SIM802. Auckland is the only exception, with relatively better income per household income in SIM801 than SIM701 even though its result in the latter simulation was already better than the national average.

Two final points on these simulations concern the labour force participation rates (LFPRs) and real wage rates. The most significant changes in regional LFPRs occur in SIM801 - see table R.28. The reduction in LFPR in the regions outside Auckland is due to the shift of households from Auckland, which had a lower average LFPR in the baseyear. ${ }^{39}$ Finally, despite the higher assumed degree of relative wage sensitivity in these long-run immigration scenarios, regional real wage rates remain significantly different. Out of the indirectly affected regions (those that don't receive the immigration shock directly), notably the real wage rate of Other North Island remains above those of the others. Neither moving from short-run to long-run nor allowing moderate household mobility substantially eliminates the estimated regional real wage differences (tables R.25

\footnotetext{
${ }^{39}$ The results in the table are percentage changes, so for example the $1.0 \%$ decrease in the LFPR of Other South Island corresponds to a 0.778 percentage-point decrease. In order to capture the effect of household mobility on LFPRs, the labour-leisure choice is turned off for these simulations. The endogenous LFPR changes from the no-mobility simulation are included in the calculation of LFPR changes that arise from the inter-regional household flows. Further changes in LFPRs due to changes in wages and consumption prices cannot be obtained from the model without a further run under the appropriate closure. In short, there is a determinacy problem with the LFPRs in a single run of the model using the household mobility algorithm and the labour-leisure choice.
} 
and Q.25).

Discussion There are two main drivers of the results from these long-run simulations increases in regional working age population and increases in capital stocks. While capital stocks were assumed to increase in the short-run simulations, the degree of increase was not allowed to vary in response to changes in demographic trends. Here, population growth stimulates capital accumulation and this is most apparent in the immigration simulations. There is therefore a positive effect of immigration on output and investment. To the extent that regional differences in capital growth lead to regional real wage rate differences, the long-run perfect capital mobility assumption adds to households' incentive to move between regions when unbalanced demographic change causes regional wage rates to diverge. The economic effects of inter-regional households flows are magnified when capital is induced to flow in the same direction. Assumptions regarding the degree of labour and capital mobility do not change some of the broad conclusions on the welfare impact of immigration to the immigrant-receiving regions, but they are important factors in determining the relative indirect effects on the other regions.

\subsubsection{Comparison with the Department of Labour Research}

Our simulation results are consistent with the conclusion of Hodgson \& Poot (2010), which is in turn consistent with other New Zealand and international empirical work, that immigration has little effect on the aggregate real wage rate in the long-term. ${ }^{40}$ These simulations do however offer the complementary idea that regional real wage rates can differ significantly even in the long-run, as we have seen in table R.25. The macro level results also mask important distributional consequences such as differences in regional output, investment, employment, and household disposable income. Internal migration can alleviate these somewhat if households (and perhaps capital) flow out of the directly affected regions (thereby reducing the excess labour supply that the regional economy has to adjust to) and into regions where the associated resources can be most efficiently

\footnotetext{
${ }^{40}$ See for example Poot \& Cochrane (2005). Our short-run immigration simulations indicate a negative effect on the real wage but in the long-run the effect is considerably less despite the higher inflow of immigrant households - compare the results for SIM601 and SIM602 in table Q.22 with those of SIM801 and SIM 802 in table R.22.
} 
utilised. $^{41}$

The overarching conclusion of the Department of Labour research programme is that immigration has a net benefit to the domestic economy. As already noted, our application of JENNIFER to the question is not directly comparable to the methods used leading to that conclusion. A simple example is that in the CGE analysis of Nana et al. (2009), productivity and trade sector developments were built into the baseline that have not been included here. This is probably the main reason our results predict that real GDP per capita falls with immigration while Nana et al. find a small positive effect. The main contribution of our simulations is to show how the regional distribution of households and industrial production, and therefore regional prices, matter for the impact across regions and industries.

\footnotetext{
${ }^{41}$ Our simulations predict some displacement of currently residing households by immigrant households (recalling that our definition of immigration is simply an inflow of households where the immigrant households are identical in characteristics to those currently residing in the destination region). There is a competing argument and associated body of research that immigration can have the opposite effect especially in urban areas - agglomeration economies, clustering, etc. Longhi, Nijkamp \& Poot (2010) discuss the empirical evidence on these and other determinants of the impact of immigration on regional labour markets.
} 


\section{Chapter 5}

\section{Conclusion}

Although New Zealand has had an active CGE modelling community since the 1980's, a multi-regional CGE model for the country has not been developed until now. In presenting the prototype JENNIFER model, this thesis has demonstrated the feasibility of developing a comprehensive model that captures the benefits of the bottom-up approach. The key findings are set out below, followed by a discussion of ways in which the model may be developed to maximise its full potential.

\subsection{The JENNIFER Model}

Chapter 2 sets out the theoretical structure of a multi-regional CGE model with bottomup micro-foundations and details a particular implementation of the model using New Zealand data. This fills an important gap in the New Zealand CGE modelling literature as none of the models in current use have a structure involving bottom-up regional modelling. The method of implementation is also a key contribution, utilising a maximum-entropy approach to overcome data shortages. An illustrative simulation of a natural disaster that strikes the Wellington central business district demonstrates the strengths of the bottomup multi-regional approach - that the model can capture differential effects across regions of shocks that occur at the regional level, and incorporates flow-on and feedback effects between regions. Sensitivity testing of the substitution elasticity between domestic sources of products reinforces the importance of empirically-estimated parameters in CGE models. 


\subsection{The Geographic Nature of Distribution}

An extension to the basic JENNIFER model is pursued in Chapter 3 to introduce the modelling of distribution services. The key structural difference is that products identified as distribution services are required to facilitate movement of other products from seller to buyer. Thus there are no opportunities to substitute away from these services if they become relatively more expensive. To implement the additional structure, sets of coefficients are specified to control technical possibilities in the usage of the distribution services. These include switches that can dictate, for example, that wholesale trade is only involved in the delivery of tangible products, that retail trade is only used by in-region purchasers, and that transport is required for moving physical products across regional borders or to exporters. That these assumptions can be integrated seamlessly into the database highlights the strength of the maximum-entropy approach discussed in chapter 2. Simulations of an oil price shock show that the regional assumptions surrounding the distribution networks can have an important bearing on simulation results even when the shock originates at the national rather than regional level.

\subsection{Partial Labour Mobility}

Chapter 4 investigates the issue of labour mobility in the short-run and long-run context. A module is added to the extended JENNIFER model of Chapter 3 to allow for a continuum of labour mobility possibilities between the usual short-run, total immobility and long-run, perfectly mobile assumptions. By varying the parameter that controls the degree of household responsiveness to regional real wage differences, labour can be made more mobile than capital in the short-run and less mobile than capital in the long-run. Additional structure is required to link populations, households, and labour market components. An important element is a link back to the endogenous labour supply theory of the basic model. Publicly available demographic and labour market data are used to implement the mobility module. The importance of a mobility response to relative real wage changes is explored in an illustrative application looking at the impact of regionallyconcentrated immigration flows. The simulations suggest that population movements can work to dissipate the welfare effects of such migration inflows. 


\subsection{Future Development}

CGE models should be based on as much actual data and empirically-estimated parameters as possible to strengthen their predictions. The JENNIFER model is designed to take maximum advantage of available sub-national GDP estimates and other regional data. At present only arbitrary assumptions are used to disaggregate exports and imports top-down by region. The use of New Zealand trade data by port would allow a more detailed description of the flows of tangible products from producing regions to ports of export, and ports of import to users. The integration of the information contained in the Auckland SAM prepared by Market Economics Ltd. would enable a richer model description of the special role Auckland plays in the economy - that of a conduit between the foreign sector and the rest of the country.

Other structural elements could be added to the model provided there was sufficient information to support them. Particularly relevant for our multi-regional model would be sub-models of the transport network or population to include efficient routing and demographic trends respectively. In this regard, GAMS is particularly useful in that it can handle different classes of model other than CGE. The output of a node-based transport sub-model could become input into the CGE model for example. Another aspect that could be considered is whether modelling activity at the regional level weakens the industrial structure micro-foundations. For higher degrees of industrial and regional disaggregation, we may wish to replace the neo-classical assumptions with imperfect competition for some industries and regions.

Yet with the existing structure there are many possibilities for improvement, particularly in the areas of elasticity estimation and investment parameters. While available data would allow more industries and regions to receive separate treatment, labour demand to be broken down into occupations, and households to be split into income groups, these are of secondary importance to improving the quality of data presently used. In any case, expanding the model along those lines will quickly raise implementation challenges if computer memory constraints were reached.

It is envisaged that such challenges will recede as computational power continues to rise. In that case however, there may be more to gain from taking the model in entirely different directions. Explicit dynamics and intertemporal behaviour could be incorporated into the model. This would be useful especially in further formalising inter-regional labour 
mobility and accounting for associated adjustment costs. The opportunity also exists to exploit the ability of GAMS to solve mixed complementarity problems. This class of problems involves optimising subject to a set of constraints, of which some may be inequalities rather than strict equalities. Thus some kind of objective could be maximised subject to the CGE model, potentially without needing all markets to clear. The most obvious example of the use of such a model would be to determine an optimum tax rate that delivers a certain outcome for employment or output across regions, or more crucially, the optimum change in such a policy in response to a given exogenous shock.

Inevitably, the direction in which the JENNIFER model develops will be strongly influenced by the real-world issues to which it is applied. At the time of writing, a significant current issue that the model could be used to investigate is the economic impact across regions of the Christchurch earthquakes of September 2010 and February 2011. For such an application, useful enhancements would include introducing different capital types, disaggregation of labour by occupation, separation of local and central government, and more detailed descriptions of the insurance industry and transportation networks for which Christchurch is an important hub. Further development of the labour mobility module would also be useful, including extending it to take into account international movements of households. 
Appendices 



\section{Appendix A}

\section{CGE Models in the Literature}

\section{New Zealand CGE Models ${ }^{1}$}

JOANNA (Wallace 1984) A comparative-static national model, solved by linearisation. Constructed roughly at the same time as ORANI, it bears many similarities to that model in its theoretical structure and implementation. A modern and up-to-date version is in current use by BERL.

JULIANNE (Stroombergen 1986) A dynamic national model, solved in the levels. This model was developed at about the same time as JOANNA, and serves well as a natural counterpoint to it.

JOANI (Nana, Hall \& Philpott 1995) A comparative static multi-regional model of NZ and Australia, solved by linearisation. In linking JOANNA and ORANI together, the model investigates the implications of the interdependence of the countries' product and factor markets.

JODY (Nana 1999) A dynamic national model incorporating forward-looking behaviour of consumers and investors, solved by linearisation. This involved adding intertemporal relationships to JOANNA and utilising the time-saving features of GEMPACK to aid the solution algorithm.

GEAR (Yeoman, Kim, McDonald \& Zhang 2009) The General Equilibrium of Auckland

\footnotetext{
${ }^{1}$ Along with the citations here, the reader is directed to Wells \& Easton (1986) and the selection of papers from the RPEP collection listed in appendix B.
} 
Region Model, a single-region model developed by Market Economics Ltd.

ORANI-NZ A New Zealand version of ORANI, operated by NZIER.

MONASH-NZ A New Zealand version of MONASH, operated by NZIER. ${ }^{2}$

NZCEM (Lennox \& van Nieuwkoop 2009) The New Zealand Climate-Economy Model developed at Landcare Research.

NZTGEM (Lennox 2010) The New Zealand Tourism General Equilibrium Model developed at LEaP.

ESSAM The Energy Substitution, Social Accounting Matrix Model developed by Infometrics. The model is probably derived from (or a variant of) JULIANNE, developed to include energy sector detail along with the complete set of financial flows captured in the SAM.

\section{Selected Single-Region Models in the International Literature}

MSG $^{3}$ (Johansen 1960) Widely considered to be the first CGE model. Essentially a multi-sectoral growth model of Norway with a national input-output table used (along with other data) to assign values to the equation coefficients. The first to propose the method of logarithmic differentiation as a means of numerically solving Walrasian general equilibrium models.

ORANI (Dixon, Parmenter, Sutton \& Vincent 1982) A comparative-static, single-region model of the Australian economy. Chapter 6 describes the ORES (ORANI Regional Equation System) top-down regional extension module. While the solution method is based on Johansen's work, the use of GEMPACK enables the linearisation errors inherent in the Johansen solution to be virtually eliminated.

\footnotetext{
${ }^{2}$ For a recent economic impact analysis that utilises this model, see Kaye-Blake, Schilling \& Zuccollo (2011).

${ }^{3}$ Not to be confused with the McKibbin-Sachs Global model (McKibbin \& Sachs 1989, known as MSG2) which is a multi-country dynamic general equilibrium model. Although Johansen did not name his model as MSG, it is often referred to as such in the literature. See for example Bergman (2005, p. 1279)
} 
ORANI-G (Horridge 2008) A more recent, generic description of ORANI for use in modelling courses run by the CoPS group. The model equations are expressed in TABLO code for GEMPACK usage. Unlike in the original treatment, the model is not introduced by way of a 'skeletal' version - this is the function of the MINIMAL model.

MINIMAL (Horridge 2001) The equivalent for the generic ORANI-G model of the miniature ORANI model set out in chapter 2 of Dixon, Parmenter, Sutton \& Vincent (1982). That is, a miniature version of ORANI-G, with the linearised model equations written in TABLO code.

MONASH (Dixon \& Rimmer 2002) A recursive-dynamic, single-region model of the Australian economy, building upon ORANI. It incorporates a top-down regional disaggregation module, also adapted from that of ORANI.

\section{Selected Multi-Regional Models in the International Literature}

ORES (Dixon, Parmenter, Sutton \& Vincent 1982, ch. 6) Although not strictly speaking a multi-regional model, instances of ORANI with this top-down regional disaggregation module provides regional results. The user specifies a division of model industries into national and local types and then various sourcing and usage assumptions are made to produce local multiplier effects. Sometimes referred to as ORANI-LMPST in the literature, since the module is based on the method used in Leontief, Morgan, Polenske, Simpson \& Tower (1965).

MRSMAE (Liew 1981) A bottom-up multi-regional CGE model based on ORANI. The multi-regional input-output database is constructed using Leontief \& Strout's (1963) gravity model. The main point of the model is to emphasise the strengths and weaknesses of the bottom-up approach relative to the top-down approach taken in ORANI's regional extension module.

FEDERAL (Madden 1990) A bottom-up comparative-static model of Australia, solved by linearisation. FEDERAL assumes the availability of multi-regional input-output data and region-level fiscal data. The cited work sets out a two region implementation of FEDERAL named TASMAIN, the two regions being Tasmania and the Rest 
of Australia. An important contribution was the detailing of how one "subtracts" a region's (Tasmania's) input-output flows from a national table (Australia's) to obtain the residual region's flows.

FEDERAL-F (Giesecke \& Madden 2003) A model based on FEDERAL with MONASHstyle dynamics. Due to FEDERAL's emphasis on modelling national and state-level government finances, it is particularly suited to fiscal policy questions.

MMRF (Centre of Policy Studies 2008) A multi-regional model developed around the same time as MONASH. It eventually received MONASH-style dynamics and has detailed modelling of greenhouse gas emissions and energy use.

TERM (Horridge, Madden \& Wittwer 2005) A static multi-regional model that uses bottom-up modelling for Australian states and then top-down modelling to obtain results at a higher level of geographic disaggregation. Using state-level input-output tables, the model uses a gravity formula to estimate interregional flows.

GTAP (Hertel 1997, Narayanan \& Walmsley 2008) A bottom-up comparative-static model of the world economy. 


\section{Appendix B}

\section{Selected RPEP Papers ${ }^{1}$}

\section{JOANNA}

IP 91 Wallace \& Philpott (1980b) The Equation Structure of the JOANNA Model

IP 98 Wallace \& Philpott (1980a) The Equation Structure of JOANNA in Short-Run Model

IP 127 Wallace \& Philpott (1982) Economy-Wide Effects of Some Alternative Fiscal Packages - A General Equilibrium Analysis Using JOANNA

IP 131 Wallace, Stroombergen \& The General Equilibrium Results of a RePhilpott (1982) duction in Textile Industry Protection

IP 141 Nana (1983)

OP 73 Nana \& Philpott (1983) The 38 Sector JOANNA Model

OP 86 Nana \& Philpott (1985) Macroeconomic and Sectoral Implications of the GST - A General Equilibrium Analysis Using the JOANNA Model

\footnotetext{
${ }^{1}$ This is a list of the RPEP papers reviewed during the preparation of this thesis. Philpott (2000) provides a useful classification of a wider selection of RPEP papers. The left-hand column shows the series number: $\mathrm{OP}=$ Occasional Paper, $\mathrm{IP}=$ Internal Paper, $\mathrm{P}=$ Paper
} 


\section{JULIANNE}

OP 59 Stroombergen \& Philpott The Julianne Semi-Dynamic General (1982) Equilibrium Model

OP 66 Stroombergen \& Philpott (1983a)

IP 153 Stroombergen \& Philpott Julianne Model Sectoral Analysis of the (1983b) Economy to 1990

OP 82 Stroombergen \& Philpott Dynamic General Equilibrium Modelling (1985) of the Effects of Protection on the New Zealand Economy 1977-84

OP 94 Philpott (1988) Julianne Model Sectoral Projections of the Economy to 1992

\section{JOANI}

OP 92 Nana \& Philpott (1988) The JOANI Two Country General Equi-

OP 93 Philpott \& Nana (1988) librium Model Quantitative Implications of AustraliaNew Zealand Free Trade

\section{JODY}

$\begin{array}{lll}\text { P 271 Philpott (1995) } & \begin{array}{l}\text { Estimating the Database for a } 1990 \text { Based } \\ \text { JODY Model }\end{array} \\ & \text { Dynamic General Equilibrium Modelling } \\ & \text { of World Agricultural Trade Impacts on } \\ & \text { New Zealand - An Appraisal of a Proto- } \\ & \text { type Model }\end{array}$




\section{Other}

\begin{tabular}{|c|c|c|}
\hline IP 133 & Stroombergen (1982) & A Critique of the Johansen-Type Model \\
\hline OP 87 & $\begin{array}{l}\text { Philpott \& Stroombergen } \\
(1986)\end{array}$ & $\begin{array}{l}\text { Analysing Flexible Labour Markets - } \\
\text { A General Equilibrium Approach Using } \\
\text { CRESH Production Functions }\end{array}$ \\
\hline OP 91 & $\begin{array}{l}\text { Poot, Nana \& Philpott } \\
(1987)\end{array}$ & $\begin{array}{l}\text { International Migration and the New } \\
\text { Zealand Economy - A Trans-Tasman } \\
\text { Comparison }\end{array}$ \\
\hline OP 98 & Philpott (1990b) & $\begin{array}{l}\text { Labour Market Flexibility in a General } \\
\text { Equilibrium Analysis of Paths to Full } \\
\text { Employment }\end{array}$ \\
\hline OP 100 & Philpott $(1990 a)$ & $\begin{array}{l}\text { Economic Growth in New Zealand - } \\
\text { Models and Experience }\end{array}$ \\
\hline OP 101 & Easton (1990) & The Real Wage Debate 1978 - 1990 \\
\hline OP 102 & Philpott (1992a) & $\begin{array}{l}\text { General Equilibrium Modelling for Pol- } \\
\text { icy Analysis and Economic Planning }\end{array}$ \\
\hline OP 105 & Philpott $(1992 b)$ & $\begin{array}{l}\text { Real Capital Stock by SNA Production } \\
\text { Group } 1950 \text { - } 1990\end{array}$ \\
\hline
\end{tabular}




\section{Appendix C}

\section{List of Model Variables}

This appendix lists the variables of the core model - measurement-type variables, where the meaning is obvious from the variable name, are not included here.

\begin{tabular}{|c|c|}
\hline Variable & Description \\
\hline$A P C_{r}^{w}$ & average propensity to consume of $\mathbf{h s h} \mathbf{h}_{r}$ (out of $w$ income) \\
\hline$A P S_{r}^{w}$ & average propensity to save of $\mathbf{h} \mathbf{s} \mathbf{h}_{r}$ (out of $w$ income) \\
\hline$C^{v}$ & aggregate private consumption expenditure (with valuation $v$ ) \\
\hline$C P I^{i}$ & consumption expenditure deflator index of type $i \in P I N D E X$ \\
\hline$C V_{r}$ & compensating variation for $\mathbf{h} \mathbf{s} \mathbf{h}_{r}$ \\
\hline$E M P \bullet$ & employment measures in IOunits \\
\hline$E N D I N C_{r}^{w, v}$ & $w$ endowment income of $\mathbf{h s h}_{r}$ (with valuation $v$ ) \\
\hline$E V_{r}$ & equivalent variation for $\mathbf{h} \mathbf{s} \mathbf{h}_{r}$ \\
\hline$E X P_{g}^{\text {for }(D)}$ & demand for exports of product $g$ by for \\
\hline$E X P_{g}^{(S)}$ & supply of exports of product $g$ by $\exp _{g}$ \\
\hline$F_{j, r}^{\text {ind }(D)}$ & demand for composite factor by ind $_{j, r}$ \\
\hline$F O R_{g}^{\operatorname{imp}(D)}$ & foreign product $g$ demanded by $\mathbf{i m p}_{g}$ \\
\hline$F O R_{g}^{(S)}$ & supply of foreign product $g$ by for \\
\hline$G^{v}$ & aggregate government consumption expenditure (with valuation $v$ ) \\
\hline$G D P D E F^{i}$ & GDP deflator index of type $i \in P I N D E X$ \\
\hline$G P I^{i}$ & government expenditure deflator index of type $i \in P I N D E X$ \\
\hline
\end{tabular}




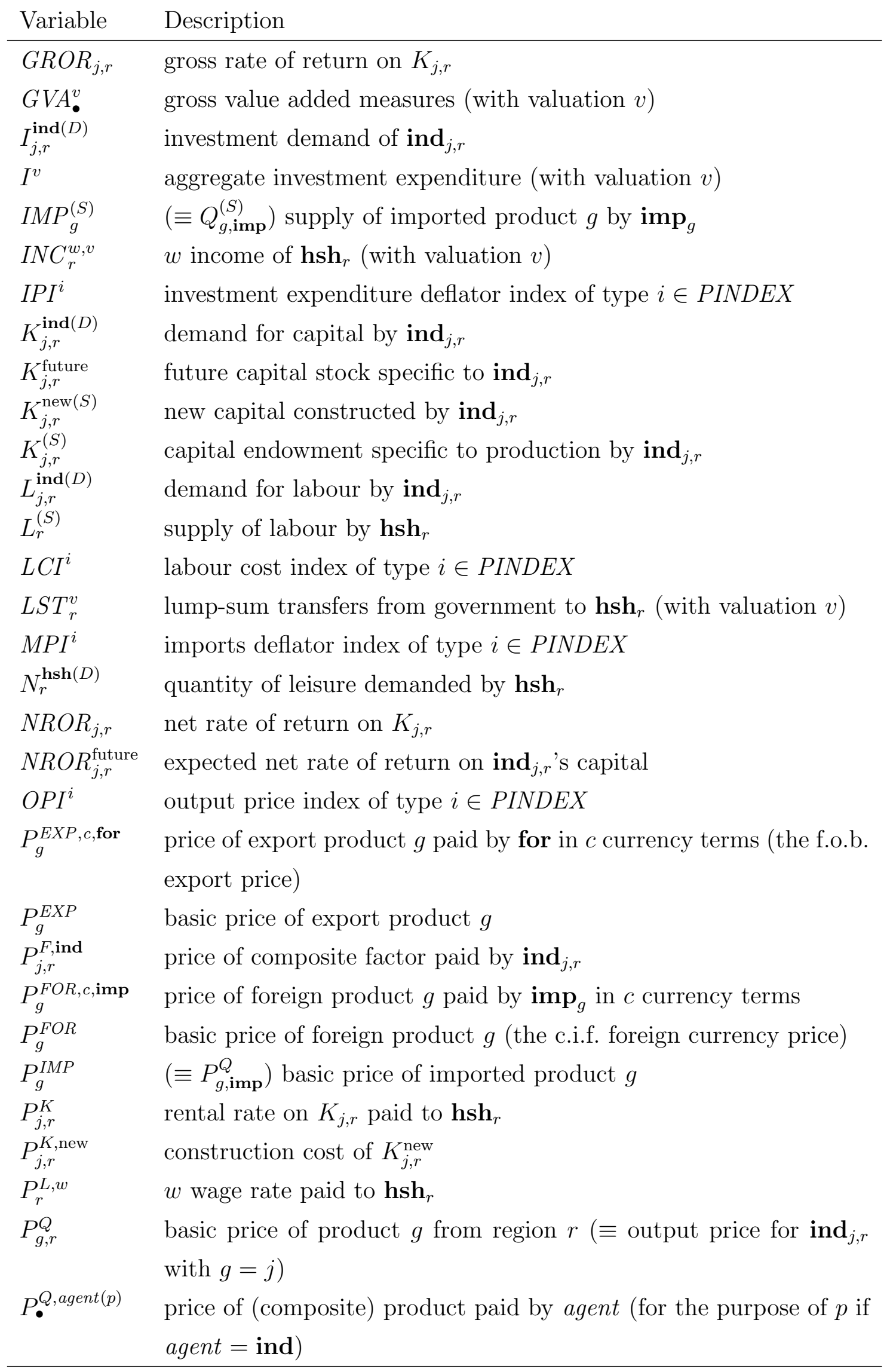




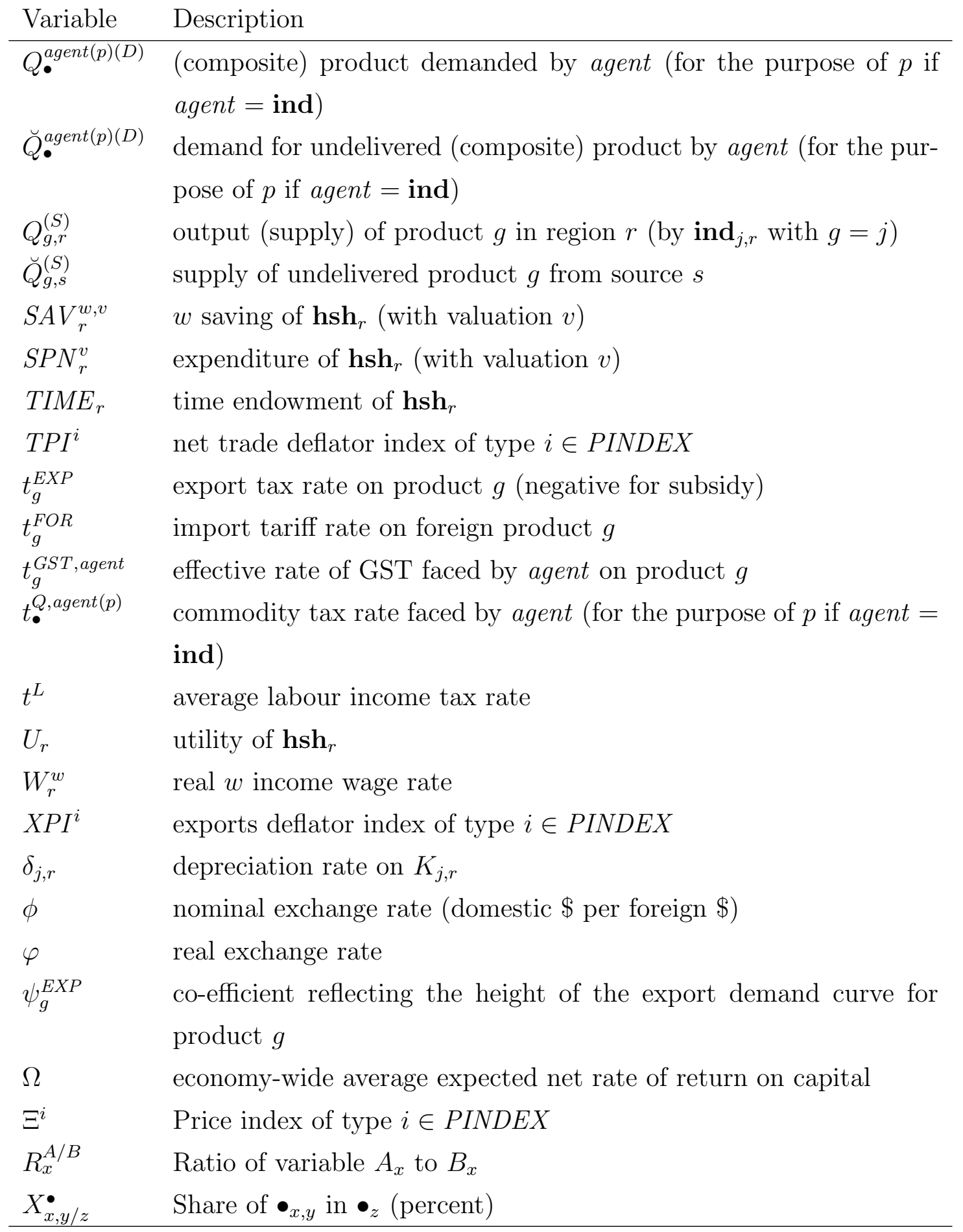




\section{Appendix D}

\section{List of Model Equations}

This appendix lists all the equations of the core model. Note that the form of the equations appear slightly different to those in the main body because these are closer to the computer-representation, while the equations in the text were simplified for clarity.

\section{Household Demand Functions}

$$
\begin{aligned}
Q_{r}^{\mathbf{h s h}(D)} & =Q_{r}^{\mathbf{h s h}}\left(P_{r}^{Q, \mathbf{h s h}}, P_{r}^{L, \text { atax }}, E N D I N C_{r}^{\text {atax }, \mathbf{c} \$}, S A V_{r}^{\text {atax }, \mathbf{c} \$} ; \sigma_{r}^{\mathbf{h s h}}\right) \\
N_{r}^{\mathbf{h s h}(D)} & =N_{r}^{\mathbf{h s h}}\left(P_{r}^{Q, \mathbf{h s h}}, P_{r}^{L, \text { atax }}, E N D I N C_{r}^{\text {atax }, \mathbf{c} \$}, S A V_{r}^{\text {atax }, \mathbf{c} \$} ; \sigma_{r}^{\mathbf{h s h}}\right) \\
Q_{g, r}^{\mathbf{h s h}(D)} & =Q_{g, r}^{\mathbf{h s h}}\left(Q_{r}^{\mathbf{h s h}(D)},\left.P_{h, r}^{Q, \mathbf{h s h}}\right|_{h} ; \sigma_{r}^{Q, \mathbf{h s h}}\right) \\
Q_{g, o, r}^{\mathbf{h s h}(D)} & =Q_{g, o, r}^{\mathbf{h s h}}\left(Q_{g, r}^{\mathbf{h s h}(D)},\left.P_{g, a, r}^{Q, \mathbf{h s h}}\right|_{a \in O R G} ; \sigma_{g, r}^{\mathbf{h s h}}\right) \\
Q_{g, x, r}^{\mathbf{h s h}(D)} & =Q_{g, x, r}^{\mathbf{h s h}}\left(Q_{g, \mathbf{d o m}, r}^{\mathbf{h s h}(D)},\left.P_{g, y, r}^{Q, \mathbf{h s h}}\right|_{y} ; \sigma_{g, \mathbf{d o m}, r}^{\mathbf{h s h}}\right)
\end{aligned}
$$

\section{Industry Demand Functions}

$$
\begin{aligned}
& Q_{j, r}^{\text {ind }(D)}=Q_{j, r}^{\text {ind }}\left(Q_{g, r}^{(S)}, P_{j, r}^{Q \text {,ind }}, P_{j, r}^{F, \text { ind }} ; \sigma_{j, r}^{\text {ind }}\right) \\
& F_{j, r}^{\text {ind }(D)}=F_{j, r}^{\text {ind }}\left(Q_{g, r}^{(S)}, P_{j, r}^{Q, \text { ind }}, P_{j, r}^{F, \text { ind }} ; \sigma_{j, r}^{\text {ind }}\right) \\
& L_{j, r}^{\operatorname{ind}(D)}=L_{j, r}^{\text {ind }}\left(F_{j, r}^{\operatorname{ind}(D)}, P_{r}^{L, \text { btax }}, P_{j, r}^{K} ; \sigma_{j, r}^{F, \text { ind }}\right) \\
& K_{j, r}^{\operatorname{ind}(D)}=K_{j, r}^{\operatorname{ind}}\left(F_{j, r}^{\operatorname{ind}(D)}, P_{r}^{L, \text { btax }}, P_{j, r}^{K} ; \sigma_{j, r}^{F, \text { ind }}\right) \\
& Q_{g, j, r}^{\operatorname{ind}(p)(D)}=Q_{g, j, r}^{\operatorname{ind}(p)}\left(Q_{j, r}^{\operatorname{ind}(D)},\left.P_{h, j, r}^{Q, \operatorname{ind}(p)}\right|_{h} ; \sigma_{j, r}^{Q, \operatorname{ind}(p)}\right) \\
& Q_{g, o, j, r}^{\operatorname{ind}(p)(D)}=Q_{g, o, j, r}^{\operatorname{ind}(p)}\left(Q_{g, j, r}^{\operatorname{ind}(p)(D)},\left.P_{g, a, j, r}^{Q, \operatorname{ind}(p)}\right|_{a \in O R G} ; \sigma_{g, j, r}^{\operatorname{ind}(p)}\right) \\
& Q_{g, x, j, r}^{\operatorname{ind}(p)(D)}=Q_{g, x, j, r}^{\operatorname{ind}(p)}\left(Q_{g, \mathbf{d o m}, j, r}^{\operatorname{ind}(p)(D)},\left.P_{g, y, j, r}^{Q, \operatorname{ind}(p)}\right|_{y} ; \sigma_{g, \mathbf{d o m}, j, r}^{\operatorname{ind}(p)}\right)
\end{aligned}
$$




\section{Government Demand Functions}

$$
\begin{aligned}
& Q_{g}^{\operatorname{gov}(D)}=Q_{g}^{\text {gov }}\left(G^{\mathbf{b} \$},\left.P_{h}^{Q, \text { gov }}\right|_{h} ; \sigma^{Q, \text { gov }}\right) \\
& Q_{g, o}^{\text {gov }(D)}=Q_{g, o}^{\text {gov }}\left(Q_{g}^{\operatorname{gov}(D)},\left.P_{g, a}^{Q, \text { gov }}\right|_{a \in O R G} ; \sigma_{g}^{\text {gov }}\right) \\
& Q_{g, x}^{\text {gov }(D)}=Q_{g, x}^{\text {gov }}\left(Q_{g, \text { dom }}^{\text {gov }(D)},\left.P_{g, y}^{Q, \text { gov }}\right|_{y} ; \sigma_{g, \text { dom }}^{\text {gov }}\right)
\end{aligned}
$$

\section{Exporting and Importing}

$$
\begin{aligned}
Q_{g}^{\exp (D)} & =E X P_{g}^{(S)} \\
Q_{g, x}^{\exp (D)} & =Q_{g, x}^{\exp }\left(Q_{g}^{\exp (D)},\left.P_{g, y}^{Q, \mathbf{e x p}}\right|_{y} ; \sigma_{g}^{\exp }\right) \\
F O R_{g}^{\mathbf{i m p}(D)} & =I M P_{g}^{(S)} \\
P_{g}^{E X P, \mathbf{f} \$, \text { for }} & =\left(E X P_{g}^{\mathbf{f o r}(D)}\right)^{-1 / \varepsilon_{g}^{E X P}} \cdot \psi_{g}^{E X P}
\end{aligned}
$$

\section{Investment Allocation}

$$
\begin{aligned}
G R O R_{j, r} & =100 \times \frac{P_{j, r}^{K}}{P_{j, r}^{K, \text { new }}} \\
N R O R_{j, r} & =G R O R_{j, r}-\delta_{j, r} \\
R_{j, r}^{G R O R / N R O R} & =\frac{G R O R_{j, r}}{N R O R_{j, r}} \\
R_{j, r}^{K \text { new } / K \text { future }} & =100 \times \frac{K_{j, r}^{\text {new }(S)}}{K_{j, r}^{\text {future }}} \\
K_{j, r}^{\text {future }} & =\left(1-\frac{\delta_{j, r}}{100}\right)^{K_{j, r}^{(S)}}+K_{j, r}^{\text {new }(S)} \\
N R O R_{j, r}^{\text {future }} & =\left(\frac{K_{j, r}^{\text {future }}}{K_{j, r}^{(S)}}\right)^{-\beta_{j, r}} \cdot N R O R_{j, r} \\
N R O R_{j, r}^{\text {future }} & =\Omega
\end{aligned}
$$




\section{Market Clearing Conditions}

$$
\begin{aligned}
\breve{Q}_{g, s}^{(S)} & =\sum_{r} \breve{Q}_{g, s, r}^{\mathbf{h s h}(D)}+\sum_{p} \sum_{j} \sum_{r} \breve{Q}_{g, s, j, r}^{\operatorname{ind}(p)(D)}+\breve{Q}_{g, s}^{\operatorname{gov}(D)}+\breve{Q}_{g, s}^{\exp (D)} \\
K_{j, r}^{(S)} & =K_{j, r}^{\operatorname{ind}(D)} \\
L_{r}^{(S)} & =\sum_{j} L_{j, r}^{\operatorname{ind}(D)} \\
K_{j, r}^{\text {new }(S)} & =I_{j, r}^{\operatorname{ind}(D)} \\
E X P_{g}^{(S)} & =E X P_{g}^{\text {for }(D)} \\
F O R_{g}^{(S)} & =F O R_{g}^{\operatorname{imp}(D)}
\end{aligned}
$$

\section{Zero Pure Profit Conditions}

$$
\begin{aligned}
P_{g, r}^{Q} \cdot \breve{Q}_{g, r}^{(S)} & =P_{j, r}^{Q, \text { ind }} \cdot Q_{j, r}^{\operatorname{ind}(D)}+P_{j, r}^{F, \text { ind }} \cdot F_{j, r}^{\text {ind }(D)} \\
P_{j, r}^{K, \text { new }} \cdot K_{j, r}^{\text {new }(S)} & =\sum_{g} P_{g, j, r}^{Q, \text { ind }(\mathbf{K})} \cdot Q_{g, j, r}^{\text {ind }(\mathbf{K})(D)} \\
P_{g}^{I M P} \cdot I M P_{g}^{(S)} & =P_{g}^{F O R, \mathbf{d} \$, \mathbf{i m p}} \cdot F O R_{g}^{\mathbf{i m p}(D)} \\
P_{g}^{E X P} \cdot E X P_{g}^{(S)} & =P_{g}^{Q, \exp } \cdot Q_{g}^{\exp (D)}
\end{aligned}
$$

\section{Purchase Prices}

$$
\begin{aligned}
P_{r}^{Q, \mathbf{h s h}} \cdot Q_{r}^{\mathbf{h s h}(D)} & =\sum_{g} P_{g, r}^{Q, \mathbf{h s h}} \cdot Q_{g, r}^{\mathbf{h s h}(D)} \\
P_{g, r}^{Q, \mathbf{h s h}} \cdot Q_{g, r}^{\mathbf{h s h}(D)} & =\sum_{o} P_{g, o, r}^{Q, \mathbf{h s h}} \cdot Q_{g, o, r}^{\mathbf{h s h}(D)} \\
P_{g, \mathbf{d o m}, r}^{Q, \mathbf{h s h}} \cdot Q_{g, \mathbf{d o m}, r}^{\mathbf{h s h}(D)} & =\sum_{x} P_{g, x, r}^{Q, \mathbf{h s h}} \cdot Q_{g, x, r}^{\mathbf{h s h}(D)} \\
P_{g, s, r}^{Q, \mathbf{h s h}} \cdot Q_{g, s, r}^{\mathbf{h s h}(D)} & =P_{g, s}^{Q} \cdot\left(1+t_{g, r}^{Q, \mathbf{h s h}}+t_{g}^{G S T, \mathbf{h s h}}\right) \cdot \breve{Q}_{g, s, r}^{\mathbf{h s h}(D)}
\end{aligned}
$$




$$
\begin{aligned}
& P_{j, r}^{Q, \text { ind }} \cdot Q_{j, r}^{\operatorname{ind}(D)}=\sum_{g} P_{g, j, r}^{Q, \operatorname{ind}(\mathbf{Q})} \cdot Q_{g, j, r}^{\operatorname{ind}(\mathbf{Q})(D)} \\
& P_{j, r}^{F, \text { ind }} \cdot F_{j, r}^{\operatorname{ind}(D)}=P_{r}^{L, \text { btax }} \cdot L_{j, r}^{\operatorname{ind}(D)}+P_{j, r}^{K} \cdot K_{j, r}^{\operatorname{ind}(D)} \\
& P_{g, j, r}^{Q, \operatorname{ind}(p)} \cdot Q_{g, j, r}^{\operatorname{ind}(p)(D)}=\sum_{o} P_{g, o, j, r}^{Q, \operatorname{ind}(p)} \cdot Q_{g, o, j, r}^{\operatorname{ind}(p)(D)} \\
& P_{g, \mathbf{d o m}, j, r}^{Q, \operatorname{ind}(p)} \cdot Q_{g, \mathbf{d o m}, j, r}^{\operatorname{ind}(p)(D)}=\sum_{x} P_{g, x, j, r}^{Q, \operatorname{ind}(p)} \cdot Q_{g, x, j, r}^{\operatorname{ind}(p)(D)} \\
& P_{g, s, j, r}^{Q, \operatorname{ind}(p)} \cdot Q_{g, s, j, r}^{\operatorname{ind}(p)(D)}=P_{g, s}^{Q} \cdot\left(1+t_{g, j, r}^{Q, \operatorname{ind}(p)}\right) \cdot \breve{Q}_{g, s, j, r}^{\operatorname{ind}(p)(D)} \\
& P_{g}^{Q, \text { gov }} \cdot Q_{g}^{\operatorname{gov}(D)}=\sum_{o} P_{g, o}^{Q, \text { gov }} \cdot Q_{g, o}^{\operatorname{gov}(D)} \\
& P_{g, \text { dom }}^{Q, \text { gov }} \cdot Q_{g, \text { dom }}^{\text {gov }(D)}=\sum_{x} P_{g, x}^{Q, \text { gov }} \cdot Q_{g, x}^{\operatorname{gov}(D)} \\
& P_{g, s}^{Q, \text { gov }} \cdot Q_{g, s}^{\operatorname{gov}(D)}=P_{g, s}^{Q} \cdot\left(1+t_{g}^{Q, \text { gov }}\right) \cdot \breve{Q}_{g, s}^{\operatorname{gov}(D)} \\
& P_{g}^{Q, \exp } \cdot Q_{g}^{\exp (D)}=\sum_{x} P_{g, x}^{Q, \exp } \cdot Q_{g, x}^{\exp (D)} \\
& P_{g, x}^{Q, \exp } \cdot Q_{g, x}^{\exp (D)}=P_{g, x}^{Q} \cdot\left(1+t_{g}^{Q, \exp }+t_{g}^{G S T, \exp }\right) \cdot \breve{Q}_{g, x}^{\exp (D)} \\
& P_{g}^{E X P}=\left(1-t_{g}^{E X P}\right) \cdot P_{g}^{E X P, \mathbf{d} \$, \text { for }} \\
& P_{g}^{F O R, \mathbf{f} \$ \mathbf{i m p}}=\left(1+t_{g}^{F O R}\right) \cdot P_{g}^{F O R} \\
& P_{g}^{E X P, \mathbf{d} \$, \text { for }}=\phi \cdot P_{g}^{E X P, \mathbf{f} \$, \text { for }} \\
& P_{g}^{F O R, \mathbf{d} \$, \mathbf{i m p}}=\phi \cdot P_{g}^{F O R, \mathbf{f} \$, \mathbf{i m p}} \\
& P_{r}^{L, \text { atax }}=P_{r}^{L, \text { btax }}\left(1-t^{L}\right) \\
& \phi=1
\end{aligned}
$$

Household Endowment Income, Expenditure, Income, and Saving

$$
\begin{aligned}
S P N_{r}^{\mathbf{c} \$} & =\frac{A P C_{r}^{w}}{100} \cdot I N C_{r}^{w, \mathbf{c} \$} \\
S P N_{r}^{\mathbf{c} \$} & =P_{r}^{Q, \mathbf{h s h}} \cdot Q_{r}^{\mathbf{h s h}(D)} \\
S P N_{r}^{\mathbf{b} \$} & =\left[P_{r}^{Q, \mathbf{h s h}}\right] \cdot Q_{r}^{\mathbf{h s h}(D)} \\
S P N_{r}^{\mathbf{b Q}} & =P_{r}^{Q, \mathbf{h s h}} \cdot\left[Q_{r}^{\mathbf{h s h}(D)}\right] \\
S P N_{\mathbf{N Z}}^{v} & =\sum_{r} S P N_{r}^{v}
\end{aligned}
$$$$
\forall r \in N Z R E G
$$ 


$$
\begin{aligned}
I N C_{r}^{w, v} & =W A G E S_{r}^{w, v}+K R E N T S_{r}^{v}+L S T_{r}^{v} \\
S A V_{r}^{w, v} & =I N C_{r}^{w, v}-S P N_{r}^{v} \\
A P C_{r}^{w} & =100-A P S_{r}^{w} \\
E N D I N C_{r}^{w, \mathbf{c} \$} & =P_{r}^{L, w} \cdot T I M E_{r}+\sum_{j} P_{j, r}^{K} \cdot K_{j, r}^{(S)}+L S T_{r}^{\mathbf{c} \$} \\
E N D I N C_{r}^{w, \mathbf{b} \$} & =\left[P_{r}^{L, w}\right] \cdot T I M E_{r}+\sum_{j}\left[P_{j, r}^{K}\right] \cdot K_{j, r}^{(S)}+L S T_{r}^{\mathbf{b} \$} \\
E N D I N C_{r}^{w, \mathbf{b Q}} & =P_{r}^{L, w} \cdot\left[T I M E_{r}\right]+\sum_{j} P_{j, r}^{K} \cdot\left[K_{j, r}^{(S)}\right]+L S T_{r}^{\mathbf{b Q}} \\
L_{r}^{(S)} & =T I M E_{r}-N_{r}^{\mathbf{h s h}(D)}
\end{aligned}
$$

$\forall r \in N Z R E G \quad$ (D.62)

$\forall r \in N Z R E G$ (D.63)

$\forall r \in N Z R E G$ (D.64)

\section{Domestic Expenditure on GDP}

$$
\begin{aligned}
C^{v} & =\sum_{r} S P N_{r}^{v} \\
I^{\mathbf{c} \$} & =\sum_{j} \sum_{r} P_{j, r}^{K, \text { new }} \cdot I_{j, r}^{\text {ind }(D)} \\
I^{\mathbf{b} \$} & =\sum_{j} \sum_{r}\left[P_{j, r}^{K, \text { new }}\right] \cdot I_{j, r}^{\text {ind }(D)} \\
I^{\mathbf{b Q}} & =\sum_{j} \sum_{r} P_{j, r}^{K, \text { new }} \cdot\left[I_{j, r}^{\text {ind }(D)}\right] \\
G^{\mathbf{c} \$} & =\sum_{g} P_{g}^{Q, \text { gov }} \cdot Q_{g}^{\operatorname{gov}(D)} \\
G^{\mathbf{b Q}} & =\sum_{g} P_{g}^{Q, \text { gov }} \cdot\left[Q_{g}^{\operatorname{gov}(D)}\right]
\end{aligned}
$$

\section{Trade Flows and the Trade Balance}

$$
\begin{aligned}
E X P^{\mathbf{c} \$, \mathbf{d} \$, \text { bas }} & =\sum_{g} P_{g}^{E X P} \cdot E X P_{g}^{(S)} \\
E X P^{\mathbf{c} \$, \mathbf{f} \$, \text { bas }} & =\frac{1}{\phi} \cdot E X P^{\mathbf{c} \$, \mathbf{d} \$, \text { bas }} \\
E X P^{\mathbf{c} \$, c, \text { fob }} & =\sum_{g} P_{g}^{E X P, c, \text { for }} \cdot E X P_{g}^{\text {for }(D)}
\end{aligned}
$$




$$
\begin{aligned}
& I M P^{\mathbf{c} \$, c, \text { bas }}=\sum_{g} P_{g}^{F O R, c, \mathbf{i m p}} \cdot F O R_{g}^{\mathbf{i m p}(D)} \\
& I M P^{\mathbf{c} \$, \mathbf{f} \$, \mathbf{c i f}}=\sum_{g} P_{g}^{F O R} \cdot F O R_{g}^{(S)} \\
& I M P^{\mathbf{c} \$, \mathbf{d} \$, \mathbf{c i f}}=\phi \cdot I M P^{\mathbf{c} \$, \mathbf{f} \$, \mathbf{c i f}} \\
& E X P^{\mathbf{b} \$, \mathbf{d} \$, \text { bas }}=\sum_{g}\left[P_{g}^{E X P}\right] \cdot E X P_{g}^{(S)} \\
& E X P^{\mathbf{b} \$, \mathbf{f} \$, \mathbf{b a s}}=\frac{1}{[\phi]} \cdot E X P^{\mathbf{c} \$, \mathbf{d} \$, \mathbf{b a s}} \\
& E X P^{\mathbf{b} \$, c, \text { fob }}=\sum_{g}\left[P_{g}^{E X P, c, \text { for }}\right] \cdot E X P_{g}^{\text {for }(D)}
\end{aligned}
$$

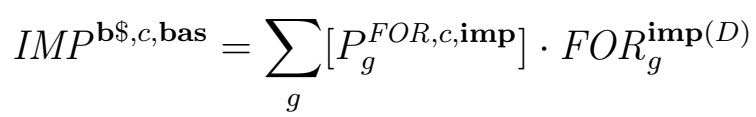

$$
\begin{aligned}
& I M P^{\mathbf{b} \$, \mathbf{f} \$, \mathbf{c i f}}=\sum_{g}\left[P_{g}^{F O R}\right] \cdot F O R_{g}^{(S)} \\
& I M P^{\mathbf{b} \$, \mathbf{d} \$, \mathbf{c i f}}=[\phi] \cdot I M P^{\mathbf{c} \$, \mathbf{f} \$, \mathbf{c i f}} \\
& T R D B A L^{v, c}=E X P^{v, c, \text { fob }}-I M P^{\mathbf{c} \$, c, \mathbf{c i f}}
\end{aligned}
$$

\section{Government Revenue and the Fiscal Balance}

$$
\begin{aligned}
I N C T A X^{\mathbf{c} \$}= & t^{L} \cdot \sum_{j} \sum_{r} P_{r}^{L, \mathbf{b t a x}} \cdot L_{j, r}^{\mathbf{i n d}(D)} \\
D U T Y^{\mathbf{c} \$}= & \sum_{g} t_{g}^{F O R} \cdot \phi \cdot P_{g}^{F O R} \cdot F O R_{g}^{\mathbf{i m p}(D)} \\
E X P S U B^{\mathbf{c} \$}= & -\sum_{g} t_{g}^{E X P} \cdot P_{g}^{E X P, \mathbf{d} \$ \text {,for }} \cdot E X P_{g}^{(S)} \\
C O M T A X^{\mathbf{c} \$}= & \sum_{g} \sum_{s} \sum_{p} \sum_{j} \sum_{r}\left(P_{g, s}^{Q} \cdot t_{g, r}^{Q, \mathbf{h s h}} \cdot \breve{Q}_{g, s, r}^{\mathbf{h s h}(D)}\right. \\
& \left.+P_{g, s}^{Q} \cdot t_{g, j, r}^{Q, \text { ind }(p)} \cdot \breve{Q}_{g, s, j, r}^{\mathbf{i n d}(p)(D)}+P_{g, s}^{Q} \cdot t_{g}^{Q, \mathbf{g o v}} \cdot \breve{Q}_{g, s}^{\operatorname{gov}(D)}+P_{g, r}^{Q} \cdot t_{g}^{Q, \mathbf{e x p}} \cdot \breve{Q}_{g, r}^{\mathbf{e x p}(D)}\right) \\
G S T^{\mathbf{c} \$}= & \sum_{g} \sum_{s} \sum_{r}\left(P_{g, s}^{Q} \cdot t_{g}^{G S T, \mathbf{h s h}} \cdot \breve{Q}_{g, s, r}^{\mathbf{h s h}(D)}+P_{g, r}^{Q} \cdot t_{g}^{G S T, \mathbf{e x p}} \cdot \breve{Q}_{g, r}^{\exp (D)}\right)
\end{aligned}
$$$$
I N C T A X^{\mathbf{b} \$}=t^{L} \cdot \sum_{j} \sum_{r}\left[P_{r}^{L, \mathbf{b t a x}}\right] L_{j, r}^{\mathrm{ind}(D)}
$$ 


$$
\begin{aligned}
\operatorname{DUTY} Y^{\mathbf{b} \$}= & \sum_{g} t_{g}^{F O R} \cdot[\phi] \cdot\left[P_{g}^{F O R}\right] \cdot F O R_{g}^{\mathbf{i m p}(D)} \\
E X P S U B^{\mathbf{b} \$}= & -\sum_{g} t_{g}^{E X P} \cdot\left[P_{g}^{E X P, \mathbf{d} \$, \mathbf{f o r}}\right] \cdot E X P_{g}^{(S)} \\
C O M T A X^{\mathbf{b} \$}= & \sum_{g} \sum_{s} \sum_{p} \sum_{j} \sum_{r}\left(\left[P_{g, s}^{Q}\right] \cdot t_{g, r}^{Q, \mathbf{h s h}} \cdot \breve{Q}_{g, s, r}^{\mathbf{h s h}(D)}\right. \\
& \left.+\left[P_{g, s}^{Q}\right] \cdot t_{g, j, r}^{Q, \mathbf{i n d}(p)} \cdot \breve{Q}_{g, s, j, r}^{\mathbf{i n d}(p)(D)}+\left[P_{g, s}^{Q}\right] \cdot t_{g}^{Q, \mathbf{g o v}} \cdot \breve{Q}_{g, s}^{\mathbf{g o v}(D)}+\left[P_{g, r}^{Q}\right] \cdot t_{g}^{Q, \exp } \cdot \breve{Q}_{g, r}^{\exp (D)}\right)
\end{aligned}
$$

$$
G S T^{\mathbf{b} \$}=\sum_{g} \sum_{s} \sum_{r}\left(\left[P_{g, s}^{Q}\right] \cdot t_{g}^{G S T, \mathbf{h s h}} \cdot \breve{Q}_{g, s, r}^{\mathbf{h s h}(D)}+\left[P_{g, r}^{Q}\right] \cdot t_{g}^{G S T, \exp } \cdot \breve{Q}_{g, r}^{\exp (D)}\right)
$$

$$
\begin{aligned}
G O V R E V^{v} & =I N C T A X^{v}+D U T Y^{v}+C O M T A X^{v}+G S T^{v}-E X P S U B^{v} \\
G O V B A L^{v} & =G O V R E V^{v}-G^{v}-\sum_{r} L S T_{r}^{v}
\end{aligned}
$$

\section{Labour Market Measures}

$$
\begin{aligned}
E M P_{j, r} & =\left[P_{r}^{L, \mathbf{b t a x}}\right] \cdot L_{j, r}^{\operatorname{ind}(D)} \\
E M P_{j, \mathbf{N Z}} & =\sum_{r} E M P_{j, r} \\
E M P_{r} & =\sum_{j} E M P_{j, r}
\end{aligned} \quad \forall r \in N Z R E G
$$

\section{Factor Incomes and Gross Value Added}

$$
\begin{aligned}
W A G E S_{j, r}^{w, \mathbf{c} \$} & =P_{r}^{L, w} \cdot L_{j, r}^{\operatorname{ind}(D)} \\
W A G E S_{j, r}^{w, \mathbf{b} \$} & =\left[P_{r}^{L, w}\right] \cdot L_{j, r}^{\operatorname{ind}(D)} \\
W A G E S_{j, r}^{w, \mathbf{b Q}} & =P_{r}^{L, w} \cdot\left[L_{j, r}^{\operatorname{ind}(D)}\right] \\
W A G E S_{j, \mathbf{N Z}}^{w, v} & =\sum_{r} W A G E S_{j, r}^{w, v} \\
W A G E S_{r}^{w, v} & =\sum_{j} W A G E S_{j, r}^{w, v}
\end{aligned}
$$

$\forall r \in N Z R E G$ 


$$
\begin{aligned}
K R E N T S_{j, r}^{\mathbf{c}} & =P_{j, r}^{K} \cdot K_{j, r}^{\mathbf{i n d}(D)} & & \\
K R E N T S_{j, r}^{\mathbf{b}} & =\left[P_{j, r}^{K}\right] \cdot K_{j, r}^{\mathbf{i n d}(D)} & & \\
K R E N T S_{j, r}^{\mathbf{b Q}} & =P_{j, r}^{K} \cdot\left[K_{j, r}^{\mathbf{i n d}(D)}\right] & & \\
K R E N T S_{j, \mathbf{N Z}}^{v} & =\sum_{r} K R E N T S_{j, r}^{v} & & \forall r \in N Z R E G \\
K R E N T S_{r}^{v} & =\sum_{j} K R E N T S_{j, r}^{v} & & \forall r \in N Z R E G \\
G V A_{j, r}^{v} & =W A G E S_{j, r}^{\mathbf{b t a x}, v}+K R E N T S_{j, r}^{v} & & \forall r \in N Z R E G \\
G V A_{r}^{v} & =W A G E S_{r}^{\mathbf{b t a x}, v}+K R E N T S_{r}^{v} & & \forall r \in \text { R }
\end{aligned}
$$

\section{Output and Investment}

$$
\begin{aligned}
& I_{j, r}^{\mathbf{c} \$}=P_{j, r}^{K, \text { new }} \cdot I_{j, r}^{\text {ind }(D)} \\
& I_{j, r}^{\mathbf{b} \$}=\left[P_{j, r}^{K, \text { new }}\right] \cdot I_{j, r}^{\text {ind }(D)} \\
& I_{j, r}^{\mathrm{bQ}}=P_{j, r}^{K, \text { new }} \cdot\left[I_{j, r}^{\mathbf{i n d}(D)}\right] \\
& I_{j, \mathbf{N Z}}^{v}=\sum_{r} I_{j, r}^{v} \\
& I_{r}^{v}=\sum_{j} I_{j, r}^{v} \\
& O U T P U T_{j, r}^{\mathbf{c} \$}=P_{g, r}^{Q} \cdot \breve{Q}_{g, r}^{(S)} \\
& \operatorname{OUTPUT} T_{j, r}^{\mathbf{b}}=\left[P_{g, r}^{Q}\right] \cdot \breve{Q}_{g, r}^{(S)} \\
& \operatorname{OUTPUT} T_{j, r}^{\mathrm{bQ}}=P_{g, r}^{Q} \cdot\left[\breve{Q}_{g, r}^{(S)}\right] \\
& \operatorname{OUTPUT}_{j, \mathbf{N Z}}^{v}=\sum_{r} \operatorname{OUTPUT}_{j, r}^{v} \\
& \operatorname{OUTPUT}_{r}^{v}=\sum_{j} \operatorname{OUTPUT}_{j, r}^{v}
\end{aligned}
$$

\section{Capital Stocks and Net Returns}

$$
\begin{aligned}
K S T O C K_{j, r} & =K_{j, r}^{(S)} \\
K S T O C K_{j, \mathbf{N Z}} & =\sum_{r} K_{j, r}^{(S)} \\
K S T O C K_{r} & =\sum_{j} K_{j, r}^{(S)}
\end{aligned}
$$




$$
\begin{aligned}
\operatorname{NRTRN}_{j, r} & =\operatorname{KRENTS}_{j, r}^{\mathbf{c}}-\frac{\delta_{j, r}}{100} \cdot P_{j, r}^{K, \text { new }} \cdot K_{j, r}^{(S)} & & \\
\operatorname{NRTRN}_{j, \mathbf{N Z}} & =\sum_{r} \operatorname{NRTRN}_{j, r} & & \\
\operatorname{NRTRN}_{r} & =\sum_{j} N R T R N_{j, r} & & \forall r \in N Z R E G \\
\operatorname{NROR}_{j, \mathbf{N Z}} & =100 \cdot \frac{N R T R N_{j, \mathbf{N Z}}}{\sum_{r} P_{j, r}^{K, \text { new }} \cdot K_{j, r}^{(S)}} & & \\
\operatorname{NROR}_{r} & =100 \cdot \frac{N R T R N_{r}}{\sum_{j} P_{j, r}^{K, \text { new }} \cdot K_{j, r}^{(S)}} & &
\end{aligned}
$$

Measures of GDP and Domestic Saving

$$
\begin{aligned}
G D P E X P^{v} & =C^{v}+I^{v}+G^{v}+T R D B A L^{v, \mathbf{d} \$} \\
G D P I N C^{v} & =\sum_{r} I N C_{r}^{\mathbf{b t a x}, v}+G O V R E V^{v}-\sum_{r} L S T_{r}^{v} \\
G D P V A^{v} & =G V A^{v}+G O V R E V^{v}-I N C T A X^{v} \\
S A V^{v} & =\sum_{r} S A V_{r}^{\text {atax }, v}+G O V B A L^{v}
\end{aligned}
$$

\section{Price Indices}

$$
\begin{aligned}
\Xi^{\mathbf{F}} & =\sqrt{\Xi^{\mathbf{P}} \cdot \Xi^{\mathbf{L}}} \\
G D P D E F^{\mathbf{P}} & =\frac{G D P E X P^{\mathbf{c} \$}}{G D P E X P^{\mathbf{b} \$}} \\
G D P D E F^{\mathbf{L}} & =\frac{G D P E X P^{\mathbf{b Q}}}{\left[G D P E X P^{\mathbf{c} \$}\right]} \\
C P I^{\mathbf{P}} & =\frac{C^{\mathbf{c} \$}}{C^{\mathbf{b} \$}} \\
C P I^{\mathbf{L}} & =\frac{C^{\mathbf{b Q}}}{\left[C^{\mathbf{c} \$}\right]} \\
I P I^{\mathbf{P}} & =\frac{I^{\mathbf{c}}}{I^{\mathbf{b} \$}} \\
I P I^{\mathbf{L}} & =\frac{I^{\mathbf{b Q}}}{\left[I^{\mathbf{c} \$}\right]}
\end{aligned}
$$




$$
\begin{aligned}
& G P I^{\mathbf{P}}=\frac{G^{\mathbf{c} \$}}{G^{\mathbf{b} \$}} \\
& G P I^{\mathbf{L}}=\frac{G^{\mathbf{b Q}}}{\left[G^{\mathbf{c} \$}\right]} \\
& X P I^{\mathbf{P}, t}=\frac{E X P^{\mathbf{c} \$, \mathbf{d} \$, t}}{E X P^{\mathbf{b} \$, \mathbf{d} \$, t}} \\
& X P I^{\mathbf{L}, t}=\frac{E X P^{\mathbf{b Q}, \mathbf{d} \$, t}}{\left[E X P^{\mathbf{c} \$, \mathbf{d} \$, t}\right]} \\
& M P I^{\mathbf{P}, t}=\frac{I M P^{\mathbf{c} \$, \mathbf{d} \$, t}}{I M P^{\mathbf{b} \$, \mathbf{d} \$, t}} \\
& M P I^{\mathbf{L}, t}=\frac{I M P^{\mathbf{b} \mathbf{Q}, \mathbf{d} \$, t}}{\left[I M P^{\mathbf{c} \$, \mathbf{d} \$, t}\right]} \\
& T P I^{\mathbf{P}}=\frac{T R D B A L^{\mathbf{c} \$, \mathbf{d} \$}}{T R D B A L^{\mathbf{b} \$, \mathbf{d} \$}} \\
& T P I^{\mathbf{L}}=\frac{T R D B A L^{\mathbf{b Q}, \mathbf{d} \$}}{\left[T R D B A L^{\mathbf{c} \$, \mathbf{d} \$}\right]} \\
& C P I_{r}^{\mathbf{P}}=\frac{S P N_{r}^{\mathbf{c} \$}}{S P N_{r}^{\mathbf{b} \$}} \\
& C P I_{r}^{\mathbf{L}}=\frac{S P N_{r}^{\mathbf{b Q}}}{\left[S P N_{r}^{\mathbf{c} \$}\right]} \\
& I P I_{r}^{\mathbf{P}}=\frac{I_{r}^{\mathbf{c} \$}}{I_{r}^{\mathbf{b} \$}} \\
& I P I_{r}^{\mathbf{L}}=\frac{I_{r}^{\mathbf{b Q}}}{\left[I_{r}^{\mathbf{c} \$}\right]} \\
& I P I_{j}^{\mathbf{P}}=\frac{I_{j}^{\mathbf{c} \$}}{I_{j}^{\mathbf{b} \$}} \\
& I P I_{j}^{\mathbf{L}}=\frac{I_{j}^{\mathbf{b Q}}}{\left[I_{j}^{\mathbf{c} \$}\right]} \\
& O P I_{r}^{\mathbf{P}}=\frac{O U T P U T_{r}^{\mathbf{c} \$}}{O U T P U T_{r}^{\mathbf{b} \$}} \\
& O P I_{r}^{\mathbf{L}}=\frac{O U T P U T_{r}^{\mathbf{b Q}}}{\left[O U T P U T_{r}^{\mathbf{c} \$}\right]} \\
& O P I_{j}^{\mathbf{P}}=\frac{O U T P U T_{j, \mathbf{N Z}}^{\mathbf{c} \$}}{O U T P U T_{j, \mathbf{N Z}}^{\mathbf{b} \$}} \\
& O P I_{j}^{\mathbf{L}}=\frac{O U T P U T_{j, \mathbf{N Z}}^{\mathbf{b Q}}}{\left[O U T P U T_{j, \mathbf{N Z}}^{\mathbf{c} \$}\right]}
\end{aligned}
$$




$$
\begin{array}{rlr}
L C I_{r}^{\mathbf{P}, w} & =\frac{W A G E S_{r}^{w, \mathbf{c} \$}}{W A G E S_{r}^{w, \mathbf{b} \$}} & \forall r \in N Z R E G \\
L C I_{r}^{\mathbf{L}, w}=\frac{W A G E S_{r}^{w, \mathbf{b Q}}}{\left[W A G E S_{r}^{w, \mathbf{c} \$}\right]} & \forall r \in N Z R E G \\
L C I_{j}^{\mathbf{P}, w}=\frac{W A G E S_{j, \mathbf{N Z}}^{w, \mathbf{} \$}}{W A G E S_{j, \mathbf{N Z}}^{w, \mathbf{b}}} & \\
L C I_{j}^{\mathbf{L}, w}=\frac{W A G E S_{j, \mathbf{N Z}}^{w, \mathbf{N Q}}}{\left[W A G E S_{j, \mathbf{N Z}}^{w, \mathbf{c} \$}\right]} &
\end{array}
$$

\section{Real Prices}

$$
\begin{aligned}
W_{r}^{w} & =\frac{L C I_{r}^{\mathbf{F}, w}}{C P I_{r}^{\mathbf{F}}} \\
\varphi & =\phi \cdot \frac{M P I^{\mathbf{F}, \mathbf{c i f}}}{G D P D E F^{\mathbf{F}}}
\end{aligned}
$$

\section{Household Welfare}

$$
\begin{aligned}
& C V_{r}=\frac{U_{r}-\left[U_{r}\right]}{U_{r}} \cdot\left(E N D I N C_{r}^{\text {atax }, \mathbf{c} \$}-S A V_{r}^{\text {atax }, \mathbf{c} \$}\right) \\
& E V_{r}=\frac{U_{r}-\left[U_{r}\right]}{\left[U_{r}\right]} \cdot\left(\left[E N D I N C_{r}^{\text {atax }, \mathbf{c} \$}\right]-\left[S A V_{r}^{\text {atax }, \mathbf{c} \$}\right]\right)
\end{aligned}
$$

\section{Selected Shares and Ratios}

$$
\begin{aligned}
X_{j, r / \text { tot }}^{I, \mathbf{b} \$} & =100 \times \frac{\left[P_{j, r}^{K, \text { new }}\right] \cdot I_{j, r}^{\text {ind }(D)}}{I^{\mathbf{b} \$}} \\
X_{C / \text { tot }}^{G D P, v} & =100 \times \frac{C^{v}}{G D P E X P} \\
X_{I / \text { tot }}^{G D P}= & =100 \times \frac{I^{v}}{G D P E X P} \\
X_{G / \text { tot }}^{G D P} & =100 \times \frac{G^{v}}{G D P E X P} \\
X_{E X P / \text { tot }}^{G D P, v} & =100 \times \frac{E X P^{v, \mathbf{d} \$ \text {,fob }}}{G D P E X P} \\
X_{I M P / \text { tot }}^{G D P, v} & =100 \times \frac{I M P^{v, \mathbf{d} \$, \mathbf{c i f}}}{G D P E X P} \\
R_{E M P_{r}}^{K S T O C K_{r}} & =\frac{K S T O C K_{r}}{E M P_{r}}
\end{aligned}
$$




\section{Appendix E}

\section{Solutions to Constrained Optimisation Problems}

This appendix lists analytical solutions to the utility maximisation problem when the objective is either Leontief, Cobb-Douglas, or Constant Elasticity of Substitution (CES), and also the expenditure minimisation problem when the constraint is any of these.

\section{Utility Maximisation}

A general form of the utility maximisation problem is:

$$
\text { Maximise } U=f\left(Q_{1}, Q_{2}, \ldots, Q_{n}\right) \text { subject to } \sum_{i=1}^{n} P_{i} Q_{i}=E
$$

\section{CES Case}

$$
U=v \cdot\left(\sum_{i=1}^{n} a_{i} \cdot Q_{i}^{\frac{\sigma-1}{\sigma}}\right)^{\frac{\sigma}{\sigma-1}} \quad\left(0<\sigma<\infty \text { but } \sigma \neq 1, \text { and } \sum_{i=1}^{n} a_{i}=1\right)
$$

where $\sigma$ is the constant elasticity of substitution.

Solution: ${ }^{1}$

$$
Q_{i}=\frac{a_{i}{ }^{\sigma} P_{i}^{-\sigma}}{\sum_{j=1}^{n} a_{j}{ }^{\sigma} P_{j}{ }^{1-\sigma}} \cdot E \quad \forall i \in\{1, \ldots, n\}
$$

\footnotetext{
${ }^{1}$ The parameter $v$ does not affect the solution; it simply scales the utility function
} 
When $\sigma=0$ or $\sigma=1$ the demand functions derived from maximising a CES objective are replaced by those from Leontief and Cobb-Douglas respectively as the CES function is not defined but converges to these latter functions for those values of $\sigma$.

\section{Leontief Case}

$$
U=v \cdot \min \left(\frac{Q_{1}}{a_{1}}, \frac{Q_{2}}{a_{2}}, \ldots, \frac{Q_{n}}{a_{n}}\right) \quad\left(\text { and } \sum_{i=1}^{n} a_{i}=1\right)
$$

Solution:

$$
Q_{i}=\frac{a_{i}}{\sum_{j=1}^{n} P_{j} a_{j}} \cdot E \quad \forall i \in\{1, \ldots, n\}
$$

\section{Cobb-Douglas Case}

$$
U=v \cdot \prod_{i=1}^{n} Q_{i}^{a_{i}} \quad\left(\text { and } \sum_{i=1}^{n} a_{i}=1\right)
$$

Solution:

$$
Q_{i}=\frac{a_{i}}{P_{i}} \cdot E \quad \forall i \in\{1, \ldots, n\}
$$

\section{Expenditure Minimisation}

A general form of the expenditure (cost) minimisation problem is:

Minimise $\sum_{i=1}^{n} P_{i} Q_{i} \quad$ subject to $Q=f\left(Q_{1}, Q_{2}, \ldots, Q_{n}\right)$

\section{CES Case}

$$
Q=v \cdot\left(\sum_{i=1}^{n} a_{i} \cdot Q_{i}^{\frac{\sigma-1}{\sigma}}\right)^{\frac{\sigma}{\sigma-1}} \quad\left(0<\sigma<\infty \text { but } \sigma \neq 1, \text { and } \sum_{i=1}^{n} a_{i}=1\right)
$$

where $\sigma$ is the constant elasticity of substitution. 
Solution: ${ }^{2}$

$$
Q_{i}=\frac{a_{i}{ }^{\sigma} P_{i}{ }^{-\sigma}}{\left(\sum_{j=1}^{n} a_{j}{ }^{\sigma} P_{j}{ }^{1-\sigma}\right)^{\frac{\sigma}{\sigma-1}}} \cdot \frac{Q}{v} \quad \forall i \in\{1, \ldots, n\}
$$

As above, these demand functions are replaced by those for Leontief and Cobb-Douglas when $\sigma=0$ and $\sigma=1$ respectively.

\section{Leontief Case}

$$
Q=v \cdot \min \left(\frac{Q_{1}}{a_{1}}, \frac{Q_{2}}{a_{2}}, \ldots, \frac{Q_{n}}{a_{n}}\right) \quad\left(\text { and } \sum_{i=1}^{n} a_{i}=1\right)
$$

Solution:

$$
Q_{i}=a_{i} \cdot \frac{Q}{v} \quad \forall i \in\{1, \ldots, n\}
$$

\section{Cobb-Douglas Case}

$$
Q=v \cdot \prod_{i=1}^{n} Q_{i}^{a_{i}} \quad\left(\text { and } \sum_{i=1}^{n} a_{i}=1\right)
$$

Solution:

$$
Q_{i}=\frac{Q}{v} \cdot \prod_{j=1}^{n}\left(\frac{a_{i} \cdot P_{j}}{a_{j} \cdot P_{i}}\right)^{a_{j}} \quad \forall i \in\{1, \ldots, n\}
$$

\footnotetext{
${ }^{2}$ An alternative expression is:

$$
Q_{i}=\frac{Q}{v} \cdot\left(\sum_{j=1}^{n} a_{j} \cdot\left(\frac{a_{i} \cdot P_{j}}{a_{j} \cdot P_{i}}\right)^{1-\sigma}\right)^{\frac{\sigma}{1-\sigma}} \quad \forall i \in\{1, \ldots, n\}
$$
}


214 APPENDIX E. SOLUTIONS TO CONSTRAINED OPTIMISATION PROBLEMS 


\section{Appendix F}

\section{Linearisation of Demand Functions}

A result from appendix $\mathrm{E}$ is that the demand functions derived from the maximisation of a CES objective function subject to an expenditure constraint are:

$$
Q_{i}=\frac{a_{i}{ }^{\sigma} P_{i}^{-\sigma}}{\sum_{j=1}^{n} a_{j}{ }^{\sigma} P_{j}{ }^{1-\sigma}} \cdot E \quad \forall i \in\{1, \ldots, n\}
$$

These demand functions can be linearised as follows:

$$
\begin{aligned}
\frac{d Q_{i}}{Q_{i}} & =\frac{d\left(a_{i}{ }^{\sigma} P_{i}{ }^{-\sigma}\right)}{a_{i}{ }^{\sigma} P_{i}{ }^{-\sigma}}-\frac{d\left(\sum_{j=1}^{n} a_{j}{ }^{\sigma} P_{j}{ }^{1-\sigma}\right)}{\sum_{j=1}^{n} a_{j}{ }^{\sigma} P_{j}{ }^{1-\sigma}}+\frac{d E}{E} & & \forall i \in\{1, \ldots, n\} \\
\Rightarrow \frac{d Q_{i}}{Q_{i}} & =-\sigma \frac{d P_{i}}{P_{i}}-\frac{\sum_{j=1}^{n} a_{j}{ }^{\sigma} P_{j}{ }^{1-\sigma} \frac{d\left(a_{j}{ }^{\sigma} P_{j}{ }^{1-\sigma}\right)}{a_{j}{ }^{1-}{ }^{1-\sigma}}}{\sum_{j=1}^{n} a_{j}{ }^{\sigma} P_{j}{ }^{1-\sigma}}+\frac{d E}{E} & & \forall i \in\{1, \ldots, n\} \\
\Rightarrow \frac{d Q_{i}}{Q_{i}} & =-\sigma \frac{d P_{i}}{P_{i}}-(1-\sigma) \sum_{k=1}^{n} \frac{a_{k}{ }^{\sigma} P_{k}{ }^{1-\sigma}}{\sum_{j=1}^{n} a_{j}{ }^{\sigma} P_{j}{ }^{1-\sigma}} \frac{d P_{k}}{P_{k}}+\frac{d E}{E} & & \forall i \in\{1, \ldots, n\} \\
\Rightarrow \frac{d Q_{i}}{Q_{i}} & =-\sigma \frac{d P_{i}}{P_{i}}-(1-\sigma) \sum_{k=1}^{n} \frac{P_{k} \cdot Q_{k}}{E} \frac{d P_{k}}{P_{k}}+\frac{d E}{E} & & \forall i \in\{1, \ldots, n\}
\end{aligned}
$$

Making the following substitutions,

$$
q_{i}=\frac{d Q_{i}}{Q_{i}} \quad p_{i}=\frac{d P_{i}}{P_{i}} \quad X_{k}=\frac{P_{k} \cdot Q_{k}}{E} \quad e=\frac{d E}{E}
$$

the linearised equation can be written:

$$
q_{i}=-\sigma p_{i}-(1-\sigma) \sum_{k=1}^{n} X_{k} p_{k}+e \quad \forall i \in\{1, \ldots, n\}
$$


Some useful alternative ways of writing the linearised demand functions are:

$$
\begin{array}{rlrl}
q_{i} & =e-\left(\sigma+(1-\sigma) X_{i}\right) p_{i}-(1-\sigma) \sum_{k \neq i} X_{k} p_{k} & \forall i \in\{1, \ldots, n\} \\
\text { and } q_{i}=e-\sum_{k=1}^{n} X_{k} p_{k}-\sigma\left(p_{i}-\sum_{k=1}^{n} X_{k} p_{k}\right) & \forall i \in\{1, \ldots, n\}
\end{array}
$$

It can easily be seen that $q_{i}=e-p_{i}$ in the Cobb-Douglas $(\sigma=1)$ case and $q_{i}=$ $e-\sum_{k=1}^{n} X_{k} p_{k}$ in the Leontief $(\sigma=0)$ case.

Appendix E also states that the demand functions derived from the minimisation of expenditure subject to a CES constraint function are:

$$
Q_{i}=\frac{a_{i}{ }^{\sigma} P_{i}{ }^{-\sigma}}{\left(\sum_{j=1}^{n} a_{j}{ }^{\sigma} P_{j}{ }^{1-\sigma}\right)^{\frac{\sigma}{\sigma-1}}} \cdot \frac{Q}{v} \quad \forall i \in\{1, \ldots, n\}
$$

The demand functions can be linearised in a similar fashion to the above:

$$
\begin{array}{rlrl}
\frac{d Q_{i}}{Q_{i}}=\frac{d\left(a_{i}{ }^{\sigma} P_{i}{ }^{-\sigma}\right)}{a_{i}{ }^{\sigma} P_{i}{ }^{-\sigma}}-\frac{d\left(\sum_{j=1}^{n} a_{j}{ }^{\sigma} P_{j}{ }^{1-\sigma}\right)^{\frac{\sigma}{\sigma-1}}}{\left(\sum_{j=1}^{n} a_{j}{ }^{\sigma} P_{j}{ }^{1-\sigma}\right)^{\frac{\sigma}{\sigma-1}}}+\frac{d Q}{Q} & \forall i \in\{1, \ldots, n\} \\
\Rightarrow \frac{d Q_{i}}{Q_{i}}=-\sigma \frac{d P_{i}}{P_{i}}-\frac{\sigma}{\sigma-1} \cdot \frac{d\left(\sum_{j=1}^{n} a_{j}{ }^{\sigma} P_{j}{ }^{1-\sigma}\right)}{\sum_{j=1}^{n} a_{j}{ }^{\sigma} P_{j}^{1-\sigma}}+\frac{d Q}{Q} & \forall i \in\{1, \ldots, n\} \\
\Rightarrow \frac{d Q_{i}}{Q_{i}}=-\sigma \frac{d P_{i}}{P_{i}}-\frac{\sigma}{\sigma-1} \cdot \frac{\sum_{j=1}^{n} a_{j}{ }^{\sigma} P_{j}{ }^{1-\sigma} \frac{d\left(a_{j}{ }^{\sigma} P_{j}{ }^{1-\sigma}\right)}{a_{j}{ }^{\sigma} P_{j}{ }^{1-\sigma}}}{\sum_{j=1}^{n} a_{j}{ }^{\sigma} P_{j}{ }^{1-\sigma}} & \frac{d Q}{Q} & \forall i \in\{1, \ldots, n\} \\
\Rightarrow \frac{d Q_{i}}{Q_{i}}=-\sigma \frac{d P_{i}}{P_{i}}+\sigma \sum_{k=1}^{n} \frac{a_{k}{ }^{\sigma} P_{k}{ }^{1-\sigma}}{\sum_{j=1}^{n} a_{j}{ }^{\sigma} P_{j}{ }^{1-\sigma}} \frac{d P_{k}}{P_{k}}+\frac{d Q}{Q} & \forall i \in\{1, \ldots, n\} \\
\Rightarrow \frac{d Q_{i}}{Q_{i}}=-\sigma \frac{d P_{i}}{P_{i}}+\sigma \sum_{k=1}^{n} \frac{P_{k} \cdot Q_{k}}{\sum_{j=1}^{n} P_{j} \cdot Q_{j}} \frac{d P_{k}}{P_{k}}+\frac{d Q}{Q} & \forall i \in\{1, \ldots, n\}
\end{array}
$$

Making the following substitutions,

$$
q_{i}=\frac{d Q_{i}}{Q_{i}} \quad p_{i}=\frac{d P_{i}}{P_{i}} \quad X_{k}=\frac{P_{k} \cdot Q_{k}}{\sum_{j=1}^{n} P_{j} \cdot Q_{j}} \quad q=\frac{d Q}{Q}
$$

the linearised equation can be written

$$
q_{i}=-\sigma p_{i}+\sigma \sum_{k=1}^{n} X_{k} p_{k}+q \quad \forall i \in\{1, \ldots, n\}
$$


Some useful alternative ways of writing the linearised demand functions are:

$$
\begin{array}{rlrl}
q_{i} & =q-\sigma\left(\left(1-X_{i}\right) p_{i}-\sum_{k \neq i} X_{k} p_{k}\right) & \forall i \in\{1, \ldots, n\} \\
\text { and } q_{i}=q-\sigma\left(p_{i}-\sum_{k=1}^{n} X_{k} p_{k}\right) & \forall i \in\{1, \ldots, n\}
\end{array}
$$

It follows that $q_{i}=q-\left(p_{i}-\sum_{k=1}^{n} X_{k} p_{k}\right)$ in the Cobb-Douglas $(\sigma=1)$ case and $q_{i}=q$ in the Leontief $(\sigma=0)$ case. 


\section{Appendix G}

\section{Labour Supply Functions}

It was shown in appendix E that if a CES utility function with substitution elasticity $\sigma$ is maximised subject to a budget constraint, the solution is:

$$
Q_{i}=\frac{a_{i}{ }^{\sigma} P_{i}^{-\sigma}}{\sum_{j=1}^{n} a_{j}{ }^{\sigma} P_{j}{ }^{1-\sigma}} \cdot E \quad \forall i \in\{1, \ldots, n\}
$$

The solution yields a labour supply curve when one of the $Q_{i}$ 's is leisure and the associated price is interpreted as the wage rate. Let leisure be $Q_{n}$ and the wage rate $P_{n}$. Given endowments of time $T$ and capital $K$, we can derive the labour supply $L$ as follows: ${ }^{1}$

Let $Q_{n}=T-L \quad$ and $\quad E=P_{n} T+P_{K} K$.

$$
\begin{aligned}
& \text { Then } T-L=\frac{a_{n}{ }^{\sigma} P_{n}{ }^{-\sigma}}{\sum_{j=1}^{n} a_{j}{ }^{\sigma} P_{j}{ }^{1-\sigma}} \cdot\left(P_{n} T+P_{K} K\right) \\
& \text { Rearranging, } L=\left(1-\frac{a_{n}{ }^{\sigma} P_{n}{ }^{1-\sigma}}{\sum_{j=1}^{n} a_{j}{ }^{\sigma} P_{j}{ }^{1-\sigma}}\right) \cdot T-\left(\frac{a_{n}{ }^{\sigma} P_{n}{ }^{-\sigma}}{\sum_{j=1}^{n} a_{j}{ }^{\sigma} P_{j}{ }^{1-\sigma}}\right) \cdot P_{K} K
\end{aligned}
$$

If the utility function is Leontief then the labour supply function is:

$$
L=\left(1-\frac{a_{n} P_{n}}{\sum_{j=1}^{n} a_{j} P_{j}}\right) \cdot T-\left(\frac{a_{n}}{\sum_{j=1}^{n} a_{j} P_{j}}\right) \cdot P_{K} K
$$

\footnotetext{
${ }^{1}$ For simplicity saving is ignored here. To consider its implications, the capital income $P_{K} K$ can be thought of as net of saving.
} 
while if it is Cobb-Douglas, the labour supply function is:

$$
L=\left(1-a_{n}\right) \cdot T-\frac{a_{n}}{P_{n}} \cdot P_{K} K
$$

The elasticity of labour supply with respect to the wage rate depends on the magnitude of the time endowment. We can see this by deriving the labour supply elasticity from the function obtained using Cobb-Douglas utility: ${ }^{2}$

$$
\begin{gathered}
\frac{\partial L}{\partial P_{n}}=\frac{a_{n}}{P_{n}^{2}} \cdot P_{K} K \\
\Rightarrow \epsilon=\frac{\partial L}{\partial P_{n}} \frac{P_{n}}{L}=a_{n} \frac{P_{K} K}{P_{n} L}=a_{n} \frac{P_{K} K}{\left(1-a_{n}\right) \cdot P_{n} T-a_{n} \cdot P_{K} K}
\end{gathered}
$$

The value of $a_{n}$ is calibrated to the benchmark equilibrium values (in square brackets) as:

$$
a_{n}=\frac{\left[P_{n}\right]\left[Q_{n}\right]}{\left[P_{n}\right][T]+\left[P_{K}\right][K]}
$$

It can easily be shown that the elasticity evaluated at the benchmark equilibrium is given by:

$$
[\epsilon]=\frac{\left[P_{K}\right][K]}{\left[P_{n}\right][T]+\left[P_{K}\right][K]} \cdot \frac{[T]-[L]}{[L]}
$$

The larger the magnitude of the time endowment relative to the capital stock, or the smaller the time endowment relative to labour supply, the lower the elasticity will be in the benchmark equilibrium. However the time endowment is measured or calculated, it should be checked that it yields a believable labour supply elasticity.

\footnotetext{
${ }^{2}$ Deriving the labour supply elasticity in equilibrium when utility is CES or Leontief involves considerably more complex and tedious algebra. Given that the proposition is proven for Cobb-Douglas utility, it is reasonable to expect the same holds for these other functional forms.
} 


\section{Appendix $\mathbf{H}$}

\section{Endogenous Investment Allocation}

It can be shown that the set of equations given in Chapter 2 that constitute the endogenous investment module reduces to an investment allocation decision given current and (equalised) expected future net rates of return, depreciation rates, and current capital stock levels. Starting off with the relevant set of equations from the chapter, with the definition of real investment shares (equation (2.31)) slightly rearranged in (H.3):

$$
\begin{aligned}
I_{j, r}^{\operatorname{ind}(D)} & =K_{j, r}^{\text {new }(S)} \\
I^{\mathbf{b} \$} & =\sum_{j} \sum_{r}\left[P_{j, r}^{K, \text { new }}\right] \cdot I_{j, r}^{\text {ind }(D)} \\
I_{j, r}^{\text {ind }(D)} & =\frac{1}{\left[P_{j, r}^{K, \text { new }}\right]} \frac{X_{j, r / \text { tot }}^{I, \mathbf{b} \$} I^{\mathbf{b} \$}}{100} \\
K_{j, r}^{\text {future }} & =\left(1-\frac{\delta_{j, r}}{100}\right) K_{j, r}^{(S)}+K_{j, r}^{\text {new }(S)} \\
\operatorname{GrossROR}_{j, r} & =100 \times \frac{P_{j, r}^{K}}{P_{j, r}^{K, \text { new }}} \\
\operatorname{NetROR}_{j, r} & =G r o s s R O R_{j, r}-\delta_{j, r}
\end{aligned}
$$




$$
\begin{aligned}
R_{j, r}^{\text {GrossROR } / \text { NetROR }} & =\frac{\text { GrossROR }_{j, r}}{N e t R O R_{j, r}} \\
R_{j, r}^{\text {Knew } / \text { Kfuture }} & =100 \times \frac{K_{j, r}^{\text {new }(S)}}{K_{j, r}^{\text {future }}} \\
N e t R O R_{j, r}^{\text {future }} & =\left(\frac{K_{j, r}^{\text {future }}}{K_{j, r}^{(S)}}\right)^{-\beta_{j, r}} \cdot \text { NetROR }_{j, r} \\
N e t R O R^{\text {future }_{j, r}} & =\Omega
\end{aligned}
$$

We substitute (H.1) into (H.2) and (H.3), (H.10) into (H.9), drop (H.8) and (H.7), and set $\left[P_{j, r}^{K, \text { new }}\right]=1$ to reduce the system to:

$$
\begin{aligned}
I^{\mathbf{b} \$} & =\sum_{j} \sum_{r} K_{j, r}^{\mathrm{new}(S)} \\
K_{j, r}^{\mathrm{new}(S)} & =\frac{X_{j, r / \mathrm{tot}}^{I, \mathbf{b}}}{100} I^{\mathbf{b} \$} \\
K_{j, r}^{\mathrm{future}} & =\left(1-\frac{\delta_{j, r}}{100}\right) K_{j, r}^{(S)}+K_{j, r}^{\mathrm{new}(S)} \\
\operatorname{GrossROR}_{j, r} & =100 \times \frac{P_{j, r}^{K}}{P_{j, r}^{K, \text { new }}} \\
\operatorname{NetROR}_{j, r} & =\operatorname{GrossROR}_{j, r}-\delta_{j, r} \\
\Omega & =\left(\frac{K_{j, r}^{\mathrm{future}}}{K_{j, r}^{(S)}}\right)^{-\beta_{j, r}} \cdot \operatorname{NetROR}_{j, r}
\end{aligned}
$$


We then substitute (H.12) into (H.11) and (H.13), and (H.14) into (H.15), then rearrange (H.16) for $K_{j, r}^{\text {future }}$. The system is now:

$$
\begin{aligned}
100 & =\sum_{j} \sum_{r} X_{j, r / \text { tot }}^{I, \mathbf{b} \$} \\
K_{j, r}^{\text {future }} & =\left(1-\frac{\delta_{j, r}}{100}\right) K_{j, r}^{(S)}+\frac{X_{j, r / \text { tot }}^{I, \mathbf{b}} I^{\mathbf{b} \$}}{100} \\
N e t R O R_{j, r} & =100 \times \frac{P_{j, r}^{K}}{P_{j, r}^{K, \text { new }}}-\delta_{j, r} \\
K_{j, r}^{\text {future }} & =\left(\frac{N e t R O R_{j, r}}{\Omega}\right)^{\frac{1}{\beta_{j, r}}} K_{j, r}^{(S)}
\end{aligned}
$$

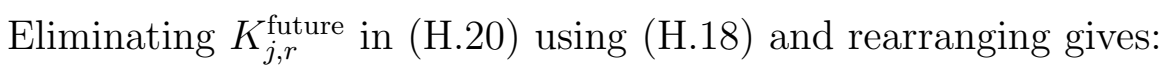

$$
\begin{aligned}
& \sum_{j} \sum_{r} X_{j, r / \mathrm{tot}}^{I, \mathbf{b} \$}=100 \\
& \operatorname{NetROR} R_{j, r}=100 \times \frac{P_{j, r}^{K}}{P_{j, r}^{K, \text { new }}}-\delta_{j, r} \\
& \frac{X_{j, r / \mathrm{tot}}^{I, \mathbf{b}}}{100}=\frac{1}{I^{\mathbf{b}}}\left(\left(\frac{N e t R O R_{j, r}}{\Omega}\right)^{\frac{1}{\beta_{j, r}}}-\left(1-\frac{\delta_{j, r}}{100}\right)\right) K_{j, r}^{(S)}
\end{aligned}
$$

Analytically, the investment allocation problem is to find a solution to (H.23) subject to (H.21) and the definition of $N e t R O R_{j, r}$ in (H.22). Even if $N e t R O R_{j, r}, \beta_{j, r}$, and $\delta_{j, r}$ do not vary over regions for a given industry $j, X_{j, r / \text { tot }}^{I, \mathbf{b} \$}$ will as long as $K_{j, r}^{(S)}$ does. The larger a region's current capital stock, the more investment is allocated to it, ceteris paribus. 


\section{Appendix I}

\section{Regional Consumption and Propensities to Save}

Regional propensities to consume and save relative to the national average depend on how consumption is distributed across regions in the initial equilibrium. Using labour shares means:

$$
\begin{aligned}
& {\left[X_{r / t o t}^{S P N}\right]=\left[X_{r / t o t}^{L A B O U R}\right]} \\
& \left.\Rightarrow \frac{\left[S P N_{r}^{\mathbf{c}}\right]}{\left[S P N_{\mathbf{N Z}}^{\mathbf{c}}\right]}=\frac{\left[W A G E S_{r}^{\text {atax }, \mathbf{c} \$}\right]}{\left[W A G E S_{\mathbf{N Z}}^{\text {atax }}, \mathbf{c} \$\right.}\right] \\
& \Rightarrow \frac{\left[S P N_{r}^{\mathbf{c} \$}\right]}{\left[W A G E S_{r}^{\mathbf{a t a x}, \mathbf{c} \$}\right]}=\frac{\left[S P N_{\mathbf{N Z}}^{\mathbf{c} \$}\right]}{\left[W A G E S_{\mathbf{N Z}}^{\mathbf{a t a x}, \mathbf{c} \$}\right]} \\
& \Rightarrow \frac{\left[S P N_{r}^{\mathbf{c} \$}\right]}{\left[I N C_{r}^{\text {atax, } \mathbf{c} \$}\right]}=\frac{\left[W A G E S_{r}^{\text {atax, }, \mathbf{c} \$}\right]}{\left[I N C_{r}^{\text {atax, } \mathbf{c} \$}\right]} \times \frac{\left[S P N_{\mathbf{N Z}}^{\mathbf{c} \$}\right]}{\left[W A G E S_{\mathbf{N Z}}^{\text {atax, } \mathbf{c} \$}\right]} \\
& \Rightarrow \frac{\left[A P C_{r}^{\text {atax }}\right]}{100}=\frac{\left[W A G E S_{r}^{\text {atax }, \mathbf{c} \$}\right]}{\left[I N C_{r}^{\text {atax, } \mathbf{c} \$}\right]} \times \frac{\left[S P N_{\mathbf{N Z}}^{\mathbf{c} \$}\right]}{\left[W A G E S_{\mathbf{N Z}}^{\mathbf{a t a x}, \mathbf{c} \$}\right]} \\
& \Rightarrow \frac{\left[A P S_{r}^{\text {atax }}\right]}{100}=1-\frac{\left[I N C_{r}^{\text {atax }, \mathbf{c} \$}\right]-\left[K R E N T S_{r}^{\mathbf{c} \$}\right]}{\left[I N C_{r}^{\text {atax, } \mathbf{c} \$}\right]} \times \frac{\left[S P N_{\mathbf{N Z}}^{\mathbf{c} \$}\right]}{\left[W A G E S_{\mathbf{N Z}}^{\text {atax,c } \$}\right]} \\
& \Rightarrow \frac{\left[A P S_{r}^{\mathbf{a t a x}}\right]}{100}=\frac{\left[W A G E S_{\mathbf{N Z}}^{\mathbf{a t a x}, \mathbf{c} \$}\right]-\left[S P N_{\mathbf{N Z}}^{\mathbf{c} \$}\right]}{\left[W A G E S_{\mathbf{N Z}}^{\mathbf{a t a x}, \mathbf{c} \$}\right]}+\frac{\left[K R E N T S_{r}^{\mathbf{c} \$}\right]}{\left[I N C_{r}^{\mathbf{a t a x}, \mathbf{c} \$}\right]} \times \frac{\left[S P N_{\mathbf{N Z}}^{\mathbf{c} \$}\right]}{\left[W A G E S_{\mathbf{N Z}}^{\mathbf{a t a x}, \mathbf{c} \$}\right]}
\end{aligned}
$$

Therefore, the higher the share of capital rents in regional income, the higher the region's propensity to save. 
226 APPENDIX I. REGIONAL CONSUMPTION AND PROPENSITIES TO SAVE 


\section{Appendix $\mathbf{J}$}

\section{Conversion Factors}

This appendix lists the factors used for converting demographic and labour market measures between persons, FTEs, and IOunits. 


\begin{tabular}{|l|ccccc|}
\hline \multicolumn{5}{|c|}{ Conversion Factors - FTEs $\rightarrow$ persons } \\
\hline \multirow{2}{*}{ Industry } & \multicolumn{5}{c|}{ Region } \\
\cline { 2 - 6 } & AKL & WLG & ONI & CAN & OSI \\
\hline AGRI & 1.1436 & 1.1380 & 1.1197 & 1.1210 & 1.1042 \\
FOLO & 1.1099 & 1.0785 & 1.0609 & 1.0924 & 1.0571 \\
FISH & 1.0787 & 1.1316 & 1.0990 & 1.0868 & 1.0813 \\
MINE & 1.0421 & 1.0606 & 1.0306 & 1.0450 & 1.0273 \\
OIGA & 1.0000 & 1.0000 & 1.0191 & 0.0000 & 0.0000 \\
PETR & 0.0000 & 0.0000 & 1.0625 & 0.0000 & 0.0000 \\
FDBT & 1.0747 & 1.1092 & 1.0559 & 1.0627 & 1.0530 \\
TWPM & 1.0759 & 1.0760 & 1.0737 & 1.0907 & 1.0921 \\
CHNM & 1.0416 & 1.0467 & 1.0328 & 1.0430 & 1.0760 \\
METL & 1.0417 & 1.0454 & 1.0435 & 1.0478 & 1.0362 \\
EQFO & 1.0473 & 1.0528 & 1.0563 & 1.0473 & 1.0518 \\
UTIL & 1.0519 & 1.0342 & 1.0518 & 1.0567 & 1.0617 \\
CONS & 1.0566 & 1.0543 & 1.0556 & 1.0523 & 1.0517 \\
ACCR & 1.2426 & 1.2658 & 1.2683 & 1.2623 & 1.2418 \\
CMIF & 1.0697 & 1.0713 & 1.1280 & 1.1136 & 1.1359 \\
PROP & 1.1222 & 1.1392 & 1.1315 & 1.1525 & 1.1441 \\
RBUS & 1.1029 & 1.0949 & 1.1311 & 1.1328 & 1.1356 \\
GOVT & 1.0441 & 1.0423 & 1.0519 & 1.0571 & 1.0696 \\
EDUC & 1.1706 & 1.1732 & 1.1837 & 1.2019 & 1.1972 \\
HEAL & 1.1474 & 1.1659 & 1.1737 & 1.1999 & 1.2064 \\
CUPE & 1.1719 & 1.1630 & 1.1850 & 1.1925 & 1.1812 \\
OWND & 0.0000 & 0.0000 & 0.0000 & 0.0000 & 0.0000 \\
WHOL & 1.0646 & 1.0728 & 1.0780 & 1.0731 & 1.0834 \\
RETT & 1.2062 & 1.2097 & 1.1959 & 1.2190 & 1.2025 \\
TRAN & 1.0632 & 1.0592 & 1.0822 & 1.0669 & 1.0718 \\
\hline All & 1.1128 & 1.1165 & 1.1289 & 1.1351 & 1.1339 \\
\hline
\end{tabular}

Table J.1: Conversion Factors - FTEs $\rightarrow$ persons 


\begin{tabular}{|l|ccccc|}
\hline \multicolumn{5}{|c|}{ Conversion } & Factors - IOunits $\rightarrow$ FTEs \\
\hline \multirow{2}{*}{ Industry } & \multicolumn{5}{c|}{ Region } \\
\cline { 2 - 6 } & AKL & WLG & ONI & CAN & OSI \\
\hline AGRI & 0.0421 & 0.0882 & 0.0253 & 0.0625 & 0.0196 \\
FOLO & 0.0113 & 0.0337 & 0.0104 & 0.0187 & 0.0122 \\
FISH & 0.0133 & 0.0579 & 0.0181 & 0.1196 & 0.0121 \\
MINE & 0.0295 & 0.1180 & 0.0215 & 0.0104 & 0.0236 \\
OIGA & 0.0090 & 0.0041 & 0.0024 & 0.0000 & 0.0000 \\
PETR & 0.0000 & 0.0000 & 0.1010 & 0.0000 & 0.0000 \\
FDBT & 0.0181 & 0.0257 & 0.0174 & 0.0330 & 0.0128 \\
TWPM & 0.0281 & 0.0361 & 0.0195 & 0.0304 & 0.0194 \\
CHNM & 0.0122 & 0.0149 & 0.0791 & 0.0149 & 0.0121 \\
METL & 0.0177 & 0.0215 & 0.0174 & 0.0167 & 0.0185 \\
EQFO & 0.0169 & 0.0167 & 0.0220 & 0.0264 & 0.0206 \\
UTIL & 0.0153 & 0.0091 & 0.0140 & 0.0265 & 0.0139 \\
CONS & 0.0321 & 0.0354 & 0.0315 & 0.0395 & 0.0290 \\
ACCR & 0.0492 & 0.0552 & 0.0488 & 0.0698 & 0.0414 \\
CMIF & 0.0171 & 0.0156 & 0.0185 & 0.0184 & 0.0176 \\
PROP & 0.0473 & 0.0565 & 0.0515 & 0.0567 & 0.0453 \\
RBUS & 0.0225 & 0.0257 & 0.0260 & 0.0294 & 0.0263 \\
GOVT & 0.0154 & 0.0112 & 0.0156 & 0.0174 & 0.0149 \\
EDUC & 0.0221 & 0.0264 & 0.0242 & 0.0271 & 0.0201 \\
HEAL & 0.0271 & 0.0272 & 0.0269 & 0.0305 & 0.0236 \\
CUPE & 0.0224 & 0.0254 & 0.0252 & 0.0290 & 0.0222 \\
OWND & 0.0000 & 0.0000 & 0.0000 & 0.0000 & 0.0000 \\
WHOL & 0.0169 & 0.0181 & 0.0197 & 0.0221 & 0.0164 \\
RETT & 0.0290 & 0.0352 & 0.0304 & 0.0395 & 0.0273 \\
TRAN & 0.0154 & 0.0179 & 0.0226 & 0.0232 & 0.0166 \\
\hline All & 0.0223 & 0.0226 & 0.0248 & 0.0300 & 0.0214 \\
\hline
\end{tabular}

Table J.2: Conversion Factors - IOunits $\rightarrow$ FTEs 


\section{Appendix K}

\section{Calibration of Demand Functions}

The demand functions presented in appendix E are calibrated by fixing the variables at their benchmark equilibrium values and solving for the coefficients.

Demands from Utility Maximisation

\begin{tabular}{|c|c|c|c|}
\hline Case & Demand function & Calibration & \\
\hline Leontief & $Q_{i}=\frac{a_{i}}{\sum_{j=1}^{n} P_{j} a_{j}} \cdot E$ & $a_{i}=\frac{\left[Q_{i}\right]}{[E]}$ & (K.1) \\
\hline Cobb - Douglas & $Q_{i}=\frac{a_{i}}{P_{i}} \cdot E$ & $a_{i}=\frac{\left[P_{i}\right]\left[Q_{i}\right]}{[E]}$ & $(\mathrm{K} .2)$ \\
\hline CES & $Q_{i}=\frac{a_{i}^{\sigma} P_{i}^{-\sigma}}{\sum_{j=1}^{n} a_{j}^{\sigma} P_{j}^{1-\sigma}} \cdot E$ & $a_{i}=\frac{\left[P_{i}\right]\left[Q_{i}\right]^{1 / \sigma}}{\sum_{j=1}^{n}\left[P_{j}\right]\left[Q_{j}\right]^{1 / \sigma}}$ & (K.3) \\
\hline
\end{tabular}

Demands from Expenditure Minimisation

\begin{tabular}{lll} 
Case & Demand function & Calibration \\
\hline Leontief & $Q_{i}=a_{i} \cdot \frac{Q}{v}$ & $a_{i}=\frac{\left[Q_{i}\right]}{[Q]}$ \\
Cobb - Douglas & $Q_{i}=\frac{Q}{v} \cdot \prod_{j=1}^{n}\left(\frac{a_{i} \cdot P_{j}}{a_{j} \cdot P_{i}}\right)^{a_{j}}$ & $a_{i}=\frac{\left[P_{i}\right]\left[Q_{i}\right]}{[Q]}$ \\
CES & $Q_{i}=\frac{a_{i}{ }^{\sigma} P_{i}^{-\sigma}}{\left(\sum_{j=1}^{n} a_{j}{ }^{\sigma} P_{j}{ }^{1-\sigma}\right)^{\frac{\sigma}{\sigma-1}}} \cdot \frac{Q}{v}$ & $a_{i}=\frac{\left[P_{i}\right]\left[Q_{i}\right]^{1 / \sigma}}{\sum_{j=1}^{n}\left[P_{j}\right]\left[Q_{j}\right]^{1 / \sigma}}$
\end{tabular}

The $v$ coefficients are calibrated consistent with the mixing function once the $a_{i}$ coef- 
ficients have been determined. For example, for the Leontief case:

$$
v=\frac{[Q]}{\min \left(\frac{\left[Q_{1}\right]}{a_{1}}, \ldots, \frac{\left[Q_{n}\right]}{a_{n}}\right)}
$$

Proofs of the above calibrating calculations use a variety of methods. The solutions K.2 and K.4 can be found through simple rearrangement. Dixon et al. (1992, p. 92) demonstrate K.5 using the first-order conditions of the expenditure minimisation problem. Solutions K.1, K.3, and K.6 can only be proven through substitution. The first of these is straight-forward but demonstrating the CES solutions requires some detailed algebra. ${ }^{1}$

Proof by substitution is as follows:

Let

$$
V=\left(\sum_{j=1}^{n}\left[P_{j}\right]\left[Q_{j}\right]^{1 / \sigma}\right)^{-1}
$$

Then,

$$
\begin{aligned}
Q_{i} & =\frac{\left[P_{i}\right]^{\sigma}\left[Q_{i}\right] V^{\sigma}\left[P_{i}\right]^{-\sigma}}{\left(\sum_{j=1}^{n}\left[P_{j}\right]^{\sigma}\left[Q_{j}\right] V^{\sigma}\left[P_{j}\right]^{1-\sigma}\right)^{\frac{\sigma}{\sigma-1}}} \cdot \frac{[Q]}{v} \\
\Rightarrow Q_{i} & =\frac{\left[Q_{i}\right] V^{\sigma}}{\left(\sum_{j=1}^{n}\left[P_{j}\right]\left[Q_{j}\right]\right)^{\frac{\sigma}{\sigma-1}} V^{\frac{\sigma^{2}}{\sigma-1}}} \cdot\left(\sum_{k=1}^{n} a_{k} \cdot Q_{k}^{\frac{\sigma-1}{\sigma}}\right)^{\frac{\sigma}{\sigma-1}} \\
\Rightarrow Q_{i} & =\frac{\left[Q_{i}\right] V^{\frac{\sigma}{1-\sigma}}}{\left(\sum_{j=1}^{n}\left[P_{j}\right]\left[Q_{j}\right]\right)^{\frac{\sigma}{\sigma-1}}} \cdot\left(\sum_{k=1}^{n}\left[P_{k}\right]\left[Q_{k}\right] V\right)^{\frac{\sigma}{\sigma-1}} \\
\Rightarrow Q_{i} & =\frac{\left[Q_{i}\right] V^{\frac{\sigma}{1-\sigma}}}{\left(\sum_{j=1}^{n}\left[P_{j}\right]\left[Q_{j}\right]\right)^{\frac{\sigma}{\sigma-1}}} \cdot \frac{\left(\sum_{k=1}^{n}\left[P_{k}\right]\left[Q_{k}\right]\right)^{\frac{\sigma}{\sigma-1}}}{V^{\frac{\sigma}{1-\sigma}}} \\
\Rightarrow Q_{i} & =\left[Q_{i}\right]
\end{aligned}
$$

That is, $a_{i}=\frac{\left[P_{i}\right]\left[Q_{i}\right]^{1 / \sigma}}{\sum_{j=1}^{n}\left[P_{j}\right]\left[Q_{j}\right]^{1 / \sigma}}$ is a valid calibration for any given set of prices $\left\{\left[P_{1}\right], \ldots,\left[P_{n}\right]\right\}$. Similar reasoning can be used for the proof of (K.3).

${ }^{1}$ The MONASH version of K.6 is found in Dixon \& Rimmer (2002, eq. 12.15, p. 127). 


\section{Appendix L}

\section{Walras' Law}

This appendix demonstrates that Walras' Law holds for the core JENNIFER model presented in Chapter 2.

First, substitution of equations (2.4), (2.3), and the household budget constraint into equation (2.107) gives: ${ }^{1}$

$$
\begin{aligned}
S A V^{\mathbf{c} \$} & =\sum_{r} S A V_{r}^{\mathbf{a t a x}, \mathbf{c} \$}+G O V B A L^{\mathbf{c} \$} \\
& =\sum_{r} I N C_{r}^{\text {atax }, \mathbf{c} \$}-\sum_{r} S P N_{r}^{\mathbf{c} \$}+G O V B A L^{\mathbf{c} \$} \\
& =\sum_{r} P_{r}^{L, \text { atax }} \cdot L_{r}^{(S)}+\sum_{j} \sum_{r} P_{j, r}^{K} \cdot K_{j, r}^{(S)}-\sum_{r} P_{r}^{Q, \mathbf{h s h}} \cdot Q_{r}^{\mathbf{h s h}(D)}+G O V B A L^{\mathbf{c} \$}
\end{aligned}
$$

Factor market clearing conditions (2.48) and (2.47) then imply:

$$
\begin{aligned}
S A V^{\mathbf{c} \$} & =\sum_{j} \sum_{r} P_{r}^{L, \operatorname{atax}} \cdot L_{j, r}^{\mathrm{ind}(D)}+\sum_{j} \sum_{r} P_{j, r}^{K} \cdot K_{j, r}^{\mathrm{ind}(D)}-\sum_{r} P_{r}^{Q, \mathbf{h s h}} \cdot Q_{r}^{\mathbf{h s h}(D)} \\
& +G O V B A L^{\mathbf{c} \$}
\end{aligned}
$$

Using the zero pure profits conditions $(2.52)$, the purchase price definition for $P_{j, r}^{F, \text { ind }}$

\footnotetext{
${ }^{1}$ An identity that emerges from the household agent's utility maximisation subject to budget constraint is:

$$
S P N_{r}^{\mathbf{c} \$}=P_{r}^{Q, \mathbf{h s h}} \cdot Q_{r}^{\mathbf{h s h}(D)}
$$
}

This definition is listed in the ninth subsection of appendix D. 
(2.60), and the definition of income tax revenue (2.42) we obtain:

$$
\begin{aligned}
S A V^{\mathbf{c} \$} & =\sum_{g} \sum_{r} P_{g, r}^{Q} \cdot \breve{Q}_{g, r}^{(S)}-\sum_{j} \sum_{r} P_{j, r}^{Q, \text { ind }} \cdot Q_{j, r}^{\operatorname{ind}(D)}-I N C T A X^{\mathbf{c} \$} \\
& -\sum_{r} P_{r}^{Q, \mathbf{h s h}} \cdot Q_{r}^{\mathbf{h s h}(D)}+G O V B A L^{\mathbf{c} \$}
\end{aligned}
$$

Substituting in the definition of government balance and using the purchase price definitions to derive an expression using basic prices gives us:

$$
\begin{aligned}
S A V^{\mathbf{c}} & =\sum_{g} \sum_{r} P_{g, r}^{Q} \cdot \breve{Q}_{g, r}^{(S)}-\sum_{g} \sum_{s} \sum_{j} \sum_{r} P_{g, s}^{Q} \cdot \breve{Q}_{g, s, j, r}^{\mathbf{i n d}(\mathbf{Q})(D)} \\
& -\sum_{g} \sum_{s} \sum_{r} P_{g, s}^{Q} \cdot \breve{Q}_{g, s, r}^{\mathbf{h s h}(D)}+D U T Y^{\mathbf{c} \$}-E X P S U B^{\mathbf{c} \$} \\
& -\sum_{g} \sum_{s} P_{g, s}^{Q} \cdot \breve{Q}_{g, s}^{\operatorname{gov}(D)}
\end{aligned}
$$

The market clearing equations (2.46) then imply:

$$
\begin{aligned}
S A V^{\mathbf{c}} & =\sum_{g} \sum_{x} \sum_{j} \sum_{r} P_{g, x}^{Q} \cdot \breve{Q}_{g, x, j, r}^{\mathbf{i n d}(\mathbf{K})(D)}+\sum_{g} \sum_{x} P_{g, x}^{Q} \cdot \breve{Q}_{g, x}^{\exp (D)} \\
& -\sum_{g} \sum_{j} \sum_{r} P_{g, \mathbf{i m p}}^{Q} \cdot \breve{Q}_{g, \mathbf{i m p}, j, r}^{\mathbf{i n d}(\mathbf{Q})(D)}-\sum_{g} \sum_{r} P_{g, \mathbf{i m p}}^{Q} \cdot \breve{Q}_{g, \mathbf{i m p}, r}^{\mathbf{h s h}(D)} \\
& -\sum_{g} P_{g, \mathbf{i m p}}^{Q} \cdot \breve{Q}_{g, \mathbf{i m p}}^{\mathbf{g o v}(D)}+D U T Y^{\mathbf{c} \$}-E X P S U B^{\mathbf{c} \$}
\end{aligned}
$$

And simplifying we obtain:

$$
\begin{aligned}
S A V^{\mathbf{c} \$} & =I^{\mathbf{c} \$}+E X P^{\mathbf{c} \$, \mathbf{d} \$, \mathbf{b a s}}-I M P^{\mathbf{c} \$, \mathbf{d} \$, \text { bas }}+D U T Y^{\mathbf{c} \$}-E X P S U B^{\mathbf{c} \$} \\
\Rightarrow S A V^{\mathbf{c} \$} & =I^{\mathbf{c} \$}+T R D B A L^{\mathbf{c} \$, \mathbf{d} \$}
\end{aligned}
$$

That is, the supply of finance equals the demand for finance. 


\section{Appendix M}

\section{Margins Demands: A Worked Example}

This appendix sets out a very simple numerical example to show the effects of different assumptions regarding margin flows.

We begin with the following information from a balanced $\mathrm{MRIO}^{\prime}$ database of an economy with one margin (M), two other goods (1 and 2), and three regions (A, B, and C):

\begin{tabular}{|c|c|c|c|c|c|}
\hline & \multirow{2}{*}{\multicolumn{2}{|c|}{$\begin{array}{l}\mathrm{MRIO}^{\prime} \\
\text { row/col }\end{array}$}} & \multicolumn{3}{|c|}{$\mathrm{CON}$} \\
\hline & & & $\mathrm{A}$ & B & $\mathrm{C}$ \\
\hline \multirow{6}{*}{ 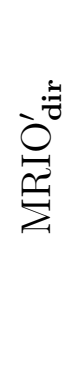 } & & $\mathrm{A}$ & 25 & 25 & 25 \\
\hline & 1 & B & 25 & 25 & 25 \\
\hline & & $\mathrm{C}$ & 0 & 0 & 0 \\
\hline & & $\mathrm{A}$ & 40 & 40 & 40 \\
\hline & 2 & B & 0 & 0 & 0 \\
\hline & & $\mathrm{C}$ & 10 & 10 & 10 \\
\hline \multirow{3}{*}{ 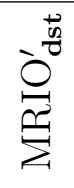 } & & $\mathrm{A}$ & 4 & 4 & 4 \\
\hline & $\mathrm{M}$ & B & 4 & 4 & 4 \\
\hline & & $\mathrm{C}$ & 4 & 4 & 4 \\
\hline \multirow{3}{*}{ 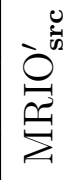 } & & A & 0 & 0 & 0 \\
\hline & $\mathrm{M}$ & B & 0 & 0 & 0 \\
\hline & & $\mathrm{C}$ & 0 & 0 & 0 \\
\hline
\end{tabular}


The margin M has been treated as destination-type but not local. Consider the margin demands of the household agent in region $\mathrm{A}$. The agent will demand $\mathrm{M}$ from region $y$ to deliver product $g$ from region $x$ as follows: ${ }^{1}$

\begin{tabular}{|cc|ccc|}
\cline { 3 - 5 } \multicolumn{1}{c|}{} & & & & \\
\multicolumn{1}{c|}{} & & $\mathrm{A}$ & $\mathrm{B}$ & $\mathrm{C}$ \\
\hline & $\mathrm{A}$ & 1 & 1 & 1 \\
1 & $\mathrm{~B}$ & 1 & 1 & 1 \\
& $\mathrm{C}$ & 0 & 0 & 0 \\
& $\mathrm{~A}$ & $8 / 5$ & $8 / 5$ & $8 / 5$ \\
2 & $\mathrm{~B}$ & 0 & 0 & 0 \\
& $\mathrm{C}$ & $2 / 5$ & $2 / 5$ & $2 / 5$ \\
\hline
\end{tabular}

The agent's demand for M from each region has simply been proportionately spread (pro-rated) over their product demands.

If $\mathrm{M}$ is a local margin then the relevant part of $\mathrm{MRIO}^{\prime}$ changes to: ${ }^{2}$

\begin{tabular}{|c|c|c|c|c|c|}
\hline & \multirow{2}{*}{\multicolumn{2}{|c|}{$\begin{array}{l}\mathrm{MRIO}^{\prime} \\
\text { row/col }\end{array}$}} & \multicolumn{3}{|c|}{$\mathrm{CON}$} \\
\hline & & & $\mathrm{A}$ & $\mathrm{B}$ & $\mathrm{C}$ \\
\hline \multirow{3}{*}{ 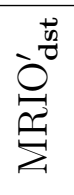 } & \multirow{3}{*}{$\mathrm{M}$} & $\mathrm{A}$ & 12 & 0 & 0 \\
\hline & & B & 0 & 12 & 0 \\
\hline & & $\mathrm{C}$ & 0 & 0 & 12 \\
\hline
\end{tabular}

Pro-rating over the product flows then gives the following margin demands for the household agent in region A:

\begin{tabular}{|cc|ccc|}
\cline { 3 - 5 } \multicolumn{1}{c|}{} & \multicolumn{3}{|c|}{ M } \\
\hline & $\mathrm{A}$ & 3 & 0 & 0 \\
1 & $\mathrm{~B}$ & 3 & 0 & 0 \\
& $\mathrm{C}$ & 0 & 0 & 0 \\
& $\mathrm{~A}$ & $24 / 5$ & 0 & 0 \\
2 & $\mathrm{~B}$ & 0 & 0 & 0 \\
& $\mathrm{C}$ & $6 / 5$ & 0 & 0 \\
\hline
\end{tabular}

${ }^{1}$ These are the benchmark values of $Q_{\mathbf{M}, y, g, s, \mathbf{A}}^{\mathbf{h s h}(D)}$

${ }^{2}$ For simplicity any RAS scaling necessitated by destabilisation of the MRIO' has been ignored. 
If the margin service $\mathrm{M}$ is treated as a source-type margin instead, the MRIO' data is:

\begin{tabular}{|c|c|c|c|c|c|}
\hline & \multirow{2}{*}{\multicolumn{2}{|c|}{$\begin{array}{l}\mathrm{MRIO}^{\prime} \\
\text { row/col }\end{array}$}} & \multicolumn{3}{|c|}{$\mathrm{CON}$} \\
\hline & & & $\mathrm{A}$ & B & $\mathrm{C}$ \\
\hline \multirow{6}{*}{$\frac{\dot{\sigma}}{\underbrace{0}}$} & & $\mathrm{~A}$ & 25 & 25 & 25 \\
\hline & 1 & B & 25 & 25 & 25 \\
\hline & & $\mathrm{C}$ & 0 & 0 & 0 \\
\hline & & $\mathrm{A}$ & 40 & 40 & 40 \\
\hline & 2 & B & 0 & 0 & 0 \\
\hline & & $\mathrm{C}$ & 10 & 10 & 10 \\
\hline \multirow{3}{*}{$\frac{0^{\frac{4}{0}}}{\underbrace{\frac{1}{2}}}$} & & $\mathrm{~A}$ & 0 & 0 & 0 \\
\hline & $\mathrm{M}$ & B & 0 & 0 & 0 \\
\hline & & $\mathrm{C}$ & 0 & 0 & 0 \\
\hline \multirow{3}{*}{ 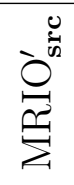 } & & A & 4 & 4 & 4 \\
\hline & $\mathrm{M}$ & B & 4 & 4 & 4 \\
\hline & & $\mathrm{C}$ & 4 & 4 & 4 \\
\hline
\end{tabular}

This time, margin from region $y$ is only used to deliver products from that region, so to determine the amount of $\mathrm{M}$ demanded from region $y$ to deliver product $g$ from region $x$, we pro-rate the agent's demand for M from $y$ over all product flows (to the agent) from region $x$ where $x=y$, and set the other demands equal to zero. Thus the demands of region A's household agent are:

\begin{tabular}{|cc|ccc|}
\cline { 3 - 5 } \multicolumn{1}{c|}{} & \multicolumn{3}{|c|}{ M } \\
\hline & A & A & B & C \\
1 & B & 0 & 4 & 0 \\
& C & 0 & 0 & 0 \\
& A & $32 / 13$ & 0 & 0 \\
2 & B & 0 & 0 & 0 \\
& C & 0 & 0 & 4 \\
\hline
\end{tabular}

If $\mathrm{M}$ is also a border margin then the source-margin rows change to: 


\begin{tabular}{|c|c|c|c|c|c|}
\hline & \multirow{2}{*}{\multicolumn{2}{|c|}{$\begin{array}{l}\text { MRIO' }^{\prime} \\
\text { row/col }\end{array}$}} & \multicolumn{3}{|c|}{$\mathrm{CON}$} \\
\hline & & & A & B & C \\
\hline \multirow{3}{*}{ 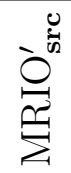 } & & A & 0 & 6 & 6 \\
\hline & $\mathrm{M}$ & B & 6 & 0 & 6 \\
\hline & & $\mathrm{C}$ & 6 & 6 & 0 \\
\hline
\end{tabular}

Then pro-rating the margin demands as source-type margins gives the following margin demands for the household agent:

\begin{tabular}{|cc|ccc|}
\cline { 3 - 5 } \multicolumn{1}{c|}{} & \multicolumn{3}{c|}{ M } \\
\hline & $\mathrm{A}$ & 0 & 0 & 0 \\
1 & $\mathrm{~B}$ & 0 & 6 & 0 \\
& $\mathrm{C}$ & 0 & 0 & 0 \\
& $\mathrm{~A}$ & 0 & 0 & 0 \\
2 & $\mathrm{~B}$ & 0 & 0 & 0 \\
& $\mathrm{C}$ & 0 & 0 & 6 \\
\hline
\end{tabular}




\section{Appendix N}

\section{The Extended MRIO Matrix}

This appendix presents the extended version of the multi-regional input-output matrix $\left(\mathrm{MRIO}^{\prime}\right)$ as developed in chapter 3 and subsequently used to calibrate the JENNIFER model for the simulations of that chapter and chapter 4. As the $\mathrm{MRIO}^{\prime}$ matrix is very large, it is divided into parts with each part shown in a separate table labelled as indicated in the diagram overleaf (table N.1).

Note that table cells showing "0.00" indicate a positive number less than 0.005 while cells that are exactly zero are left empty. Zero rows and columns of the matrix are omitted from the tables.

An electronic copy of the MRIO' matrix is available in the Research Archive of the Victoria University of Wellington Library at http://researcharchive.vuw.ac.nz/, alongside the electronic copy of this thesis Two versions are provided: one is formatted for Microsoft Office Excel 2007, and has separate sheets displaying the gross value flows, the shares of those flows in their column total, and the shares of those flows in their row total; the other version has only the gross value flows and is formatted as comma separated values for importing into other software. In each version, the MRIO' matrix is presented as a single table of values rounded to four decimal places, with row and column labels as shown in table N.1. 


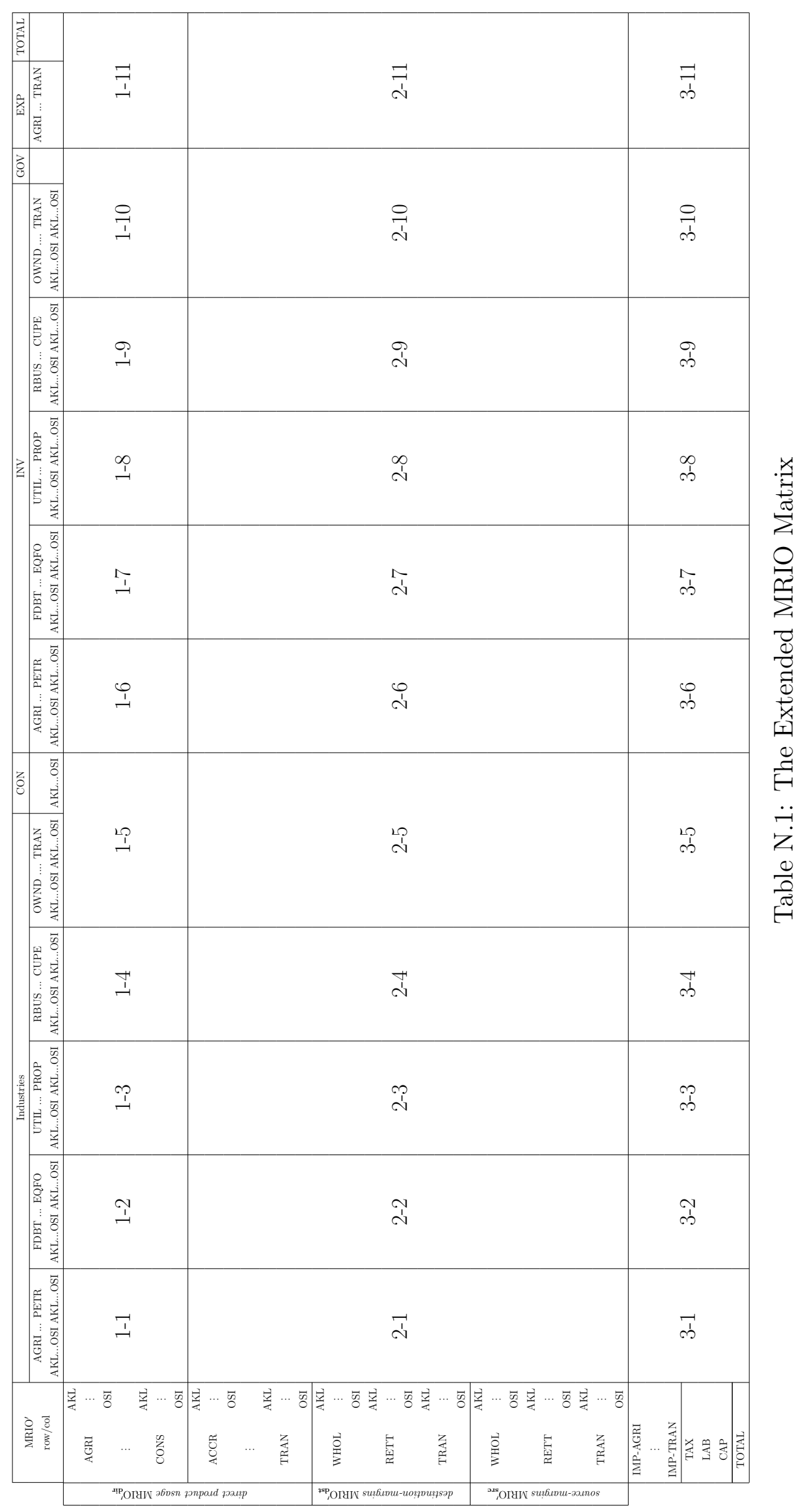




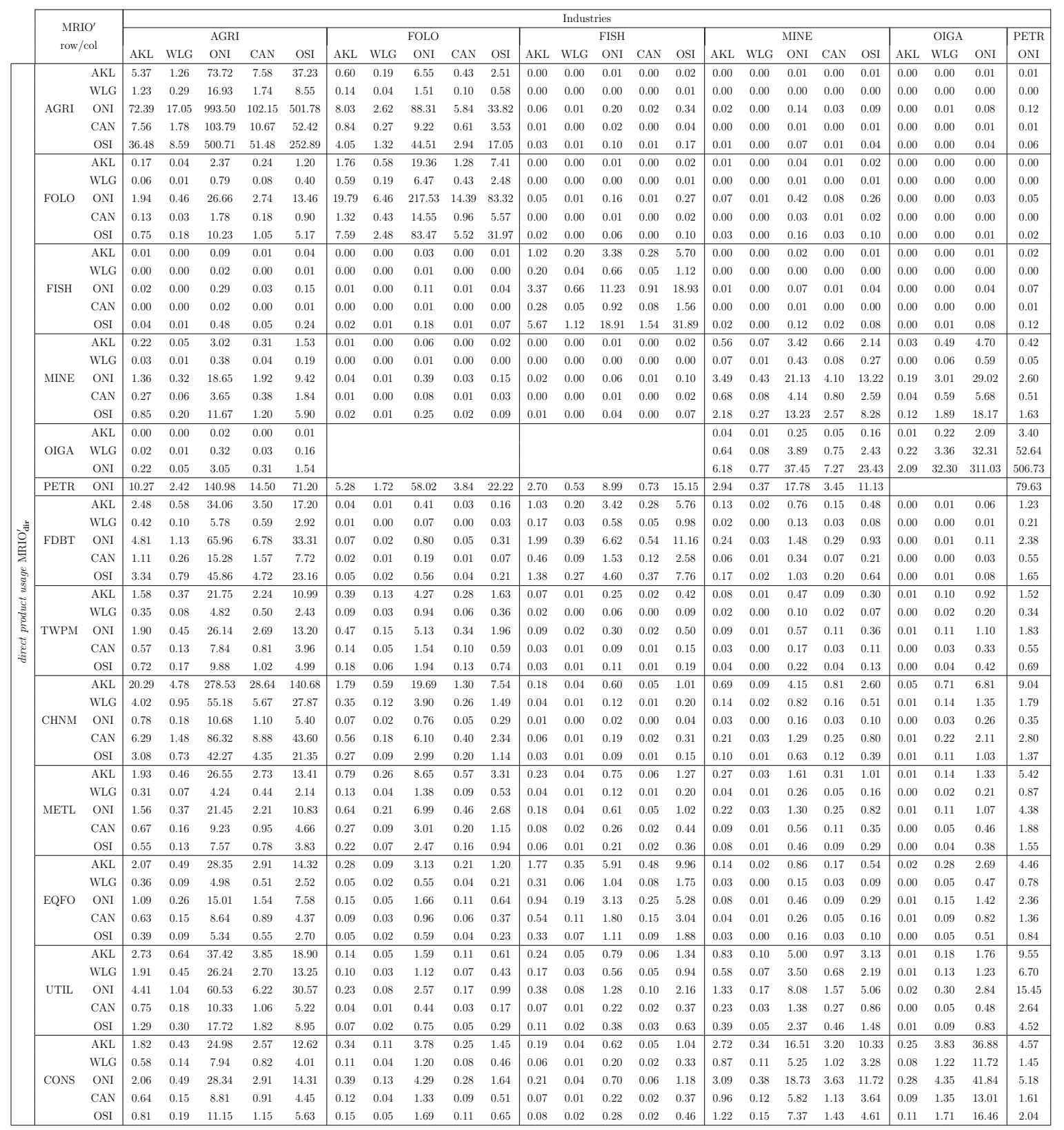

Extended MRIO Table - Part 1-1 


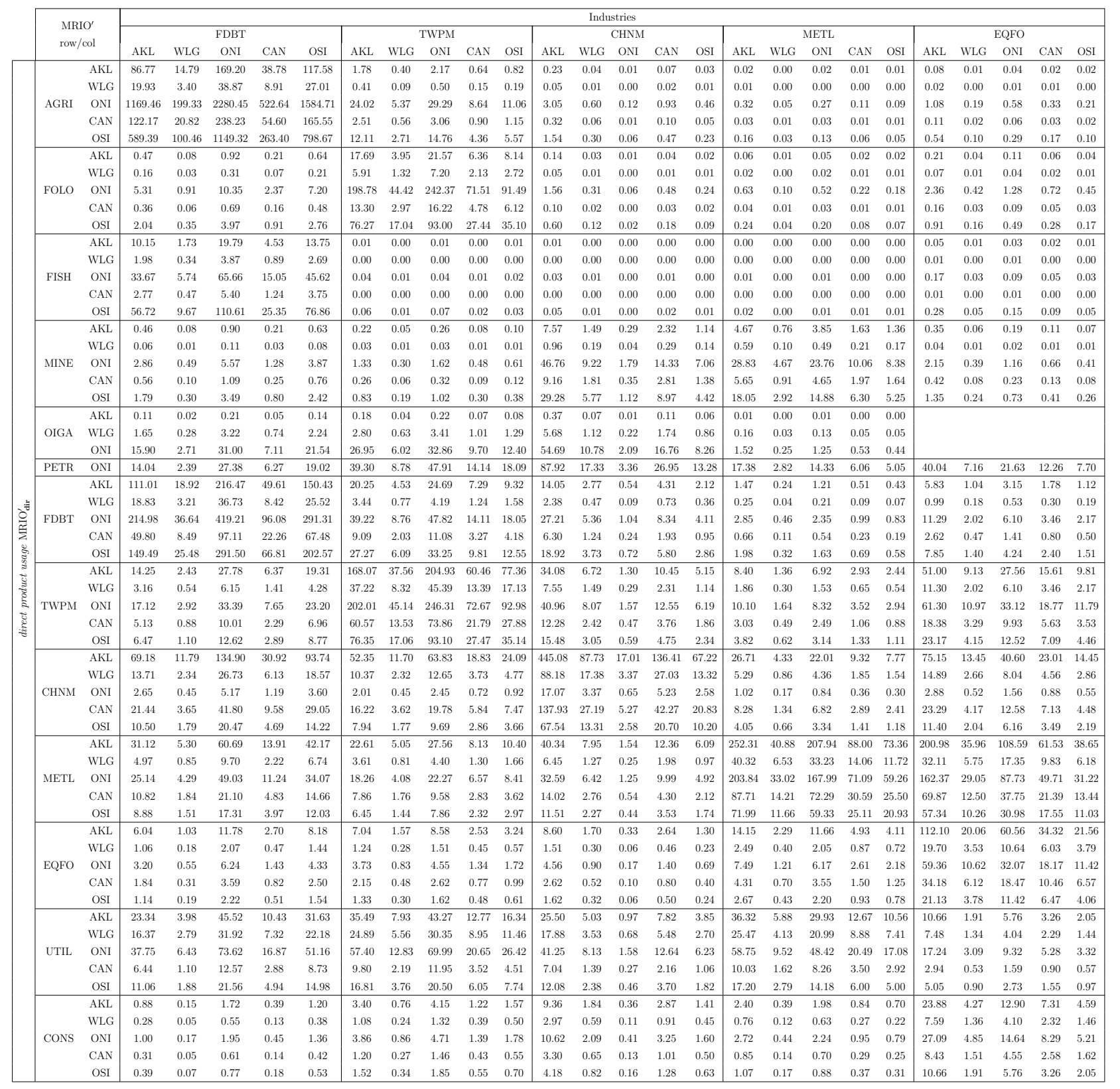

Extended MRIO Table - Part 1-2 


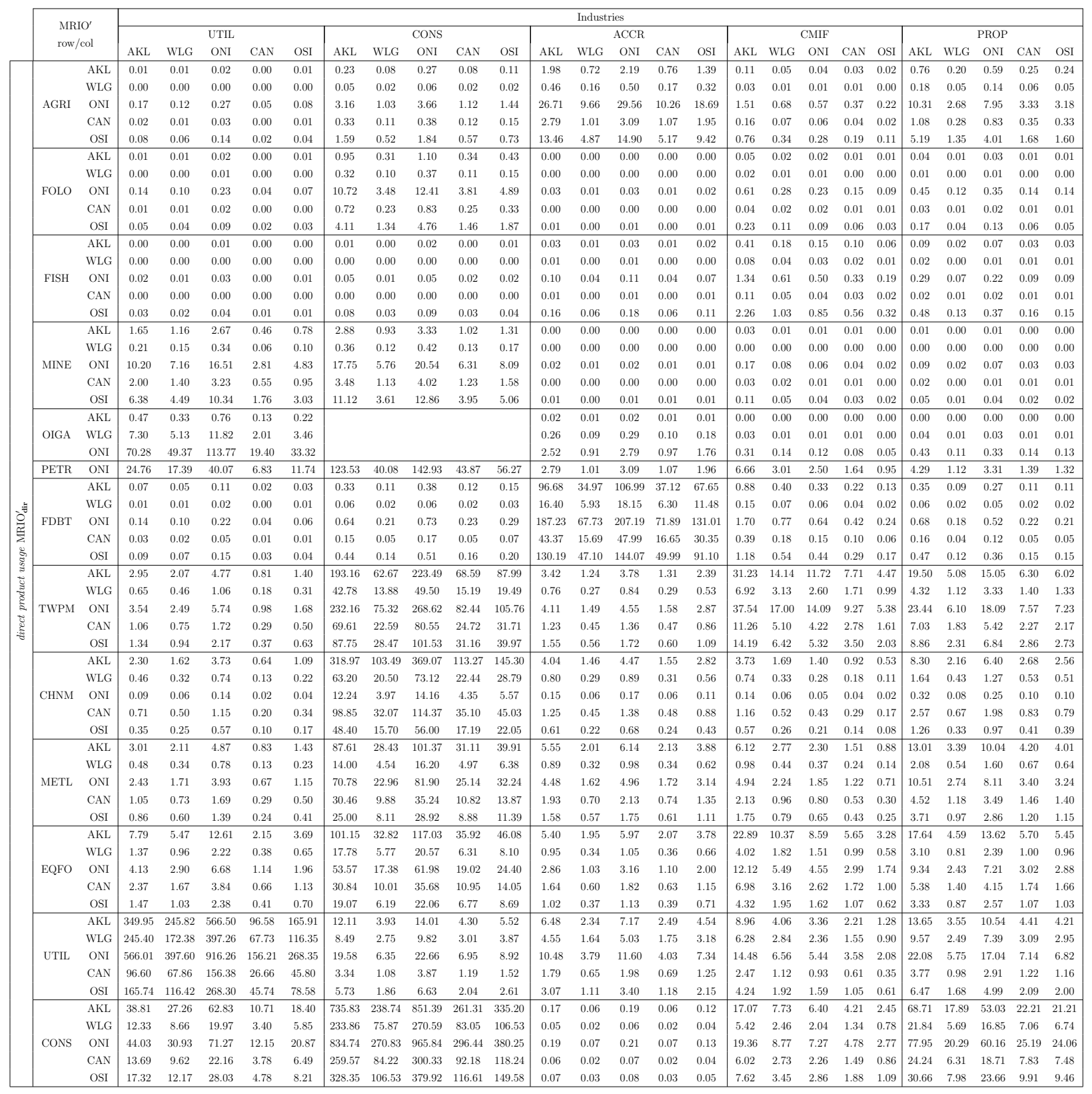

Extended MRIO Table - Part 1-3 


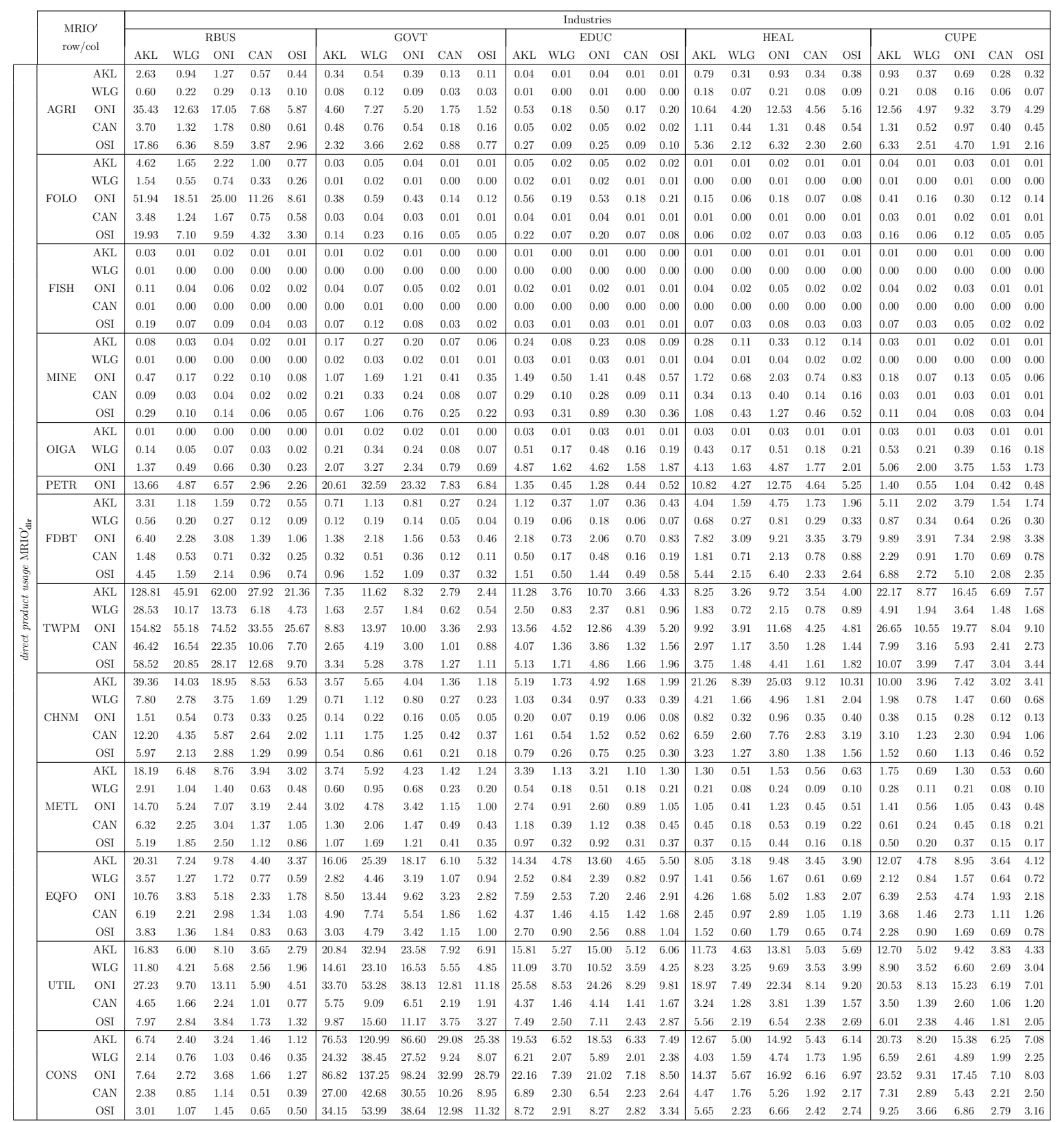

Extended MRIO Table - Part 1-4 


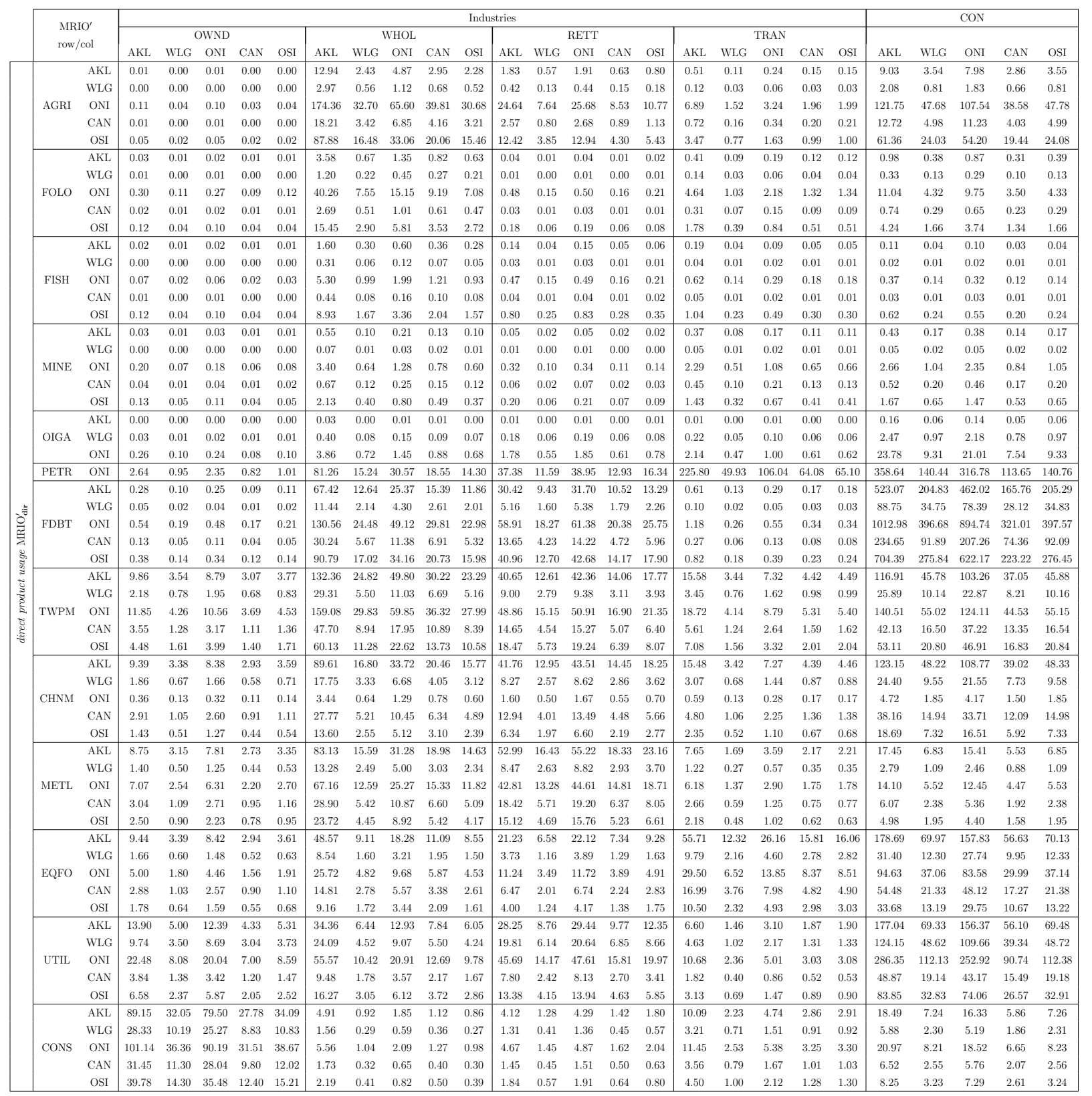

Extended MRIO Table - Part 1-5 


\begin{tabular}{|c|c|c|c|c|c|c|c|c|c|c|c|c|c|c|c|c|c|c|c|c|c|c|c|c|c|c|}
\hline & & \multicolumn{24}{|c|}{ INV } \\
\hline & & & \multicolumn{5}{|c|}{ AGRI } & \multicolumn{5}{|c|}{ FOLO } & & & FISH & & & & & MINE & & & & OIGA & & PETR \\
\hline & & & AKL & WLG & ONI & CAN & OSI & AKL & WLG & ONI & CAN & OSI & AKL & WLG & ONI & CAN & OSI & AKL & WLG & ONI & CAN & OSI & AKL & WLG & ONI & ONI \\
\hline & & AKL & 0.01 & 0.00 & 0.16 & 0.02 & 0.08 & \begin{tabular}{|l|l|}
0.00 \\
\end{tabular} & 0.00 & 0.02 & 0.00 & 0.01 & \begin{tabular}{|l|l|}
0.00 \\
\end{tabular} & 0.00 & 0.00 & 0.00 & 0.01 & 0.00 & 0.00 & 0.02 & $\begin{array}{l}0.00 \\
\end{array}$ & 0.01 & 0.00 & 0.02 & 0.17 & 0.02 \\
\hline & & WLG & 0.00 & 0.00 & 0.04 & 0.00 & 0.02 & 0.00 & 0.00 & 0.00 & 0.00 & 0.00 & \begin{tabular}{|l|l|} 
& 0.00
\end{tabular} & 0.00 & 0.00 & 0.00 & 0.00 & \begin{tabular}{|l|l}
0.00 \\
\end{tabular} & 0.00 & 0.01 & 0.00 & 0.00 & \begin{tabular}{|l|l|} 
\\
\end{tabular} & 0.00 & 0.04 & .00 \\
\hline & AGRI & ONI & 0.16 & 0.04 & 2.20 & 0.23 & 1.11 & 0.02 & 0.01 & 0.26 & 0.02 & 0.10 & $\mid 0.01$ & 0.00 & 0.04 & 0.00 & 0.07 & 0.05 & 0.01 & 0.30 & 0.06 & 0.19 & \begin{tabular}{|l|l} 
& 0.01
\end{tabular} & 0.24 & 2.29 & 0.26 \\
\hline & & CAN & 0.02 & 0.00 & 0.23 & 0.02 & 0.12 & \begin{tabular}{|l|l|} 
& 0.00 \\
\end{tabular} & 0.00 & 0.03 & 0.00 & 0.01 & $\mid 0.00$ & 0.00 & 0.00 & 0.00 & 0.01 & 0.00 & 0.00 & 0.03 & 0.01 & 0.02 & \begin{tabular}{|l|l|} 
\\
\end{tabular} & 0.02 & 0.24 & 0.03 \\
\hline & & OSI & 0.08 & 0.02 & 1.11 & 0.11 & 0.56 & 0.01 & 0.00 & 0.13 & 0.01 & 0.05 & 0.01 & 0.00 & 0.02 & 0.00 & 0.04 & \begin{tabular}{|l|l} 
& 0.02
\end{tabular} & 0.00 & 0.15 & 0.03 & 0.09 & \begin{tabular}{|l|l} 
\\
\end{tabular} & 0.12 & 1.15 & 0.13 \\
\hline & & AKL & 0.01 & 0.00 & 0.14 & 0.01 & 0.07 & 0.00 & 0.00 & 0.02 & $\begin{array}{l}0.00 \\
\end{array}$ & 0.01 & \begin{tabular}{|l|l|}
0.00 \\
\end{tabular} & 0.00 & 0.00 & 0.00 & 0.00 & \begin{tabular}{|l|l|}
0.00 \\
\end{tabular} & 0.00 & 0.02 & $\begin{array}{l}0.00 \\
\end{array}$ & 0.01 & 0 & 0.01 & 0.14 & 0.02 \\
\hline & & WLG & 00 & 0.00 & 0.05 & 0.00 & 0.02 & 0.00 & 0.00 & 0.01 & 0.00 & 0.00 & 0.00 & 0.00 & 0.00 & 0.00 & 0.00 & 0.00 & 0.00 & 0.01 & 0.00 & 0.00 & \begin{tabular}{|l|l|} 
\\
\end{tabular} & 0.00 & 0.05 & 0.01 \\
\hline & FOLO & ONI & 0.11 & 0.03 & 1.53 & 0.16 & 0.77 & 0.02 & 0.01 & 0.18 & 0.01 & 0.07 & 0.01 & 0.00 & 0.03 & 0.00 & 0.05 & \begin{tabular}{|l|l} 
& 0.03
\end{tabular} & 0.00 & 0.21 & 0.04 & 0.13 & \begin{tabular}{|l|} 
\\
\end{tabular} & 0.17 & 1.59 & 0.18 \\
\hline & & CAN & 0.01 & 0.00 & 0.10 & 0.01 & 0.05 & 0.00 & 0.00 & 0.01 & 0.00 & 0.00 & 0.00 & 0.00 & 0.00 & 0.00 & 0.00 & 0.00 & 0.00 & 0.01 & 0.00 & 0.01 & \begin{tabular}{|l|l|} 
\\
\end{tabular} & 0.01 & 0.11 & 0.01 \\
\hline & & OSI & 0.04 & 0.01 & 0.59 & 0.06 & 0.30 & 0.01 & 0.00 & 0.07 & 0.00 & 0.03 & \begin{tabular}{|l|l|} 
& 0.00
\end{tabular} & 0.00 & 0.01 & 0.00 & 0.02 & \begin{tabular}{|l|l} 
& 0.01
\end{tabular} & 0.00 & 0.08 & 0.02 & 0.05 & \begin{tabular}{|l|l|} 
\\
\end{tabular} & 0.06 & 0.61 & 0.07 \\
\hline & & AKL & \begin{tabular}{|l|l|}
0.00 \\
\end{tabular} & 0.00 & 0.01 & 0.00 & 0.01 & \begin{tabular}{|l|l|}
0.00 \\
\end{tabular} & 0.00 & 0.00 & 0.00 & 0.00 & \begin{tabular}{|l|l|}
0.00 \\
\end{tabular} & 0.00 & 0.00 & 0.00 & 0.00 & \begin{tabular}{|l|l|}
0.00 \\
\end{tabular} & 0.00 & 0.00 & $\begin{array}{l}0.00 \\
\end{array}$ & 0.00 & 0 & 0.00 & 0.01 & 0.00 \\
\hline & & WLG & 00 & 0.00 & 0.00 & 0.00 & 0.00 & 0.00 & 0.00 & 0.00 & 0.00 & 0.00 & $\mid 0.00$ & 0.00 & 0.00 & 0.00 & 0.00 & \begin{tabular}{|l|l} 
& 0.00
\end{tabular} & 0.00 & 0.00 & 0.00 & 0.00 & \begin{tabular}{|l|l|} 
\\
\end{tabular} & 0.00 & 0.00 & 0.00 \\
\hline & FISH & ONI & 0.00 & 0.00 & 0.03 & 0.00 & 0.02 & 0.00 & 0.00 & 0.00 & 0.00 & 0.00 & $\mid 0.00$ & 0.00 & 0.00 & 0.00 & 0.00 & 0.00 & 0.00 & 0.00 & 0.00 & 0.00 & \begin{tabular}{|l|l|} 
\\
\end{tabular} & 0.00 & 0.04 & 0.00 \\
\hline & & CAN & 0.00 & 0.00 & 0.00 & 0.00 & 0.00 & 0.00 & 0.00 & 0.00 & 0.00 & 0.00 & 0.00 & 0.00 & 0.00 & 0.00 & 0.00 & 0.00 & 0.00 & 0.00 & 0.00 & 0.00 & \begin{tabular}{|l|l|} 
\\
\end{tabular} & 0.00 & 0.00 & 0.00 \\
\hline & & OSI & 0.00 & 0.00 & 0.06 & 0.01 & 0.03 & 0.00 & 0.00 & 0.01 & 0.00 & 0.00 & 0.00 & 0.00 & 0.00 & 0.00 & 0.00 & 0.00 & 0.00 & 0.01 & 0.00 & 0.00 & \begin{tabular}{|l|l|} 
\\
\end{tabular} .00 & 0.01 & 0.06 & 0.01 \\
\hline & & AKL & \begin{tabular}{|l|l}
0.00 \\
\end{tabular} & 0.00 & 0.06 & 0.01 & 0.03 & .00 & 0.00 & 0.01 & 0.00 & 0.00 & \begin{tabular}{|l|l|}
0.00 \\
\end{tabular} & 0.00 & 0.00 & 0.00 & 0.00 & 0.00 & 0.00 & 0.01 & 0.00 & 0.01 & \begin{tabular}{|l|l|}
0.00 \\
\end{tabular} & 0.01 & 0.06 & 0.01 \\
\hline & & WLG & .00 & 0.00 & 0.01 & 0.00 & 0.00 & 0.00 & 0.00 & 0.00 & 0.00 & 0.00 & $\mid 0.00$ & 0.00 & 0.00 & 0.00 & 0.00 & 0.00 & 0.00 & 0.00 & 0.00 & 0.00 & \begin{tabular}{|l|l|} 
\\
\end{tabular} & 0.00 & 0.01 & .00 \\
\hline & MINE & ONI & 0.03 & 0.01 & 0.38 & 0.04 & 0.19 & 0.00 & 0.00 & 0.05 & 0.00 & 0.02 & $\mid 0.00$ & 0.00 & 0.01 & 0.00 & 0.01 & \begin{tabular}{|l|l} 
& 0.01
\end{tabular} & 0.00 & 0.05 & 0.01 & 0.03 & $\mid 0.00$ & 0.04 & 0.40 & 0.05 \\
\hline & & CAN & 0.01 & 0.00 & 0.08 & 0.01 & 0.04 & 0.00 & 0.00 & 0.01 & 0.00 & 0.00 & 0.00 & 0.00 & 0.00 & 0.00 & 0.00 & 0.00 & 0.00 & 0.01 & 0.00 & 0.01 & \begin{tabular}{|l|l|} 
\\
\end{tabular} & 0.01 & 0.08 & 0.01 \\
\hline & & OSI & 0.02 & 0.00 & 0.24 & 0.02 & 0.12 & 0.00 & 0.00 & 0.03 & 0.00 & 0.01 & 0.00 & 0.00 & 0.00 & 0.00 & 0.01 & \begin{tabular}{|l|l} 
& 0.01
\end{tabular} & 0.00 & 0.03 & 0.01 & 0.02 & $|0.00|$ & 0.03 & 0.25 & 0.03 \\
\hline & & AKL & 0.00 & 0.00 & 0.02 & 0.00 & 0.01 & \begin{tabular}{|l|l|}
0.00 \\
\end{tabular} & 0.00 & 0.00 & $\begin{array}{l}0.00 \\
\end{array}$ & 0.00 & \begin{tabular}{|l|l|}
0.00 \\
\end{tabular} & 0.00 & 0.00 & 0.00 & 0.00 & 0.00 & 0.00 & 0.00 & 0.00 & 0.00 & \begin{tabular}{|l|l|}
0.00 \\
\end{tabular} & 0.00 & 0.03 & 0.00 \\
\hline & OIGA & WLG & 03 & 0.01 & 0.37 & 0.04 & 0.19 & 0.00 & 0.00 & 0.04 & 0.00 & 0.02 & $\mid \begin{array}{l}0.00 \\
\end{array}$ & 0.00 & 0.01 & 0.00 & 0.01 & 0.01 & 0.00 & 0.05 & 0.01 & 0.03 & \begin{tabular}{|l|l|} 
\\
\end{tabular} & 0.04 & 0.39 & 0.04 \\
\hline & & ONI & 0.26 & 0.06 & 3.59 & 0.37 & 1.81 & \begin{tabular}{|l} 
\\
\end{tabular} & 0.01 & 0.43 & 0.03 & 0.16 & \begin{tabular}{|l|l} 
& 0.02
\end{tabular} & 0.00 & 0.07 & 0.01 & 0.12 & 0.08 & 0.01 & 0.48 & 0.09 & 0.30 & 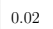 & 0.39 & 3.73 & 0.42 \\
\hline & PETR & ONI & \begin{tabular}{|l|l|}
0.09 \\
\end{tabular} & 0.02 & $\begin{array}{l}1.19 \\
\end{array}$ & 0.12 & 0.60 & 0.01 & 0.00 & 0.14 & 0.01 & 0.05 & \begin{tabular}{|l|l|}
0.01 \\
\end{tabular} & 0.00 & 0.02 & 0.00 & 0.04 & \begin{tabular}{|l|l|}
0.03 \\
\end{tabular} & 0.00 & 0.16 & $\begin{array}{l}0.03 \\
\end{array}$ & 0.10 & \begin{tabular}{|l|l|}
0.01 \\
\end{tabular} & 0.13 & 1.24 & 0.14 \\
\hline & & AKL & 0.03 & 0.01 & 0.48 & 0.05 & 0.24 & 0.00 & 0.00 & 0.06 & $\begin{array}{l}0.00 \\
\end{array}$ & $\begin{array}{ll}0.02 \\
\end{array}$ & \begin{tabular}{|l|l|}
0.00 \\
\end{tabular} & 0.00 & 0.01 & 0.00 & 0.02 & 0.01 & 0.00 & 0.06 & 0.01 & 0.04 & \begin{tabular}{|l|l|}
0.00 \\
\end{tabular} & 0.05 & 0.50 & 0.06 \\
\hline & & WLG & .01 & 0.00 & 0.08 & 0.01 & 0.04 & 0.00 & 0.00 & 0.01 & 0.00 & 0.00 & | 0.00 & 0.00 & 0.00 & 0.00 & 0.00 & 0.00 & 0.00 & 0.01 & 0.00 & 0.01 & $\mid 0.00$ & 0.01 & 0.09 & 0.01 \\
\hline & FDBT & ONI & 0.07 & 0.02 & 0.93 & 0.10 & 0.47 & 0.01 & 0.00 & 0.11 & 0.01 & 0.04 & 0.01 & 0.00 & 0.02 & 0.00 & 0.03 & \begin{tabular}{|l|l} 
& 0.02
\end{tabular} & 0.00 & 0.13 & 0.02 & 0.08 & \begin{tabular}{|l|} 
\\
\end{tabular} & 0.10 & 0.97 & 0.11 \\
\hline & & CAN & 0.02 & 0.00 & 0.22 & 0.02 & 0.11 & 0.00 & 0.00 & 0.03 & 0.00 & 0.01 & $\mid 0.00$ & 0.00 & 0.00 & 0.00 & 0.01 & 0.00 & 0.00 & 0.03 & 0.01 & 0.02 & \begin{tabular}{|l|l|} 
\\
\end{tabular} & 0.02 & .23 & 0.03 \\
\hline & & OSI & 0.05 & 0.01 & 0.65 & 0.07 & 0.33 & 0.01 & 0.00 & 0.08 & 0.01 & 0.03 & 0.00 & 0.00 & 0.01 & 0.00 & 0.02 & \begin{tabular}{|l|l} 
& 0.01
\end{tabular} & 0.00 & 0.09 & 0.02 & 0.05 & 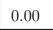 & 0.07 & 0.68 & 0.08 \\
\hline & & AKL & \begin{tabular}{|l|l|}
0.02 \\
\end{tabular} & 0.00 & 0.29 & 0.03 & 0.15 & \begin{tabular}{|l|l|}
0.00 \\
\end{tabular} & 0.00 & 0.03 & 0.00 & 0.01 & \begin{tabular}{|l|l|}
0.00 \\
\end{tabular} & 0.00 & 0.01 & 0.00 & 0.01 & 0.01 & 0.00 & 0.04 & 0.01 & 0.02 & 0.00 & 0.03 & 0.30 & 0.03 \\
\hline & & WLG & 0.00 & 0.00 & 0.06 & 0.01 & 0.03 & \begin{tabular}{|l|l|} 
\\
\end{tabular} .00 & 0.00 & 0.01 & 0.00 & 0.00 & \begin{tabular}{|l|l|} 
& 0.00
\end{tabular} & 0.00 & 0.00 & 0.00 & 0.00 & 0.00 & 0.00 & 0.01 & 0.00 & 0.01 & \begin{tabular}{|l|l|} 
\\
\end{tabular} & 0.01 & 0.07 & 0.01 \\
\hline & TWPM & ONI & 0.03 & 0.01 & 0.35 & 0.04 & 0.18 & 0.00 & 0.00 & 0.04 & 0.00 & 0.02 & 0.00 & 0.00 & 0.01 & 0.00 & .01 & 0.01 & 0.00 & .05 & 0.01 & 0.03 & \begin{tabular}{|l|l|} 
\\
\end{tabular} & 0.04 & 0.37 & 0.04 \\
\hline & & CAN & 0.01 & 0.00 & 0.11 & 0.01 & 0.05 & 0.00 & 0.00 & 0.01 & 0.00 & 0.00 & \begin{tabular}{|l|l|} 
& 0.00
\end{tabular} & 0.00 & 0.00 & 0.00 & 0.00 & 0.00 & 0.00 & .01 & 0.00 & 0.01 & \begin{tabular}{|l|l|} 
\\
\end{tabular} & 0.01 & 0.11 & 0.01 \\
\hline & & OSI & 0.01 & 0.00 & 0.13 & 0.01 & 0.07 & 0.00 & 0.00 & 0.02 & 0.00 & 0.01 & $\begin{array}{l}0.00 \\
\end{array}$ & 0.00 & 0.00 & 0.00 & 0.00 & 0.00 & 0.00 & 0.02 & 0.00 & 0.01 & \begin{tabular}{|l|l|} 
\\
\end{tabular} .00 & 0.01 & 0.14 & 0.02 \\
\hline & & AKL & 09 & 0.02 & 1.19 & 0.12 & 0.60 & \begin{tabular}{|l|l|}
0.01 \\
\end{tabular} & 0.0 & 0.14 & 0.01 & 0.05 & \begin{tabular}{|l|l|}
0.01 \\
\end{tabular} & 0.00 & 0.02 & 0.00 & 0.04 & .03 & 0.00 & 0.16 & 0.03 & 0.10 & 0.01 & 0.13 & 1.24 & 0.14 \\
\hline & & WLG & 02 & 0.00 & 0.24 & 0.02 & 0.1 & 0.00 & 0. & 0.0 & 0.00 & 0.01 & 0.6 & 0.00 & 0 & 0.00 & 01 & 0.01 & 00 & 03 & 0.01 & 0.02 & 00 & .03 & 0.25 & .03 \\
\hline & CHNM & ONI & 0. & 0.00 & 0.0 & 0.00 & 0.02 & 00 & 0.00 & 0.01 & 0.00 & 0.00 & 0.00 & 0.00 & 0.00 & 0.00 & .00 & \begin{tabular}{|l|l} 
& 0.00
\end{tabular} & 00 & 01 & 0.00 & 0.00 & 00 & 0.00 & 05 & 0.01 \\
\hline & & CAN & 0.03 & 0.01 & 0.37 & 0.04 & 0.19 & 0.00 & 0.00 & 0.04 & 0.00 & 0.02 & 0.00 & 0.00 & 0.01 & 0.00 & 0.01 & 0.01 & .00 & .05 & 0.01 & 0.03 & .00 & 0.04 & 0.38 & 0.04 \\
\hline & & OSI & 0.01 & 0.00 & 0.18 & 0.02 & 0.09 & 0.00 & 0.00 & 0.02 & 0.00 & 0.01 & \begin{tabular}{|l|l|} 
& 0.00 \\
\end{tabular} & 0.00 & 0.00 & 0.00 & 0.01 & 0.00 & 0.00 & 0.02 & 0.00 & 0.02 & \begin{tabular}{|l|l|} 
\\
\end{tabular} & 0.02 & 0.19 & 0.02 \\
\hline & & $\overline{\mathrm{Al}}$ & 0.13 & 0.0 & 1. & 0.19 & 0.93 & 0.02 & 0. & 0 & 0. & 0. & $\begin{array}{ll} \\
\end{array}$ & 0. & 0 & 0 & 76 & 0.04 & 0.01 & 0.25 & 0.05 & 0.16 & 01 & 0.20 & 1.92 & 22 \\
\hline & & WLG & 0.02 & 0.0 & 0.3 & 0.0 & 0 & 0.00 & 0. & 0.0 & 0.00 & 0.01 & 0. & .00 & 0.0 & 0.00 & 0.01 & 0.01 & 00 & 04 & 0.01 & 0. & .00 & .03 & .31 & 0.03 \\
\hline & METL & ONI & 0 & 0.03 & 1.4 & 0.1 & 0.7 & 0 & 0.0 & 0.1 & 0, & 0.07 & 0.0 & $0.0 \mathrm{~s}$ & 0.0 & 0.00 & 0.05 & 0.03 & .00 & 20 & 0.04 & 0.1 & .01 & 0.16 & 1.55 & 1.18 \\
\hline & & CAN & 0.05 & 0.01 & 0.64 & 0.07 & 0.32 & 0.01 & 0.00 & 0.08 & 0.01 & 0.03 & 0.00 & 0.00 & 0.01 & 0.00 & 0.02 & 0.01 & 0.00 & 0.09 & 0.02 & 0.05 & \begin{tabular}{|l|l|} 
\\
\end{tabular} & 0.07 & 0.67 & 0.08 \\
\hline & & OSI & 0.04 & 0.01 & 0.53 & 0.05 & 0.27 & 0.01 & 0.00 & 0.06 & 0.00 & 0.02 & \begin{tabular}{|l|l|} 
\\
\end{tabular} & 0.00 & 0.01 & 0.00 & 0.02 & 0.01 & 0.00 & 0.07 & 0.01 & 0.04 & 0.00 & 0.06 & 0.55 & 0.06 \\
\hline & & & 1. & 0.35 & 20.65 & 2.12 & 10.42 & 0.21 & 0.07 & 2.4 & 0.16 & 0.95 & \begin{tabular}{|l|l|}
0.12 \\
\end{tabular} & 0.02 & 0.39 & 03 & 67 & 0.44 & 0.06 & 78 & 0.5 & 1. & 14 & .23 & 21.46 & 43 \\
\hline & & WLG & & 0.0 & & 0. & 1. & 0 & 0 & & 0.03 & 0.17 & 0. & 0.00 & 0. & 0.01 & 12 & 0.08 & 0.01 & 49 & 0.09 & 31 & 02 & 39 & .77 & 43 \\
\hline & EQFO & ONI & \begin{tabular}{|l|l}
0.79 \\
\end{tabular} & 0.19 & 10.94 & 1.12 & 5.52 & 0.11 & 0.0 & 1.31 & 0.09 & 0.50 & \begin{tabular}{|l|l|} 
& 0.06
\end{tabular} & 0.01 & 0. & 0.02 & .35 & .23 & 0.03 & 47 & 0.28 & 0.92 & \begin{tabular}{|l|l|} 
& 0.07
\end{tabular} & 1.18 & 11.37 & 1.29 \\
\hline & & CAN & \begin{tabular}{|l|l}
0.45 \\
\end{tabular} & 0.11 & 6.30 & 0.65 & 3.18 & 0.07 & 0.02 & 0.75 & 0.05 & 0.29 & \begin{tabular}{|l|l|} 
& 0.04
\end{tabular} & 0.01 & 0.12 & 0.01 & 0.20 & 0.13 & 0.02 & .85 & 0.16 & 0.53 & \begin{tabular}{|l|l|} 
& 0.04
\end{tabular} & 0.68 & 6.54 & 0.74 \\
\hline & & OSI & 0.28 & 0.0 & 3.8 & 0.40 & 1.5 & 0.04 & 0.0 & 0. & 0.0 & 0.18 & 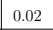 & 0.00 & 0.07 & 0.01 & 0.13 & 0.08 & .01 & .52 & 0.10 & 0.33 & .03 & 0.42 & 4.05 & .46 \\
\hline & & & & 0. & 1. & 0 . & 0. & & 0. & & 0 & 0 . & 0 & & 0. & 00 & 0 & 02 & 0.00 & 16 & 0.03 & 0.10 & 01 & 13 & 1.21 & 14 \\
\hline & & WLG & 0.06 & 0.01 & 0. & $0 . c$ & 0.4 & 0.01 & 0.0 & 0.10 & 01 & 0.04 & 0.00 & 0.00 & 0.02 & 0.00 & .03 & 0.02 & .00 & 11 & 0.02 & 0.07 & \begin{tabular}{|l|l} 
& 0.01
\end{tabular} & 0.09 & 0.85 & 0.10 \\
\hline & UTIL & ONI & 0.14 & 0.03 & 1.88 & 0.19 & 0.95 & 0.02 & 0.01 & 0.22 & 0.01 & 0.09 & 0.01 & 0.00 & 0.04 & 0.00 & 0.06 & 0.04 & 0.01 & 25 & 0.05 & 0.16 & \begin{tabular}{|l|} 
\\
\end{tabular} & 0.20 & 1.95 & 0.22 \\
\hline & & CAN & 0.02 & 0.0 & 0.3 & 0.0 & 0.16 & 0.00 & 0.00 & 0.04 & 0.00 & 01 & 00 & 0.00 & 0.01 & 0.00 & 0.01 & .01 & 00 & 04 & 0.01 & 0.03 & 00 & 0.03 & 33 & .04 \\
\hline & & OSI & 0. & 0.01 & & 0. & 0. & 0.01 & 0. & 0.07 & .00 & 0.03 & $\begin{array}{l}0.00 \\
\end{array}$ & 00 & 0.01 & .00 & 0.02 & 0.01 & 00 & 07 & 0.01 & 0.05 & 00 & .06 & .57 & .06 \\
\hline & & $\mathrm{Al}$ & \begin{tabular}{|l|l|}
6.56 \\
\end{tabular} & 1.55 & 9 & 9.3 & 4 & 0. & 0. & 10.85 & 71 & $\begin{array}{l}4.17 \\
\end{array}$ & \begin{tabular}{|l|l}
0.51 \\
\end{tabular} & 0.10 & 74 & 0.14 & 93 & \begin{tabular}{|l|l}
1.93 \\
\end{tabular} & 25 & 12.24 & 2.36 & 7.67 & 62 & 9.82 & $\begin{array}{l}94.46 \\
\end{array}$ & 10.72 \\
\hline & & WLG & 2.09 & 0.49 & 28.88 & 2.97 & 14.57 & 0.30 & 0.10 & 3.45 & 0.23 & 1.32 & 0.16 & 0.03 & 0.55 & 0.04 & 0.93 & 0.61 & 0.08 & 3.89 & 0.75 & 2.44 & 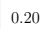 & 3.12 & 30.02 & 3.41 \\
\hline & CONS & ONI & 7.44 & 1.76 & 103.09 & 10.59 & 2 & 1.0 & 0.3 & 12.31 & 0.81 & 4.73 & 0.58 & 0. & 1. & 0.16 & 3.32 & 2.19 & 0.28 & 3.88 & 2.67 & 8.70 & .70 & 11.14 & 107.16 & 2.16 \\
\hline & & CAN & 2.3 & 0.5 & 32 & 3.2 & 16. & 0.33 & 0.1 & 3.8 & 0.25 & 1. & \begin{tabular}{|l|l|} 
& 0.18
\end{tabular} & 0.04 & 0.61 & 0.05 & 1.03 & 0.68 & 0.09 & 4.32 & .83 & .71 & 22 & 46 & 32 & .78 \\
\hline & & OSI & 2.93 & 0.69 & 40.55 & 4.16 & 20.46 & 0.42 & 0.14 & 4.84 & 0.32 & 1.86 & \begin{tabular}{|l|l|}
0.23 \\
\end{tabular} & 0.05 & 0.77 & 0.06 & 31 & .86 & 0.11 & 5.46 & 1.05 & 3.42 & 28 & 4.38 & 42.15 & 4.78 \\
\hline
\end{tabular}

Extended MRIO Table - Part 1-6 


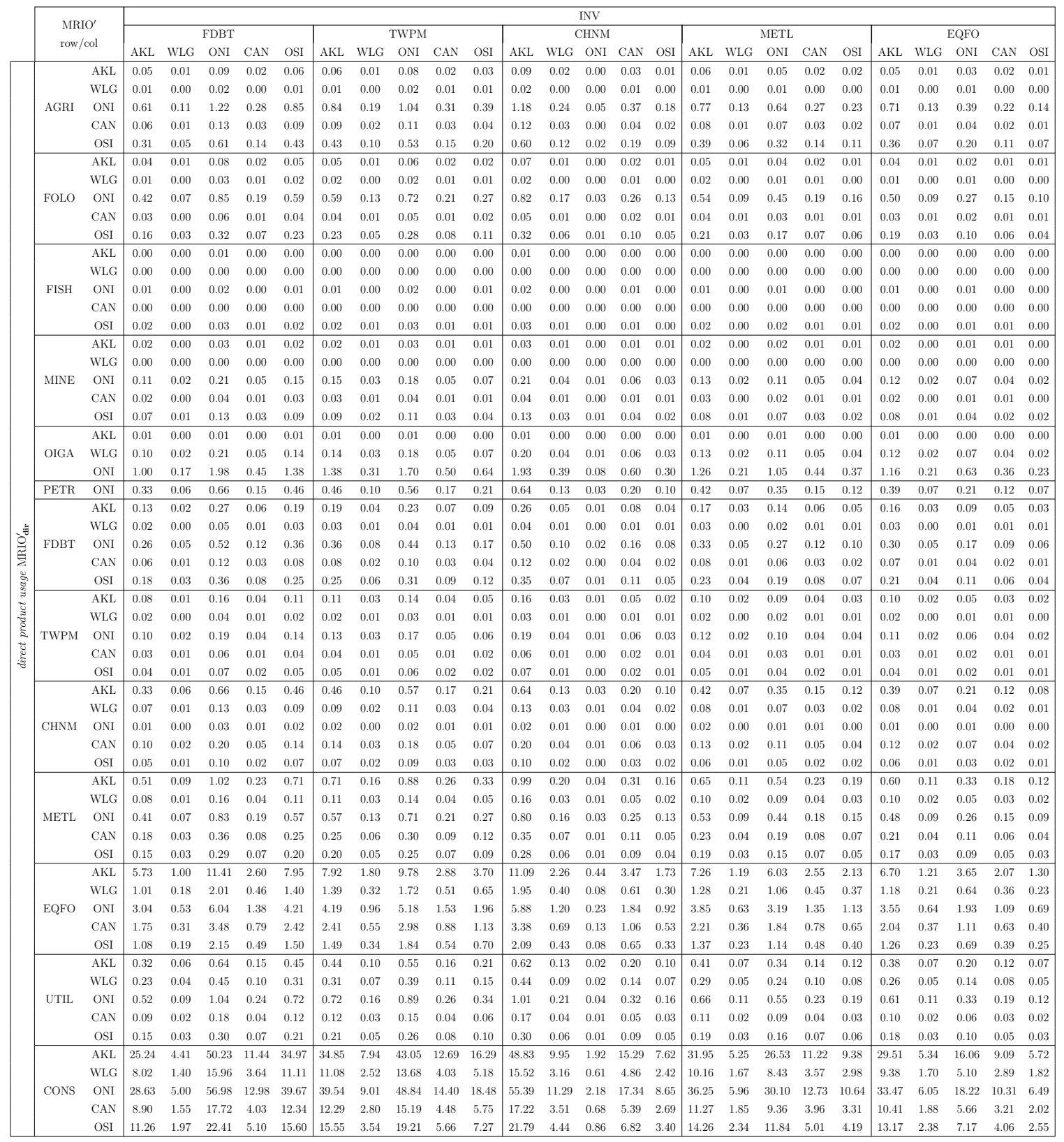

Extended MRIO Table — Part 1-7 


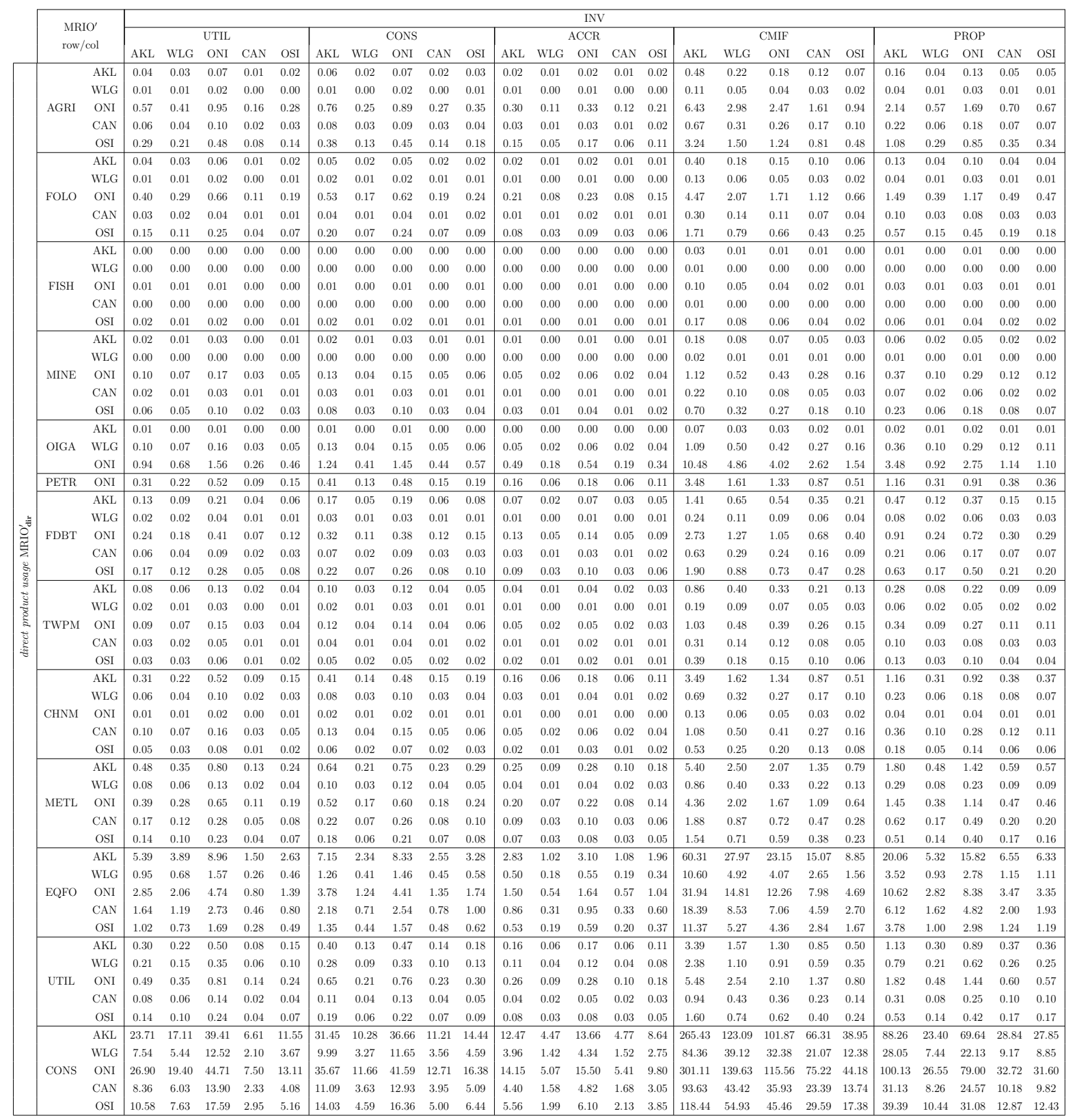

Extended MRIO Table - Part 1-8 


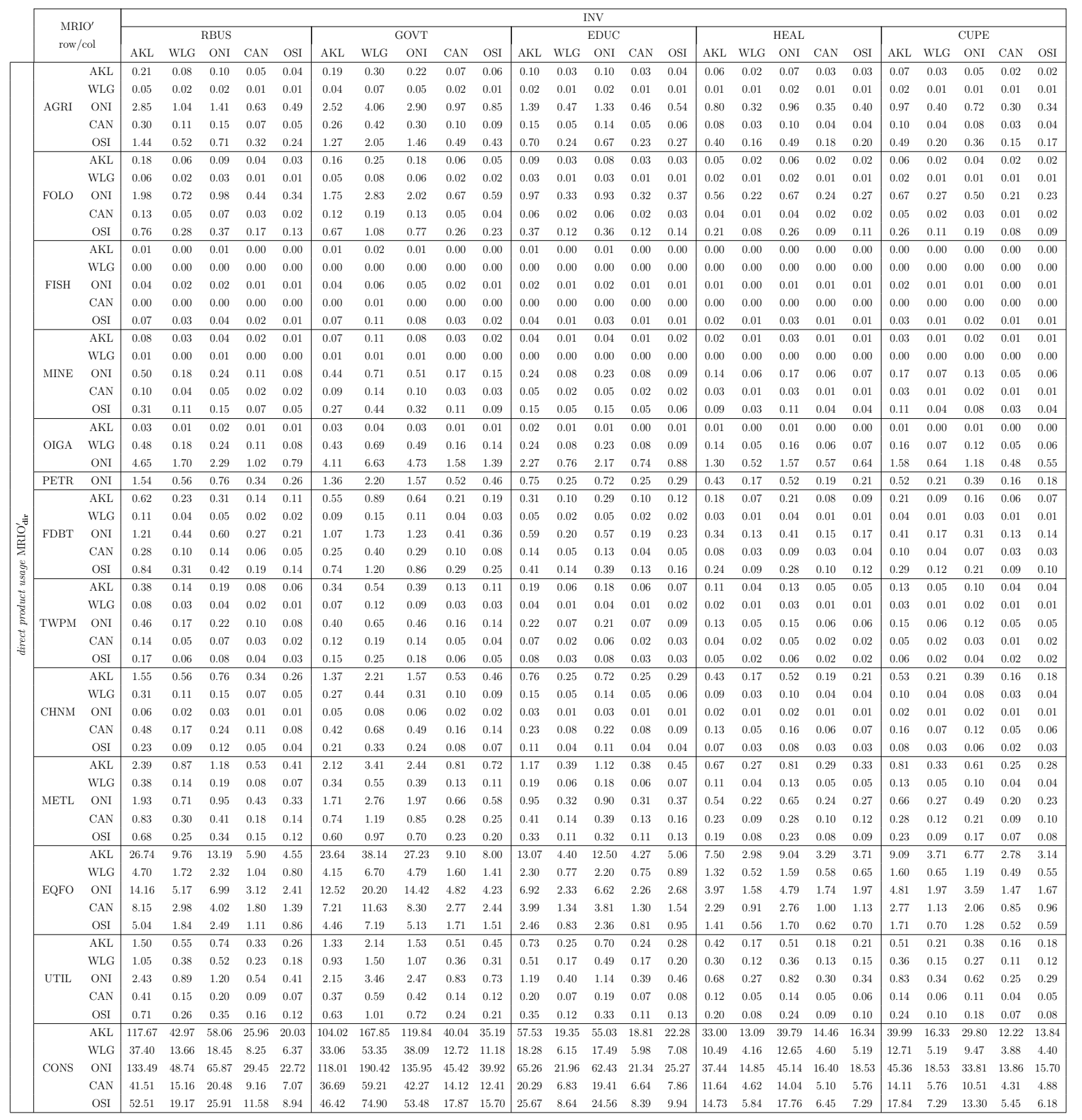

Extended MRIO Table - Part 1-9 


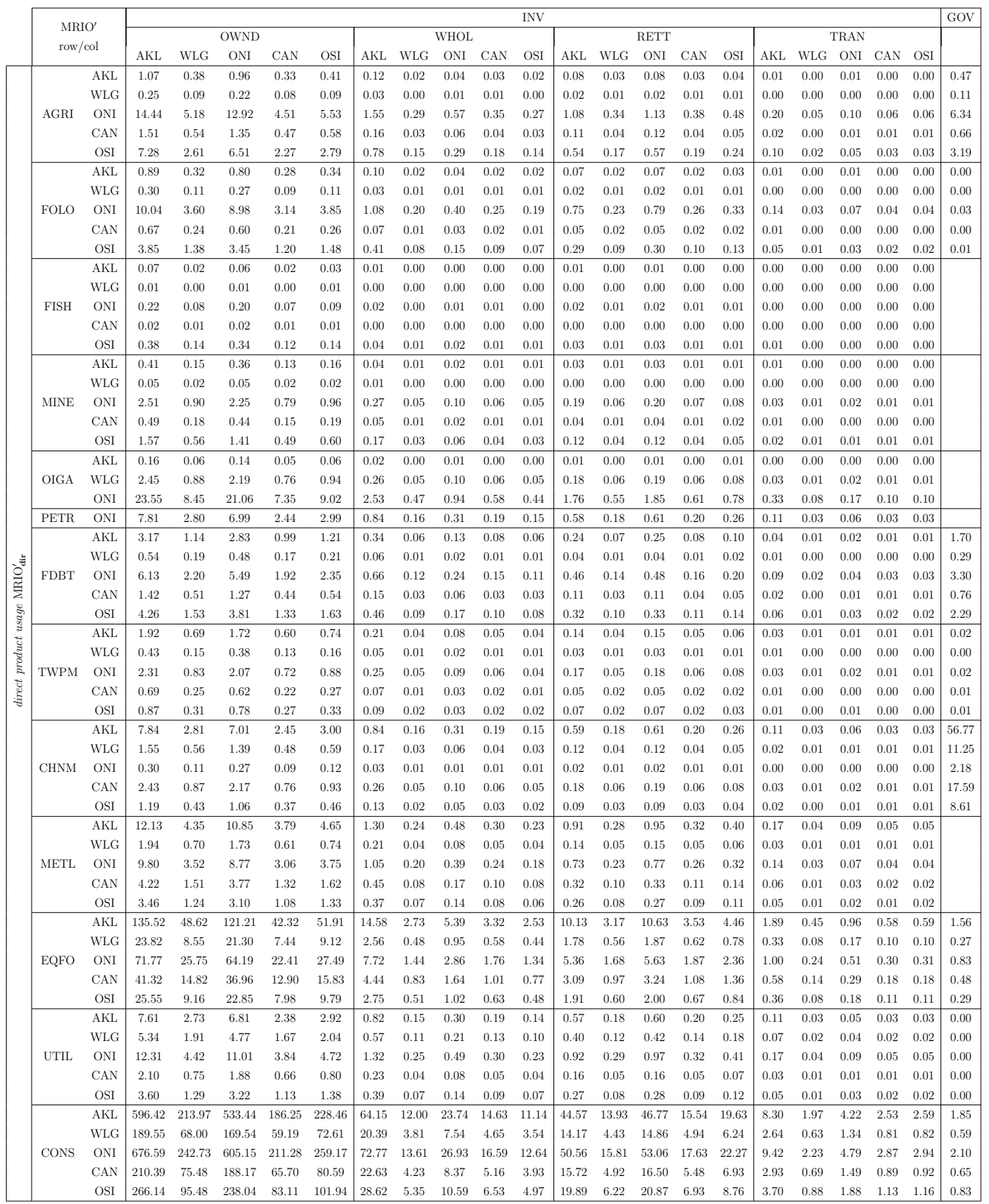

Extended MRIO Table — Part 1-10 


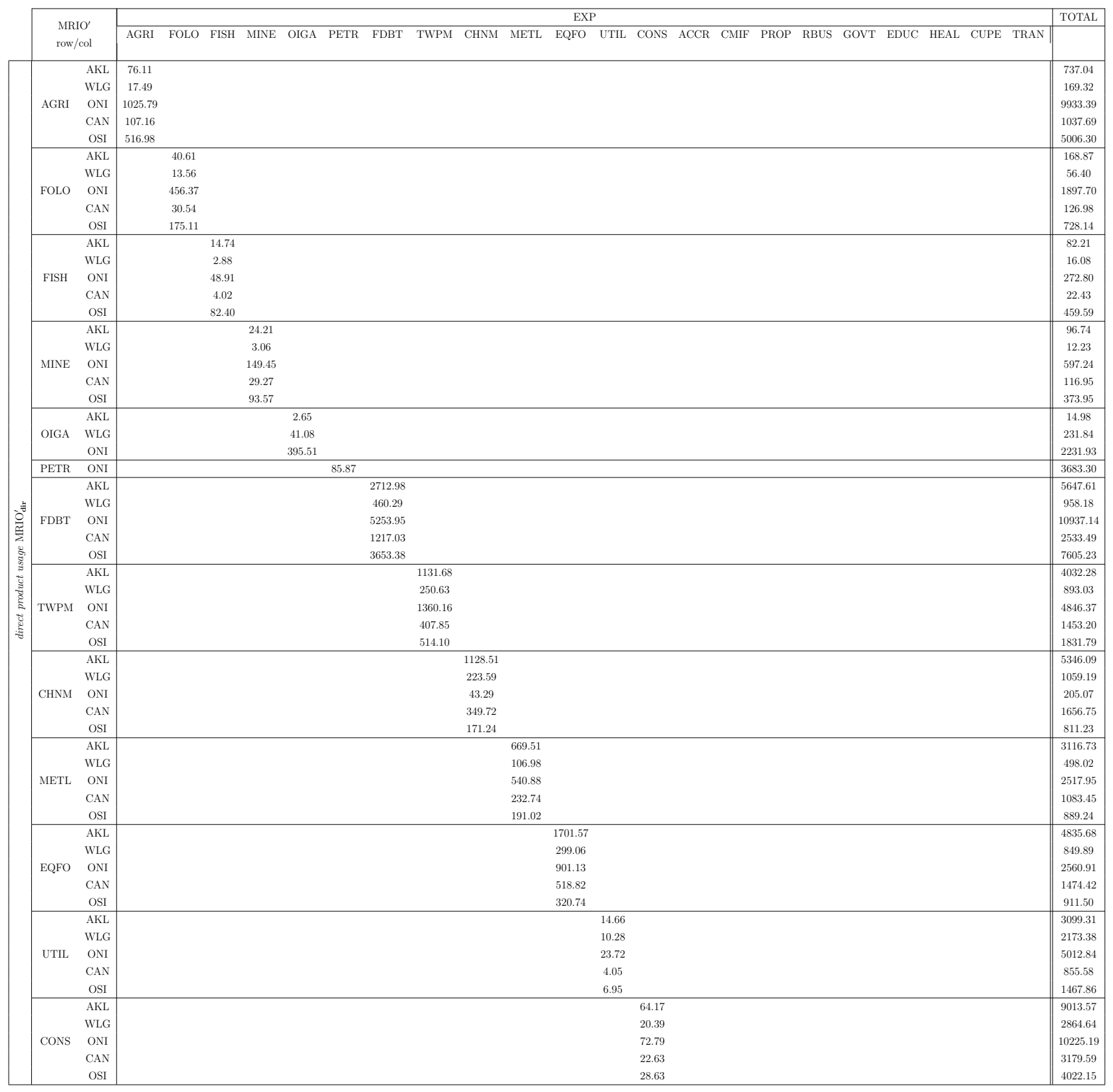

Extended MRIO Table - Part 1-11 


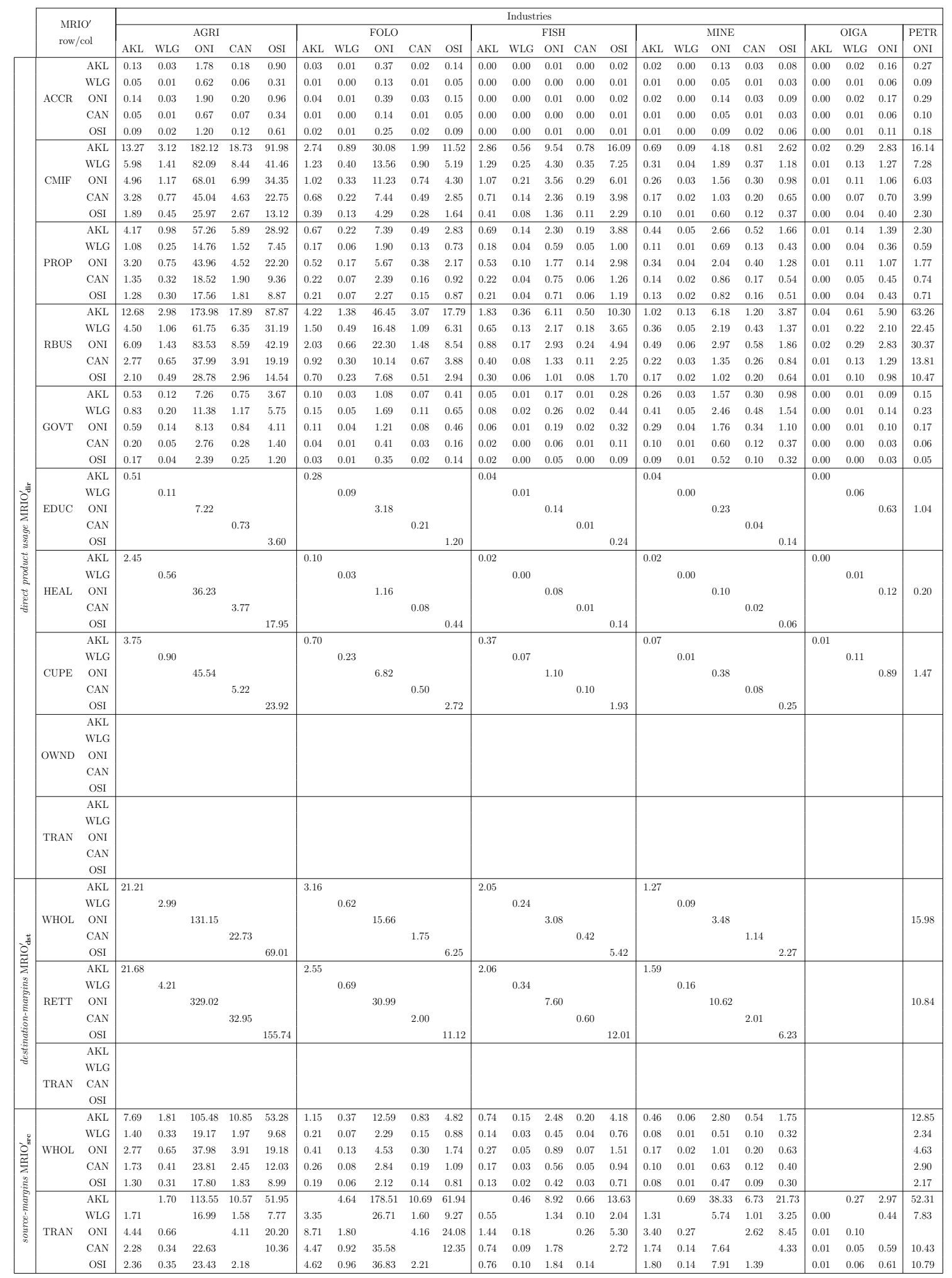

Extended MRIO Table - Part 2-1 


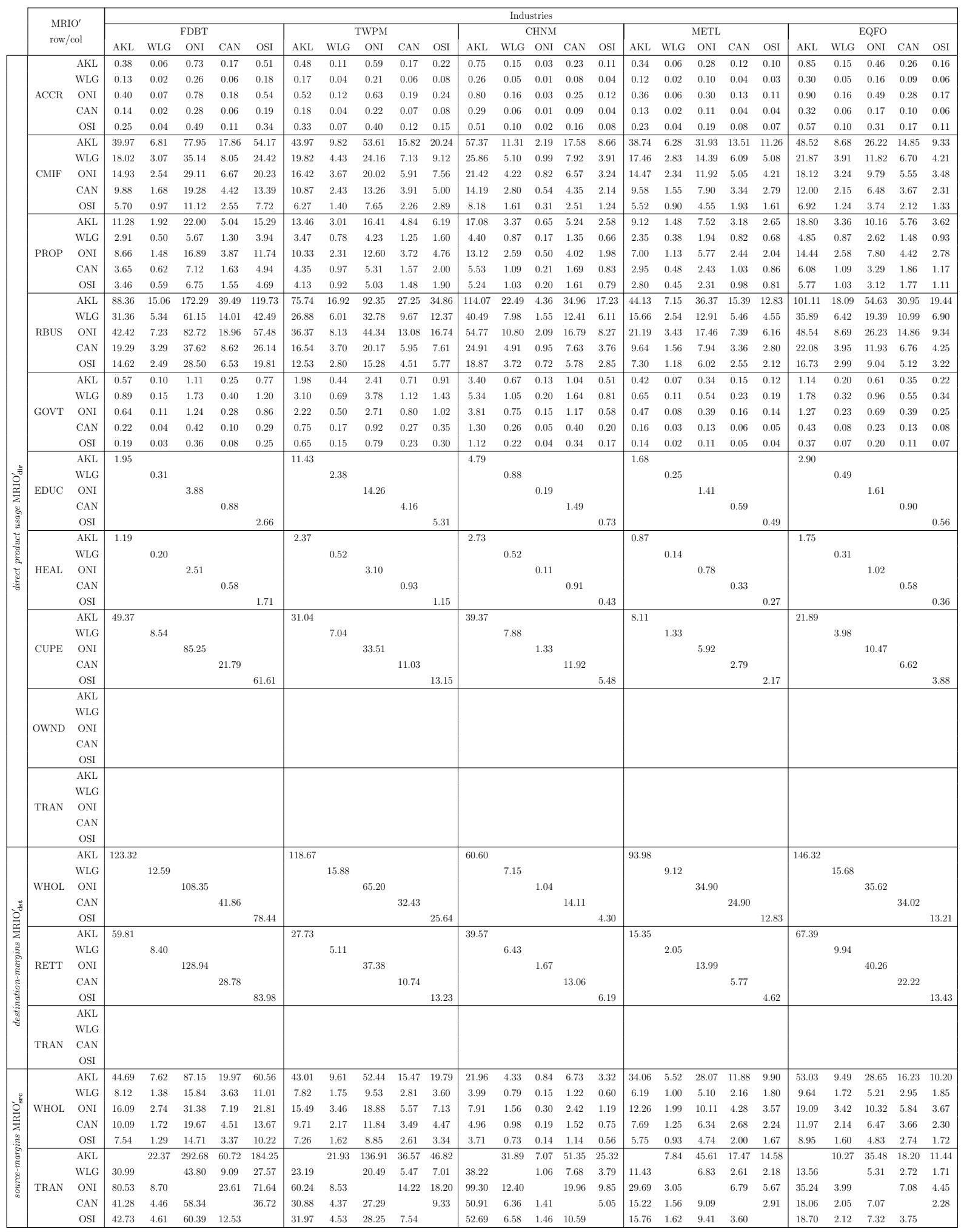

Extended MRIO Table - Part 2-2 


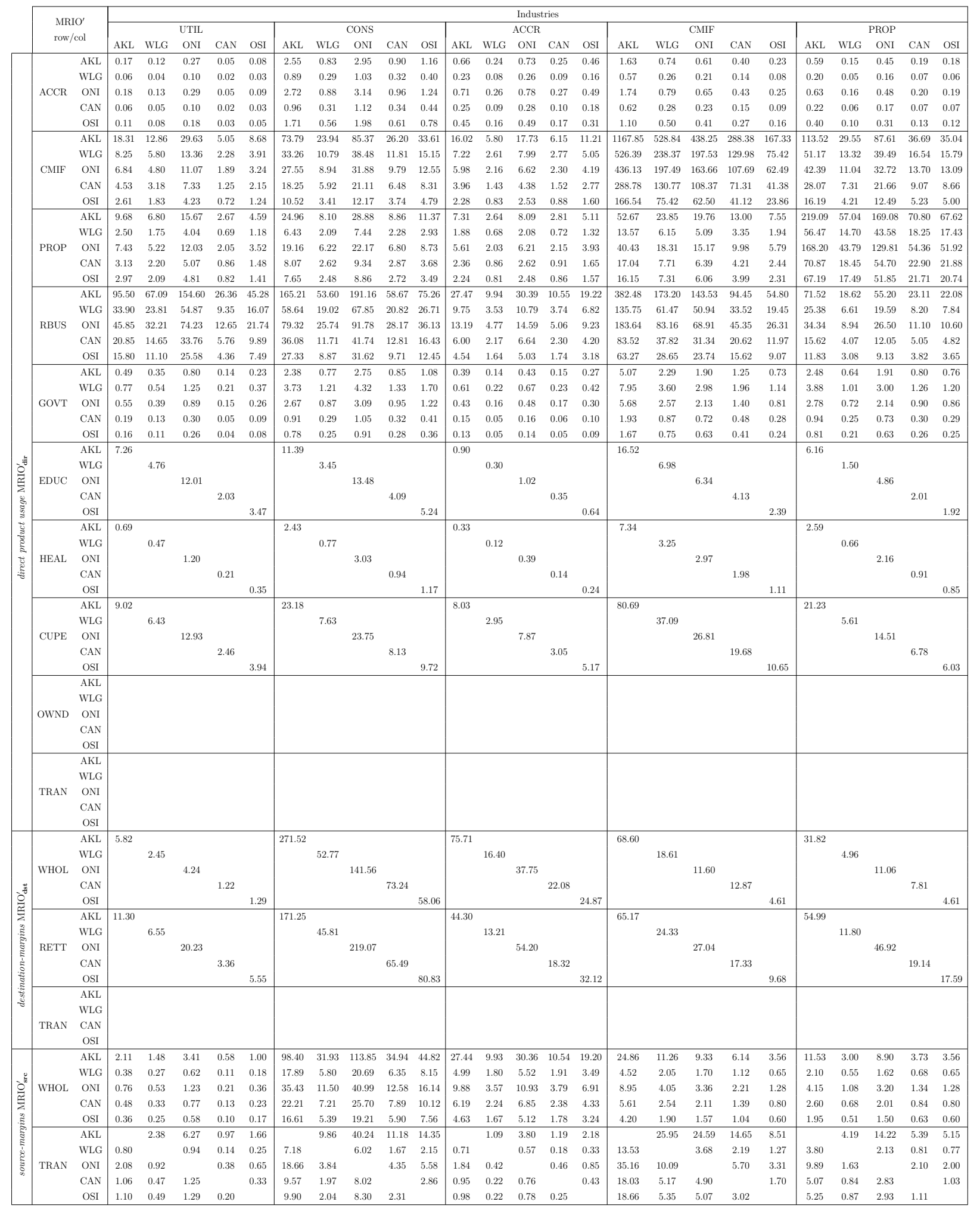

Extended MRIO Table - Part 2-3 


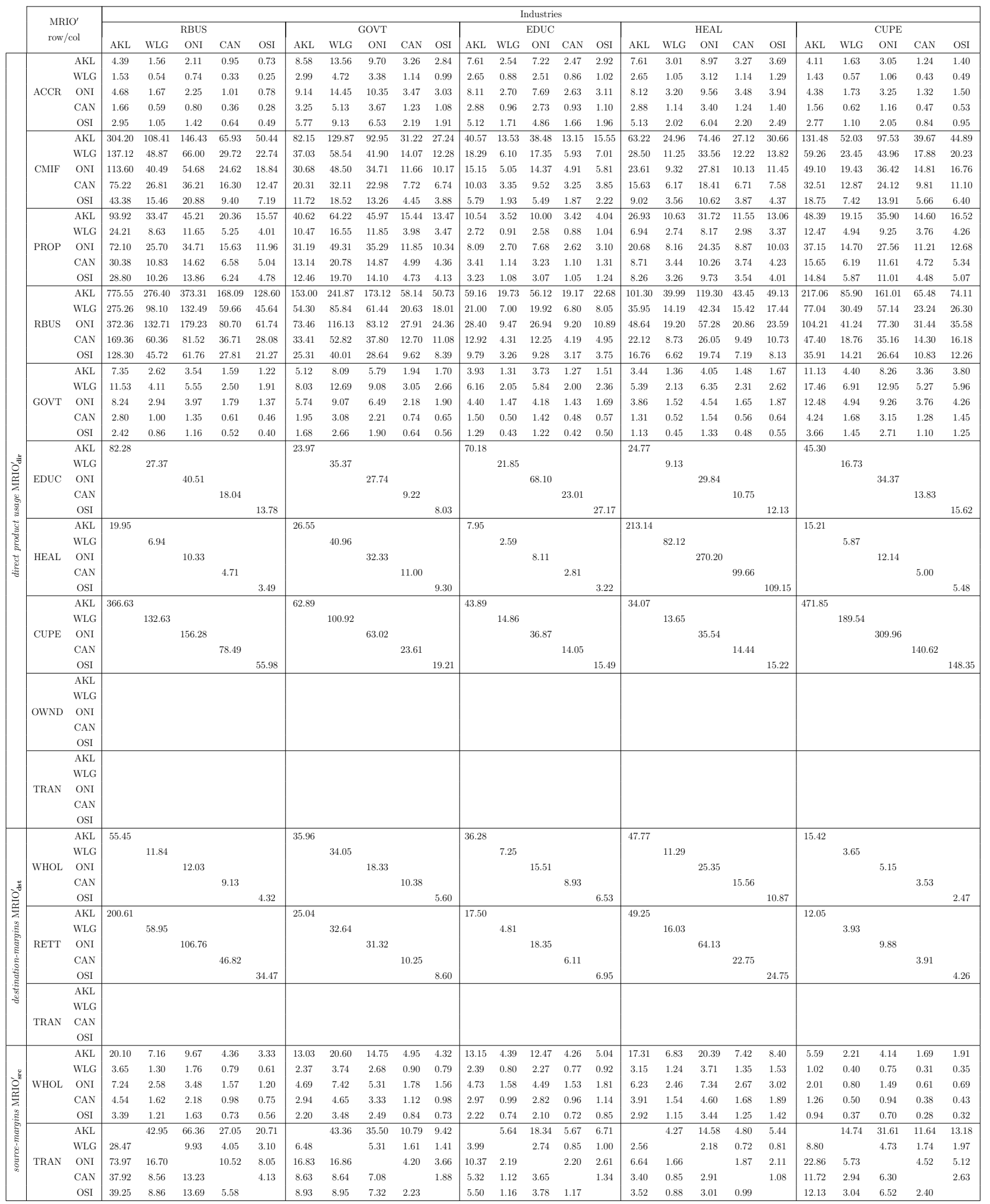

Extended MRIO Table - Part 2-4 


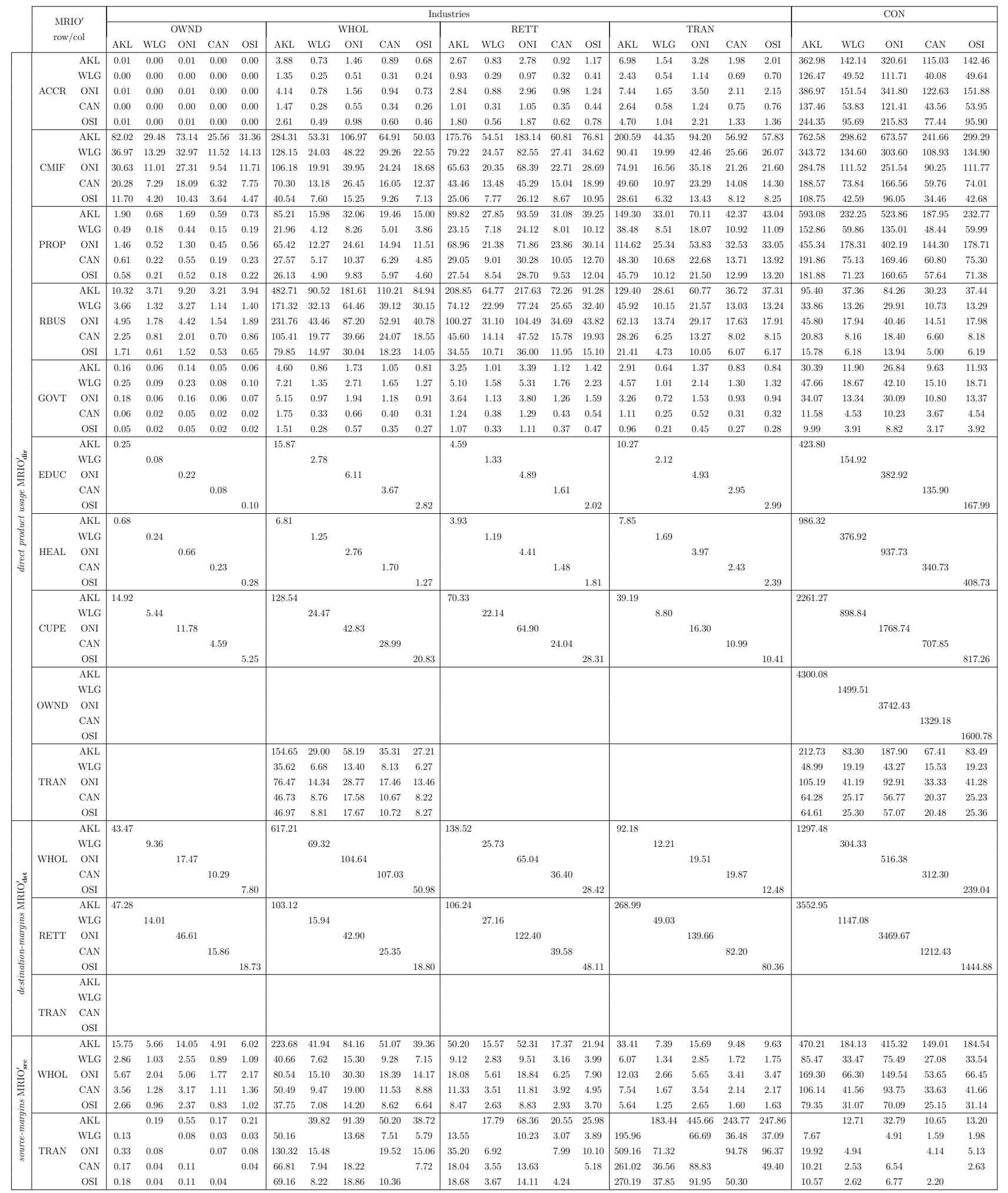

Extended MRIO Table - Part 2-5 


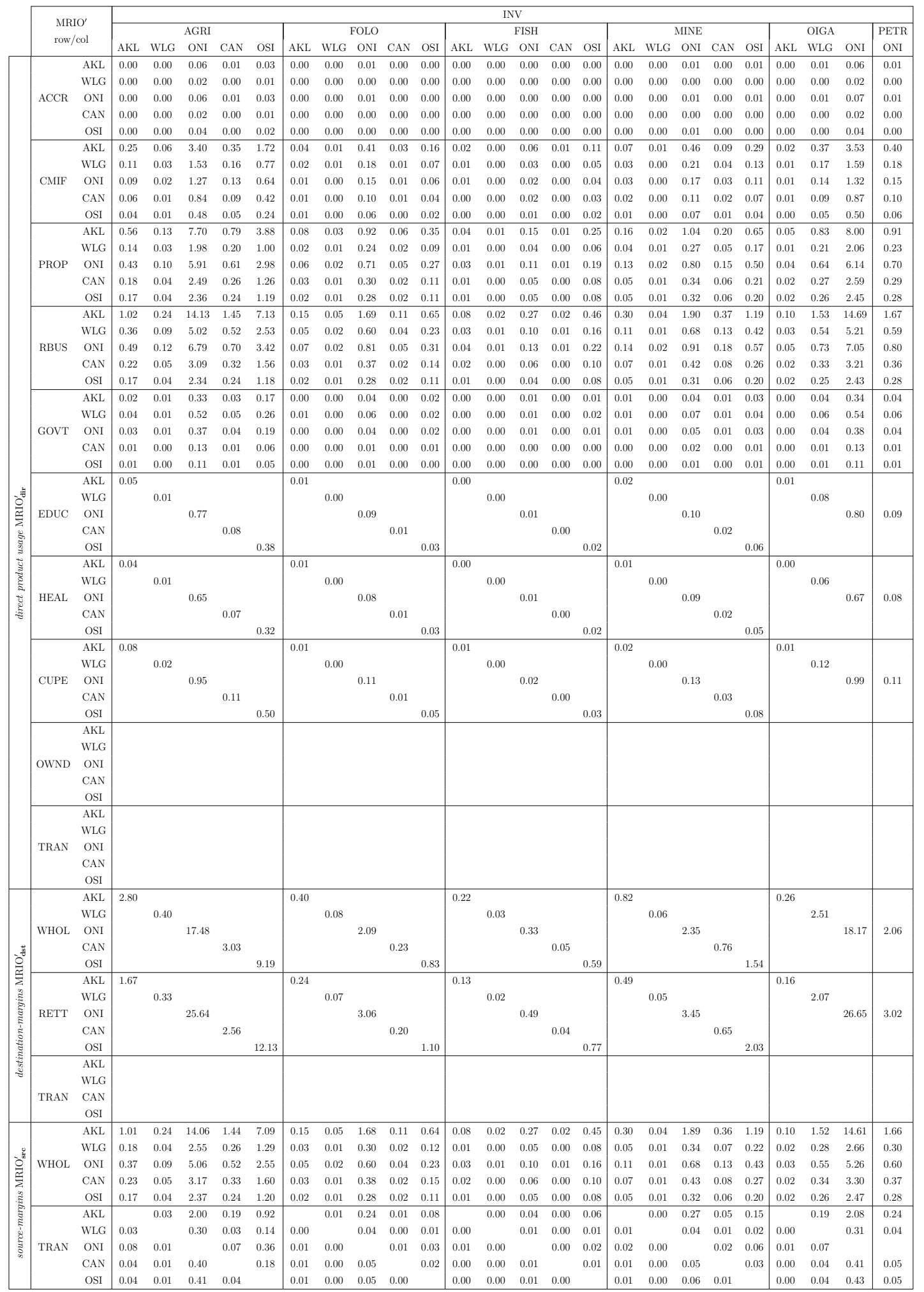

Extended MRIO Table - Part 2-6 


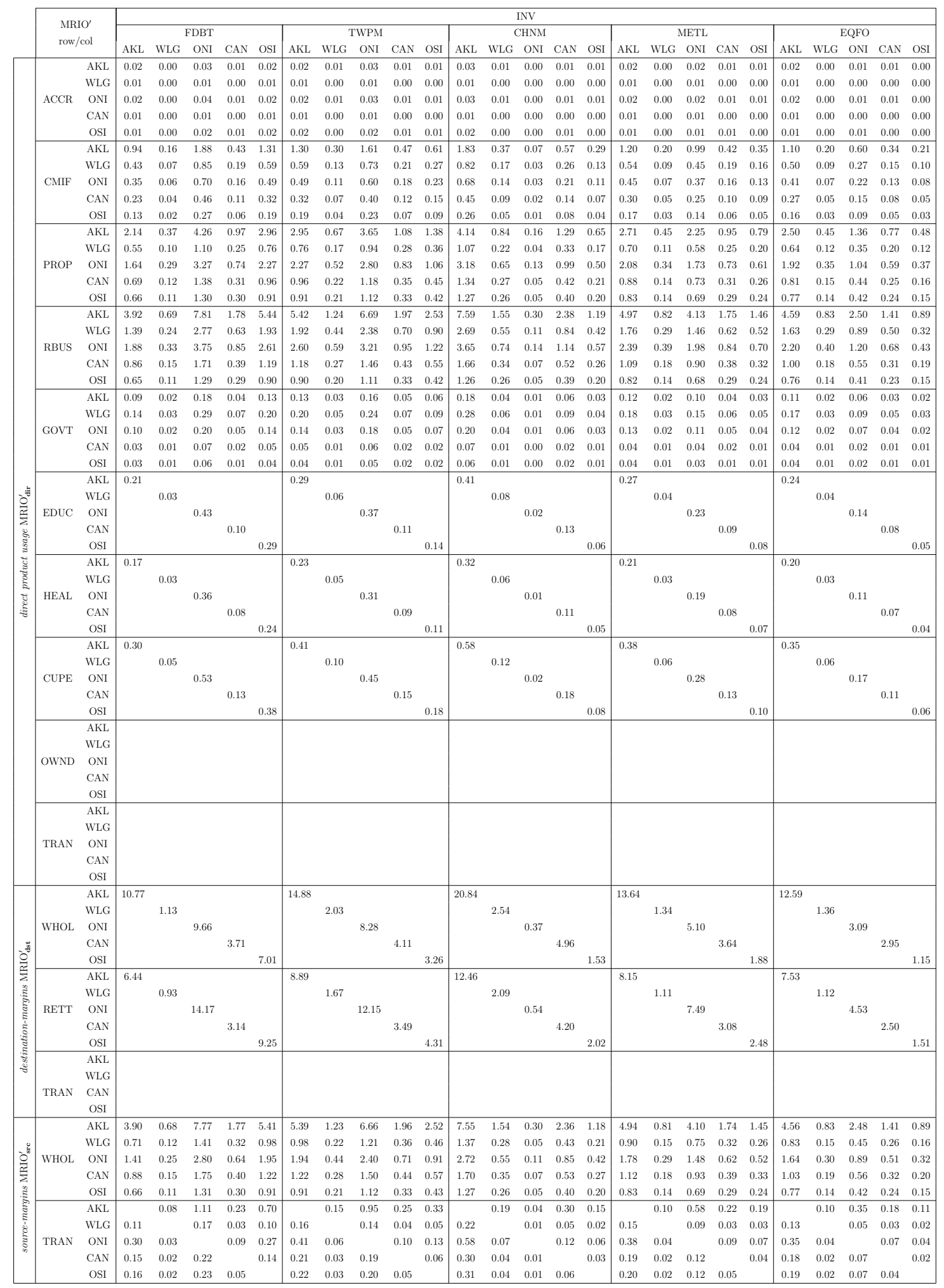

Extended MRIO Table - Part 2-7 


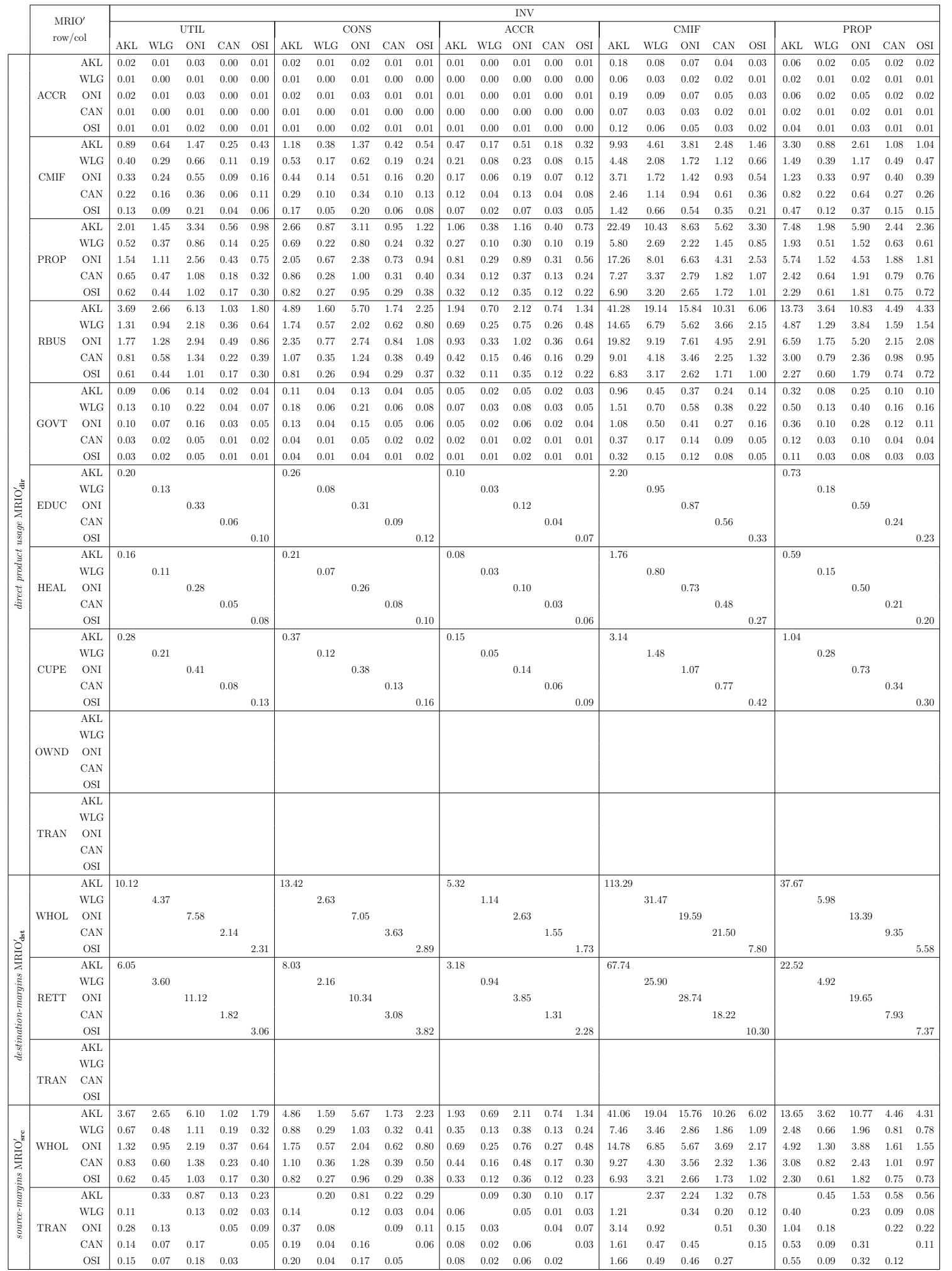

Extended MRIO Table - Part 2-8 


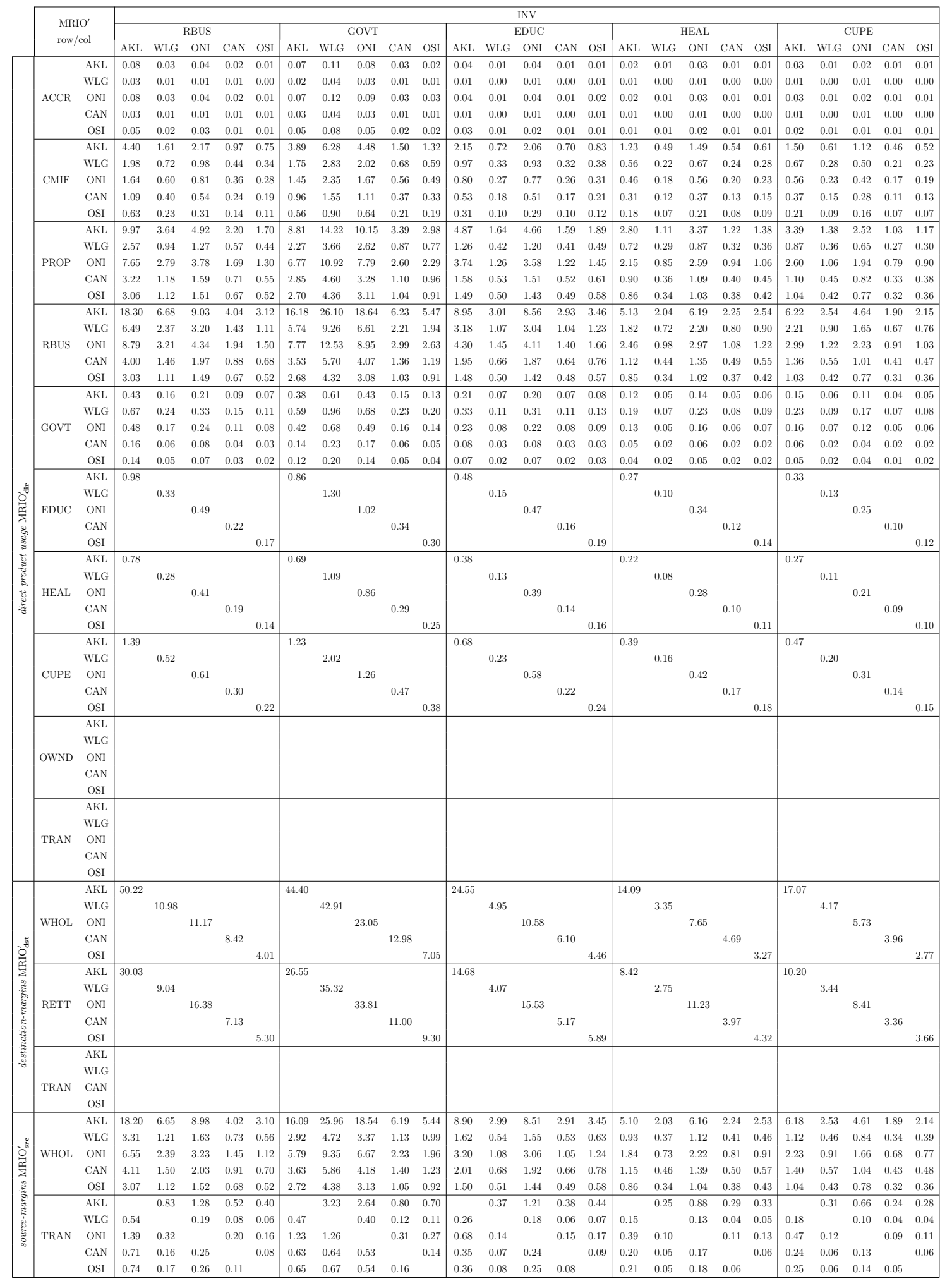

Extended MRIO Table - Part 2-9 


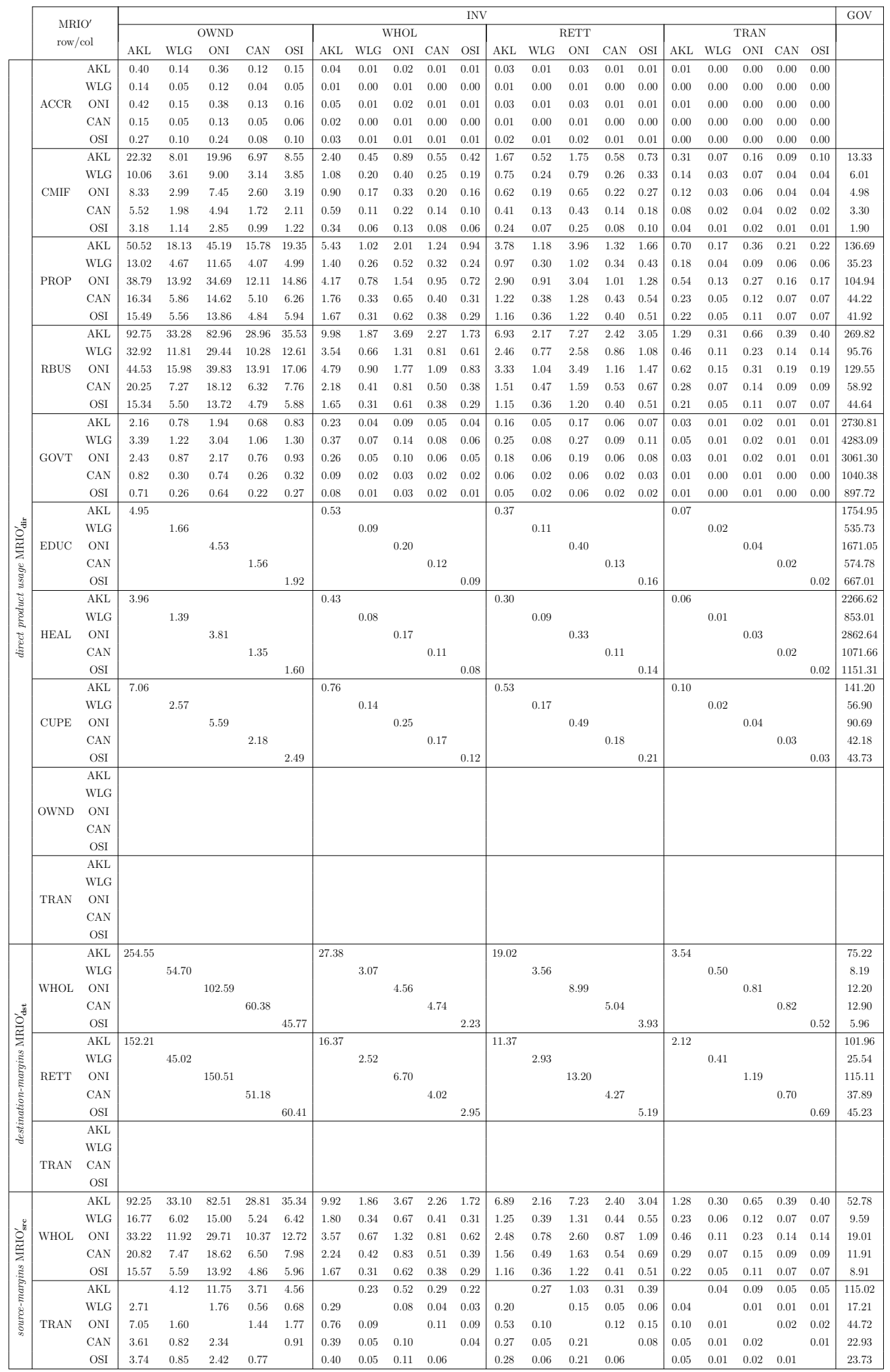

Extended MRIO Table - Part 2-10 


\begin{tabular}{|c|c|c|c|c|c|c|c|c|c|c|c|c|c|c|c|c|c|c|c|c|c|c|}
\hline & & & & & & & & & & & & & & $\mathrm{XP}$ & & & & & & & & \\
\hline & $\begin{array}{l}\text { MRI } \\
\text { row/s }\end{array}$ & & \begin{tabular}{|l|l|} 
AGRI \\
\end{tabular} & FOLO & FISH & MINE & OIGA & PETR & FDBT & TWPM & CHNM & METL & EQFO & UTIL & CONS & $\mathrm{ACCR}$ & CMIF & PROP & RBUS & GOVT & EDUC & HEAL \\
\hline & & AKL & & & & & & & & & & & & & & 606.50 & & & & & & \\
\hline & & WLG & & & & & & & & & & & & & & 211.31 & & & & & & \\
\hline & $\mathrm{ACCR}$ & ONI & & & & & & & & & & & & & & 646.58 & & & & & & \\
\hline & & CAN & & & & & & & & & & & & & & 229.67 & & & & & & \\
\hline & & OSI & & & & & & & & & & & & & & 408.29 & & & & & & \\
\hline & & AKL & & & & & & & & & & & & & & & 493.74 & & & & & \\
\hline & & WLG & & & & & & & & & & & & & & & 222.55 & & & & & \\
\hline & CMIF & ONI & & & & & & & & & & & & & & & 184.38 & & & & & \\
\hline & & CAN & & & & & & & & & & & & & & & 122.09 & & & & & \\
\hline & & OSI & & & & & & & & & & & & & & & 70.41 & & & & & \\
\hline & & AKL & & & & & & & & & & & & & & & & 200.58 & & & & \\
\hline & & WLG & & & & & & & & & & & & & & & & 51.70 & & & & \\
\hline & PROP & ONI & & & & & & & & & & & & & & & & 154.00 & & & & \\
\hline & & CAN & & & & & & & & & & & & & & & & 64.89 & & & & \\
\hline & & OSI & & & & & & & & & & & & & & & & 61.51 & & & & \\
\hline & & AKL & & & & & & & & & & & & & & & & & 501.79 & & & \\
\hline & & WLG & & & & & & & & & & & & & & & & & 178.09 & & & \\
\hline & RBUS & ONI & & & & & & & & & & & & & & & & & 240.92 & & & \\
\hline & & CAN & & & & & & & & & & & & & & & & & 109.57 & & & \\
\hline & & OSI & & & & & & & & & & & & & & & & & 83.01 & & & \\
\hline & & AKL & & & & & & & & & & & & & & & & & & 34.25 & & \\
\hline & & WLG & & & & & & & & & & & & & & & & & & 53.71 & & \\
\hline & GOVT & ONI & & & & & & & & & & & & & & & & & & 38.39 & & \\
\hline & & CAN & & & & & & & & & & & & & & & & & & 13.05 & & \\
\hline & & OSI & & & & & & & & & & & & & & & & & & 11.26 & & \\
\hline & & AKL & & & & & & & & & & & & & & & & & & & 246.34 & \\
\hline $0^{\frac{2}{6}}$ & & WLG & & & & & & & & & & & & & & & & & & & 75.20 & \\
\hline $\overrightarrow{\tilde{a}}$ & EDUC & ONI & & & & & & & & & & & & & & & & & & & 234.56 & \\
\hline$z$ & & CAN & & & & & & & & & & & & & & & & & & & 80.68 & \\
\hline 总 & & OSI & & & & & & & & & & & & & & & & & & & 93.63 & \\
\hline 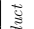 & & AKL & & & & & & & & & & & & & & & & & & & & 22.43 \\
\hline 总 & & WLG & & & & & & & & & & & & & & & & & & & & 8.44 \\
\hline E & HEAL & ONI & & & & & & & & & & & & & & & & & & & & 28.32 \\
\hline 复 & & CAN & & & & & & & & & & & & & & & & & & & & 10.60 \\
\hline & & OSI & & & & & & & & & & & & & & & & & & & & 11.39 \\
\hline & & AKL & & & & & & & & & & & & & & & & & & & & \\
\hline & & WLG & & & & & & & & & & & & & & & & & & & & \\
\hline & CUPE & ONI & & & & & & & & & & & & & & & & & & & & \\
\hline & & CAN & & & & & & & & & & & & & & & & & & & & \\
\hline & & OSI & & & & & & & & & & & & & & & & & & & & \\
\hline & & AKL & & & & & & & & & & & & & & & & & & & & \\
\hline & & WLG & & & & & & & & & & & & & & & & & & & & \\
\hline & OWND & ONI & & & & & & & & & & & & & & & & & & & & \\
\hline & & CAN & & & & & & & & & & & & & & & & & & & & \\
\hline & & OSI & & & & & & & & & & & & & & & & & & & & \\
\hline & & AKL & & & & & & & & & & & & & & & & & & & & \\
\hline & & WLG & & & & & & & & & & & & & & & & & & & & \\
\hline & TRAN & ONI & & & & & & & & & & & & & & & & & & & & \\
\hline & & CAN & & & & & & & & & & & & & & & & & & & & \\
\hline & & OSI & & & & & & & & & & & & & & & & & & & & \\
\hline & & AKL & \begin{tabular}{|l|}
69.22 \\
\end{tabular} & 28.31 & 6.06 & 11.85 & 17.40 & 6.82 & 524.53 & 144.93 & $\begin{array}{l}75.92 \\
\end{array}$ & $\begin{array}{ll}68.87 \\
\end{array}$ & 147.94 & & & & & & & & & \\
\hline & & WLG & 7.54 & 3.08 & 0.66 & 1.29 & 1.89 & 0.74 & 57.11 & 15.78 & 8.27 & 7.50 & 16.11 & & & & & & & & & \\
\hline & WHOL & ONI & 11.23 & 4.59 & 0.98 & 1.92 & 2.82 & 0.55 & 85.10 & 23.51 & 12.32 & 11.17 & 24.00 & & & & & & & & & \\
\hline & & CAN & 11.87 & 4.85 & 1.04 & 2.03 & 5.97 & 1.17 & 89.93 & 24.85 & 13.02 & 11.81 & 25.36 & & & & & & & & & \\
\hline 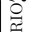 & & OSI & 5.48 & 2.24 & 0.48 & 0.94 & 2.76 & 0.54 & 41.55 & 11.48 & 6.01 & 5.46 & 11.72 & & & & & & & & & \\
\hline 光 & & AKL & \begin{tabular}{|l|}
16.08 \\
\end{tabular} & 6.58 & 1.41 & 2.75 & & & $\begin{array}{l}121.89 \\
\end{array}$ & 33.68 & 17.64 & 16.00 & 34.38 & 0.55 & 1.92 & 19.39 & 10.08 & 4.91 & $\begin{array}{l}10.26 \\
\end{array}$ & 1.38 & 6.71 & 0.75 \\
\hline 韵 & & WLG & 4.03 & 1.65 & 0.35 & 0.69 & & & 30.54 & 8.44 & 4.42 & 4.01 & 8.61 & 0.14 & 0.48 & 4.86 & 2.52 & 1.23 & 2.57 & 0.35 & 1.68 & 0.19 \\
\hline है & RETT & ONI & 18.16 & 7.43 & 1.59 & 3.11 & & & 137.60 & 38.02 & 19.92 & 18.07 & 38.81 & 0.62 & 2.17 & 21.89 & 11.38 & 5.55 & 11.58 & 1.56 & 7.57 & 0.84 \\
\hline 咅 & & CAN & 5.98 & 2.44 & 0.52 & 1.02 & & & 45.29 & 12.51 & 6.56 & 5.95 & 12.77 & 0.20 & 0.72 & 7.20 & 3.74 & 1.83 & 3.81 & 0.51 & 2.49 & 0.28 \\
\hline 焉 & & OSI & 7.13 & 2.92 & 0.62 & 1.22 & & & 54.06 & 14.94 & 7.83 & 7.10 & 15.25 & 0.24 & 0.85 & 8.60 & 4.47 & 2.18 & 4.55 & 0.61 & 2.97 & 0.33 \\
\hline 䔅 & & AKL & & & & & & 2.41 & & & & & & & & & & & & & & \\
\hline$\theta$ & & WLG & & & & & & 0.55 & & & & & & & & & & & & & & \\
\hline & TRAN & CAN & & & & & 3.70 & 0.73 & & & & & & & & & & & & & & \\
\hline & & OSI & & & & & 3.72 & 0.73 & & & & & & & & & & & & & & \\
\hline & & AKL & \begin{tabular}{|l|l|}
48.57 \\
\end{tabular} & 19.87 & 4.25 & 8.32 & 12.21 & & 368.08 & $\begin{array}{l}101.70 \\
\end{array}$ & 53.28 & $\begin{array}{l}48.32 \\
\end{array}$ & 103.81 & & & & & & & & & \\
\hline & & WLG & 8.83 & 3.61 & 0.77 & 1.51 & 2.22 & & 66.90 & 18.48 & 9.68 & 8.78 & 18.87 & & & & & & & & & \\
\hline $0^{\pi}$ & WHOL & ONI & 17.49 & 7.15 & 1.53 & 3.00 & 4.40 & 0.86 & 132.53 & 36.62 & 19.18 & 17.40 & 37.38 & & & & & & & & & \\
\hline 妾 & & CAN & 10.96 & 4.48 & 0.96 & 1.88 & & & 83.08 & 22.96 & 12.03 & 10.91 & 23.43 & & & & & & & & & \\
\hline 实 & & OSI & 8.20 & 3.35 & 0.72 & 1.40 & & & 62.11 & 17.16 & 8.99 & 8.15 & 17.52 & & & & & & & & & \\
\hline 焉 & & AKL & \begin{tabular}{|l|l|}
59.75 \\
\end{tabular} & 24.43 & 5.23 & 10.23 & $\begin{array}{l}15.02 \\
\end{array}$ & & $\begin{array}{l}452.73 \\
\end{array}$ & $\begin{array}{l}125.09 \\
\end{array}$ & $\begin{array}{l}65.53 \\
\end{array}$ & $\begin{array}{l}59.44 \\
\end{array}$ & 127.69 & & & & & & & & & \\
\hline 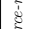 & & WLG & 8.94 & 3.66 & 0.78 & 1.53 & 2.25 & & 67.75 & 18.72 & 9.81 & 8.89 & 19.11 & & & & & & & & & \\
\hline 部 & TRAN & ONI & 23.23 & 9.50 & 2.03 & 3.98 & 5.84 & 1.14 & 176.03 & 48.64 & 25.48 & 23.11 & 49.65 & & & & & & & & & \\
\hline & & CAN & 11.91 & 4.87 & 1.04 & 2.04 & & & 90.24 & 24.93 & 13.06 & 11.85 & 25.45 & & & & & & & & & \\
\hline & & OSI & 12.33 & 5.04 & 1.08 & 2.11 & & & 93.41 & 25.81 & 13.52 & 12.26 & 26.35 & & & & & & & & & \\
\hline
\end{tabular}

Extended MRIO Table - Part 2-11 


\begin{tabular}{|c|c|c|c|c|c|c|c|c|c|c|c|c|c|c|c|c|c|c|c|c|c|c|c|c|}
\hline \multirow{3}{*}{$\begin{array}{l}\text { MRIO' } \\
\text { row/col }\end{array}$} & \multicolumn{24}{|c|}{ Industries } \\
\hline & \multicolumn{5}{|c|}{ AGRI } & \multicolumn{5}{|c|}{ FOLO } & \multicolumn{5}{|c|}{ FISH } & \multicolumn{5}{|c|}{ MINE } & \multicolumn{3}{|c|}{ OIGA } & \multirow{2}{*}{\begin{tabular}{|c|} 
PETR \\
ONI
\end{tabular}} \\
\hline & AKL & WLG & ONI & CAN & OSI & AKL & WLG & ONI & CAN & OSI & AKL & WLG & ONI & $\mathrm{CAN}$ & OSI & AKL & WLG & $\mathrm{ONI}$ & CAN & OSI & AKL & WLG & $\mathrm{ONI}$ & \\
\hline IMP-AGRI & 5.45 & 1.28 & 74.81 & 7.69 & 37.78 & 0.51 & 0.17 & 5.62 & 0.37 & 2.15 & \begin{tabular}{|l|l|}
0.00 \\
\end{tabular} & 0.00 & 0.01 & 0.00 & 0.02 & 0.00 & 0.00 & 0.00 & 0.00 & 0.00 & 0.00 & 0.00 & 0.01 & \\
\hline IMP-FOLO & 0.04 & 0.01 & 0.54 & 0.06 & 0.27 & 0.50 & 0.16 & 5.49 & 0.36 & 2.10 & 0.00 & 0.00 & 0.00 & 0.00 & 0.01 & 0.00 & 0.00 & 0.00 & 0.00 & 0.00 & 0.00 & 0.00 & 0.00 & \\
\hline IMP-FISH & 0.01 & 0.00 & 0.10 & 0.01 & 0.05 & 0.01 & 0.00 & 0.06 & 0.00 & 0.02 & 1.45 & 0.29 & 4.83 & 0.39 & 8.14 & 0.00 & 0.00 & 0.01 & 0.00 & 0.01 & 0.00 & 0.00 & 0.04 & \\
\hline IMP-MINE & 0.37 & 0.09 & 5.11 & 0.53 & 2.58 & 0.01 & 0.00 & 0.13 & 0.01 & 0.05 & 0.00 & 0.00 & 0.02 & 0.00 & 0.03 & 0.27 & 0.03 & 1.63 & 0.32 & 1.02 & 0.04 & 0.69 & 6.61 & \\
\hline IMP-OIGA & & & & & & & & & & & & & & & & & & & & & & & & 1180.04 \\
\hline IMP-PETR & 0.13 & 0.03 & 1.82 & 0.19 & 0.92 & 0.11 & 0.04 & 1.20 & 0.08 & 0.46 & 0.53 & 0.10 & 1.77 & 0.14 & 2.98 & 3.59 & 0.45 & 21.77 & 4.22 & 13.62 & & & & 95.04 \\
\hline IMP-FDBT & 1.86 & 0.44 & 25.53 & 2.63 & 12.90 & 0.03 & 0.01 & 0.36 & 0.02 & 0.14 & 0.90 & 0.18 & 3.01 & 0.24 & 5.07 & 0.01 & 0.00 & 0.05 & 0.01 & 0.03 & 0.00 & 0.02 & 0.16 & \\
\hline IMP-TWPM & 1.44 & 0.34 & 19.78 & 2.03 & 9.99 & 0.39 & 0.13 & 4.32 & 0.29 & 1.65 & 0.13 & 0.03 & 0.44 & 0.04 & 0.74 & 0.02 & 0.00 & 0.11 & 0.02 & 0.07 & 0.01 & 0.15 & 1.43 & \\
\hline IMP-CHNM & 20.98 & 4.94 & 287.90 & 29.60 & 145.41 & 2.55 & 0.83 & 27.98 & 1.85 & 10.72 & 0.23 & 0.04 & 0.76 & 0.06 & 1.27 & 0.16 & 0.02 & 0.95 & 0.18 & 0.59 & & & & 9.98 \\
\hline IMP-METL & 1.60 & 0.38 & 21.96 & 2.26 & 11.09 & 0.70 & 0.23 & 7.71 & 0.51 & 2.95 & $\mid 0.19$ & 0.04 & 0.62 & 0.05 & 1.04 & 0.07 & 0.01 & 0.40 & 0.08 & 0.25 & & & & 3.50 \\
\hline IMP-EQFO & 9.65 & 2.27 & 132.50 & 13.62 & 66.92 & 1.40 & 0.46 & 15.36 & 1.02 & 5.88 & 8.07 & 1.59 & 26.91 & 2.19 & 45.37 & 0.32 & 0.04 & 1.95 & 0.38 & 1.22 & 0.04 & 0.66 & 6.36 & 28.49 \\
\hline IMP-UTIL & 0.01 & 0.00 & 0.12 & 0.01 & 0.06 & 0.00 & 0.00 & 0.01 & 0.00 & 0.00 & 0.00 & 0.00 & 0.00 & 0.00 & 0.00 & 0.00 & 0.00 & 0.00 & 0.00 & 0.00 & 0.00 & 0.00 & 0.03 & 0.00 \\
\hline IMP-CONS & 0.01 & 0.00 & 0.13 & 0.01 & 0.07 & 0.00 & 0.00 & 0.02 & 0.00 & 0.01 & 0.00 & 0.00 & 0.00 & 0.00 & 0.00 & 0.00 & 0.00 & 0.02 & 0.00 & 0.01 & & & & 0.18 \\
\hline IMP-ACCR & 0.00 & 0.00 & 0.00 & 0.00 & 0.00 & 0.00 & 0.00 & 0.00 & 0.00 & 0.00 & 0.00 & 0.00 & 0.00 & 0.00 & 0.00 & 0.00 & 0.00 & 0.00 & 0.00 & 0.00 & & & & 0.00 \\
\hline IMP-CMIF & 1.00 & 0.23 & 13.67 & 1.41 & 6.90 & 0.22 & 0.07 & 2.37 & 0.16 & 0.91 & 0.14 & 0.03 & 0.46 & 0.04 & 0.78 & 0.02 & 0.00 & 0.10 & 0.02 & 0.06 & & & & 1.58 \\
\hline IMP-PROP & 0.66 & 0.15 & 9.00 & 0.92 & 4.54 & 0.30 & 0.10 & 3.34 & 0.22 & 1.28 & 0.30 & 0.06 & 1.01 & 0.08 & 1.70 & 0.05 & 0.01 & 0.33 & 0.06 & 0.20 & & & & 0.70 \\
\hline IMP-RBUS & 2.18 & 0.51 & 29.94 & 3.08 & 15.12 & 0.98 & 0.32 & 10.82 & 0.72 & 4.14 & 0.30 & 0.06 & 1.00 & 0.08 & 1.69 & 0.05 & 0.01 & 0.28 & 0.06 & 0.18 & & & & 7.03 \\
\hline IMP-EDUC & 0.00 & 0.00 & 0.01 & 0.00 & 0.01 & 0.00 & 0.00 & 0.01 & 0.00 & 0.01 & 0.00 & 0.00 & 0.00 & 0.00 & 0.00 & 0.00 & 0.00 & 0.00 & 0.00 & 0.00 & & & & 0.00 \\
\hline IMP-HEAL & 0.00 & 0.00 & 0.01 & 0.00 & 0.00 & 0.00 & 0.00 & 0.00 & 0.00 & 0.00 & 0.00 & 0.00 & 0.00 & 0.00 & 0.00 & 0.00 & 0.00 & 0.00 & 0.00 & 0.00 & & & & 0.00 \\
\hline IMP-CUPE & 0.03 & 0.01 & 0.44 & 0.05 & 0.22 & 0.00 & 0.00 & 0.04 & 0.00 & 0.02 & 0.00 & 0.00 & 0.00 & 0.00 & 0.00 & 0.00 & 0.00 & 0.00 & 0.00 & 0.00 & & & & 0.02 \\
\hline IMP-WHOL & 0.18 & 0.04 & 2.44 & 0.25 & 1.23 & 0.09 & 0.03 & 1.04 & 0.07 & 0.40 & 0.03 & 0.01 & 0.11 & 0.01 & 0.19 & 0.01 & 0.00 & 0.05 & 0.01 & 0.03 & & & & 0.37 \\
\hline IMP-RETT & 6.16 & 1.45 & 84.58 & 8.70 & 42.72 & 0.46 & 0.15 & 5.06 & 0.33 & 1.94 & 0.48 & 0.09 & 1.58 & 0.13 & 2.67 & 0.14 & 0.02 & 0.82 & 0.16 & 0.51 & & & & 1.75 \\
\hline IMP-TRAN & 0.39 & 0.09 & 5.31 & 0.55 & 2.68 & 0.83 & 0.27 & 9.14 & 0.60 & 3.50 & 0.42 & 0.08 & 1.38 & 0.11 & 2.33 & 0.31 & 0.04 & 1.86 & 0.36 & 1.16 & & & & 7.85 \\
\hline TAX & \begin{tabular}{|l|}
20.49 \\
\end{tabular} & 4.71 & 276.18 & 28.85 & 139.19 & 1.12 & 0.37 & 12.57 & 0.84 & 4.82 & 0.08 & 0.02 & 0.25 & 0.02 & 0.43 & 2.63 & 0.33 & 16.22 & 3.18 & 10.15 & 0.69 & 10.74 & 103.37 & \begin{tabular}{|l|l|l}
1049.00 \\
\end{tabular} \\
\hline LAB & 182.89 & 42.02 & 2464.88 & 257.49 & 1242.27 & 25.42 & 8.49 & 285.66 & 19.11 & 109.60 & 10.07 & 1.97 & 33.41 & 2.75 & 56.29 & 13.27 & 1.68 & 81.93 & 16.04 & 51.30 & 0.67 & 10.37 & 99.83 & 64.11 \\
\hline CAP & 117.15 & 26.91 & 1578.86 & 164.93 & 795.73 & 26.36 & 8.80 & 296.23 & 19.82 & 113.66 & 11.87 & 2.32 & 39.40 & 3.24 & 66.37 & 25.31 & 3.20 & 156.27 & 30.60 & 97.85 & 9.59 & 148.41 & 1428.70 & 162.07 \\
\hline $\begin{array}{l}\text { TOTAL } \\
\end{array}$ & 737.04 & 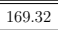 & (9933.39 & "1037.69 & (5006.30 & 168.87 & $\begin{array}{l}56.40 \\
\end{array}$ & (1897.70 & $\begin{array}{ll}126.98 \\
\end{array}$ & \begin{tabular}{ll|l}
728.14 \\
\end{tabular} & 82.21 & $\begin{array}{ll}16.08 \\
\end{array}$ & 272.80 & $\begin{array}{l}22.43 \\
\end{array}$ & (459.59 & 96.74 & $\begin{array}{l}12.23 \\
\end{array}$ & (597.24 & 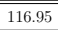 & (373.95 & $\begin{array}{ll}14.98 \\
\end{array}$ & 231.84 & 2231.93 & 3683.30 \\
\hline
\end{tabular}

Extended MRIO Table - Part 3-1

\begin{tabular}{|c|c|c|c|c|c|c|c|c|c|c|c|c|c|c|c|c|c|c|c|c|c|c|c|c|c|}
\hline \multirow{3}{*}{$\begin{array}{l}\mathrm{MRIO}^{\prime} \\
\mathrm{row} / \mathrm{col}\end{array}$} & \multicolumn{25}{|c|}{ Industries } \\
\hline & \multicolumn{5}{|c|}{ FDBT } & \multicolumn{5}{|c|}{ TWPM } & \multicolumn{5}{|c|}{ CHNM } & \multicolumn{5}{|c|}{ METL } & \multicolumn{5}{|c|}{ EQFO } \\
\hline & AKL & WLG & ONI & CAN & OSI & AKL & WLG & ONI & CAN & OSI & AKL & WLG & ONI & CAN & OSI & AKL & WLG & ONI & CAN & OSI & AKL & WLG & ONI & CAN & OSI \\
\hline IMP-AGRI & \begin{tabular}{|l|l|}
39.95 \\
\end{tabular} & 6.81 & 77.90 & 17.85 & 54.13 & 2.96 & 0.66 & 3.61 & 1.06 & 1.36 & 0.28 & 0.05 & 0.01 & 0.09 & 0.04 & 0.01 & 0.00 & 0.01 & 0.01 & 0.00 & 0.06 & 0.01 & 0.03 & 0.02 & 0.01 \\
\hline IMP-FOLO & 0.08 & 0.01 & 0.17 & 0.04 & 0.11 & 3.49 & 0.78 & 4.26 & 1.26 & 1.61 & 0.05 & 0.01 & 0.00 & 0.01 & 0.01 & 0.01 & 0.00 & 0.01 & 0.00 & 0.00 & 0.05 & 0.01 & 0.03 & 0.02 & 0.01 \\
\hline IMP-FISH & 17.77 & 3.03 & 34.64 & 7.94 & 24.07 & 0.02 & 0.00 & 0.02 & 0.01 & 0.01 & 0.02 & 0.00 & 0.00 & 0.01 & 0.00 & 0.00 & 0.00 & 0.00 & 0.00 & 0.00 & 0.09 & 0.02 & 0.05 & 0.03 & 0.02 \\
\hline IMP-MINE & 0.65 & 0.11 & 1.27 & 0.29 & 0.88 & 0.40 & 0.09 & 0.49 & 0.14 & 0.18 & 14.23 & 2.81 & 0.54 & 4.36 & 2.15 & 9.32 & 1.51 & 7.68 & 3.25 & 2.71 & 0.70 & 0.13 & 0.38 & 0.22 & 0.14 \\
\hline IMP-OIGA & & & & & & & & & & & & & & & & & & & & & & & & & \\
\hline IMP-PETR & 14.12 & 2.41 & 27.54 & 6.31 & 19.14 & 29.26 & 6.54 & 35.67 & 10.53 & 13.47 & 58.73 & 11.58 & 2.24 & 18.00 & 8.87 & 7.86 & 1.27 & 6.48 & 2.74 & 2.28 & 9.29 & 1.66 & 5.02 & 2.84 & 1.79 \\
\hline IMP-FDBT & 60.48 & 10.31 & 117.93 & 27.03 & 81.95 & 4.06 & 0.91 & 4.96 & 1.46 & 1.87 & 5.77 & 1.14 & 0.22 & 1.77 & 0.87 & 0.36 & 0.06 & 0.29 & 0.12 & 0.10 & 1.59 & 0.28 & 0.86 & 0.49 & 0.30 \\
\hline IMP-TWPM & 14.05 & 2.40 & 27.40 & 6.28 & 19.04 & 272.04 & 60.79 & 331.70 & 97.87 & 125.21 & 42.29 & 8.34 & 1.62 & 12.96 & 6.39 & 4.95 & 0.80 & 4.08 & 1.72 & 1.44 & 53.36 & 9.55 & 28.83 & 16.34 & 10.26 \\
\hline IMP-CHNM & 101.54 & 17.31 & 198.01 & 45.38 & 137.60 & 67.31 & 15.04 & 82.07 & 24.21 & 30.98 & 682.68 & 134.57 & 26.09 & 209.23 & 103.11 & 18.98 & 3.08 & 15.65 & 6.62 & 5.52 & 87.64 & 15.68 & 47.35 & 26.83 & 16.85 \\
\hline IMP-METL & 29.81 & 5.08 & 58.13 & 13.32 & 40.39 & 17.82 & 3.98 & 21.73 & 6.41 & 8.20 & 286.82 & 56.54 & 10.96 & 87.91 & 43.32 & 293.04 & 47.48 & 241.51 & 102.20 & 85.20 & $\mid 265.82$ & 47.56 & 143.62 & 81.37 & 51.12 \\
\hline IMP-EQFO & 30.04 & 5.12 & 58.58 & 13.42 & 40.71 & 35.08 & 7.84 & 42.77 & 12.62 & 16.15 & 56.26 & 11.09 & 2.15 & 17.24 & 8.50 & 48.46 & 7.85 & 39.94 & 16.90 & 14.09 & \begin{tabular}{|l|l|} 
& 434.37 \\
\end{tabular} & 77.72 & 234.68 & 132.97 & 83.53 \\
\hline IMP-UTIL & 0.05 & 0.01 & 0.11 & 0.02 & 0.07 & 0.12 & 0.03 & 0.15 & 0.04 & 0.06 & 0.12 & 0.02 & 0.00 & 0.04 & 0.02 & 0.15 & 0.02 & 0.12 & 0.05 & 0.04 & 0.03 & 0.01 & 0.02 & 0.01 & 0.01 \\
\hline IMP-CONS & 0.01 & 0.00 & 0.01 & 0.00 & 0.01 & 0.02 & 0.00 & 0.02 & 0.01 & 0.01 & 0.05 & 0.01 & 0.00 & 0.02 & 0.01 & 0.01 & 0.00 & 0.01 & 0.00 & 0.00 & 0.12 & 0.02 & 0.07 & 0.04 & 0.02 \\
\hline IMP-ACCR & 0.00 & 0.00 & 0.00 & 0.00 & 0.00 & 0.00 & 0.00 & 0.00 & 0.00 & 0.00 & 0.00 & 0.00 & 0.00 & 0.00 & 0.00 & 0.00 & 0.00 & 0.00 & 0.00 & 0.00 & 0.00 & 0.00 & 0.00 & 0.00 & 0.00 \\
\hline IMP-CMIF & 2.39 & 0.41 & 4.66 & 1.07 & 3.24 & 3.48 & 0.78 & 4.24 & 1.25 & 1.60 & 6.41 & 1.26 & 0.25 & 1.97 & 0.97 & 0.59 & 0.10 & 0.48 & 0.20 & 0.17 & 3.80 & 0.68 & 2.05 & 1.16 & 0.73 \\
\hline IMP-PROP & 2.34 & 0.40 & 4.56 & 1.04 & 3.17 & 2.80 & 0.62 & 3.41 & 1.01 & 1.29 & 5.35 & 1.05 & 0.20 & 1.64 & 0.81 & 0.89 & 0.14 & 0.74 & 0.31 & 0.26 & 3.02 & 0.54 & 1.63 & 0.93 & 0.58 \\
\hline IMP-RBUS & 16.95 & 2.89 & 33.06 & 7.58 & 22.97 & 14.87 & 3.32 & 18.14 & 5.35 & 6.85 & 31.85 & 6.28 & 1.22 & 9.76 & 4.81 & 5.21 & 0.84 & 4.30 & 1.82 & 1.52 & 17.37 & 3.11 & 9.39 & 5.32 & 3.34 \\
\hline IMP-EDUC & 0.01 & 0.00 & 0.01 & 0.00 & 0.01 & 0.03 & 0.01 & 0.04 & 0.01 & 0.01 & 0.03 & 0.01 & 0.00 & 0.01 & 0.00 & 0.00 & 0.00 & 0.00 & 0.00 & 0.00 & 0.01 & 0.00 & 0.00 & 0.00 & 0.00 \\
\hline IMP-HEAL & 0.00 & 0.00 & 0.00 & 0.00 & 0.00 & 0.00 & 0.00 & 0.00 & 0.00 & 0.00 & 0.00 & 0.00 & 0.00 & 0.00 & 0.00 & 0.00 & 0.00 & 0.00 & 0.00 & 0.00 & 0.00 & 0.00 & 0.00 & 0.00 & 0.00 \\
\hline IMP-CUPE & 0.31 & 0.05 & 0.60 & 0.14 & 0.42 & 0.27 & 0.06 & 0.33 & 0.10 & 0.12 & 0.63 & 0.12 & 0.02 & 0.19 & 0.10 & 0.04 & 0.01 & 0.03 & 0.01 & 0.01 & 0.20 & 0.04 & 0.11 & 0.06 & 0.04 \\
\hline IMP-WHOL & 0.61 & 0.10 & 1.18 & 0.27 & 0.82 & 1.62 & 0.36 & 1.98 & 0.58 & 0.75 & 1.78 & 0.35 & 0.07 & 0.55 & 0.27 & 1.52 & 0.25 & 1.25 & 0.53 & 0.44 & 1.64 & 0.29 & 0.89 & 0.50 & 0.32 \\
\hline IMP-RETT & 10.57 & 1.80 & 20.61 & 4.72 & 14.32 & 5.67 & 1.27 & 6.91 & 2.04 & 2.61 & 13.61 & 2.68 & 0.52 & 4.17 & 2.06 & 1.93 & 0.31 & 1.59 & 0.67 & 0.56 & 12.87 & 2.30 & 6.95 & 3.94 & 2.47 \\
\hline IMP-TRAN & 10.35 & 1.76 & 20.17 & 4.62 & 14.02 & 12.38 & 2.77 & 15.09 & 4.45 & 5.70 & 20.82 & 4.10 & 0.80 & 6.38 & 3.14 & 3.53 & 0.57 & 2.91 & 1.23 & 1.03 & 6.45 & 1.15 & 3.49 & 1.98 & 1.24 \\
\hline TAX & 327.80 & 55.62 & 634.82 & 147.05 & 441.43 & 23.90 & 5.29 & 28.73 & 8.61 & 10.86 & 51.32 & 10.17 & 1.97 & 15.90 & 7.79 & \begin{tabular}{|l|l|}
19.42 \\
\end{tabular} & 3.10 & 15.69 & 6.75 & 5.54 & 16.73 & 2.94 & 8.86 & 5.10 & 3.15 \\
\hline LAB & 638.60 & 108.35 & 1236.72 & 286.47 & 859.96 & 824.51 & 182.60 & 990.97 & 297.14 & 374.56 & 847.08 & 167.83 & 32.49 & 262.51 & 128.54 & 557.60 & 89.10 & 450.47 & 193.83 & 159.09 & $\mid 1141.78$ & 200.67 & 604.67 & 348.13 & 215.22 \\
\hline CAP & 454.57 & 77.12 & 880.32 & 203.92 & 612.14 & 590.95 & 130.88 & 710.25 & 212.97 & 268.46 & 903.01 & 178.91 & 34.64 & 279.84 & 137.03 & 672.86 & 107.52 & 543.60 & 233.90 & 191.98 & \begin{tabular}{|l}
698.61 \\
\end{tabular} & 122.78 & 369.98 & 213.01 & 131.68 \\
\hline TOTAL & \begin{tabular}{|l|l}
5647.6 \\
\end{tabular} & $\begin{array}{l}958.2 \\
\end{array}$ & 10937.1 & 2533.5 & 7605.2 & \begin{tabular}{|l|l|}
4032.3 \\
\end{tabular} & $\begin{array}{l}893.0 \\
\end{array}$ & 4846.4 & 1453.2 & 1831.8 & 5346.1 & 1059.2 & 205.1 & 1656.8 & 811.2 & \begin{tabular}{|l|l|}
3116.7 \\
\end{tabular} & 498.0 & 2518.0 & 1083.5 & 889.2 & 4835.7 & 849.9 & 2560.9 & 1474.4 & 911.5 \\
\hline
\end{tabular}

Extended MRIO Table - Part 3-2 


\begin{tabular}{|c|c|c|c|c|c|c|c|c|c|c|c|c|c|c|c|c|c|c|c|c|c|c|c|c|c|}
\hline \multirow{3}{*}{$\begin{array}{l}\mathrm{MRIO}^{\prime} \\
\mathrm{row} / \mathrm{col}\end{array}$} & \multicolumn{25}{|c|}{ Industries } \\
\hline & \multicolumn{5}{|c|}{ UTIL } & \multicolumn{5}{|c|}{ CONS } & \multicolumn{5}{|c|}{ ACCR } & \multicolumn{5}{|c|}{ CMIF } & \multicolumn{5}{|c|}{ PROP } \\
\hline & AKL & WLG & ONI & CAN & OSI & AKL & WLG & $\mathrm{ONI}$ & CAN & OSI & AKL & WLG & ONI & CAN & OSI & AKL & WLG & ONI & $\mathrm{CAN}$ & OSI & AKL & WLG & ONI & CAN & OSI \\
\hline IMP-AGRI & 0.01 & 0.00 & 0.01 & 0.00 & 0.00 & 0.18 & 0.06 & 0.20 & 0.06 & 0.08 & 4.97 & 1.80 & 5.50 & 1.91 & 3.48 & 0.17 & 0.08 & 0.07 & 0.04 & 0.02 & 1.15 & 0.30 & 0.88 & 0.37 & 0.35 \\
\hline IMP-FOLO & 0.00 & 0.00 & 0.00 & 0.00 & 0.00 & 0.14 & 0.05 & 0.16 & 0.05 & 0.06 & 0.00 & 0.00 & 0.00 & 0.00 & 0.00 & 0.01 & 0.01 & 0.01 & 0.00 & 0.00 & 0.01 & 0.00 & 0.01 & 0.00 & 0.00 \\
\hline IMP-FISH & 0.00 & 0.00 & 0.01 & 0.00 & 0.00 & 0.01 & 0.00 & 0.02 & 0.01 & 0.01 & 0.06 & 0.02 & 0.06 & 0.02 & 0.04 & 0.67 & 0.30 & 0.25 & 0.17 & 0.10 & 0.13 & 0.03 & 0.10 & 0.04 & 0.04 \\
\hline IMP-MINE & 1.65 & 1.16 & 2.67 & 0.45 & 0.78 & 3.45 & 1.12 & 3.99 & 1.22 & 1.57 & 0.01 & 0.00 & 0.01 & 0.00 & 0.01 & 0.06 & 0.03 & 0.02 & 0.01 & 0.01 & 0.02 & 0.01 & 0.02 & 0.01 & 0.01 \\
\hline IMP-OIGA & & & & & & & & & & & & & & & & & & & & & & & & & \\
\hline IMP-PETR & 36.36 & 25.54 & 58.86 & 10.03 & 17.24 & 9.24 & 3.00 & 10.70 & 3.28 & 4.21 & 0.29 & 0.11 & 0.33 & 0.11 & 0.21 & 2.32 & 1.05 & 0.87 & 0.57 & 0.33 & 0.56 & 0.15 & 0.43 & 0.18 & 0.17 \\
\hline IMP-FDBT & 0.04 & 0.03 & 0.06 & 0.01 & 0.02 & 0.16 & 0.05 & 0.18 & 0.06 & 0.07 & 44.89 & 16.24 & 49.68 & 17.24 & 31.41 & 0.60 & 0.27 & 0.22 & 0.15 & 0.09 & 0.22 & 0.06 & 0.17 & 0.07 & 0.07 \\
\hline IMP-TWPM & 1.49 & 1.05 & 2.41 & 0.41 & 0.71 & 43.83 & 14.22 & 50.71 & 15.56 & 19.97 & 3.38 & 1.22 & 3.74 & 1.30 & 2.37 & 26.82 & 12.15 & 10.07 & 6.62 & 3.84 & 22.58 & 5.88 & 17.43 & 7.30 & 6.97 \\
\hline IMP-CHNM & 1.88 & 1.32 & 3.04 & 0.52 & 0.89 & 204.41 & 66.32 & 236.52 & 72.59 & 93.12 & 8.61 & 3.11 & 9.53 & 3.31 & 6.02 & 6.38 & 2.89 & 2.40 & 1.58 & 0.91 & 8.15 & 2.12 & 6.29 & 2.63 & 2.52 \\
\hline IMP-METL & 1.87 & 1.32 & 3.03 & 0.52 & 0.89 & 112.62 & 36.54 & 130.30 & 39.99 & 51.30 & 5.34 & 1.93 & 5.91 & 2.05 & 3.73 & 5.35 & 2.42 & 2.01 & 1.32 & 0.77 & 9.36 & 2.44 & 7.23 & 3.03 & 2.89 \\
\hline IMP-EQFO & 23.73 & 16.67 & 38.41 & 6.55 & 11.25 & 353.19 & 114.59 & 408.66 & 125.43 & 160.89 & 21.11 & 7.64 & 23.36 & 8.11 & 14.77 & 177.01 & 80.16 & 66.42 & 43.71 & 25.36 & 48.32 & 12.58 & 37.29 & 15.62 & 14.92 \\
\hline IMP-UTIL & 0.67 & 0.47 & 1.09 & 0.19 & 0.32 & 0.02 & 0.01 & 0.02 & 0.01 & 0.01 & 0.03 & 0.01 & 0.03 & 0.01 & 0.02 & 0.03 & 0.01 & 0.01 & 0.01 & 0.00 & 0.01 & 0.00 & 0.01 & 0.00 & 0.00 \\
\hline IMP-CONS & 0.12 & 0.08 & 0.19 & 0.03 & 0.06 & 38 & 0.77 & 2.75 & 0.84 & 1.08 & 0.00 & 0.00 & 0.00 & 0.00 & 0.00 & 0.08 & 0.04 & 0.03 & 0.02 & 0.01 & 0.29 & 0.08 & 0.22 & 0.09 & 0.09 \\
\hline IMP-ACCR & 0.00 & 0.00 & 0.00 & 0.00 & 0.00 & 0.00 & 0.00 & 0.00 & 0.00 & 0.00 & 0.00 & 0.00 & 0.00 & 0.00 & 0.00 & 0.00 & 0.00 & 0.00 & 0.00 & 0.00 & 0.00 & 0.00 & 0.00 & 0.00 & 0.00 \\
\hline IMP-CMIF & 0.67 & 0.47 & 1.08 & 0.18 & 0.32 & 4.58 & 1.49 & 5.30 & 1.63 & 2.09 & 0.97 & 0.35 & 1.07 & 0.37 & 0.68 & 57.96 & 26.25 & 21.75 & 14.31 & 8.30 & 5.77 & 1.50 & 4.45 & 1.86 & 1.78 \\
\hline IMP-PROP & 1.15 & 0.81 & 1.86 & 0.32 & 0.54 & 2. & 0.73 & 2.61 & 0.80 & 1.03 & 0.74 & 0.27 & 0.82 & 0.28 & 0.52 & 7.43 & 3.36 & 2.79 & 1.83 & 1.06 & 12.19 & 3.17 & 9.41 & 94 & 3.76 \\
\hline IMP-RBUS & 7.18 & 5.05 & 11.63 & 1.98 & 3.41 & 19.81 & 6.43 & 22.92 & 7.04 & 9.02 & 6.77 & 2.45 & 7.49 & 2.60 & 4.74 & 76.23 & 34.52 & 28.61 & 18.82 & 10.92 & 13.02 & 3.39 & 10.05 & 4.21 & 4.02 \\
\hline IMP-EDUC & 0.01 & 0.01 & 0.02 & 0.00 & 0.01 & 0.03 & 0.01 & 0.03 & 0.01 & 0.01 & 0.00 & 0.00 & 0.00 & 0.00 & 0.00 & 0.07 & 0.03 & 0.03 & 0.02 & 0.01 & 0.02 & 0.00 & 0.01 & 0.01 & 0.01 \\
\hline IMP-HEAL & 0.00 & 0.00 & 0.00 & 0.00 & 0.00 & 0.00 & 0.00 & 0.00 & 0.00 & 0.00 & 0.00 & 0.00 & 0.00 & 0.00 & 0.00 & 0.00 & 0.00 & 0.00 & 0.00 & 0.00 & 0.00 & 0.00 & 0.00 & 0.00 & 0.00 \\
\hline IMP-CUPE & 0.03 & 0.02 & 0.04 & 0.01 & 0.01 & 0.09 & 0.03 & 0.11 & 0.03 & 0.04 & 0.10 & 0.04 & 0.1 & 0.04 & 0.07 & 1.01 & 0.46 & 0.38 & 0.25 & 0.15 & 0.16 & 0.04 & 0.12 & 05 & 0.05 \\
\hline IMP-WHOL & 0.14 & 0.10 & 0.23 & 0.04 & 0.07 & 1. & 0.46 & 1.65 & 0.51 & 0.65 & 0.24 & 0.09 & 0.26 & 0.09 & 0.17 & 0.74 & 0.33 & 0.28 & 0.18 & 0.11 & 0.16 & 0.04 & 0.13 & 0.05 & 0.05 \\
\hline IMP-RETT & 2.00 & 1.41 & 3.24 & 0.55 & 0.95 & 33.01 & 10.71 & 38.19 & 11.72 & 15.04 & 7.00 & 2.53 & 7.75 & 2.69 & 4.90 & 24.22 & 10.97 & 9.09 & 5.98 & 3.47 & 15.33 & 3.99 & 11.83 & 4.95 & 4.73 \\
\hline IMP-TRAN & 0.32 & 0.22 & 0.51 & 0.09 & 0.15 & 1.67 & 0.54 & 1.94 & 0.59 & 0.76 & 0.74 & 0.27 & 0.82 & 0.28 & 0.52 & 12.60 & 5.70 & 4.73 & 3.11 & 1.81 & 2.68 & 0.70 & 2.07 & 0.87 & 0.83 \\
\hline TAX & 15.89 & 11.14 & 25.69 & 4.39 & 7.52 & 108.52 & $\begin{array}{ll}34.49 \\
\end{array}$ & 123.11 & 38.28 & 48.43 & 43.47 & 15.14 & 46.34 & 16.46 & 29.26 & \begin{tabular}{|l|l|}
548.49 \\
\end{tabular} & 247.22 & 204.83 & 135.63 & 78.22 & 385.64 & 99.39 & $\begin{array}{ll}296.08 \\
\end{array}$ & 124.75 & 118.27 \\
\hline LAB & 158.88 & 111.41 & 6.97 & 43.86 & .25 & 36.42 & 424.73 & 1516.06 & 471.43 & 596.35 & 506.36 & 176.42 & 539.83 & 191.75 & 340.88 & 2206.00 & 994.33 & 823.82 & 545.49 & 314.59 & 359.99 & 92.78 & 276.38 & 116.45 & 110.40 \\
\hline CAP & 808.70 & 567.09 & 1307.99 & 223.24 & 383.01 & 1202.98 & 382.33 & 1364.69 & 424.36 & 536.81 & 303.62 & 105.78 & 323.68 & 114.98 & 204.39 & 3438.66 & 1549.93 & 1284.15 & 850.30 & 490.37 & 2634.79 & 679.07 & 2022.86 & 852.34 & 808.03 \\
\hline TOTAL & 3099.3 & 2173.4 & $\begin{array}{l}5012.8 \\
\end{array}$ & 855.6 & 1467.9 & 9013.6 & 2864.6 & 10225.2 & $\begin{array}{l}3179.6 \\
\end{array}$ & \begin{tabular}{|l|}
4022.2 \\
\end{tabular} & \begin{tabular}{|l|l|}
1861.8 \\
\end{tabular} & 648.7 & $\begin{array}{ll}1984.8 \\
\end{array}$ & 705.0 & \begin{tabular}{|l|}
1253.3 \\
\end{tabular} & \begin{tabular}{|l|l|}
10852.0 \\
\end{tabular} & 4891.4 & 4052.6 & 2683.4 & \begin{tabular}{|l|}
1547.5 \\
\end{tabular} & 5143.0 & $\begin{array}{l}1325.5 \\
\end{array}$ & $\begin{array}{l}3948.6 \\
\end{array}$ & $\begin{array}{l}1663.7 \\
\end{array}$ & 1577.2 \\
\hline
\end{tabular}

Extended MRIO Table - Part 3-3

\begin{tabular}{|c|c|c|c|c|c|c|c|c|c|c|c|c|c|c|c|c|c|c|c|c|c|c|c|c|c|}
\hline \multirow{3}{*}{$\begin{array}{c}\mathrm{MRIO}^{\prime} \\
\mathrm{row} / \mathrm{col}\end{array}$} & \multicolumn{25}{|c|}{ Industries } \\
\hline & \multicolumn{5}{|c|}{ RBUS } & \multicolumn{5}{|c|}{ GOVT } & \multicolumn{5}{|c|}{ EDUC } & \multicolumn{5}{|c|}{ HEAL } & \multicolumn{5}{|c|}{ CUPE } \\
\hline & AKL & WLG & ONI & CAN & OSI & AKL & WLG & ONI & CAN & OSI & AKL & WLG & ONI & CAN & OSI & AKL & WLG & $\mathrm{ONI}$ & CAN & OSI & AKL & WLG & ONI & CAN & OSI \\
\hline IMP-AGRI & 1.81 & 0.65 & 0.87 & 0.39 & 0.30 & 0.31 & 0.49 & 0.35 & 0.12 & 0.10 & 0.04 & 0.01 & 0.03 & 0.01 & 0.01 & 2.11 & 0.83 & 2.48 & 0.90 & 1.02 & 2.02 & 0.80 & 1.50 & 0.61 & 0.69 \\
\hline IMP-FOLO & 1.09 & 0.39 & 0.53 & 0.24 & 0.18 & 0.01 & 0.01 & 0.01 & 0.00 & 0.00 & 0.01 & 0.00 & 0.01 & 0.00 & 0.00 & 0.00 & 0.00 & 0.01 & 0.00 & 0.00 & 0.01 & 0.01 & 0.01 & 0.00 & 0.00 \\
\hline IMP-FISH & 0.06 & 0.02 & 0.03 & 0.01 & 0.01 & 0.01 & 0.02 & 0.02 & 0.01 & 0.00 & 0.01 & 0.00 & 0.01 & 0.00 & 0.00 & 0.03 & 0.01 & 0.03 & 0.01 & 0.01 & 0.03 & 0.01 & 0.02 & 0.01 & 0.01 \\
\hline $\begin{array}{l}\text { IMP-MINE } \\
\text { IMP-OIGA }\end{array}$ & 0.14 & 0.05 & 0.07 & 0.03 & 0.02 & 0.14 & 0.22 & 0.16 & 0.05 & 0.05 & 0.36 & 0.12 & 0.34 & 0.12 & 0.14 & 0.69 & 0.27 & 0.81 & 0.29 & 0.33 & 0.08 & 0.03 & 0.06 & 0.02 & 0.03 \\
\hline IMP-PETR & 15.05 & 5.36 & 7.24 & 3.26 & 2.49 & 12.94 & 20.46 & 14.64 & 4.92 & 4.29 & 3.72 & 1.24 & 3.53 & 1.21 & 1.43 & 6.33 & 2.50 & 7.45 & 2.71 & 3.07 & 0.83 & 0.33 & 0.62 & 0.25 & 0.29 \\
\hline IMP-FDBT & 1.04 & 0.37 & 0.50 & 0.23 & 0.17 & 0.19 & 0.31 & 0.22 & 0.07 & 0.06 & 0.25 & 0.08 & 0.23 & 0.08 & 0.09 & 4.86 & 1.92 & 5.72 & 2.08 & 2.36 & 4.60 & 1.82 & 3.41 & 1.39 & 1.57 \\
\hline IMP-TWPM & 103.23 & 36.79 & 49.69 & 22.37 & 17.12 & 6.60 & 10.43 & 7.46 & 2.51 & 2.19 & 8.94 & 2.98 & 8.48 & 2.90 & 3.43 & 10.60 & 4.18 & 12.48 & 4.55 & 5.14 & 28.83 & 11.41 & 21.39 & 8.70 & 9.84 \\
\hline IMP-METL & 14.21 & 5.06 & 6.84 & 3.08 & 2.36 & 2.56 & 4.05 & 2.90 & 0.97 & 0.85 & 2.55 & 0.85 & 2.42 & 0.83 & 0.98 & 1.70 & 0.67 & 2.01 & 0.73 & 0.83 & 2.78 & 1.10 & 2.06 & 0.84 & 0.95 \\
\hline IMP-EQFO & 120.76 & 43.04 & 58.13 & 26.17 & 20.02 & 58.88 & 93.09 & 66.63 & 22.38 & 19.53 & 59.87 & 19.97 & 56.79 & 19.40 & 22.95 & 60.45 & 23.87 & 71.19 & 25.93 & 29.32 & 90.08 & 35.65 & 66.82 & 27.18 & 30.76 \\
\hline IMP-UTIL & 0.06 & 0.02 & 0.03 & 0.01 & 0.01 & 0.03 & 0.04 & 0.03 & 0.01 & 0.01 & 0.04 & 0.01 & 0.04 & 0.01 & 0.02 & 0.04 & 0.02 & 0.05 & 0.02 & 0.02 & 0.05 & 0.02 & 0.04 & 0.02 & 0.02 \\
\hline IMP-CONS & 0.03 & 0.01 & 0.02 & 0.01 & 0.01 & 0.20 & 0.32 & 0.23 & 0.08 & 0.07 & 0.07 & 0.02 & 0.06 & 0.02 & 0.02 & 0.08 & 0.03 & 0.09 & 0.03 & 0.04 & 0.14 & 0.06 & 0.10 & 0.04 & 0.05 \\
\hline IMP-ACCR & 0.00 & 0.00 & 0.00 & 0.00 & 0.00 & 0.00 & 0.01 & 0.00 & 0.00 & 0.00 & 0.00 & 0.00 & 0.00 & 0.00 & 0.00 & 0.01 & 0.00 & 0.01 & 0.00 & 0.00 & 0.00 & 0.00 & 0.00 & 0.00 & 0.00 \\
\hline IMP-CMIF & 22.21 & 7.92 & 10.69 & 4.81 & 3.68 & 4.30 & 6.79 & 4.86 & 1.63 & 1.43 & 2.64 & 0.88 & 2.51 & 0.86 & 1.01 & 6.87 & 2.71 & 8.09 & 2.95 & 3.33 & 13.61 & 5.38 & 10.09 & 4.10 & 4.65 \\
\hline IMP-PROP & 17.67 & 6.30 & 8.50 & 3.83 & 2.93 & 5.10 & 8.07 & 5.77 & 1.94 & 1.69 & 1.71 & 0.57 & 1.63 & 0.56 & 0.66 & 6.01 & 2.37 & 7.08 & 2.58 & 2.92 & 10.87 & 4.30 & 8.07 & 3.28 & 3.71 \\
\hline IMP-RBUS & 201.52 & 71.82 & 97.00 & 43.68 & 33.41 & 20.60 & 32.56 & 23.30 & 7.83 & 6.83 & 9.97 & 3.33 & 9.46 & 3.23 & 3.82 & 27.88 & 11.01 & 32.83 & 11.96 & 13.52 & 66.87 & 26.46 & 49.60 & 20.17 & 22.83 \\
\hline IMP-EDUC & 0.34 & 0.12 & 0.17 & 0.07 & 0.06 & 0.08 & 0.12 & 0.09 & 0.03 & 0.03 & 0.21 & 0.07 & 0.20 & 0.07 & 0.08 & 0.14 & 0.06 & 0.17 & 0.06 & 0.07 & 0.22 & 0.09 & 0.16 & 0.07 & 0.07 \\
\hline IMP-HEAL & 0.00 & 0.00 & 0.00 & 0.00 & 0.00 & 0.00 & 0.01 & 0.00 & 0.00 & 0.00 & 0.00 & 0.00 & 0.00 & 0.00 & 0.00 & 0.07 & 0.03 & 0.09 & 0.03 & 0.04 & 0.01 & 0.00 & 0.00 & 0.00 & 0.00 \\
\hline IMP-RETT & 64.72 & 23.07 & 31.15 & 14.03 & 10.73 & 7.04 & 11.13 & 7.97 & 2.68 & 2.34 & 5.06 & 1.69 & 4.80 & 1.64 & 1.94 & 22.28 & 8.80 & 26.24 & 9.56 & 10.81 & 24.04 & 9.51 & 17.83 & 7.25 & 8.21 \\
\hline IMP-TRAN & 23.78 & 8.47 & 11.45 & 5.15 & 3.94 & 4.26 & 6.73 & 4.82 & 1.62 & 1.41 & 1.48 & 0.49 & 1.40 & 0.48 & 0.57 & 1.75 & 0.69 & 2.07 & 0.75 & 0.85 & 8.41 & 3.33 & 6.24 & 2.54 & 2.87 \\
\hline TAX & 47.41 & 16.83 & 22.76 & 10.35 & 7.84 & 33.20 & 52.08 & 37.22 & 12.65 & 10.92 & 17.96 & 5.87 & 16.72 & 5.81 & 6.76 & 6.35 & 2.45 & 7.45 & 2.75 & 3.06 & 132.58 & 52.63 & 96.16 & 40.09 & 44.59 \\
\hline LAB & 304.15 & 1279.17 & 1730.43 & 787.03 & 596.24 & 1250.63 & 1961.54 & 1401.99 & 476.46 & 411.13 & 1728.89 & 565.36 & 1609.29 & 559.58 & 650.60 & 1752.31 & 675.76 & 2056.04 & 760.00 & 842.97 & $\mid 1102.87$ & 437.80 & 799.89 & 333.52 & 370.94 \\
\hline CAP & 2281.06 & 809.58 & 1095.19 & 498.11 & 377.36 & 279.99 & 439.15 & 313.88 & 106.67 & 92.04 & 181.94 & 59.49 & 169.35 & 58.89 & 68.46 & \begin{tabular}{|l|l|}
526.43 \\
\end{tabular} & 203.01 & 617.68 & 228.32 & 253.25 & \begin{tabular}{|l|l|}
858.24 \\
\end{tabular} & 340.69 & 622.47 & 259.54 & 288.67 \\
\hline TOTAL & 11056.1 & $\begin{array}{l}3924.0 \\
\end{array}$ & "5308.3 & 2414.3 & $\begin{array}{ll}1829.0 \\
\end{array}$ & \begin{tabular}{|l|l|}
3059.6 \\
\end{tabular} & (4798.8 & $\begin{array}{l}3429.9 \\
\end{array}$ & $\begin{array}{lll}1165.6 \\
\end{array}$ & $\begin{array}{lll}1005.8 \\
\end{array}$ & 2782.2 & $\begin{array}{l}909.8 \\
\end{array}$ & 2589.8 & $\begin{array}{l}900.5 \\
\end{array}$ & | & 3613.4 & (1393.5 & 4239.7 & 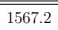 & $\begin{array}{lll}1738.3 \\
\end{array}$ & $\begin{array}{l}4260.7 \\
\end{array}$ & 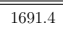 & $\begin{array}{l}3090.2 \\
\end{array}$ & (1288.5 & 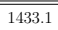 \\
\hline
\end{tabular}

Extended MRIO Table - Part 3-4 


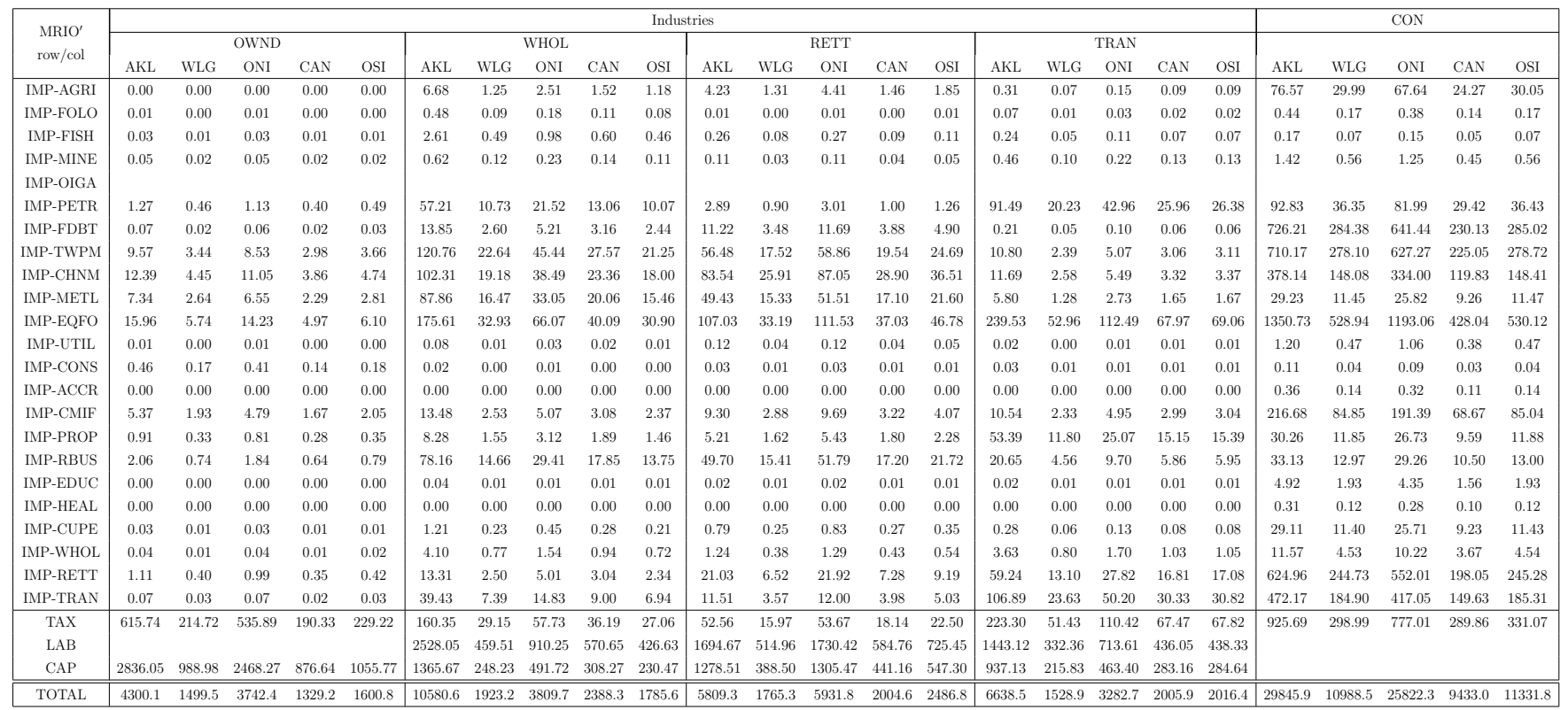

Extended MRIO Table - Part 3-5

\begin{tabular}{|c|c|c|c|c|c|c|c|c|c|c|c|c|c|c|c|c|c|c|c|c|c|c|c|c|}
\hline \multirow{3}{*}{$\begin{array}{c}\mathrm{MRIO}^{\prime} \\
\mathrm{row} / \mathrm{col}\end{array}$} & \multicolumn{24}{|c|}{ INV } \\
\hline & \multicolumn{5}{|c|}{ AGRI } & \multicolumn{5}{|c|}{ FOLO } & \multicolumn{5}{|c|}{ FISH } & \multicolumn{5}{|c|}{ MINE } & \multicolumn{3}{|c|}{ OIGA } & \multirow{2}{*}{$\begin{array}{c}\text { PETR } \\
\text { ONI }\end{array}$} \\
\hline & AKL & WLG & ONI & CAN & OSI & AKL & WLG & ONI & CAN & OSI & AKL & WLG & ONI & CAN & OSI & AKL & WLG & ONI & CAN & OSI & AKL & WLG & $\mathrm{ONI}$ & \\
\hline IMP-AGRI & \begin{tabular}{|l|l|}
0.01 \\
\end{tabular} & 0.00 & 0.14 & 0.01 & 0.07 & \begin{tabular}{|l|l|}
0.00 \\
\end{tabular} & 0.00 & 0.02 & 0.00 & 0.01 & 0.00 & 0.00 & 0.00 & 0.00 & 0.00 & \begin{tabular}{|l|l|}
0.00 \\
\end{tabular} & 0.00 & 0.02 & 0.00 & 0.01 & \begin{tabular}{|l|l|}
0.00 \\
\end{tabular} & 0.01 & 0.14 & 0.02 \\
\hline IMP-FOLO & 0.01 & 0.00 & 0.18 & 0.02 & 0.09 & 0.00 & 0.00 & 0.02 & 0.00 & 0.01 & 0.00 & 0.00 & 0.00 & 0.00 & 0.01 & 0.00 & 0.00 & 0.02 & 0.00 & 0.02 & 0.00 & 0.02 & 0.19 & 0.02 \\
\hline IMP-FISH & 0.00 & 0.00 & 0.03 & 0.00 & 0.02 & 0.00 & 0.00 & 0.00 & 0.00 & 0.00 & 0.00 & 0.00 & 0.00 & 0.00 & 0.00 & 0.00 & 0.00 & 0.00 & 0.00 & 0.00 & 0.00 & 0.00 & 0.04 & 0.00 \\
\hline IMP-MINE & 0.04 & 0.01 & 0.56 & 0.06 & 0.28 & $\mid 0.01$ & 0.00 & 0.07 & 0.00 & 0.03 & 0.00 & 0.00 & 0.01 & 0.00 & 0.02 & $\mid 0.01$ & 0.00 & 0.08 & 0.01 & 0.05 & 0.00 & 0.06 & 0.58 & 0.07 \\
\hline IMP-OIGA & 0.63 & 0.15 & 8.67 & 0.89 & 4.37 & 0.09 & 0.03 & 1.03 & 0.07 & 0.40 & 0.05 & 0.01 & 0.17 & 0.01 & 0.28 & 0.18 & 0.02 & 1.17 & 0.22 & 0.73 & 0.06 & 0.94 & 9.01 & 1.02 \\
\hline IMP-PETR & 0.26 & 0.06 & 3.67 & 0.38 & 1.85 & 0.04 & 0.01 & 0.44 & 0.03 & 0.17 & 0.02 & 0.00 & 0.07 & 0.01 & 0.12 & 0.08 & 0.01 & 0.49 & 0.10 & 0.31 & 0.02 & 0.40 & 3.81 & 0.43 \\
\hline IMP-FDBT & 0.02 & 0.00 & 0.25 & 0.03 & 0.13 & 0.00 & 0.00 & 0.03 & 0.00 & 0.01 & 0.00 & 0.00 & 0.00 & 0.00 & 0.01 & $\mid 0.01$ & 0.00 & 0.03 & 0.01 & 0.02 & 0.00 & 0.03 & 0.26 & 0.03 \\
\hline IMP-TWPM & 0.05 & 0.01 & 0.64 & 0.07 & 0.32 & 0.01 & 0.00 & 0.08 & 0.01 & 0.03 & 0.00 & 0.00 & 0.01 & 0.00 & 0.02 & $\mid 0.01$ & 0.00 & 0.09 & 0.02 & 0.05 & $\mid 0.00$ & 0.07 & 0.66 & 0.08 \\
\hline IMP-CHNM & 0.18 & 0.04 & 2.46 & 0.25 & 1.24 & 0.03 & 0.01 & 0.29 & 0.02 & 0.11 & 0.01 & 0.00 & 0.05 & 0.00 & 0.08 & 0.05 & 0.01 & 0.33 & 0.06 & 0.21 & 0.02 & 0.27 & 2.55 & 0.29 \\
\hline IMP-METL & 0.17 & 0.04 & 2.33 & 0.24 & 1.18 & 0.02 & 0.01 & 0.28 & 0.02 & 0.11 & 0.01 & 0.00 & 0.04 & 0.00 & 0.07 & 0.05 & 0.01 & 0.31 & 0.06 & 0.20 & 0.02 & 0.25 & 2.42 & 0.27 \\
\hline IMP-EQFO & 9.73 & 2.30 & 134.77 & 13.84 & 68.01 & 1.39 & 0.48 & 16.09 & 1.06 & 6.18 & 0.75 & 0.15 & 2.57 & 0.21 & 4.34 & 2.86 & 0.37 & 18.15 & 3.49 & 11.38 & 0.91 & 14.57 & 140.09 & 15.89 \\
\hline IMP-UTIL & 0.00 & 0.00 & 0.01 & 0.00 & 0.00 & 0.00 & 0.00 & 0.00 & 0.00 & 0.00 & 0.00 & 0.00 & 0.00 & 0.00 & 0.00 & 0.00 & 0.00 & 0.00 & 0.00 & 0.00 & 0.00 & 0.00 & 0.01 & 0.00 \\
\hline IMP-CONS & 0.03 & 0.01 & 0.40 & 0.04 & 0.20 & 0.00 & 0.00 & 0.05 & 0.00 & 0.02 & 0.00 & 0.00 & 0.01 & 0.00 & 0.01 & $\mid 0.01$ & 0.00 & 0.05 & 0.01 & 0.03 & 0.00 & 0.04 & 0.42 & 0.05 \\
\hline IMP-ACCR & 0.00 & 0.00 & 0.00 & 0.00 & 0.00 & 0.00 & 0.00 & 0.00 & 0.00 & 0.00 & 0.00 & 0.00 & 0.00 & 0.00 & 0.00 & 0.00 & 0.00 & 0.00 & 0.00 & 0.00 & 0.00 & 0.00 & 0.00 & 0.00 \\
\hline IMP-CMIF & 0.02 & 0.01 & 0.34 & 0.04 & 0.17 & 0.00 & 0.00 & 0.04 & 0.00 & 0.02 & 0.00 & 0.00 & 0.01 & 0.00 & 0.01 & 0.01 & 0.00 & 0.05 & 0.01 & 0.03 & 1.00 & 0.04 & .36 & 0.04 \\
\hline IMP-PROP & 0.02 & 0.01 & 0.34 & 0.04 & 0.17 & 0.00 & 0.00 & 0.04 & 0.00 & 0.02 & 0.00 & 0.00 & 0.01 & 0.00 & 0.01 & 0.01 & 0.00 & 0.05 & 0.01 & 0.03 & 0.00 & 0.04 & 0.36 & 0.04 \\
\hline IMP-RBUS & 0.16 & 0.04 & 2.23 & 0.23 & 1.13 & 0.02 & 0.01 & 0.27 & 0.02 & 0.10 & 0.01 & 0.00 & 0.04 & 0.00 & 0.07 & 0.05 & 0.01 & 0.30 & 0.06 & 0.19 & 0.02 & 0.24 & 2.32 & 0.26 \\
\hline IMP-EDUC & 0.00 & 0.00 & 0.00 & 0.00 & 0.00 & 0.00 & 0.00 & 0.00 & 0.00 & 0.00 & 0.00 & 0.00 & 0.00 & 0.00 & 0.00 & 0.00 & 0.00 & 0.00 & 0.00 & 0.00 & 0.00 & 0.00 & 0.00 & 0.00 \\
\hline IMP-HEAL & 0.00 & 0.00 & 0.00 & 0.00 & 0.00 & 0.00 & 0.00 & 0.00 & 0.00 & 0.00 & 0.00 & 0.00 & 0.00 & 0.00 & 0.00 & 0.00 & 0.00 & 0.00 & 0.00 & 0.00 & 0.00 & 0.00 & 0.00 & 0.00 \\
\hline IMP-CUPE & 0.00 & 0.00 & 0.01 & 0.00 & 0.01 & 0.00 & 0.00 & 0.00 & 0.00 & 0.00 & 0.00 & 0.00 & 0.00 & 0.00 & 0.00 & $\mid 0.00$ & 0.00 & 0.00 & 0.00 & 0.00 & 0.00 & 0.00 & 0.01 & 0.00 \\
\hline IMP-WHOL & 0.01 & 0.00 & 0.13 & 0.01 & 0.07 & 0.00 & 0.00 & 0.02 & 0.00 & 0.01 & 0.00 & 0.00 & 0.00 & 0.00 & 0.00 & 0.00 & 0.00 & 0.02 & 0.00 & 0.01 & 0.00 & 0.01 & 0.14 & 0.02 \\
\hline IMP-RETT & 0.20 & 0.05 & 2.78 & 0.29 & 1.40 & 0.03 & 0.01 & 0.33 & 0.02 & 0.13 & 0.02 & 0.00 & 0.05 & 0.00 & 0.09 & 0.06 & 0.01 & 0.37 & 0.07 & 0.23 & 0.02 & 0.30 & 2.89 & 0.33 \\
\hline IMP-TRAN & 0.04 & 0.01 & 0.50 & 0.05 & 0.25 & 0.01 & 0.00 & 0.06 & 0.00 & 0.02 & 0.00 & 0.00 & 0.01 & 0.00 & 0.02 & 0.01 & 0.00 & 0.07 & 0.01 & 0.04 & 0.00 & 0.05 & 0.52 & 0.06 \\
\hline $\begin{array}{l}\text { TAX } \\
\text { LAB } \\
\text { CAP }\end{array}$ & 1.26 & 0.29 & 16.98 & 1.77 & 8.56 & \begin{tabular}{|l|l|}
0.18 \\
\end{tabular} & 0.06 & 2.03 & 0.14 & 0.78 & 0.10 & 0.02 & 0.32 & 0.03 & 0.55 & \begin{tabular}{|l|l|}
0.37 \\
\end{tabular} & 0.05 & 2.29 & 0.45 & 1.43 & \begin{tabular}{|l|l|}
0.12 \\
\end{tabular} & 1.83 & 17.65 & 2.00 \\
\hline TOTAL & 50.61 & 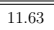 & 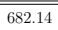 & 71.26 & \begin{tabular}{ll|l}
343.79 \\
\end{tabular} & 7.25 & 2.42 & 81.43 & 25.45 & 31.25 & 3.93 & 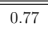 & $\begin{array}{ll}13.02 \\
\end{array}$ & 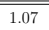 & 21.94 & 14.88 & 1.88 & $\begin{array}{lll}91.86 \\
\end{array}$ & $\begin{array}{lll}17.99 \\
\end{array}$ & \begin{tabular}{c|c|}
57.52 \\
\end{tabular} & 4.76 & 73.65 & |709.07 & 80.43 \\
\hline
\end{tabular}

Extended MRIO Table - Part 3-6 


\begin{tabular}{|c|c|c|c|c|c|c|c|c|c|c|c|c|c|c|c|c|c|c|c|c|c|c|c|c|c|}
\hline \multirow{3}{*}{$\begin{array}{c}\mathrm{MRIO}^{\prime} \\
\mathrm{row} / \mathrm{col}\end{array}$} & \multicolumn{25}{|c|}{ INV } \\
\hline & \multicolumn{5}{|c|}{ FDBT } & \multicolumn{5}{|c|}{ TWPM } & \multicolumn{5}{|c|}{ CHNM } & \multicolumn{5}{|c|}{ METL } & \multicolumn{5}{|c|}{ EQFO } \\
\hline & AKL & WLG & ONI & CAN & OSI & AKL & WLG & ONI & CAN & OSI & AKL & WLG & $\mathrm{ONI}$ & CAN & OSI & AKL & WLG & $\mathrm{ONI}$ & CAN & OSI & AKL & WLG & $\mathrm{ONI}$ & CAN & OSI \\
\hline IMP-AGRI & 0.04 & 0.01 & 0.08 & 0.02 & 0.05 & 0.05 & 0.01 & 0.07 & 0.02 & 0.02 & 0.07 & 0.02 & 0.00 & 0.02 & 0.01 & 0.05 & 0.01 & 0.04 & 0.02 & 0.01 & 0.04 & 0.01 & 0.02 & 0.01 & 0.01 \\
\hline IMP-FOLO & 0.05 & 0.01 & 0.10 & 0.02 & 0.07 & 0.07 & 0.02 & 0.08 & 0.02 & 0.03 & 0.10 & 0.02 & 0.00 & 0.03 & 0.01 & 0.06 & 0.01 & 0.05 & 0.02 & 0.02 & 0.06 & 0.01 & 0.03 & 0.02 & 0.01 \\
\hline IMP-FISH & 0.01 & 0.00 & 0.02 & 0.00 & 0.01 & 0.01 & 0.00 & 0.02 & 0.00 & 0.01 & 0.02 & 0.00 & 0.00 & 0.01 & 0.00 & 0.01 & 0.00 & 0.01 & 0.00 & 0.00 & 0.01 & 0.00 & 0.01 & 0.00 & 0.00 \\
\hline IMP-MINE & 0.15 & 0.03 & 0.31 & 0.07 & 0.21 & 0.21 & 0.05 & 0.26 & 0.08 & 0.10 & 0.30 & 0.06 & 0.01 & 0.09 & 0.05 & 0.20 & 0.03 & 0.16 & 0.07 & 0.06 & 0.18 & 0.03 & 0.10 & 0.06 & 0.04 \\
\hline IMP-OIGA & 2.41 & 0.42 & 4.79 & 1.09 & 3.34 & 3.32 & 0.76 & 4.11 & 1.21 & 1.55 & 4.66 & 0.95 & 0.18 & 1.46 & 0.73 & 3.05 & 0.50 & 2.53 & 1.07 & 0.89 & 2.81 & 0.51 & 1.53 & 0.87 & 0.55 \\
\hline IMP-PETR & 1.02 & 0.18 & 2.03 & 0.46 & 1.41 & 1.41 & 0.32 & 1.74 & 0.51 & 0.66 & 1.97 & 0.40 & 0.08 & 0.62 & 0.31 & 1.29 & 0.21 & 1.07 & 45 & 0.38 & 1.19 & 0.22 & 0.65 & 0.37 & 0.23 \\
\hline IMP-FDBT & 0.07 & 0.01 & 0.14 & 0.03 & 0.10 & 0.10 & 0.02 & 0.12 & 0.04 & 0.05 & 0.14 & 0.03 & 0.01 & 0.04 & 0.02 & 0.09 & 0.01 & 0.07 & 0.03 & 0.03 & 0.08 & 0.01 & 0.04 & 0.03 & 0.02 \\
\hline IMP-TWPM & 0.18 & 0.03 & 0.35 & 0.08 & 0.25 & 0.24 & 0.06 & 0.30 & 0.09 & 0.11 & 0.34 & 0.07 & 0.01 & 0.11 & 0.05 & 0.22 & 0.04 & 0.19 & 0.08 & 0.07 & 0.21 & 0.04 & 0.11 & 0.06 & 0.04 \\
\hline IMP-CHNM & 0.68 & 0.12 & 1.36 & 0.31 & 0.95 & 0.94 & 0.21 & 1.16 & 0.34 & 0.44 & 1.32 & 0.27 & 0.05 & 0.41 & 0.21 & 0.86 & 0.14 & 0.72 & 0.30 & 0.25 & 0.80 & 0.14 & 0.43 & 0.25 & 0.15 \\
\hline IMP-METL & 0.65 & 0.11 & 1.29 & 0.29 & 0.90 & 0.89 & 0.20 & 1.10 & 0.33 & 0.42 & 1.25 & 0.26 & 0.05 & 0.39 & 0.20 & 0.82 & 0.13 & 0.68 & 0.29 & 0.24 & 0.76 & 0.14 & 0.41 & 0.23 & 0.15 \\
\hline IMP-EQFO & 37.43 & 6.53 & 74.49 & 16.96 & 51.86 & 51.69 & 11.78 & 63.85 & 18.82 & 24.16 & 72.41 & 14.76 & 2.85 & 22.67 & 11.31 & \begin{tabular}{|l|l}
47.39 \\
\end{tabular} & 7.79 & 39.35 & 16.64 & 13.91 & 43.76 & 7.91 & 23.82 & 13.48 & 8.49 \\
\hline IMP-UTIL & 0.00 & 0.00 & 0.00 & 0.00 & 0.00 & 0.00 & 0.00 & 0.00 & 0.00 & 0.00 & 0.00 & 0.00 & 0.00 & 0.00 & 0.00 & 0.00 & 0.00 & 0.00 & 0.00 & 0.00 & 0.00 & 0.00 & 0.00 & 0.00 & 0.00 \\
\hline IMP-CONS & 0.11 & 0.02 & 0.22 & 0.05 & 0.16 & 0.15 & 0.04 & 0.19 & 0.06 & 0.07 & 0.22 & 0.04 & 0.01 & 0.07 & 0.03 & 0.14 & 0.02 & 0.12 & 0.05 & 0.04 & 0.13 & 0.02 & 0.07 & 0.04 & 0.03 \\
\hline IMP-ACCR & 0.00 & 0.00 & 0.00 & 0.00 & 0.00 & 0.00 & 0.00 & 0.00 & 0.00 & 0.00 & 0.00 & 0.00 & 0.00 & 0.00 & 0.00 & 0.00 & 0.00 & 0.00 & 0.00 & 0.00 & 0.00 & 0.00 & 0.00 & 0.00 & 0.00 \\
\hline IMP-CMIF & 0.10 & 0.02 & 0.19 & 0.04 & 0.13 & 0.13 & 0.03 & 0.16 & 0.05 & 0.06 & 0.19 & 0.04 & 0.01 & 0.06 & 0.03 & 0.12 & 0.02 & 0.10 & 0.04 & 0.04 & 0.11 & 0.02 & 0.06 & 0.03 & 0.02 \\
\hline IMP-PROP & 0.10 & 0.02 & 0.19 & 0.04 & 0.13 & 0.13 & 0.03 & 0.16 & 0.05 & 0.06 & 0.18 & 0.04 & 0.01 & 0.06 & 0.03 & 0.12 & 0.02 & 0.10 & .04 & 0.04 & 0.11 & .02 & 0.06 & 0.03 & 0.02 \\
\hline IMP-RBUS & 0.62 & 0.11 & 1.23 & 0.28 & 0.86 & 0.86 & 0.20 & 1.06 & 0.31 & 0.40 & 1.20 & 0.24 & 0.05 & 0.38 & 0.19 & 0.78 & 0.13 & 0.65 & 0.28 & 0.23 & 0.72 & 0.13 & 0.39 & 0.22 & 0.14 \\
\hline IMP-EDUC & 0.00 & 0.00 & 0.00 & 0.00 & 0.00 & 0.00 & 0.00 & 0.00 & 0.00 & 0.00 & 0.00 & 0.00 & 0.00 & 0.00 & 0.00 & 0.00 & 0.00 & 0.00 & 0.00 & 0.00 & 0.00 & 0.00 & 0.00 & 0.00 & 0.00 \\
\hline IMP-HEAL & 0.00 & 0.00 & 0.00 & 0.00 & 0.00 & 0.00 & 0.00 & 0.00 & 0.00 & 0.00 & 0.00 & 0.00 & 0.00 & 0.00 & 0.00 & 0.00 & 0.00 & 0.00 & 0.00 & 0.00 & 0.00 & .00 & 0.00 & 0.00 & 0.00 \\
\hline IMP-CUPE & 0.00 & 0.00 & 0.01 & 0.00 & 0.01 & 0.0 & 0.00 & 0.01 & 0.00 & 0.00 & 0.01 & 0.00 & 0.00 & 0.00 & 0.00 & 0.00 & 0.00 & 0.00 & .00 & 0.00 & 0.00 & .00 & 0.00 & 0.00 & 0.00 \\
\hline IMP-WHOL & 0.04 & 0.01 & 0.07 & 0.02 & 0. & 0.6 & 0 & 0.06 & 0.02 & 0. & 0.07 & 0.0 & 0.00 & 0.02 & 0.01 & 0.05 & 0.01 & .04 & .02 & 0.01 & 0.04 & .01 & 0.02 & 0.01 & 0.01 \\
\hline IMP-RETT & 0.77 & 0.13 & 1.54 & 0.35 & 1.07 & 1.07 & 0.24 & 1.32 & 0.39 & 0.50 & 1.50 & 0.30 & 0.06 & 0.47 & 0.23 & 0.98 & 0.16 & 0.81 & 0.34 & 0.29 & 0.90 & 0.16 & 0.49 & 0.28 & 0.18 \\
\hline IMP-TRAN & 0.14 & 0.02 & 0.28 & 0.06 & 0.19 & 0.19 & 0.04 & 0.24 & 0.07 & 0.09 & 0.27 & 0.05 & 0.01 & 0.08 & 0.04 & 0.18 & 0.03 & 0.15 & 0.06 & 0.05 & 0.16 & 0.03 & 0.09 & 0.05 & 0.03 \\
\hline $\begin{array}{l}\text { TAX } \\
\text { LAB } \\
\text { CAP }\end{array}$ & 4.85 & 0.82 & 9.39 & 2.17 & 6.53 & 6.69 & 1.48 & 8.04 & 2.41 & 3.04 & 9.38 & 1.86 & 0.36 & 2.91 & 1.42 & 6.14 & 0.98 & 4.96 & 2.13 & 1.75 & 5.67 & 1.00 & 3.00 & 1.73 & 1.07 \\
\hline " & \begin{tabular}{l|l|l}
194.70 \\
\end{tabular} & 3.03 & $\begin{array}{l}377.05 \\
\end{array}$ & .34 & 18 & 8.88 & .55 & 16 & 96.90 & 2.15 & (6.66 & 74.63 & 14.45 & .73 & "57.16 & (46.51 & 39.39 & 199.15 & $\begin{array}{c}85.69 \\
\end{array}$ & 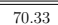 & 2227.63 & 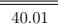 & 120.55 & 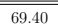 & $\overline{422.91}$ \\
\hline
\end{tabular}

Extended MRIO Table - Part 3-7

\begin{tabular}{|c|c|c|c|c|c|c|c|c|c|c|c|c|c|c|c|c|c|c|c|c|c|c|c|c|c|}
\hline \multirow{3}{*}{$\begin{array}{l}\mathrm{MRIO}^{\prime} \\
\mathrm{row} / \mathrm{col}\end{array}$} & \multicolumn{25}{|c|}{ INV } \\
\hline & \multicolumn{5}{|c|}{ UTIL } & \multicolumn{5}{|c|}{ CONS } & \multicolumn{5}{|c|}{$\mathrm{ACCR}$} & \multicolumn{5}{|c|}{ CMIF } & \multicolumn{5}{|c|}{ PROP } \\
\hline & AKL & WLG & ONI & CAN & OSI & AKL & WLG & ONI & CAN & OSI & AKL & WLG & ONI & CAN & OSI & AKL & WLG & ONI & CAN & OSI & AKL & WLG & ONI & CAN & OSI \\
\hline IMP-AGRI & 0.04 & 0.03 & 0.06 & 0.01 & 0.02 & 0.05 & $\begin{array}{ll}0.02 \\
\end{array}$ & 0.06 & 0.02 & 0.02 & 0.02 & 0.01 & 0.02 & 0.01 & 0.01 & 0.40 & 0.19 & 0.15 & 0.10 & 0.06 & 0.13 & 0.04 & 0.11 & 0.04 & 0.04 \\
\hline IMP-FOLO & 0.05 & 0.03 & 0.08 & 0.01 & 0.02 & 0.06 & 0.02 & 0.07 & 0.02 & 0.03 & 0.02 & 0.01 & 0.03 & 0.01 & 0.02 & 0.52 & 0.24 & 0.20 & 0.13 & 0.08 & 0.17 & 0.05 & 0.14 & 0.06 & 0.05 \\
\hline IMP-FISH & 0.01 & 0.01 & 0.01 & 0.00 & 0.00 & 0.01 & 0.00 & 0.01 & 0.00 & 0.01 & 0.00 & 0.00 & 0.01 & 0.00 & 0.00 & 0.10 & 0.05 & 0.04 & 0.02 & 0.01 & 0.03 & 0.01 & 0.03 & 0.01 & 0.01 \\
\hline IMP-MINE & 0.15 & 0.11 & 0.24 & 0.04 & 0.07 & 0.19 & 0.06 & 0.23 & 0.07 & 0.09 & 0.08 & 0.03 & 0.08 & 0.03 & 0.05 & 1.63 & 0.76 & 0.63 & 0.41 & 0.24 & 0.54 & 0.14 & 0.43 & 0.18 & 0.17 \\
\hline IMP-OIGA & 2.26 & 1.63 & 3.76 & 0.63 & 1.10 & 3.00 & 0.98 & 3.50 & 1.07 & 1.38 & 1.19 & 0.43 & 1.30 & 0.46 & 0.82 & 25.32 & 11.74 & 9.72 & 6.33 & 3.72 & 8.42 & 2.23 & 6.64 & 2.75 & 2.66 \\
\hline IMP-PETR & 0.96 & 0.69 & 1.59 & 0.27 & 0.47 & 1.27 & 0.42 & 1.48 & 0.45 & 0.58 & 0.50 & 0.18 & 0.55 & 0.19 & 0.35 & 10.72 & 4.97 & 4.11 & 2.68 & 1.57 & 3.56 & 0.94 & 2.81 & 1.16 & 1.12 \\
\hline IMP-FDBT & 0.07 & 0.05 & 0.11 & 0.02 & 0.03 & 0.09 & 0.03 & 0.10 & 0.03 & 0.04 & 0.03 & 0.01 & 0.04 & 0.01 & 0.02 & 0.74 & 0.34 & 0.28 & 0.18 & 0.11 & 0.24 & 0.06 & 0.19 & 0.08 & 0.08 \\
\hline IMP-TWPM & 0.17 & 0.12 & 0.28 & 0.05 & 0.08 & 0.22 & 0.07 & 0.26 & 0.08 & 0.10 & 0.09 & 0.03 & 0.10 & 0.03 & 0.06 & 1.87 & 0.86 & 0.72 & 0.47 & 0.27 & 0.62 & 0.16 & 0.49 & 0.20 & 0.20 \\
\hline IMP-CHNM & 0.64 & 0.46 & 1.07 & 0.18 & 0.31 & 0.85 & 0.28 & 0.99 & 0.30 & 0.39 & 0.34 & 0.12 & 0.37 & 0.13 & 0.23 & 7.17 & 3.33 & 2.75 & 1.79 & 1.05 & 2.39 & 0.63 & 1.88 & 0.78 & 0.75 \\
\hline IMP-METL & 0.61 & 0.44 & 1.01 & 0.17 & 0.30 & 0.81 & 0.26 & 0.94 & 0.29 & 0.37 & 0.32 & 0.11 & 0.35 & 0.12 & 0.22 & 6.80 & 3.15 & 2.61 & 1.70 & 1.00 & 2.26 & 0.60 & 1.78 & 0.74 & 0.71 \\
\hline IMP-EQFO & 35.16 & 25.37 & 58.45 & 9.81 & 17.14 & 46.64 & 15.25 & 54.37 & 16.62 & 21.41 & 18.49 & 6.63 & 20.26 & 7.08 & 12.81 & 393.66 & 182.55 & 151.08 & 98.34 & 57.76 & 130.90 & 34.71 & 103.28 & 42.78 & 41.31 \\
\hline IMP-UTIL & 0.00 & 0.00 & 0.00 & 0.00 & 0.00 & 0.00 & 0.00 & 0.00 & 0.00 & 0.00 & 0.00 & 0.00 & 0.00 & 0.00 & 0.00 & 0.02 & 0.01 & 0.01 & 0.00 & 0.00 & 0.01 & 0.00 & 0.00 & 0.00 & 0.00 \\
\hline IMP-CONS & 0.11 & 0.08 & 0.18 & 0.03 & 0.05 & 0.14 & 0.05 & 0.16 & 0.05 & 0.06 & 0.06 & 0.02 & 0.06 & 0.02 & 0.04 & 1.18 & 0.55 & 0.45 & 0.29 & 0.17 & 0.39 & 0.10 & 0.31 & 0.13 & 0.12 \\
\hline IMP-ACCR & 0.00 & 0.00 & 0.00 & 0.00 & 0.00 & 0.00 & 0.00 & 0.00 & 0.00 & 0.00 & 0.00 & 0.00 & 0.00 & 0.00 & 0.00 & 0.00 & 0.00 & 0.00 & 0.00 & 0.00 & 0.00 & 0.00 & 0.00 & 0.00 & 0.00 \\
\hline IMP-CMIF & 0.09 & 0.06 & 0.15 & 0.03 & 0.04 & 0.12 & 0.04 & 0.14 & 0.04 & 0.05 & 0.05 & 0.02 & 0.05 & 0.02 & 0.03 & 1.01 & 0.47 & 0.39 & 0.25 & 0.15 & 0.33 & 0.09 & 0.26 & 0.11 & 0.11 \\
\hline IMP-PROP & 0.09 & 0.06 & 0.15 & 0.02 & 0.04 & 0.12 & 0.04 & 0.14 & 0.04 & 0.05 & 0.05 & 0.02 & 0.05 & 0.02 & 0.03 & 1.00 & 0.46 & 0.38 & 0.25 & 0.15 & 0.33 & 0.09 & 0.26 & 0.11 & 0.10 \\
\hline IMP-RBUS & 0.58 & 0.42 & 0.97 & 0.16 & 0.28 & 0.77 & 0.25 & 0.90 & 0.28 & 0.35 & 0.31 & 0.11 & 0.34 & 0.12 & 0.21 & 6.52 & 3.02 & 2.50 & 1.63 & 0.96 & 2.17 & 0.57 & 1.71 & 0.71 & 0.68 \\
\hline IMP-EDUC & 0.00 & 0.00 & 0.00 & 0.00 & 0.00 & 0.00 & 0.00 & 0.00 & 0.00 & 0.00 & 0.00 & 0.00 & 0.00 & 0.00 & 0.00 & 0.01 & 0.01 & 0.00 & 0.00 & 0.00 & 0.00 & 0.00 & 0.00 & 0.00 & 0.00 \\
\hline IMP-HEAL & 0.00 & 0.00 & 0.00 & 0.00 & 0.00 & 0.00 & 0.00 & 0.00 & 0.00 & 0.00 & 0.00 & 0.00 & 0.00 & 0.00 & 0.00 & 0.00 & 0.00 & 0.00 & 0.00 & 0.00 & 0.00 & 0.00 & 0.00 & 0.00 & 0.00 \\
\hline IMP-CUPE & 0.00 & 0.00 & 0.01 & 0.00 & 0.00 & 0.00 & 0.00 & 0.01 & 0.00 & 0.00 & 0.00 & 0.00 & 0.00 & 0.00 & 0.00 & 0.04 & 0.02 & 0.01 & 0.01 & 0.01 & 0.01 & 0.00 & 0.01 & 0.00 & 0.00 \\
\hline IMP-WHOL & 0.03 & 0.02 & 0.06 & 0.01 & 0.02 & 0.05 & 0.01 & 0.05 & 0.02 & 0.02 & 0.02 & 0.01 & 0.02 & 0.01 & 0.01 & 0.38 & 0.18 & 0.15 & 0.10 & 0.06 & 0.13 & 0.03 & 0.10 & 0.04 & 0.04 \\
\hline IMP-RETT & 0.73 & 0.52 & 1.21 & 0.20 & 0.35 & 0.96 & 0.31 & 1.12 & 0.34 & 0.44 & 0.38 & 0.14 & 0.42 & 0.15 & 0.26 & 8.13 & 3.77 & 3.12 & 2.03 & 1.19 & 2.70 & 0.72 & 2.13 & 0.88 & 0.85 \\
\hline IMP-TRAN & 0.13 & 0.09 & 0.22 & 0.04 & 0.06 & 0.17 & 0.06 & 0.20 & 0.06 & 0.08 & 0.07 & 0.02 & 0.08 & 0.03 & 0.05 & 1.46 & 0.68 & 0.56 & 0.37 & 0.21 & 0.49 & 0.13 & 0.38 & 0.16 & 0.15 \\
\hline TAX & 4.55 & 3.19 & 7.36 & 1.26 & 2.16 & 6.04 & 1.92 & 6.85 & 2.13 & 2.69 & 2.39 & 0.83 & 2.55 & 0.91 & 1.61 & 50.97 & 22.98 & 19.04 & 12.60 & 7.27 & 16.95 & 4.37 & 13.01 & 5.48 & 5.20 \\
\hline LAB & & & & & & & & & & & & & & & & & & & & & & & & & \\
\hline CAP & & & & & & & & & & & & & & & & & & & & & & & & & \\
\hline TOTAL & 182.91 & 128.26 & 295.83 & 50.49 & 86.63 & 242.59 & 77.10 & 275.20 & 85.58 & 108.25 & 96.20 & 33.52 & 102.56 & 36.43 & 64.76 & 2047.65 & 922.95 & 764.68 & 506.34 & 292.01 & \begin{tabular}{|l|l|}
680.89 \\
\end{tabular} & 175.49 & 522.75 & 220.26 & 208.81 \\
\hline
\end{tabular}

Extended MRIO Table - Part 3-8 


\begin{tabular}{|c|c|c|c|c|c|c|c|c|c|c|c|c|c|c|c|c|c|c|c|c|c|c|c|c|c|}
\hline \multirow{3}{*}{$\begin{array}{l}\mathrm{MRIO}^{\prime} \\
\mathrm{row} / \mathrm{col}\end{array}$} & \multicolumn{25}{|c|}{ INV } \\
\hline & \multicolumn{5}{|c|}{ RBUS } & \multicolumn{5}{|c|}{ GOVT } & \multicolumn{5}{|c|}{ EDUC } & \multicolumn{5}{|c|}{ HEAL } & \multicolumn{5}{|c|}{ CUPE } \\
\hline & AKL & WLG & ONI & CAN & OSI & AKL & WLG & ONI & CAN & OSI & AKL & WLG & ONI & CAN & OSI & AKL & WLG & ONI & CAN & OSI & AKL & WLG & ONI & CAN & OSI \\
\hline IMP-AGRI & 0.18 & 0.07 & 0.09 & 0.04 & 0.03 & 0.16 & 0.25 & 0.18 & 0.06 & 0.05 & 0.09 & 0.03 & 0.08 & 0.03 & 0.03 & 0.05 & 0.02 & 0.06 & 0.02 & 0.02 & 0.06 & 0.02 & 0.05 & 0.02 & 0.02 \\
\hline IMP-FOLO & 0.23 & 0.08 & 0.11 & 0.05 & 0.04 & 0.20 & 0.33 & 0.24 & 0.08 & 0.07 & 0.11 & 0.04 & 0.11 & 0.04 & 0.04 & 0.06 & 0.03 & 0.08 & 0.03 & 0.03 & 0.08 & 0.03 & 0.06 & 0.02 & 0.03 \\
\hline IMP-FISH & 0.04 & 0.02 & 0.02 & 0.01 & 0.01 & 0.04 & 0.06 & 0.04 & 0.01 & 0.01 & 0.02 & 0.01 & 0.02 & 0.01 & 0.01 & 0.01 & 0.00 & 0.01 & 0.01 & 0.01 & 0.01 & 0.01 & 0.01 & 0.00 & 0.01 \\
\hline IMP-MINE & 0.72 & 0.26 & 0.36 & 0.16 & 0.12 & 0.64 & 1.03 & 0.74 & 0.25 & 0.22 & 0.35 & 0.12 & 0.34 & 0.12 & 0.14 & 0.20 & 0.08 & 0.24 & 0.09 & 0.10 & 0.25 & 0.10 & 0.18 & 0.07 & 0.08 \\
\hline IMP-OIGA & 11.23 & 4.10 & 5.54 & 2.48 & 1.91 & 9.92 & 16.01 & 11.43 & 3.82 & 3.36 & 5.49 & 1.85 & 5.25 & 1.79 & 2.13 & 3.15 & 1.25 & 3.80 & 1.38 & 1.56 & 3.81 & 1.56 & 2.84 & 1.17 & 1.32 \\
\hline IMP-PETR & 4.75 & 1.73 & 2.34 & 1.05 & 0.81 & 4.20 & 6.78 & 4.84 & 1.62 & 1.42 & 2.32 & 0.78 & 2.22 & 0.76 & 0.90 & 1.33 & 0.53 & 1.61 & 0.58 & 0.66 & 1.61 & 0.66 & 1.20 & 0.49 & 0.56 \\
\hline IMP-FDBT & 0.33 & 0.12 & 0.16 & 0.07 & 0.06 & 0.29 & 0.47 & 0.33 & 0.11 & 0.10 & 0.16 & 0.05 & 0.15 & 0.05 & 0.06 & 0.09 & 0.04 & 0.11 & 0.04 & 0.05 & 0.11 & 0.05 & 0.08 & 0.03 & 0.04 \\
\hline IMP-TWPM & 0.83 & 0.30 & 0.41 & 0.18 & 0.14 & 0.73 & 1.18 & 0.84 & 0.28 & 0.25 & 0.40 & 0.14 & 0.39 & 0.13 & 0.16 & 0.23 & 0.09 & 0.28 & 0.10 & 0.11 & 0.28 & 0.11 & 0.21 & 0.09 & 0.10 \\
\hline IMP-CHNM & 3.18 & 1.16 & 1.57 & 0.70 & 0.54 & 2.81 & 4.54 & 3.24 & 1.08 & 0.95 & 1.55 & 0.52 & 1.49 & 0.51 & 0.60 & 0.89 & 0.35 & 1.08 & 0.39 & 0.44 & 1.08 & 0.44 & 0.81 & 0.33 & 0.37 \\
\hline IMP-METL & 3.02 & 1.10 & 1.49 & 0.67 & 0.51 & 2.67 & 4.30 & 3.07 & 1.03 & 0.90 & 1.47 & 0.50 & 1.41 & 0.48 & 0.57 & 0.85 & 0.34 & 1.02 & 0.37 & 0.42 & 1.02 & 0.42 & 0.76 & 0.31 & 0.35 \\
\hline IMP-EQFO & 174.52 & 63.72 & 86.11 & 38.50 & 29.71 & 154.27 & 248.94 & 177.73 & 59.38 & 52.18 & 85.32 & 28.70 & 81.62 & 27.90 & 33.04 & 48.94 & 19.42 & 59.02 & 21.45 & 24.23 & 59.30 & 24.22 & 44.20 & 18.12 & 20.52 \\
\hline IMP-UTIL & 0.01 & 0.00 & 0.00 & 0.00 & 0.00 & 0.01 & 0.01 & 0.01 & 0.00 & 0.00 & 0.00 & 0.00 & 0.00 & 0.00 & 0.00 & 0.00 & 0.00 & 0.00 & 0.00 & 0.00 & 0.00 & 0.00 & 0.00 & 0.00 & 0.00 \\
\hline IMP-CONS & 0.52 & 0.19 & 0.26 & 0.12 & 0.09 & 0.46 & 0.75 & 0.53 & 0.18 & 0.16 & 0.26 & 0.09 & 0.24 & 0.08 & 0.10 & 0.15 & 0.06 & 0.18 & 0.06 & 0.07 & 0.18 & 0.07 & 0.13 & 0.05 & 0.06 \\
\hline IMP-ACCR & 0.00 & 0.00 & 0.00 & 0.00 & 0.00 & 0.00 & 0.00 & 0.00 & 0.00 & 0.00 & 0.00 & 0.00 & 0.00 & 0.00 & 0.00 & 0.00 & 0.00 & 0.00 & 0.00 & 0.00 & 0.00 & 0.00 & 0.00 & 0.00 & 0.00 \\
\hline IMP-CMIF & 0.45 & 0.16 & 0.22 & 0.10 & 0.08 & 0.39 & 0.64 & 0.45 & 0.15 & 0.13 & 0.22 & 0.07 & 0.21 & 0.07 & 0.08 & 0.13 & 0.05 & 0.15 & 0.05 & 0.06 & 0.15 & 0.06 & 0.11 & 0.05 & 0.05 \\
\hline IMP-PROP & 0.44 & 0.16 & 0.22 & 0.10 & 0.08 & 0.39 & 0.63 & 0.45 & 0.15 & 0.13 & 0.22 & 0.07 & 0.21 & 0.07 & 0.08 & 0.12 & 0.05 & 0.15 & 0.05 & 0.06 & 0.15 & 0.06 & 0.11 & 0.05 & 0.05 \\
\hline IMP-RBUS & 2.89 & 1.06 & 1.43 & 0.64 & 0.49 & 2.56 & 4.12 & 2.94 & 0.98 & 0.86 & 1.41 & 0.48 & 1.35 & 0.46 & 0.55 & 0.81 & 0.32 & 0.98 & 0.36 & 0.40 & 0.98 & 0.40 & 0.73 & 0.30 & 0.34 \\
\hline IMP-EDUC & 0.01 & 0.00 & 0.00 & 0.00 & 0.00 & 0.00 & 0.01 & 0.01 & 0.00 & 0.00 & 0.00 & 0.00 & 0.00 & 0.00 & 0.00 & 0.00 & 0.00 & 0.00 & 0.00 & 0.00 & 0.00 & 0.00 & 0.00 & 0.00 & 0.00 \\
\hline IMP-HEAL & 0.00 & 0.00 & 0.00 & 0.00 & 0.00 & 0.00 & 0.00 & 0.00 & 0.00 & 0.00 & 0.00 & 0.00 & 0.00 & 0.00 & 0.00 & 0.00 & 0.00 & 0.00 & 0.00 & 0.00 & 0.00 & 0.00 & 0.00 & 0.00 & 0.00 \\
\hline IMP-CUPE & 0.02 & 0.01 & 0.01 & 0.00 & 0.00 & 0.02 & 0.02 & 0.02 & 0.01 & 0.01 & 0.01 & 0.00 & 0.01 & 0.00 & 0.00 & 0.00 & 0.00 & 0.01 & 0.00 & 0.00 & 0.01 & 0.00 & 0.00 & 0.00 & 0.00 \\
\hline IMP-WHOL & 0.17 & 0.06 & 0.08 & 0.04 & 0.03 & 0.15 & 0.24 & 0.17 & 0.06 & 0.05 & 0.08 & 0.03 & 0.08 & 0.03 & 0.03 & 0.05 & 0.02 & 0.06 & 0.02 & 0.02 & 0.06 & 0.02 & 0.04 & 0.02 & 0.02 \\
\hline IMP-RETT & 3.60 & 1.32 & 1.78 & 0.80 & 0.61 & 3.19 & 5.14 & 3.67 & 1.23 & 1.08 & 1.76 & 0.59 & 1.69 & 0.58 & 0.68 & 1.01 & 0.40 & 1.22 & 0.44 & 0.50 & 1.22 & 0.50 & 0.91 & 0.37 & 0.42 \\
\hline IMP-TRAN & 0.65 & 0.24 & 0.32 & 0.14 & 0.11 & 0.57 & 0.92 & 0.66 & 0.22 & 0.19 & 0.32 & 0.11 & 0.30 & 0.10 & 0.12 & 0.18 & 0.07 & 0.22 & 0.08 & 0.09 & 0.22 & 0.09 & 0.16 & 0.07 & 0.08 \\
\hline TAX & 22.60 & 8.02 & 10.85 & 4.93 & 3.74 & 19.98 & 31.33 & 22.39 & 7.61 & 6.57 & 11.05 & 3.61 & 10.28 & 3.58 & 4.16 & 6.34 & 2.44 & 7.44 & 2.75 & 3.05 & 7.68 & 3.05 & 5.57 & 2.32 & 2.58 \\
\hline LAB & & & & & & & & & & & & & & & & & & & & & & & & & \\
\hline CAP & & & & & & & & & & & & & & & & & & & & & & & & & \\
\hline TOTAL & \begin{tabular}{|l|l}
907.79 \\
\end{tabular} & 322.19 & 435.85 & 198.23 & 150.18 & \begin{tabular}{|l|l|}
802.47 \\
\end{tabular} & 1258.63 & 899.59 & 305.72 & 263.80 & 443.79 & 145.12 & 413.09 & 143.64 & 167.00 & \begin{tabular}{|l}
254.59 \\
\end{tabular} & 98.18 & 298.72 & 110.42 & 122.47 & \begin{tabular}{|l|l|}
308.48 \\
\end{tabular} & 122.45 & 223.73 & 93.29 & 103.76 \\
\hline
\end{tabular}

Extended MRIO Table - Part 3-9

\begin{tabular}{|c|c|c|c|c|c|c|c|c|c|c|c|c|c|c|c|c|c|c|c|c|c|}
\hline \multirow{3}{*}{$\begin{array}{l}\mathrm{MRIO}^{\prime} \\
\mathrm{row} / \mathrm{col}\end{array}$} & \multicolumn{20}{|c|}{ INV } & \multirow[t]{3}{*}{ GOV } \\
\hline & \multicolumn{5}{|c|}{ OWND } & \multicolumn{5}{|c|}{ WHOL } & \multicolumn{5}{|c|}{ RETT } & \multicolumn{5}{|c|}{ TRAN } & \\
\hline & AKL & WLG & ONI & CAN & OSI & AKL & WLG & ONI & CAN & OSI & AKL & WLG & ONI & CAN & OSI & AKL & WLG & ONI & CAN & OSI & \\
\hline IMP-AGRI & 0.91 & 0.32 & 0.81 & 0.28 & 0.35 & 0.10 & 0.02 & 0.04 & 0.02 & 0.02 & 0.07 & 0.02 & 0.07 & 0.02 & 0.03 & 0.01 & 0.00 & 0.01 & 0.00 & \begin{tabular}{l|l|}
0.00 & \\
\end{tabular} & 0.39 \\
\hline IMP-FOLO & 1.17 & 0.42 & 1.05 & 0.37 & 0.45 & 0.13 & 0.02 & 0.05 & 0.03 & 0.02 & 0.09 & 0.03 & 0.09 & 0.03 & 0.04 & 0.02 & 0.00 & 0.01 & 0.00 & 0.01 & 0.00 \\
\hline IMP-FISH & 0.22 & 0.08 & 0.20 & 0.07 & 0.08 & 0.02 & 0.00 & 0.01 & 0.01 & 0.00 & 0.02 & 0.01 & 0.02 & 0.01 & 0.01 & 0.00 & 0.00 & 0.00 & 0.00 & 0.00 & \\
\hline IMP-MINE & 3.66 & 1.31 & 3.27 & 1.14 & 1.40 & 0.39 & 0.07 & 0.15 & 0.09 & 0.07 & 0.27 & 0.09 & 0.29 & 0.10 & 0.12 & 0.05 & 0.01 & 0.03 & 0.02 & 0.02 & \\
\hline IMP-OIGA & 56.90 & 20.41 & 50.89 & 17.77 & 21.79 & 6.12 & 1.14 & 2.26 & 1.40 & 1.06 & 4.25 & 1.33 & 4.46 & 1.48 & 1.87 & 0.79 & 0.19 & 0.40 & 0.24 & 0.25 & \\
\hline IMP-PETR & 24.08 & 8.64 & 21.54 & 7.52 & 9.22 & 2.59 & 0.48 & 0.96 & 0.59 & 0.45 & 1.80 & 0.56 & 1.89 & 0.63 & 0.79 & 0.34 & 0.08 & 0.17 & 0.10 & 0.10 & \\
\hline IMP-FDBT & 1.65 & 0.59 & 1.48 & 0.52 & 0.63 & 0.18 & 0.03 & 0.07 & 0.04 & 0.03 & 0.12 & 0.04 & 0.13 & 0.04 & 0.05 & 0.02 & 0.01 & 0.01 & 0.01 & 0.01 & 2.62 \\
\hline IMP-TWPM & 4.19 & 1.50 & 3.75 & 1.31 & 1.61 & 0.45 & 0.08 & 0.17 & 0.10 & 0.08 & 0.31 & 0.10 & 0.33 & 0.11 & 0.14 & 0.06 & 0.01 & 0.03 & 0.02 & 0.02 & 0.03 \\
\hline IMP-CHNM & 16.12 & 5.78 & 14.42 & 5.03 & 6.17 & 1.73 & 0.32 & 0.64 & 0.40 & 0.30 & 1.20 & 0.38 & 1.26 & 0.42 & 0.53 & 0.22 & 0.05 & 0.11 & 0.07 & 0.07 & 163.85 \\
\hline IMP-METL & 15.28 & 5.48 & 13.67 & 4.77 & 5.85 & 1.64 & 0.31 & 0.61 & 0.37 & 0.29 & 1.14 & 0.36 & 1.20 & 0.40 & 0.50 & 0.21 & 0.05 & 0.11 & 0.06 & 0.07 & \\
\hline IMP-EQFO & 884.54 & 317.34 & 791.14 & 276.22 & 338.82 & 95.13 & 17.79 & 35.20 & 21.69 & 16.52 & 66.10 & 20.67 & 69.37 & 23.04 & 29.12 & 12.31 & 2.92 & 6.26 & 3.76 & 3.85 & 14.20 \\
\hline IMP-UTIL & 0.03 & 0.01 & 0.03 & 0.01 & 0.01 & 0.00 & 0.00 & 0.00 & 0.00 & 0.00 & 0.00 & 0.00 & 0.00 & 0.00 & 0.00 & 0.00 & 0.00 & 0.00 & 0.00 & 0.00 & \\
\hline IMP-CONS & 2.65 & 0.95 & 2.37 & 0.83 & 1.02 & 0.29 & 0.05 & 0.11 & 0.07 & 0.05 & 0.20 & 0.06 & 0.21 & 0.07 & 0.09 & 0.04 & 0.01 & 0.02 & 0.01 & 0.01 & 0.00 \\
\hline IMP-ACCR & 0.00 & 0.00 & 0.00 & 0.00 & 0.00 & 0.00 & 0.00 & 0.00 & 0.00 & 0.00 & 0.00 & 0.00 & 0.00 & 0.00 & 0.00 & 0.00 & 0.00 & 0.00 & 0.00 & 0.00 & \\
\hline IMP-CMIF & 2.26 & 0.81 & 2.02 & 0.71 & 0.87 & 0.24 & 0.05 & 0.09 & 0.06 & 0.04 & 0.17 & 0.05 & 0.18 & 0.06 & 0.07 & 0.03 & 0.01 & 0.02 & 0.01 & 0.01 & 0.64 \\
\hline IMP-PROP & 2.25 & 0.81 & 2.01 & 0.70 & 0.86 & 0.24 & 0.05 & 0.09 & 0.06 & 0.04 & 0.17 & 0.05 & 0.18 & 0.06 & 0.07 & 0.03 & 0.01 & 0.02 & 0.01 & 0.01 & 2.78 \\
\hline IMP-RBUS & 14.65 & 5.26 & 13.10 & 4.58 & 5.61 & 1.58 & 0.29 & 0.58 & 0.36 & 0.27 & 1.09 & 0.34 & 1.15 & 0.38 & 0.48 & 0.20 & 0.05 & 0.10 & 0.06 & 0.06 & 56.32 \\
\hline IMP-EDUC & 0.03 & 0.01 & 0.02 & 0.01 & 0.01 & 0.00 & 0.00 & 0.00 & 0.00 & 0.00 & 0.00 & 0.00 & 0.00 & 0.00 & 0.00 & 0.00 & 0.00 & 0.00 & 0.00 & 0.00 & 11.20 \\
\hline IMP-HEAL & 0.00 & 0.00 & 0.00 & 0.00 & 0.00 & 0.00 & 0.00 & 0.00 & 0.00 & 0.00 & 0.00 & 0.00 & 0.00 & 0.00 & 0.00 & 0.00 & 0.00 & 0.00 & 0.00 & 0.00 & 1.29 \\
\hline IMP-CUPE & 0.09 & 0.03 & 0.08 & 0.03 & 0.03 & 0.01 & 0.00 & 0.00 & 0.00 & 0.00 & 0.01 & 0.00 & 0.01 & 0.00 & 0.00 & 0.00 & 0.00 & 0.00 & 0.00 & 0.00 & 5.64 \\
\hline IMP-WHOL & 0.86 & 0.31 & 0.77 & 0.27 & 0.33 & 0.09 & 0.02 & 0.03 & 0.02 & 0.02 & 0.06 & 0.02 & 0.07 & 0.02 & 0.03 & 0.01 & 0.00 & 0.01 & 0.00 & 0.00 & 0.08 \\
\hline IMP-RETT & 18.27 & 6.55 & 16.34 & 5.70 & 7.00 & 1.96 & 0.37 & 0.73 & 0.45 & 0.34 & 1.37 & 0.43 & 1.43 & 0.48 & 0.60 & 0.25 & 0.06 & 0.13 & 0.08 & 0.08 & 46.60 \\
\hline IMP-TRAN & 3.28 & 1.18 & 2.94 & 1.03 & 1.26 & 0.35 & 0.07 & 0.13 & 0.08 & 0.06 & 0.25 & 0.08 & 0.26 & 0.09 & 0.11 & 0.05 & 0.01 & 0.02 & 0.01 & 0.01 & 4.95 \\
\hline $\begin{array}{l}\text { TAX } \\
\text { LAB }\end{array}$ & 114.54 & 39.94 & 99.68 & 35.40 & 42.64 & 12.32 & 2.24 & 4.44 & 2.78 & 2.08 & 8.56 & 2.60 & 8.74 & 2.95 & 3.66 & 1.59 & 0.37 & 0.79 & 0.48 & \begin{tabular}{l|l}
0.48 \\
\end{tabular} & 671.39 \\
\hline $\begin{array}{c}\text { CAP } \\
\text { TOTAL }\end{array}$ & 01.02 & 1604.45 & 400435 & 2221 & 1712.81 & 494.85 & 89.94 & 818 & 170 & 83.51 & 34384 & 4.48 & 351.09 & 11864 & 147.19 & 64.04 & 14.75 & 31.67 & 19.35 & 19.45 & 2866100 \\
\hline
\end{tabular}

Extended MRIO Table - Part 3-10 


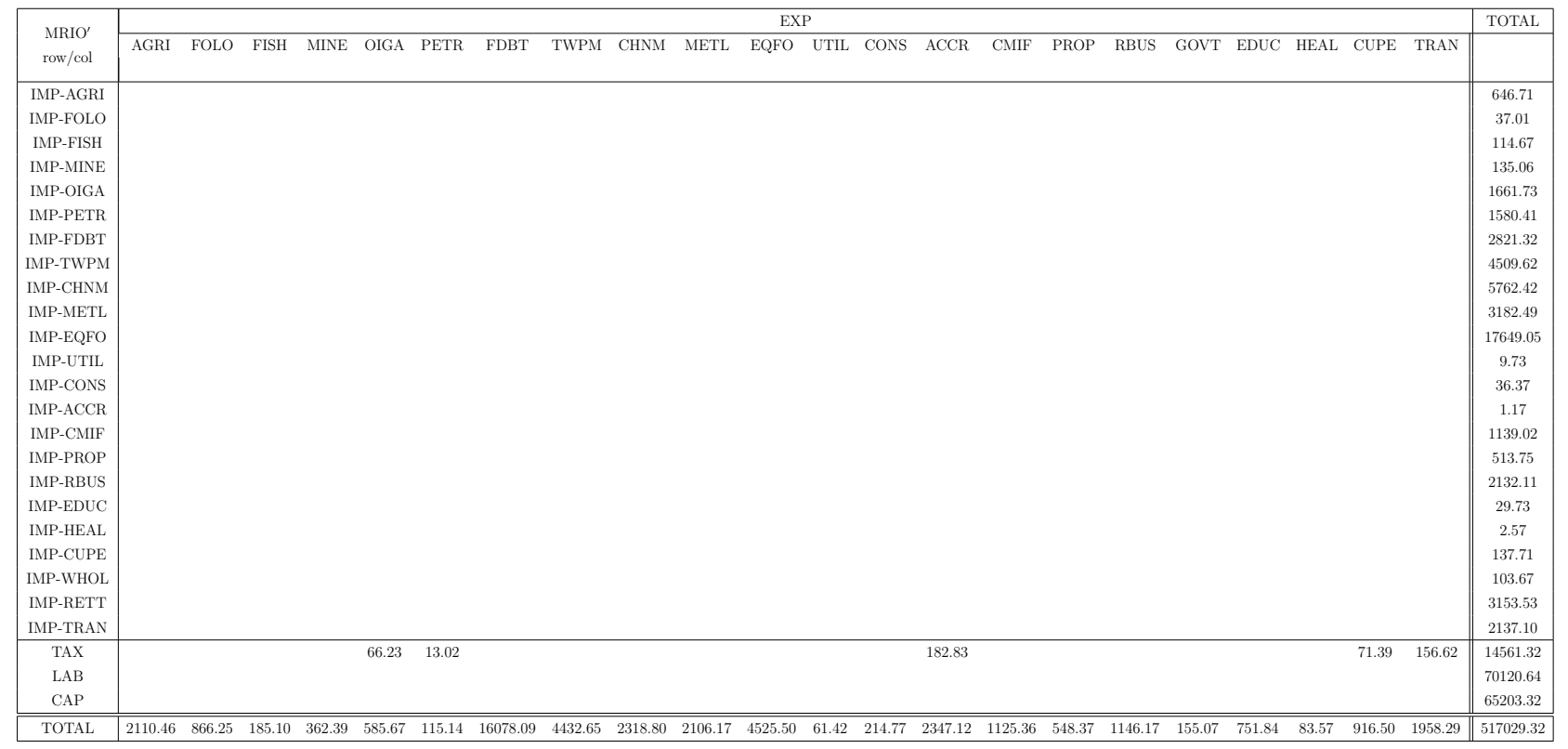

Extended MRIO Table — Part 3-11 


\section{Appendix $\mathrm{O}$}

\section{Simulation Results - Disaster Scenario}

This appendix provides the results for the illustrative simulations discussed in chapter 2 .

\begin{tabular}{|l|c|ccc|}
\hline \multicolumn{5}{|c|}{ Regional Capital Stocks } \\
\hline \multirow{2}{*}{ Region } & BASEYEAR $(\$ \mathrm{M})$ & \multicolumn{4}{|c|}{$\%$ ON BASEYEAR } \\
\cline { 3 - 5 } & & SIM101 & SIM102 & SIM103 \\
\hline AKL & 154208 & 0.0 & 0.0 & 0.0 \\
WLG & 62648 & -8.2 & -8.2 & -8.2 \\
ONI & 144170 & 0.0 & 0.0 & 0.0 \\
CAN & 49536 & 0.0 & 0.0 & 0.0 \\
OSI & 59263 & 0.0 & 0.0 & 0.0 \\
\hline NZ & 469826 & -1.1 & -1.1 & -1.1 \\
\hline
\end{tabular}

Table O.1: Regional Capital Stocks 


\begin{tabular}{|l|c|ccc|}
\hline \multicolumn{5}{|c|}{ Regional Output } \\
\hline \multirow{2}{*}{ Region } & BASEYEAR $(\$ \mathrm{M})$ & \multicolumn{4}{|c|}{$\Delta$ ON BASEYEAR } \\
\cline { 3 - 5 } & & SIM101 & SIM102 & SIM103 \\
\hline AKL & 106148 & -0.1 & -0.1 & -0.2 \\
WLG & 36082 & -2.6 & -2.5 & -2.1 \\
ONI & 100332 & -0.1 & -0.2 & -0.2 \\
CAN & 33657 & -0.1 & -0.2 & -0.2 \\
OSI & 43428 & -0.1 & -0.2 & -0.2 \\
\hline NZ & 319647 & -0.4 & -0.4 & -0.4 \\
\hline
\end{tabular}

Table O.2: Regional Output

\begin{tabular}{|l|ccc|}
\hline \multicolumn{4}{|c|}{ Regional Output Price Index } \\
\hline \multirow{2}{*}{ Region } & \multicolumn{1}{c|}{$\Delta$ ON BASEYEAR } \\
\cline { 2 - 4 } & SIM101 & SIM102 & SIM103 \\
\hline AKL & 0.2 & 0.2 & 0.1 \\
WLG & 1.4 & 1.9 & 3.3 \\
ONI & 0.0 & 0.0 & 0.0 \\
CAN & 0.1 & 0.1 & 0.0 \\
OSI & 0.0 & 0.0 & 0.0 \\
\hline NZ & 0.2 & 0.3 & 0.4 \\
\hline
\end{tabular}

Table O.3: Regional Output Price Index 


\begin{tabular}{|l|c|ccc|}
\hline \multicolumn{4}{|c|}{ Industry Capital Stocks } \\
\hline \multirow{2}{*}{ Industry } & BASEYEAR $(\$ \mathrm{M})$ & \multicolumn{3}{c|}{$\Delta$ ON BASEYEAR } \\
\cline { 2 - 5 } & & SIM101 & SIM102 & SIM103 \\
\hline AGRI & 14374 & 0.0 & 0.0 & 0.0 \\
FOLO & 1351 & 0.0 & 0.0 & 0.0 \\
FISH & 962 & 0.0 & 0.0 & 0.0 \\
MINE & 878 & 0.0 & 0.0 & 0.0 \\
OIGA & 4450 & 0.0 & 0.0 & 0.0 \\
PETR & 455 & 0.0 & 0.0 & 0.0 \\
FDBT & 11491 & 0.0 & 0.0 & 0.0 \\
TWPM & 8052 & 0.0 & 0.0 & 0.0 \\
CHNM & 4959 & 0.0 & 0.0 & 0.0 \\
METL & 3412 & 0.0 & 0.0 & 0.0 \\
EQFO & 5892 & 0.0 & 0.0 & 0.0 \\
UTIL & 23050 & -0.2 & -0.2 & -0.2 \\
CONS & 5578 & -0.1 & -0.1 & -0.1 \\
ACCR & 5251 & -0.5 & -0.5 & -0.5 \\
CMIF & 15121 & -2.0 & -2.0 & -2.0 \\
PROP & 83623 & -1.0 & -1.0 & -1.0 \\
RBUS & 6376 & -1.6 & -1.6 & -1.6 \\
GOVT & 43300 & -5.3 & -5.3 & -5.3 \\
EDUC & 16100 & -1.1 & -1.1 & -1.1 \\
HEAL & 10847 & -1.1 & -1.1 & -1.1 \\
CUPE & 11086 & -0.7 & -0.7 & -0.7 \\
OWND & 163680 & -0.6 & -0.6 & -0.6 \\
WHOL & 8281 & -0.1 & -0.1 & -0.1 \\
RETT & 7265 & -0.5 & -0.5 & -0.5 \\
TRAN & 13992 & -1.0 & -1.0 & -1.0 \\
\hline All & 469826 & -1.1 & -1.1 & -1.1 \\
\hline
\end{tabular}

Table O.4: Industry Capital Stocks 


\begin{tabular}{|l|c|ccc|}
\hline \multicolumn{5}{|c|}{ Industrial Output } \\
\hline \multirow{2}{*}{ Industry } & BASEYEAR $(\$ \mathrm{M})$ & $\%$ ON BASEYEAR \\
\cline { 2 - 5 } & & SIM101 & SIM102 & SIM103 \\
\hline AGRI & 16884 & -0.2 & -0.2 & -0.1 \\
FOLO & 2978 & -0.3 & -0.3 & -0.3 \\
FISH & 853 & -0.3 & -0.3 & -0.3 \\
MINE & 1197 & -0.1 & -0.1 & -0.1 \\
OIGA & 2479 & 0.0 & 0.0 & 0.0 \\
PETR & 3683 & -0.3 & -0.3 & -0.3 \\
FDBT & 27682 & -0.1 & -0.1 & -0.1 \\
TWPM & 13057 & -0.2 & -0.2 & -0.2 \\
CHNM & 9078 & -0.2 & -0.2 & -0.2 \\
METL & 8105 & -0.1 & -0.1 & -0.2 \\
EQFO & 10632 & -0.1 & -0.1 & -0.1 \\
UTIL & 12609 & -0.3 & -0.3 & -0.3 \\
CONS & 29305 & -0.4 & -0.4 & -0.4 \\
ACCR & 6454 & -0.2 & -0.2 & -0.2 \\
CMIF & 24027 & -1.0 & -1.0 & -1.0 \\
PROP & 13658 & -0.7 & -0.7 & -0.7 \\
RBUS & 24532 & -0.6 & -0.6 & -0.6 \\
GOVT & 13460 & -0.4 & -0.5 & -0.5 \\
EDUC & 8229 & -0.3 & -0.3 & -0.3 \\
HEAL & 12552 & -0.4 & -0.4 & -0.4 \\
CUPE & 11764 & -0.5 & -0.5 & -0.5 \\
OWND & 12472 & -0.5 & -0.5 & -0.5 \\
WHOL & 20487 & -0.3 & -0.3 & -0.4 \\
RETT & 17998 & -0.4 & -0.4 & -0.4 \\
TRAN & 15472 & -0.7 & -0.7 & -0.7 \\
\hline All & 319647 & -0.4 & -0.4 & -0.4 \\
\hline
\end{tabular}

Table O.5: Industrial Output 


\begin{tabular}{|l|c|ccc|}
\hline & Output By Industry - Region WLG \\
\hline \multirow{2}{*}{ Industry } & BASEYEAR $(\$ \mathrm{M})$ & \multicolumn{3}{c|}{$\Delta$ ON BASEYEAR } \\
\cline { 2 - 5 } & & SIM101 & SIM102 & SIM103 \\
\hline AGRI & 169 & 1.1 & 0.5 & -0.4 \\
FOLO & 56 & 0.6 & 0.2 & -0.5 \\
FISH & 16 & 0.4 & 0.1 & -0.4 \\
MINE & 12 & 0.5 & 0.2 & -0.3 \\
OIGA & 232 & 0.0 & 0.0 & 0.0 \\
PETR & 0 & 0.0 & 0.0 & 0.0 \\
FDBT & 958 & 0.7 & 0.3 & -0.2 \\
TWPM & 893 & 1.1 & 0.5 & -0.4 \\
CHNM & 1059 & 0.6 & 0.2 & -0.4 \\
METL & 498 & 0.8 & 0.4 & -0.3 \\
EQFO & 850 & 1.4 & 0.7 & -0.4 \\
UTIL & 2173 & -0.8 & -0.8 & -0.8 \\
CONS & 2865 & -0.1 & -0.4 & -0.7 \\
ACCR & 649 & -1.4 & -1.6 & -1.8 \\
CMIF & 4891 & -6.4 & -5.9 & -5.0 \\
PROP & 1326 & -8.4 & -8.0 & -7.0 \\
RBUS & 3924 & -4.7 & -4.4 & -3.8 \\
GOVT & 4799 & -2.1 & -1.9 & -1.7 \\
EDUC & 910 & -0.1 & -0.6 & -0.8 \\
HEAL & 1393 & -2.1 & -1.9 & -1.5 \\
CUPE & 1691 & -1.9 & -1.5 & -0.6 \\
OWND & 1500 & -3.7 & -3.5 & -2.9 \\
WHOL & 1923 & -0.6 & -0.4 & 0.3 \\
RETT & 1765 & -1.8 & -1.5 & -0.4 \\
TRAN & 1529 & -4.3 & -4.0 & -3.3 \\
\hline All & 36082 & -2.6 & -2.5 & -2.1 \\
\hline
\end{tabular}

Table O.6: Output By Industry — Region WLG 


\begin{tabular}{|l|c|ccc|}
\hline \multicolumn{5}{|c|}{ Output By Industry - Region AKL } \\
\hline Industry & BASEYEAR $(\$ \mathrm{M})$ & \multicolumn{3}{c|}{$\Delta$ ON BASEYEAR } \\
\cline { 2 - 5 } & & SIM101 & SIM102 & SIM103 \\
\hline AGRI & 737 & -0.3 & -0.3 & -0.2 \\
FOLO & 169 & -0.3 & -0.3 & -0.3 \\
FISH & 82 & -0.3 & -0.3 & -0.3 \\
MINE & 97 & -0.2 & -0.2 & -0.2 \\
OIGA & 15 & 0.0 & 0.0 & 0.0 \\
PETR & 0 & 0.0 & 0.0 & 0.0 \\
FDBT & 5648 & -0.2 & -0.2 & -0.1 \\
TWPM & 4032 & -0.3 & -0.3 & -0.2 \\
CHNM & 5346 & -0.3 & -0.3 & -0.2 \\
METL & 3117 & -0.2 & -0.2 & -0.2 \\
EQFO & 4836 & -0.2 & -0.2 & -0.1 \\
UTIL & 3099 & -0.3 & -0.3 & -0.2 \\
CONS & 9014 & -0.5 & -0.5 & -0.4 \\
ACCR & 1862 & -0.2 & -0.1 & -0.1 \\
CMIF & 10852 & 0.4 & 0.3 & 0.1 \\
PROP & 5143 & 0.1 & 0.0 & -0.1 \\
RBUS & 11056 & 0.1 & 0.1 & 0.0 \\
GOVT & 3060 & 0.4 & 0.3 & 0.2 \\
EDUC & 2782 & -0.4 & -0.3 & -0.3 \\
HEAL & 3613 & -0.2 & -0.2 & -0.3 \\
CUPE & 4261 & -0.2 & -0.3 & -0.5 \\
OWND & 4300 & -0.1 & -0.1 & -0.2 \\
WHOL & 10581 & -0.3 & -0.3 & -0.4 \\
RETT & 5809 & -0.2 & -0.2 & -0.4 \\
TRAN & 6639 & -0.3 & -0.4 & -0.5 \\
\hline All & 106148 & -0.1 & -0.1 & -0.2 \\
\hline
\end{tabular}

Table O.7: Output By Industry — Region AKL 


\begin{tabular}{|l|c|ccc|}
\hline \multicolumn{5}{|c|}{ Output By Industry - Region CAN } \\
\hline \multirow{2}{*}{ Industry } & BASEYEAR $(\$ \mathrm{M})$ & \multicolumn{3}{c|}{$\Delta$ ON BASEYEAR } \\
\cline { 2 - 5 } & & SIM101 & SIM102 & SIM103 \\
\hline AGRI & 1038 & -0.2 & -0.2 & -0.2 \\
FOLO & 127 & -0.3 & -0.3 & -0.3 \\
FISH & 22 & -0.3 & -0.3 & -0.3 \\
MINE & 117 & -0.1 & -0.1 & -0.1 \\
OIGA & 0 & 0.0 & 0.0 & 0.0 \\
PETR & 0 & 0.0 & 0.0 & 0.0 \\
FDBT & 2533 & -0.2 & -0.1 & -0.1 \\
TWPM & 1453 & -0.3 & -0.2 & -0.2 \\
CHNM & 1657 & -0.2 & -0.2 & -0.2 \\
METL & 1083 & -0.2 & -0.2 & -0.2 \\
EQFO & 1474 & -0.2 & -0.2 & -0.1 \\
UTIL & 856 & -0.3 & -0.3 & -0.2 \\
CONS & 3180 & -0.5 & -0.4 & -0.4 \\
ACCR & 705 & -0.1 & -0.1 & -0.1 \\
CMIF & 2683 & 0.4 & 0.3 & 0.1 \\
PROP & 1664 & 0.1 & 0.0 & -0.1 \\
RBUS & 2414 & 0.1 & 0.1 & 0.0 \\
GOVT & 1166 & 0.4 & 0.3 & 0.2 \\
EDUC & 901 & -0.3 & -0.3 & -0.3 \\
HEAL & 1567 & -0.2 & -0.2 & -0.3 \\
CUPE & 1288 & -0.2 & -0.3 & -0.5 \\
OWND & 1329 & -0.1 & -0.1 & -0.2 \\
WHOL & 2388 & -0.3 & -0.3 & -0.4 \\
RETT & 2005 & -0.2 & -0.3 & -0.4 \\
TRAN & 2006 & -0.3 & -0.3 & -0.5 \\
\hline All & 33657 & -0.1 & -0.2 & -0.2 \\
\hline
\end{tabular}

Table O.8: Output By Industry — Region CAN 


\begin{tabular}{|c|c|c|c|c|}
\hline \multicolumn{5}{|c|}{ Industry Employment (Full-Time Equivalents) } \\
\hline \multirow{2}{*}{ Industry } & \multirow{2}{*}{ BASEYEAR (000s) } & \multicolumn{3}{|c|}{$\% \Delta$ ON BASEYEAR } \\
\hline & & SIM101 & SIM102 & SIM103 \\
\hline AGRI & 114 & 0.0 & 0.0 & 0.1 \\
\hline FOLO & 5 & 0.0 & 0.0 & 0.0 \\
\hline FISH & 2 & 0.0 & 0.0 & 0.1 \\
\hline MINE & 4 & 0.1 & 0.1 & 0.1 \\
\hline OIGA & 0 & 0.2 & 0.1 & 0.1 \\
\hline PETR & 6 & -0.2 & -0.2 & -0.1 \\
\hline FDBT & 56 & 0.1 & 0.1 & 0.2 \\
\hline TWPM & 65 & 0.1 & 0.0 & 0.0 \\
\hline CHNM & 21 & 0.1 & 0.0 & -0.1 \\
\hline METL & 26 & 0.1 & 0.0 & 0.0 \\
\hline EQFO & 50 & 0.1 & 0.1 & 0.1 \\
\hline UTIL & 9 & -0.2 & -0.2 & -0.1 \\
\hline CONS & 142 & -0.3 & -0.3 & -0.3 \\
\hline ACCR & 88 & 0.0 & 0.0 & 0.0 \\
\hline CMIF & 84 & 0.2 & 0.2 & 0.2 \\
\hline PROP & 48 & -0.1 & -0.1 & 0.0 \\
\hline RBUS & 198 & 0.0 & 0.0 & 0.0 \\
\hline GOVT & 77 & 0.6 & 0.5 & 0.4 \\
\hline EDUC & 120 & -0.1 & -0.1 & -0.1 \\
\hline HEAL & 164 & -0.1 & -0.1 & -0.1 \\
\hline CUPE & 74 & 0.0 & 0.0 & -0.1 \\
\hline OWND & 0 & 0.0 & 0.0 & 0.0 \\
\hline WHOL & 89 & -0.1 & -0.1 & -0.1 \\
\hline RETT & 163 & -0.1 & -0.1 & 0.0 \\
\hline TRAN & 62 & -0.3 & -0.3 & -0.3 \\
\hline All & 1668 & 0.0 & 0.0 & 0.0 \\
\hline
\end{tabular}

Table O.9: Industry Employment 


\begin{tabular}{|c|c|c|c|c|}
\hline \multicolumn{5}{|c|}{ Employment By Industry — Region WLG (FTEs) } \\
\hline \multirow{2}{*}{ Industry } & \multirow{2}{*}{ BASEYEAR (000s) } & \multicolumn{3}{|c|}{$\% \Delta$ ON BASEYEAR } \\
\hline & & SIM101 & SIM102 & SIM103 \\
\hline AGRI & 4 & 2.6 & 1.5 & -0.4 \\
\hline FOLO & 0 & 2.2 & 1.3 & -0.5 \\
\hline FISH & 0 & 2.1 & 1.2 & -0.4 \\
\hline MINE & 0 & 2.1 & 1.3 & -0.3 \\
\hline OIGA & 0 & 1.3 & 0.9 & 0.0 \\
\hline PETR & 0 & 0.0 & 0.0 & 0.0 \\
\hline FDBT & 3 & 2.3 & 1.4 & -0.2 \\
\hline TWPM & 7 & 2.6 & 1.6 & -0.4 \\
\hline CHNM & 3 & 2.1 & 1.3 & -0.4 \\
\hline METL & 2 & 2.3 & 1.5 & -0.4 \\
\hline EQFO & 3 & 2.9 & 1.7 & -0.4 \\
\hline UTIL & 1 & 0.8 & 0.4 & -0.7 \\
\hline CONS & 15 & 1.4 & 0.7 & -0.6 \\
\hline $\mathrm{ACCR}$ & 10 & 0.4 & 0.0 & -1.0 \\
\hline CMIF & 16 & -3.1 & -2.7 & -2.0 \\
\hline PROP & 5 & -5.1 & -4.5 & -3.2 \\
\hline RBUS & 33 & -2.1 & -1.9 & -1.8 \\
\hline GOVT & 22 & 0.5 & 0.5 & 0.1 \\
\hline EDUC & 15 & 1.3 & 0.6 & 0.0 \\
\hline HEAL & 18 & 0.3 & 0.3 & 0.4 \\
\hline CUPE & 11 & 0.2 & 0.5 & 1.1 \\
\hline OWND & 0 & 0.0 & 0.0 & 0.0 \\
\hline WHOL & 8 & 0.8 & 0.7 & 0.6 \\
\hline RETT & 18 & 0.3 & 0.5 & 1.1 \\
\hline TRAN & 6 & -2.0 & -1.8 & -1.9 \\
\hline All & 200 & -0.2 & -0.3 & -0.5 \\
\hline
\end{tabular}

Table O.10: Employment By Industry - Region WLG 


\begin{tabular}{|l|c|ccc|}
\hline \multicolumn{5}{|c|}{ Industrial Investment } \\
\hline \multirow{2}{*}{ Industry } & BASEYEAR $(\$ \mathrm{M})$ & \multicolumn{3}{c|}{$\Delta$ ON BASEYEAR } \\
\cline { 2 - 5 } & & SIM101 & SIM102 & SIM103 \\
\hline AGRI & 1159 & -0.5 & -0.5 & -0.5 \\
FOLO & 128 & -0.1 & -0.1 & -0.2 \\
FISH & 41 & -1.8 & -1.8 & -1.8 \\
MINE & 184 & -0.5 & -0.6 & -0.6 \\
OIGA & 787 & -0.6 & -0.6 & -0.7 \\
PETR & 80 & -0.9 & -0.9 & -1.0 \\
FDBT & 954 & -1.3 & -1.3 & -1.2 \\
TWPM & 871 & -0.5 & -0.6 & -0.6 \\
CHNM & 640 & -0.5 & -0.6 & -0.7 \\
METL & 641 & -0.4 & -0.4 & -0.4 \\
EQFO & 500 & -0.3 & -0.4 & -0.4 \\
UTIL & 744 & -4.2 & -4.2 & -3.9 \\
CONS & 789 & -0.2 & -0.2 & -0.2 \\
ACCR & 333 & -0.4 & -0.4 & -0.4 \\
CMIF & 4534 & 0.0 & 0.1 & 0.2 \\
PROP & 1808 & -0.3 & -0.3 & -0.3 \\
RBUS & 2014 & -0.8 & -0.8 & -0.8 \\
GOVT & 3530 & -0.4 & -0.4 & -0.4 \\
EDUC & 1313 & -0.4 & -0.4 & -0.4 \\
HEAL & 884 & -0.4 & -0.4 & -0.4 \\
CUPE & 852 & -0.7 & -0.7 & -0.7 \\
OWND & 13345 & -0.4 & -0.4 & -0.4 \\
WHOL & 958 & -0.4 & -0.4 & -0.4 \\
RETT & 1065 & -0.5 & -0.5 & -0.5 \\
TRAN & 149 & 18.9 & 17.1 & 13.4 \\
\hline All & -0.4 & -0.4 & -0.4 \\
\hline
\end{tabular}

Table O.11: Industrial Investment 


\begin{tabular}{|l|c|ccc|}
\hline \multicolumn{4}{|c|}{ Investment By Industry - Region WLG } \\
\hline \multirow{2}{*}{ Industry } & BASEYEAR (\$M) & \multicolumn{3}{c|}{$\Delta$ ON BASEYEAR } \\
\cline { 2 - 5 } & & SIM101 & SIM102 & SIM103 \\
\hline AGRI & 12 & 1.1 & 0.2 & -0.9 \\
FOLO & 2 & 0.1 & 0.0 & -0.2 \\
FISH & 1 & 1.4 & -0.5 & -2.8 \\
MINE & 2 & 0.4 & -0.1 & -0.9 \\
OIGA & 74 & -0.9 & -0.8 & -0.7 \\
PETR & 0 & 0.0 & 0.0 & 0.0 \\
FDBT & 33 & 2.3 & 0.2 & -2.1 \\
TWPM & 60 & 1.1 & 0.2 & -1.0 \\
CHNM & 75 & 0.3 & -0.2 & -0.8 \\
METL & 39 & 0.4 & 0.0 & -0.6 \\
EQFO & 40 & 1.1 & 0.3 & -0.7 \\
UTIL & 128 & -1.3 & -1.3 & -0.8 \\
CONS & 77 & -0.9 & -1.0 & -1.0 \\
ACCR & 34 & -0.5 & -0.6 & -0.4 \\
CMIF & 923 & -3.5 & -2.5 & -0.4 \\
PROP & 175 & -6.1 & -4.9 & -2.2 \\
RBUS & 322 & -5.4 & -5.0 & -4.1 \\
GOVT & 1259 & -0.4 & -0.4 & -0.4 \\
EDUC & 145 & -0.4 & -0.4 & -0.4 \\
HEAL & 98 & -0.4 & -0.4 & -0.4 \\
CUPE & 122 & -3.8 & -3.6 & -3.0 \\
OWND & 1604 & -0.4 & -0.4 & -0.4 \\
WHOL & 90 & -1.0 & -0.8 & 0.0 \\
RETT & 154 & -3.3 & -3.0 & -2.1 \\
TRAN & 407.7 & 443.0 & 507.2 \\
\hline All & -0.4 & -0.1 & 0.6 \\
\hline
\end{tabular}

Table O.12: Investment By Industry — Region WLG 


\begin{tabular}{|l|c|ccc|}
\hline \multicolumn{5}{|c|}{ F.O.B. Exports } \\
\hline Product & BASEYEAR & $\% \Delta$ ON BASEYEAR \\
\cline { 3 - 5 } & NZ\$M) & SIM101 & SIM102 & SIM103 \\
\hline AGRI & 1757 & 0.1 & 0.1 & 0.2 \\
FOLO & 721 & 0.0 & 0.0 & 0.0 \\
FISH & 154 & -0.4 & -0.4 & -0.5 \\
MINE & 302 & 0.3 & 0.4 & 0.4 \\
OIGA & 475 & 1.2 & 1.2 & 1.2 \\
PETR & 93 & 0.2 & 0.2 & 0.1 \\
FDBT & 13384 & 0.0 & 0.0 & 0.1 \\
TWPM & 3690 & 0.3 & 0.3 & 0.3 \\
CHNM & 1930 & 0.3 & 0.3 & 0.2 \\
METL & 1753 & 0.3 & 0.4 & 0.4 \\
EQFO & 3767 & 0.3 & 0.3 & 0.3 \\
UTIL & 60 & 1.1 & 0.9 & 0.4 \\
CONS & 209 & 0.7 & 0.7 & 0.5 \\
ACCR & 2252 & -0.1 & -0.2 & -0.3 \\
CMIF & 1093 & -5.9 & -6.4 & -7.3 \\
PROP & 533 & -3.6 & -3.9 & -4.5 \\
RBUS & 1114 & -2.2 & -2.5 & -3.1 \\
GOVT & 151 & -3.1 & -3.7 & -5.3 \\
EDUC & 730 & 0.5 & 0.4 & 0.2 \\
HEAL & 81 & -0.4 & -0.6 & -0.8 \\
CUPE & 879 & -1.0 & -1.2 & -1.8 \\
OWND & 0 & 0.0 & 0.0 & 0.0 \\
WHOL & 3226 & -0.2 & -0.3 & -0.4 \\
RETT & 1076 & -0.5 & -0.5 & -0.6 \\
TRAN & 3859 & -1.2 & -1.3 & -1.5 \\
\hline All & 43290 & -0.3 & -0.4 & -0.5 \\
\hline
\end{tabular}

Table O.13: F.O.B. Exports 


\begin{tabular}{|l|c|ccc|}
\hline \multicolumn{5}{|c|}{ C.I.F. Imports } \\
\hline \multirow{2}{*}{ Product } & BASEYEAR & \multicolumn{1}{c|}{$\Delta$ ON BASEYEAR } \\
\cline { 2 - 5 } & (NZ\$M) & SIM101 & SIM102 & SIM103 \\
\hline AGRI & 647 & -0.3 & -0.3 & -0.2 \\
FOLO & 37 & -0.3 & -0.3 & -0.4 \\
FISH & 115 & 0.0 & -0.1 & -0.1 \\
MINE & 135 & -0.5 & -0.5 & -0.4 \\
OIGA & 1662 & -0.4 & -0.4 & -0.4 \\
PETR & 1580 & -0.5 & -0.4 & -0.4 \\
FDBT & 2821 & -0.3 & -0.2 & -0.2 \\
TWPM & 4510 & -0.4 & -0.4 & -0.3 \\
CHNM & 5762 & -0.4 & -0.4 & -0.3 \\
METL & 3182 & -0.4 & -0.4 & -0.4 \\
EQFO & 17649 & -0.4 & -0.4 & -0.4 \\
UTIL & 10 & -1.0 & -0.7 & -0.4 \\
CONS & 36 & -0.7 & -0.6 & -0.5 \\
ACCR & 1 & -0.2 & -0.2 & -0.1 \\
CMIF & 1139 & 1.9 & 1.2 & 0.3 \\
PROP & 514 & 1.1 & 0.6 & 0.1 \\
RBUS & 2132 & 0.5 & 0.2 & 0.0 \\
GOVT & 0 & 0.0 & 0.0 & 0.0 \\
EDUC & 30 & -0.5 & -0.4 & -0.3 \\
HEAL & 3 & -0.2 & -0.2 & -0.3 \\
CUPE & 138 & 0.0 & -0.1 & -0.1 \\
OWND & 0 & 0.0 & 0.0 & 0.0 \\
WHOL & 104 & -0.3 & -0.3 & -0.3 \\
RETT & 3154 & -0.1 & -0.2 & -0.2 \\
TRAN & 2137 & 0.0 & -0.1 & -0.2 \\
\hline All & 47497 & -0.3 & -0.3 & -0.3 \\
\hline
\end{tabular}

Table O.14: C.I.F. Imports 


\begin{tabular}{|l|ccc|}
\hline \multicolumn{3}{|c|}{ Industry Output Price Index } \\
\hline \multirow{2}{*}{ Industry } & $\%$ ON BASEYEAR \\
\cline { 2 - 4 } & SIM101 & SIM102 & SIM103 \\
\hline AGRI & 0.0 & 0.0 & -0.1 \\
FOLO & 0.0 & 0.0 & 0.0 \\
FISH & 0.1 & 0.1 & 0.1 \\
MINE & -0.1 & -0.1 & -0.1 \\
OIGA & -0.3 & -0.3 & -0.3 \\
PETR & 0.0 & 0.0 & 0.0 \\
FDBT & 0.0 & 0.0 & 0.0 \\
TWPM & -0.1 & -0.1 & -0.1 \\
CHNM & -0.1 & -0.1 & 0.0 \\
METL & -0.1 & -0.1 & -0.1 \\
EQFO & -0.1 & -0.1 & -0.1 \\
UTIL & -0.3 & -0.2 & -0.1 \\
CONS & -0.2 & -0.2 & -0.1 \\
ACCR & 0.0 & 0.0 & 0.1 \\
CMIF & 1.5 & 1.7 & 1.9 \\
PROP & 0.9 & 1.0 & 1.2 \\
RBUS & 0.6 & 0.6 & 0.8 \\
GOVT & 0.8 & 1.0 & 1.4 \\
EDUC & -0.1 & -0.1 & 0.0 \\
HEAL & 0.1 & 0.2 & 0.2 \\
CUPE & 0.2 & 0.3 & 0.4 \\
OWND & 0.6 & 0.7 & 0.8 \\
WHOL & 0.0 & 0.1 & 0.1 \\
RETT & 0.1 & 0.2 & 0.2 \\
TRAN & 0.3 & 0.3 & 0.4 \\
\hline All & 0.2 & 0.3 & 0.4 \\
\hline
\end{tabular}

Table O.15: Industry Output Price Index 


\begin{tabular}{|l|ccc|}
\hline \multicolumn{3}{|c|}{ Output } & Prices - Region WLG \\
\hline \multirow{2}{*}{ Industry } & \% & ON BASEYEAR \\
\cline { 2 - 4 } & SIM101 & sIM102 & SIM103 \\
\hline AGRI & -0.4 & -0.3 & 0.2 \\
FOLO & -0.2 & -0.2 & 0.1 \\
FISH & -0.1 & 0.0 & 0.3 \\
MINE & -0.2 & -0.2 & 0.0 \\
OIGA & -0.6 & -0.5 & -0.2 \\
PETR & 0.0 & 0.0 & 0.0 \\
FDBT & -0.2 & -0.2 & 0.1 \\
TWPM & -0.4 & -0.3 & 0.1 \\
CHNM & -0.3 & -0.3 & 0.1 \\
METL & -0.3 & -0.3 & 0.0 \\
EQFO & -0.4 & -0.3 & 0.1 \\
UTIL & -0.2 & -0.1 & 0.2 \\
CONS & -0.3 & -0.2 & 0.3 \\
ACCR & 0.4 & 0.7 & 1.6 \\
CMIF & 3.5 & 4.4 & 6.4 \\
PROP & 3.8 & 5.1 & 8.3 \\
RBUS & 2.1 & 2.7 & 4.3 \\
GOVT & 1.8 & 2.4 & 3.8 \\
EDUC & -0.6 & 0.0 & 1.8 \\
HEAL & 1.4 & 2.1 & 4.0 \\
CUPE & 1.0 & 1.6 & 3.4 \\
OWND & 5.7 & 6.8 & 9.9 \\
WHOL & -0.6 & -0.2 & 0.9 \\
RETT & 0.9 & 1.5 & 3.3 \\
TRAN & 1.6 & 2.0 & 3.1 \\
\hline All & 1.4 & 1.9 & 3.3 \\
\hline
\end{tabular}

\begin{tabular}{|l|ccc|}
\hline \multicolumn{3}{|c|}{ Output Prices - Region ONI } \\
\hline \multirow{2}{*}{ Industry } & $\% \Delta$ ON BASEYEAR \\
\cline { 2 - 4 } & SIM101 & SIM102 & SIM103 \\
\hline AGRI & 0.0 & 0.0 & -0.1 \\
FOLO & 0.0 & 0.0 & 0.0 \\
FISH & 0.1 & 0.1 & 0.1 \\
MINE & -0.1 & -0.1 & -0.1 \\
OIGA & -0.3 & -0.3 & -0.3 \\
PETR & 0.0 & 0.0 & 0.0 \\
FDBT & 0.0 & 0.0 & 0.0 \\
TWPM & -0.1 & -0.1 & -0.1 \\
CHNM & -0.1 & -0.1 & -0.1 \\
METL & -0.1 & -0.1 & -0.1 \\
EQFO & 0.0 & -0.1 & -0.1 \\
UTIL & -0.3 & -0.3 & -0.2 \\
CONS & -0.2 & -0.2 & -0.2 \\
ACCR & 0.0 & -0.1 & -0.1 \\
CMIF & 1.0 & 1.0 & 0.8 \\
PROP & 0.6 & 0.6 & 0.4 \\
RBUS & 0.3 & 0.2 & 0.1 \\
GOVT & 0.2 & 0.1 & 0.1 \\
EDUC & -0.1 & -0.2 & -0.3 \\
HEAL & -0.1 & -0.1 & -0.3 \\
CUPE & 0.1 & 0.0 & -0.1 \\
OWND & -0.2 & -0.3 & -0.5 \\
WHOL & 0.1 & 0.1 & 0.0 \\
RETT & 0.0 & 0.0 & -0.1 \\
TRAN & 0.2 & 0.1 & 0.1 \\
\hline All & 0.0 & 0.0 & 0.0 \\
\hline
\end{tabular}

Table O.16: Output Prices - Region WLG and ONI 


\begin{tabular}{|l|ccc|}
\hline \multicolumn{3}{|c|}{ Industry Capital Rents Index } \\
\hline \multirow{2}{*}{ Industry } & \multicolumn{1}{c|}{$\Delta$ ON BASEYEAR } \\
\cline { 2 - 4 } & SIM101 & SIM102 & SIM103 \\
\hline AGRI & -0.4 & -0.4 & -0.4 \\
FOLO & -0.5 & -0.5 & -0.6 \\
FISH & -0.4 & -0.4 & -0.4 \\
MINE & -0.3 & -0.3 & -0.4 \\
OIGA & -0.3 & -0.4 & -0.4 \\
PETR & -0.7 & -0.7 & -0.7 \\
FDBT & -0.2 & -0.2 & -0.2 \\
TWPM & -0.4 & -0.4 & -0.5 \\
CHNM & -0.4 & -0.5 & -0.5 \\
METL & -0.3 & -0.4 & -0.4 \\
EQFO & -0.2 & -0.3 & -0.3 \\
UTIL & -0.6 & -0.6 & -0.4 \\
CONS & -0.8 & -0.8 & -0.8 \\
ACCR & 0.6 & 0.6 & 0.7 \\
CMIF & 3.8 & 4.0 & 4.4 \\
PROP & 1.4 & 1.5 & 1.7 \\
RBUS & 2.7 & 2.8 & 3.0 \\
GOVT & 12.6 & 12.9 & 13.4 \\
EDUC & 1.7 & 1.7 & 1.8 \\
HEAL & 1.8 & 1.8 & 1.9 \\
CUPE & 0.8 & 0.8 & 1.0 \\
OWND & 0.8 & 0.8 & 1.0 \\
WHOL & -0.4 & -0.4 & -0.4 \\
RETT & 0.4 & 0.4 & 0.5 \\
TRAN & 1.0 & 1.0 & 1.1 \\
\hline All & 1.1 & 1.2 & 1.3 \\
\hline
\end{tabular}

Table O.17: Industry Capital Rents Index 


\begin{tabular}{|l|ccc|}
\hline \multicolumn{3}{|c}{ Capital Rental Rates - Region WLG } \\
\hline \multirow{2}{*}{ Industry } & \multicolumn{1}{c}{$\%$ ON BASEYEAR } \\
\cline { 2 - 4 } & SIM101 & SIM102 & SIM103 \\
\hline AGRI & 1.8 & 0.6 & -0.7 \\
FOLO & 1.1 & 0.2 & -0.8 \\
FISH & 0.7 & 0.0 & -0.6 \\
MINE & 0.7 & 0.2 & -0.6 \\
OIGA & -0.7 & -0.6 & -0.3 \\
PETR & 0.0 & 0.0 & 0.0 \\
FDBT & 1.2 & 0.4 & -0.4 \\
TWPM & 1.8 & 0.7 & -0.8 \\
CHNM & 0.8 & 0.1 & -0.6 \\
METL & 1.3 & 0.5 & -0.6 \\
EQFO & 2.4 & 1.0 & -0.6 \\
UTIL & 0.3 & 0.4 & 0.7 \\
CONS & 1.4 & 1.0 & 0.8 \\
ACCR & 8.1 & 8.1 & 8.7 \\
CMIF & 12.0 & 14.1 & 18.6 \\
PROP & 7.5 & 9.9 & 15.8 \\
RBUS & 14.5 & 16.0 & 19.2 \\
GOVT & 35.2 & 36.3 & 38.8 \\
EDUC & 22.4 & 22.0 & 23.6 \\
HEAL & 20.1 & 21.2 & 24.6 \\
CUPE & 7.7 & 9.2 & 13.3 \\
OWND & 8.5 & 10.3 & 14.9 \\
WHOL & 0.3 & 1.0 & 3.4 \\
RETT & 7.7 & 9.2 & 13.5 \\
TRAN & 14.7 & 16.2 & 18.9 \\
\hline All & 9.6 & 10.8 & 13.8 \\
\hline
\end{tabular}

\begin{tabular}{|l|ccc|}
\hline \multicolumn{3}{|c}{ Capital Rental Rates - Region ONI } \\
\hline \multirow{2}{*}{ Industry } & \multicolumn{3}{|c|}{$\%$ ON BASEYEAR } \\
\cline { 2 - 4 } & SIM101 & SIM102 & SIM103 \\
\hline AGRI & -0.4 & -0.4 & -0.4 \\
FOLO & -0.5 & -0.5 & -0.6 \\
FISH & -0.5 & -0.4 & -0.4 \\
MINE & -0.3 & -0.3 & -0.4 \\
OIGA & -0.3 & -0.3 & -0.4 \\
PETR & -0.7 & -0.7 & -0.7 \\
FDBT & -0.3 & -0.2 & -0.1 \\
TWPM & -0.5 & -0.5 & -0.4 \\
CHNM & -0.5 & -0.5 & -0.5 \\
METL & -0.4 & -0.4 & -0.4 \\
EQFO & -0.3 & -0.3 & -0.3 \\
UTIL & -0.8 & -0.7 & -0.6 \\
CONS & -1.0 & -1.0 & -1.0 \\
ACCR & -0.2 & -0.2 & -0.2 \\
CMIF & 1.9 & 1.6 & 0.9 \\
PROP & 0.8 & 0.7 & 0.3 \\
RBUS & 0.7 & 0.5 & 0.1 \\
GOVT & 1.2 & 0.9 & 0.5 \\
EDUC & -0.7 & -0.7 & -0.8 \\
HEAL & -0.4 & -0.5 & -0.8 \\
CUPE & -0.5 & -0.6 & -1.1 \\
OWND & -0.5 & -0.6 & -1.0 \\
WHOL & -0.6 & -0.7 & -0.9 \\
RETT & -0.5 & -0.6 & -1.0 \\
TRAN & -0.3 & -0.5 & -0.8 \\
\hline All & -0.1 & -0.2 & -0.4 \\
\hline
\end{tabular}

Table O.18: Capital Rental Rates - Regions WLG and ONI 


\begin{tabular}{|l|c|ccc|}
\hline \multicolumn{4}{|c|}{ Industry Average Net Rates of Return } \\
\hline Industry & BASEYEAR (\%) & \multicolumn{4}{c|}{$\Delta$ ON BASEYEAR } \\
\cline { 2 - 5 } & & SIM101 & SIM102 & SIM103 \\
\hline AGRI & 10.4 & -0.7 & -0.7 & -0.7 \\
FOLO & 25.9 & -0.7 & -0.7 & -0.8 \\
FISH & 4.9 & -1.1 & -1.2 & -1.1 \\
MINE & 21.5 & -0.5 & -0.6 & -0.7 \\
OIGA & 27.3 & -0.4 & -0.5 & -0.5 \\
PETR & 27.3 & -0.9 & -0.9 & -1.0 \\
FDBT & 10.6 & -0.4 & -0.4 & -0.4 \\
TWPM & 14.1 & -0.7 & -0.7 & -0.9 \\
CHNM & 22.3 & -0.6 & -0.7 & -0.8 \\
METL & 41.8 & -0.4 & -0.4 & -0.6 \\
EQFO & 19.5 & -0.3 & -0.4 & -0.5 \\
UTIL & 10.7 & -0.8 & -0.8 & -0.6 \\
CONS & 57.2 & -1.0 & -1.0 & -1.0 \\
ACCR & 14.5 & 0.8 & 0.8 & 0.8 \\
CMIF & 35.7 & 5.3 & 5.5 & 5.9 \\
PROP & 5.6 & 2.1 & 2.2 & 2.4 \\
RBUS & 64.1 & 3.2 & 3.3 & 3.5 \\
GOVT & -0.7 & 47.2 & 47.9 & 49.6 \\
EDUC & 0.0 & 139.0 & 137.9 & 138.2 \\
HEAL & 12.0 & 2.4 & 2.4 & 2.5 \\
CUPE & 13.9 & 1.2 & 1.2 & 1.4 \\
OWND & 3.4 & 1.1 & 1.2 & 1.3 \\
WHOL & 22.5 & -0.6 & -0.6 & -0.7 \\
RETT & 44.0 & 0.4 & 0.5 & 0.5 \\
TRAN & 8.0 & 1.9 & 1.9 & 1.8 \\
\hline All & 9.4 & 2.0 & 2.1 & 2.2 \\
\hline
\end{tabular}

Table O.19: Industry Average Net Rates of Return 


\begin{tabular}{|l|c|ccc|}
\hline \multicolumn{3}{|c|}{ Current Net Rates of Return - Region WLG } \\
\hline Industry & BASEYEAR (\%) & \multicolumn{3}{c|}{$\%$ ON BASEYEAR } \\
\cline { 2 - 5 } & & SIM101 & SIM102 & SIM103 \\
\hline AGRI & 10.4 & 3.4 & 1.1 & -1.6 \\
FOLO & 25.9 & 1.5 & 0.3 & -1.3 \\
FISH & 4.9 & 1.9 & 0.1 & -2.1 \\
MINE & 21.5 & 1.3 & 0.3 & -1.3 \\
OIGA & 27.3 & -0.9 & -0.8 & -0.6 \\
PETR & 0.0 & 0.0 & 0.0 & 0.0 \\
FDBT & 10.6 & 2.3 & 0.7 & -1.0 \\
TWPM & 14.1 & 3.1 & 1.1 & -1.6 \\
CHNM & 22.3 & 1.1 & 0.1 & -1.1 \\
METL & 41.8 & 1.6 & 0.6 & -1.0 \\
EQFO & 19.5 & 3.2 & 1.3 & -1.1 \\
UTIL & 10.7 & 0.5 & 0.5 & 0.6 \\
CONS & 57.2 & 1.8 & 1.2 & 0.8 \\
ACCR & 14.5 & 11.2 & 11.1 & 11.7 \\
CMIF & 35.7 & 17.0 & 19.9 & 26.0 \\
PROP & 5.6 & 11.4 & 14.9 & 23.4 \\
RBUS & 64.1 & 18.0 & 19.7 & 23.5 \\
GOVT & -0.7 & 138.3 & 142.4 & 151.1 \\
EDUC & 0.0 & 1926.4 & 1886.1 & 2002.7 \\
HEAL & 12.0 & 28.2 & 29.8 & 34.3 \\
CUPE & 13.9 & 11.8 & 14.0 & 20.1 \\
OWND & 3.4 & 12.6 & 15.0 & 21.6 \\
WHOL & 22.5 & 0.5 & 1.3 & 4.6 \\
RETT & 44.0 & 9.6 & 11.3 & 16.4 \\
TRAN & 8.0 & 28.8 & 31.5 & 36.4 \\
\hline
\end{tabular}

Table O.20: Current Net Rates of Return - Region WLG 


\begin{tabular}{|l|ccc|}
\hline \multicolumn{3}{|c|}{ Regional Nominal After-Tax Wage } \\
\hline \multirow{2}{*}{ Region } & \multicolumn{3}{c|}{$\%$ ON BASEYEAR } \\
\cline { 2 - 4 } & SIM101 & SIM102 & SIM103 \\
\hline AKL & -0.1 & -0.1 & -0.4 \\
WLG & -3.3 & -2.4 & 0.1 \\
ONI & -0.2 & -0.3 & -0.6 \\
CAN & -0.1 & -0.2 & -0.5 \\
OSI & -0.3 & -0.4 & -0.6 \\
\hline
\end{tabular}

Table O.21: Regional Nominal After-Tax Wage

\begin{tabular}{|l|ccc|}
\hline \multicolumn{4}{|c|}{ Regional Consumer Price Index } \\
\hline \multirow{2}{*}{ Region } & \multicolumn{3}{|c|}{$\%$ ON BASEYEAR } \\
\cline { 2 - 4 } & SIM101 & SIM102 & SIM103 \\
\hline AKL & 0.2 & 0.2 & 0.1 \\
WLG & 1.0 & 1.4 & 2.3 \\
ONI & 0.1 & 0.1 & 0.1 \\
CAN & 0.2 & 0.2 & 0.1 \\
OSI & 0.1 & 0.1 & 0.1 \\
\hline
\end{tabular}

Table O.22: Regional Consumer Price Index

\begin{tabular}{|l|ccc|}
\hline \multicolumn{4}{|c|}{ Regional Real After-Tax Wage } \\
\hline \multirow{2}{*}{ Region } & \multicolumn{3}{c|}{$\% \Delta$ ON BASEYEAR } \\
\cline { 2 - 4 } & SIM101 & SIM102 & SIM103 \\
\hline AKL & -0.2 & -0.3 & -0.6 \\
WLG & -4.3 & -3.7 & -2.1 \\
ONI & -0.4 & -0.5 & -0.7 \\
CAN & -0.3 & -0.4 & -0.6 \\
OSI & -0.4 & -0.5 & -0.7 \\
\hline
\end{tabular}

Table O.23: Regional Real After-Tax Wage 


\begin{tabular}{|l|c|ccc|}
\hline \multicolumn{5}{|c|}{ Household Real Disposable Income } \\
\hline \multirow{2}{*}{ Region } & BASEYEAR $(\$ \mathrm{M})$ & \multicolumn{3}{|c|}{$\%$ ON BASEYEAR } \\
\cline { 3 - 5 } & & SIM101 & SIM102 & SIM103 \\
\hline AKL & 41312 & 0.0 & 0.0 & 0.0 \\
WLG & 14759 & -3.6 & -3.7 & -3.8 \\
ONI & 36391 & 0.0 & 0.0 & 0.0 \\
CAN & 12853 & 0.0 & 0.0 & 0.0 \\
OSI & 15213 & 0.0 & 0.0 & 0.0 \\
\hline NZ & 120529 & -0.4 & -0.4 & -0.4 \\
\hline
\end{tabular}

Table O.24: Household Real Disposable Income

\begin{tabular}{|l|c|ccc|}
\hline \multicolumn{5}{|c|}{ Household Real Spending } \\
\hline \multirow{2}{*}{ Region } & BASEYEAR $(\$ \mathrm{M})$ & \multicolumn{4}{|c|}{$\Delta$ ON BASEYEAR } \\
\cline { 3 - 5 } & & SIM101 & SIM102 & SIM103 \\
\hline AKL & 31830 & -0.1 & -0.2 & -0.4 \\
WLG & 11765 & -1.6 & -0.9 & 0.7 \\
ONI & 27565 & -0.3 & -0.4 & -0.5 \\
CAN & 10063 & -0.2 & -0.3 & -0.5 \\
OSI & 12108 & -0.3 & -0.4 & -0.6 \\
\hline NZ & 93331 & -0.4 & -0.4 & -0.3 \\
\hline
\end{tabular}

Table O.25: Household Real Spending 


\begin{tabular}{|l|c|ccc|}
\hline \multicolumn{5}{|c|}{ Household Real Saving (After-Tax) } \\
\hline \multirow{2}{*}{ Region } & BASEYEAR $(\$ \mathrm{M})$ & \multicolumn{3}{|c|}{$\%$ ON BASEYEAR } \\
\cline { 3 - 5 } & & SIM101 & SIM102 & SIM103 \\
\hline AKL & 9483 & 0.4 & 0.7 & 1.5 \\
WLG & 2994 & -11.7 & -14.3 & -21.2 \\
ONI & 8826 & 1.0 & 1.2 & 1.8 \\
CAN & 2790 & 0.7 & 1.0 & 1.8 \\
OSI & 3105 & 1.3 & 1.6 & 2.3 \\
\hline NZ & 27198 & -0.6 & -0.7 & -0.8 \\
\hline
\end{tabular}

Table O.26: Household Real Saving (After-Tax)

\begin{tabular}{|c|c|c|c|}
\hline \multicolumn{4}{|c|}{ Compensating Variation } \\
\hline \multirow{2}{*}{ Region } & \multicolumn{3}{|c|}{$\Delta$ IN INCOME $(\$ M)$} \\
\hline & SIM101 & SIM102 & SIM103 \\
\hline AKL & -39.0 & -66.8 & -144.3 \\
\hline WLG & -171.9 & -91.7 & 117.5 \\
\hline ONI & -86.1 & -107.6 & -159.5 \\
\hline CAN & -18.7 & -27.3 & -50.3 \\
\hline OSI & -39.9 & -49.5 & -72.3 \\
\hline \multicolumn{4}{|c|}{ Equivalent Variation } \\
\hline \multirow{2}{*}{ Region } & \multicolumn{3}{|c|}{$\Delta$ IN INCOME $(\$ M)$} \\
\hline & SIM101 & SIM102 & SIM103 \\
\hline AKL & -38.9 & -66.8 & -144.3 \\
\hline WLG & -171.2 & -90.9 & 115.2 \\
\hline ONI & -86.1 & -107.5 & -159.6 \\
\hline CAN & -18.7 & -27.3 & -50.3 \\
\hline OSI & -39.9 & -49.4 & -72.3 \\
\hline
\end{tabular}

Table O.27: CV and EV Measures 


\begin{tabular}{|l|c|ccc|}
\hline \multicolumn{5}{|c|}{ Real Macro Measures } \\
\hline \multirow{2}{*}{ Variable } & BASEYEAR $(\$ \mathrm{M})$ & \multicolumn{3}{|c|}{$\Delta$ ON BASEYEAR } \\
\cline { 2 - 5 } & & SIM101 & SIM102 & SIM103 \\
\hline GDP (Expenditure) & 156090 & -0.4 & -0.4 & -0.4 \\
GDP (Income) & 156090 & -0.4 & -0.4 & -0.4 \\
Private Consumption & 93331 & -0.4 & -0.4 & -0.3 \\
Investment & 38305 & -0.4 & -0.4 & -0.4 \\
Government Consumption & 28661 & -0.4 & -0.4 & -0.4 \\
F.O.B. Exports & 43290 & -0.3 & -0.4 & -0.5 \\
C.I.F. Imports & 47497 & -0.3 & -0.3 & -0.3 \\
Trade Balance & -4207 & -0.2 & -0.5 & -1.3 \\
Government Balance & 6900 & 0.5 & 0.5 & 0.6 \\
Domestic Private Saving & 27198 & -0.6 & -0.7 & -0.8 \\
Domestic Saving & 34098 & -0.4 & -0.4 & -0.5 \\
\hline
\end{tabular}

Table O.28: Macro Measures

\begin{tabular}{|l|ccc|}
\hline \multicolumn{4}{|c|}{ Economy-wide Price Measures } \\
\hline \multirow{2}{*}{ Variable } & \multicolumn{4}{|c|}{$\Delta$ ON BASEYEAR } \\
\cline { 2 - 4 } & SIM101 & SIM102 & SIM103 \\
\hline GDP Deflator & 0.2 & 0.3 & 0.4 \\
Consumer Price Index & 0.3 & 0.3 & 0.4 \\
Investment Price Index & 0.0 & 0.0 & 0.1 \\
Government Price Index & 0.4 & 0.5 & 0.7 \\
Export Price Index (F.O.B.) & 0.1 & 0.1 & 0.1 \\
Import Price Index (C.I.F.) & 0.0 & 0.0 & 0.0 \\
Trade Price Index & -0.8 & -0.9 & -1.2 \\
Real Exchange Rate & -0.2 & -0.3 & -0.4 \\
Nominal After-Tax Wage & -0.6 & -0.5 & -0.4 \\
Real After-Tax Wage & -0.8 & -0.8 & -0.8 \\
Capital Rents Index & 1.1 & 1.2 & 1.3 \\
Output Price Index & 0.2 & 0.3 & 0.4 \\
\hline Current Net Rate of Return (Average) & 2.0 & 2.1 & 2.2 \\
Expected Future Net Rate of Return & 0.5 & 0.6 & 0.6 \\
\hline
\end{tabular}

Table O.29: Economy-wide Price Measures 


\section{Appendix $\mathbf{P}$}

\section{Simulation Results — Oil Price Rise Scenario}

This appendix provides the results for the illustrative simulations discussed in chapter 3 .

\begin{tabular}{|l|c|ccc|}
\hline \multicolumn{5}{|c|}{ Regional Capital Stocks } \\
\hline \multirow{2}{*}{ Region } & BASEYEAR $(\$ \mathrm{M})$ & \multicolumn{4}{|c|}{$\%$ ON BASEYEAR } \\
\cline { 3 - 5 } & & SIM201 & SIM301 & SIM401 \\
\hline AKL & 154208 & -2.7 & -3.0 & -2.9 \\
WLG & 62648 & -3.3 & -3.4 & -3.4 \\
ONI & 144170 & -3.4 & -3.4 & -3.5 \\
CAN & 49536 & -2.9 & -3.1 & -3.0 \\
OSI & 59263 & -2.9 & -3.0 & -3.0 \\
\hline NZ & 469826 & -3.0 & -3.2 & -3.2 \\
\hline
\end{tabular}

Table P.1: Regional Capital Stocks 

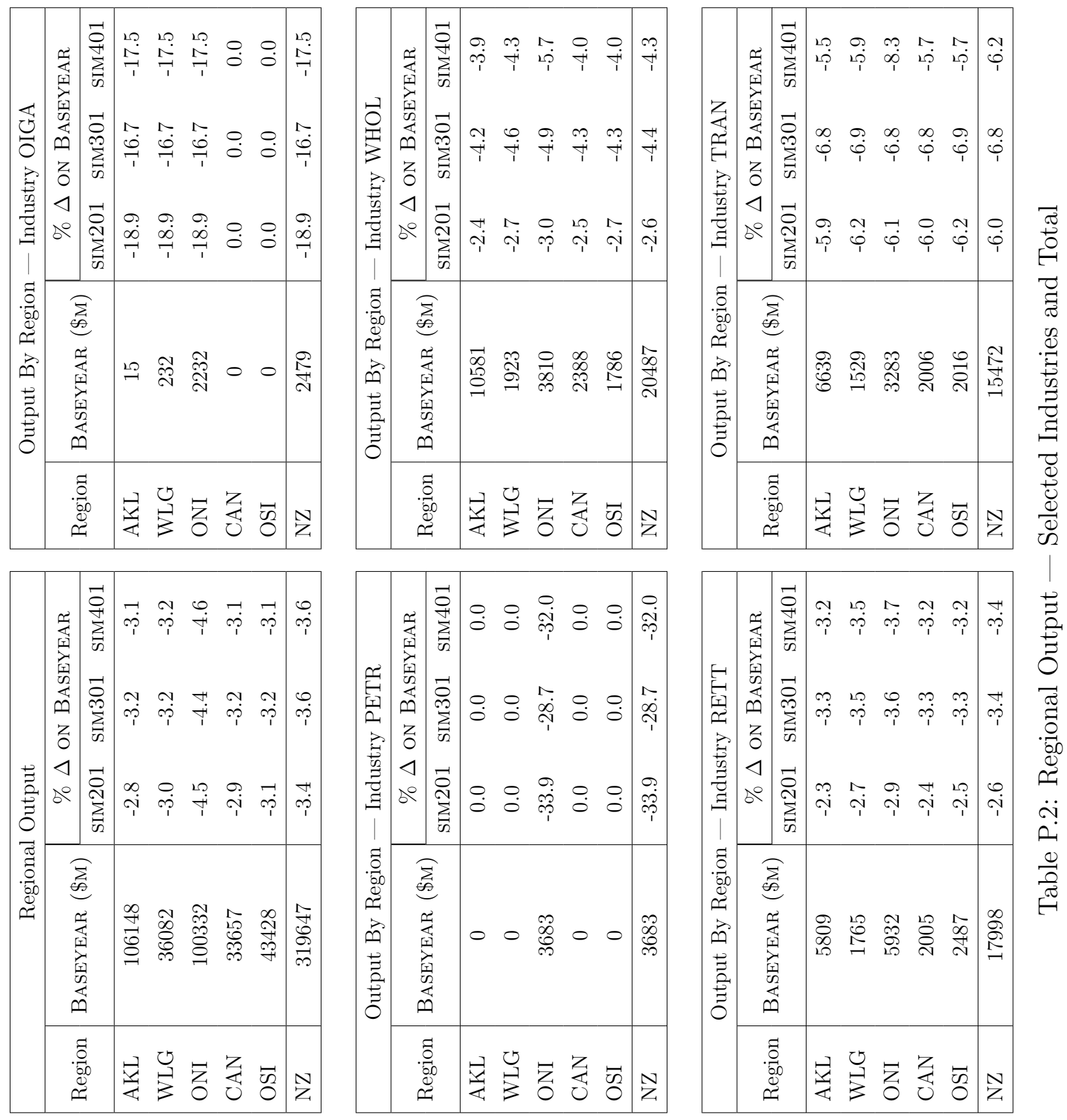

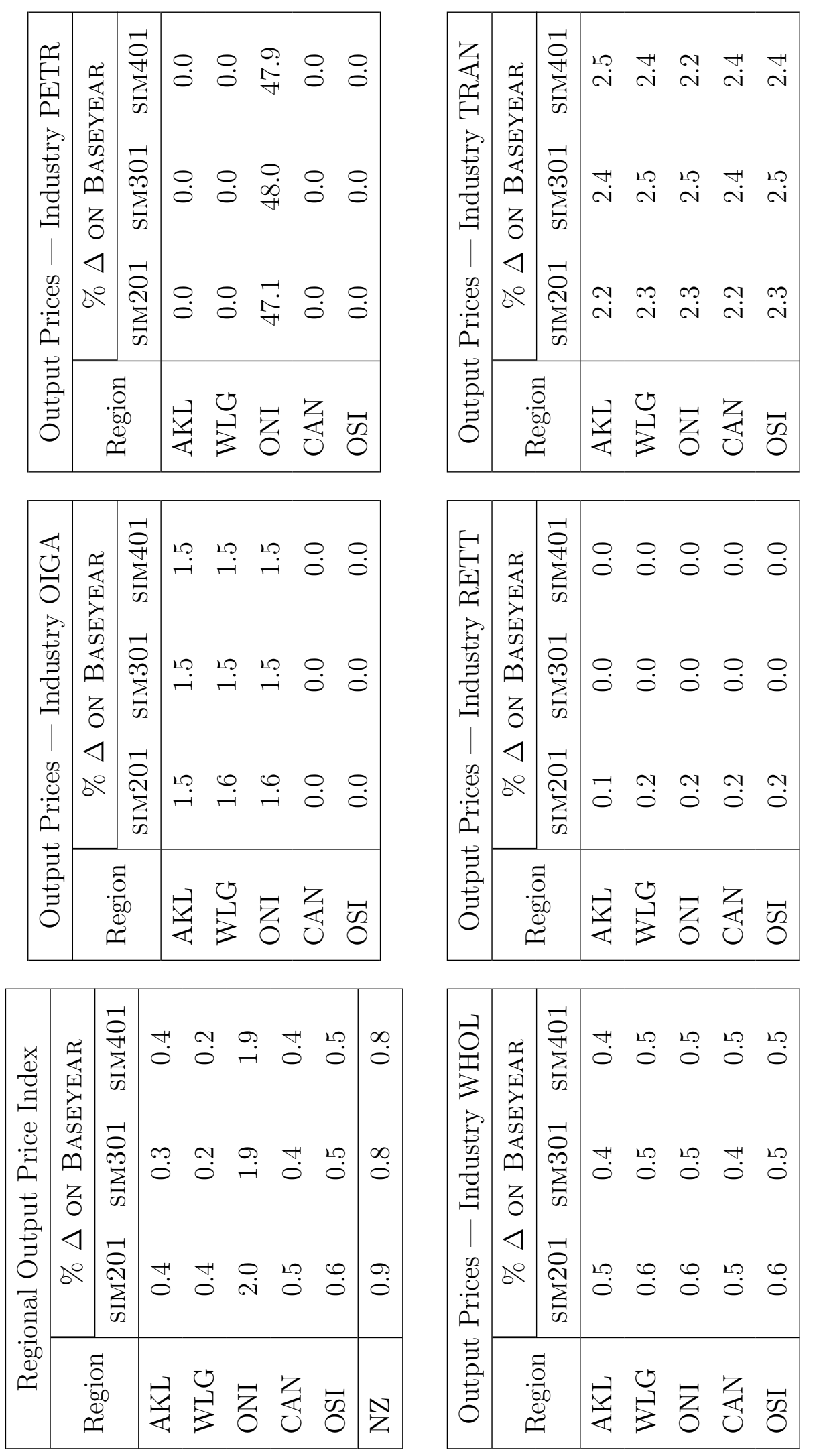


\begin{tabular}{|l|c|ccc|}
\hline \multicolumn{5}{|c|}{ Industrial Output } \\
\hline \multirow{2}{*}{ Industry } & BASEYEAR $(\$ \mathrm{M})$ & \multicolumn{4}{c|}{$\Delta$ ON BASEYEAR } \\
\cline { 2 - 5 } & & SIM201 & SIM301 & SIM401 \\
\hline AGRI & 16884 & -2.8 & -2.9 & -2.8 \\
FOLO & 2978 & -5.3 & -5.2 & -5.0 \\
FISH & 853 & -5.3 & -5.1 & -5.1 \\
MINE & 1197 & -9.7 & -8.9 & -8.9 \\
OIGA & 2479 & -18.9 & -16.7 & -17.5 \\
PETR & 3683 & -33.9 & -28.7 & -32.0 \\
FDBT & 27682 & -2.9 & -2.9 & -2.8 \\
TWPM & 13057 & -3.8 & -3.8 & -3.8 \\
CHNM & 9078 & -4.7 & -4.5 & -4.5 \\
METL & 8105 & -3.2 & -3.3 & -3.3 \\
EQFO & 10632 & -2.2 & -2.2 & -2.2 \\
UTIL & 12609 & -4.8 & -4.9 & -4.9 \\
CONS & 29305 & -2.4 & -2.4 & -2.4 \\
ACCR & 6454 & -1.8 & -1.7 & -1.7 \\
CMIF & 24027 & -2.6 & -2.9 & -2.8 \\
PROP & 13658 & -3.7 & -3.9 & -3.8 \\
RBUS & 24532 & -2.0 & -2.1 & -2.1 \\
GOVT & 13460 & -2.9 & -3.0 & -3.0 \\
EDUC & 8229 & -1.1 & -1.1 & -1.1 \\
HEAL & 12552 & -2.3 & -2.4 & -2.4 \\
CUPE & 11764 & -2.2 & -2.4 & -2.4 \\
OWND & 12472 & -1.2 & -1.3 & -1.3 \\
WHOL & 20487 & -2.6 & -4.4 & -4.3 \\
RETT & 17998 & -2.6 & -3.4 & -3.4 \\
TRAN & 15472 & -6.0 & -6.8 & -6.2 \\
\hline All & 319647 & -3.4 & -3.6 & -3.6 \\
\hline
\end{tabular}

Table P.4: Industrial Output 


\begin{tabular}{|l|ccc|}
\hline \multicolumn{3}{|c|}{ Industry Output Price Index } \\
\hline \multirow{2}{*}{ Industry } & \multicolumn{2}{c|}{ \% ON BASEYEAR } \\
\cline { 2 - 4 } & SIM201 & SIM301 & SIM401 \\
\hline AGRI & 0.6 & 0.5 & 0.5 \\
FOLO & 2.0 & 2.0 & 1.9 \\
FISH & 2.1 & 2.0 & 2.0 \\
MINE & 3.9 & 4.0 & 3.9 \\
OIGA & 1.6 & 1.5 & 1.5 \\
PETR & 47.1 & 48.0 & 47.9 \\
FDBT & 0.6 & 0.5 & 0.5 \\
TWPM & 1.0 & 0.9 & 0.9 \\
CHNM & 1.5 & 1.5 & 1.5 \\
METL & 0.7 & 0.6 & 0.6 \\
EQFO & 0.3 & 0.2 & 0.2 \\
UTIL & 2.4 & 2.3 & 2.3 \\
CONS & 1.0 & 0.9 & 0.9 \\
ACCR & 0.0 & -0.2 & -0.2 \\
CMIF & 0.2 & 0.0 & 0.1 \\
PROP & 1.1 & 1.0 & 1.0 \\
RBUS & -0.2 & -0.4 & -0.4 \\
GOVT & -0.2 & -0.4 & -0.4 \\
EDUC & -1.3 & -1.6 & -1.5 \\
HEAL & -0.7 & -1.0 & -0.9 \\
CUPE & -0.2 & -0.4 & -0.3 \\
OWND & -1.5 & -1.8 & -1.7 \\
WHOL & 0.5 & 0.4 & 0.4 \\
RETT & 0.2 & 0.0 & 0.0 \\
TRAN & 2.2 & 2.4 & 2.4 \\
\hline All & 0.9 & 0.8 & 0.8 \\
\hline
\end{tabular}

Table P.5: Industry Output Price Index 


\begin{tabular}{|l|c|ccc|}
\hline \multicolumn{5}{|c|}{ F.O.B. Exports } \\
\hline Product & BASEYEAR & $\%$ & \multicolumn{1}{c|}{ ON BASEYEAR } \\
\cline { 2 - 5 } & (NZ\$M) & SIM201 & SIM301 & SIM401 \\
\hline AGRI & 1757 & -1.8 & 18.2 & 18.1 \\
FOLO & 721 & -7.5 & 11.9 & 12.1 \\
FISH & 154 & -7.8 & 11.7 & 11.8 \\
MINE & 302 & -14.1 & 4.9 & 5.1 \\
OIGA & 475 & -6.1 & 16.4 & 16.3 \\
PETR & 93 & -78.6 & -69.2 & -69.0 \\
FDBT & 13384 & -2.5 & 17.4 & 17.3 \\
TWPM & 3690 & -3.9 & 15.8 & 15.8 \\
CHNM & 1930 & -5.8 & 13.8 & 13.7 \\
METL & 1753 & -2.9 & 16.9 & 16.8 \\
EQFO & 3767 & -1.4 & 18.5 & 18.4 \\
UTIL & 60 & -8.9 & -5.7 & -5.8 \\
CONS & 209 & -3.9 & -0.5 & -0.6 \\
ACCR & 2252 & 0.1 & 11.0 & 10.9 \\
CMIF & 1093 & -0.8 & 2.8 & 2.7 \\
PROP & 533 & -4.3 & -1.1 & -1.1 \\
RBUS & 1114 & 1.0 & 4.7 & 4.6 \\
GOVT & 151 & 0.9 & 4.6 & 4.5 \\
EDUC & 730 & 5.3 & 9.5 & 9.3 \\
HEAL & 81 & 3.0 & 6.9 & 6.8 \\
CUPE & 879 & 0.7 & 11.6 & 11.5 \\
OWND & 0 & 0.0 & 0.0 & 0.0 \\
WHOL & 3226 & -2.1 & -100.0 & -100.0 \\
RETT & 1076 & -0.6 & -100.0 & -100.0 \\
TRAN & 3859 & -8.5 & -51.3 & -51.1 \\
\hline All & 43290 & -3.1 & -2.7 & -2.7 \\
\hline
\end{tabular}

Table P.6: F.O.B. Exports 


\begin{tabular}{|l|c|cc|}
\hline \multicolumn{4}{|c|}{ F.O.B. Exports } \\
\hline Commodity & BASEYEAR & $\% \Delta$ ON & BASEYEAR \\
\cline { 2 - 4 } & NZ\$M $)$ & BSLN3 & BSLN4 \\
\hline AGRI & 1757 & 20.1 & 20.1 \\
FOLO & 721 & 20.1 & 20.1 \\
FISH & 154 & 20.1 & 20.1 \\
MINE & 302 & 20.1 & 20.1 \\
OIGA & 475 & 23.4 & 23.4 \\
PETR & 93 & 23.4 & 23.4 \\
FDBT & 13384 & 20.1 & 20.1 \\
TWPM & 3690 & 20.1 & 20.1 \\
CHNM & 1930 & 20.1 & 20.1 \\
METL & 1753 & 20.1 & 20.1 \\
EQFO & 3767 & 20.1 & 20.1 \\
UTIL & 60 & 2.9 & 2.9 \\
CONS & 209 & 2.9 & 2.9 \\
ACCR & 2252 & 10.1 & 10.1 \\
CMIF & 1093 & 2.9 & 2.9 \\
PROP & 533 & 2.9 & 2.9 \\
RBUS & 1114 & 2.9 & 2.9 \\
GOVT & 151 & 2.9 & 2.9 \\
EDUC & 730 & 2.9 & 2.9 \\
HEAL & 81 & 2.9 & 2.9 \\
CUPE & 879 & 10.1 & 10.1 \\
OWND & 0 & 0.0 & 0.0 \\
WHOL & 3226 & -100.0 & -100.0 \\
RETT & 1076 & -100.0 & -100.0 \\
TRAN & 3859 & -46.3 & -46.3 \\
\hline All & 43290 & 0.0 & 0.0 \\
\hline
\end{tabular}

Table P.7: F.O.B. Exports — benchmark differences 


\begin{tabular}{|l|c|ccc|}
\hline \multicolumn{5}{|c|}{ C.I.F. Imports } \\
\hline \multirow{2}{*}{ Product } & BASEYEAR & $\% \Delta$ ON BASEYEAR \\
\cline { 3 - 5 } & (NZ\$M) & SIM201 & SIM301 & SIM401 \\
\hline AGRI & 647 & -1.8 & -2.5 & -2.6 \\
FOLO & 37 & 1.0 & -0.2 & -0.7 \\
FISH & 115 & -0.3 & -1.0 & -1.3 \\
MINE & 135 & 2.4 & 1.4 & 1.3 \\
OIGA & 1662 & -42.0 & -36.3 & -38.6 \\
PETR & 1580 & -37.3 & -30.3 & -26.9 \\
FDBT & 2821 & -1.8 & -2.6 & -2.6 \\
TWPM & 4510 & -1.6 & -2.4 & -2.4 \\
CHNM & 5762 & -1.8 & -2.4 & -2.4 \\
METL & 3182 & -1.5 & -2.1 & -2.1 \\
EQFO & 17649 & -1.6 & -2.1 & -2.1 \\
UTIL & 10 & 1.3 & 0.7 & 0.7 \\
CONS & 36 & -0.8 & -1.0 & -1.0 \\
ACCR & 1 & -2.7 & -3.2 & -3.2 \\
CMIF & 1139 & -2.4 & -2.9 & -2.9 \\
PROP & 514 & -1.9 & -2.5 & -2.3 \\
RBUS & 2132 & -2.6 & -3.1 & -3.1 \\
GOVT & 0 & 0.0 & 0.0 & 0.0 \\
EDUC & 30 & -3.3 & -3.7 & -3.6 \\
HEAL & 3 & -3.2 & -3.6 & -3.5 \\
CUPE & 138 & -2.8 & -3.3 & -3.2 \\
OWND & 0 & 0.0 & 0.0 & 0.0 \\
WHOL & 104 & -2.0 & -2.9 & -2.8 \\
RETT & 3154 & -2.4 & -2.9 & -2.8 \\
TRAN & 2137 & -1.3 & -2.4 & -2.3 \\
\hline All & 47497 & -4.3 & -4.5 & -4.4 \\
\hline
\end{tabular}

Table P.8: C.I.F. Imports 


\begin{tabular}{|l|c|ccc|}
\hline \multicolumn{5}{|c|}{ Industrial Investment } \\
\hline \multirow{2}{*}{ Industry } & BASEYEAR $(\$ \mathrm{M})$ & \multicolumn{1}{c|}{$\Delta$ ON BASEYEAR } \\
\cline { 2 - 5 } & & SIM201 & SIM301 & SIM401 \\
\hline AGRI & 1159 & -2.7 & -2.8 & -2.7 \\
FOLO & 128 & -4.9 & -4.7 & -4.6 \\
FISH & 41 & -1.9 & -1.4 & -1.4 \\
MINE & 184 & -6.4 & -5.3 & -5.4 \\
OIGA & 787 & -17.5 & -15.2 & -16.0 \\
PETR & 80 & -2.7 & 5.9 & 0.9 \\
FDBT & 954 & 0.1 & 0.4 & 0.4 \\
TWPM & 871 & -3.2 & -3.2 & -3.1 \\
CHNM & 640 & -3.4 & -3.2 & -3.2 \\
METL & 641 & -3.1 & -3.1 & -3.1 \\
EQFO & 500 & -2.4 & -2.4 & -2.4 \\
UTIL & 744 & 5.3 & 6.0 & 5.9 \\
CONS & 789 & -3.1 & -3.1 & -3.1 \\
ACCR & 333 & -2.1 & -2.1 & -2.1 \\
CMIF & 4534 & -2.7 & -3.0 & -2.9 \\
PROP & 1808 & -3.0 & -3.2 & -3.2 \\
RBUS & 2014 & -3.0 & -3.2 & -3.2 \\
GOVT & 3530 & -2.7 & -2.8 & -2.8 \\
EDUC & 1313 & -2.7 & -2.8 & -2.8 \\
HEAL & 884 & -2.7 & -2.8 & -2.8 \\
CUPE & 852 & -3.8 & -4.1 & -4.1 \\
OWND & 13345 & -2.7 & -2.8 & -2.8 \\
WHOL & 958 & -3.0 & -4.9 & -4.7 \\
RETT & 1065 & -3.6 & -4.5 & -4.4 \\
TRAN & 149 & 51.8 & 55.1 & 56.0 \\
\hline All & 38305 & -2.7 & -2.8 & -2.8 \\
\hline
\end{tabular}

Table P.9: Industrial Investment 


\begin{tabular}{|l|c|ccc|}
\hline \multicolumn{4}{|c|}{ Industry Capital Stocks } \\
\hline \multirow{2}{*}{ Industry } & BASEYEAR $(\$ \mathrm{M})$ & \multicolumn{2}{c|}{$\Delta$ ON BASEYEAR } \\
\cline { 2 - 5 } & & SIM201 & SIM301 & SIM401 \\
\hline FGRI & 14374 & -4.0 & -4.3 & -4.1 \\
FOLO & 1351 & -5.3 & -5.1 & -5.0 \\
FISH & 962 & -5.1 & -5.0 & -5.0 \\
OIGA & 878 & -8.0 & -7.0 & -7.1 \\
PETR & 4450 & -19.1 & -17.0 & -17.8 \\
FDBT & 455 & -4.7 & 3.6 & -1.2 \\
TWPM & 11491 & -4.1 & -4.2 & -4.1 \\
CHNM & 4052 & -4.6 & -4.7 & -4.7 \\
METL & 3959 & -5.0 & -4.8 & -4.8 \\
EQFO & 5892 & -4.3 & -4.4 & -4.4 \\
UTIL & 23050 & -3.7 & -3.8 & -3.8 \\
CONS & 5578 & -4.4 & -4.5 & -4.5 \\
ACCR & 5251 & -3.3 & -3.3 & -3.3 \\
CMIF & 15121 & -3.6 & -3.7 & -3.7 \\
PROP & 83623 & -4.4 & -4.6 & -4.5 \\
RBUS & 6376 & -4.0 & -4.3 & -4.6 \\
GOVT & 43300 & -4.9 & -5.1 & -5.1 \\
EDUC & 16100 & -4.2 & -4.4 & -4.4 \\
HEAL & 10847 & -4.8 & -5.1 & -5.1 \\
CUPE & 11086 & -4.2 & -4.5 & -4.5 \\
OWND & 163680 & 0.0 & 0.0 & 0.0 \\
WHOL & 8281 & -3.8 & -5.7 & -5.6 \\
RETT & 7265 & -4.2 & -5.2 & -5.1 \\
TRAN & 13992 & -5.7 & -6.3 & -5.7 \\
\hline All & 469826 & -3.0 & -3.2 & -3.2 \\
\hline
\end{tabular}

Table P.10: Industry Capital Stocks 


\begin{tabular}{|l|ccc|}
\hline \multicolumn{4}{|c|}{ Industry Capital Rents Index } \\
\hline \multirow{2}{*}{ Industry } & $\%$ ON BASEYEAR \\
\cline { 2 - 4 } & SIM201 & SIM301 & SIM401 \\
\hline AGRI & 1.9 & 1.9 & 1.9 \\
FOLO & 1.9 & 1.9 & 1.9 \\
FISH & 1.9 & 1.9 & 1.9 \\
MINE & 1.9 & 1.9 & 1.9 \\
OIGA & 1.9 & 1.9 & 1.9 \\
PETR & 1.9 & 1.9 & 1.9 \\
FDBT & 1.9 & 1.9 & 1.9 \\
TWPM & 1.9 & 1.8 & 1.9 \\
CHNM & 1.8 & 1.8 & 1.9 \\
METL & 1.9 & 1.8 & 1.9 \\
EQFO & 1.9 & 1.8 & 1.9 \\
UTIL & 1.9 & 1.8 & 1.9 \\
CONS & 1.9 & 1.8 & 1.9 \\
ACCR & 1.9 & 1.8 & 1.9 \\
CMIF & 1.9 & 1.8 & 1.9 \\
PROP & 1.9 & 1.8 & 1.9 \\
RBUS & 1.9 & 1.8 & 1.9 \\
GOVT & 1.9 & 1.8 & 1.9 \\
EDUC & 1.9 & 1.8 & 1.9 \\
HEAL & 1.9 & 1.8 & 1.9 \\
CUPE & 1.9 & 1.8 & 1.9 \\
OWND & -2.7 & -3.1 & -3.0 \\
WHOL & 1.9 & 1.8 & 1.9 \\
RETT & 1.9 & 1.8 & 1.9 \\
TRAN & 1.9 & 1.8 & 1.9 \\
\hline All & 1.3 & 1.2 & 1.2 \\
\hline
\end{tabular}

Table P.11: Industry Capital Rents Index 


\begin{tabular}{|c|c|c|c|c|}
\hline \multicolumn{5}{|c|}{ Industry Employment (Full-Time Equivalents) } \\
\hline \multirow{2}{*}{ Industry } & \multirow{2}{*}{ BASEYEAR (000s) } & \multicolumn{3}{|c|}{$\% \Delta$ ON BASEYEAR } \\
\hline & & SIM201 & SIM301 & $\operatorname{SIM} 401$ \\
\hline AGRI & 114 & 0.4 & 0.5 & 0.5 \\
\hline FOLO & 5 & -0.9 & -0.4 & -0.4 \\
\hline FISH & 2 & -0.8 & -0.3 & -0.3 \\
\hline MINE & 4 & -3.8 & -2.4 & -2.5 \\
\hline OIGA & 0 & -15.4 & -12.8 & -13.7 \\
\hline PETR & 6 & -0.2 & 8.8 & 3.7 \\
\hline FDBT & 56 & 0.3 & 0.6 & 0.6 \\
\hline TWPM & 65 & -0.3 & 0.0 & 0.0 \\
\hline CHNM & 21 & -0.7 & -0.1 & -0.2 \\
\hline METL & 26 & 0.1 & 0.3 & 0.2 \\
\hline EQFO & 50 & 0.7 & 1.0 & 1.0 \\
\hline UTIL & 9 & 0.0 & 0.2 & 0.2 \\
\hline CONS & 142 & 1.1 & 1.5 & 1.5 \\
\hline ACCR & 88 & 0.8 & 1.1 & 1.1 \\
\hline CMIF & 84 & 0.1 & 0.1 & 0.1 \\
\hline PROP & 48 & -0.1 & 0.1 & 0.1 \\
\hline RBUS & 198 & 0.4 & 0.4 & 0.4 \\
\hline GOVT & 77 & -0.6 & -0.4 & -0.5 \\
\hline EDUC & 120 & 0.2 & 0.3 & 0.3 \\
\hline HEAL & 164 & -0.5 & -0.4 & -0.4 \\
\hline CUPE & 74 & 0.2 & 0.2 & 0.2 \\
\hline OWND & 0 & 0.0 & 0.0 & 0.0 \\
\hline WHOL & 89 & 0.5 & -1.1 & -1.0 \\
\hline RETT & 163 & 0.1 & -0.5 & -0.5 \\
\hline TRAN & 62 & -1.4 & -1.6 & -1.2 \\
\hline All & 1668 & 0.0 & 0.0 & 0.0 \\
\hline
\end{tabular}

Table P.12: Industry Employment 


\begin{tabular}{|c|c|c|c|c|}
\hline \multicolumn{5}{|c|}{ Regional Employment (Full-Time Equivalents) } \\
\hline \multirow{2}{*}{ Region } & \multirow{2}{*}{ BASEYEAR (000s) } & \multicolumn{3}{|c|}{$\% \Delta$ ON BASEYEAR } \\
\hline & & SIM201 & SIM301 & $\operatorname{SIM} 401$ \\
\hline AKL & 533 & 0.2 & 0.0 & 0.1 \\
\hline WLG & 200 & -0.1 & -0.1 & -0.1 \\
\hline ONI & 514 & 0.0 & 0.1 & 0.0 \\
\hline CAN & 227 & 0.1 & 0.0 & 0.1 \\
\hline OSI & 195 & 0.0 & 0.1 & 0.2 \\
\hline $\mathrm{NZ}$ & 1668 & 0.0 & 0.0 & 0.0 \\
\hline
\end{tabular}

Table P.13: Regional Employment

\begin{tabular}{|c|c|c|c|}
\hline \multicolumn{4}{|c|}{ Compensating Variation } \\
\hline \multirow{2}{*}{ Region } & \multicolumn{3}{|c|}{$\Delta$ IN INCOME $(\$ M)$} \\
\hline & SIM201 & SIM301 & SIM401 \\
\hline AKL & -972.9 & -1113.5 & -1081.3 \\
\hline WLG & -418.3 & -450.8 & -446.0 \\
\hline ONI & -1101.4 & -1120.4 & -1150.1 \\
\hline $\mathrm{CAN}$ & -318.1 & -353.6 & -346.1 \\
\hline OSI & -394.5 & -423.0 & -409.3 \\
\hline \multicolumn{4}{|c|}{ Equivalent Variation } \\
\hline \multirow{2}{*}{ Region } & \multicolumn{3}{|c|}{$\Delta \mathrm{IN} \operatorname{INCOME}(\$ \mathrm{M})$} \\
\hline & SIM201 & SIM301 & $\operatorname{SIM} 401$ \\
\hline AKL & -971.8 & -1113.8 & -1081.1 \\
\hline WLG & -417.2 & -450.2 & -445.2 \\
\hline ONI & -1100.1 & -1120.3 & -1150.1 \\
\hline CAN & -317.4 & -353.2 & -345.7 \\
\hline OSI & -393.5 & -422.4 & -408.7 \\
\hline
\end{tabular}

Table P.14: CV and EV Measures 


\begin{tabular}{|l|c|ccc|}
\hline \multicolumn{5}{|c|}{ Household Real Disposable Income } \\
\hline \multirow{2}{*}{ Region } & BASEYEAR $(\$ \mathrm{M})$ & \multicolumn{3}{|c|}{$\%$ ON BASEYEAR } \\
\cline { 3 - 5 } & & SIM201 & SIM301 & SIM401 \\
\hline AKL & 41312 & -1.9 & -2.2 & -2.1 \\
WLG & 14759 & -2.2 & -2.3 & -2.3 \\
ONI & 36391 & -2.7 & -2.7 & -2.8 \\
CAN & 12853 & -1.9 & -2.1 & -2.1 \\
OSI & 15213 & -2.0 & -2.1 & -2.0 \\
\hline NZ & 120529 & -2.2 & -2.3 & -2.3 \\
\hline
\end{tabular}

Table P.15: Household Real Disposable Income

\begin{tabular}{|l|c|ccc|}
\hline \multicolumn{5}{|c|}{ Household Real Spending } \\
\hline \multirow{2}{*}{ Region } & BASEYEAR $(\$ \mathrm{M})$ & \multicolumn{3}{|c|}{$\%$ ON BASEYEAR } \\
\cline { 3 - 5 } & & SIM201 & SIM301 & SIM401 \\
\hline AKL & 31830 & -3.1 & -3.5 & -3.4 \\
WLG & 11765 & -3.5 & -3.8 & -3.7 \\
ONI & 27565 & -3.9 & -4.0 & -4.1 \\
CAN & 10063 & -3.2 & -3.5 & -3.4 \\
OSI & 12108 & -3.2 & -3.5 & -3.4 \\
\hline NZ & 93331 & -3.4 & -3.7 & -3.6 \\
\hline
\end{tabular}

Table P.16: Household Real Spending

\begin{tabular}{|c|c|c|c|}
\hline \multicolumn{4}{|c|}{ Purchase Prices - Commodity PETR } \\
\hline \multirow{2}{*}{ Region } & \multicolumn{3}{|c|}{$\% \Delta$ ON BASEYEAR } \\
\hline & SIM201 & SIM301 & SIM401 \\
\hline AKL & 47.6 & 33.0 & 34.9 \\
\hline WLG & 47.6 & 36.8 & 37.2 \\
\hline ONI & 47.6 & 36.7 & 36.6 \\
\hline $\mathrm{CAN}$ & 47.6 & 34.5 & 35.5 \\
\hline OSI & 47.6 & 36.9 & 36.8 \\
\hline
\end{tabular}

Table P.17: Household Purchase Prices - Commodity PETR 


\begin{tabular}{|l|c|ccc|}
\hline \multicolumn{5}{|c|}{ Real Macro Measures } \\
\hline \multirow{2}{*}{ Variable } & BASEYEAR $(\$ \mathrm{M})$ & \multicolumn{3}{|c|}{$\Delta$ ON BASEYEAR } \\
\cline { 2 - 5 } & & SIM201 & SIM301 & SIM401 \\
\hline GDP (Expenditure) & 156090 & -2.7 & -2.8 & -2.8 \\
GDP (Income) & 156090 & -2.4 & -2.5 & -2.5 \\
Private Consumption & 93331 & -3.4 & -3.7 & -3.6 \\
Investment & 38305 & -2.7 & -2.8 & -2.8 \\
Government Consumption & 28661 & -2.7 & -2.8 & -2.8 \\
F.O.B. Exports & 43290 & -3.1 & -2.7 & -2.7 \\
C.I.F. Imports & 47497 & -4.3 & -4.5 & -4.4 \\
Trade Balance & -4207 & 17.7 & 23.0 & 21.8 \\
Government Balance & 6900 & -4.4 & -3.9 & -4.3 \\
Domestic Private Saving & 27198 & 1.9 & 2.4 & 2.3 \\
Domestic Saving & 34098 & 0.7 & 1.1 & 1.0 \\
\hline
\end{tabular}

Table P.18: Macro Measures

\begin{tabular}{|l|ccc|}
\hline \multicolumn{4}{|c|}{ Economy-wide Price Measures } \\
\hline \multirow{2}{*}{ Variable } & \multicolumn{1}{|c|}{$\Delta$ ON BASEYEAR } \\
\cline { 2 - 4 } GDP Deflator & SIM201 & SIM301 & SIM401 \\
Consumer Price Index & -0.3 & -0.4 & -0.4 \\
Investment Price Index & 0.7 & 0.6 & 0.7 \\
Government Price Index & 1.9 & 1.8 & 1.9 \\
Export Price Index (F.O.B.) & -0.5 & -0.7 & -0.7 \\
Import Price Index (C.I.F.) & 0.8 & 0.7 & 0.7 \\
Trade Price Index & 4.2 & 4.4 & 4.3 \\
Real Exchange Rate & 41.0 & 45.7 & 45.1 \\
Nominal After-Tax Wage & 4.4 & 4.8 & 4.7 \\
Real After-Tax Wage & -2.6 & -3.0 & -2.9 \\
Capital Rents Index & -3.2 & -3.6 & -3.5 \\
Output Price Index & 1.3 & 1.2 & 1.2 \\
\hline Current Net Rate of Return (Average) & -2.0 & -2.1 & -2.1 \\
Expected Future Net Rate of Return & -3.3 & -3.5 & -3.5 \\
\hline
\end{tabular}

Table P.19: Economy-wide Price Measures 


\section{Appendix Q}

\section{Simulation Results - Short-Run Immigration Scenarios}

This appendix provides the short-run results for the illustrative simulations discussed in chapter 4 .

\begin{tabular}{|l|c|cccccc|}
\hline \multicolumn{7}{|c|}{ Number of Households } \\
\hline \multirow{2}{*}{ Region } & \multirow{2}{*}{ BASEYEAR (000s) } & \multicolumn{7}{|c|}{$\% \Delta$ ON BASEYEAR } \\
\cline { 2 - 8 } & & BSLN5 & SIM501 & SIM502 & BSLN6 & SIM601 & SIM602 \\
\hline AKL & 434 & 21.0 & 44.1 & 21.0 & 19.4 & 34.2 & 21.7 \\
WLG & 167 & 13.0 & 13.0 & 13.0 & 13.5 & 18.7 & 18.4 \\
ONI & 482 & 12.0 & 12.0 & 12.0 & 12.4 & 14.4 & 14.2 \\
CAN & 200 & 14.0 & 14.0 & 39.0 & 14.5 & 18.9 & 33.4 \\
OSI & 172 & 10.0 & 10.0 & 39.1 & 11.7 & 17.2 & 32.6 \\
\hline NZ & 1454 & 14.8 & 21.7 & 21.7 & 14.8 & 21.7 & 21.7 \\
\hline
\end{tabular}

Table Q.1: Number of Households 


\begin{tabular}{|l|c|cccccc|}
\hline \multicolumn{7}{|c|}{ Total Population } \\
\hline \multirow{2}{*}{ Region } & BASEYEAR (000s) & \multicolumn{7}{|c|}{$\% \Delta$ ON BASEYEAR } \\
\cline { 2 - 8 } & & BSLN5 & SIM501 & SIM502 & BSLN6 & SIM601 & SIM602 \\
\hline AKL & 1371 & 17.0 & 39.3 & 17.0 & 15.5 & 29.7 & 17.5 \\
WLG & 466 & 9.0 & 9.0 & 9.0 & 9.6 & 15.2 & 14.1 \\
ONI & 1348 & 7.0 & 7.0 & 7.0 & 7.5 & 9.6 & 9.1 \\
CAN & 540 & 10.0 & 10.0 & 34.1 & 10.6 & 15.6 & 28.8 \\
OSI & 459 & 6.0 & 6.0 & 34.0 & 7.9 & 14.1 & 27.7 \\
\hline NZ & 4184 & 10.8 & 18.1 & 17.0 & 10.8 & 18.1 & 17.0 \\
\hline
\end{tabular}

Table Q.2: Total Population

\begin{tabular}{|l|c|cccccc|}
\hline \multicolumn{7}{|c|}{ Working-Age Population } \\
\hline \multirow{2}{*}{ Region } & \multirow{2}{*}{ BASEYEAR (000s) } & \multicolumn{7}{|c|}{$\% \Delta$ ON BASEYEAR } \\
\cline { 3 - 8 } & & BSLN5 & SIM501 & SIM502 & BSLN6 & SIM601 & SIM602 \\
\hline AKL & 849 & 15.2 & 37.1 & 15.2 & 13.6 & 27.7 & 15.6 \\
WLG & 301 & 6.3 & 6.3 & 6.3 & 6.8 & 12.1 & 11.2 \\
ONI & 814 & 3.7 & 3.7 & 3.7 & 4.2 & 6.3 & 5.8 \\
CAN & 347 & 6.1 & 6.1 & 29.4 & 6.7 & 11.4 & 24.2 \\
OSI & 296 & 2.2 & 2.2 & 29.2 & 3.9 & 9.8 & 23.1 \\
\hline NZ & 2607 & 7.9 & 15.0 & 14.0 & 7.9 & 15.0 & 14.0 \\
\hline
\end{tabular}

Table Q.3: Working-Age Population

\begin{tabular}{|l|c|cccccc|}
\hline \multicolumn{7}{|c|}{ Non-Working Age Population } \\
\hline \multirow{2}{*}{ Region } & \multirow{2}{*}{ BASEYEAR (000s) } & \multicolumn{7}{|c|}{$\% \Delta$ ON BASEYEAR } \\
\cline { 3 - 8 } & & BSLN5 & SIM501 & SIM502 & BSLN6 & SIM601 & SIM602 \\
\hline AKL & 522 & 20.0 & 42.9 & 20.0 & 18.4 & 33.1 & 20.4 \\
WLG & 165 & 14.0 & 14.0 & 14.0 & 14.7 & 20.9 & 19.5 \\
ONI & 534 & 12.0 & 12.0 & 12.0 & 12.5 & 14.5 & 14.0 \\
CAN & 193 & 17.0 & 17.0 & 42.7 & 17.7 & 23.1 & 36.9 \\
OSI & 163 & 13.0 & 13.0 & 42.9 & 15.1 & 21.9 & 36.2 \\
\hline NZ & 1577 & 15.6 & 23.1 & 21.8 & 15.6 & 23.1 & 21.8 \\
\hline
\end{tabular}

Table Q.4: Non-Working Age Population 


\begin{tabular}{|l|c|cccccc|}
\hline \multicolumn{7}{|c|}{ Regional Employment (Full-Time Equivalents) } \\
\hline \multirow{2}{*}{ Region } & \multirow{2}{*}{ BASEYEAR (000s) } & \multicolumn{5}{|c|}{$\% \Delta$ ON BASEYEAR } \\
\cline { 3 - 8 } & BSLN5 & SIM501 & SIM502 & BSLN6 & SIM601 & SIM602 \\
\hline AKL & 533 & 15.8 & 38.6 & 15.5 & 14.3 & 29.1 & 16.1 \\
WLG & 200 & 6.2 & 6.0 & 6.0 & 6.8 & 11.6 & 10.9 \\
ONI & 514 & 3.4 & 3.1 & 3.2 & 3.9 & 5.7 & 5.4 \\
CAN & 227 & 6.0 & 5.8 & 30.0 & 6.6 & 10.8 & 24.8 \\
OSI & 195 & 1.8 & 1.6 & 29.7 & 3.5 & 8.8 & 23.6 \\
\hline NZ & 1668 & 7.9 & 15.0 & 14.2 & 7.9 & 14.9 & 14.2 \\
\hline
\end{tabular}

Table Q.5: Regional Employment

\begin{tabular}{|l|c|cccccc|}
\hline \multicolumn{7}{|c|}{ Regional Capital Stocks } \\
\hline \multirow{2}{*}{ Region } & \multirow{2}{*}{ BASEYEAR $(\$ \mathrm{M})$} & \multicolumn{7}{|c|}{$\% \Delta$ ON BASEYEAR } \\
\cline { 2 - 8 } & & BSLN5 & SIM501 & SIM502 & BSLN6 & SIM601 & SIM602 \\
\hline AKL & 154208 & 18.4 & 18.4 & 18.4 & 18.4 & 18.4 & 18.4 \\
WLG & 62648 & 8.5 & 8.5 & 8.5 & 8.5 & 8.5 & 8.5 \\
ONI & 144170 & 6.0 & 6.0 & 6.0 & 6.0 & 6.0 & 6.0 \\
CAN & 49536 & 8.7 & 8.7 & 8.7 & 8.7 & 8.7 & 8.7 \\
OSI & 59263 & 4.3 & 4.3 & 4.3 & 4.3 & 4.3 & 4.3 \\
\hline NZ & 469826 & 10.5 & 10.5 & 10.5 & 10.5 & 10.5 & 10.5 \\
\hline
\end{tabular}

Table Q.6: Regional Capital Stocks 


\begin{tabular}{|l|c|cccccc|}
\hline \multicolumn{7}{|c|}{ Regional Output } \\
\hline \multirow{2}{*}{ Region } & \multirow{2}{*}{ BASEYEAR $(\$ \mathrm{M})$} & \multicolumn{7}{|c|}{$\% \Delta$ ON BASEYEAR } \\
\cline { 3 - 8 } & & BSLN5 & SIM501 & SIM502 & BSLN6 & SIM601 & SIM602 \\
\hline AKL & 106148 & 14.2 & 23.0 & 14.6 & 13.6 & 19.8 & 14.8 \\
WLG & 36082 & 6.6 & 7.1 & 6.9 & 6.8 & 9.3 & 8.9 \\
ONI & 100332 & 4.5 & 5.1 & 4.9 & 4.7 & 6.1 & 5.8 \\
CAN & 33657 & 6.4 & 7.0 & 15.8 & 6.7 & 9.0 & 14.1 \\
OSI & 43428 & 3.1 & 3.6 & 13.8 & 3.8 & 6.6 & 11.8 \\
\hline NZ & 319647 & 8.0 & 11.3 & 10.7 & 7.9 & 11.4 & 10.8 \\
\hline
\end{tabular}

Table Q.7: Regional Output

\begin{tabular}{|l|c|cccccc|}
\hline \multicolumn{7}{|c|}{ Regional Investment } \\
\hline \multirow{2}{*}{ Region } & \multirow{2}{*}{ BASEYEAR $(\$ \mathrm{M})$} & \multicolumn{7}{|c|}{$\% \Delta$ ON BASEYEAR } \\
\cline { 2 - 8 } & & BSLN5 & SIM501 & SIM502 & BSLN6 & SIM601 & SIM602 \\
\hline AKL & 12867 & 9.5 & 16.1 & 10.7 & 9.1 & 14.3 & 10.9 \\
WLG & 5434 & 7.6 & 9.4 & 9.2 & 7.7 & 10.7 & 10.3 \\
ONI & 11490 & 7.6 & 9.3 & 8.8 & 7.7 & 9.9 & 9.5 \\
CAN & 3974 & 7.7 & 8.9 & 14.7 & 7.8 & 10.5 & 13.7 \\
OSI & 4540 & 7.5 & 8.9 & 16.3 & 8.1 & 11.3 & 15.0 \\
\hline NZ & 38305 & 8.2 & 11.5 & 11.0 & 8.2 & 11.7 & 11.2 \\
\hline
\end{tabular}

Table Q.8: Regional Investment

\begin{tabular}{|l|cccccc|}
\hline \multicolumn{7}{|c|}{ Regional Output Price Index } \\
\hline \multirow{2}{*}{ Region } & \multicolumn{7}{|c|}{$\% \Delta$ ON BASEYEAR } \\
\cline { 2 - 7 } & BSLN5 & SIM501 & SIM502 & BSLN6 & SIM601 & SIM602 \\
\hline AKL & -4.7 & -7.6 & -3.8 & -4.4 & -6.3 & -4.0 \\
WLG & -1.7 & -1.3 & -0.6 & -1.8 & -2.3 & -1.9 \\
ONI & -0.5 & 0.3 & 0.2 & -0.6 & -0.3 & -0.3 \\
CAN & -1.6 & -1.2 & -4.6 & -1.6 & -2.0 & -4.0 \\
OSI & 0.0 & 0.6 & -3.6 & -0.3 & -0.7 & -2.9 \\
\hline NZ & -2.1 & -2.8 & -2.3 & -2.1 & -2.8 & -2.5 \\
\hline
\end{tabular}

Table Q.9: Regional Output Price Index 


\begin{tabular}{|l|cccccc|}
\hline \multicolumn{7}{|c|}{ Regional Investment Price Index } \\
\hline \multirow{2}{*}{ Region } & \multicolumn{5}{|c|}{$\% \Delta$ ON BASEYEAR } \\
\cline { 2 - 7 } & BSLN5 & SIM501 & SIM502 & BSLN6 & SIM601 & SIM602 \\
\hline AKL & -1.6 & -2.6 & -1.7 & -1.6 & -2.4 & -1.8 \\
WLG & -1.4 & -1.9 & -1.4 & -1.3 & -1.9 & -1.6 \\
ONI & -1.3 & -1.8 & -1.3 & -1.3 & -1.8 & -1.5 \\
CAN & -1.4 & -1.9 & -1.9 & -1.3 & -1.9 & -1.9 \\
OSI & -1.2 & -1.8 & -1.8 & -1.3 & -1.8 & -1.8 \\
\hline
\end{tabular}

Table Q.10: Regional Investment Price Index

\begin{tabular}{|l|cccccc|}
\hline \multicolumn{7}{|c|}{ Regional Capital Rents Index } \\
\hline \multirow{2}{*}{ Region } & \multicolumn{5}{|c|}{$\%$ ON BASEYEAR } \\
\cline { 2 - 7 } & BSLN5 & SIM501 & SIM502 & BSLN6 & SIM601 & SIM602 \\
\hline AKL & -9.0 & 1.4 & -6.9 & -9.6 & -1.8 & -6.8 \\
WLG & -2.5 & -0.6 & -0.1 & -2.2 & 2.2 & 2.0 \\
ONI & -0.2 & 2.5 & 1.9 & 0.0 & 3.6 & 2.9 \\
CAN & -2.4 & -0.5 & 10.6 & -2.2 & 2.1 & 8.3 \\
OSI & 1.0 & 3.4 & 16.9 & 1.9 & 7.3 & 14.1 \\
\hline NZ & -3.7 & 1.5 & 1.2 & -3.7 & 1.8 & 1.3 \\
\hline
\end{tabular}

Table Q.11: Regional Capital Rents Index

\begin{tabular}{|l|c|cccccc|}
\hline \multicolumn{7}{|c|}{ Regional Average Net Rates of Return } \\
\hline \multirow{2}{*}{ Region } & \multirow{2}{*}{ BASEYEAR (\%) } & \multicolumn{7}{|c|}{$\% \Delta$ ON BASEYEAR } \\
\cline { 3 - 8 } & & BSLN5 & SIM501 & SIM502 & BSLN6 & SIM601 & SIM602 \\
\hline AKL & 10.1 & -11.8 & 4.8 & -8.8 & -12.7 & -0.2 & -8.4 \\
WLG & 8.2 & -3.6 & 0.2 & 0.1 & -3.2 & 4.4 & 3.6 \\
ONI & 9.5 & -0.2 & 4.4 & 2.8 & 0.0 & 6.1 & 4.6 \\
CAN & 9.5 & -3.6 & 0.0 & 16.3 & -3.2 & 3.9 & 13.0 \\
OSI & 9.0 & 1.5 & 5.9 & 26.2 & 2.9 & 11.8 & 22.0 \\
\hline NZ & 9.4 & -4.9 & 4.0 & 2.4 & -4.9 & 4.2 & 2.7 \\
\hline
\end{tabular}

Table Q.12: Regional Average Net Rates of Return 


\begin{tabular}{|c|c|c|c|c|c|c|c|}
\hline \multicolumn{8}{|c|}{ Industry Employment (Full-Time Equivalents) } \\
\hline \multirow{2}{*}{ Industry } & \multirow{2}{*}{ BASEYEAR (000S) } & \multicolumn{6}{|c|}{$\% \Delta$ ON BASEYEAR } \\
\hline & & BSLN5 & SIM501 & SIM502 & BSLN6 & SIM601 & SIM602 \\
\hline AGRI & 114 & 5.4 & 8.3 & 14.3 & 5.9 & 10.9 & 13.9 \\
\hline FOLO & 5 & 5.6 & 8.1 & 13.5 & 6.1 & 10.9 & 13.5 \\
\hline FISH & 2 & 4.8 & 6.9 & 17.7 & 5.5 & 10.7 & 16.3 \\
\hline MINE & 4 & 6.3 & 9.5 & 14.1 & 6.7 & 11.7 & 13.8 \\
\hline OIGA & 0 & 5.4 & 7.0 & 6.5 & 5.8 & 9.0 & 8.4 \\
\hline PETR & 6 & 5.9 & 7.0 & 7.3 & 6.3 & 9.2 & 9.0 \\
\hline FDBT & 56 & 5.7 & 10.5 & 15.1 & 6.0 & 12.3 & 14.6 \\
\hline TWPM & 65 & 9.4 & 18.7 & 15.5 & 9.2 & 17.7 & 15.7 \\
\hline CHNM & 21 & 9.7 & 20.3 & 15.7 & 9.4 & 18.5 & 15.8 \\
\hline METL & 26 & 9.9 & 19.1 & 15.3 & 9.7 & 17.9 & 15.5 \\
\hline EQFO & 50 & 10.3 & 21.2 & 17.8 & 10.1 & 19.7 & 17.7 \\
\hline UTIL & 9 & 7.9 & 16.6 & 15.9 & 7.9 & 17.0 & 16.1 \\
\hline CONS & 142 & 8.3 & 15.2 & 14.5 & 8.3 & 15.3 & 14.6 \\
\hline ACCR & 88 & 7.1 & 14.1 & 15.3 & 7.2 & 14.7 & 15.0 \\
\hline CMIF & 84 & 8.6 & 18.0 & 13.5 & 8.4 & 16.7 & 13.9 \\
\hline PROP & 48 & 8.0 & 16.4 & 14.2 & 7.9 & 16.0 & 14.4 \\
\hline RBUS & 198 & 8.3 & 16.6 & 13.6 & 8.1 & 15.8 & 13.9 \\
\hline GOVT & 77 & 8.2 & 14.3 & 13.5 & 8.2 & 14.3 & 13.6 \\
\hline EDUC & 120 & 8.1 & 15.4 & 14.0 & 8.1 & 15.1 & 14.1 \\
\hline HEAL & 164 & 8.1 & 14.3 & 13.6 & 8.1 & 14.2 & 13.6 \\
\hline CUPE & 74 & 8.0 & 15.3 & 13.9 & 7.9 & 15.2 & 14.1 \\
\hline OWND & 0 & 0.0 & 0.0 & 0.0 & 0.0 & 0.0 & 0.0 \\
\hline WHOL & 89 & 8.5 & 17.2 & 13.8 & 8.3 & 16.0 & 13.8 \\
\hline RETT & 163 & 7.4 & 13.6 & 13.6 & 7.4 & 13.9 & 13.6 \\
\hline TRAN & 62 & 8.1 & 16.0 & 15.1 & 8.0 & 15.8 & 14.9 \\
\hline All & 1668 & 7.9 & 15.0 & 14.2 & 7.9 & 14.9 & 14.2 \\
\hline
\end{tabular}

Table Q.13: Industry Employment 


\begin{tabular}{|c|c|c|c|c|c|c|c|}
\hline \multicolumn{8}{|c|}{ Industry Capital Stocks } \\
\hline \multirow{2}{*}{ Industry } & \multirow{2}{*}{ BASEYEAR $(\$ M)$} & \multicolumn{6}{|c|}{$\% \Delta$ ON BASEYEAR } \\
\hline & & BSLN5 & SIM501 & SIM502 & BSLN6 & SIM601 & SIM602 \\
\hline AGRI & 14374 & 4.5 & 4.5 & 4.5 & 4.5 & 4.5 & 4.5 \\
\hline FOLO & 1351 & 5.4 & 5.4 & 5.4 & 5.4 & 5.4 & 5.4 \\
\hline FISH & 962 & 4.3 & 4.3 & 4.3 & 4.3 & 4.3 & 4.3 \\
\hline MINE & 878 & 5.8 & 5.8 & 5.8 & 5.8 & 5.8 & 5.8 \\
\hline OIGA & 4450 & 5.4 & 5.4 & 5.4 & 5.4 & 5.4 & 5.4 \\
\hline PETR & 455 & 5.9 & 5.9 & 5.9 & 5.9 & 5.9 & 5.9 \\
\hline FDBT & 11491 & 5.3 & 5.3 & 5.3 & 5.3 & 5.3 & 5.3 \\
\hline TWPM & 8052 & 8.2 & 8.2 & 8.2 & 8.2 & 8.2 & 8.2 \\
\hline CHNM & 4959 & 10.8 & 10.8 & 10.8 & 10.8 & 10.8 & 10.8 \\
\hline METL & 3412 & 9.9 & 9.9 & 9.9 & 9.9 & 9.9 & 9.9 \\
\hline EQFO & 5892 & 11.5 & 11.5 & 11.5 & 11.5 & 11.5 & 11.5 \\
\hline UTIL & 23050 & 7.6 & 7.6 & 7.6 & 7.6 & 7.6 & 7.6 \\
\hline CONS & 5578 & 8.2 & 8.2 & 8.2 & 8.2 & 8.2 & 8.2 \\
\hline ACCR & 5251 & 7.0 & 7.0 & 7.0 & 7.0 & 7.0 & 7.0 \\
\hline CMIF & 15121 & 8.7 & 8.7 & 8.7 & 8.7 & 8.7 & 8.7 \\
\hline PROP & 83623 & 8.3 & 8.3 & 8.3 & 8.3 & 8.3 & 8.3 \\
\hline RBUS & 6376 & 8.9 & 8.9 & 8.9 & 8.9 & 8.9 & 8.9 \\
\hline GOVT & 43300 & 8.1 & 8.1 & 8.1 & 8.1 & 8.1 & 8.1 \\
\hline EDUC & 16100 & 8.2 & 8.2 & 8.2 & 8.2 & 8.2 & 8.2 \\
\hline HEAL & 10847 & 8.1 & 8.1 & 8.1 & 8.1 & 8.1 & 8.1 \\
\hline CUPE & 11086 & 8.2 & 8.2 & 8.2 & 8.2 & 8.2 & 8.2 \\
\hline OWND & 163680 & 15.2 & 15.2 & 15.2 & 15.2 & 15.2 & 15.2 \\
\hline WHOL & 8281 & 8.7 & 8.7 & 8.7 & 8.7 & 8.7 & 8.7 \\
\hline RETT & 7265 & 7.4 & 7.4 & 7.4 & 7.4 & 7.4 & 7.4 \\
\hline TRAN & 13992 & 8.6 & 8.6 & 8.6 & 8.6 & 8.6 & 8.6 \\
\hline All & 469826 & 10.5 & 10.5 & 10.5 & 10.5 & 10.5 & 10.5 \\
\hline
\end{tabular}

Table Q.14: Industry Capital Stocks 


\begin{tabular}{|c|c|c|c|c|c|c|c|}
\hline \multicolumn{8}{|c|}{ Industrial Output } \\
\hline \multirow{2}{*}{ Industry } & \multirow{2}{*}{ BASEYEAR $(\$ M)$} & \multicolumn{6}{|c|}{$\% \Delta$ ON BASEYEAR } \\
\hline & & BSLN5 & SIM501 & SIM502 & BSLN6 & SIM601 & SIM602 \\
\hline AGRI & 16884 & 5.2 & 6.9 & 9.3 & 5.4 & 8.2 & 9.3 \\
\hline FOLO & 2978 & 6.3 & 8.5 & 8.9 & 6.4 & 9.2 & 9.2 \\
\hline FISH & 853 & 5.2 & 6.9 & 9.6 & 5.4 & 8.2 & 9.4 \\
\hline MINE & 1197 & 6.4 & 7.9 & 8.5 & 6.5 & 8.4 & 8.6 \\
\hline OIGA & 2479 & 5.6 & 6.0 & 5.9 & 5.6 & 6.2 & 6.1 \\
\hline PETR & 3683 & 6.7 & 8.3 & 8.2 & 6.8 & 8.6 & 8.5 \\
\hline FDBT & 27682 & 5.4 & 7.6 & 9.8 & 5.7 & 8.8 & 9.8 \\
\hline TWPM & 13057 & 8.0 & 11.8 & 10.7 & 8.0 & 11.8 & 11.0 \\
\hline CHNM & 9078 & 9.5 & 14.1 & 12.0 & 9.4 & 13.5 & 12.1 \\
\hline METL & 8105 & 9.5 & 13.2 & 11.5 & 9.4 & 12.9 & 11.7 \\
\hline EQFO & 10632 & 10.8 & 17.1 & 13.7 & 10.5 & 16.2 & 14.0 \\
\hline UTIL & 12609 & 7.6 & 10.6 & 10.0 & 7.6 & 10.7 & 10.1 \\
\hline CONS & 29305 & 8.1 & 11.4 & 10.8 & 8.1 & 11.5 & 10.9 \\
\hline ACCR & 6454 & 6.8 & 10.4 & 10.8 & 6.9 & 10.9 & 10.9 \\
\hline CMIF & 24027 & 8.4 & 11.8 & 10.3 & 8.3 & 11.6 & 10.5 \\
\hline PROP & 13658 & 8.2 & 10.0 & 9.5 & 8.1 & 10.0 & 9.6 \\
\hline RBUS & 24532 & 8.5 & 12.9 & 10.9 & 8.4 & 12.5 & 11.2 \\
\hline GOVT & 13460 & 8.2 & 11.8 & 11.0 & 8.2 & 11.8 & 11.2 \\
\hline EDUC & 8229 & 8.2 & 13.9 & 12.5 & 8.1 & 13.6 & 12.6 \\
\hline HEAL & 12552 & 8.1 & 11.8 & 11.2 & 8.1 & 11.9 & 11.3 \\
\hline CUPE & 11764 & 8.0 & 11.8 & 10.6 & 8.0 & 11.7 & 10.8 \\
\hline OWND & 12472 & 12.5 & 13.6 & 13.3 & 12.5 & 13.6 & 13.4 \\
\hline WHOL & 20487 & 8.0 & 11.8 & 10.6 & 8.0 & 11.6 & 10.7 \\
\hline RETT & 17998 & 7.4 & 10.5 & 10.1 & 7.4 & 10.6 & 10.2 \\
\hline TRAN & 15472 & 8.2 & 12.3 & 11.1 & 8.1 & 12.1 & 11.2 \\
\hline All & 319647 & 8.0 & 11.3 & 10.7 & 7.9 & 11.4 & 10.8 \\
\hline
\end{tabular}

Table Q.15: Industrial Output 


\begin{tabular}{|c|c|c|c|c|c|c|c|}
\hline \multicolumn{8}{|c|}{ Industrial Investment } \\
\hline \multirow{2}{*}{ Industry } & \multirow{2}{*}{ BASEYEAR $(\$ M)$} & \multicolumn{6}{|c|}{$\% \Delta$ ON BASEYEAR } \\
\hline & & BSLN5 & SIM501 & SIM502 & BSLN6 & SIM601 & SIM602 \\
\hline AGRI & 1159 & 7.0 & 7.9 & 9.1 & 7.2 & 8.9 & 9.4 \\
\hline FOLO & 128 & 5.9 & 6.0 & 6.0 & 6.0 & 6.1 & 6.1 \\
\hline FISH & 41 & 12.3 & 16.8 & 26.0 & 13.3 & 22.0 & 26.1 \\
\hline MINE & 184 & 8.5 & 9.1 & 9.3 & 8.5 & 9.5 & 9.5 \\
\hline OIGA & 787 & 8.5 & 8.4 & 8.2 & 8.6 & 8.5 & 8.4 \\
\hline PETR & 80 & 9.3 & 9.0 & 9.4 & 9.4 & 9.5 & 9.7 \\
\hline FDBT & 954 & 9.5 & 13.1 & 19.8 & 10.2 & 17.2 & 20.1 \\
\hline TWPM & 871 & 8.5 & 11.2 & 9.6 & 8.4 & 11.2 & 10.1 \\
\hline CHNM & 640 & 9.2 & 12.0 & 10.0 & 9.1 & 11.4 & 10.2 \\
\hline METL & 641 & 9.9 & 11.1 & 9.9 & 9.8 & 10.8 & 10.1 \\
\hline EQFO & 500 & 10.9 & 14.5 & 11.6 & 10.7 & 13.8 & 11.9 \\
\hline UTIL & 744 & 12.5 & 36.4 & 33.3 & 12.4 & 36.5 & 33.3 \\
\hline CONS & 789 & 8.3 & 8.4 & 8.3 & 8.3 & 8.4 & 8.3 \\
\hline ACCR & 333 & 7.5 & 8.9 & 9.2 & 7.6 & 9.4 & 9.3 \\
\hline CMIF & 4534 & 7.9 & 9.3 & 8.9 & 7.9 & 9.3 & 9.0 \\
\hline PROP & 1808 & 8.1 & 10.3 & 9.7 & 8.1 & 10.3 & 9.8 \\
\hline RBUS & 2014 & 8.5 & 8.9 & 8.8 & 8.5 & 8.9 & 8.8 \\
\hline GOVT & 3530 & 8.2 & 11.5 & 11.0 & 8.2 & 11.7 & 11.2 \\
\hline EDUC & 1313 & 8.2 & 11.5 & 11.0 & 8.2 & 11.7 & 11.2 \\
\hline HEAL & 884 & 8.2 & 11.5 & 11.0 & 8.2 & 11.7 & 11.2 \\
\hline CUPE & 852 & 8.1 & 8.6 & 8.4 & 8.1 & 8.6 & 8.4 \\
\hline OWND & 13345 & 8.2 & 11.5 & 11.0 & 8.2 & 11.7 & 11.2 \\
\hline WHOL & 958 & 8.2 & 8.5 & 8.6 & 8.2 & 8.6 & 8.6 \\
\hline RETT & 1065 & 7.6 & 7.8 & 7.9 & 7.7 & 7.8 & 7.9 \\
\hline TRAN & 149 & -23.7 & 121.6 & 68.8 & -27.6 & 115.5 & 77.6 \\
\hline All & 38305 & 8.2 & 11.5 & 11.0 & 8.2 & 11.7 & 11.2 \\
\hline
\end{tabular}

Table Q.16: Industrial Investment 


\begin{tabular}{|c|c|c|c|c|c|c|}
\hline \multicolumn{7}{|c|}{ Industry Output Price Index } \\
\hline \multirow{2}{*}{ Industry } & \multicolumn{6}{|c|}{$\% \Delta$ ON BASEYEAR } \\
\hline & BSLN5 & SIM501 & SIM502 & BSLN6 & SIM601 & SIM602 \\
\hline AGRI & -0.1 & 0.5 & -1.8 & -0.4 & -0.6 & -1.8 \\
\hline FOLO & -0.6 & -0.3 & -1.4 & -0.7 & -0.9 & -1.4 \\
\hline FISH & -0.6 & -0.5 & -1.6 & -0.7 & -0.9 & -1.4 \\
\hline MINE & -0.4 & 0.6 & -0.6 & -0.5 & 0.1 & -0.6 \\
\hline OIGA & 0.8 & 3.9 & 3.2 & 0.7 & 3.8 & 3.2 \\
\hline PETR & -0.1 & 0.6 & 0.7 & -0.1 & 0.6 & 0.6 \\
\hline FDBT & -0.7 & -0.7 & -2.0 & -0.8 & -1.3 & -1.9 \\
\hline TWPM & -1.4 & -2.1 & -2.0 & -1.5 & -2.2 & -2.1 \\
\hline CHNM & -2.4 & -3.5 & -2.5 & -2.3 & -3.1 & -2.5 \\
\hline METL & -1.9 & -2.2 & -1.9 & -1.9 & -2.1 & -1.9 \\
\hline EQFO & -2.1 & -3.5 & -2.5 & -2.1 & -3.2 & -2.6 \\
\hline UTIL & -1.4 & 2.4 & 2.3 & -1.4 & 2.4 & 2.1 \\
\hline CONS & -1.6 & -2.4 & -2.0 & -1.6 & -2.4 & -2.2 \\
\hline ACCR & -1.3 & -2.2 & -2.7 & -1.4 & -2.5 & -2.7 \\
\hline CMIF & -3.0 & -3.8 & -1.9 & -2.8 & -3.3 & -2.3 \\
\hline PROP & -2.3 & 0.5 & 0.5 & -2.3 & 0.7 & 0.5 \\
\hline RBUS & -2.7 & -5.5 & -2.9 & -2.6 & -4.9 & -3.3 \\
\hline GOVT & -1.8 & -3.5 & -2.6 & -1.8 & -4.4 & -3.7 \\
\hline EDUC & -2.0 & -6.1 & -5.1 & -2.0 & -6.3 & -5.5 \\
\hline HEAL & -1.6 & -4.1 & -4.3 & -1.7 & -4.7 & -4.6 \\
\hline CUPE & -2.2 & -3.5 & -2.6 & -2.1 & -3.5 & -2.9 \\
\hline OWND & -10.1 & -7.4 & -7.3 & -10.1 & -7.2 & -7.4 \\
\hline WHOL & -2.5 & -5.2 & -3.1 & -2.4 & -4.6 & -3.3 \\
\hline RETT & -1.8 & -2.8 & -2.4 & -1.8 & -3.0 & -2.6 \\
\hline TRAN & -2.2 & -3.6 & -2.7 & -2.1 & -3.3 & -2.8 \\
\hline All & -2.1 & -2.8 & -2.3 & -2.1 & -2.8 & -2.5 \\
\hline
\end{tabular}

Table Q.17: Industry Output Price Index 


\begin{tabular}{|l|cccccc|}
\hline \multicolumn{7}{|c}{ Industry Investment Price Index } \\
\hline \multirow{5}{*}{ Andustry } & \multicolumn{5}{c|}{$\%$ ON BASEYEAR } \\
\cline { 2 - 6 } FOLI & BSLN5 & SIM501 & SIM502 & BSLN6 & SIM601 & SIM602 \\
FISH & -1.3 & -1.8 & -1.5 & -1.3 & -1.8 & -1.6 \\
MINE & -1.3 & -1.8 & -1.5 & -1.3 & -1.8 & -1.6 \\
OIGA & -1.3 & -1.9 & -1.6 & -1.3 & -1.9 & -1.7 \\
PETR & -1.3 & -1.8 & -1.3 & -1.3 & -1.8 & -1.5 \\
FDBT & -1.3 & -1.8 & -1.3 & -1.3 & -1.8 & -1.5 \\
TWPM & -1.3 & -2.0 & -1.6 & -1.3 & -1.9 & -1.7 \\
CHNM & -1.4 & -2.1 & -1.6 & -1.4 & -2.0 & -1.7 \\
METL & -1.5 & -2.3 & -1.7 & -1.5 & -2.2 & -1.8 \\
EQFO & -1.4 & -2.2 & -1.6 & -1.4 & -2.1 & -1.7 \\
UTIL & -1.5 & -2.2 & -1.6 & -1.4 & -2.1 & -1.7 \\
CONS & -1.4 & -2.0 & -1.5 & -1.3 & -2.0 & -1.7 \\
ACCR & -1.4 & -2.1 & -1.6 & -1.4 & -2.0 & -1.7 \\
CMIF & -1.4 & -2.1 & -1.6 & -1.4 & -2.0 & -1.7 \\
PROP & -1.4 & -2.2 & -1.6 & -1.4 & -2.1 & -1.7 \\
RBUS & -1.5 & -2.2 & -1.6 & -1.4 & -2.1 & -1.7 \\
GOVT & -1.4 & -2.0 & -1.5 & -1.4 & -2.0 & -1.7 \\
EDUC & -1.4 & -2.1 & -1.6 & -1.4 & -2.0 & -1.7 \\
HEAL & -1.4 & -2.1 & -1.6 & -1.4 & -2.0 & -1.7 \\
CUPE & -1.4 & -2.1 & -1.6 & -1.4 & -2.1 & -1.7 \\
OWND & -1.4 & -2.1 & -1.6 & -1.4 & -2.0 & -1.7 \\
WHOL & -1.5 & -2.3 & -1.6 & -1.4 & -2.1 & -1.7 \\
RETT & -1.4 & -2.1 & -1.6 & -1.4 & -2.0 & -1.7 \\
\hline & -1.3 & -2.2 & -1.7 & -1.2 & -2.0 & -1.7 \\
\hline
\end{tabular}

Table Q.18: Industry Investment Price Index 


\begin{tabular}{|l|cccccc|}
\hline \multicolumn{5}{|c}{ Industry } & \multicolumn{5}{c|}{ Industry Capital Rents Index } \\
\cline { 2 - 7 } & BSLN5 & SIM501 & SIM502 & BSLN6 & SIM601 & SIM602 \\
\hline AGRI & 1.3 & 5.2 & 7.8 & 1.5 & 6.7 & 7.7 \\
FOLO & 1.1 & 5.6 & 5.6 & 1.2 & 6.4 & 6.0 \\
FISH & 1.1 & 4.4 & 8.9 & 1.5 & 6.7 & 8.7 \\
MINE & 0.8 & 4.5 & 4.7 & 0.9 & 5.1 & 4.9 \\
OIGA & 1.2 & 5.1 & 4.3 & 1.2 & 5.3 & 4.5 \\
PETR & 1.5 & 5.2 & 5.2 & 1.6 & 5.9 & 5.5 \\
FDBT & -0.4 & 3.5 & 7.0 & 0.0 & 5.4 & 6.9 \\
TWPM & -1.7 & 4.1 & 3.2 & -1.7 & 4.4 & 3.5 \\
CHNM & -4.5 & 2.3 & 0.0 & -4.7 & 1.8 & 0.1 \\
METL & -2.5 & 3.5 & 1.4 & -2.6 & 3.2 & 1.7 \\
EQFO & -3.2 & 6.3 & 2.1 & -3.4 & 5.2 & 2.5 \\
UTIL & -1.3 & 8.2 & 7.0 & -1.3 & 8.4 & 7.0 \\
CONS & -1.7 & 3.4 & 2.9 & -1.7 & 3.6 & 3.0 \\
ACCR & -1.5 & 3.8 & 5.1 & -1.3 & 4.7 & 5.0 \\
CMIF & -3.4 & 1.6 & 1.3 & -3.4 & 1.9 & 1.3 \\
PROP & -2.4 & 3.8 & 2.8 & -2.5 & 4.0 & 3.0 \\
RBUS & -3.3 & 1.1 & 1.4 & -3.2 & 1.5 & 1.4 \\
GOVT & -1.6 & 2.8 & 3.0 & -1.6 & 2.2 & 2.1 \\
EDUC & -2.0 & 3.0 & 2.8 & -2.0 & 3.0 & 2.7 \\
HEAL & -1.6 & 2.2 & 1.5 & -1.6 & 2.0 & 1.4 \\
CUPE & -2.3 & 2.9 & 2.1 & -2.3 & 3.0 & 2.2 \\
OWND & -14.2 & -9.9 & -10.1 & -14.2 & -9.7 & -10.2 \\
WHOL & -3.6 & 0.3 & 0.5 & -3.6 & 0.7 & 0.5 \\
RETT & -1.8 & 2.7 & 2.5 & -1.8 & 2.9 & 2.5 \\
TRAN & -2.8 & 3.0 & 2.1 & -2.9 & 3.1 & 2.2 \\
\hline All & -3.7 & 1.5 & 1.2 & -3.7 & 1.8 & 1.3 \\
\hline
\end{tabular}

Table Q.19: Industry Capital Rents Index 


\begin{tabular}{|c|c|c|c|c|c|c|c|}
\hline \multicolumn{8}{|c|}{ Industry Average Net Rates of Return } \\
\hline \multirow{2}{*}{ Industry } & \multirow{2}{*}{ BASEYEAR (\%) } & \multicolumn{6}{|c|}{$\% \Delta$ ON BASEYEAR } \\
\hline & & BSLN5 & SIM501 & SIM502 & BSLN6 & SIM601 & SIM602 \\
\hline AGRI & 10.4 & 4.6 & 12.9 & 16.8 & 5.0 & 15.7 & 16.9 \\
\hline FOLO & 25.9 & 3.2 & 10.1 & 9.4 & 3.3 & 11.1 & 10.1 \\
\hline FISH & 4.9 & 6.2 & 16.9 & 27.7 & 7.3 & 22.8 & 27.4 \\
\hline MINE & 21.5 & 3.5 & 10.8 & 10.4 & 3.5 & 11.7 & 10.9 \\
\hline OIGA & 27.3 & 3.3 & 9.2 & 7.4 & 3.3 & 9.5 & 8.0 \\
\hline PETR & 27.3 & 3.7 & 9.3 & 8.6 & 3.8 & 10.2 & 9.3 \\
\hline FDBT & 10.6 & 1.6 & 10.2 & 15.6 & 2.2 & 13.7 & 15.6 \\
\hline TWPM & 14.1 & -0.8 & 11.0 & 7.7 & -0.9 & 11.0 & 8.4 \\
\hline CHNM & 22.3 & -4.3 & 6.7 & 2.1 & -4.7 & 5.6 & 2.5 \\
\hline METL & 41.8 & -1.5 & 7.2 & 3.4 & -1.7 & 6.5 & 4.0 \\
\hline EQFO & 19.5 & -2.6 & 12.0 & 4.6 & -3.0 & 10.0 & 5.2 \\
\hline UTIL & 10.7 & 0.0 & 13.7 & 11.4 & -0.1 & 13.9 & 11.6 \\
\hline CONS & 57.2 & -0.5 & 6.9 & 5.4 & -0.5 & 7.0 & 5.7 \\
\hline ACCR & 14.5 & -0.3 & 8.4 & 8.9 & -0.2 & 9.4 & 9.1 \\
\hline CMIF & 35.7 & -3.0 & 5.6 & 3.8 & -3.0 & 5.7 & 4.1 \\
\hline PROP & 5.6 & -1.8 & 8.9 & 6.4 & -1.9 & 9.0 & 6.9 \\
\hline RBUS & 64.1 & -2.4 & 4.5 & 3.3 & -2.4 & 4.5 & 3.5 \\
\hline GOVT & -0.7 & -1.1 & 19.5 & 17.3 & -1.4 & 16.9 & 14.7 \\
\hline EDUC & 0.0 & -62.0 & 465.9 & 362.7 & -64.3 & 438.7 & 363.2 \\
\hline HEAL & 12.0 & -0.4 & 6.2 & 4.1 & -0.5 & 5.7 & 4.2 \\
\hline CUPE & 13.9 & -1.5 & 7.8 & 5.5 & -1.6 & 7.8 & 5.9 \\
\hline OWND & 3.4 & -19.2 & -11.8 & -12.9 & -19.2 & -11.6 & -12.8 \\
\hline WHOL & 22.5 & -3.2 & 3.7 & 2.9 & -3.2 & 4.1 & 3.1 \\
\hline RETT & 44.0 & -0.6 & 6.0 & 5.1 & -0.6 & 6.2 & 5.2 \\
\hline TRAN & 8.0 & -2.9 & 10.3 & 7.1 & -3.1 & 10.2 & 7.6 \\
\hline All & 9.4 & -4.9 & 4.0 & 2.4 & -4.9 & 4.2 & 2.7 \\
\hline
\end{tabular}

Table Q.20: Industry Average Net Rates of Return 


\begin{tabular}{|l|c|cccccc|}
\hline \multicolumn{7}{|c|}{ Real Macro Measures } \\
\hline \multirow{2}{*}{ Variable } & BASEYEAR $(\$ \mathrm{M})$ & \multicolumn{7}{c|}{$\%$ ON BASEYEAR } \\
\cline { 3 - 8 } & & BSLN5 & SIM501 & SIM502 & BSLN6 & SIM601 & SIM602 \\
\hline GDP (Expenditure) & 156090 & 8.2 & 11.5 & 11.0 & 8.2 & 11.7 & 11.2 \\
GDP (Income) & 156090 & 8.4 & 12.2 & 11.4 & 8.3 & 12.0 & 11.5 \\
Private Consumption & 93331 & 8.0 & 10.8 & 10.4 & 8.0 & 10.9 & 10.5 \\
Investment & 38305 & 8.2 & 11.5 & 11.0 & 8.2 & 11.7 & 11.2 \\
Government Consumption & 28661 & 8.2 & 11.5 & 11.0 & 8.2 & 11.7 & 11.2 \\
F.O.B. Exports & 43290 & 6.3 & 9.9 & 9.4 & 6.3 & 10.3 & 9.7 \\
C.I.F. Imports & 47497 & 6.1 & 8.7 & 8.4 & 6.1 & 8.8 & 8.4 \\
Trade Balance & -4207 & -4.3 & 4.4 & 1.9 & -4.2 & 6.6 & 4.4 \\
Government Balance & 6900 & 5.3 & 17.0 & 14.5 & 5.3 & 15.9 & 14.3 \\
Domestic Private Saving & 27198 & 10.3 & 16.3 & 14.4 & 10.3 & 15.4 & 14.4 \\
Domestic Saving & 34098 & 9.3 & 16.4 & 14.4 & 9.2 & 15.5 & 14.4 \\
\hline
\end{tabular}

Table Q.21: Macro Measures

\begin{tabular}{|l|cccccc|}
\hline \multicolumn{7}{|c|}{ Economy-wide Price Measures } \\
\hline \multirow{2}{*}{ Variable } & \multicolumn{7}{c}{$\%$ ON BASEYEAR } \\
\cline { 2 - 7 } & BSLN5 & SIM501 & SIM502 & BSLN6 & SIM601 & SIM602 \\
\hline GDP Deflator & -2.7 & -3.5 & -3.1 & -2.7 & -3.6 & -3.3 \\
Consumer Price Index & -2.6 & -2.7 & -2.4 & -2.6 & -2.7 & -2.5 \\
Investment Price Index & -1.4 & -2.1 & -1.6 & -1.4 & -2.0 & -1.7 \\
Government Price Index & -1.8 & -4.1 & -3.5 & -1.8 & -4.6 & -4.1 \\
Export Price Index (F.O.B.) & -1.5 & -2.3 & -2.2 & -1.5 & -2.4 & -2.3 \\
Import Price Index (C.I.F.) & 0.0 & 0.0 & 0.0 & 0.0 & 0.0 & 0.0 \\
Trade Price Index & 15.8 & 25.8 & 24.3 & 15.9 & 27.2 & 25.4 \\
Real Exchange Rate & 2.7 & 3.6 & 3.2 & 2.7 & 3.8 & 3.4 \\
Nominal After-Tax Wage & -2.1 & -8.7 & -7.5 & -2.1 & -9.1 & -8.0 \\
Real After-Tax Wage & 0.5 & -6.1 & -5.2 & 0.5 & -6.6 & -5.6 \\
Capital Rents Index & -3.7 & 1.5 & 1.2 & -3.7 & 1.8 & 1.3 \\
Output Price Index & -2.1 & -2.8 & -2.3 & -2.1 & -2.8 & -2.5 \\
\hline Current Net Rate of Return (Average) & -4.9 & 4.0 & 2.4 & -4.9 & 4.2 & 2.7 \\
Expected Future Net Rate of Return & -1.6 & 4.2 & 2.9 & -1.7 & 4.3 & 3.1 \\
\hline
\end{tabular}

Table Q.22: Economy-wide Price Measures 


\begin{tabular}{|l|cccccc|}
\hline \multicolumn{7}{|c|}{ Regional Nominal After-Tax Wage } \\
\hline \multirow{2}{*}{ Region } & \multicolumn{5}{|c|}{$\%$ ON BASEYEAR } \\
\cline { 2 - 7 } & BSLN5 & SIM501 & SIM502 & BSLN6 & SIM601 & SIM602 \\
\hline AKL & -7.5 & -26.8 & -6.2 & -5.9 & -19.3 & -6.9 \\
WLG & -1.0 & -0.2 & 0.4 & -1.6 & -6.7 & -5.7 \\
ONI & 1.5 & 3.1 & 2.4 & 0.8 & -0.4 & -0.4 \\
CAN & -0.7 & 0.3 & -23.3 & -1.4 & -5.6 & -19.2 \\
OSI & 2.7 & 4.2 & -24.6 & 0.5 & -4.8 & -19.7 \\
\hline
\end{tabular}

Table Q.23: Regional Nominal After-Tax Wage

\begin{tabular}{|l|cccccc|}
\hline \multicolumn{7}{|c|}{ Regional Consumer Price Index } \\
\hline \multirow{2}{*}{ Region } & \multicolumn{5}{|c|}{$\%$ ON BASEYEAR } \\
\cline { 2 - 7 } & BSLN5 & SIM501 & SIM502 & BSLN6 & SIM601 & SIM602 \\
\hline AKL & -3.9 & -4.9 & -3.3 & -3.8 & -4.4 & -3.4 \\
WLG & -2.2 & -2.0 & -1.6 & -2.3 & -2.3 & -2.1 \\
ONI & -1.8 & -1.3 & -1.3 & -1.9 & -1.5 & -1.5 \\
CAN & -2.4 & -2.0 & -3.2 & -2.4 & -2.3 & -3.1 \\
OSI & -1.5 & -1.0 & -2.5 & -1.6 & -1.5 & -2.3 \\
\hline
\end{tabular}

Table Q.24: Regional Consumer Price Index

\begin{tabular}{|l|cccccc|}
\hline \multicolumn{7}{|c|}{ Regional Real After-Tax Wage } \\
\hline \multirow{2}{*}{ Region } & \multicolumn{5}{|c|}{$\% \Delta$ ON BASEYEAR } \\
\cline { 2 - 7 } & BSLn5 & SIM501 & SIM502 & BSLN6 & SIM601 & SIM602 \\
\hline AKL & -3.7 & -23.0 & -2.9 & -2.1 & -15.6 & -3.6 \\
WLG & 1.3 & 1.8 & 2.0 & 0.7 & -4.5 & -3.7 \\
ONI & 3.4 & 4.4 & 3.8 & 2.8 & 1.1 & 1.2 \\
CAN & 1.7 & 2.4 & -20.8 & 1.0 & -3.3 & -16.6 \\
OSI & 4.2 & 5.2 & -22.7 & 2.1 & -3.4 & -17.8 \\
\hline
\end{tabular}

Table Q.25: Regional Real After-Tax Wage 


\begin{tabular}{|c|c|c|c|c|c|c|c|}
\hline \multicolumn{8}{|c|}{ Real GDP Per Capita } \\
\hline \multirow{2}{*}{ Region } & \multirow{2}{*}{ BASEYEAR $(\$)$} & \multicolumn{6}{|c|}{$\% \Delta$ ON BASEYEAR } \\
\hline & & BSLN5 & SIM501 & SIM502 & BSLN6 & SIM601 & SIM602 \\
\hline NZ & 37306 & -2.3 & -5.6 & -5.1 & -2.3 & -5.4 & -5.0 \\
\hline \multicolumn{8}{|c|}{ Real Disposable Income Per Household } \\
\hline \multirow{2}{*}{ Region } & \multirow{2}{*}{ BASEYEAR $(\$)$} & \multicolumn{6}{|c|}{$\% \Delta$ ON BASEYEAR } \\
\hline & & BSLN5 & SIM501 & SIM502 & BSLN6 & SIM601 & SIM602 \\
\hline AKL & 95271 & -3.7 & -11.9 & -3.8 & -3.0 & -8.7 & -4.2 \\
\hline WLG & 88394 & -5.8 & -5.9 & -5.9 & -6.0 & -8.1 & -8.2 \\
\hline $\mathrm{ONI}$ & 75572 & -7.3 & -7.4 & -7.4 & -7.5 & -8.3 & -8.3 \\
\hline $\mathrm{CAN}$ & 64292 & -6.7 & -6.7 & -15.4 & -6.9 & -8.6 & -13.7 \\
\hline OSI & 88548 & -7.2 & -7.3 & -17.1 & -7.9 & -10.0 & -15.2 \\
\hline NZ & 82902 & -5.5 & -8.0 & -8.5 & -5.5 & -8.1 & -8.5 \\
\hline \multicolumn{8}{|c|}{ Real Spending Per Household } \\
\hline \multirow{2}{*}{ Region } & \multirow{2}{*}{ BASEYEAR $(\$)$} & \multicolumn{6}{|c|}{$\% \triangle$ ON BASEYEAR } \\
\hline & & BSLN5 & SIM501 & SIM502 & BSLN6 & SIM601 & SIM602 \\
\hline AKL & 73403 & -8.1 & -19.1 & -7.1 & -7.2 & -14.2 & -7.6 \\
\hline WLG & 70464 & -5.3 & -4.4 & -4.2 & -5.7 & -8.0 & -7.9 \\
\hline ONI & 57243 & -5.0 & -3.6 & -4.2 & -5.3 & -5.2 & -5.5 \\
\hline $\mathrm{CAN}$ & 50335 & -6.0 & -5.0 & -18.6 & -6.3 & -7.9 & -15.9 \\
\hline OSI & 70474 & -4.1 & -2.8 & -19.4 & -5.3 & -7.4 & -16.2 \\
\hline $\mathrm{NZ}$ & 64195 & -5.9 & -9.0 & -9.3 & -5.9 & -8.9 & -9.2 \\
\hline \multicolumn{8}{|c|}{ Real Saving Per Household } \\
\hline \multirow{2}{*}{ Region } & \multirow{2}{*}{ BASEYEAR $(\$)$} & \multicolumn{6}{|c|}{$\% \Delta$ ON BASEYEAR } \\
\hline & & BSLN5 & SIM501 & SIM502 & BSLN6 & SIM601 & SIM602 \\
\hline AKL & 21868 & 11.1 & 12.2 & 7.1 & 10.8 & 10.0 & 7.1 \\
\hline WLG & 17930 & -7.4 & -11.7 & -12.3 & -7.2 & -8.7 & -9.5 \\
\hline ONI & 18329 & -14.3 & -19.2 & -17.3 & -14.1 & -17.9 & -16.8 \\
\hline $\mathrm{CAN}$ & 13957 & -9.0 & -13.1 & -4.0 & -8.8 & -11.2 & -5.7 \\
\hline OSI & 18074 & -19.1 & -24.6 & -8.2 & -18.1 & -20.1 & -11.2 \\
\hline NZ & 18707 & -3.9 & -4.4 & -6.0 & -4.0 & -5.2 & -6.0 \\
\hline
\end{tabular}

Table Q.26: Welfare Measures 


\begin{tabular}{|l|c|cccccc|}
\hline \multicolumn{7}{|c|}{ Unemployment } \\
\hline \multirow{2}{*}{ Region } & \multirow{2}{*}{ BASEYEAR (000s) } & \multicolumn{7}{|c|}{$\% \Delta$ ON BASEYEAR } \\
\cline { 3 - 8 } & & BSLN5 & SIM501 & SIM502 & BSLN6 & SIM601 & SIM602 \\
\hline AKL & 37 & 15.8 & 38.6 & 15.5 & 14.3 & 29.1 & 15.7 \\
WLG & 13 & 6.2 & 6.0 & 6.0 & 6.8 & 12.2 & 9.9 \\
ONI & 36 & 3.4 & 3.1 & 3.2 & 3.9 & 5.7 & 4.8 \\
CAN & 11 & 6.0 & 5.8 & 30.0 & 6.9 & 13.3 & 24.8 \\
OSI & 9 & 1.8 & 1.6 & 29.7 & 4.3 & 12.3 & 23.6 \\
\hline NZ & 106 & 8.2 & 16.0 & 13.0 & 8.2 & 16.0 & 13.0 \\
\hline
\end{tabular}

Table Q.27: Unemployment

\begin{tabular}{|l|c|cccccc|}
\hline \multicolumn{7}{|c|}{ Labour Force Participation Rate } \\
\hline \multirow{2}{*}{ Region } & \multirow{2}{*}{ BASEYEAR $(\%)$} & \multicolumn{7}{c|}{$\%$ ON BASEYEAR } \\
\cline { 3 - 8 } & & BSLN5 & SIM501 & SIM502 & BSLN6 & SIM601 & SIM602 \\
\hline AKL & 74.2 & 0.6 & 1.1 & 0.3 & 0.6 & 1.1 & 0.4 \\
WLG & 78.4 & 0.0 & -0.2 & -0.2 & 0.0 & -0.4 & -0.3 \\
ONI & 75.7 & -0.3 & -0.6 & -0.5 & -0.3 & -0.6 & -0.4 \\
CAN & 77.5 & 0.0 & -0.3 & 0.5 & -0.1 & -0.4 & 0.5 \\
OSI & 77.8 & -0.3 & -0.6 & 0.4 & -0.4 & -0.8 & 0.4 \\
\hline NZ & 76.0 & 0.0 & -0.1 & 0.1 & 0.0 & -0.1 & 0.1 \\
\hline
\end{tabular}

Table Q.28: Labour Force Participation Rate 


\begin{tabular}{|l|cccccc|}
\hline \multicolumn{7}{|c|}{ Compensating Variation } \\
\hline \multirow{2}{*}{ Region } & \multicolumn{7}{|c|}{$\Delta$ IN INCOME (\$M) } \\
\cline { 2 - 7 } & BSLN5 & SIM501 & SIM502 & BSLN6 & SIM601 & SIM602 \\
\hline AKL & 4266.0 & 6767.1 & 4738.4 & 4095.6 & 5952.3 & 4779.1 \\
WLG & 926.7 & 1074.1 & 1098.4 & 953.5 & 1323.9 & 1282.7 \\
ONI & 1986.9 & 2484.4 & 2296.7 & 2030.3 & 2735.6 & 2538.3 \\
CAN & 815.6 & 947.3 & 1673.6 & 838.8 & 1152.5 & 1520.8 \\
OSI & 727.2 & 919.0 & 1894.7 & 810.5 & 1267.2 & 1688.1 \\
\hline \multirow{7}{*}{ Region } & BSLN5 & SIM501 & SIM502 & BSLN6 & SIM601 & SIM602 \\
\cline { 2 - 7 } & Equalent Variation & & \\
\hline AKL & 4471.1 & 7439.7 & 4926.6 & 4274.4 & 6409.7 & 4982.0 \\
WLG & 946.2 & 1092.5 & 1113.1 & 974.6 & 1364.1 & 1317.3 \\
ONI & 2012.8 & 2499.0 & 2312.4 & 2059.7 & 2773.2 & 2573.0 \\
CAN & 833.2 & 963.3 & 1788.6 & 857.9 & 1186.0 & 1611.0 \\
OSI & 733.4 & 921.0 & 2015.3 & 820.8 & 1292.8 & 1776.9 \\
\hline
\end{tabular}

Table Q.29: CV and EV Measures 


\section{Appendix R}

\section{Simulation Results - Long-Run Immigration Scenarios}

This appendix provides the long-run results for the illustrative simulations discussed in chapter 4 .

\begin{tabular}{|l|c|cccccc|}
\hline \multicolumn{7}{|c|}{ Number of Households } \\
\hline \multirow{2}{*}{ Region } & \multirow{2}{*}{ BASEYEAR (000s) } & \multicolumn{7}{|c|}{$\%$ ON BASEYEAR } \\
\cline { 2 - 8 } & & BSLN7 & SIM701 & SIM702 & BSLN8 & SIM801 & SIM802 \\
\hline AKL & 434 & 55.0 & 112.7 & 55.0 & 43.6 & 75.5 & 51.0 \\
WLG & 167 & 28.0 & 28.0 & 28.0 & 32.8 & 48.8 & 45.3 \\
ONI & 482 & 25.0 & 25.0 & 25.0 & 28.1 & 34.1 & 32.8 \\
CAN & 200 & 31.0 & 31.0 & 93.5 & 32.9 & 45.6 & 78.0 \\
OSI & 172 & 20.0 & 20.0 & 92.8 & 33.2 & 51.1 & 82.2 \\
\hline NZ & 1454 & 34.5 & 51.7 & 51.7 & 34.5 & 51.7 & 51.7 \\
\hline
\end{tabular}

Table R.1: Number of Households 


\begin{tabular}{|l|c|cccccc|}
\hline \multicolumn{7}{|c|}{ Total Population } \\
\hline \multirow{2}{*}{ Region } & \multirow{2}{*}{ BASEYEAR (000s) } & \multicolumn{7}{|c|}{$\% \Delta$ ON BASEYEAR } \\
\cline { 3 - 8 } & & BSLN7 & SIM701 & SIM702 & BSLN8 & SIM801 & SIM802 \\
\hline AKL & 1371 & 42.0 & 94.8 & 42.0 & 31.6 & 60.7 & 38.2 \\
WLG & 466 & 16.0 & 16.0 & 16.0 & 21.1 & 37.7 & 32.2 \\
ONI & 1348 & 12.0 & 12.0 & 12.0 & 15.1 & 21.4 & 19.3 \\
CAN & 540 & 21.0 & 21.0 & 78.8 & 23.4 & 37.0 & 64.4 \\
OSI & 459 & 8.0 & 8.0 & 73.5 & 21.8 & 41.2 & 64.0 \\
\hline NZ & 4184 & 23.0 & 40.3 & 37.6 & 23.0 & 40.3 & 37.6 \\
\hline
\end{tabular}

Table R.2: Total Population

\begin{tabular}{|c|c|c|c|c|c|c|c|}
\hline \multicolumn{8}{|c|}{ Working-Age Population } \\
\hline \multirow{2}{*}{ Region } & \multirow{2}{*}{ BASEYEAR (000s) } & \multicolumn{6}{|c|}{$\% \Delta$ ON BASEYEAR } \\
\hline & & BSLN7 & SIM701 & SIM702 & BSLN8 & SIM 801 & $\operatorname{SIM} 802$ \\
\hline AKL & 849 & 31.5 & 80.5 & 31.5 & 21.9 & 48.9 & 28.0 \\
\hline WLG & 301 & 5.0 & 5.0 & 5.0 & 9.5 & 24.3 & 19.3 \\
\hline ONI & 814 & -1.8 & -1.8 & -1.8 & 1.2 & 7.2 & 5.1 \\
\hline $\mathrm{CAN}$ & 347 & 8.2 & 8.2 & 59.8 & 10.4 & 22.5 & 47.0 \\
\hline OSI & 296 & -6.8 & -6.8 & 49.6 & 5.4 & 22.6 & 41.5 \\
\hline $\mathrm{NZ}$ & 2607 & 10.6 & 26.5 & 23.9 & 10.6 & 26.5 & 23.9 \\
\hline
\end{tabular}

Table R.3: Working-Age Population

\begin{tabular}{|l|c|cccccc|}
\hline \multicolumn{7}{|c|}{ Non-Working Age Population } \\
\hline \multirow{2}{*}{ Region } & \multirow{2}{*}{ BASEYEAR (000s) } & \multicolumn{7}{|c|}{$\%$ ON BASEYEAR } \\
\cline { 3 - 8 } & & BSLN7 & SIM701 & SIM702 & BSLN8 & SIM801 & SIM802 \\
\hline AKL & 522 & 59.0 & 118.1 & 59.0 & 47.3 & 80.0 & 54.7 \\
WLG & 165 & 36.0 & 36.0 & 36.0 & 42.2 & 62.2 & 55.6 \\
ONI & 534 & 33.0 & 33.0 & 33.0 & 36.3 & 43.1 & 40.9 \\
CAN & 193 & 44.0 & 44.0 & 112.7 & 46.9 & 63.1 & 95.7 \\
OSI & 163 & 35.0 & 35.0 & 116.9 & 51.7 & 75.0 & 105.0 \\
\hline NZ & 1577 & 43.5 & 63.0 & 60.3 & 43.5 & 63.0 & 60.3 \\
\hline
\end{tabular}

Table R.4: Non-Working Age Population 


\begin{tabular}{|l|c|cccccc|}
\hline \multicolumn{7}{|c|}{ Regional Employment (Full-Time Equivalents) } \\
\hline \multirow{2}{*}{ Region } & BASEYEAR (000s) & \multicolumn{5}{|c|}{$\% \Delta$ ON BASEYEAR } \\
\cline { 2 - 8 } & & BSLN7 & SIM701 & SIM702 & BSLN8 & SIM801 & SIM802 \\
\hline AKL & 533 & 31.6 & 80.7 & 31.6 & 22.0 & 49.1 & 28.1 \\
WLG & 200 & 5.0 & 5.1 & 5.0 & 9.3 & 23.2 & 19.0 \\
ONI & 514 & -1.8 & -1.8 & -1.9 & 1.2 & 7.0 & 5.1 \\
CAN & 227 & 8.2 & 8.2 & 59.9 & 10.1 & 21.5 & 47.1 \\
OSI & 195 & -6.9 & -6.9 & 49.7 & 4.7 & 20.9 & 41.6 \\
\hline NZ & 1668 & 10.5 & 26.1 & 24.1 & 10.4 & 26.0 & 24.1 \\
\hline
\end{tabular}

Table R.5: Regional Employment

\begin{tabular}{|l|c|cccccc|}
\hline \multicolumn{7}{|c|}{ Regional Capital Stocks } \\
\hline \multirow{2}{*}{ Region } & \multirow{2}{*}{ BASEYEAR $(\$ \mathrm{M})$} & \multicolumn{7}{|c|}{$\% \Delta$ ON BASEYEAR } \\
\cline { 3 - 8 } & & BSLN7 & SIM701 & SIM702 & BSLN8 & SIM801 & SIM802 \\
\hline AKL & 154208 & 28.4 & 60.6 & 33.3 & 23.1 & 44.0 & 31.5 \\
WLG & 62648 & 14.5 & 20.7 & 19.8 & 16.5 & 30.0 & 27.2 \\
ONI & 144170 & 12.8 & 18.5 & 17.4 & 14.2 & 23.2 & 21.2 \\
CAN & 49536 & 16.5 & 22.1 & 50.0 & 17.4 & 29.4 & 43.1 \\
OSI & 59263 & 10.2 & 15.6 & 48.7 & 16.7 & 31.3 & 44.0 \\
\hline NZ & 469826 & 18.2 & 32.6 & 30.3 & 18.1 & 32.6 & 30.6 \\
\hline
\end{tabular}

Table R.6: Regional Capital Stocks 


\begin{tabular}{|l|c|cccccc|}
\hline \multicolumn{7}{|c|}{ Regional Output } \\
\hline \multirow{2}{*}{ Region } & \multirow{2}{*}{ BASEYEAR $(\$ \mathrm{M})$} & \multicolumn{7}{|c|}{$\% \Delta$ ON BASEYEAR } \\
\cline { 3 - 8 } & & BSLN7 & SIM701 & SIM702 & BSLN8 & SIM801 & SIM802 \\
\hline AKL & 106148 & 18.7 & 44.4 & 24.2 & 14.8 & 33.0 & 23.0 \\
WLG & 36082 & 7.6 & 13.5 & 12.6 & 9.6 & 22.5 & 19.5 \\
ONI & 100332 & 4.7 & 10.8 & 9.6 & 6.0 & 15.3 & 13.1 \\
CAN & 33657 & 8.9 & 14.8 & 36.2 & 9.8 & 21.5 & 31.4 \\
OSI & 43428 & 2.0 & 7.6 & 31.6 & 7.4 & 21.1 & 28.7 \\
\hline NZ & 319647 & 9.8 & 22.2 & 20.6 & 9.9 & 23.4 & 21.2 \\
\hline
\end{tabular}

Table R.7: Regional Output

\begin{tabular}{|l|c|cccccc|}
\hline \multicolumn{7}{|c|}{ Regional Investment } \\
\hline \multirow{2}{*}{ Region } & \multirow{2}{*}{ BASEYEAR $(\$ \mathrm{M})$} & \multicolumn{7}{|c|}{$\% \Delta$ ON BASEYEAR } \\
\cline { 3 - 8 } & & BSLN7 & SIM701 & SIM702 & BSLN8 & SIM801 & SIM802 \\
\hline AKL & 12867 & 13.4 & 30.1 & 23.1 & 12.3 & 27.8 & 23.2 \\
WLG & 5434 & 9.8 & 20.5 & 19.6 & 10.5 & 24.3 & 22.0 \\
ONI & 11490 & 9.0 & 19.8 & 18.4 & 9.6 & 22.3 & 20.0 \\
CAN & 3974 & 10.2 & 20.7 & 26.7 & 10.7 & 24.1 & 25.7 \\
OSI & 4540 & 8.1 & 18.7 & 25.5 & 10.1 & 24.3 & 25.1 \\
\hline NZ & 38305 & 10.6 & 23.3 & 21.8 & 10.8 & 24.9 & 22.6 \\
\hline
\end{tabular}

Table R.8: Regional Investment

\begin{tabular}{|l|cccccc|}
\hline \multicolumn{7}{|c|}{ Regional Output Price Index } \\
\hline \multirow{2}{*}{ Region } & \multicolumn{5}{|c|}{$\%$ ON BASEYEAR } \\
\cline { 2 - 7 } & BSLN7 & SIM701 & SIM702 & BSLN8 & SIM801 & SIM802 \\
\hline AKL & -5.9 & -11.9 & -5.4 & -4.3 & -8.2 & -5.0 \\
WLG & -1.5 & -1.6 & -0.7 & -2.2 & -4.6 & -3.6 \\
ONI & -0.2 & -0.3 & 0.0 & -0.7 & -1.8 & -1.4 \\
CAN & -2.0 & -2.2 & -9.0 & -2.2 & -4.2 & -7.6 \\
OSI & 0.8 & 0.7 & -7.5 & -1.3 & -4.0 & -6.7 \\
\hline NZ & -2.4 & -4.7 & -4.0 & -2.3 & -4.9 & -4.3 \\
\hline
\end{tabular}

Table R.9: Regional Output Price Index 


\begin{tabular}{|l|cccccc|}
\hline \multicolumn{7}{|c|}{ Regional Investment Price Index } \\
\hline \multirow{2}{*}{ Region } & \multicolumn{7}{|c|}{ \% ON BASEYEAR } \\
\cline { 2 - 7 } & BSLN7 & SIM701 & SIM702 & BSLN8 & SIM801 & SIM802 \\
\hline AKL & -1.8 & -4.0 & -2.7 & -1.6 & -3.6 & -2.8 \\
WLG & -1.4 & -3.1 & -2.3 & -1.3 & -3.2 & -2.6 \\
ONI & -1.3 & -3.0 & -2.2 & -1.2 & -3.0 & -2.5 \\
CAN & -1.5 & -3.1 & -3.0 & -1.4 & -3.2 & -3.0 \\
OSI & -1.2 & -2.9 & -2.8 & -1.3 & -3.2 & -2.9 \\
\hline
\end{tabular}

Table R.10: Regional Investment Price Index

\begin{tabular}{|l|cccccc|}
\hline \multicolumn{7}{|c|}{ Regional Capital Rents Index } \\
\hline \multirow{2}{*}{ Region } & \multicolumn{7}{|c|}{$\%$ ON BASEYEAR } \\
\cline { 2 - 7 } & BSLN7 & SIM701 & SIM702 & BSLN8 & SIM801 & SIM802 \\
\hline AKL & -5.6 & -9.7 & -5.7 & -4.7 & -7.5 & -5.6 \\
WLG & -3.6 & -4.4 & -3.7 & -3.8 & -5.8 & -5.2 \\
ONI & -3.3 & -4.1 & -3.5 & -3.5 & -4.7 & -4.2 \\
CAN & -3.8 & -4.6 & -8.1 & -3.9 & -5.6 & -7.3 \\
OSI & -3.1 & -3.8 & -8.3 & -4.1 & -6.1 & -7.8 \\
\hline NZ & -4.2 & -6.3 & -5.4 & -4.0 & -6.1 & -5.6 \\
\hline
\end{tabular}

Table R.11: Regional Capital Rents Index

\begin{tabular}{|l|c|cccccc|}
\hline \multicolumn{7}{|c|}{ Regional Average Net Rates of Return } \\
\hline \multirow{2}{*}{ Region } & \multirow{2}{*}{ BASEYEAR (\%) } & \multicolumn{5}{|c|}{$\%$ ON BASEYEAR } \\
\cline { 3 - 8 } & & BSLN7 & SIM701 & SIM702 & BSLN8 & SIM801 & SIM802 \\
\hline AKL & 10.1 & -13.4 & -20.7 & -10.9 & -10.9 & -14.1 & -9.9 \\
WLG & 8.2 & -7.6 & -5.9 & -5.6 & -8.3 & -8.8 & -8.6 \\
ONI & 9.5 & -7.0 & -4.3 & -4.7 & -7.7 & -5.9 & -6.2 \\
CAN & 9.5 & -8.3 & -5.9 & -17.7 & -8.6 & -8.3 & -14.9 \\
OSI & 9.0 & -6.3 & -3.6 & -18.9 & -9.4 & -10.0 & -16.9 \\
\hline NZ & 9.4 & -9.3 & -10.8 & -10.4 & -9.1 & -9.9 & -10.2 \\
\hline
\end{tabular}

Table R.12: Regional Average Net Rates of Return 


\begin{tabular}{|c|c|c|c|c|c|c|c|}
\hline \multicolumn{8}{|c|}{ Industry Employment (Full-Time Equivalents) } \\
\hline \multirow{2}{*}{ Industry } & \multirow{2}{*}{ BASEYEAR (000s) } & \multicolumn{6}{|c|}{$\% \Delta$ ON BASEYEAR } \\
\hline & & BSLN7 & SIM701 & SIM702 & BSLN8 & $\operatorname{SIM} 801$ & SIM802 \\
\hline AGRI & 114 & 1.4 & 5.5 & 19.3 & 4.5 & 14.9 & 19.6 \\
\hline FOLO & 5 & 0.8 & 4.9 & 17.9 & 4.6 & 15.8 & 19.4 \\
\hline FISH & 2 & 0.7 & 4.1 & 26.7 & 5.2 & 17.1 & 25.3 \\
\hline MINE & 4 & 2.6 & 10.0 & 21.0 & 6.1 & 19.3 & 21.8 \\
\hline OIGA & 0 & 1.3 & 3.9 & 3.0 & 3.7 & 12.0 & 9.6 \\
\hline PETR & 6 & 0.5 & 3.0 & 3.4 & 3.1 & 11.4 & 9.6 \\
\hline FDBT & 56 & 4.9 & 14.1 & 22.8 & 6.8 & 19.7 & 22.3 \\
\hline TWPM & 65 & 12.8 & 33.4 & 25.9 & 11.6 & 29.1 & 25.5 \\
\hline CHNM & 21 & 16.0 & 38.9 & 29.0 & 13.6 & 32.6 & 27.7 \\
\hline METL & 26 & 13.3 & 35.2 & 26.2 & 11.9 & 30.2 & 25.7 \\
\hline EQFO & 50 & 14.8 & 38.1 & 30.7 & 12.9 & 32.0 & 28.9 \\
\hline UTIL & 9 & 8.8 & 21.9 & 21.9 & 9.4 & 24.0 & 22.7 \\
\hline CONS & 142 & 11.3 & 26.9 & 25.6 & 11.6 & 28.1 & 26.2 \\
\hline ACCR & 88 & 8.7 & 22.7 & 24.7 & 9.2 & 24.2 & 24.2 \\
\hline CMIF & 84 & 14.3 & 35.2 & 24.6 & 12.6 & 30.4 & 24.9 \\
\hline PROP & 48 & 11.2 & 27.5 & 23.9 & 10.9 & 26.9 & 24.1 \\
\hline RBUS & 198 & 13.3 & 33.8 & 25.0 & 12.1 & 29.6 & 25.1 \\
\hline GOVT & 77 & 10.8 & 24.6 & 22.1 & 11.2 & 26.5 & 23.9 \\
\hline EDUC & 120 & 11.4 & 28.0 & 24.4 & 11.1 & 27.0 & 24.5 \\
\hline HEAL & 164 & 10.7 & 25.8 & 24.9 & 10.8 & 26.1 & 24.7 \\
\hline CUPE & 74 & 11.1 & 27.6 & 23.9 & 10.8 & 26.9 & 24.3 \\
\hline OWND & 0 & 0.0 & 0.0 & 0.0 & 0.0 & 0.0 & 0.0 \\
\hline WHOL & 89 & 12.9 & 31.3 & 24.2 & 11.5 & 27.4 & 23.5 \\
\hline RETT & 163 & 9.2 & 22.5 & 22.5 & 9.6 & 24.1 & 22.8 \\
\hline TRAN & 62 & 10.4 & 25.6 & 24.6 & 10.0 & 25.1 & 23.8 \\
\hline All & 1668 & 10.5 & 26.1 & 24.1 & 10.4 & 26.0 & 24.1 \\
\hline
\end{tabular}

Table R.13: Industry Employment 


\begin{tabular}{|c|c|c|c|c|c|c|c|}
\hline \multicolumn{8}{|c|}{ Industry Capital Stocks } \\
\hline \multirow{2}{*}{ Industry } & \multirow{2}{*}{ BASEYEAR $(\$ M)$} & \multicolumn{6}{|c|}{$\% \triangle$ ON BASEYEAR } \\
\hline & & BSLN7 & $\operatorname{siM} 701$ & SIM702 & BSLN8 & SIM 801 & SIM802 \\
\hline AGRI & 14374 & 5.7 & 14.0 & 15.5 & 6.6 & 17.4 & 16.4 \\
\hline FOLO & 1351 & 6.9 & 16.6 & 16.0 & 7.4 & 19.0 & 17.1 \\
\hline FISH & 962 & 5.6 & 14.2 & 16.8 & 6.7 & 18.1 & 17.4 \\
\hline MINE & 878 & 7.8 & 18.4 & 17.9 & 8.2 & 20.5 & 18.6 \\
\hline OIGA & 4450 & 7.8 & 17.9 & 16.1 & 7.9 & 19.2 & 17.0 \\
\hline PETR & 455 & 8.2 & 19.2 & 17.8 & 8.6 & 21.2 & 19.0 \\
\hline FDBT & 11491 & 6.1 & 15.0 & 16.3 & 6.9 & 18.1 & 17.0 \\
\hline TWPM & 8052 & 8.8 & 20.8 & 18.5 & 8.8 & 21.8 & 19.2 \\
\hline CHNM & 4959 & 10.1 & 23.4 & 19.9 & 9.7 & 23.2 & 20.2 \\
\hline METL & 3412 & 10.1 & 23.7 & 19.9 & 9.7 & 23.7 & 20.5 \\
\hline EQFO & 5892 & 11.6 & 27.3 & 21.5 & 10.8 & 25.9 & 21.9 \\
\hline UTIL & 23050 & 8.7 & 20.1 & 18.7 & 8.9 & 21.4 & 19.2 \\
\hline CONS & 5578 & 10.2 & 22.6 & 21.1 & 10.4 & 24.1 & 21.8 \\
\hline ACCR & 5251 & 7.6 & 18.1 & 17.7 & 8.0 & 20.0 & 18.3 \\
\hline CMIF & 15121 & 9.0 & 20.7 & 18.7 & 9.0 & 21.6 & 19.2 \\
\hline PROP & 83623 & 9.0 & 20.5 & 18.9 & 9.0 & 21.6 & 19.4 \\
\hline RBUS & 6376 & 9.4 & 21.5 & 19.2 & 9.3 & 22.3 & 19.7 \\
\hline GOVT & 43300 & 10.1 & 22.4 & 20.6 & 10.1 & 22.7 & 20.6 \\
\hline EDUC & 16100 & 9.9 & 22.2 & 20.1 & 9.8 & 22.6 & 20.3 \\
\hline HEAL & 10847 & 9.8 & 21.9 & 20.2 & 9.8 & 22.4 & 20.3 \\
\hline CUPE & 11086 & 9.1 & 20.8 & 18.8 & 9.0 & 21.6 & 19.3 \\
\hline OWND & 163680 & 35.7 & 55.6 & 51.7 & 35.2 & 53.5 & 51.7 \\
\hline WHOL & 8281 & 7.6 & 17.5 & 16.9 & 7.9 & 18.9 & 17.3 \\
\hline RETT & 7265 & 8.3 & 19.2 & 18.0 & 8.5 & 20.3 & 18.4 \\
\hline TRAN & 13992 & 8.0 & 18.7 & 17.5 & 8.1 & 19.7 & 17.8 \\
\hline All & 469826 & 18.2 & 32.6 & 30.3 & 18.1 & 32.6 & 30.6 \\
\hline
\end{tabular}

Table R.14: Industry Capital Stocks 


\begin{tabular}{|c|c|c|c|c|c|c|c|}
\hline \multicolumn{8}{|c|}{ Industrial Output } \\
\hline \multirow{2}{*}{ Industry } & \multirow{2}{*}{ BASEYEAR $(\$ M)$} & \multicolumn{6}{|c|}{$\% \Delta$ ON BASEYEAR } \\
\hline & & BSLN7 & SIM701 & SIM702 & BSLN8 & SIM801 & SIM802 \\
\hline AGRI & 16884 & 3.6 & 10.0 & 15.9 & 5.6 & 16.3 & 16.9 \\
\hline FOLO & 2978 & 5.9 & 14.7 & 16.2 & 7.0 & 18.7 & 17.5 \\
\hline FISH & 853 & 4.6 & 12.6 & 18.0 & 6.5 & 18.0 & 18.4 \\
\hline MINE & 1197 & 7.0 & 17.0 & 18.5 & 7.9 & 20.4 & 19.3 \\
\hline OIGA & 2479 & 7.4 & 17.1 & 15.4 & 7.7 & 18.9 & 16.7 \\
\hline PETR & 3683 & 7.4 & 17.2 & 16.2 & 7.8 & 19.3 & 17.4 \\
\hline FDBT & 27682 & 4.8 & 12.8 & 17.0 & 6.4 & 17.8 & 17.8 \\
\hline TWPM & 13057 & 8.9 & 21.6 & 19.4 & 9.0 & 22.6 & 20.1 \\
\hline CHNM & 9078 & 11.3 & 26.0 & 21.5 & 10.4 & 24.7 & 21.3 \\
\hline METL & 8105 & 10.7 & 25.6 & 21.0 & 10.1 & 24.8 & 21.4 \\
\hline EQFO & 10632 & 12.8 & 30.8 & 23.2 & 11.5 & 27.8 & 23.2 \\
\hline UTIL & 12609 & 8.8 & 20.4 & 18.8 & 9.0 & 21.9 & 19.6 \\
\hline CONS & 29305 & 10.5 & 23.3 & 21.9 & 10.7 & 25.0 & 22.7 \\
\hline ACCR & 6454 & 7.4 & 18.2 & 19.4 & 8.2 & 21.1 & 20.0 \\
\hline CMIF & 24027 & 10.5 & 24.4 & 20.1 & 10.1 & 24.2 & 20.8 \\
\hline PROP & 13658 & 9.3 & 21.4 & 19.5 & 9.3 & 22.4 & 20.0 \\
\hline RBUS & 24532 & 11.4 & 26.9 & 21.2 & 10.7 & 25.7 & 21.8 \\
\hline GOVT & 13460 & 10.5 & 23.0 & 20.7 & 10.7 & 24.8 & 22.1 \\
\hline EDUC & 8229 & 11.1 & 26.5 & 23.1 & 10.9 & 26.0 & 23.2 \\
\hline HEAL & 12552 & 10.1 & 23.3 & 22.4 & 10.3 & 24.4 & 22.6 \\
\hline CUPE & 11764 & 10.0 & 23.5 & 20.4 & 9.9 & 23.8 & 21.0 \\
\hline OWND & 12472 & 26.0 & 42.5 & 39.6 & 25.8 & 42.1 & 40.0 \\
\hline WHOL & 20487 & 9.2 & 21.3 & 19.0 & 9.0 & 21.6 & 19.2 \\
\hline RETT & 17998 & 8.7 & 20.4 & 19.2 & 8.9 & 21.7 & 19.7 \\
\hline TRAN & 15472 & 9.0 & 21.0 & 19.3 & 8.9 & 21.6 & 19.4 \\
\hline All & 319647 & 9.8 & 22.2 & 20.6 & 9.9 & 23.4 & 21.2 \\
\hline
\end{tabular}

Table R.15: Industrial Output 


\begin{tabular}{|c|c|c|c|c|c|c|c|}
\hline \multicolumn{8}{|c|}{ Industrial Investment } \\
\hline \multirow{2}{*}{ Industry } & \multirow{2}{*}{ BASEYEAR $(\$ M)$} & \multicolumn{6}{|c|}{$\% \Delta$ ON BASEYEAR } \\
\hline & & BSLN7 & SIM701 & SIM702 & BSLN8 & SIM801 & SIM 802 \\
\hline AGRI & 1159 & 6.8 & 16.0 & 17.6 & 7.8 & 19.6 & 18.5 \\
\hline FOLO & 128 & 7.2 & 17.2 & 16.6 & 7.8 & 19.6 & 17.7 \\
\hline FISH & 41 & 8.6 & 19.1 & 22.1 & 9.9 & 23.7 & 22.9 \\
\hline MINE & 184 & 9.3 & 20.8 & 20.4 & 9.7 & 23.2 & 21.3 \\
\hline OIGA & 787 & 9.6 & 20.9 & 19.2 & 9.8 & 22.6 & 20.2 \\
\hline PETR & 80 & 10.0 & 22.2 & 20.9 & 10.5 & 24.6 & 22.2 \\
\hline FDBT & 954 & 9.9 & 21.2 & 22.9 & 10.9 & 25.2 & 24.0 \\
\hline TWPM & 871 & 10.1 & 23.0 & 20.8 & 10.2 & 24.3 & 21.7 \\
\hline CHNM & 640 & 11.6 & 25.8 & 22.4 & 11.2 & 25.9 & 22.8 \\
\hline METL & 641 & 11.2 & 25.7 & 21.9 & 10.9 & 25.9 & 22.6 \\
\hline EQFO & 500 & 12.8 & 29.3 & 23.6 & 12.0 & 28.2 & 24.1 \\
\hline UTIL & 744 & 17.8 & 35.1 & 34.4 & 18.4 & 38.3 & 35.7 \\
\hline CONS & 789 & 10.4 & 22.8 & 21.4 & 10.6 & 24.4 & 22.1 \\
\hline ACCR & 333 & 9.0 & 20.3 & 20.0 & 9.5 & 22.6 & 20.8 \\
\hline CMIF & 4534 & 10.4 & 23.0 & 21.1 & 10.5 & 24.2 & 21.7 \\
\hline PROP & 1808 & 10.2 & 22.6 & 21.1 & 10.4 & 23.9 & 21.7 \\
\hline RBUS & 2014 & 10.3 & 23.1 & 20.8 & 10.3 & 24.0 & 21.5 \\
\hline GOVT & 3530 & 10.6 & 23.3 & 21.8 & 10.8 & 24.9 & 22.6 \\
\hline EDUC & 1313 & 10.6 & 23.3 & 21.8 & 10.8 & 24.9 & 22.6 \\
\hline HEAL & 884 & 10.6 & 23.3 & 21.8 & 10.8 & 24.9 & 22.6 \\
\hline CUPE & 852 & 9.4 & 21.4 & 19.4 & 9.4 & 22.3 & 19.9 \\
\hline OWND & 13345 & 10.6 & 23.3 & 21.8 & 10.8 & 24.9 & 22.6 \\
\hline WHOL & 958 & 8.4 & 18.8 & 18.2 & 8.7 & 20.3 & 18.7 \\
\hline RETT & 1065 & 8.9 & 20.2 & 19.0 & 9.1 & 21.4 & 19.5 \\
\hline TRAN & 149 & 62.0 & 107.5 & 110.5 & 64.9 & 119.8 & 115.2 \\
\hline All & 38305 & 10.6 & 23.3 & 21.8 & 10.8 & 24.9 & 22.6 \\
\hline
\end{tabular}

Table R.16: Industrial Investment 


\begin{tabular}{|c|c|c|c|c|c|c|}
\hline \multicolumn{7}{|c|}{ Industry Output Price Index } \\
\hline \multirow{2}{*}{ Industry } & \multicolumn{6}{|c|}{$\% \Delta$ ON BASEYEAR } \\
\hline & BSLN7 & SIM701 & SIM702 & BSLN8 & SIM801 & SIM 802 \\
\hline AGRI & 0.7 & 0.6 & -2.6 & -0.4 & -2.2 & -3.0 \\
\hline FOLO & -0.4 & -1.4 & -2.5 & -0.9 & -2.9 & -2.9 \\
\hline FISH & -0.4 & -1.6 & -3.5 & -1.0 & -3.1 & -3.5 \\
\hline MINE & -0.7 & -1.9 & -3.0 & -1.1 & -3.1 & -3.2 \\
\hline OIGA & -1.0 & -2.4 & -1.7 & -1.0 & -2.8 & -2.2 \\
\hline PETR & -0.6 & -1.4 & -0.9 & -0.6 & -1.5 & -1.1 \\
\hline FDBT & -0.2 & -1.2 & -3.1 & -0.9 & -3.0 & -3.3 \\
\hline TWPM & -1.5 & -3.6 & -3.2 & -1.5 & -3.8 & -3.4 \\
\hline CHNM & -2.6 & -5.5 & -3.9 & -2.1 & -4.6 & -3.7 \\
\hline METL & -2.0 & -4.5 & -3.4 & -1.8 & -4.1 & -3.4 \\
\hline EQFO & -2.5 & -5.6 & -3.7 & -2.1 & -4.7 & -3.7 \\
\hline UTIL & -1.6 & -3.5 & -2.5 & -1.5 & -3.7 & -3.0 \\
\hline CONS & -1.7 & -3.8 & -3.1 & -1.6 & -4.0 & -3.4 \\
\hline ACCR & -1.1 & -2.9 & -3.8 & -1.5 & -4.0 & -4.0 \\
\hline CMIF & -2.9 & -6.1 & -3.7 & -2.4 & -5.4 & -4.0 \\
\hline PROP & -1.8 & -4.1 & -3.0 & -1.7 & -3.9 & -3.2 \\
\hline RBUS & -3.2 & -6.9 & -4.0 & -2.6 & -5.8 & -4.3 \\
\hline GOVT & -1.7 & -3.3 & -2.3 & -1.9 & -4.8 & -3.8 \\
\hline EDUC & -2.3 & -5.2 & -4.4 & -2.3 & -5.6 & -4.8 \\
\hline HEAL & -1.6 & -3.6 & -3.9 & -1.8 & -4.6 & -4.4 \\
\hline CUPE & -2.3 & -5.0 & -3.7 & -2.1 & -5.0 & -4.0 \\
\hline OWND & -14.8 & -17.4 & -16.7 & -14.6 & -17.2 & -16.9 \\
\hline WHOL & -3.0 & -6.4 & -4.2 & -2.5 & -5.5 & -4.3 \\
\hline RETT & -1.8 & -4.0 & -3.5 & -1.8 & -4.4 & -3.8 \\
\hline TRAN & -2.4 & -5.2 & -4.0 & -2.1 & -4.9 & -4.0 \\
\hline All & -2.4 & -4.7 & -4.0 & -2.3 & -4.9 & -4.3 \\
\hline
\end{tabular}

Table R.17: Industry Output Price Index 


\begin{tabular}{|c|c|c|c|c|c|c|}
\hline \multicolumn{7}{|c|}{ Industry Investment Price Index } \\
\hline \multirow{2}{*}{ Industry } & \multicolumn{6}{|c|}{$\% \Delta$ ON BASEYEAR } \\
\hline & BSLN7 & SIM701 & SIM702 & BSLN8 & SIM801 & SIM 802 \\
\hline AGRI & -1.3 & -3.0 & -2.5 & -1.3 & -3.1 & -2.7 \\
\hline FOLO & -1.3 & -3.1 & -2.4 & -1.3 & -3.1 & -2.6 \\
\hline FISH & -1.3 & -3.1 & -2.6 & -1.3 & -3.2 & -2.7 \\
\hline MINE & -1.4 & -3.1 & -2.5 & -1.3 & -3.2 & -2.7 \\
\hline OIGA & -1.3 & -3.0 & -2.2 & -1.3 & -3.1 & -2.5 \\
\hline PETR & -1.3 & -3.0 & -2.2 & -1.2 & -3.0 & -2.5 \\
\hline FDBT & -1.4 & -3.2 & -2.6 & -1.3 & -3.2 & -2.7 \\
\hline TWPM & -1.5 & -3.4 & -2.6 & -1.4 & -3.3 & -2.7 \\
\hline CHNM & -1.7 & -3.6 & -2.7 & -1.5 & -3.5 & -2.8 \\
\hline METL & -1.5 & -3.4 & -2.6 & -1.4 & -3.3 & -2.7 \\
\hline EQFO & -1.6 & -3.5 & -2.6 & -1.4 & -3.4 & -2.8 \\
\hline UTIL & -1.5 & -3.3 & -2.5 & -1.4 & -3.2 & -2.7 \\
\hline CONS & -1.5 & -3.3 & -2.5 & -1.4 & -3.3 & -2.7 \\
\hline ACCR & -1.5 & -3.3 & -2.6 & -1.4 & -3.3 & -2.7 \\
\hline CMIF & -1.6 & -3.5 & -2.6 & -1.4 & -3.4 & -2.7 \\
\hline PROP & -1.5 & -3.4 & -2.6 & -1.4 & -3.3 & -2.7 \\
\hline RBUS & -1.6 & -3.5 & -2.6 & -1.4 & -3.4 & -2.7 \\
\hline GOVT & -1.5 & -3.3 & -2.5 & -1.4 & -3.3 & -2.7 \\
\hline EDUC & -1.5 & -3.4 & -2.6 & -1.4 & -3.3 & -2.7 \\
\hline HEAL & -1.5 & -3.3 & -2.5 & -1.4 & -3.3 & -2.7 \\
\hline CUPE & -1.5 & -3.4 & -2.6 & -1.4 & -3.3 & -2.7 \\
\hline OWND & -1.5 & -3.4 & -2.6 & -1.4 & -3.3 & -2.7 \\
\hline WHOL & -1.6 & -3.6 & -2.6 & -1.4 & -3.4 & -2.8 \\
\hline RETT & -1.5 & -3.3 & -2.6 & -1.4 & -3.3 & -2.7 \\
\hline TRAN & -1.6 & -3.5 & -2.6 & -1.4 & -3.4 & -2.8 \\
\hline
\end{tabular}

Table R.18: Industry Investment Price Index 


\begin{tabular}{|c|c|c|c|c|c|c|}
\hline \multicolumn{7}{|c|}{ Industry Capital Rents Index } \\
\hline \multirow{2}{*}{ Industry } & \multicolumn{6}{|c|}{$\% \Delta$ ON BASEYEAR } \\
\hline & BSLN7 & $\operatorname{SIM} 701$ & SIM702 & BSLN 8 & SIM801 & SIM802 \\
\hline AGRI & -1.3 & -3.0 & -2.5 & -1.3 & -3.1 & -2.7 \\
\hline FOLO & -1.3 & -3.1 & -2.4 & -1.3 & -3.1 & -2.6 \\
\hline FISH & -1.3 & -3.1 & -2.6 & -1.3 & -3.2 & -2.7 \\
\hline MINE & -1.4 & -3.1 & -2.5 & -1.3 & -3.2 & -2.7 \\
\hline OIGA & -1.3 & -3.0 & -2.2 & -1.3 & -3.1 & -2.5 \\
\hline PETR & -1.3 & -3.0 & -2.2 & -1.2 & -3.0 & -2.5 \\
\hline FDBT & -1.4 & -3.2 & -2.6 & -1.3 & -3.2 & -2.7 \\
\hline TWPM & -1.5 & -3.4 & -2.6 & -1.4 & -3.3 & -2.7 \\
\hline CHNM & -1.7 & -3.6 & -2.7 & -1.5 & -3.5 & -2.8 \\
\hline METL & -1.5 & -3.4 & -2.6 & -1.4 & -3.3 & -2.7 \\
\hline EQFO & -1.6 & -3.5 & -2.6 & -1.4 & -3.4 & -2.8 \\
\hline UTIL & -1.5 & -3.3 & -2.5 & -1.4 & -3.2 & -2.7 \\
\hline CONS & -1.5 & -3.3 & -2.5 & -1.4 & -3.3 & -2.7 \\
\hline ACCR & -1.5 & -3.3 & -2.6 & -1.4 & -3.3 & -2.7 \\
\hline CMIF & -1.6 & -3.5 & -2.6 & -1.4 & -3.4 & -2.7 \\
\hline PROP & -1.5 & -3.4 & -2.6 & -1.4 & -3.3 & -2.7 \\
\hline RBUS & -1.6 & -3.5 & -2.6 & -1.4 & -3.4 & -2.7 \\
\hline GOVT & -1.5 & -3.3 & -2.5 & -1.4 & -3.3 & -2.7 \\
\hline EDUC & -1.5 & -3.4 & -2.6 & -1.4 & -3.3 & -2.7 \\
\hline HEAL & -1.5 & -3.3 & -2.6 & -1.4 & -3.3 & -2.7 \\
\hline CUPE & -1.5 & -3.4 & -2.6 & -1.4 & -3.3 & -2.7 \\
\hline OWND & -20.7 & -23.5 & -22.9 & -20.5 & -23.2 & -23.2 \\
\hline WHOL & -1.6 & -3.6 & -2.6 & -1.4 & -3.4 & -2.8 \\
\hline RETT & -1.5 & -3.3 & -2.6 & -1.4 & -3.3 & -2.7 \\
\hline TRAN & -1.6 & -3.5 & -2.6 & -1.4 & -3.4 & -2.8 \\
\hline All & -4.2 & -6.3 & -5.4 & -4.0 & -6.1 & -5.6 \\
\hline
\end{tabular}

Table R.19: Industry Capital Rents Index 


\begin{tabular}{|c|c|c|c|c|c|c|c|}
\hline \multicolumn{8}{|c|}{ Industry Average Net Rates of Return } \\
\hline \multirow{2}{*}{ Industry } & \multirow{2}{*}{ BASEYEAR $(\%)$} & \multicolumn{6}{|c|}{$\% \Delta$ ON BASEYEAR } \\
\hline & & BSLN7 & SIM701 & SIM702 & BSLN8 & SIM801 & SIM802 \\
\hline AGRI & 10.4 & 0.0 & 0.0 & 0.0 & 0.0 & 0.0 & 0.0 \\
\hline FOLO & 25.9 & 0.0 & 0.0 & 0.0 & 0.0 & 0.0 & 0.0 \\
\hline FISH & 4.9 & 0.0 & 0.0 & 0.0 & 0.0 & 0.0 & 0.0 \\
\hline MINE & 21.5 & 0.0 & 0.0 & 0.0 & 0.0 & 0.0 & 0.0 \\
\hline OIGA & 27.3 & 0.0 & 0.0 & 0.0 & 0.0 & 0.0 & 0.0 \\
\hline PETR & 27.3 & 0.0 & 0.0 & 0.0 & 0.0 & 0.0 & 0.0 \\
\hline FDBT & 10.6 & 0.0 & 0.0 & 0.0 & 0.0 & 0.0 & 0.0 \\
\hline TWPM & 14.1 & 0.0 & 0.0 & 0.0 & 0.0 & 0.0 & 0.0 \\
\hline CHNM & 22.3 & 0.0 & 0.0 & 0.0 & 0.0 & 0.0 & 0.0 \\
\hline METL & 41.8 & 0.0 & 0.0 & 0.0 & 0.0 & 0.0 & 0.0 \\
\hline EQFO & 19.5 & 0.0 & 0.0 & 0.0 & 0.0 & 0.0 & 0.0 \\
\hline UTIL & 10.7 & 0.0 & 0.0 & 0.0 & 0.0 & 0.0 & 0.0 \\
\hline CONS & 57.2 & 0.0 & 0.0 & 0.0 & 0.0 & 0.0 & 0.0 \\
\hline ACCR & 14.5 & 0.0 & 0.0 & 0.0 & 0.0 & 0.0 & 0.0 \\
\hline CMIF & 35.7 & 0.0 & 0.0 & 0.0 & 0.0 & 0.0 & 0.0 \\
\hline PROP & 5.6 & 0.0 & 0.0 & 0.0 & 0.0 & 0.0 & 0.0 \\
\hline RBUS & 64.1 & 0.0 & 0.0 & 0.0 & 0.0 & 0.0 & 0.0 \\
\hline GOVT & -0.7 & 0.0 & 0.0 & 0.0 & 0.0 & 0.0 & 0.0 \\
\hline EDUC & 0.0 & 0.0 & 0.0 & 0.0 & 0.0 & 0.0 & 0.0 \\
\hline HEAL & 12.0 & 0.0 & 0.0 & 0.0 & 0.0 & 0.0 & 0.0 \\
\hline CUPE & 13.9 & 0.0 & 0.0 & 0.0 & 0.0 & 0.0 & 0.0 \\
\hline OWND & 3.4 & -29.1 & -33.2 & -31.9 & -28.6 & -30.8 & -31.5 \\
\hline WHOL & 22.5 & 0.0 & 0.0 & 0.0 & 0.0 & 0.0 & 0.0 \\
\hline RETT & 44.0 & 0.0 & 0.0 & 0.0 & 0.0 & 0.0 & 0.0 \\
\hline TRAN & 8.0 & 0.0 & 0.0 & 0.0 & 0.0 & 0.0 & 0.0 \\
\hline All & 9.4 & -9.3 & -10.8 & -10.4 & -9.1 & -9.9 & -10.2 \\
\hline
\end{tabular}

Table R.20: Industry Average Net Rates of Return 


\begin{tabular}{|l|c|cccccc|}
\hline \multicolumn{7}{|c|}{ Real Macro Measures } \\
\hline \multirow{2}{*}{ Variable } & BASEYEAR $(\$ \mathrm{M})$ & \multicolumn{5}{c|}{$\%$ ON BASEYEAR } \\
\cline { 3 - 8 } & & BSLN7 & SIM701 & SIM702 & BSLN8 & SIM801 & SIM802 \\
\hline GDP (Expenditure) & 156090 & 10.6 & 23.3 & 21.8 & 10.8 & 24.9 & 22.6 \\
GDP (Income) & 156090 & 11.1 & 25.4 & 22.7 & 11.1 & 25.5 & 23.1 \\
Private Consumption & 93331 & 10.9 & 22.9 & 21.6 & 11.1 & 24.2 & 22.1 \\
Investment & 38305 & 10.6 & 23.3 & 21.8 & 10.8 & 24.9 & 22.6 \\
Government Consumption & 28661 & 10.6 & 23.3 & 21.8 & 10.8 & 24.9 & 22.6 \\
F.O.B. Exports & 43290 & 6.3 & 16.0 & 15.0 & 6.6 & 17.7 & 15.8 \\
C.I.F. Imports & 47497 & 7.2 & 15.9 & 15.2 & 7.4 & 17.0 & 15.6 \\
Trade Balance & -4207 & -16.8 & -14.8 & -17.6 & -16.0 & -9.3 & -13.2 \\
Government Balance & 6900 & 5.2 & 23.2 & 17.4 & 4.7 & 19.0 & 16.5 \\
Domestic Private Saving & 27198 & 13.9 & 36.7 & 28.8 & 13.0 & 32.4 & 28.5 \\
Domestic Saving & 34098 & 12.2 & 34.0 & 26.5 & 11.3 & 29.7 & 26.1 \\
\hline
\end{tabular}

Table R.21: Macro Measures

\begin{tabular}{|l|cccccc|}
\hline \multicolumn{7}{|c|}{ Economy-wide Price Measures } \\
\hline \multirow{2}{*}{ Variable } & \multicolumn{7}{c|}{$\%$ ON BASEYEAR } \\
\cline { 2 - 7 } & BSLN7 & SIM701 & SIM702 & BSLN8 & SIM801 & SIM802 \\
\hline GDP Deflator & -3.1 & -5.6 & -5.0 & -3.1 & -6.0 & -5.4 \\
Consumer Price Index & -3.3 & -5.3 & -4.8 & -3.3 & -5.4 & -5.0 \\
Investment Price Index & -1.5 & -3.4 & -2.5 & -1.4 & -3.3 & -2.7 \\
Government Price Index & -1.7 & -3.7 & -3.3 & -1.9 & -4.8 & -4.1 \\
Export Price Index (F.O.B.) & -1.5 & -3.6 & -3.5 & -1.6 & -4.0 & -3.6 \\
Import Price Index (C.I.F.) & 0.0 & 0.0 & 0.0 & 0.0 & 0.0 & 0.0 \\
Trade Price Index & 14.8 & 37.6 & 35.3 & 15.6 & 43.0 & 37.8 \\
Real Exchange Rate & 3.2 & 6.0 & 5.3 & 3.2 & 6.4 & 5.7 \\
Nominal After-Tax Wage & -2.6 & -6.0 & -5.3 & -2.7 & -6.7 & -5.9 \\
Real After-Tax Wage & 0.8 & -0.8 & -0.6 & 0.6 & -1.4 & -1.0 \\
Capital Rents Index & -4.2 & -6.3 & -5.4 & -4.0 & -6.1 & -5.6 \\
Output Price Index & -2.4 & -4.7 & -4.0 & -2.3 & -4.9 & -4.3 \\
\hline Current Net Rate of Return (Average) & -9.3 & -10.8 & -10.4 & -9.1 & -9.9 & -10.2 \\
Expected Future Net Rate of Return & -2.7 & -4.0 & -4.2 & -2.8 & -4.5 & -4.4 \\
\hline
\end{tabular}

Table R.22: Economy-wide Price Measures 


\begin{tabular}{|l|cccccc|}
\hline \multicolumn{7}{|c|}{ Regional Nominal After-Tax Wage } \\
\hline \multirow{2}{*}{ Region } & \multicolumn{5}{|c|}{$\%$ ON BASEYEAR } \\
\cline { 2 - 7 } & BSLN7 & SIM701 & SIM702 & BSLN8 & SIM801 & SIM802 \\
\hline AKL & -14.9 & -29.4 & -10.5 & -9.8 & -17.9 & -8.7 \\
WLG & 1.0 & 6.4 & 6.5 & -1.8 & -5.1 & -3.1 \\
ONI & 6.3 & 12.2 & 11.4 & 4.0 & 5.4 & 5.9 \\
CAN & -1.3 & 3.9 & -22.2 & -2.5 & -4.2 & -17.3 \\
OSI & 10.3 & 16.3 & -18.5 & 1.2 & -3.8 & -15.0 \\
\hline
\end{tabular}

Table R.23: Regional Nominal After-Tax Wage

\begin{tabular}{|l|cccccc|}
\hline \multicolumn{7}{|c|}{ Regional Consumer Price Index } \\
\hline \multirow{2}{*}{ Region } & \multicolumn{5}{|c|}{$\%$ ON BASEYEAR } \\
\cline { 2 - 7 } & BSLN7 & SIM701 & SIM702 & BSLN8 & SIM801 & SIM802 \\
\hline AKL & -5.4 & -9.9 & -5.4 & -4.3 & -7.4 & -5.2 \\
WLG & -2.5 & -3.0 & -2.6 & -3.0 & -5.0 & -4.4 \\
ONI & -2.1 & -2.4 & -2.1 & -2.4 & -3.5 & -3.1 \\
CAN & -2.9 & -3.4 & -8.1 & -3.1 & -4.8 & -7.2 \\
OSI & -1.5 & -1.9 & -7.7 & -2.9 & -5.1 & -7.1 \\
\hline
\end{tabular}

Table R.24: Regional Consumer Price Index

\begin{tabular}{|l|cccccc|}
\hline \multicolumn{7}{|c|}{ Regional Real After-Tax Wage } \\
\hline \multirow{2}{*}{ Region } & \multicolumn{5}{|c|}{$\%$ ON BASEYEAR } \\
\cline { 2 - 7 } & BSLN7 & SIM701 & SIM702 & BSLN8 & SIM801 & SIM802 \\
\hline AKL & -10.0 & -21.6 & -5.4 & -5.7 & -11.3 & -3.6 \\
WLG & 3.6 & 9.8 & 9.4 & 1.2 & -0.1 & 1.3 \\
ONI & 8.5 & 15.0 & 13.8 & 6.5 & 9.3 & 9.3 \\
CAN & 1.7 & 7.5 & -15.4 & 0.7 & 0.7 & -10.9 \\
OSI & 12.0 & 18.5 & -11.7 & 4.2 & 1.4 & -8.5 \\
\hline
\end{tabular}

Table R.25: Regional Real After-Tax Wage 


\begin{tabular}{|c|c|c|c|c|c|c|c|}
\hline \multicolumn{8}{|c|}{ Real GDP Per Capita } \\
\hline \multirow{2}{*}{ Region } & \multirow{2}{*}{ BASEYEAR $(\$)$} & \multicolumn{6}{|c|}{$\% \Delta$ ON BASEYEAR } \\
\hline & & BSLN7 & SIM701 & SIM702 & BSLN8 & SIM801 & $\operatorname{SIM} 802$ \\
\hline NZ & 37306 & -10.1 & -12.1 & -11.5 & -9.9 & -11.0 & -11.0 \\
\hline \multicolumn{8}{|c|}{ Real Disposable Income Per Household } \\
\hline \multirow{2}{*}{ Region } & \multirow{2}{*}{ BASEYEAR $(\$)$} & \multicolumn{6}{|c|}{$\% \Delta$ ON BASEYEAR } \\
\hline & & BSLN7 & SIM701 & SIM702 & BSLN8 & SIM801 & $\operatorname{SIM} 802$ \\
\hline AKL & 95271 & -19.6 & -24.9 & -17.4 & -17.5 & -20.1 & -16.6 \\
\hline WLG & 88394 & -15.9 & -13.2 & -13.4 & -16.8 & -16.9 & -17.2 \\
\hline ONI & 75572 & -17.0 & -13.9 & -14.5 & -17.5 & -15.3 & -15.9 \\
\hline CAN & 64292 & -16.2 & -13.5 & -24.1 & -16.5 & -15.8 & -22.0 \\
\hline OSI & 88548 & -16.6 & -13.7 & -26.7 & -19.0 & -18.8 & -25.3 \\
\hline NZ & 82902 & -17.1 & -16.9 & -18.8 & -17.1 & -16.9 & -18.5 \\
\hline \multicolumn{8}{|c|}{ Real Spending Per Household } \\
\hline \multirow{2}{*}{ Region } & \multirow{2}{*}{ BASEYEAR (\$) } & \multicolumn{6}{|c|}{$\% \Delta$ ON BASEYEAR } \\
\hline & & BSLN7 & SIM701 & SIM702 & BSLN8 & SIM801 & SIM 802 \\
\hline AKL & 73403 & -23.8 & -33.7 & -19.8 & -20.0 & -24.8 & -18.3 \\
\hline WLG & 70464 & -15.1 & -10.1 & -10.4 & -16.8 & -17.4 & -17.1 \\
\hline ONI & 57243 & -14.6 & -9.5 & -10.3 & -15.8 & -12.6 & -13.2 \\
\hline CAN & 50335 & -16.1 & -11.3 & -30.3 & -16.7 & -16.0 & -26.6 \\
\hline OSI & 70474 & -13.0 & -7.9 & -31.6 & -18.1 & -18.9 & -29.1 \\
\hline NZ & 64195 & -17.6 & -19.0 & -19.8 & -17.4 & -18.2 & -19.5 \\
\hline \multicolumn{8}{|c|}{ Real Saving Per Household } \\
\hline \multirow{2}{*}{ Region } & \multirow{2}{*}{ BASEYEAR $(\$)$} & \multicolumn{6}{|c|}{$\% \Delta$ ON BASEYEAR } \\
\hline & & BSLN7 & SIM701 & SIM702 & BSLN8 & SIM801 & SIM802 \\
\hline AKL & 21868 & -5.4 & 4.5 & -9.4 & -9.1 & -4.4 & -10.9 \\
\hline WLG & 17930 & -19.2 & -25.3 & -25.4 & -16.7 & -14.8 & -17.2 \\
\hline ONI & 18329 & -24.3 & -27.6 & -27.4 & -22.8 & -23.7 & -24.3 \\
\hline CAN & 13957 & -16.8 & -21.5 & -1.4 & -15.8 & -15.2 & -5.6 \\
\hline OSI & 18074 & -30.5 & -36.2 & -7.5 & -22.6 & -18.6 & -10.8 \\
\hline NZ & 18707 & -15.3 & -9.9 & -15.1 & -16.0 & -12.8 & -15.3 \\
\hline
\end{tabular}

Table R.26: Welfare Measures 


\begin{tabular}{|l|c|cccccc|}
\hline \multicolumn{7}{|c|}{ Unemployment } \\
\hline \multirow{2}{*}{ Region } & \multirow{2}{*}{ BASEYEAR (000s) } & \multicolumn{7}{|c|}{$\% \Delta$ ON BASEYEAR } \\
\cline { 2 - 8 } & & BSLN7 & SIM701 & SIM702 & BSLN8 & SIM801 & SIM802 \\
\hline AKL & 37 & 31.6 & 80.7 & 31.6 & 22.0 & 49.1 & 27.7 \\
WLG & 13 & 5.0 & 5.1 & 5.0 & 9.7 & 25.0 & 17.3 \\
ONI & 36 & -1.8 & -1.8 & -1.9 & 1.1 & 7.0 & 3.9 \\
CAN & 11 & 8.2 & 8.2 & 59.9 & 12.0 & 28.6 & 47.1 \\
OSI & 9 & -6.9 & -6.9 & 49.7 & 9.7 & 33.8 & 41.6 \\
\hline NZ & 106 & 11.3 & 28.5 & 21.6 & 11.3 & 28.5 & 21.6 \\
\hline
\end{tabular}

Table R.27: Unemployment

\begin{tabular}{|l|c|cccccc|}
\hline \multicolumn{7}{|c|}{ Labour Force Participation Rate } \\
\hline \multirow{2}{*}{ Region } & \multirow{2}{*}{ BASEYEAR $(\%)$} & \multicolumn{7}{|c|}{$\%$ ON BASEYEAR } \\
\cline { 2 - 8 } & & BSLN7 & SIM701 & SIM702 & BSLN8 & SIM801 & SIM802 \\
\hline AKL & 74.2 & 0.1 & 0.1 & 0.0 & 0.1 & 0.1 & 0.1 \\
WLG & 78.4 & 0.0 & 0.1 & 0.0 & -0.2 & -0.8 & -0.3 \\
ONI & 75.7 & 0.0 & -0.1 & -0.1 & -0.1 & -0.2 & 0.0 \\
CAN & 77.5 & 0.0 & 0.0 & 0.1 & -0.1 & -0.5 & 0.1 \\
OSI & 77.8 & 0.0 & 0.0 & 0.1 & -0.4 & -1.0 & 0.1 \\
\hline NZ & 76.0 & -0.2 & -0.4 & 0.1 & -0.2 & -0.4 & 0.1 \\
\hline
\end{tabular}

Table R.28: Labour Force Participation Rate 


\begin{tabular}{|l|cccccc|}
\hline \multicolumn{7}{|c|}{ Compensating Variation } \\
\hline \multirow{2}{*}{ Region } & \multicolumn{7}{|c|}{$\Delta$ IN InCOME (\$M) } \\
\cline { 2 - 7 } & BSLN7 & SIM701 & SIM702 & BSLN8 & SIM801 & SIM802 \\
\hline AKL & 7349.6 & 15850.4 & 9308.5 & 5911.3 & 12218.9 & 8825.6 \\
WLG & 1095.4 & 1828.6 & 1799.5 & 1399.4 & 3091.4 & 2695.7 \\
ONI & 1721.2 & 3488.0 & 3204.8 & 2204.1 & 5053.3 & 4381.0 \\
CAN & 1115.7 & 1735.1 & 4074.5 & 1243.9 & 2562.8 & 3577.7 \\
OSI & 373.9 & 1114.0 & 4433.0 & 1227.1 & 3143.3 & 4045.7 \\
\hline \multirow{7}{*}{ Region } & BSLN7 & SIM701 & SIM702 & BSLN8 & SIM801 & SIM802 \\
\hline AKL & 7920.1 & 18379.3 & 9942.2 & 6244.7 & 13490.8 & 9373.4 \\
WLG & 1118.1 & 1859.7 & 1822.9 & 1439.7 & 3253.8 & 2813.1 \\
ONI & 1733.4 & 3491.9 & 3204.1 & 2234.4 & 5160.3 & 4455.8 \\
CAN & 1146.6 & 1775.9 & 4547.5 & 1282.6 & 2690.1 & 3922.1 \\
OSI & 373.1 & 1106.7 & 4892.2 & 1255.6 & 3304.5 & 4414.2 \\
\hline
\end{tabular}

Table R.29: CV and EV Measures 


\section{Bibliography}

Auckland Regional Council (2009), Economic Futures for the Auckland Region. Part 2: Scenarios for Economic Development, Auckland Regional Council \& Market Economics Ltd., Auckland, New Zealand.

Bacharach, M. (1970), Biproportional Matrices and Input-Output Change, Cambridge University Press.

Batten, D. F. (1982), 'The interregional linkages between national and regional inputoutput models', International Regional Science Review 7(1), 53-67.

Bergman, L. (2005), CGE modeling of environmental policy and resource management, in K.-G. Maler \& J. R. Vincent, eds, 'Handbook of Environmental Economics: Economywide and International Environmental Issues', Vol. 3, Elsevier B.V., Amsterdam, chapter 24, pp. 1273-1306.

Burton, B. G. (1985), A multi-regional modelling framework for policy analysis in New Zealand, Technical report, Town and Country Planning Directorate, Ministry of Works and Development, Wellington.

Centre of Policy Studies (2008), MMRF: Monash Multi-Regional Forecasting Model, Centre of Policy Studies, Monash University, Melbourne. Available at URL below, inside archive TPPA0090.zip.

URL: http://www.monash.edu.au/policy/mmrf.htm

Centre of Policy Studies (2010), Numerical Accuracy, Centre of Policy Studies, Monash University, Melbourne.

URL: http://www.monash.edu.au/policy/gpnumacc.htm 
Dixon, P. B., Parmenter, B. R., Powell, A. A. \& Wilcoxen, P. J. (1992), Notes and Problems in Applied General Equilibrium Economics, North-Holland, New York.

Dixon, P. B., Parmenter, B. R., Sutton, J. \& Vincent, D. P. (1982), ORANI: A Multisectoral Model of the Australian Economy, North-Holland, New York.

Dixon, P. B. \& Rimmer, M. T. (2002), Dynamic General Equilibrium Modelling for Forecasting and Policy: A Practical Guide and Documentation of MONASH, Elsevier, Amsterdam.

Donaghy, K. P. (2009), CGE modeling in space: a survey, in R. Capello \& P. Nijkamp, eds, 'Handbook of Regional Growth and Development Theories', Edward Elgar, Cheltenham, UK and Northampton, MA, USA, chapter 20, pp. 389-422.

Easton, B. (1990), The Real Wage Debate 1978 - 1990, RPEP OP 101, Research Project on Economic Planning, Victoria University of Wellington, Wellington.

Enterprise North Shore (2010), Economic Futures Report for North Shore City: Full Report, Enterprise North Shore \& Market Economics Ltd., Auckland, New Zealand.

Fofana, I., Lemelin, A. \& Cockburn, J. (2005), Balancing a Social Accounting Matrix: Theory and Application, Centre Interuniversitaire sur le Risque, les Politiques Économiques et L'Emploi (CIRPÉE), Université Laval, Québec, Canada.

URL: http://www.pep-net.org/fileadmin/medias/pdf/sambal.pdf

GAMS Development Corp. (2008), GAMS The Solver Manuals, GAMS Development Corporation, Washington DC. Chapter 24: PATH 4.6, pp. 337-375.

GAMS Development Corp. (2011), GAMS User Guide, GAMS Development Corporation, Washington DC.

URL: http://www . gams . com/dd/docs/bigdocs/GAMSUsersGuide.pdf

Giesecke, J. A. \& Madden, J. R. (2003), 'A large-scale dynamic multi-regional CGE model with an illustrative application', Review of Urban and Regional Development Studies 15(1).

Gillig, D. \& McCarl, B. A. (n.d.), Notes on Formulating and Solving Computable General Equilibrium Models within GAMS, draft edn, Department of Agricultural Economics, 
Texas A\&M University, College Station, Texas, USA.

URL: http://agecon2.tamu .edu/people/faculty/mccarl-bruce/685/957.pdf

Hall, V. B. (1992), Long-run concepts in New Zealand macroeconometric and CGE models, in C. P. Hargreaves, ed., 'Macroeconomic Modelling of the Long Run', Edward Elgar Publishing Ltd., chapter 5, pp. 95-118.

Hall, V. B. \& McDermott, C. J. (2007), 'Regional business cycles in New Zealand: Do they exist? What might drive them?', Papers in Regional Science 86(2), 167-191.

Hall, V. B. \& McDermott, C. J. (2011), 'Unobserved components business cycles for New Zealand. What are they, and what might drive them?', The Economic Record 87(277), 294-317.

Harrison, J., Horridge, M., Jerie, M. \& Pearson, K. (2012), GEMPACK manual, Centre of Policy Studies, Monash University, Melbourne.

URL: http://www.monash.edu.au/policy/gpmanual.htm

Hertel, T. W., ed. (1997), Global Trade Analysis: Modeling and Applications, Cambridge University Press.

Hertel, T. W., Horridge, J. M. \& Pearson, K. R. (1992), 'Mending the family tree: A reconciliation of the linearization and levels schools of age modelling', Economic Modelling 9(4), 385 - 407.

Hodgson, R. \& Poot, J. (2010), New Zealand Research on the Economic Impacts of Immigration 2005-2010: Synthesis and Research Agenda, IMSED Research, Department of Labour and University of Waikato.

Horridge, J. M. (2001), MINIMAL: A Simplified General Equilibrium Model, Centre of Policy Studies, Monash University, Melbourne.

Horridge, J. M. (2008), ORANI-G: A General Equilibrium Model of the Australian Economy, 2006 edn, Centre of Policy Studies, Monash University, Melbourne.

Horridge, J. M., Madden, J. R. \& Wittwer, G. (2005), 'The impact of the 2002-2003 drought on Australia', Journal of Policy Modeling 27(3), 285-308. 
Horridge, J. M. \& Pearson, K. R. (2011), Solution Software for CGE Modeling, CoPS / IMPACT Project General Paper No. G-214, Centre of Policy Studies, Monash University, Melbourne.

Hosoe, N. (2004), Computable General Equilibrium Modeling with GAMS, Published on World Wide Web.

URL: http://sun.grips.ac.jp/cge-gams20040209.pdf

IMF (2004), Producer Price Index Manual, International Monetary Fund, Washington, D.C.

Jensen, R. C. (1980), 'The concept of accuracy in regional input-output models', International Regional Science Review 5(2), 139-154.

Jensen, R. C., Mandeville, T. D. \& Karunaratne, N. D. (1979), Regional Economic Planning: Generation of Regional Input-Output Analysis, Croom Helm, London.

Jensen, R. C. \& West, G. R. (1989), 'On objectivity in economic research: a response to Willis', Environment and Planning A 21(3), 405-407.

Johansen, L. (1960), A Multi-Sectoral Study of Economic Growth, North Holland, Amsterdam.

Jones, R. \& Whalley, J. (1989), 'A Canadian regional general equilibrium model and some applications', Journal of Urban Economics 25(3), 368-405.

Kaye-Blake, B., Schilling, C. \& Zuccollo, J. (2011), The Economic Impact of Increased Irrigation: A Dynamic Computable General Equilibrium Analysis of Increased Irrigation in New Zealand, NZIER, Wellington.

Keyzer, M. (1997), Building Applied General Equilibrium Models in GAMS, MIT Press.

Klein, L. R. \& Glickman, N. J. (1977), 'Econometric model-building at regional level', Regional Science and Urban Economics 7(1-2), 3-23.

Lahr, M. L. (2001), A strategy for producing hybrid regional input-output tables, in M. L. Lahr \& E. Dietzenbacher, eds, 'Input-Output Analysis: Frontiers and Extensions', Palgrave Macmillan, London, chapter 11 draft (available at the URL below). 
URL:

http://policy.rutgers.edu/faculty/lahr/Lahr StrategyforProducingHybridRegional Input-OutputTables.pdf

Lennox, J. A. (2010), The New Zealand Tourism General Equilibrium Model (NZTGEM): Technical Documentation, Lincoln University, Canterbury, NZ. Land Environment and People Research Report no. 14.

Lennox, J. \& van Nieuwkoop, R. (2009), Affordable and Effective Climate Change Mitigation Policies for New Zealand, Landcare Research. Presented at the $15^{\text {th }}$ Annual International Sustainable Development Research Conference, Utrecht, The Netherlands, 5-8 July, 2009.

Leontief, W., Morgan, A., Polenske, K., Simpson, D. \& Tower, E. (1965), 'The economic impact-industrial and regional-of an arms cut', The Review of Economics and Statistics $47(3), 217-241$.

Leontief, W. \& Strout, A. (1963), Multiregional input-output analysis, in T. Barna, ed., 'Structural Interdependence and Economic Development', Macmillan, London, chapter 7 , pp. 119-150.

Liew, L. H. (1981), A Multi-Regional, Multi-Sectoral General Equilibrium Model of the Australian Economy, PhD thesis, Monash University, Melbourne, Victoria, Australia.

Liew, L. H. (1984a), 'A Johansen model for regional analysis', Regional Science and Urban Economics 14(1), 129-146.

Liew, L. H. (1984b), "'tops-down" versus "bottoms-up" approaches to regional modeling', Journal of Policy Modeling 6(3), 351-367.

Löfgren, H., Harris, R. L. \& Robinson, S. (2002), A Standard Computable General Equilibrium (CGE) Model in GAMS, International Food Policy Research Institute, Washington D.C., USA.

Longhi, S., Nijkamp, P. \& Poot, J. (2010), 'Joint impacts of immigration on wages and employment: Review and meta-analysis', Journal of Geographical Systems 12(4), 355 $-387$. 
Madden, J. R. (1990), FEDERAL: A Two-Region Multisectoral Fiscal Model of the Australian Economy, PhD thesis, University of Tasmania, Australia.

McCarl, B. A. \& GAMS Development Corp. (2011), McCarl Expanded GAMS User Guide. Published on World Wide Web.

URL: http://www.gams.com/mccarl/mccarlhtml/

McDougall, R. (1999), Entropy Theory and RAS are Friends, GTAP Working Paper 300, Center for Global Trade Analysis, Department of Agricultural Economics, Purdue University.

URL: http://ideas.repec.org/p/gta/workpp/300.html

McKibbin, W. J. \& Sachs, J. D. (1989), The McKibbin-Sachs global model: Theory and specifications, Working Paper 3100, National Bureau of Economic Research.

URL: http://www. nber .org/papers/w3100

Miller, R. E. \& Blair, P. D. (2009), Input-Output Analysis: Foundations and Extensions, $2^{\text {nd }}$ edn, Cambridge University Press.

Nana, G. (1983), A (Neo)-Keynesian Version of the Short-Run JOANNA Model, RPEP IP 141, Research Project on Economic Planning, Victoria University of Wellington, Wellington.

Nana, G. (1999), A Multi-industry Computable General Equilibrium Model with Dynamic Investor and Consumer Behaviour, PhD thesis, Victoria University of Wellington.

Nana, G., Hall, V. \& Philpott, B. (1995), 'Trans-tasman CGE modelling: Some illustrative results from the JOANI model', Economic Modelling 12(4), 377-389.

Nana, G. \& Philpott, B. (1983), The 38 Sector JOANNA Model, RPEP OP 73, Research Project on Economic Planning, Victoria University of Wellington, Wellington.

Nana, G. \& Philpott, B. (1985), Macroeconomic and Sectoral Implications of the GST A General Equilibrium Analysis Using the JOANNA Model, RPEP OP 86, Research Project on Economic Planning, Victoria University of Wellington, Wellington.

Nana, G. \& Philpott, B. (1988), The JOANI Two Country General Equilibrium Model, RPEP OP 92, Research Project on Economic Planning, Victoria University of Wellington, Wellington. 
Nana, G. \& Poot, J. (1996), 'A study of trade liberalisation and factor mobility with a CGE model of Australia and New Zealand', Studies in Regional Science 26(2).

Nana, G., Sanderson, K. \& Hodgson, R. (2009), Economic Impacts of Immigration: Scenarios Using a Computable General Equilibrium Model of the New Zealand Economy, Business and Economic Research Ltd. and IMSED Research, Department of Labour.

Narayanan, G. B. \& Walmsley, T. L., eds (2008), Global Trade, Assistance, and Production: The GTAP 7 Data Base, Center for Global Trade Analysis, Purdue University.

Paltsev, S. (1999), Moving from Static to Dynamic General Equilibrium Economic Models (Notes for a beginner in MPSGE), Joint Program on the Science and Policy of Global Change, Massachusetts Institute of Technology, Cambridge, Massachusetts, USA. Revised April 2004.

Partridge, M. D. \& Rickman, D. S. (2010), 'Computable general equilibrium (CGE) modelling for regional economic development analysis', Regional Studies 44(10), 13111328.

Philpott, B. (1988), Julianne Model Sectoral Projections of the Economy to 1992, RPEP OP 94, Research Project on Economic Planning, Victoria University of Wellington, Wellington.

Philpott, B. (1990a), Economic Growth in New Zealand - Models and Experience, RPEP OP 100, Research Project on Economic Planning, Victoria University of Wellington, Wellington.

Philpott, B. (1990b), Labour Market Flexibility in a General Equilibrium Analysis of Paths to Full Employment, RPEP OP 98, Research Project on Economic Planning, Victoria University of Wellington, Wellington.

Philpott, B. (1992a), General Equilibrium Modelling for Policy Analysis and Economic Planning, RPEP OP 102, Research Project on Economic Planning, Victoria University of Wellington, Wellington.

Philpott, B. (1992b), Real Capital Stock by SNA Production Group 1950 - 1990, RPEP OP 105, Research Project on Economic Planning, Victoria University of Wellington, Wellington. 
Philpott, B. (1995), Estimating the Database for a 1990 Based JODY Model, RPEP Paper 271, Research Project on Economic Planning, Victoria University of Wellington, Wellington.

Philpott, B. (2000), 'The Research Project on Economic Planning: A brief account of its 30 years of work, 1970-2000', Victoria Economic Commentaries (2).

Philpott, B. \& Nana, G. (1988), Quantitative Implications of Australia-New Zealand Free Trade, RPEP OP 93, Research Project on Economic Planning, Victoria University of Wellington, Wellington.

Philpott, B. \& Nana, G. (1995), Dynamic General Equilibrium Modelling of World Agricultural Trade Impacts on New Zealand - An Appraisal of a Prototype Model, RPEP Paper 272, Research Project on Economic Planning, Victoria University of Wellington, Wellington.

Philpott, B. \& Stroombergen, A. (1986), Analysing Flexible Labour Markets - A General Equilibrium Approach Using CRESH Production Functions, RPEP OP 87, Research Project on Economic Planning, Victoria University of Wellington, Wellington.

Poot, J. (1984), Models of New Zealand Internal Migration and Residential Mobility, PhD thesis, Victoria University of Wellington.

Poot, J. \& Cochrane, B. (2005), Measuring the Economic Impact of Immigration: A Scoping Paper, Population Studies Centre, University of Waikato, Hamilton, New Zealand. Population Studies Centre Discussion Paper No.48.

URL: http://hdl . handle.net/10289/823

Poot, J., Nana, G. \& Philpott, B. (1987), International Migration and the New Zealand Economy: A Trans-Tasman Comparison, RPEP OP 91, Research Project on Economic Planning, Victoria University of Wellington, Wellington.

Robinson, S., Cattaneo, A. \& El-Said, M. (2001), 'Updating and estimating a social accounting matrix using cross entropy methods', Economic Systems Research 13(1), 47-64. 
Rutherford, T. F. (1999), 'Applied general equilibrium modeling with MPSGE as a GAMS subsystem: An overview of the modeling framework and syntax', Computational Economics 14(1-2), 1-46.

Rutherford, T. F. (2003), 'Using linear or nonlinear programming to balance a social accounting matrix', Published on World Wide Web (MPSGE Homepage).

URL: http://www.mpsge.org/tza/tzabal.htm

Rutherford, T. F. (n.d.), 'RASing a Matrix', Published on World Wide Web (GAMS Support Wiki).

URL: $\quad$ http://support.gams-software.com/doku.php?id=gams:rasing_a_ matrix

Shore, J. E. \& Johnson, R. W. (1980), 'Axiomatic derivation of the principle of maximum entropy and the principle of minimum cross-entropy', IEEE Transactions on Information Theory IT-26(1).

Statistics NZ (2001), Inter-Industry Study 1996, Statistics New Zealand, Wellington. (and associated input-output tables).

Statistics NZ (2006), Research Report on Regional Gross Domestic Product, Statistics New Zealand, Wellington. (and associated regional data tables).

Stroombergen, A. (1982), A Critique of The Johansen-Type Model, RPEP IP 133, Research Project on Economic Planning, Victoria University of Wellington, Wellington.

Stroombergen, A. (1986), Development and Application of a New Zealand General Equilibrium Model, PhD thesis, Victoria University of Wellington.

Stroombergen, A. (2008), ESSAM General Equilibrium Model: Estimation of 2005/06 Input-Output Tables, Motu Economic and Public Policy Research. Motu Working Paper 08-01, available at URL below.

URL: http://www.motu.org.nz/publications/working-papers/2008

Stroombergen, A. \& Philpott, B. (1982), The Julianne Semi-Dynamic General Equilibrium Model, RPEP OP 59, Research Project on Economic Planning, Victoria University of Wellington, Wellington. 
Stroombergen, A. \& Philpott, B. (1983a), The Julianne Dynamic Model, RPEP OP 66, Research Project on Economic Planning, Victoria University of Wellington, Wellington.

Stroombergen, A. \& Philpott, B. (1983b), Julianne Model Sectoral Analysis of the Economy to 1990, RPEP IP 153, Research Project on Economic Planning, Victoria University of Wellington, Wellington.

Stroombergen, A. \& Philpott, B. (1985), Dynamic General Equilibrium Modelling of the Effects of Protection on the New Zealand Economy 1977-84, RPEP OP 82, Research Project on Economic Planning, Victoria University of Wellington, Wellington.

Tipper, A. (2011), One for all? The capital-labour substitution elasticity in New Zealand. Paper prepared for the $52^{\text {nd }}$ New Zealand Association of Economists Conference, Wellington, New Zealand.

Varian, H. R. (1992), Microeconomic Analysis, $3^{\text {rd }}$ edn, W. W. Norton \& Co., New York.

Wallace, R. (1984), A Johansen type general equilibrium model of New Zealand with some applications, Master's thesis, Victoria University of Wellington, Wellington. New Zealand.

Wallace, R. \& Philpott, B. (1980a), The Equation Structure of JOANNA in Short-Run Mode, RPEP IP 98, Research Project on Economic Planning, Victoria University of Wellington, Wellington.

Wallace, R. \& Philpott, B. (1980b), The Equation Structure of the JOANNA Model, RPEP IP 91, Research Project on Economic Planning, Victoria University of Wellington, Wellington.

Wallace, R. \& Philpott, B. (1982), Economy-wide Effects of Some Alternative Fiscal Packages - A General Equilibirum Analysis Using JOANNA, RPEP IP 127, Research Project on Economic Planning, Victoria University of Wellington, Wellington.

Wallace, R., Stroombergen, A. \& Philpott, B. (1982), The General Equilibrium Results of a Reduction in Textile Industry Protection, RPEP IP 127, Research Project on Economic Planning, Victoria University of Wellington, Wellington. 
Wells, G. \& Easton, B. (1986), Economy Wide Models of New Zealand, Research paper No. 33, New Zealand Institute of Economic Research, Wellington.

West, G. R. (1980), 'Generation of regional input-output tables (GRIT): An introspection', Economic Analysis and Policy 10(1-2), 71-86.

West, G. R., Morison, J. B. \& Jensen, R. C. (1984), 'A method for the estimation of hybrid interregional input-output tables', Regional Studies 18(5), 413-421.

Yeoman, R., Kim, J., McDonald, G. \& Zhang, J. (2009), Modelling regional general equilibrium for the Auckland region, NZCEE Research Monograph Series No. 19, New Zealand Centre for Ecological Economics, Palmerston North, New Zealand. ISBN 978-0-9582949-6-6. ISSN 1176-7251 (print) ISSN 1179-1179 (online).

Zhang, Y., McDonald, G., Nixon, P. \& Smith, N. (2008), Development of a regional social accounting framework for New Zealand, NZCEE Research Monograph Series No. 15, New Zealand Centre for Ecological Economics, Palmerston North, New Zealand. ISBN 978-0-9582949-2-8. ISSN 1176-7251 (print) ISSN 1179-1179 (online). 